\title{
Pig interorgan balance studies in health and disease
}

Citation for published version (APA):

ten Have, G. A. M. (2017). Pig interorgan balance studies in health and disease. [Doctoral Thesis, Maastricht University]. Maastricht University. https://doi.org/10.26481/dis.20170913gth

Document status and date:

Published: 01/01/2017

DOI:

10.26481/dis.20170913gth

Document Version:

Publisher's PDF, also known as Version of record

\section{Please check the document version of this publication:}

- A submitted manuscript is the version of the article upon submission and before peer-review. There can be important differences between the submitted version and the official published version of record.

People interested in the research are advised to contact the author for the final version of the publication, or visit the DOI to the publisher's website.

- The final author version and the galley proof are versions of the publication after peer review.

- The final published version features the final layout of the paper including the volume, issue and page numbers.

Link to publication

\footnotetext{
General rights rights.

- You may freely distribute the URL identifying the publication in the public portal. please follow below link for the End User Agreement:

www.umlib.nl/taverne-license

Take down policy

If you believe that this document breaches copyright please contact us at:

repository@maastrichtuniversity.nl

providing details and we will investigate your claim.
}

Copyright and moral rights for the publications made accessible in the public portal are retained by the authors and/or other copyright owners and it is a condition of accessing publications that users recognise and abide by the legal requirements associated with these

- Users may download and print one copy of any publication from the public portal for the purpose of private study or research.

- You may not further distribute the material or use it for any profit-making activity or commercial gain

If the publication is distributed under the terms of Article $25 \mathrm{fa}$ of the Dutch Copyright Act, indicated by the "Taverne" license above, 




\section{Pig interorgan balance studies in health and disease}

Gabriëlla A.M. ten Have 


\section{Colophon}

Pig interorgan balance studies in health and disease

Dissertation, Maastricht University, Maastricht, The Netherlands

Author

Gabriëlla A.M. ten Have

Design

Eva M.C. Oosterlaken

Printing

Blurb, San Francisco, USA

Copyright (c) 2017 Gabriëlla A.M. ten Have, Montgomery, TX, United States.

All rights reserved. No part of this publication may be reproduced or transmitted in any form by any means, without written permission of the author.

Studies presented in this thesis were conducted at University of Arkansas and Medical Science (Little Rock, AR, USA), Arkansas Children Hospital (Little Rock, AR, USA), University Hospital of North Norway (Tromsø, Norway), University of Tromsø (Norway), Texas A\&M University (USA), University College London (UK) and Maastricht University.

Part of the studies was funded by DMV Campina, Unilever, DSM Food Specialties, SenterNovem (Dutch Ministry of Economic Affairs) and National Institute of Health (USA).

Financial support for the printing of this thesis was kindly provided by Cambridge Isotope Laboratories Inc, Trouw Nutreco, Nutricia Research and Maastricht University.

\section{trouw nutrition}

a Nutreco company 


\title{
Pig interorgan balance studies in health and disease
}

\author{
Proefschrift \\ Ter verkrijging van de graad van doctor \\ aan de Universiteit van Maastricht \\ op gezag van de Rector Magnificus \\ Prof. Dr. Rianne M. Letschert
}

volgens het besluit van het College van Decanen

in het openbaar te verdedigen

op woensdag 13 september 2017 om 12:00 uur

door

Gabriëlla Adriana Maria ten Have 


\section{Promotores}

Prof. dr. S.W.M. Olde Damink

Prof. dr. N.E.P. Deutz (Texas A\&M University, College Station, TX, USA)

\section{Copromotor}

Dr. M.P.K.J. Engelen (Texas A\&M University, College Station, TX, USA)

\section{Beoordelingscommissie}

Prof. dr. W.H. Lamers (voorzitter)

Prof. dr. L.J.C. van Loon

Dr. M. van de Poll

Prof. dr. O. Rooijackers (Karolinska Institutet, Stockholm, Sweden)

Dr. M.R. Soeters (Academisch Medisch Centrum, Amsterdam) 



\section{Table of contents}

8

\section{Chapter 1}

Short introduction

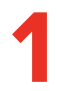

Interorgan balance studies during feeding

16

Chapter 2

Absorption kinetics of amino acids, peptides and intact proteins

\section{4}

\section{Chapter 3}

Simultaneous measurement of metabolic flux in portally-drained viscera, liver, spleen, kidney and hindquarter in the conscious pig

\section{2}

\section{Chapter 4}

Absence of post-prandial gut anabolism after intake of a low quality protein meal

\section{6}

\section{Chapter 5}

Enhanced lacto-tri-peptide bio-availability by co-Ingestion of macronutrients
Use of isotopes in pig sepsis models

106

\section{Chapter 6}

Characteristics of a Pseudomonas aeruginosa induced porcine sepsis model for multi-organ metabolic flux measurements

\section{2}

\section{Chapter 7}

Phenylalanine isotope pulse method to measure effect of sepsis on protein breakdown and membrane transport in the pig 
Interorgan balance studies in disease models

158

Chapter 8

Arginine de novo and nitric oxide production in disease states

188

\section{Chapter 9}

Nitric oxide and L-arginine metabolism in a devascularized porcine model of acute liver failure

\section{8}

\section{Chapter 10}

Inhibited jejunal protein synthesis and breakdown in Pseudomonas aeruginosa induced severe sepsis pig model

\section{4}

\section{Chapter 11}

Altered transorgan protein kinetics during a Pseudomonas aeruginosa induced severe sepsis in the pig
Summary and conclusions

258

Chapter 12

Summary and future perspectives

280

Chapter 13

Samenvatting

286

Chapter 14

Valorization

292

Biography

294

Publications

298

Dankwoord / Thank you 
CHAPTER 1 


\section{Short introduction}

A healthy human body need nutrients to be kept in metabolic balance. To keep balance, complex interaction between multiple specialized organs for transportation, digestion, absorption, redistribution, utilization, conversion, breakdown of nutritional substrates during post-prandial and/or post-absorptive stage must occur. These interactions are highly dynamic and are disturbed in many pathophysiological conditions. For instance, amino acid substrate wasting in critical illness and other diseases in humans result in severe muscle wasting and is a major problem for their recovery/rehabilitation (1-7). Sepsis, a life-threatening organ dysfunction caused by a dysregulated host response to infection in critical illness, has symptoms like gut atrophy and stimulation of liver acute phase response, indicating complex interactions between organs, in addition to muscle wasting (8-10).

In another example, when an important metabolic organ like the liver is failing, metabolic routes between organs are disturbed and are the cause of progressive (but reversible) multi-organ failures (11-13). Therefore, it is critical to gain more knowledge about inter/trans organ fluxes of nutritional substrates in health and disease to ensure that improved nutritional strategies or other interventions can be developed for a variety of pathophysiological conditions $(6,7)$.

Measuring concentrations by collecting blood sample of substrates in plasma is relatively easy in humans. However, to measure complex fluxes or trafficking (amounts in time) of substrates over and in organs is extremely difficult in humans due to the multitude of ethical issues and the limitations of invasive procedures. In pathophysiological conditions, like sepsis, it is especially difficult to obtain transorgan measurements as these patients are very unstable and have coagulation abnormality in addition to ethical problems (1, 4). Therefore, substrate studies in humans usually are done by measuring substrate flux on a whole body level with the use of stable tracers (14-19) or performing arterial-venous balance studies across the arm or leg as proxy for a muscle compartment (20). Additionally, by adding stable tracers to food, it is possible to get flux information across the splanchnic area (21-23). We also measured fluxes across individual organs, during surgery or catheterization for diagnostic purposes $(24,25)$.

Therefore, clinically relevant animal models are essential to study (patho) physiological interorgan metabolism in a controlled, repeatable way. Animal models also allow for the use of invasive techniques, providing multiple measurements and mechanistic data within the same animal in several nutritional/pathological stages. Due to the fact that the pig is an omnivorous mammal and have remarkable 
similarity to humans in gastrointestinal tract anatomy, physiology, biochemistry (and even pathology), this species is often used in the biomedical research and more generally in the field of nutrition and associated metabolic disease states (26).

In this dissertation, I present different aspects of interorgan research studies in the pig. The dissertation describes interorgan/transorgan measurement of mainly protein/amino acid metabolism, their metabolites, and amino acid interaction with other macronutrients using advanced stable isotope tracer methods of measurement in health and disease models with multiorgan disturbed metabolism. The main focus is on a conscious disease model with acute severe sepsis induced by Pseudomonas aeruginosa bacteremia. In addition, I show a study in an unconscious devascularized acute liver failure model.

\section{Thesis outline}

\section{Section 1 interorgan balance studies during feeding}

Chapter 2 and 3 are an extension of the introduction of the present dissertation. Chapter 2 is an introductory review on the several kinetic aspects of the absorption of amino acids, peptides and intact proteins in the small intestines (27). The small intestine acts as an interface and regulator between the gut lumen and the rest of the body. The small intestine also controls the degree and rate of transport of amino acids coming from dietary protein via the portal vein to the liver and the systemic circulation. In this review, we discuss the factors that lead to enhanced, reduced, or modified absorption kinetics. To study these factors, a sophisticated model in multi catheterized pigs along with the use of isotopes that can calculate absorption and gut metabolism is necessary. In chapter 3, we describe the technical part of the multi catheterized pig model that is used throughout this dissertation (28).

Chapter 4 and 5 describe in more detail interorgan balance studies to determine transorgan bioavailability of amino acids/peptides for potential anabolic/bioactive response. In chapter 4 , we state that intake of low quality food plays a key role in e.g. environmental enteropathy, a subclinical chronic situation present in developing countries. However, limited data were available on acute effects of low quality foods. Therefore, we determined the acute effects of a low quality food on gut metabolism and the consequences for liver metabolism (29). In the last chapter in this section, chapter 5 , we hypothesize that potential benefits on health of food-derived bioactive peptides depends on the availability on the site of action. Therefore, we determined transorgan availability/kinetics of food-derived peptides and the influence of other macronutrients in the food (30).

\section{Section 2: Use of isotopes in pig sepsis models}

In chapter 6, we describe a newly developed clinically relevant disease model of severe sepsis induced by intravenous administration of Pseudomonas aeruginosa 
(31). This is a pathophysiological condition in which severe amino acid substrate wasting is related to high mortality (32). With this model, in chapter 7, we describe an example of the use of stable isotopes tracers in metabolic research. We compared two different stable isotope methods/models to determine whole body protein breakdown, on their sensitivity to detect physiological differences, their accuracy, precision, practicality in animal models and discuss their potency for human studies (33).

\section{Section 3: Interorgan balance studies in disease models}

Amino acid metabolism is not only involved in protein synthesis and breakdown, but also involved in many other metabolic routes. A multiorgan research approach is especially needed for advancement in studying disturbances in those routes with interorgan substrate trafficking. For instance, arginine, derived from dietary protein intake, body protein breakdown, or endogenous de novo arginine production, may be linked to the availability of citrulline, which is the immediate precursor of arginine and the limiting factor for de novo arginine production. Arginine metabolism is highly compartmentalized due to the expression of the enzymes involved in arginine metabolism in various organs. A small fraction of arginine enters the NO synthase (NOS) pathway. In chapter 8, we review the existing literature concerning the ARG-NO pathway in disease states (34). And in chapter 9, we studied this pathway in an unconscious multiorgan catheterized pig model with an acute liver failure. The aim of the study was to evaluate the evolution of disturbances in NO metabolism in relation to its regulators, L-arginine, asymmetric dimethylarginine, and arginase, in the first $6 \mathrm{~h}$ of acute liver failure using stable isotope technology (35).

In chapter 10 and 11, multi-tissue and organ protein metabolism in Pseudomonas induced severe sepsis, are described in more detail and using different isotope models. Chapter 10 is focused on alterations of fractional synthesis and breakdown rates in splanchnic tissues in comparison with muscle and lung tissue. In chapter 11, we try to unravel/explain protein turnover alterations on whole body level with changes of net balances and protein synthesis/breakdown on organ level using multiple protein turnover related isotope tracers.

\section{Section 4: Summary and conclusions}

In chapter 12 we summarize the findings of the present dissertation with some key data figures and discuss future perspectives in this type of research. In chapter 13 we made a short summary in Dutch. Finally in chapter 14 we valorized certain findings of the present dissertation. 


\section{References}

1. Rosenblatt S, Clowes GH, Jr., George BC, Hirsch E, Lindberg B. Exchange of amino acids by muscle and liver in sepsis. Arch Surg. 1983;118(2):167-75.

2. Hartl WH, Jauch KW. Metabolic self-destruction in critically ill patients: origins, mechanisms and therapeutic principles. Nutrition. 2014;30(3):261-7.

3. Klaude M, Mori M, Tjader I, Gustafsson T, Wernerman J, Rooyackers O. Protein metabolism and gene expression in skeletal muscle of critically ill patients with sepsis. Clin Sci (Lond). 2012;122(3):133-42.

4. Rooyackers O, Kouchek-Zadeh R, Tjader I, Norberg A, Klaude M, Wernerman J. Whole body protein turnover in critically ill patients with multiple organ failure. Clin Nutr. 2015;34(1):95-100.

5. Coker RH, Wolfe RR. Bedrest and sarcopenia. Curr Opin Clin Nutr Metab Care. 2012;15(1):7-11.

6. Heyland DK, Stapleton RD, Mourtzakis M, Hough CL, Morris P, Deutz NE, et al. Combining nutrition and exercise to optimize survival and recovery from critical illness: Conceptual and methodological issues. Clin Nutr. 2016;35(5):1196-206.

7. Deutz NE, Matheson EM, Matarese LE, Luo M, Baggs GE, Nelson JL, et al. Readmission and mortality in malnourished, older, hospitalized adults treated with a specialized oral nutritional supplement: A randomized clinical trial. Clin Nutr. 2016;35(1):18-26.

8. Song J, Wolf SE, Herndon DN, Wu XW, Jeschke MG. Second hit post burn increased proximal gut mucosa epithelial cells damage. Shock. 2008;30(2):184-8.

9. Ding LA, Li JS. Intestinal failure: pathophysiological elements and clinical diseases. World J Gastroenterol. 2004;10(7):930-3.

10. Strnad P, Tacke F, Koch A, Trautwein C. Liver - guardian, modifier and target of sepsis. Nat Rev Gastroenterol Hepatol. 2017;14(1):55-66.

11. Jalan R, Olde Damink SW, Hayes PC, Deutz NE, Lee A. Pathogenesis of intracranial hypertension in acute liver failure: inflammation, ammonia and cerebral blood flow. J Hepatol. 2004;41(4):613-20.

12. Olde Damink SW, Deutz NE, Dejong CH, Soeters PB, Jalan R. Interorgan ammonia metabolism in liver failure. Neurochem Int. 2002;41(2-3):177-88.

13. Ytrebo LM, Sen S, Rose C, Ten Have GA, Davies NA, Hodges S, et al. Interorgan ammonia, glutamate, and glutamine trafficking in pigs with acute liver failure. Am J Physiol Gastrointest Liver Physiol. 2006;291(3):G373-81.

14. Luiking YC, Poeze M, Ramsay G, Deutz NE. Reduced citrulline production in sepsis is related to diminished de novo arginine and nitric oxide production. Am J Clin Nutr. 2009;89(1):142-52.

15. Liebau F, Wernerman J, van Loon LJ, Rooyackers O. Effect of initiating enteral protein feeding on whole-body protein turnover in critically ill patients. Am J Clin Nutr. 2015;101(3):549-57.

16. Jonker R, Deutz NEP, Erbland ML, Anderson PJ, Engelen MPKJ. Hydrolyzed casein and whey protein meals comparably stimulate net whole-body protein synthesis in COPD patients with nutritional depletion without an additional effect of leucine co-ingestion. Clinical Nutrition. 2013(0).

17. Jonker R, Deutz NE, Erbland ML, Anderson PJ, Engelen MP. Alterations in whole-body arginine metabolism in chronic obstructive pulmonary disease. Am J Clin Nutr. 2016;103(6):1458-64.

18. Mason A, Engelen MP, Ivanov I, Toffolo GM, Deutz NE. A Four Compartments Compartmental Model to Assess Net Whole Body Protein Breakdown Using a Pulse of 
Phenylalanine and Tyrosine Stable Isotopes in Humans. Am J Physiol Endocrinol Metab. 2017:ajpendo 003622016.

19. Engelen MP, Klimberg VS, Allasia A, Deutz NE. Presence of early stage cancer does not impair the early protein metabolic response to major surgery. J Cachexia Sarcopenia Muscle. 2017.

20. Koopman R, Walrand S, Beelen M, Gijsen AP, Kies AK, Boirie Y, et al. Dietary protein digestion and absorption rates and the subsequent postprandial muscle protein synthetic response do not differ between young and elderly men. J Nutr. 2009;139(9):1707-13.

21. Jonker R, Deutz NE, Erbland ML, Anderson PJ, Engelen MP. Effectiveness of essential amino acid supplementation in stimulating whole body net protein anabolism is comparable between COPD patients and healthy older adults. Metabolism. 2017;69:120-9.

22. Engelen MP, Com G, Anderson PJ, Deutz NE. New stable isotope method to measure protein digestibility and response to pancreatic enzyme intake in cystic fibrosis. Clin Nutr. 2014;33(6):1024-32.

23. Boirie Y, Gachon P, Beaufrere B. Splanchnic and whole-body leucine kinetics in young and elderly men. Am J Clin Nutr. 1997;65(2):489-95.

24. Olde Damink SW, Jalan R, Redhead DN, Hayes PC, Deutz NE, Soeters PB. Interorgan ammonia and amino acid metabolism in metabolically stable patients with cirrhosis and a TIPSS. Hepatology. 2002;36(5):1163-71.

25. van de Poll MC, Siroen MP, van Leeuwen PA, Soeters PB, Melis GC, Boelens PG, et al. Interorgan amino acid exchange in humans: consequences for arginine and citrulline metabolism. Am J Clin Nutr. 2007;85(1):167-72.

26. Guilloteau P, Zabielski R, Hammon HM, Metges CC. Nutritional programming of gastrointestinal tract development. Is the pig a good model for man? Nutr Res Rev. 2010;23(1):4-22.

27. Ten Have GA, Engelen MP, Luiking YC, Deutz NE. Absorption kinetics of Amino Acids, Peptides, and Intact Proteins. Int J Sport Nutr Exerc Metab. 2007;17(Supplement):S23-S36.

28. Ten Have GA, Bost MC, Suyk-Wierts JC, van den Bogaard AE, Deutz NE. Simultaneous measurement of metabolic flux in portally-drained viscera, liver, spleen, kidney and hindquarter in the conscious pig. Lab Anim. 1996;30(4):347-58.

29. Ten Have GA, Engelen MP, Soeters PB, Deutz NE. Absence of post-prandial gut anabolism after intake of a low quality protein meal. Clin Nutr. 2011.

30. Ten Have GA, van der Pijl PC, Kies AK, Deutz NE. Enhanced Lacto-Tri-Peptide BioAvailability by Co-Ingestion of Macronutrients. PLoS One. 2015;10(6):e0130638.

31. Ten Have GAM, Deutz RCI, Engelen M, Wolfe RR, Deutz NEP. Characteristics of a Pseudomonas aeruginosa induced porcine sepsis model for multi-organ metabolic flux measurements. Lab Anim. 2017:23677217718003.

32. Hermans $G$, Van den Berghe $G$. Clinical review: intensive care unit acquired weakness. Crit Care. 2015;19:274.

33. Ten Have GA, Engelen MP, Wolfe RR, Deutz NE. Phenylalanine isotope pulse method to measure effect of sepsis on protein breakdown and membrane transport in the pig. Am J Physiol Endocrinol Metab. 2017:ajpendo 003512016.

34. Luiking YC, Ten Have GA, Wolfe RR, Deutz NE. Arginine de novo and nitric oxide production in disease states. Am J Physiol Endocrinol Metab. 2012;303(10):E1177-89.

35. Sharma V, Ten Have GA, Ytrebo L, Sen S, Rose CF, Dalton RN, et al. Nitric Oxide and L-Arginine metabolism in a devascularized porcine model of Acute Liver Failure. American journal of physiology Gastrointestinal and liver physiology. 2012. 



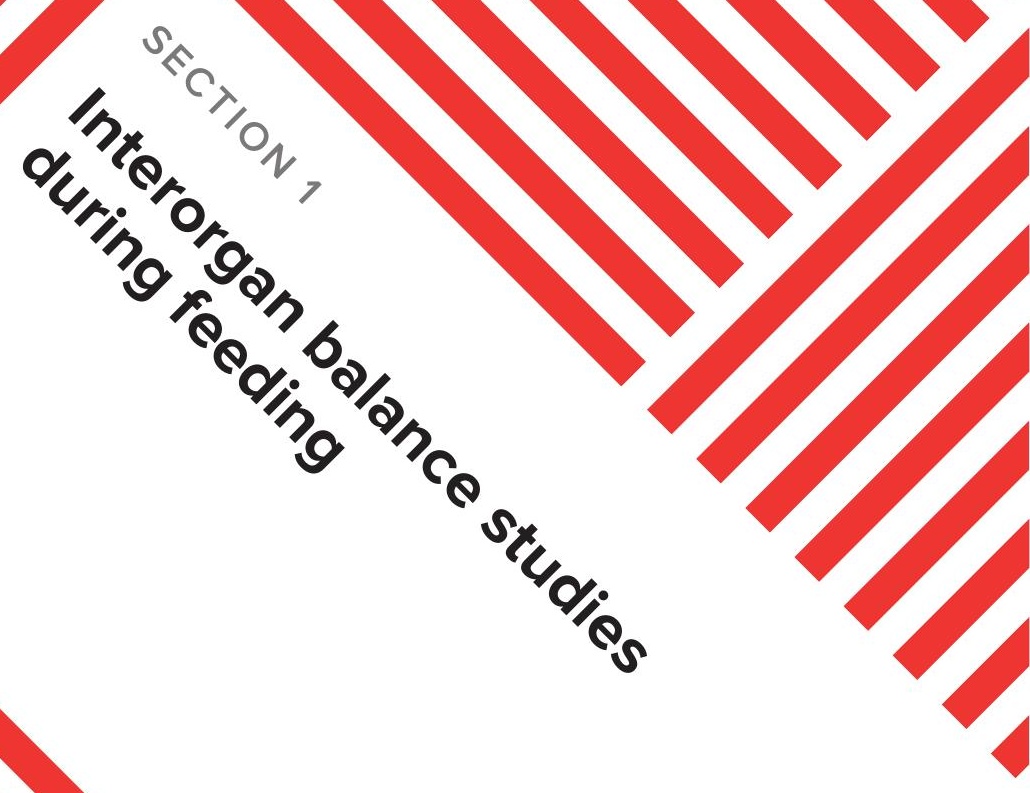


CHAPTER 2 


\title{
Absorption kinetics of amino acids, peptides and intact proteins
}

\author{
Gabriella A.M. Ten Have, Marielle P.K.J. Engelen, Yvette \\ C. Luiking, Nicolaas E.P. Deutz \\ International Journal of Sport Nutrition and Exercise Metabolism, \\ 2007, 17, S23- 54
}

\section{Abstract}

The small intestine acts as interface and regulator between the gut lumen and the rest of the body and controls the degree and rate of transport of amino acids coming from dietary protein via the portal vein to the liver and the systemic circulation. To measure protein absorption, kinetics multi-catheter animal (pig) models in combination with amino acid tracer technology are available. Dietary factors influence the absorption rates from the lumen to the gut, metabolism of dietary component in the gut, and the release of amino acids to the portal circulation from digested protein. In a balanced-protein meal, the gut dietary amino acid utilization (30-50\%) for gut protein synthesis will result in a labile protein pool in the gut that can be beneficial during the post absorptive state. To enhance gut retention, amount and quality of protein and the presence of carbohydrate are major factors. Besides this the use of a slowly digestible protein or the presence of fibre in the meal can increase retention further. During the absorption of low quality protein meals, fewer amino acids are utilized by the gut, resulting in higher amounts of amino acid release to the portal circulation. Malnutrition or starvation, protein depletion, deficiencies of specific nutrients, or illness such as sepsis all inhibit the growth and change protein turnover of the intestinal mucosa and therefore affect absorption kinetics. Therefore, the kind of protein meal that has the most optimal absorption kinetics (the most beneficial) for gut and for the rest of the body depends on these (patho) physiological circumstances. Despite the absence of different absorption kinetics between protein, peptides, and amino acids, they could be beneficial in specific circumstances. 


\section{Introduction}

The gastrointestinal (Gl) tract is the primary organ in the control of food digestion, absorption of food components, and release of food components to the rest of the body. Furthermore, the gut has a barrier function that protects the body against invasion (translocation) of endogenous luminary microorganisms and/or toxins (1).

To cover this variety of functions, the structure of the small intestine is characterized by a large luminal surface area (mucosa), through mucosal folding with the presence of villi and microvilli on top and crypts at the bottom. The intestinal mucosal layer is one of the most rapidly replicating tissues in the body, with a continuous process of differentiation of stem cells into specialized cells for absorption (enterocytes or epithelial cells), excretion (enteroendocrine cells), and mucus (water and glycoproteins) secretion (Goblet cells) (2).

Digestion of dietary protein begins in the stomach, but occurs mainly in the small intestine. While approx. 95\% of dietary protein is absorbed in the small intestine (3), the remaining amino acids, undigested proteins and unabsorbed peptides, of either dietary or endogenous origin, can enter the large intestine. In the large intestine these components are subject to digestion and metabolism by the intestinal micro flora (4).

The small intestine itself is the absorption organ for amino acids coming from dietary protein and is the first pass for amino acids to the circulation. Therefore, the small intestine acts as interface and regulator between the gut lumen and the rest of the body and controls the degree and rate of transport of amino acids coming from dietary protein via the portal vein to the liver and the systemic circulation. However, not all amino acids that pass the gut enter the circulation, since a part of the amino acids is used for local metabolism (e.g. oxidation, protein synthesis). The present review will focus on the influence of several dietary factors on gut amino acid absorption, metabolism and release to the portal system.

\section{Gut as a metabolic active organ}

Besides the organ that has direct contact with ingested food, the gut is also a very metabolic active organ (5). Supply of amino acids to the gut cells does not rely solely on luminal supply during feeding. There is also a substantial use from arterial supply and as such the gut is "competing" with other tissues for amino acid utilization (figure 1). The high level of metabolic activity in the gut is also demonstrated by the fact that rates of protein synthesis in the intestinal mucosa are among the highest in the body (6). 


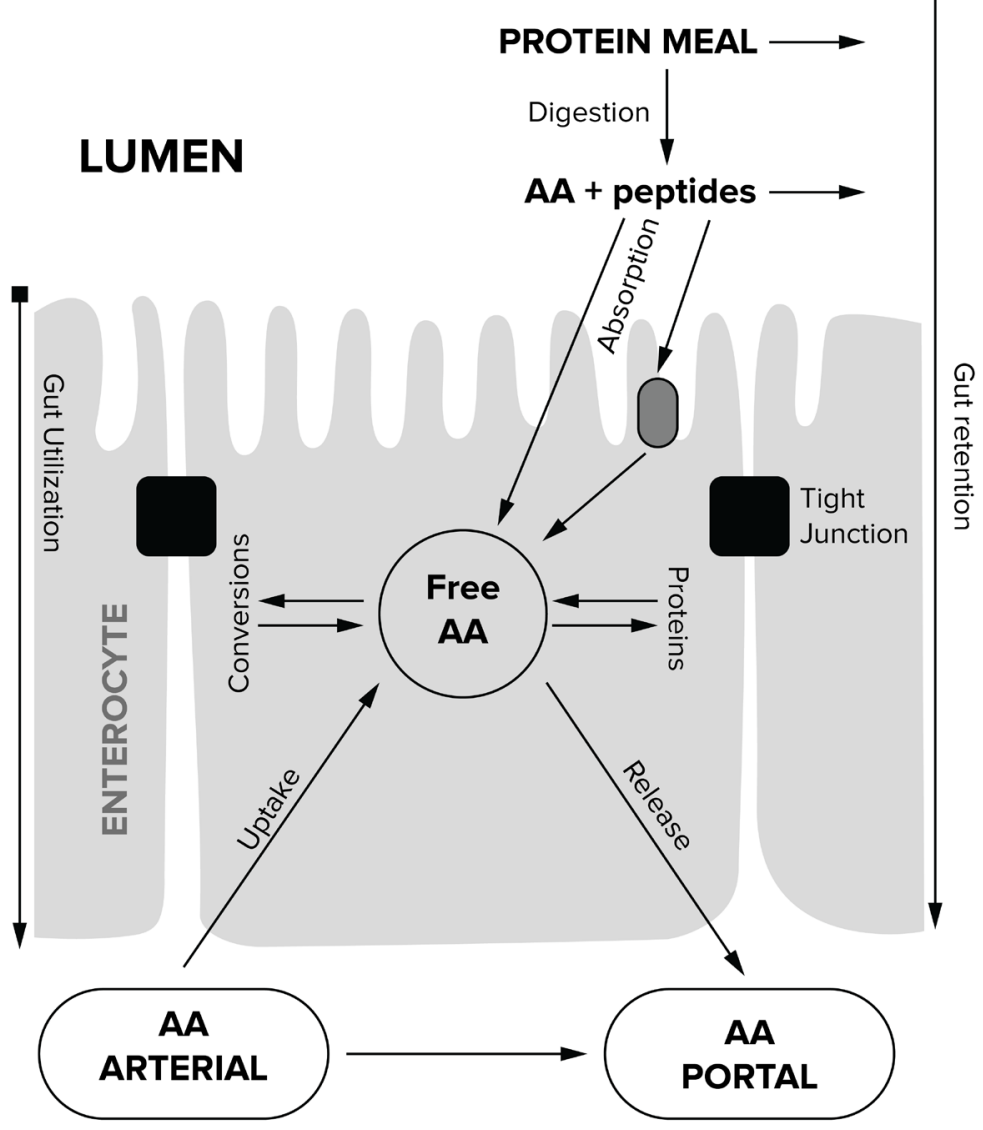

\section{BLOOD}

Figure 1. Model that explains net amino acid uptake and release into the circulation after a protein-peptide-amino acid meal. AA indicates amino acid.

\section{During feeding}

Luminal contact with nutrients is very important in the regulation of intestinal protein metabolism (7-9). Feeding is known to rapidly stimulate protein synthesis in the gut. Approximately $50 \%$ of dietary amino acid intake is used by the Portal Drained Viscera (PDV), but this percentage varies between different amino acids (10, 11). It is known that mucosal protein turnover is high and that amino acids with a different origin, e.g. lumen and circulation contribute to protein synthesis at a different level during feeding (11). 
However, mucosal protein synthesis rate is not affected by feeding and is less sensitive to short-term variations in nutritional status (12). In neonatal pigs, less than $20 \%$ of intestinal amino acid utilization is used for constitutive gut growth by the intestinal mucosa (13). It appears that an enhanced secretory (glyco) protein synthesis rate in these neonatal pigs contributes to the increased gut protein synthesis during feeding (11). Approximately $26 \%$ of the dietary protein intake reappeared in the portal vein by way of recycling of digested (glyco)-proteins. Therefore, intestinal recycling of amino acids apparently contributes to their overall systemic availability. The protein synthesizing capacity, however, depends on the dietary protein source used, as will be discussed later.

\section{During fasting}

Malnutrition or starvation, protein depletion or deficiencies of specific nutrients all inhibit growth and turnover of the intestinal mucosa $(1,14,15)$. Interestingly during fasting, gut protein is considered a (labile) pool (16). After a meal there is a net accumulation of protein in the gut whereas in the post-absorptive state a net loss of protein takes place. The hypothesis is that net retention of amino acids as protein in the gut serves to 'buffer' a bolus meal containing protein. When a protein would be rapidly digested, absorbed and directly released to the portal system, the large flux of highly concentrated amino acids in the portal vein would give rise to a high rate of urea production, gluconeogenesis and amino acid oxidation (17). A more gradual release of amino acids from the gut would ensure a more prolonged supply of amino acids in the portal vein, resulting in lower plasma concentrations in the portal vein and a lower urea production and potentially more muscle anabolism (16). During prolonged fasting, the intestine is taking up amino acids from the arterial side. Especially glutamine coming from the muscle compartment is utilized as a major energy substrate by intestinal mucosal and immune cells $(14,18)$.

\section{Measurement of gut amino acid absorption kinetics in vivo}

The in- and outflow (flux) of amino acids, protein, and nitrogen in the gut exhibits a complicated pattern (19). The digestion of many dietary proteins by the gut is incomplete. Besides dietary supply, there is also a continuous (but variable) entry into the intestinal lumen of endogenous protein and amino acid nitrogen that is subject to digestion. To get insight in the absorption of a meal, methods are used that examine the digestion characteristics of a protein meal in the gut lumen, while other techniques are based on amino acid oxidation and the use of stable isotope techniques. In order to measure absorption and fluxes of amino acids across the gut, a multi-catheter technique is needed (20). Combination of catherization of 
multiple organs and stable isotopes techniques enables measurement of in vivo gut metabolism and the interaction with other organs (10).

\section{In humans}

Multi-organ catheter techniques are often not applicable in humans. In humans, measurements across the portal drained viscera (PDV: the GI tract, pancreas, spleen, and associated adipose tissue) are only possible during surgery (21-23). Therefore, in studies in healthy conscious subjects, mostly measurement of splanchnic extraction of alimentary amino acids, which represents the sum of PDV and liver amino acid uptake and utilization, is done (24-31). Introduction of this doubly labeled stable isotope technique has enabled a more detailed study of splanchnic conversions and amino acid extraction $(32,33)$ in which two tracers of the same amino acid but with a different label are used. Simultaneously, one tracer is infused intra-gastric or orally, and the other intravenously. A multi-compartmental computing simulation model has been developed to describe dietary nitrogen postprandial distribution and metabolism in humans. The model is using experimental data on dietary $\mathrm{N}$ kinetics in certain accessible pools of the intestine, blood, and urine in healthy adults fed a [15N]-labeled protein meal $(34,35)$. However, in this model specialized information on the absorption/utilization of dietary amino acids in the gut is limited.

\section{Large and small animal models}

Small animals like rodents are considered suitable for investigation of the mechanisms of absorption and bio-availability (14), whereas larger animals are generally used to assess absorption kinetics $(19,20)$. Pigs are often used in metabolic research as pigs are very similar to humans with respect to renal, cardiovascular and digestive anatomy and physiology, (36), (37-39). Furthermore, larger species like the pig are also useful when multiple blood samples have to be taken in time.

\section{Multi-compartmental modeling}

Studying amino acid absorption and utilization by the gut is difficult. The complicating factor is that the intestinal mucosa receives nutrients from 2 sources, the diet (brush border membrane) and the systemic circulation (basolateral membrane). Flux of amino acids from the lumen to the portal system is mainly studied in animal models using multi-catheter techniques. This enables the sampling of blood entering (i.e. afferent blood vessel) and leaving the organ (i.e. efferent blood vessel) for measurement of concentration differences across the organ with simultaneous measurement of organ blood flow (20) (see figure 1). Net balance studies of amino acids across an organ, however, only provide information on the net uptake (net anabolism) or release (net catabolism) and dynamic processes (eg disposal and production) cannot be quantified. 


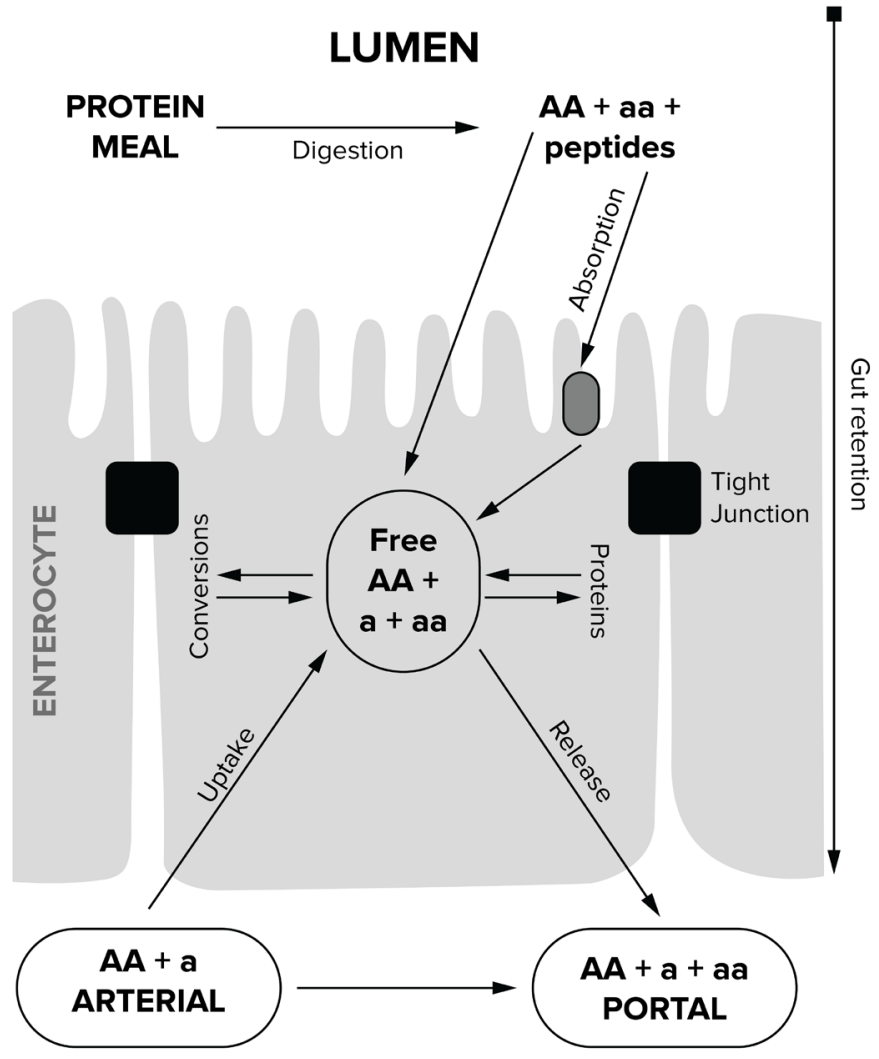

BLOOD

Figure 2. Model to measure amino acid uptake, metabolism, and release into the circulation after a protein-peptide-amino acid meal by using isotopes of amino acids (AA) that are present in protein.

Extension of substrate (tracee) studies by the use of isotopes (tracers) enables measurements of amino acid disposal and production across the organ. The principle is based on the dilution principle of Fick. During primed-continuous intravenous tracer infusion any change in the enrichment over time occurs as a consequence of dilution from the unlabeled tracee. The rate of appearance (Ra) of an amino acid is determined by measuring the change of the tracer/tracee ratio (TTR) in relation to the isotope infusion rate (40). 
In the gut, phenylalanine disposal and production (turnover) are related to protein synthesis and protein breakdown and are measured by calculating tracer disappearance rate in that organ and the net balance of tracee. To assess phenylalanine measured protein turnover, TTR measurements on the arterial and portal side are needed (figure 2). This is a so-called "two-compartment" model, with plasma and interstitial fluid representing the two compartments. A "three-compartment" model can also be applied when tissues or biopsies are available (third compartment represents the free tissue pool). Combination of several tracers makes it possible to get quantitative information of multiple metabolic pathways in the gut.

Flux of a single amino acid across the gut coming from a protein meal represents absorption, utilization and release to the portal system. To get more insight in the absorption rate in time of a single amino acid, two tracers of this amino acid are used (figure 2). One is done intravenously via a primed-continuous infusion protocol and the other is given orally, added to a protein meal.

\section{Dietary factors that affect gut amino acid absorption rate, metabolism and release}

The amount of protein, the protein source, and the presence of other macro nutrients in the meal influence the absorption rate and metabolism of amino acids in the gut and affect the release rate of amino acids to the portal system. All these factors determine the anabolic capacity of a meal.

\section{Protein amount}

The amount of protein in a diet has a major effect on the magnitude of change of protein metabolism in the gut and in the rest of the body. With a balanced meal approximately $90 \%$ of the dietary amino acids are absorbed by the gut. About $30-50 \%$ will be utilized by the intestine itself and the remainder released to the portal system. An excessive amount of protein intake potentially could lead to limitation of gut absorption, and thus to a reduction of the percentage protein absorption. However, it is more likely that the maximum that the gut cells can utilize for own metabolism is reached faster and that consequently higher protein intake will reduce the percentage of protein extracted. The amino acids that are not absorbed and undigested proteins will flow into the colon. During low protein intake, the percentage protein extracted by the gut will be higher, although the intestine can adapt to reduced protein intake by reducing its amino acid oxidation(41) (see Table 1). 


\begin{tabular}{|c|c|c|}
\hline Dietary factor & $\begin{array}{l}\text { Effect on percent- } \\
\text { age gut retention }\end{array}$ & $\begin{array}{l}\text { Effect on percentage } \\
\text { portal appearance }\end{array}$ \\
\hline Increasing amount of Protein & Decrease & Increase \\
\hline Excessive amount of Protein & Decrease & Decrease \\
\hline Lowering amount of Protein & Minor increase & Minor decrease \\
\hline Improving the quality of Protein & Increase & Decrease \\
\hline $\begin{array}{l}\text { Slowing of the rate of protein } \\
\text { digestion }\end{array}$ & Increase & Decrease \\
\hline $\begin{array}{l}\text { Adding Carbohydrates to the } \\
\text { meal }\end{array}$ & Increase & Decrease \\
\hline Adding Fats to the meal & Minor increase & Minor decrease \\
\hline $\begin{array}{l}\text { Adding soluble Fiber to the } \\
\text { meal (prolonged) }\end{array}$ & Increase & Decrease \\
\hline $\begin{array}{l}\text { Partially hydrolyze protein } \\
\text { ( }{ }^{\sim} \text { peptides) }\end{array}$ & No change & No change \\
\hline $\begin{array}{l}\text { Fully hydrolyse protein ( }{ }^{\sim} \text { free } \\
\text { amino acids) }\end{array}$ & No change & No change \\
\hline
\end{tabular}

Table 1. Summary of the effects of dietary factors on percentage gut retention and portal appearance after bolus feedings of proteins, peptides, and amino acids under healthy conditions.

\section{Protein quality}

The quality (nutritional value) of a dietary protein is related to both the bio-availability of ingested nitrogen and amino acids and the efficiency of their metabolic utilization to meet nitrogen and amino acid requirements for growth and renewal of body proteins (42-45). It is postulated that a high quality dietary protein source stimulates amino acid utilization in the gut and is therefore of benefit for the gut and for the rest of the body $(11,16)$. We compared in a pig study, soy (low quality) and casein (high quality) protein and observed that liver urea production and net release of essential amino acids by the gut was higher with soy (10). This was confirmed in a study in healthy subjects, demonstrating a lower net protein synthesis and higher ureagenesis after a soy containing meal (25). This suggests that amino acid retention across the PDV is higher after a casein protein meal. Possible explanations are the fact that soy is deficient in the essential amino acids methionine and lysine and contains less branched-chain amino acids and the difference in the digestion rate between both proteins (46). In addition, the high biological value of casein protein potentially is related to the release of peptides that have a local trophic effect on the gut and that can increases mucine production (47).

Metabolic utilization of amino acids in the gut thus depends on the composition of the meal with respect to the presence or absence of (in) dispensable essential amino acids. Complete lack of an amino acid in a protein meal makes the protein of inferior quality. Previous studies in pigs showed that ingestion of an isoleucine-lacking 


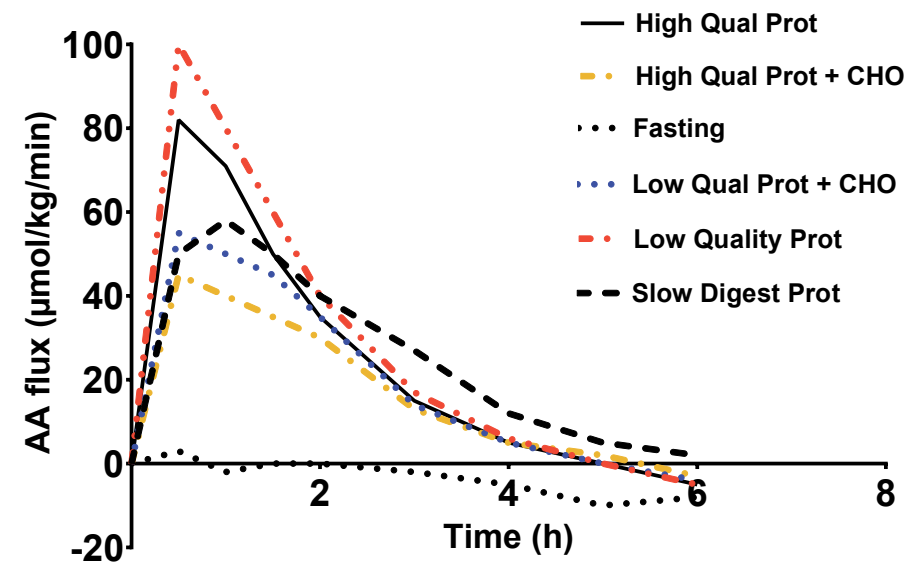

Figure 3. Pattern of PDV amino acid (AA) efflux during bolus feeding with proteins.

blood protein meal resulted in an elevated urea production. Concomitant intravenous isoleucine infusion lowered the increase of urea and promoted amino acid retention in the gut (17) (see figure 3). In conclusion, high quality proteins stimulate amino acid utilization in the gut and therefore induce more gut amino acid retention (see table 1).

\section{Slow versus fast proteins}

A difference in the speed of protein digestion and absorption in the gut, the so called slow vs. fast protein concept, also affects the quality of a protein (48). Postprandial plasma amino acid profile shows a rapid high increase with whey protein and a prolonged plateau of moderate hyperaminoacidemia with casein protein (49), resulting in a more prolonged positive net protein balance after casein than after whey intake in young healthy males $(48,49)$ (figure 3$)$. The discussion whether a slow digestible protein (e.g. casein) is better than a fast digestible protein (e.g. whey) depends on the (patho) physiological situation. The 'labile' gut protein pool hypothesis suggests that prolonged amino acid release from the gut is better for the body in the long term. Recent evidence indicates that the optimal dietary protein source and feeding pattern changes with age. Stimulation of muscle protein synthesis in the elderly necessary to counteract sarcopenia is higher with fast proteins like whey, with protein hydrolyzates or with a protein feeding pulse pattern (50). Therefore factors like amino acid profile, digestibility and absorption rate of a protein can all influence gut retention of that protein. 


\section{Role of adding carbohydrates to the meal}

In a multi-catheterized pig study, pigs were given a bolus meal consisting of high quality proteins with and without carbohydrates $(\mathrm{CHO})$. Addition of carbohydrates to a protein meal resulted in an increased intestinal amino acid retention (Figure 3), lower urea production and increased gut GLN uptake and ALA release, indicating stimulated gut metabolism (3). This suggests that addition of carbohydrate to a protein meal improves the anabolic quality of a protein meal in the gut. Recent studies showed that adding high quality protein to a carbohydrate meal will have an insulinotropic effect (51). Intake of free leucine, phenylalanine and arginine further augmented the insulin response. More recent studies show that co ingestion of a protein hydrolysate with additional free leucine will maximize the insulinotropic response (in both type 2 diabetes patients as well as in normoglycemic controls (52, 53). Simultaneous ingestion of these amino acids and carbohydrates resulted in a 100\% higher insulin response than with carbohydrate only (51).

\section{Role of adding fat to the meal}

Until now, the effect of fat intake on amino acid retention in the gut has not been studied directly. Studying splanchnic nitrogen retention after adding fat vs $\mathrm{CHO}$ to a protein meal using a simulation model revealed that fat enhances splanchnic dietary $\mathrm{N}$ anabolism only transiently, without significantly affecting the global kinetics of splanchnic retention and peripheral uptake (54). This suggests that after fat co-intake the absorption rate can be reduced in the lumen side but that no major gut metabolic changes are expected.

\section{Role of adding fiber to the meal}

Fiber is a well-known dietary ingredient that stimulates long-term mucosal growth, especially in the colon. The magnitude of growth depends on the type of fiber ingested (2). It is expected that, if the structural changes only take place in the colon, it will not influence the amino acid absorption in the small intestine. However, extra food retention due to the higher viscosity of a fiber containing meal can lead to more protein digestion retention in the intestinal lumen. Recent studies showed that prolonged ingestion of soluble dietary fiber like Pectin leads to structural changes in the small intestine (55). The total protein amount of the intestine was enhanced, which resulted in higher protein turnover, and consequently in higher energy and amino acid requirements for the digestive tract. It is expected that the enhanced mucosa amount will result in an increased absorption capacity and rate.

\section{Absorption kinetics of peptides}

Small peptides can be actively absorbed by the enterocytes via specific transporters. The peptides in the enterocyte are converted to single amino acids which 
are released to the portal system or utilized by the gut itself. Another possibility is that the peptides are directly released to the portal system. In a study with healthy young pigs, the gut absorption kinetics of intact proteins, hydrolyzates and free amino acids mixture were found to be comparable (56). In this study the selected protein was a fast protein, with no digestion limitation in the gut lumen itself. This suggests that amino acid absorption coming from hydrolyzates can only be higher than that of intact protein, if these hydrolyzates come from a slow protein or are in a mixed meal that slowed digestion. This hypothesis is confirmed in a study with young man in which hydrolyzates of a fast and slow protein were compared with the intact protein (57).

Modification of the structure of dietary proteins by hydrolysis has been reported to improve the nutritional value of a meal in several studies under specific (patho) physiological circumstances. For instance in a post-exercise situation where hydrolyzate works insulinotropic (58). In another study it is suggested that the fact that milk peptide hydrolyzate solutions elicit greater release of glucose-dependent insulinotropic polipeptide (GIP) than the complete protein from which they are derived, might be beneficial in clinical situations where there is insulin resistance and glucose intolerance (57). Also in conditions of reduced digestibility as in criticalill patients, a meal with hydrolyzed protein or peptides might be beneficial (59).

It has been suggested that some proteins also contain bioactive sequences which may affect gut absorption and/or metabolism. Oligopeptides derived from casein for example stimulate mucin release from the Globlet cells in an isolated vascularly perfused rate jejunum (47).

\section{Absorption kinetics of amino acids}

As mentioned in the previous paragraph, the gut absorption rate of free amino acids and intact proteins coming from the same native protein are identical (56). This does not mean that the flux rate of all amino acids are identical. The flux rate depends whether or not these amino acids can be used by the gut itself (protein synthesis, oxidation) or converted in the gut to other amino acids that can be beneficial to the rest of the body (figure 1). The quantitative and qualitative utilization of amino acids by the gut is dependent on the amino acid profile of the dietary protein, the added macro-nutrients and the interaction with other organs.

\section{How is absorption influenced by gut amino acid metabolism?}

Involvement of several amino acids in metabolic pathways in the gut is reflected by their large intestinal extraction during enteral feeding: glutamate (96\%), glutamine (64\%), threonine (57\%), arginine (65\%), cysteine (44\%), BCAA (50-60\%) (10, 11 , 
60-63). Recently it was observed that the gut also uses threonine, sulfur amino acids (cysteine, methionine) and branched-chain amino acids (leucine, isoleucine and valine) to a higher extent than anticipated from the requirements for protein synthesis in the gut. Apparently gut extracted amino acids are involved in different kind of pathways. Limiting these amino acids in a protein meal (low quality protein meal) will lead to a less than optimal amino acid profile for the gut. Because of this, amino acid flux can be changed into more release to the portal system (17). A single amino acid deficient in a protein meal can be compensated by extra intravenous infusion of this amino acid, which normalized the gut metabolism (17). Threonine is important for the structural protein mucus layer and that probably is the reason that in neonatal piglets, more than two thirds of the enteral threonine intake was used by the PDV. By this threonine metabolism can influence the absorption rate (11).

\section{How is absorption influenced by illness?}

Recently, pigs are studied pigs that were made septic by $24 \mathrm{~h}$ infusion of endotoxins $(18,64)$. When these pigs were fed a meal with high quality proteins, the appearance of amino acids in the portal circulation was increased (figure 4), indicating that amino acid utilization of the gut was reduced in the septic pigs. Tracer studies revealed that protein synthesis was not changed but protein breakdown was enhanced in the septic pigs. Gut metabolism was clearly affected in the septic pigs as gut glutamine consumption was greatly reduced. So an altered gut metabolism caused by illness can change the amount of gut amino acid absorption and release to the circulation but also the profile of amino acid released to the gut.

The amount of protein received by continuous infusion was comparable between the groups. FedpostS= after $30 \mathrm{~h}$ of starvation; FedpostS+LPS= after $30 \mathrm{~h}$ of starvation

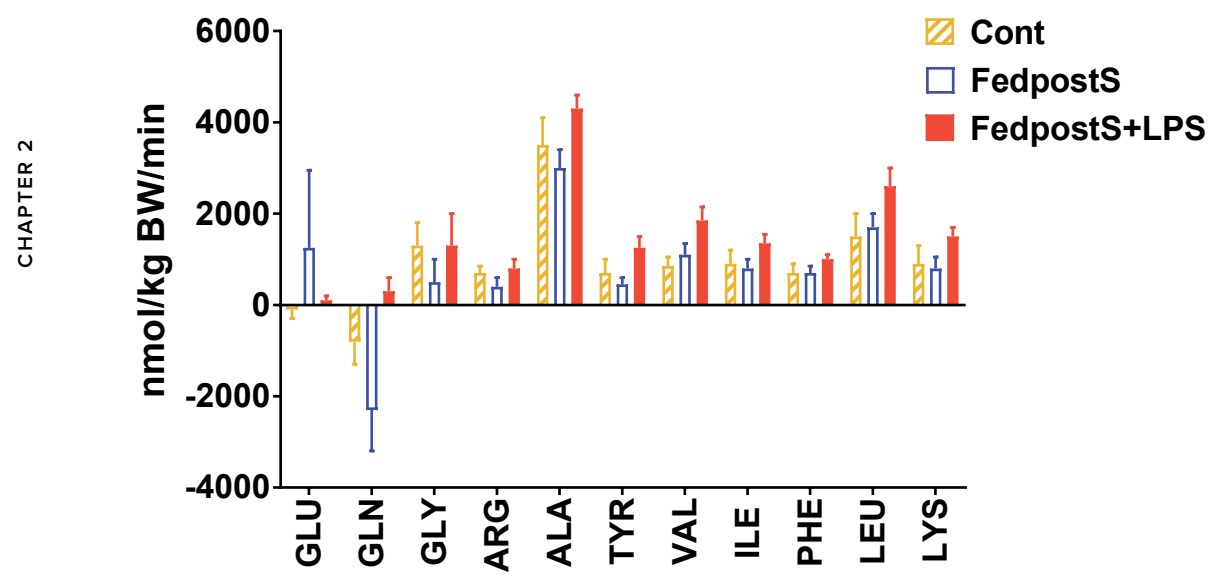

Figure 4. The effect of sepsis on the portal appearance of amino acids in the pig (net PDV flux). 
and $24 \mathrm{~h}$ of endotoxin (endotoxin) infusion. No change in Protein Synthesis was observed, while Protein Breakdown was increased. Percentage absorption was not different. PDV indicates portal-drained viscera.

\section{Conclusion}

In this review, we have discussed the factors that lead to enhanced, reduced or modified absorption kinetics. To study these factors, a sophisticated model in multicatheterized pigs that with the use of isotopes can calculate absorption and gut metabolism, is necessary.

\section{References}

1. Ziegler TR, Evans ME, Fernandez-Estivariz C, Jones DP. Trophic and cytoprotective nutrition for intestinal adaptation, mucosal repair, and barrier function. Annu Rev Nutr. 2003;23:229-61.

2. Johnson LR. Regulation of gastrointestinal mucosal growth. Physiological reviews. 1988;68(2):456-502.

3. Deutz NE, Ten Have GA, Soeters PB, Moughan PJ. Increased intestinal amino-acid retention from the addition of carbohydrates to a meal. Clin Nutr. 1995;14(6):354-64.

4. Zebrowska T, Simon O, Munchmeyer R, Bergner H. [Secretion of endogenous amino acids in the gastrointestinal tract and amino acid resorption in the swine]. Arch Tierernahr. 1976;26(2):69-82.

5. Wu G. Intestinal mucosal amino acid catabolism. J Nutr. 1998;128(8):1249-52.

6. McNurlan MA, Garlick PJ. Contribution of rat liver and gastrointestinal tract to wholebody protein synthesis in the rat. Biochem J. 1980;186(1):381-3.

7. Dudley MA, Wykes LJ, Dudley AW, Jr., Burrin DG, Nichols BL, Rosenberger J, et al. Parenteral nutrition selectively decreases protein synthesis in the small intestine. Am J Physiol. 1998;274(1 Pt 1):G131-7.

8. Frankel W, Zhang W, Singh A, Bain A, Satchithanandam S, Klurfeld D, et al. Fiber: effect on bacterial translocation and intestinal mucin content. World J Surg. 1995;19(1):144-8; discussion 8-9.

9. Sax HC, Illig KA, Ryan CK, Hardy DJ. Low-dose enteral feeding is beneficial during total parenteral nutrition. Am J Surg. 1996;171(6):587-90.

10. Deutz NE, Bruins MJ, Soeters PB. Infusion of soy and casein protein meals affects interorgan amino acid metabolism and urea kinetics differently in pigs. J Nutr. 1998;128(12):2435-45.

11. Van Der Schoor SR, Reeds PJ, Stoll B, Henry JF, Rosenberger JR, Burrin DG, et al. The high metabolic cost of a functional gut. Gastroenterology. 2002;123(6):1931-40.

12. Bouteloup-Demange C, Boirie Y, Dechelotte P, Gachon P, Beaufrere B. Gut mucosal protein synthesis in fed and fasted humans. Am J Physiol. 1998;274(3 Pt 1):E541-6.

13. Stoll B, Henry J, Reeds PJ, Yu H, Jahoor F, Burrin DG. Catabolism dominates the first-pass intestinal metabolism of dietary essential amino acids in milk protein-fed piglets. J Nutr. 1998;128(3):606-14.

14. de Blaauw I, Deutz NE, Von Meyenfeldt MF. In vivo amino acid metabolism of gut 
and liver during short and prolonged starvation. Am J Physiol. 1996;270(2 Pt 1):G298-306.

15. Katayama M, Xu D, Specian RD, Deitch EA. Role of bacterial adherence and the mucus barrier on bacterial translocation: effects of protein malnutrition and endotoxin in rats. Ann Surg. 1997;225(3):317-26.

16. Soeters PB, de Jong $\mathrm{CH}$, Deutz NE. The protein sparing function of the gut and the quality of food protein. Clin Nutr. 2001;20(2):97-9.

17. Deutz NE, Reijven PL, Bost MC, van Berlo CL, Soeters PB. Modification of the effects of blood on amino acid metabolism by intravenous isoleucine. Gastroenterology. 1991;101(6):1613-20.

18. Bruins MJ, Deutz NE, Soeters PB. Aspects of organ protein, amino acid and glucose metabolism in a porcine model of hypermetabolic sepsis. Clin Sci (Lond). 2003;104(2):127-41.

19. Fuller MF, Tome D. In vivo determination of amino acid bioavailability in humans and model animals. J AOAC Int. 2005;88(3):923-34.

20. Ten Have GA, Bost MC, Suyk-Wierts JC, van den Bogaard AE, Deutz NE. Simultaneous measurement of metabolic flux in portally-drained viscera, liver, spleen, kidney and hindquarter in the conscious pig. Lab Anim. 1996;30(4):347-58.

21. Felig $\mathrm{P}$, Wahren J. Amino acid metabolism in exercising man. J Clin Invest. 1971;50(12):2703-14.

22. Olde Damink SW, Jalan R, Redhead DN, Hayes PC, Deutz NE, Soeters PB. Interorgan ammonia and amino acid metabolism in metabolically stable patients with cirrhosis and a TIPSS. Hepatology. 2002;36(5):1163-71.

23. van de Poll MC, Siroen MP, van Leeuwen PA, Soeters PB, Melis GC, Boelens PG, et al. Interorgan amino acid exchange in humans: consequences for arginine and citrulline metabolism. Am J Clin Nutr. 2007;85(1):167-72.

24. Engelen MP, Rutten EP, De Castro CL, Wouters EF, Schols AM, Deutz NE. Altered interorgan response to feeding in patients with chronic obstructive pulmonary disease. Am J Clin Nutr. 2005;82(2):366-72.

25. Luiking YC, Deutz NE, Jakel M, Soeters PB. Casein and soy protein meals differentially affect whole-body and splanchnic protein metabolism in healthy humans. J Nutr. 2005;135(5):1080-7.

26. Boza JJ, Dangin M, Moennoz D, Montigon F, Vuichoud J, Jarret A, et al. Free and protein-bound glutamine have identical splanchnic extraction in healthy human volunteers. Am J Physiol Gastrointest Liver Physiol. 2001;281(1):G267-74.

27. Crenn P, Thuillier F, Rakatoambinina B, Rongier M, Darmaun D, Messing B. Duodenal vs. gastric administration of labeled leucine for the study of splanchnic metabolism in humans. Journal of applied physiology. 2000;89(2):573-80.

28. Kriengsinyos W, Wykes LJ, Ball RO, Pencharz PB. Oral and intravenous tracer protocols of the indicator amino acid oxidation method provide the same estimate of the lysine requirement in healthy men. J Nutr. 2002;132(8):2251-7.

29. Uusaro A, Ruokonen E, Takala J. Estimation of splanchnic blood flow by the Fick principle in man and problems in the use of indocyanine green. Cardiovasc Res. 1995;30(1):106-12.

30. Boirie Y, Gachon P, Beaufrere B. Splanchnic and whole-body leucine kinetics in young and elderly men. Am J Clin Nutr. 1997;65(2):489-95.

31. Volpi E, Mittendorfer B, Wolf SE, Wolfe RR. Oral amino acids stimulate muscle protein anabolism in the elderly despite higher first-pass splanchnic extraction. Am J Physiol. 1999;277(3 Pt 1):E513-20.

32. Hoerr RA, Matthews DE, Bier DM, Young VR. Leucine kinetics from [2H3]- and [13C] leucine infused simultaneously by gut and vein. Am J Physiol. 1991;260(1 Pt 1):E111-7. 

and glutamic acid in humans. Am J Physiol. 1993;264(6 Pt 1):E848-54.

34. Fouillet H, Gaudichon C, Mariotti F, Mahe S, Lescoat P, Huneau JF, et al. Compartmental modeling of postprandial dietary nitrogen distribution in humans. Am J Physiol Endocrinol Metab. 2000;279(1):E161-75.

35. Juillet B, Saccomani MP, Bos C, Gaudichon C, Tome D, Fouillet H. Conceptual, methodological and computational issues concerning the compartmental modeling of a complex biological system: Postprandial inter-organ metabolism of dietary nitrogen in humans. Math Biosci. 2006;204(2):282-309

36. Miller ER, Ullrey DE. The pig as a model for human nutrition. Annu Rev Nutr. 1987;7:361-82

37. Rowan AM, Moughan PJ, Wilson MN, Maher K, Tasman-Jones C. Comparison of the ileal and faecal digestibility of dietary amino acids in adult humans and evaluation of the pig as a model animal for digestion studies in man. Br J Nutr. 1994;71(1):29-42.

38. Darragh AJ, Moughan PJ. The three-week-old piglet as a model animal for studying protein digestion in human infants. J Pediatr Gastroenterol Nutr. 1995;21(4):387-93.

39. Kararli TT. Comparison of the gastrointestinal anatomy, physiology, and biochemistry of humans and commonly used laboratory animals. Biopharm Drug Dispos. 1995;16(5):351-80.

40. Miller S, Chinkes D, MacLean DA, Gore D, Wolfe RR. In vivo muscle amino acid transport involves two distinct processes. Am J Physiol Endocrinol Metab. 2004;287(1):E136-41.

41. van Goudoever JB, van der Schoor SR, Stoll B, Burrin DG, Wattimena D, Schierbeek $\mathrm{H}$, et al. Intestinal amino acid metabolism in neonates. Nestle Nutr Workshop Ser Pediatr Program. 2006(58):95-102; discussion -8.

42. Pellet PL. Protein requirements in humans. Am J Clin Nutr. 1990;51(5):723-37.

43. Young VR, Marchini JS. Mechanisms and nutritional significance of metabolic responses to altered intakes of protein and amino acids, with reference to nutritional adaptation in humans. Am J Clin Nutr. 1990;51(2):270-89.

44. Hiramatsu T, Cortiella J, Marchini JS, Chapman TE, Young VR. Source and amount of dietary nonspecific nitrogen in relation to whole-body leucine, phenylalanine, and tyrosine kinetics in young men. Am J Clin Nutr. 1994;59(6):1347-55.

45. Millward J. Can we define indispensable amino acid requirements and assess protein quality in adults? J Nutr. 1994;124(8 Suppl):1509S-16S.

46. Bos C, Metges CC, Gaudichon C, Petzke KJ, Pueyo ME, Morens C, et al. Postprandial kinetics of dietary amino acids are the main determinant of their metabolism after soy or milk protein ingestion in humans. J Nutr. 2003;133(5):1308-15.

47. Claustre J, Toumi F, Trompette A, Jourdan G, Guignard H, Chayvialle JA, et al. Effects of peptides derived from dietary proteins on mucus secretion in rat jejunum. Am $J$ Physiol Gastrointest Liver Physiol. 2002;283(3):G521-8.

48. Dangin M, Boirie Y, Garcia-Rodenas C, Gachon P, Fauquant J, Callier P, et al. The digestion rate of protein is an independent regulating factor of postprandial protein retention. Am J Physiol Endocrinol Metab. 2001;280(2):E340-8.

49. Boirie Y, Dangin M, Gachon P, Vasson MP, Maubois JL, Beaufrere B. Slow and fast dietary proteins differently modulate postprandial protein accretion. Proc Natl Acad Sci U S A. 1997;94(26):14930-5.

50. Walrand S, Boirie Y. Optimizing protein intake in aging. Curr Opin Clin Nutr Metab Care. 2005;8(1):89-94.

51. Van Loon LJ, Saris WH, Verhagen H, Wagenmakers AJ. Plasma insulin responses after ingestion of different amino acid or protein mixtures with carbohydrate. Am J Clin Nutr. 
2000;72(1):96-105.

52. Manders RJ, Wagenmakers AJ, Koopman R, Zorenc AH, Menheere PP, Schaper NC, et al. Co-ingestion of a protein hydrolysate and amino acid mixture with carbohydrate improves plasma glucose disposal in patients with type 2 diabetes. Am J Clin Nutr. 2005;82(1):76-83.

53. Manders RJ, Koopman R, Sluijsmans WE, van den Berg R, Verbeek K, Saris WH, et al. Co-ingestion of a protein hydrolysate with or without additional leucine effectively reduces postprandial blood glucose excursions in Type 2 diabetic men. J Nutr. 2006;136(5):1294-9.

54. Fouillet H, Gaudichon C, Mariotti F, Bos C, Huneau JF, Tome D. Energy nutrients modulate the splanchnic sequestration of dietary nitrogen in humans: a compartmental analysis. Am J Physiol Endocrinol Metab. 2001;281(2):E248-60.

55. Pirman T, Ribeyre MC, Mosoni L, Remond D, Vrecl M, Salobir J, et al. Dietary pectin stimulates protein metabolism in the digestive tract. Nutrition. 2007;23(1):69-75.

56. Deutz NE, Welters CF, Soeters PB. Intragastric bolus feeding of meals containing elementary, partially hydrolyzed or intact protein causes comparable changes in interorgan substrate flux in the pig. Clin Nutr. 1996;15(3):119-28.

57. Calbet JA, Holst JJ. Gastric emptying, gastric secretion and enterogastrone response after administration of milk proteins or their peptide hydrolysates in humans. Eur J Nutr. 2004;43(3):127-39.

58. Van Loon LJ, Saris WH, Kruijshoop M, Wagenmakers AJ. Maximizing postexercise muscle glycogen synthesis: carbohydrate supplementation and the application of amino acid or protein hydrolysate mixtures. Am J Clin Nutr. 2000;72(1):106-11.

59. Heimburger DC, Geels VJ, Bilbrey J, Redden DT, Keeney C. Effects of small-peptide and whole-protein enteral feedings on serum proteins and diarrhea in critically ill patients: a randomized trial. JPEN J Parenter Enteral Nutr. 1997;21(3):162-7.

60. Reeds PJ, Burrin DG. The gut and amino acid homeostasis. Nutrition. 2000;16(7-8):666-8.

61. Burrin DG, Davis TA. Proteins and amino acids in enteral nutrition. Curr Opin Clin Nutr Metab Care. 2004;7(1):79-87.

62. Engelen MP, Rutten EP, De Castro CL, Wouters EF, Schols AM, Deutz NE. Supplementation of soy protein with branched-chain amino acids alters protein metabolism in healthy elderly and even more in patients with chronic obstructive pulmonary disease. Am J Clin Nutr. 2007;85(2):431-9.

63. Welters CF, Dejong CH, Deutz NE, Heineman E. Intestinal function and metabolism in the early adaptive phase after massive small bowel resection in the rat. J Pediatr Surg. 2001;36(12):1746-51.

64. Bruins MJ, Soeters PB, Deutz NE. Endotoxemia affects organ protein metabolism differently during prolonged feeding in pigs. J Nutr. 2000;130(12):3003-13. 
T
D
D
血
N 


\section{CHAPTER 3}




\title{
Simultaneous measurement of metabolic flux in portally-drained viscera, liver, spleen, kidney and hindquarter in the conscious pig
}

\author{
G. A. M. Ten Have, M. C. F. Bost, J. C. A.W. Suyk-Wierts A. \\ E. J. M. van den Bogaard, N. E. P. Deutz
}

Laboratory Animals (1996) 30, 347-358

\section{Abstract}

A method was developed to measure metabolic fluxes simultaneously across the portally drained viscera (PDV), liver, spleen, kidney and hindquarter (HQ) in the conscious pig (20-25 kg). For this purpose, sampling catheters were implanted in the abdominal artery, portal vein, hepatic vein, splenic vein, renal vein and caval vein. Further, two extra infusion catheters were implanted in the splenic vein and abdominal artery. These allow continuous infusion of para-aminohippuric acid $(\mathrm{PAH})$, providing a method for estimating the plasma flow of the liver, PDV, spleen, kidney and $\mathrm{HQ}$.

To minimize the postoperative recovery period of the pigs, great attention was paid to the housing conditions. After a recovery period of seven days, pigs were used for experiments twice a week. During the three weeks experimental period, food intake, body temperature, weight gain, blood gas data and plasma flow were monitored. Mean plasma flow was: liver $52 \pm 6$, PDV $40 \pm 5, \mathrm{HQ} 20 \pm 2$, spleen $4 \pm 1$ and kidneys $15 \pm 2 \mathrm{ml} / \mathrm{kg}$ body weight/min. These data were characteristic for a pig in a conscious normal resting and unstressed state. The long term patency rate of the sampling catheters was very high (ranging from $75 \%$ to 100\%). This was probably due to the prevention of catheter-related infections using a gentamicin (20 mg/ $\mathrm{ml})$, a-chymotrypsin $(225 \mathrm{U} / \mathrm{ml})$ solution as catheter filling. We conclude that this model enables simultaneously liver, PDV, intestine, spleen, liver, kidney and HQ flux measurement of many metabolic substances in the conscious pig. 


\section{Introduction}

The pig has become an accepted animal model in the research of human disease (1-10). Further, the pig allows a variety of surgical interventions that cannot be contemplated in humans or are not feasible in smaller animals like the rat. Finally, the large size of the animal enables multiple sampling of relatively large volumes enabling many parameters to be analysed in blood simultaneously and over a prolonged period.

Measurement of arterial-venous differences, repeatedly and over time is only possible when catheters are present in the blood vessels supplying and draining an organ. To calculate the production or consumption across an organ, it is necessary to measure the plasma flow. For flow measurement, it is preferable to use a method with minor extra techniques involved and which is capable of measuring plasma flow over a prolonged period. For this purpose, an indicator-dilution technique with para-aminohippuric acid (PAH) may be used which is well-known in animal research (11-16). Also, PAH can be used for the measurement of kidney flow, using PAH extraction by the kidney $(17,18)$. In our model, we used these techniques for plasma flow measurement of the splanchnic area, portally-drained viscera (PDV), spleen and hindquarter $(\mathrm{HQ})$ and the renal flow measurement.

For our research, several organ fluxes have to be measured in the same animal. Although several catheter implantation techniques in large animals have been described $(3,5,10,19-21)$, no reports are available in which all these techniques have been combined in the same animal. Therefore, we have developed a technique to measure plasma flow and arterial-venous differences of several substances simultaneously for portally drained viscera, spleen, liver, kidney and hindquarter $(\mathrm{HQ})$ in the conscious pig.

\section{Materials and Methods}

\section{Animals}

Female crossbred piglets (Dutch land-race x Yorkshire, 20-25 kg, age 8-12 weeks) were obtained from a commercial breeder. At least one week before surgery, the animals were allowed to adapt to individual housing in galvanized bar runs $(2 \times 3$ $\mathrm{m})$, equipped with drinking nipples and isolated floors. Environmental temperature was held at $22-25^{\circ} \mathrm{C}(22)$, humidity level was $55 \%$, light was on for $12 \mathrm{~h}$ per day (from 07:00 to 19:00 h). A radio set was switched on during the light period for them to get accustomed to human voices and noise. Light and music were switched on and off gradually. A ball was provided for the piglets to play with. 
The piglets were fed maintenance pellets for adult sows (Landbouwbelang, Roermond, The Netherlands; 16\% raw protein). Pigs used in laboratories should be fed balanced diets formulated for research-oriented maintenance rather than market production of meat, hence food intake was restricted to a maximum of $1 \mathrm{~kg}$ per day $(2,22)$. Water was available ad libitum.

\section{Operation procedure}

Before operation, the animals were fasted for $16 \mathrm{~h}$. About 45 min after premedication with azoperone $\left(8 \mathrm{mg} / \mathrm{kg}\right.$ bw, i.m., Stresnil ${ }^{\circledR}$, Janssen Pharmaceutica, Tilburg, The Netherlands), anaesthesia was induced with N20/02 (2:1) and halothane (4\%, Trofield Surgicals AG, Zug, Switzerland) mixture by a face mask. Anaesthesia was maintained after oral endotracheal intubation (tube diameter $7 \mathrm{~mm}$ ) with N20/02 and halothane (0.8\%) mixture. Immediately before surgery, lincomycin and spectinomycin $(6.25 \mathrm{mg} / \mathrm{kg}$ bw and $12.5 \mathrm{mg} / \mathrm{kg}$ bw respectively, $2.5 \mathrm{ml}$ dissolved in $100 \mathrm{ml}$ saline $(0.9 \% \mathrm{NaCl} w / \mathrm{v})$; Lincospectin ${ }^{\circledR}$, Upjohn, Ede, The Netherlands) were given as antimicrobial prophylaxis via the ear vein. Also, flunixine-meglumine $(2.5 \mathrm{mg} / \mathrm{kg}$ bw, i.v., Finadyne ${ }^{\circledR}$, Mycofarm Nederland, De Bilt, The Netherlands), a prostaglandinesterase inhibitor, was given as anticoagulant to avoid activation of the haemostatic system and for postoperative analgesia. During surgery, Ringer's lactate (500 to $100 \mathrm{ml}$ i.v., Lactetrol ${ }^{\circledR}$, Aesculaap nv/sa, Boxtel, The Netherlands) was given via the same ear vein.

Through a midline abdominal incision, several $80 \mathrm{~cm}$ long Tygon ${ }^{\circledR}$ catheters (ID 0.040», OD 0.070», Norton Co. Akron, OH 44305, USA) were implanted.

1. Catheters were inserted via the left and right iliac circumflex profunda artery into the abdominal artery with the tip approximately $5 \mathrm{~cm}$ above the bifurcation (A1: Figure 1) and above the level of the right renal artery (A2: Figure 1), respectively.

2. Catheters were inserted via the left and right iliac circumflex profunda vein into the inferior caval vein with the tip approximately $5 \mathrm{~cm}$ above the bifurcation (VI: Figure 1) and above the level of the right renal vein (V2: Figure 1), respectively.

3. A catheter (R: Figure 1) was placed through a purse string suture into the kidney hilus of the left renal vein by puncturing the renal vein with a hollow needle containing the catheter. The catheter tip was placed approximately $1.5 \mathrm{~cm}$ before the entrance of the inferior caval vein. Finally, the catheter was fixated with two ligatures on the left abdominal wall. This procedure was found to ensure stable position of the renal catheter. 


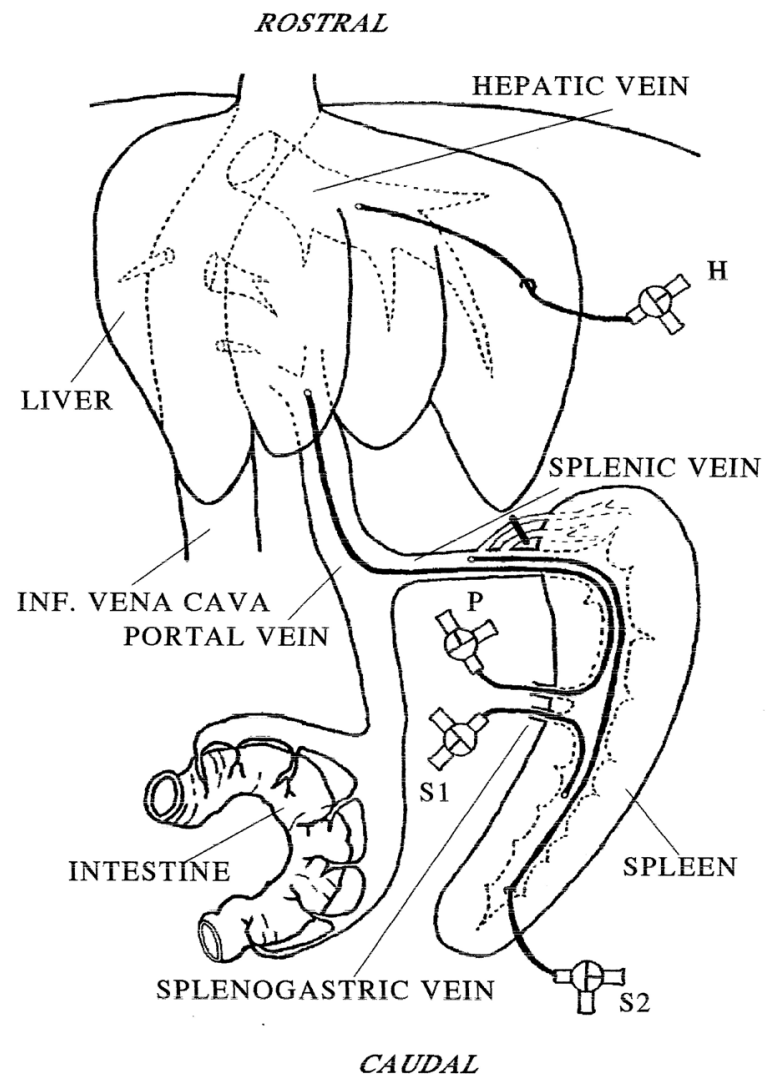

Figure 1. Location of catheters in the hindquarter and kidney area

The long arterial catheter (A2), renal (R) and short caval vein (V1) catheter are used for sampling blood. The short arterial catheter (A1) and long caval vein catheter (V2) are infusing catheters.

4. Two catheters were placed via the two branches of the splenogastric vein: one with its tip about $5 \mathrm{~cm}$ into the distal splenic part of the splenic vein (S1: Figure 2), and the portal vein catheter via the splenic vein with its tip positioned in the free liver hilum (P: Figure 2). Although the Tygon catheter tends to enter the cranial mesenteric vein rather than the portal vein, by manipulating the catheter in the portal vein gently, the tip position is easily located in the liver hilum.

5. A splenic vein catheter was placed through a purse string suture in the vein by puncturing the splenic vein with a hollow needle containing the catheter. Although the diameter of the splenic vein at this level is small, it is relatively easy to do. The catheter tip was positioned in the proximal part of the splenic vein approximately $7 \mathrm{~cm}$ away from the spleen (S2: Figure 2). Several 
small branches of the splenic veins from the proximal part of the spleen were ligated to direct all venous blood via the large splenic vein. Normal arterial blood flow remained unaltered.

6. In the pig many small and large hepatic veins drain into the inferior cava vein. A large hepatic vein in the pig is the left common hepatic vein, draining the left lateral and central hepatic lobes. This vein can be felt as a groove in the left liver lobe. To puncture this vein, the liver was penetrated into this groove with a hollow needle containing the catheter as described by Imamura and Clowes (1975) (3). After successful puncturing, the needle was then withdrawn, leaving the catheter in place. Its tip was positioned $2 \mathrm{~cm}$ from the entrance of the hepatic vein in the inferior cava vein. The catheter entry was fixated with two ligatures in the liver lobe $(\mathrm{H}$ : Figure 2$)$.

\section{ROSTRAL}

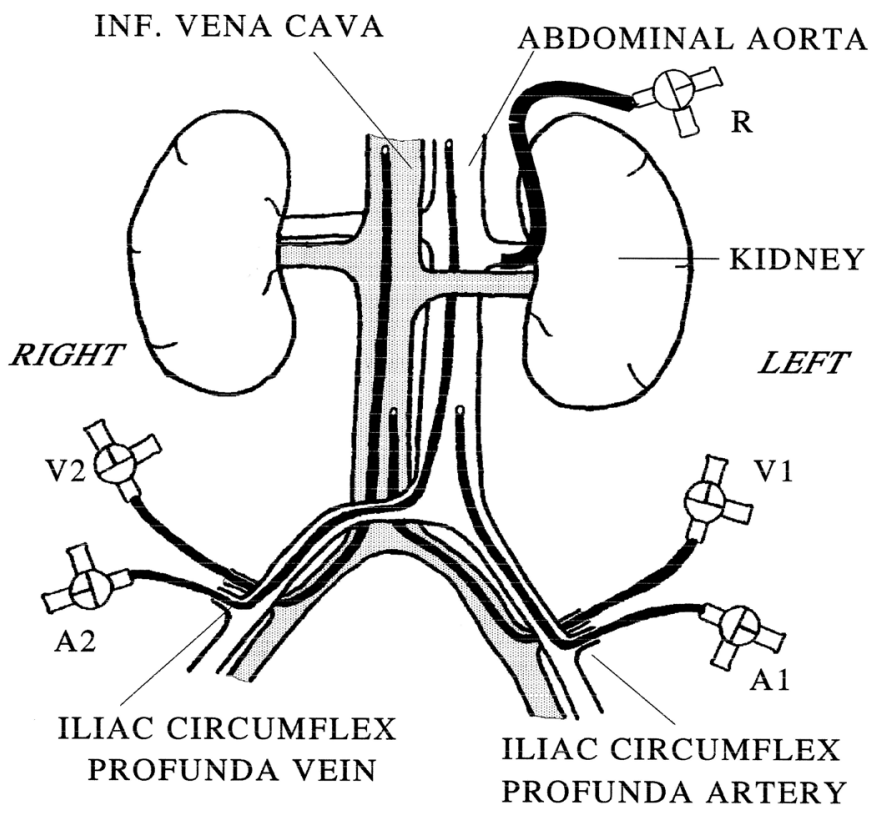


All the catheters were filled with heparinized saline solution (50 U/ml Heparin Leo ${ }^{\circledR}$ Pharmaceutical Products BV, Weesp, The Netherlands, 0.9\% NaCl w/v). The position of the catheter tips was checked during operation using fluoroscopy and iodinecontaining contrast solution (Urografin ${ }^{\circledR} 76 \%$, Shering AG, Germany). Finally, all catheters were secured in place by sutures and alpha-cyanoacrylate glue (Cyanolit ${ }^{\circledR}$ 201, Rubberhuis, Maastricht, The Netherlands). Catheters were tunnelled through the left abdominal wall and subcutaneously. The exteriorized catheters were connected to two-way stopcocks (Baxter Healthcare Corporation, Uden, Holland). The abdominal incision was closed in three layers with a continuous stitch using Mersilene ${ }^{\circledR}$, catgut and finally Vicry ${ }^{\circledR}$ (Ethicon, Norderstedt, Germany). The skin wound is decontaminated with Aureomycin ${ }^{\circledR}$ spray (chlorotetracycline $2 \%$, gentian violet $0.5 \%$, Cyanamid animal health, Etten-Leur, The Netherlands). Total operation time was approximately six hours. At the end of the operation, the catheters were flushed with saline solution to remove the heparin and filled with a solution containing a mixture of gentamicin (20 mg/ ml, Gentamicine $5 \%{ }^{\circledR}$, Cuyk, The Netherlands) and a-chymotrypsin (225 U/ml, Merck, Darmstadt, Germany) (23). The pigs were fitted with a canvas harness to protect the catheters and to allow easy handling of the animals. After the operation, $2.5 \mathrm{ml}$ Lincospectin ${ }^{\circledR}$ was given i.m. and the animal was given $100 \mathrm{~g}$ of feed.

\section{Postoperative care}

On the first two days after the operation the animals were given $100-200 \mathrm{~g}$ feed twice daily for reactivation of intestinal activity. This was usually accepted. Also, 2.5 $\mathrm{ml}$ Lincospectin ${ }^{\circledR}$ dissolved in $20 \mathrm{ml}$ saline was given i.v. twice a day during the first two days. In the first week the animals were housed individually in a galvanized pen with straw bedding. Daily, the catheters were flushed with saline solution, the patency checked and again filled with the gentamicin-chymotrypsin solution. In order to minimize any risk of systemic toxicity of gentamicin, meticulous attention was paid to filling the catheter lumen only. Before flushing or one day before an experiment the contents were withdrawn. After the first week, the catheters were flushed twice a week and the animals were housed again in the galvanized bar runs.

\section{Experiments}

Experiments were started after seven days recovery. In this period, food and water intake had returned to preoperative levels. The animals were trained to become accustomed to a small movable cage $(0.9 \times 0.5 \times 0.3 \mathrm{~m})$. By this, the experiments could be done in unrestrained, conditioned, conscious animals, standing or lying in the small movable cage. During the experiment, the catheters were filled with a heparinized $(50 \mathrm{U} / \mathrm{mL})$ saline solution. After the experiment the catheters were filled again with the gentamicin-chymotrypsin solution. 
Blood flows were determined by infusion of a measurable non-toxic indicator, paraaminohippuric acid (PAH, 25 mM, Sigma Chemicals Co, St Louis, USA). The PAH solution was made iso-osmolar (300 mOsm) by addition of $\mathrm{NaCl}$ and adjusted to $\mathrm{pH}$ 7.4 by titration of $\mathrm{NaOH}(1 \mathrm{~N})$. The solution was sterilized before the experiment by filtration through a 0.22 pin filter (Millipore, Molsheim, France). The evening before an experiment, food was withheld. On the morning of an experiment (08:00h), a bolus of $5 \mathrm{ml}$ PAH solution was given. This was followed by continuous infusion of $\mathrm{PAH}$ at a rate of $30 \mathrm{~mL} / \mathrm{h} /$ catheter via the catheters in the short splenic vein (S1) and in the abdominal aorta (A1) during the experimental period. After one hour, steady state PAH concentrations were obtained (not shown).

Hereafter, blood samples (approximately $2 \mathrm{ml}$ ) were taken from the arterial catheter $(A 2)$, portal $(P)$ and hepatic $(H)$, splenic $(S 2)$, renal $(R)$ catheter and from the catheter in the caval vein (V1) for the determinations of amino acids, ammonia, urea, glucose, lactate, PAH and blood gas analysis.

Experiments were done twice a week for a period of 21 days. After this period the animals were anaesthetized with $10-20 \mathrm{mg} / \mathrm{kg}$ sodium thiopental (Nesdonal ${ }^{\circledR}$, Rhone Merieux, Lyon, France). The position of all catheter tips was checked using fluoroscopy and iodine containing contrast solution. Finally, the animals were euthanized with $200 \mathrm{mg} / \mathrm{kg}$ pentobarbital (Euthanasate ${ }^{\circledR}$, Apharmo, Arnhem, The Netherlands) i.v.

\section{Analytical procedures}

During all procedures, accomplished promptly after collection of blood, the samples were heparinized and kept on ice. Hematocrit was measured by centrifugation in a micro centrifuge. All centrifugations were done at $4^{\circ} \mathrm{C}$ for $5 \mathrm{~min}$ at $8500 \mathrm{~g}$ in an Eppendorf 5413 centrifuge. Blood gas analysis was done immediately after sampling in a blood gas analyzer (Acid Base Laboratory (ABL3), Radiometer, Copenhagen, Denmark), after correction for temperature.

For PAH determinations, $300 \mu$ l of whole blood was added to $600 \mu \mathrm{l}$ of $0.73 \mathrm{M}$ trichloroacetic acid (TCA) solution, thoroughly mixed for deproteinization and centrifuged after which the supernatant was collected. To obtain plasma, blood was centrifuged. $500 \mu$ l plasma was collected and deproteinized with $50 \mu \mathrm{l}$ of $2.5 \mathrm{M} \mathrm{TCA}$ solution, thoroughly mixed for ammonia, urea, lactate and glucose determination. For amino acid analysis, $100 \mu \mathrm{l}$ of plasma was deproteinized with $4 \mathrm{mg}$ sulfosalicylic acid. After these preparations samples were frozen in liquid nitrogen and stored at $-80^{\circ} \mathrm{C}$ until further analysis.

Plasma ammonia, urea, glucose and lactate were determined spectrophotometrically by standard enzymatic methods on an analyzer system (Cobas Mira, Hoffmann-La 
Roche, Basel, Switzerland) using commercial kits (Ureum kit UV-800 of Bio Merieux, Mary L'Etoile, France; Ammonia Reagent kit of Raichem, San Diego, CA 92111, USA; glucose HK Unimate 5 of Hoffman-La Roche, Basel, Switzerland). Urea values were corrected for ammonia Plasma lactate was determined by a standard enzymatic method according to Pesce et al. (1975), Westgard et al. (1972) $(24,25)$. For PAH determination the method according to Brun (1951) was adapted for small volumes (26). Because the liver and kidney acetylate PAH to a certain content, PAH supernatant was deacetylated by heating for $45 \mathrm{~min}$ at $100^{\circ} \mathrm{C}$. By this, the determined PAH is total PAH, and the flow measurement is independent of the acetylated grade. Hereafter, the PAH supernatant was measured at $465 \mathrm{~nm}$ on a Cobas Mira, using Ehrlich's reagent (1 g p-dimethylaminobenzaldehyde (Merck, Darmstadt, Germany), $35 \mathrm{ml}$ alcohol 96\%, $4 \mathrm{ml}$ TCA 2N, $61 \mathrm{ml}$ aqua bidest) and a solution of 35\% alcohol. After precolumn derivatization with o-phthaldialdehyde, plasma amino acids were determined with a fully automated HPLC system $(27,28)$.

\section{Calculations and statistics}

All calculations were performed using plasma flows and plasma concentrations. For plasma flow determination we used PAH in two ways. First (Figure 3, top), by calculating the dilution of $\mathrm{PAH}$, plasma flow is measured. In this situation, the amount of $\mathrm{PAH}$ entering the vessel by the infusion and by blood equals the amount leaving the vessel:

Equation(1) IN = OUT

Equation (2) IN $=($ Infusion rate $x[\mathrm{PAH}]$ infused $)+($ Flow $\times[\mathrm{PAH}]$ pre $)$

Equation (3) OUT $=$ Flow $\times[P A H]$ post

Using the formulae (1), (2) and (3), calculation of plasma flows is easily made.

Equation (4) Flow $=($ Infusion rate $x[\mathrm{PAH}]$ infused $) /(\mathrm{PAH}$ post $-[\mathrm{PAH}]$ pre $)$

$[\mathrm{PAH}]$ infused is the concentration of $\mathrm{PAH}$ in the infused solution, $[\mathrm{PAH}]$ post is the plasma concentration of $\mathrm{PAH}$ after the organ and $[\mathrm{PAH}]$ pre before.

This model is used in the measurement of splenic, PDV and liver plasma flow (11, 13, 29). The catheter in the splenic vein (S1: Figure 2) was used for PAH infusion and by measuring the PAH concentration in the S2 catheter (first dilution), portal vein (second dilution) and liver vein (third dilution), plasma flow can be calculated. Also, measurement of $\mathrm{HQ}$ was by dilution of $\mathrm{PAH}$ infused in the abdominal aorta (A1: Figure 1) and measurement of the PAH dilution in the caval vein (V1). 

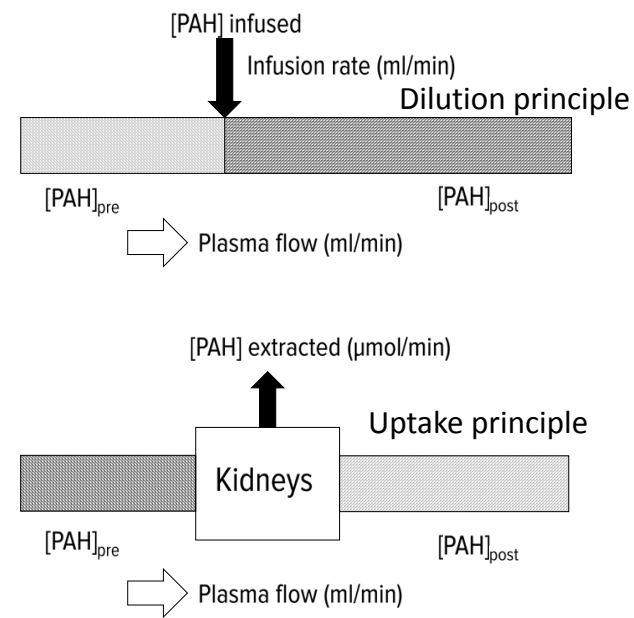

Figure 3. Mechanistic model of infused PAH transport

$[\mathrm{PAH}]$ is the para-aminohippuric acid concentration in $\mu \mathrm{M}$. Top: dilution principle used for the calculation of splenic, portal drained viscera, liver and $\mathrm{HQ}$ plasma flows. Bottom: uptake principle used for the calculation of kidney plasma flow.

Second (Figure 3, bottom), by measuring the extraction of PAH by the kidney, kidney flow can be calculated. In this situation, PAH is extracted by the kidney. The amount extracted equals the amount infused as the system is in steady state:

Equation (5) $\quad \mathrm{PAH}$ extracted $=$ Infusion rate $\mathrm{x}[\mathrm{PAH}]$ infused

Further, the amount extracted plus the amount leaving the kidney by the renal vein equals the amount entering the kidney:

$$
\begin{array}{ll}
\text { Equation (6) } & \text { IN }=\text { OUT } \\
\text { Equation (7) } & \text { IN = Flow } \times[\mathrm{PAH}] \text { pre } \\
\text { Equation (8) } & \text { OUT = PAH extracted + (Flow } \times[\mathrm{PAH}] \text { post) }
\end{array}
$$

Using the formulae (5), (6), (7) and (8), the formula for calculating the kidney flow is easily made.

Equation (9) Flow $=($ Infusion rate $\times[\mathrm{PAH}]$ infused $) /[\mathrm{PAH}]$ arterial $-[\mathrm{PAH}]$ renal

Plasma flow is expressed as $\mathrm{ml} / \mathrm{kg} \mathrm{bw} / \mathrm{min}$. 
Portally drained viscera (PDV) flux was calculated by multiplication of the portal-arterial difference with the portal plasma flow. The calculation for the liver flux is more complex, because of the portal and arterial routes of blood delivery. However, the total flux across the splanchnic region (liver+PDV) is easy to calculate as hepatic-arterial difference times splanchnic plasma flow (splanchnic plasma flow=liver plasma flow). Therefore, liver flux was calculated by subtracting PDV flux from the splanchnic flux. Splenic flux was calculated by multiplication of the splenic plasma flow and the splenic-arterial difference. Intestinal flux was calculated by subtracting splenic flux from the PDV flux. HQ plasma differences mainly represent plasma differences across muscle tissue. HQ flux was calculated by multiplication of the HQ plasma flow with the caval vein-arterial difference. Renal flux for two kidneys was calculated by multiplication of the renal plasma flow with the renal-arterial difference.

The Wilcoxon test was used to test significance from zero $(P<0.05)$. For clarity reasons results are presented as means \pm SEM.

\section{Results}

Seventeen animals were operated: two animals died in the recovery phase of a splenic bleeding and an intestinal obstruction respectively. Fifteen animals were used for the experiments. The catheters A1, A2, V1, V2, S1, P and H were implanted in all animals, and in at least four animals catheters $\mathrm{S} 2$ and $\mathrm{R}$ were also implanted. Control data of two experiments per animal were used for the results of this article. Experiments were done randomly during the experimental period of 21 days. The number of observations are variable because samples were not taken every time from all catheters.

The first two days after the operation food was restricted to $200 \mathrm{~g}$ twice daily. After the third day the food intake was increased gradually until day eight, when the food intake was normalized (more than $900 \mathrm{~g}$ and a maximum of $1 \mathrm{~kg}$ a day). There was no weight gain, but also no weight loss during the recovery phase in the first week $(0.08 \pm 0.17 \mathrm{~kg} /$ week). After recovery, weight gain was $2.15 \pm 0.21 \mathrm{~kg} /$ week with the restricted food intake of $1 \mathrm{~kg}$. Body temperature during the experimental period was $38.2 \pm 0.1^{\circ} \mathrm{C}$. No signs of infection were observed like catheter-related infections, wound infections, intestinal infections as diarrhea or unexplained high body temperature. Characteristic patterns in $\mathrm{pCO}_{2}, \mathrm{pO}_{2}$ and $\mathrm{O}_{2}$ saturation of arterial, portal vein, hepatic vein, inferior caval vein, splenic vein and renal vein blood were observed (Table 1). For example, in the splanchnic region, percentage oxygen extraction (37\%) is relatively high compared to other organs or regions. On the other hand, oxygen consumption of the spleen is very low (9\%). 
Table 1: Blood gas data of blood in the artery, portal vein, hepatic vein, inferior caval vein, splenic vein, and in the renal vein

\begin{tabular}{|c|c|c|c|c|c|c|}
\hline & Artery & Portal vein & $\begin{array}{l}\text { Hepatic } \\
\text { vein }\end{array}$ & $\begin{array}{l}\text { Inf. caval } \\
\text { vein }\end{array}$ & $\begin{array}{l}\text { Splenic } \\
\text { vein }\end{array}$ & Renal vein \\
\hline $\mathrm{Hct}$ & 29.3(0.7) & 29.6(0.6) & 30.1(0.6) & 29.1(0.7) & 29.1(1.1) & $28.3(0.8)$ \\
\hline $\mathrm{pH}$ & 7.46(0.01) & 7.44(0.03) & 7.41(0.01) & 7.42(0.01) & 7.44(0.01) & $7.42(0.01)$ \\
\hline pCO2 & $5.42(0.11)$ & $6.07(0.38)$ & 6.51(0.15) & $6.13(0.15)$ & $5.68(0.17)$ & $6.23(0.08)$ \\
\hline pO2 & $12.33(0.3)$ & $5.49(0.34)$ & $4.47(0.24)$ & 5.38(0.18) & $7.65(0.35)$ & 7.36(0.17) \\
\hline $\mathrm{HCO} 3$ & 28.6(0.6) & $30(0.6)$ & $30.3(0.7)$ & $29(0.8)$ & $28.5(0.9)$ & $29.2(0.5)$ \\
\hline SAT & $97(0.1)$ & $74(2)$ & $60(3)$ & $71(2)$ & $88(2)$ & $87(3)$ \\
\hline
\end{tabular}

$\mathrm{HCO}_{3}$ in $\mathrm{mmol} / \mathrm{l}, \mathrm{pO}_{2}$ and $\mathrm{pCO}_{2}$ in $\mathrm{kPa}$ and $\mathrm{O}_{2}$ saturation (Sat) in \%

The percentage of patent sampling catheters 28 days after the implantation is plotted in Figure 4. A patency rate less than $100 \%$ was only caused by a dislocation within the vessel of the catheter tip as a result of the increased size of the animals.

Plasma flows after the recovery time and under post-absorptive conditions are calculated and plotted in Table 2. Total plasma flow of the splanchnic area is $52.5 \pm 5.9$ $\mathrm{ml} / \mathrm{kg} \mathrm{bw} / \mathrm{min}$. By subtracting the portal plasma flow from the liver plasma flow, we found a hepatic artery plasma flow of $13.0 \mathrm{ml} / \mathrm{kg}$ bw/min. Variance was comparable for the liver, portal, $\mathrm{HQ}$ and renal plasma flow, but higher for the splenic plasma flow.

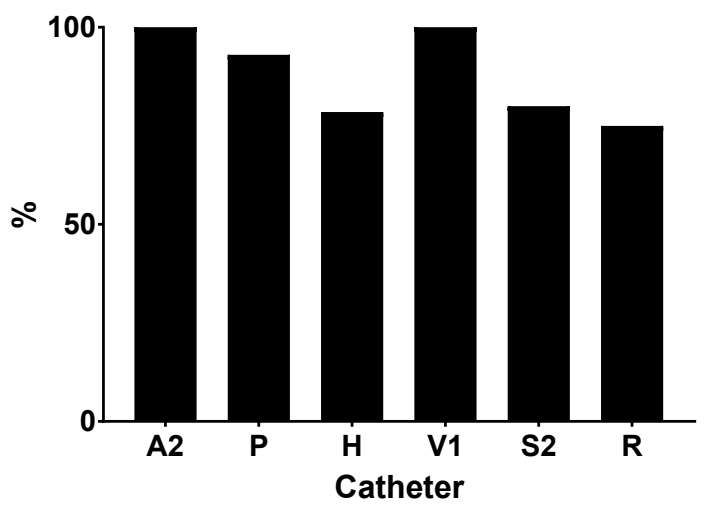

Figure 4. Patency rates

Patency rates 28 days after operation (in \%) of the long arterial ( $A 2, n=15)$, portal ( $P, n=14)$, hepatic $(H, n=14)$, short caval vein $(V 1, n=15)$, long splenic $(S 2, n=5)$ and renal $(R, n=4)$ sampling catheters. 
Table 2. Post absorptive plasma flows

\begin{tabular}{lll} 
& $\begin{array}{l}\text { Plasma flow } \\
(\mathrm{ml} / \mathrm{kg} \text { bw/min) }\end{array}$ & $\mathrm{N}$ \\
\hline Portally-drained viscera & $39.5(5.0)$ & 18 \\
Liver & $52.5(5.9)$ & 25 \\
Hindquarter & $19.7(1.8)$ & 16 \\
Spleen & $4.4(1.3)$ & 6 \\
Kidneys & $15.2(1.6)$ & 8 \\
\hline
\end{tabular}

Values are mean(SEM)

Three examples of plasma concentration differences across organs are given in Figure 5. In addition, the fluxes are given in Figure 6. For ammonia, the PDV produces ammonia (Figures 5A and 6A) while the liver takes it up. Calculation of the splanchnic ammonia flux shows that ammonia does not escape from the splanchnic region (Figures 5A and 6A). Also, renal production was observed. However, neither production nor consumption was observed in the HQ. Glutamine (Figures 5B and $6 \mathrm{~B})$ is a non-essential amino acid, consumed by the intestine and produced by the spleen and $\mathrm{HQ}$. Branched-chain amino acids (BCAA, Figures 5C and 6C) are calculated as the sum of the essential amino acids valine, leucine and isoleucine. These are neither produced nor consumed by the intestine, liver and spleen during postabsorptive conditions. However, there is a consumption of BCAA by the HQ and the spleen and production of BCAA by the kidneys.

\section{Discussion}

To study combined portally-drained viscera, liver, spleen, kidney and hindquarter flux measurement in the conscious pig, it is important that the recovery period be as short as possible to minimize the influence of the catheter implantation operation on subsequent experiments. Thus, a meticulous surgical technique and careful postoperative care is mandatory. First, the housing conditions were kept stable. Secondly, straw bedding was used in the bar runs, so that the pig can adapt her own body temperature changes to her environment during the recovery period. Straw bedding also stimulates the normal rooting behavior. Further, gradual reactivating of the intestinal functions by feeding small portions postoperatively during the first two days prevents intestinal complications like ileus or stasis. The operation and the reduced food intake in the first seven postoperative days were probably the reasons for the observed unchanged body weight. After the first week, when food intake was normalized ( $1 \mathrm{~kg} /$ day), a weight gain of about $2 \mathrm{~kg} /$ week was obtained. This is the normal weight gain, which might be expected in pigs with food restriction to about $5 \%$ of the body weight for growing pigs with a body weight of $20 \mathrm{~kg}(22)$. 
Figure 5

A

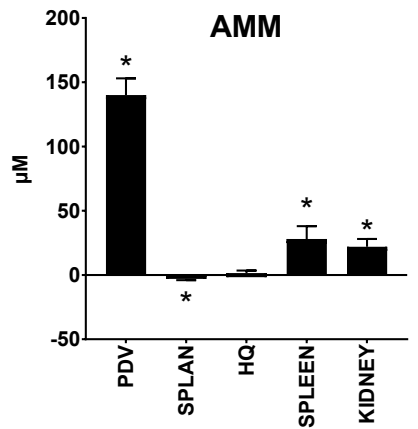

B

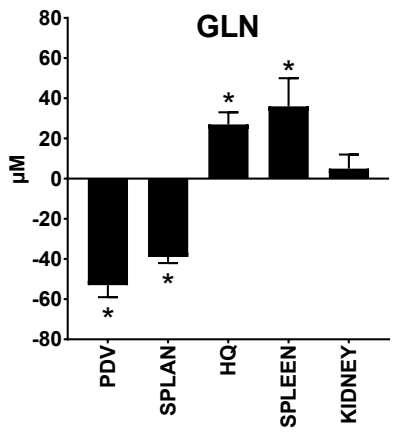

C

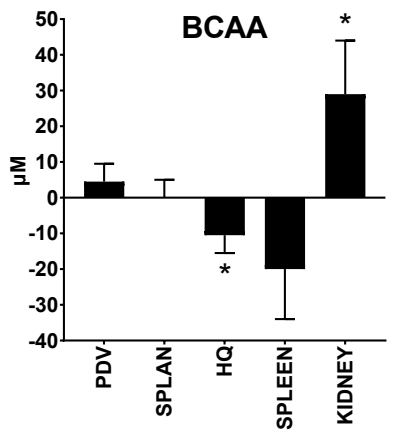

Figure 6

A

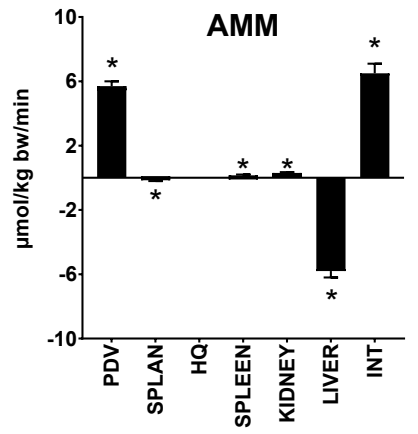

B

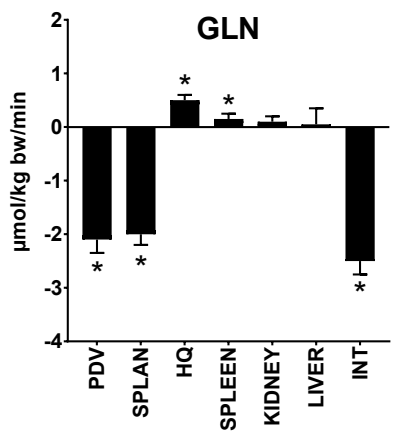

C

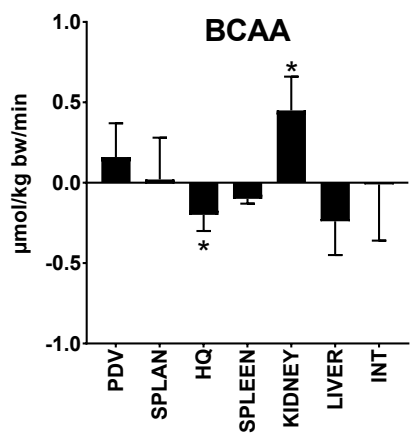

Figure 5. Plasma concentration differences across organs

Post-absorptive ammonia (Fig 5A), glutamine (Fig 5B) and branched chain amino acids (BCAA, Fig 5C) plasma concentrations differences across the portally drained viscera (PDV, $n=25$ ), splanchnic ( $S P L, n=26)$, hindquarter $(H Q, n=17)$, spleen $(n=8)$, kidneys $(n=6)$. In $\mu M$ values are mean $\pm \mathrm{SEM}$; *) is significant compared to zero ( $p<0.05$, Wilcoxon).

Figure 6. Metabolic fluxes of different organs

Post-absorptive ammonia (Fig 6A), glutamine (Fig 6B) and branched chain amino acids (BCAA, Fig $6 C$ ) fluxes of the portally drained viscera (PDV, $n=25)$, splanchnic region (SPL, $n=26$ ), hindquarter $(H Q, n=17)$, spleen ( $n=8)$, kidneys (kidney*2, $n=6)$, liver $(n=23)$, intestine (INT, $n=8)$.Values are in $\mu \mathrm{mol} / \mathrm{kg}$ body weight $/ \mathrm{min}$ and mean $\pm \mathrm{SEM}$; $\left.{ }^{*}\right)$ is significant compared to zero $(\mathrm{P}<0.05$, Wilcoxon). 
Mean arterial pressure (Table 1) is comparable with literature values (2, 30-32). Also, the oxygen saturation values in the portal, hepatic and caval vein are comparable with literature values $(30,33)$.

We observed very high patency rates (Figure 4), compared to previously reported results $(10,20,21)$. There are several reasons for this high patency rate. First, it is important to use a catheter with a very smooth and inert inner and outer wall, such as Tygon ${ }^{\circledR}$. Secondly, at the beginning of the operation, flunixine, a prostaglandinesterase inhibitor, is given to lower platelet (thrombocyte) adhesion to minimize activation of the haemostatic system. Thirdly, regular catheter flushing, using strictly aseptic procedures, especially during the first week, might have prevented catheter-related infections and subsequent clotting at the catheter tips. In our opinion, a very important factor influencing catheter patency is the use of the gentamicin chymotrypsin solution as catheter-filling. This solution was found to be very successful in dogs (23), but experience was lacking in pigs. Before 1992, our average patency rates at day 28 after the operation were about 60\% (not published), with the use of a saline solution with $50 \mathrm{U} / \mathrm{ml}$ lithium heparin as catheter filling. Also, the patency values in the literature for implanted catheters in the pig are relatively low (10, 20, 21). However, after the introduction of the gentamicin-chymotrypsin solution, average patency rates increased by $27 \%$ to nearly $100 \%$. Catheter tip infections are probably the most important cause of occlusion.

Although transport of metabolic substances across the erythrocyte was shown to be slow (29), several other factors could influence this transport in vivo (e.g. duration of capillary contact, flow velocity). These make a valid estimation of the contribution of metabolic substance exchange via the erythrocyte impossible. Therefore, we assumed plasma exchange only to be an indicator of whole-blood exchange and all calculations were performed using plasma flow and plasma concentrations (13).

Plasma flow measurement is needed for determining the flux of different substances across an organ or body region. For measuring plasma flow, it is important that the experimental conditions are as constant as possible. It is known that the plasma flow in an organ or body region is dependent on the cardiac output (CO). For example, $\mathrm{CO}$ is related to the body posture: in humans, a standing position increases the CO by $20 \%$ and exercise increases CO by $200 \%$ (34). Thus, conditions and procedures during the experiment have to be stable and the conscious pig must not be stressed. Therefore, the pigs were studied while they were resting. In fact, the trained animals were normally sleeping while we were taking blood samples. Another factor for a reliable plasma flow measurement is the use of a technique which does not interfere with the physiological functioning of the vessels. Therefore, the position of the catheter insertion site has to be a safe distance from 
the catheter tip position, to minimize interference with the normal contraction and relaxation pattern of the vessel.

The blood flow measurement in the splanchnic area and HQ was based on the general principle applied in physiology by Adolph Fick more than a century ago. The principle is, in effect, a restatement of the law of conservation of mass for an indicator. The indicator may be an endogenous substance like oxygen or an exogenous one introduced by the investigator. The Fick dilution method is one of the oldest yet most accurate ways of measuring cardiac output (34). The Fick dilution principle is also the basis of many procedures for determining regional blood flow using an exogenous indicator like $\mathrm{PAH}$ or indocyanine green.

Various lines of evidence suggest that the error in the indicator-dilution methods is probably about 5\% (34). However, this may be overestimated, because its reproducibility in successive determinations can rarely be made better than approximately $7 \%$, even with the most careful attention to technical details $(3,15,22,30,34,35)$. We observed an average variation of plasma flow across several organs (excluding splenic flow) of about $11 \%$, and is therefore acceptable to us.

It is very difficult to compare absolute plasma flow values with literature values, because the procedures and conditions of the experiments are very variable or un-known. For this purpose CO-distribution can be used. For comparing our plasma flows with the literature we have calculated the splanchnic, $\mathrm{HQ}$ and renal plasma flow, in percentages of normal resting plasma $\mathrm{CO}$ in piglets of approximately $20 \mathrm{~kg}$ (105 ml/ min/kg) (36). Splanchnic, HQ and renal percentages of plasma CO (50.0 \pm $5.6 \%, 18.8 \pm 1.7 \%$ and $14.5 \pm 1.5 \%$, respectively) are characteristic for pigs in a resting condition $(3,36)$ and comparable with humans in a resting condition (34). Also the ratio of portal/splanchnic flow is comparable with the literature (3).

Figures 5 and 6 are examples of measurement of metabolic substances in our piglet model. It illustrates a few of the many possibilities of this model. For example, the low oxygen consumption (Table 1) and a small glutamine production in the spleen suggests a non-activated immune system, because an activated immune system, which is a normal physiological response during the first three postoperative days, causes an enhanced utilization of glucose and glutamine (13). The advantage of this model is the possibility of studying metabolic substances in different areas of the body in a conscious animal.

In conclusion, multiple catheterization of pigs enables simultaneous measurement of liver, PDV, spleen, kidney and HQ flux in a conscious, unstressed animal over a prolonged time. The refinement of this model by optimizing housing conditions, the 
use of a postoperative feeding schedule and meticulous attention to catheter care reduces the number of animals required for a certain experiment.

\section{Acknowledgements}

The authors wish to express many thanks to $\mathrm{Mr} \mathrm{H}$. M. H. van Eijk, BSc and Mr D. R. Rooyakkers, BSc for their accurate amino acid determinations and C. H. C. Dejong, $\mathrm{MD}, \mathrm{PhD}$ for helping with the development of the surgical technique for the kidney catheter.

\section{References}

1. 1987;7:361-82.

2. Tumbleson ME. Swine in biomedical research. New York: Plenum Press; 1986

3. Imamura M, Clowes $\mathrm{GH}$, Jr. Hepatic blood flow and oxygen consumption in starvation, sepsis and septic shock. Surg Gynecol Obstet. 1975;141(1):27-34.

4. Dodds WJ. The pig model for biomedical research. Federation Proceedings. 1982;41:247-56.

5. Moritz MW, Dawe EJ, Holliday JF, Elliott S, Mattei JA, Thomas AL. Chronic central vein catheterization for intraoperative and long-term venous access in swine. Lab Anim Sci. 1989;39(2):153-5.

6. $\quad$ Dantzer R. The pig as a model for behavioral research. Lab Anim Sci. 1986;36(4):362-5.

7. Dantzer R. Behavioral, physiological and functional aspects of stereotyped behavior: a review and a re-interpretation. J Anim Sci. 1986;62(6):1776-86.

8. Glauser EM. Advantages of piglets as experimental animals in pediatric research. Exp Med Surg. 1966;24(2):181-90.

9. Book SA, Bustad LK. The fetal and neonatal pig in biomedical research. J Anim Sci. 1974;38(5):997-1002.

10. Mustard RA, Lipohar C, Finley RJ, Duff JH, Radziuk J, Nohr C. A simplified method for metabolic studies in conscious swine. Lab Anim Sci. 1986;36(4):393-5.

11. Katz ML, Bergman EN. Simultaneous measurements of hepatic and portal venous blood flow in the sheep and dog. Am J Physiol. 1969;216(4):946-52.

12. Souba WW, Wilmore DW. Postoperative alteration of arteriovenous exchange of amino acids across the gastrointestinal tract. Surgery. 1983;94(2):342-50.

13. Deutz NE, Reijven PL, Athanasas G, Soeters PB. Post-operative changes in hepatic, intestinal, splenic and muscle fluxes of amino acids and ammonia in pigs. Clin Sci (Lond). 1992;83(5):607-14.

14. Dejong $\mathrm{CH}$, Kampman MT, Deutz NE, Soeters PB. Altered glutamine metabolism in rat portal drained viscera and hindquarter during hyperammonemia. Gastroenterology. 1992;102(3):936-48.

15. Yen JT, Nienaber JA, Hill DA, Pond WG. Oxygen consumption by portal veindrained organs and by whole animal in conscious growing swine. Proc Soc Exp Biol Med. 1989;190(4):393-8.

16. Deutz NE, Reijven PL, Bost MC, van Berlo CL, Soeters PB. Modification of the effects of blood on amino acid metabolism by intravenous isoleucine. Gastroenterology. 1991;101(6):1613-20. 

without urine collection. A critical evaluation of the constant-infusion technic for determination of inulin and para-aminohippurate. N Engl J Med. 1972;287(22):1109-14.

18. Rose BD. Clinical Physiology of acid-base and electrolyte disorders. Tokyo: McGraw-Hill Kogakusha Ltd; 1977.

19. Rerat A, Chayvialle JA, Kande J, Vaissade P, Vaugelade P, Bourrier T. Metabolic and hormonal effects of test meals with various protein contents in pigs. Can J Physiol Pharmacol. 1985;63(12):1547-59.

20. van Berlo CL, van den Bogaard AE, Bost MC, Soeters PB. A technique to study splanchnic metabolism in the unrestrained conscious pig. Lab Anim Sci. 1988;38(4):463-6.

21. Yen JT, Killefer J. A method for chronically quantifying net absorption of nutrients and gut metabolites into hepatic portal vein in conscious swine. J Anim Sci. 1987;64(3):923-34.

22. Mount LE, Ingram DL. The pig as a laboratory animal. London: Academic Press; 1971.

23. Palm U, Boemke W, Bayerl D, Schnoy N, Juhr NC, Reinhardt HW. Prevention of catheter-related infections by a new, catheter-restricted antibiotic filling technique. Lab Anim. 1991;25(2):142-52.

24. Pesce MA, Bodourian SH, Nicholson JF. Rapid kinetic measurement of lactate in plasma with a centrifugal analyzer. Clin Chem. 1975;21(13):1932-4.

25. Westgard JO, Lahmeyer BL, Birnbaum ML. Use of the Du Pont "automatic clinical analyzer" in direct determination of lactic acid in plasma stabilized with sodium fluoride. Clin Chem. 1972;18(11):1334-8.

26. Brun C. A rapid method for the determination of para-aminohippuric acid in kidney function tests. J Lab Clin Med. 1951;37(6):955-8.

27. van Eijk HM, Rooyakkers DR, Deutz NE. Rapid routine determination of amino acids in plasma by high-performance liquid chromatography with a 2-3 microns Spherisorb ODS ॥ column. J Chromatogr. 1993;620(1):143-8.

28. van Eijk HMH, MAH vdH, van Berlo CLH, Soeters PB. Fully automated liquid-chromatographic determination of amino acids. ClinChem. 1988;34:2510-3.

29. Schaefer A, Piquard F, Haberey P. The effects of changes in plasma amino acid concentrations on erythrocyte amino acid content. Clin Biochem. 1990;23(3):237-40.

30. Arvidsson D, Rasmussen I, Almqvist P, Niklasson F, Haglund U. Splanchnic oxygen consumption in septic and hemorrhagic shock. Surgery. 1991;109(2):190-7.

31. Hacker TA, Hall JL, Stone CK, Stanley WC. Alanine, glutamate, and ammonia exchanges in acutely ischemic swine myocardium. Basic Res Cardiol. 1992;87(2):184-92.

32. Salzman AL, Wang H, Wollert PS, Vandermeer TJ, Compton CC, Denenberg AG, et al. Endotoxin-induced ileal mucosal hyperpermeability in pigs: role of tissue acidosis. Am J Physiol. 1994;266(4 Pt 1):G633-46.

33. Fink MP, Cohn SM, Lee PC, Rothschild HR, Deniz YF, Wang H, et al. Effect of lipopolysaccharide on intestinal intramucosal hydrogen ion concentration in pigs: evidence of gut ischemia in a normodynamic model of septic shock. Crit Care Med. 1989;17(7):641-6.

34. Milnor WR. Cardiovascular physiology. Oxford: Oxford University Press; 1990.

35. Munoz S, Walser M. Effect of experimental liver disease on the utilization for protein synthesis of orally administered alpha-ketoisocaproate. Hepatology. 1986;6(3):472-6.

36. Hannon JP, Bossone CA, Wade CE. Normal physiological values for conscious pigs used in biomedical research. In: research DoMt, editor. San Francisco, CA, USA: Letterman Army Institute of Reserach; 1989. p. 1-20. 


\section{CHAPTER 4}




\title{
Absence of post-prandial gut anabolism after intake of a low quality protein meal
}

\author{
Gabriella A.M. Ten Have, Mariëlle P.K.J. Engelen, Peter B. \\ Soeters, Nicolaas E.P. Deutz
}

Clinical Nutrition. 2012 Apr;31(2):273-82

\section{Abstract}

Background \& aims: Gut health relates to a diet with a high digestibility and quality. Limited data are available on the acute effects of low quality foods on gut metabolism and the consequences for liver metabolism.

Methods: A meal with the low quality protein gelatin (tryptophan deficient and low amount of essential amino acids) was compared to a meal with the high quality protein Whey and a tryptophan supplemented gelatin meal (Gel+TRP) in healthy pigs with chronic implanted catheters. In a conscious state, amino acid, ammonia, urea, glucose and lactate fluxes across the portal drained viscera (PDV) and liver were studied for 6 hours after administration of the protein meal.

Results: The average net portal appearance of amino acids was $99.8 \pm 14.6 \%$ of the intake in the Gel group as compared to $61.4 \pm 9.0 \%(p=0.022)$ in the Whey group. In addition, a net portal appearance of tryptophan was observed in the Gel group $(p=0.005)$ of about $42 \%$ of tryptophan released in the Whey group. Intestinal energy metabolism and citrulline production was not affected. Adding tryptophan to the Gel meal diminished net portal AA appearance to $41.6 \pm 24.0 \%$ of the intake $(p=0.012)$, but did not reduce the stimulated liver urea production.

Conclusions: In the postprandial phase after intake of a low protein quality meal, net anabolism in the healthy intestine is absent. It is likely that the intestine responds with a net breakdown of endogenous (labile) proteins to secure amino acid availability for the body. Addition of the first limiting essential amino acid to this meal improved protein anabolism in the intestine. Protein quality of a meal is related to the anabolic response of the intestine during the meal. 


\section{Introduction}

A healthy gut is important for overall body health. The gut controls digestion and absorption of dietary protein and amino acids (AA), and therefore regulates the availability of substrates to the liver, muscle and other organs. Although the intestine in the prandial phase receives AA both from the small intestinal luminal side and via the systemic circulation, it has been observed that the intestine preferentially utilizes AA from the diet for its own metabolism. Luminal contact with nutrients is very important in the regulation of intestinal protein metabolism. Feeding is known to rapidly stimulate protein synthesis in the intestine $(1,2)$ as reflected by the large intestinal extraction during enteral feeding. Approximately $50 \%$ of dietary amino acid intake is used by the portal-drained viscera, but this percentage varies between different amino acids and diets (3-9).

Stimulation of overall protein anabolism by protein meals is reflected by the rapid stimulation of intestinal protein synthesis $(6,10)$. This means that the protein synthesizing capacity of the intestine depends on the same factors as whole body anabolism like the quality of the dietary protein source and the presence of carbohydrates in the protein meal $(4,9)$. We postulated that a high-quality dietary protein source stimulates amino acid utilization in the intestine for protein synthesis (11). After temporary retention of dietary protein as newly synthesized protein in the intestine, a tapered release of AA after the meal will ensure a more prolonged supply of AA to other tissues of the body. In this way, the intestine can act as a "buffer" or what is called, the labile protein pool (11).

A low quality protein meal is defined as a meal containing a normal amount of macronutrients and a protein source with low digestibility or a deficient/low amount of essential (indispensible) amino acids (EAA). In disease states, a meal with a low amount of functional and/or conditional EAA is also considered as low quality (5, $12,13)$. It is hypothesized that intake of low quality food plays a key role in e.g. environmental enteropathy, a subclinical chronic situation often present in developing countries (14-17).

Previous studies examining the effects of low quality food mainly focused on whole body growth (18-20). Limited data are available of the direct effects of low quality foods on gut metabolism and the consequences for liver metabolism. Previously, we showed that intake of soy protein, characterized by low levels of essential amino acids, resulted in a higher net release of essential amino acids into the portal system, higher levels of liver urea production and lower values for net protein synthesis than high quality casein protein $(10,21)$. The different digestion and absorption rate of the two protein meals could partly explain this observation. The splanchnic tissues like gut and liver potentially can act as a gate keeper to dietary amino acid delivery to peripheral tissues. Furthermore, we (3) observed that intake of a pure blood protein 
mixture, deficient of the essential amino acid isoleucine, resulted in increased net portal appearance of AA. Subsequent intravenous infusion of isoleucine was able to normalize the portal appearance of AA.

Information is still lacking whether the relative portal release of $A A$ is increased in low quality protein meals with comparable digestion and absorption rates, and if Iow quality protein meals indeed will not stimulate intestinal net protein synthesis. Furthermore, it is unknown whether adding of the deficient AA to the meal will normalize the intestinal response to the meal.

In the present study, we examined the metabolic responses in the portal drained viscera (PDV) and liver to a gelatin protein meal (with low levels of EAA and TRP deficient) as compared to a high quality whey protein meal. In addition, we studied the effects of TRP supplementation to the gelatin protein meal. We hypothesize that adding tryptophan to a gelatin meal will mainly improve intestinal metabolism. The experiments were performed in healthy pigs with chronic implanted catheters, as models in pigs are viewed to be an excellent model for human gastro-intestinal tract and nutrition research in relation to gut and liver metabolism (4, 22-24).

\section{Materials and Methods}

\section{Animals}

Ten female crossbred (Yorkshire $\times$ Dutch Landrace: $20-22 \mathrm{~kg}$ ) piglets were used. They were housed individually inside galvanized bar runs ( 2 by $3 \mathrm{~m}$ ) equipped with an automated watering device and PVC-coated floors. During the whole experimental period, the piglets were fed sow feed (Landbouwbelang, Roermond, the Netherlands; $16 \%$ protein) on which the pigs grow at a rate of about $2 \mathrm{~kg} /$ week. This study was approved by the Animal Ethics Committee of the Maastricht University.

\section{Surgical procedure}

To perform cross-organ balances, several catheters are implanted under anesthesia as previously described $(13,22)$. Catheters for blood sampling were placed in the abdominal aorta, the portal vein and the hepatic vein. Catheters for the infusions of post-operative medication or para-aminohippuric acid solution (PAH) for flow measurement were placed in the central vein and splenic vein. Finally, a gastric catheter was placed.

\section{Postoperative care}

Postoperative care was standardized as previously described $(13,22)$ and the pigs remained healthy without signs of infection. During the recovery period the animals 
became accustomed to a small movable cage $(0.9 \times 0.5 \times 0.3 \mathrm{~m})$. After a recovery period of 7-10 days, this cage training ensures that the testing of the experimental meals was done in conditioned and conscious animals, standing or recumbent in the small cage.

\section{Experimental protocol}

After a recovery period, four different experimental diets were tested in each pig in random order (Table 1 and 2). The period between the tests was 2-3 days. The following meals were given: an high quality protein meal with a relative high amount of EAA, high digestion and fast absorption rate: Whey protein meal (Whey). Secondly, a low quality protein meal with relative low EAA's, a deficient EAA (TRP) but comparable digestion and absorption rates as the Whey meal: gelatin protein meal (Gel). Third, a gelatin protein meal to which the limiting amino acid tryptophan (Gel+TRP) was added in a sufficient amount to make tryptophan not the limiting EAA (25). All the protein meals were iso-nitrogen, iso-ionic (for sodium, chloride, potassium and calcium), iso-tonic and contained a similar amount of maltodextrins. The amount of protein and carbohydrates in the test meal represents about $30 \%$ of the normal daily intake of the pig of this age. The control test mixture did not contain any macronutrients and was used as control for volume, osmolarity and ion intake. This test mixture was either a solution of electrolytes, iso-osmolar to the protein meals (ContOsm) or an iso-ionic solution for sodium, potassium and calcium ions (Control). Each control mixture was given in 5 animals.

Food was withdrawn 16 hours prior to each test day. On the morning of a test day, a primed-continuous infusion of para-aminohippuric acid via the splenic catheter (PAH 25mM, iso-tonic, Sigma Chemicals CO, St. Louis, U.S.A.) at a rate of $60 \mathrm{ml} / \mathrm{h}$ after an initial bolus of $5 \mathrm{ml}$ PAH solution was given. After taking initial blood samples from the arterial, portal and hepatic vein catheter, the liquid test meal ( $20 \mathrm{ml} / \mathrm{kg} \mathrm{bw})$ was given though the gastric catheter within 5 minutes. Blood samples were taken at the time intervals as indicated in the figures (Figure 1, 2 and 3), and used for amino acid and $\mathrm{PAH}$ concentrations analysis.

\section{Sample processing}

Immediately after collection, the blood samples were placed on ice and hematocrit $(\mathrm{Ht})$ was measured. All samples were centrifuged at $4^{\circ} \mathrm{C}$ for $5 \mathrm{~min}$ at $8500 \mathrm{~g}$ in an Eppendorf 5413 centrifuge to obtain plasma. For glucose and lactate determinations, $300 \mu \mathrm{l}$ of whole blood was centrifuged in sodium fluoride containing tubes (Microvette FH, Sarstedt, Nümbrecht, Germany). For PAH determinations, $300 \mu \mathrm{l}$ of whole blood was added to $600 \mu$ of $12 \%$ Tri-chloroacetic acid solution, thoroughly mixed and centrifuged. For ammonia and urea determination $200 \mu$ plasma was collected, and. for amino acid analysis, $100 \mu \mathrm{l}$ of plasma was deproteinized 
Table 1. Composition of experimental test meals**

\begin{tabular}{|c|c|c|c|c|c|c|}
\hline & & Control* & & Whey ${ }^{+}$ & $\mathrm{Gel}^{\ddagger}$ & Gel+TRP§ \\
\hline Substance & Unit & Control & ContOsm & & & \\
\hline Nitrogen & $\mathrm{g} / \mathrm{kg}$ bw & 0.0 & 0.0 & 0.1875 & 0.1875 & 0.1875 \\
\hline Protein & $\mathrm{g} / \mathrm{kg} \mathrm{bw}$ & 0.0 & 0.0 & 1.44 & 1.11 & 1.11 \\
\hline Tryptophan & $\mathrm{mg} / \mathrm{kg}$ bw & 0.0 & 0.0 & 0.0 & 0.0 & 8.83 \\
\hline Maltodextrin & $\mathrm{g} / \mathrm{kg}$ bw & 0.0 & 0.0 & 4.69 & 4.69 & 4.69 \\
\hline Sodium & $\mathrm{mM}$ & 42.5 & 148.4 & 42.5 & 42.5 & 42.5 \\
\hline Chloride & $\mathrm{mM}$ & 61.1 & 167.0 & 45.0 & 45.0 & 45.0 \\
\hline Potassium & $\mathrm{mM}$ & 0.73 & 0.73 & 0.73 & 0.73 & 0.73 \\
\hline Calcium & $\mathrm{mM}$ & 8.92 & 8.92 & 8.92 & 8.92 & 8.92 \\
\hline Osmolariteit & mOsm & 113.25 & 325.05 & 300.66 & 325.04 & 325.04 \\
\hline
\end{tabular}

*) The control test meal (Control) was either a solution of electrolytes iso-osmolair to the other test meals (ContOsm) or a solution with similar amount of sodium, potassium and calcium ions (Contlon).

$\left.{ }^{\dagger}\right)$ Whey protein meal (Whey): Whey Protein Isolate, 90\% w/W protein, supplied by DMV International, Veghel, The Netherlands,

‡) Gelatin protein meal (Gel): Gelatin, 97\% w/w protein, (SoluGel P®) supplied by PB Gelatines, Tessenderloo, Belgium.

§) Gelatin protein meal with added amino acid tryptophan (Gel+TRP). Total amount of tryptophan added (T 0254, Sigma Chemicals CO, St. Louis, U.S.A.: $8.83 \mathrm{mg}=43.2 \mu \mathrm{mol}$ ) is sufficient to make tryptophan not the limiting amino acid (Cortamira et al 1991).

**) Total nitrogen of the protein was determined by an elementary analyzer (CHN O RAPID, Heraeus, Hanau, F.R.G.). All the protein meals were iso-nitrogen, iso-ionic (for sodium, chloride, potassium and calcium) and iso-tonic. They contained a similar amount of maltodextrins: Maldex 20, supplied by Amylum NV, Aalst, Belgium, is $28 \mathrm{mmol}$ glucose/kg bw. Test meal ingredients were dissolved in trice distillate water (MilliQ) and electrolytes $(\mathrm{NaCl}, \mathrm{KCl}$ and $\mathrm{CaCl} 2)$ were added to the indicated concentrations. The test meals were prepared the day before the experiment, kept over-night at $4^{\circ} \mathrm{C}$ and heated up in a warm water bath to $37^{\circ} \mathrm{C}$ just before the intra-gastric administration. Test meal volume was $20 \mathrm{ml} / \mathrm{kg}$ bw.

with $4 \mathrm{mg}$ dry sulfosalicylic acid. All prepared samples were subsequently snap frozen in liquid nitrogen and stored at $-70^{\circ} \mathrm{C}$ until further analysis.

\section{Biochemical analysis}

Plasma ammonia, urea, glucose and lactate were determined spectrophotometrically by standard enzymatic methods as described previously $(4,26,27)$ with commercial available kits on a centrifugal analyzer system (Cobas Bio, Hoffmann-La Roche, Basel, Switzerland). Urea values were corrected for ammonia. PAH concentrations were determined spectrophotometrically after deacetylation of the supernatant at $100^{\circ} \mathrm{C}$ for $45 \mathrm{~min}$ as previously described $(4,22,26)$. Plasma AA were 
Table 2. The amino acid content of the Gelatine and Whey protein isolate

\begin{tabular}{|c|c|c|}
\hline & Gelatin* & Whey** \\
\hline Amino Acid & $\begin{array}{l}\mu \mathrm{mol} / \mathrm{kg} \text { bw given } \\
\text { in the meal }\end{array}$ & $\begin{array}{l}\mu \mathrm{mol} / \mathrm{kg} \text { bw given } \\
\text { in the meal }\end{array}$ \\
\hline ASP & 180 & 573 \\
\hline ASN & 253 & 363 \\
\hline GLU & 431 & 1035 \\
\hline GLN & 320 & 713 \\
\hline SER & 178 & 535 \\
\hline GLY & 3168 & 250 \\
\hline THR (essential) & 226 & 688 \\
\hline HIS (essential) & 83 & 106 \\
\hline ALA & 1249 & 738 \\
\hline ARG & 552 & 195 \\
\hline TYR & 52 & 207 \\
\hline VAL (essential) & 280 & 640 \\
\hline MET (essential) & 45 & 213 \\
\hline ILE (essential) & 179 & 616 \\
\hline PHE (essential) & 155 & 227 \\
\hline TRP (essential) & 0 & 92 \\
\hline LEU (essential) & 268 & 1044 \\
\hline LYS (essential) & 305 & 770 \\
\hline Sum amino acids & 7924 & 9007 \\
\hline Sum N of amino acids & 9767 & 10774 \\
\hline $\begin{array}{l}\text { Sum N of non-ess amino } \\
\text { acids }\end{array}$ & 8105 & 6286 \\
\hline Sum $N$ of ess amino acids & 1662 & 4488 \\
\hline CYS§ & 28 & 233 \\
\hline PRO & 1150 & 753 \\
\hline HydroxyPRO & 1090 & 0 \\
\hline HydroxyLYS & 50 & 0 \\
\hline
\end{tabular}

The amino acid content of the protein is determined in triplicate with methods, suggested by the FAO/WHO (FAO/WHO 1991) and were determined by the manufacturer.

*) The content of glutamine and asparagines in the Gelatine are calculated from amino acid compositions (from protein sequencing) given by Genome Net (Kyoto University Bioinformatics Center, Japan), assuming that the Gelatine used contains mainly collagen, type V, alpha 2 [Sus scrofa] (code AB191304.1, National Center for biotechnology Information, USA).

${ }^{* *}$ ) The content of glutamine and asparagines in the Whey protein isolate are calculated from amino acid compositions (from protein sequencing) given in Table A-6 from Walstra and Jennes (Walstar and Jennes 1984) for individual milk proteins, assuming that the (bovine) 
Whey protein isolate contains $85 \%$ of $\beta$-lacto globulin and $15 \%$ of $\alpha$-lactalbumin (data DMV international, Veghel, The Netherlands).

§) The amounts of cysteine, proline, hydroxyproline and hydroxylysine are not included in the total amount of amino acids, because these amino acids were undetected in the amino acid analysis, and the values given are tabulated values.

determined with a fully automated HPLC system after pre-column derivatization with o-phthaldialdehyde (28).

\section{Calculations}

Plasma flow was calculated by means of an indicator-dilution technique as described $(4,13,22)$.

\section{Plasma flow $=$ infusion rate $*[\mathrm{PAH}$-infused $] /([\mathrm{PAH}]$ post $-[\mathrm{PAH}]$ pre $)$}

[PAH-infused] is the concentration of PAH in the infused solution, [PAH]post is the concentration in plasma after the organ and [PAH]pre in plasma before the organ. Plasma PAH concentrations were used and calculated as [blood PAH]* (100/ (100-Hematocrit)). Plasma flow was expressed as ml×kg BW-1×min-1. Organ plasma flow is highly dynamic and depends on the high variance in cardiac output in conscious subjects (29). This means that measuring individual plasma flow can have a high variance. Therefore, multiple measurements were done in time and transorgan fluxes were calculated with the mean plasma flow for each time point of each experimental group. PDV flux was calculated by multiplication of the portal-arterial concentration difference with the portal plasma flow. Liver flux was calculated by the subtracting PDV flux from the splanchnic flux (hepatic-arterial concentration difference* liver plasma flow). Total net balance (net release or net uptake) of an organ was calculated by the area under the curve (AUC) of the respective flux time course from $\mathrm{t}=\mathrm{Oh}$ to the time, indicated. A positive value of flux or net balance means net release. A negative value means net uptake.

In the present study, comparisons of groups of amino acids with their nitrogen contents were done between protein meals of a different quality and quantity of amino acid but comparable amounts of nitrogen. Amino acid nitrogen (AA_N) was calculated as the sum of $\mathrm{N}$ of all $\mathrm{AA}$ that were detected in the amino acid analyses and therefore cysteine, proline, hydroxyproline and hydroxylysine were not included (Table 2).The essential amino acid nitrogen (EAA_N) and the non-essential amino acid nitrogen (non-EAA_N) were calculated as the sum of $N$ of EAA and nonessential amino acids (non-EAA), respectively.

\section{Statistics}

Results are presented as means \pm SEM. Levels of significance were set at $\mathrm{P}<0.05$ unless stated otherwise. For statistical analysis of variance, the two-way ANOVA 
test was used to compare time courses of plasma flows, arterial concentrations and the organ fluxes of the test groups. Student's t-test was used to compare the total amount of net release or net uptake of metabolites over the $6 \mathrm{~h}$ experimental period (= total net balances). One sample t-test was used to determine if data are different from zero or from 100\%. We used Prism 5.04 (Graphpad.com) for statistical analysis.

\section{Results}

\section{Main}

Overall, catheters remained patent. Incidentally, some minor technical problems were present with the hepatic catheter, resulting in a reduced number of liver data points (especially in the Gel+TRP group). In this case, hepatic observations were under-powered. As the results of the ContOsm group and Control group were not significantly different, the results of these groups were combined and discussed below as Control group.

\section{Plasma flow}

PDV plasma flow in time (not shown) after administration of the protein meals Gel and Whey was higher compared to the post-absorptive (Control) group (resp. $p=0.005$ and $p=0.014$ ). There were no differences in liver plasma flow. Mean plasma flows over the $6 \mathrm{~h}$ experimental period per group are given in table 3 .

Table 3. Mean plasma flow during the experimental period (6h)

\begin{tabular}{lcccccccc}
\hline $\begin{array}{l}\text { Organ plasmaflow } \\
\text { ml/kg bw/min }\end{array}$ & Control & & Whey & & Gel & & Gel+TRP \\
\hline Portal drained viscera & 8 & $38(9)$ & 7 & $44(9)$ & 6 & $48(9)$ & 7 & $37(7)$ \\
Liver & 8 & $58(12)$ & 9 & $55(9)$ & 6 & $55(8)$ & 7 & $45(10)$ \\
\hline
\end{tabular}

Mean plasma flow during the experimental period (6h) was calculated by integration of the respective plasma flow time courses from $\mathrm{t}=0 \mathrm{~h}$ to $\mathrm{t}=6 \mathrm{~h}$ (area under the curve divided by time). Values are mean \pm SEM. Significance tested with Student t-test: no significance differences were observed.

\section{Nitrogen balances}

\section{Amino acids}

In the present study, nitrogen balances provided overall information about the effects of protein meals with a different quality and quantity of amino acid contents but comparable amounts of nitrogen. Arterial AA_N (Figure 1A) increased from approx. $3.7 \mathrm{mM}$ at $\mathrm{T}=0$ to a maximum of $6.2 \mathrm{mM}$ at $\mathrm{t}=1$ hour after Whey feeding, and to a maximum of $5.5 \mathrm{mM}$ in both Gel-groups at $\mathrm{t}=2$ hour. The levels of the Gelgroups did not return to baseline values at the end of the experiment (Gel: $p=0.002$, 
Gel+TRP:p=0.033). The shapes of the arterial AA_N time courses of the Whey and Gel-group were significantly different (ANOVA Interaction: $p=0.010$ ). In the control group, the PDV was taken up AA (Figure 2A and 3A). Despite the fact that the Gel meals contained comparable amounts of nitrogen, the Gel group showed a significantly higher net release of AA_N by the PDV over a six hour period as compared to the Gel+TRP group ( $p=0.035)$ and was finished after 6 hours. There were no differences between the meals in AA_N fluxes across the whole splanchnic area.

The total amount of measured AA_N in the Whey and Gel meals were slightly different. Therefore PDV AA_N net balances were expressed as percentage of AA_N intake in Figure 4. Thirty-nine percent $(p=0.022)$ of the $A A \_N$ intake in the Wheygroup was not released to the portal system. This is in contrast to the Gel-group where $99.8 \pm 14.6 \%$ of the intake was released into the portal system. The Gel+TRPgroup and Whey-group showed a comparable release of AA_N.

\section{Essential amino acids}

The total net amounts of EAA_N (Figure 3B) released by the PDV after a test meal were different in all groups except between Gel and Gel+TRP group where only a tendency $(p=0.097)$ was observed. Seventeen percent of the intake (Figure 4 , $p=0.033$ ) in the Whey-group was not released to the portal system. The released amount of EAA_N as \% of intake was in the Gel group higher $(p=0.046)$ than in the Whey group. A tendancy was observed that it was higher $(124 \pm 20 \%, p=0.12)$ than the intake. The released amount as \% of intake in the Gel+TRP group was not significantly different as compared to the Whey-group. The arterial EAA_N time courses (Figure 1B) in the Gel-groups were not different from the level in the post-absorptive state, although the effect on the arterial concentrations in time where significantly different (ANOVA Interaction: Gel p=0.001, Gel+TRP $p<0.0001$ ). In the first two hours the arterial EAA_N concentrations in the Gel-group were higher than in the Controlgroup, but in the last 3 hours of the experiment the concentrations were below the Control-group (post-absorptive) time course (Gel:p=0.003, Gel+TRP:p<0.0001).

\section{Non-Essential amino acids (Figure 1C, 2C, 3C and 4)}

As percentage of intake (Figure 4), different amounts of non-essential amino acid nitrogen (nonEAA_N) were released by the PDV over the 6 hours feeding period. The Whey-group showed that the equivalent of $54 \%$ of the intake $(p<0.0006)$ of nonEAA_N was not released to the portal system. This percentage was significantly lower in the Gel-group (5\% of the intake, $p<0.012$ ). The release of nonEAA_N in the Gel+TRP group was comparable to that of the Whey-group. Although the total absolute splanchnic release (Figure $3 \mathrm{C}$ ) was comparable between all protein meals groups, arterial nonEAA_N concentration time courses (Figure 1C) of the Gel groups were significantly higher $(p<0.0001)$, than the Whey-group and did not reach baseline values at the end of the experiment (Gel:p=0.003, Gel+TRP=0.029). 

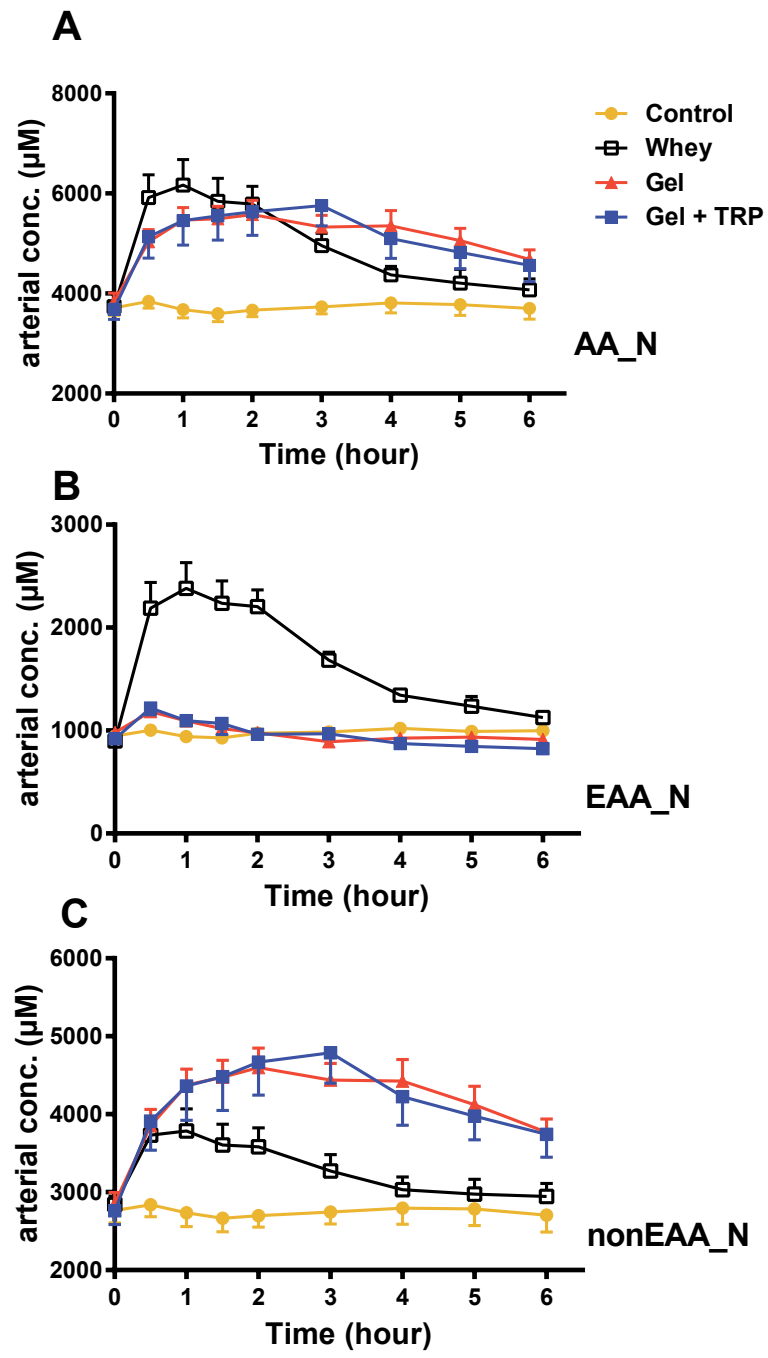

Figure 1. Arterial amino acid nitrogen (AA_N)(A), essential amino acid nitrogen (EAA_N) (B) and amino acid nitrogen (NonEAA_N)(C) concentrations, in $\mu \mathrm{M}$, in post-absorptive state (Control, $n=9$ ) and during 6 hours after administration of Whey protein (Whey, $n=8$ ), gelatin (Gel, $n=9)$ or gelatin + tryptophan (Gel+TRP, $n=9$ ) meals. Values are mean \pm SEM. ANOVA tested, significant meal effect ( $<<0.05)$ : (A) protein meals vs. Control; (B) Whey vs. all other test meals; (C) proteins vs. control, Whey vs. Gel groups. Significant interaction ( $<<0.05)$ : (A) Control vs. protein meals, Gel vs. Whey;(B) Control vs. all protein meals, Whey vs. all test meals; (C) Control vs. Gel groups, Gel vs. Whey. 

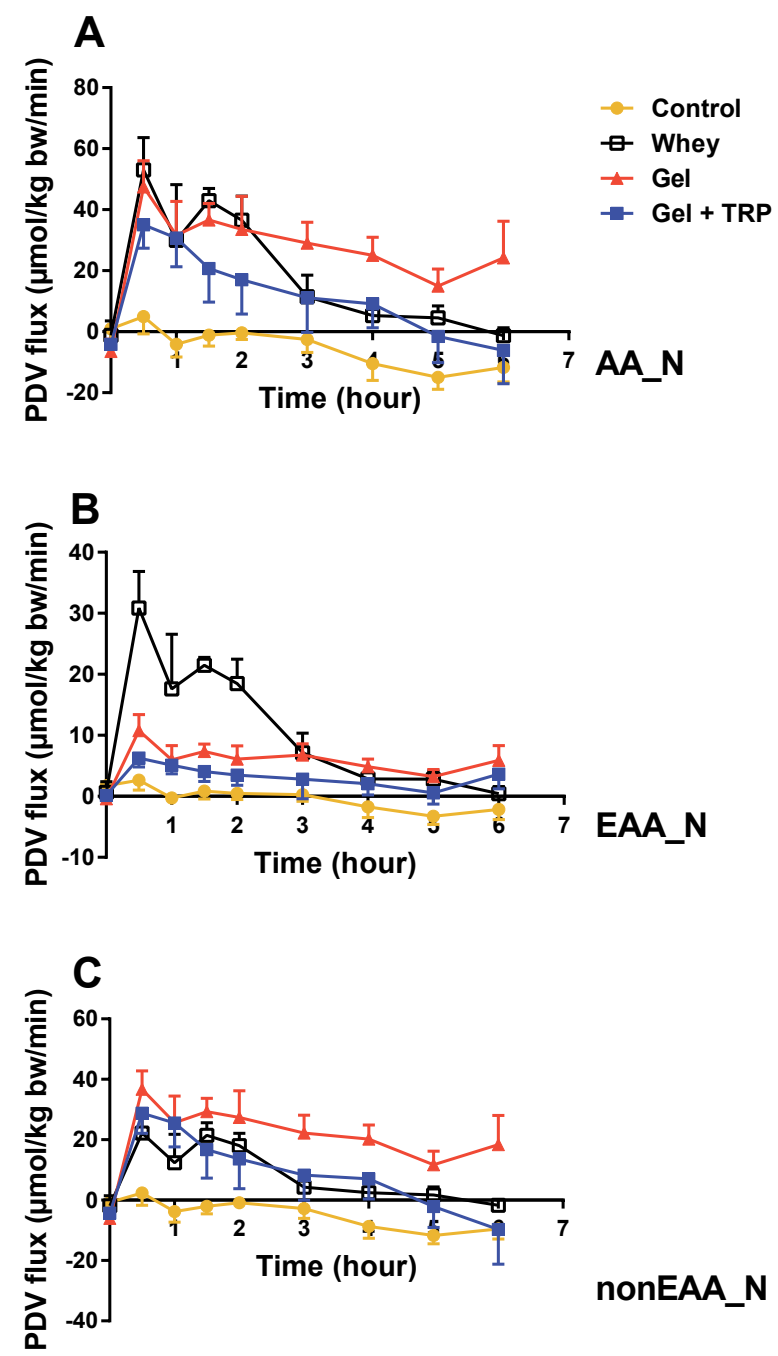

Figure 2. PDV fluxes of amino acid nitrogen (AA_N)(A), essential amino acid nitrogen (EAA_N) (B) and amino acid nitrogen (NonEAA_N)(C) in $\mu \mathrm{mol} / \mathrm{kg} \mathrm{bw} / \mathrm{min}$, in post-absorptive state (Control, $n=8$ ) and during 6 hours after administration of Whey protein (Whey, $n=7$ ), gelatin $(G e l, n=9)$ or gelatin + tryptophan (Gel+TRP, n=6) meals. Values are mean \pm SEM. ANOVA tested, significant meal effect ( $p<0.05)$ : (A) Control vs. all protein meals; Gel vs. Gel+TRP; (B) Control vs. all protein meals; Whey vs. Gel meals; Gel vs. Gel+TRP; (C) Control vs. all protein meals; Whey vs. Gel; Gel vs. Gel+TRP. Significant interaction ( $p<0.05)$ : (A) Control vs. Gel; Control vs. Whey; (B) Whey vs. all other meals; (C) Control vs. Gel. Positive values = release to portal sytem. Negative values = uptake from portal system. 

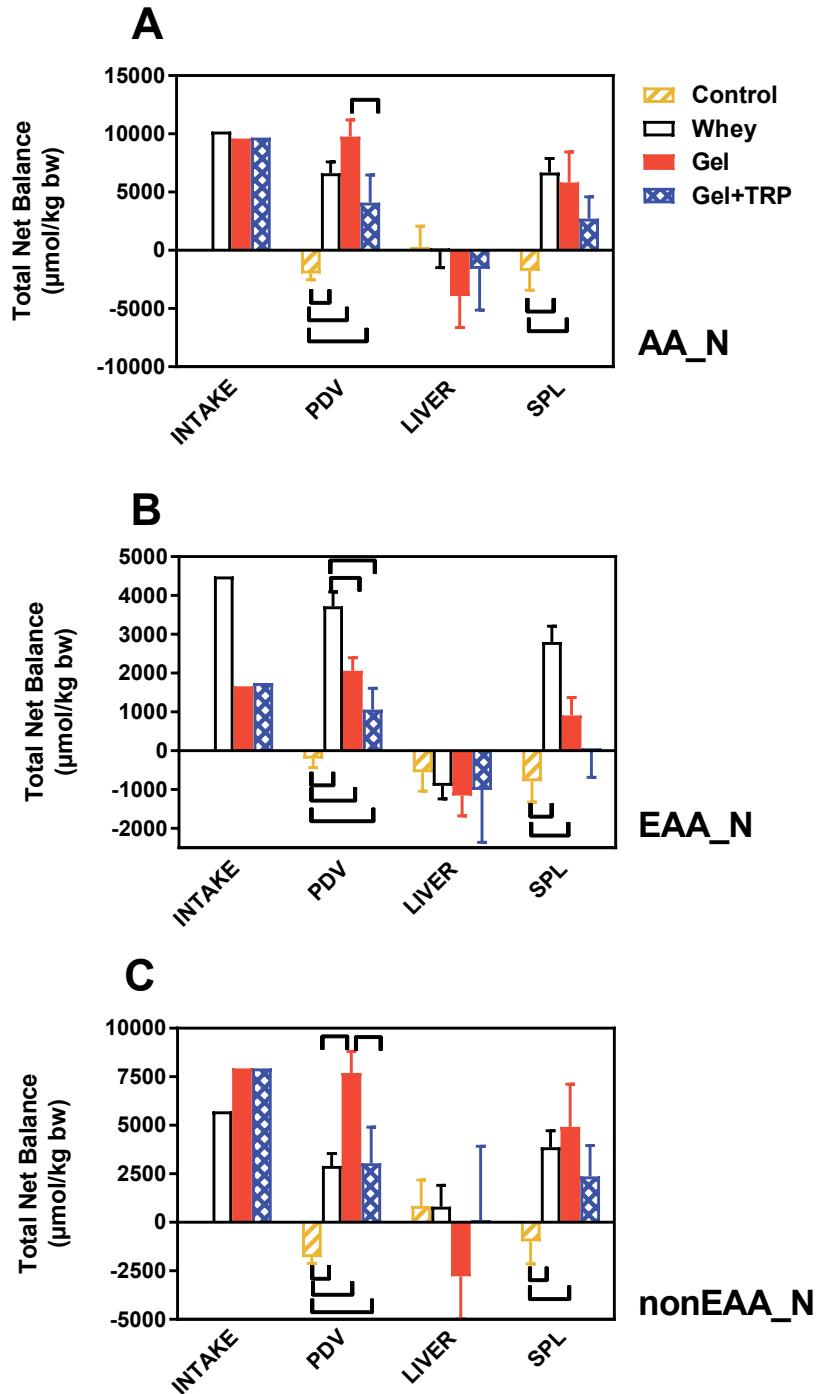

Figure 3. Total net balances across organs comparing with intake amounts of amino acid nitrogen $\left(A A \_N\right)(A)$, essential amino acid nitrogen (EAA_N)(B) and amino acid nitrogen (nonEAA_N)(C), in $\mu \mathrm{mol} / \mathrm{kg}$ bodyweight, over 6 hours after administration of Whey protein (Whey, $n=8$ ), gelatin (Gel, $n=9$ ) or gelatin + tryptophan (Gel+TRP, $n=9$ ) meals. Organs showed are portal drained viscera (PDV), liver and total splanchnic area (SPL = PDV + Liver). Values are mean \pm SEM. Significance with Student t-test: $P<0.05$, indicated with hooks. Positive values = release. Negative values $=$ uptake. 


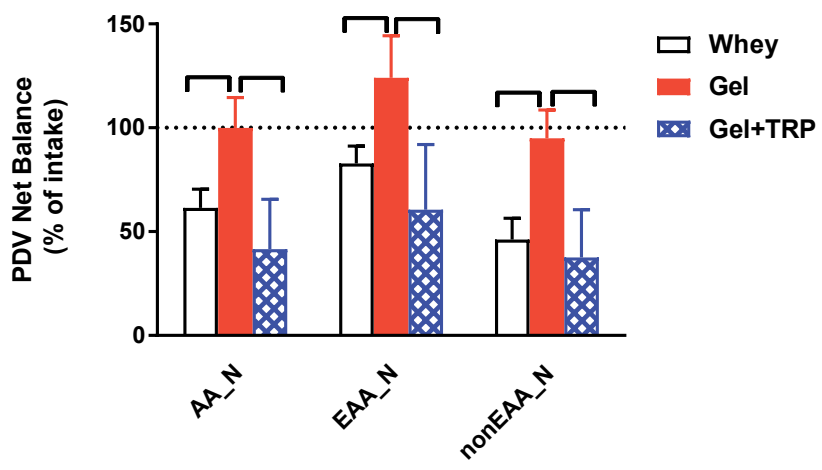

Figure 4. Total net balances across the gut of amino acid nitrogen (AA_N), essential amino acid nitrogen (EAA_N) and amino acid nitrogen (nonEAA_N) as percentage of the intake, over 6 hours after administration of Whey protein (Whey, $n=8$ ), gelatin (Gel, $n=9$ ) or gelatin + tryptophan (Gel+TRP, $n=9$ ) meals. Values are mean \pm SEM. Significance with Student t-test: $p$ $<0.05$, indicated with hooks.

\section{Ammonia}

Ammonia $\left(\mathrm{NH}_{3}\right)$ (Figure $5 \mathrm{~A}$ ) is a nitrogen-carrier mainly coming from protein breakdown and production by the intestinal lumen bacteria followed by $\mathrm{NH}_{3}$ uptake of the gut on the lumen side and release into the portal system. Arterial $\mathrm{NH}_{3}$ was increased after meal intake, but no significant differences were observed between the different meals. At organ level, an increased release of $\mathrm{NH}_{3}$ was seen by the PDV after administration of a protein meal and $\mathrm{NH}_{3}$ uptake by the liver was increased (Table 4). As a consequence no net splanchnic release of $\mathrm{NH}_{3}$ was observed over the 6 hour experimental period.

\section{Urea}

Urea (Figure 5B, Table 4) is the carrier of nitrogen coming from amino acid breakdown and ammonia absorption, is mainly produced by the liver. Arterial time course of urea concentration and net urea production by the splanchnic area was enhanced in the Gel groups in comparing to the Control group (Arterial time course: Gel: $p=0.030$, Gel+TRP: $p=0.010$ ) during the total experimental period (6 hours), which was mainly due to the increased liver urea production.

\section{The deficient essential amino acid: Tryptophan}

TRP (Figure 6) was deficient in the Gel group but was added to the meal in the amount of $43.2 \mu \mathrm{mol}$ TRP per kg bodyweight in the Gel+TRP group. The TRP PDV flux in the Gel group was biphasic: in the first hour it was not different from the 
control group. But in the last four hours of the experiment it was significant higher than the control group $(p<0.0001)$ and comparable with the Whey and Gel+TRP group. In the Gel+TRP group, the PDV flux in the first hour was higher than the Gel group ( $p=0.033$ ) but not different from the Whey group.

Remarkably this phenomenon resulted in a net PDV release (Different from zero: $\mathrm{p}=0.005$ ) of TRP (Figure 6C) in the TRP-deficient Gel group. The amount was $42 \%$ of the release in the Whey group. Due to the biphasic shape of the PDV flux time course in the Gel+Trp group, the PDV net balance was not different from zero $(p=0.551)$. Splanchnic flux time courses showed the same patterns (data not shown).

As in the PDV and splanchnic fluxes, the group differences in arterial TRP time courses (Figure 6) were comparable in the first hour. But in the last four hours arterial time courses didn't reflect the PDV fluxes: the time course of the Gel-group was significant lower $(p<0.0001)$ than the time course of the control group. Gel +TRP group was not different with the Whey group.
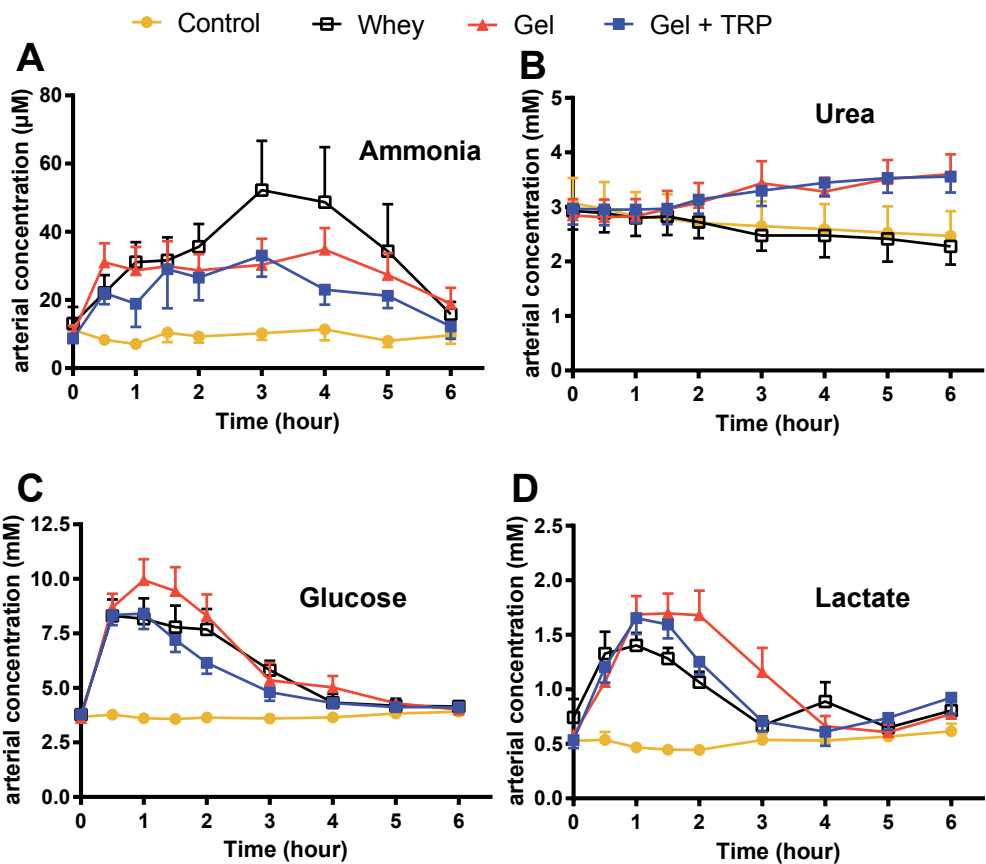

Figure 5. Arterial ammonia(A), urea(B), glucose(C), lactate(D), in post-absorptive state (Control, $n=9$ ) and during 6 hours after administration of Whey protein (Whey, $n=8$ ), gelatin (Gel, $n=9)$ or gelatin + tryptophan (Gel+TRP, $n=9)$ meals. Values are mean \pm SEM. ANOVA tested, significant meal effect $(p<0.05)$ : $(A)(C)(D)$ protein meals vs. Control $(A)$ Whey vs. Gel + TRP; (B) Whey vs Gel meals; (C) Gel vs. Gel+TRP; (D) Gel vs. Whey only on T=2h. No time courses were different. 


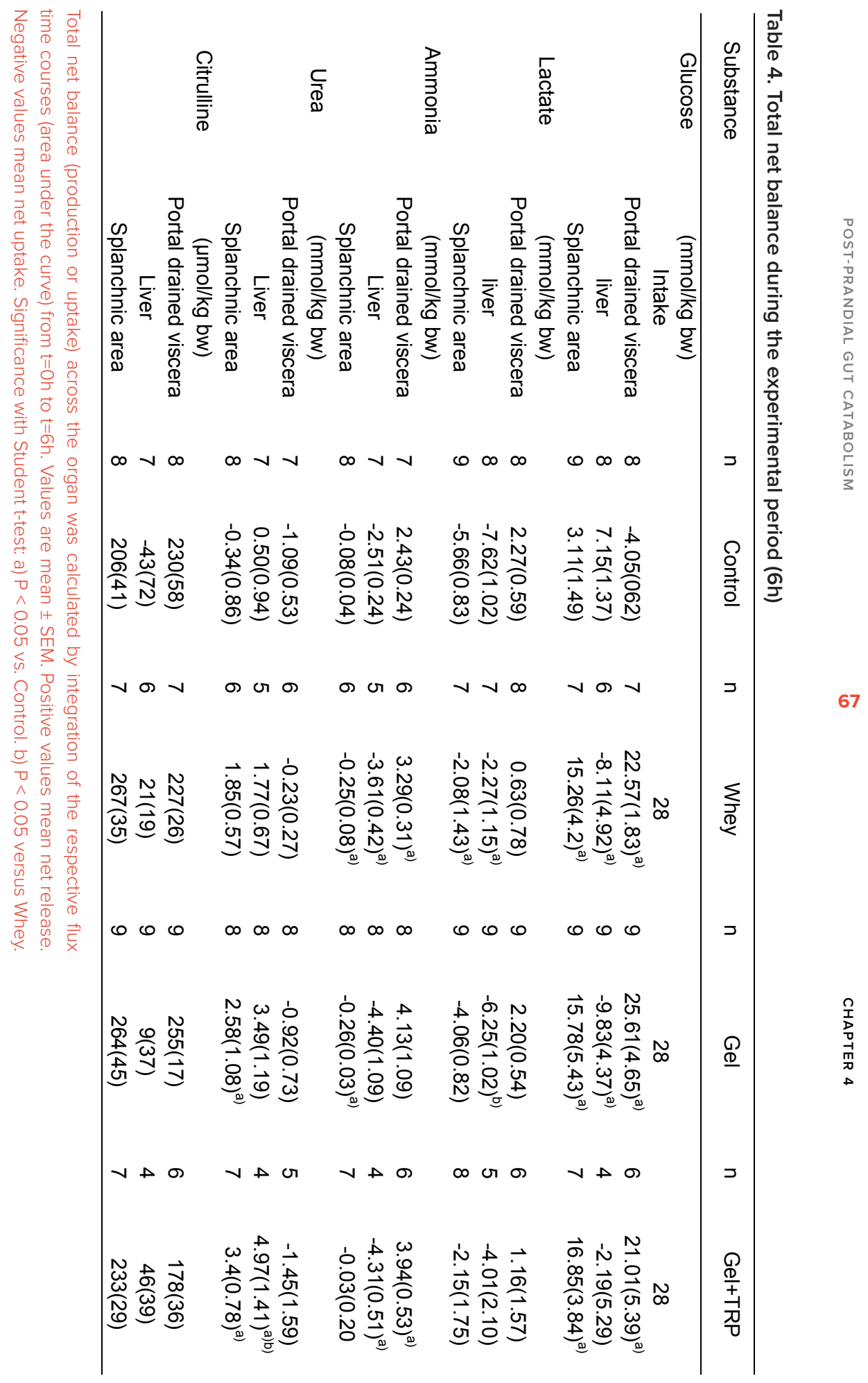



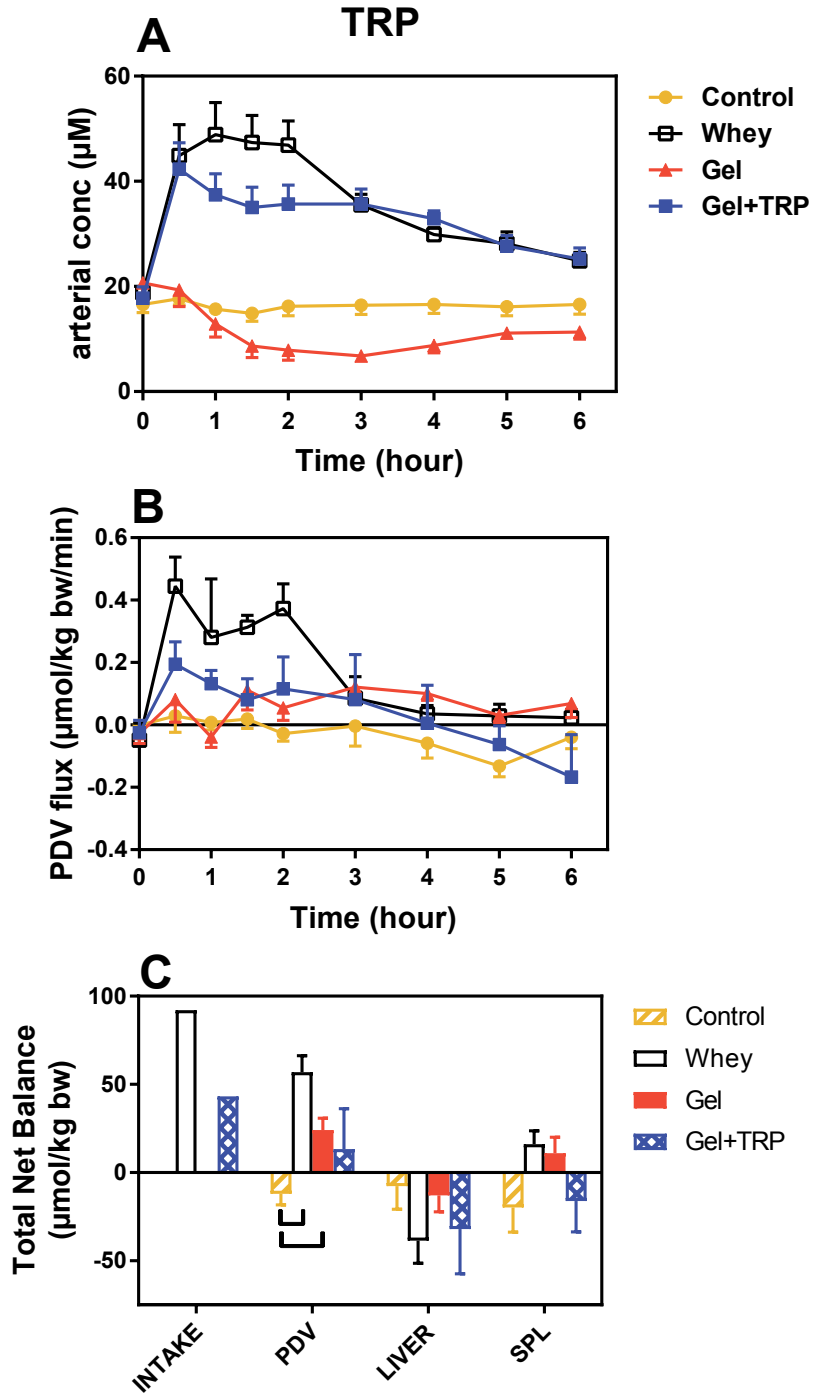

Figure 6. Arterial tryptophan concentrations (A),gut fluxes of tryptophan (B) and total net balances (C), in post-absorptive state (Control) and during 6 hours after administration of Whey protein (Whey), gelatin (Gel) or gelatin + tryptophan (Gel+TRP) meals. Values are mean \pm SEM. (A) and (B) time courses are tested with ANOVA. Significant meal effect $(p<0.05)$ : (A) All time courses were different (B) Control vs. all meals; Whey vs. all meals. (C): Significance with Student t-test: $p<0.05$, indicated with hooks. 


\section{Energy parameters}

\section{Glucose}

Despite equal amounts provided, the arterial glucose concentration time course of the Gel group was significant higher comparing to the Gel+TRP group ( $p=0.003$ ) with a maximum of $9.9 \mathrm{mM}$ at $\mathrm{T}=1 \mathrm{~h}$ (Figure $5 \mathrm{C}$ ). Furthermore in all protein test meals, a similar net PDV release of glucose, net uptake by the liver, resulting in net splanchnic release was seen (Table 4). The mean percentage of glucose released by the PDV was $82 \%$ of the glucose intake during the 6 hour feeding period.

\section{Lactate}

In a post-absorptive state, the systemic levels of lactate (Figure 5D) were around $0.5 \mathrm{mM}$ in the control group. The PDV was releasing lactate and the liver was taking up lactate, with net uptake across the splanchnic region (Table 4). Lactate in the Gel group stayed longer at the maximum level then in the Whey and Gel+TRP group, and returned to baseline values at $T=4$ hours (ANOVA between 1 and $4 \mathrm{~h}$ : resp. $p=0.003$ and $p=0.034$ ). There were no significant differences in lactate production by the PDV between the test meals, although the uptake of lactate by the liver was lower in the Whey group in comparison with the Gel group $(p=0.015)$.

\section{Intestinal function parameter: Citrulline}

The PDV production of Citrulline is related to intestinal function (30, 31). The PDV net production (Table 4) was comparable between the post-prandial period and the post-absorptive state. Also on the splanchnic level there were no net differences in CIT release between the groups.

\section{Discussion}

In the present study, we examined the effects of a low quality protein meal (gelatin protein in a protein-carbohydrate meal) on intestinal metabolism, and whether improving the biological value of this meal (by adding tryptophan) would affect intestinal, hepatic metabolism and systemic availability of nutrients. PDV metabolism was studied and assumed to be representative of intestinal metabolism. We observed that after intake of a low protein quality meal (Gel), the healthy intestine released into the portal vein an equivalent amount of amino acids that was given with the meal together with a substantial amount of the deficient TRP. We also observed a tendency that more EAA's were released to the portal system then consumed. It is likely that the intestine responds to such a meal by net breakdown of endogenous (labile) gut protein. Moreover at whole body level, post-prandial systemic EAA levels were below post-absorptive levels, suggesting that net peripheral protein synthesis is stimulated by meal intake itself. Finally, adding the deficient amino acid TRP to the meal resulted in normalized intestinal net balance but not the systemic 
EAA and urea levels, suggesting improved intestinal metabolism without improved whole body response.

\section{Quality of the model used}

The studies were done in a normal physiological condition. We found that plasma portal flow values were not different between the protein meals suggesting that the differences in portal flow will not play a role in the observed differences in net balance. Net balance data of amino acids across an organ only provide information on the net uptake (net anabolism) or net release (net catabolism) of those amino acids, and reflects the net result of dynamic processes like (arterial) disposal, production, recycling of endogenous secretions, synthesis, breakdown etc. These dynamic processes cannot be quantified without amino acid isotope tracer technology. In a post-prandial condition, net release of EAA to the portal system (PDV net balance) does not reflect net catabolism as the PDV absorbs amino acids from the luminal side. The PDV is in a net catabolic state when release of EAA is more than $100 \%$ of its intake.

The percentage of protein digestion by the intestinal lumen and absorption of AA by the gut tissue itself was not measured in the present study. Based on the literature we assumed that in healthy intestine, the percentage of protein digestion and amino acid absorption is nearly the same (around 95\%) in the two test meals (32). This is supported by the fact that gut ammonia production was comparable between the test meals, suggesting that the amount of amino acids available for intra luminal bacteria was comparable. Also, $t_{50}$ of total net portal appearance was not different between the gelatin and the whey protein meal (data not shown), suggesting that the digestion rates of gelatin and whey protein are comparable. Therefore, gelatin protein is the most optimal candidate to study the effects of a low quality protein meal on gut and liver metabolism. As the changes in PDV metabolism were mainly representing changes in intestinal metabolism, we hypothesize that the difference between net intake and observed net portal release of $A A$ is the net fraction of $A A$ that was utilized/oxidized by the intestine itself.

\section{Nitrogen metabolism}

\section{The absence of Portal Drained Viscera anabolic state after a low quality Gel meal}

The observed fraction of intestinal amino acid utilization/oxidation after intake of the high quality Whey protein meal is in line with previous studies $(6,10)$. Our present data show the absence of AA utilization/oxidation after a low quality meal. In addition, the substantial release of TRP by the intestine in the TRP deficient test group and the tendency to release more EAA than was ingested (124\% of the intake) indicate that a single bolus meal of a very low-quality meal with gelatin protein can 
induce net catabolism in the intestine. Further studies with tracer amino acids are needed to confirm this hypothesis.

Remarkably, after 6 hours the intestine was still releasing AA in the Gel-group in contrast to the high quality meal and the Gel+TRP-group, suggesting that a catabolic state can be anticipated in a more extended experimental period. Adding the deficient amino acid TRP to the Gel-meal was enough to prevent the non-anabolic state of the intestine. This suggests that in order to induce intestinal anabolism, a protein meal should contain a complete essential amino acid profile or even maybe need a complete 20 AA's profile (20). TRP is for the intestine beside an EAA for protein synthesis also a functional amino acid as it is a precursor for melatonin and serotonin production $(33,34)$. It is therefore unlikely that non-anabolic responsiveness of the intestine is exclusively a TRP-phenomenon. Previously we showed (3) that a protein mixture without the essential amino acid isoleucine was less utilized/ oxidized by the intestine and therefore is of less or no benefit to the intestine.

\section{Enhanced urea production by the in low quality meals}

AA and ammonia released into the portal system are known to stimulate urea production in the liver. Intestinal ammonia production was comparable between all protein meals and therefore cannot explain the enhanced splanchnic urea production during both Gel meals, resulting in enhanced systemic urea concentrations. The total net intestinal amino acid nitrogen release was elevated only in the Gel meal but not in the TRP supplemented Gel-meal. Although the time courses of the systemic AA_N concentration were different between the high and low quality meals, these observations do not explain the enhanced urea production. Interestingly, the difference between the systemic post-prandial increase in nonEAA_N and EAA_N in the low quality meals compared to the high quality meals was completely different. The ratio nonEAA_N/EAA_N of the post-prandial increase in the low quality meals was $9.5 \pm 1.7$ (Gel) and $8.0 \pm 1.7$ (Gel+TRP) versus $0.7 \pm 0.2$ in the high quality meal, one hour after the meal administration. This was not reflected by the ratio in the meals itself: ratio nonEAA_N/EAA_N in low quality meal was $4.8(\mathrm{Gel}: \mathrm{p}=0.029$, Gel+TRP: $p=0.001)$ versus $1.3(p=0.029)$ in the high quality meal. Therefore, it is more likely that the enhanced systemic Non EAA_N concentration has driven the urea production. The ratio was still very high and did not return to baseline values at the end of the experiment. Furthermore, this hypothesis is supported by the shape of the systemic urea time course. This time course was still increasing at the end of the experimental period in the low quality experimental groups.

\section{Effects of splanchnic release of limited EAA_N on post-prandial peripheral metabolism}

Systemic (arterial) post-prandial amino acid concentrations are the result of net splanchnic release of $A A$ and whole body use of $A A$ for net protein synthesis. 
Post-prandial whole body protein synthesis is stimulated by AA appearing into the systemic circulation but also via the hormonal action of insulin $(4,35)$. Splanchnic extraction of AA coming from a meal is a major factor determining systemic availability of $A A$ for protein synthesis in the periphery. The systemic AA_N, EAA_N and nonEAA_N time courses were not different between the low quality meals. Remarkably, the EAA_N time courses were below baseline after $\mathrm{T}=2 \mathrm{~h}$ (ANOVA $T=2-6 h$. Gel:p=0.011, Gel+TRP:p=0.005) . This suggests that, although the intestinal amino acid metabolism was normalized by the TRP supplementation and systemic TRP concentration were elevated, other EAA_N amino acids in the Gel protein meals became limited for the stimulated whole body net protein synthesis, due to the fact that the ratio nonEAA_N/EAA_N was still not optimal in these meals. The below baseline values of the EAA_N, suggest that the demand for EAA_N is higher than the splanchnic area can deliver.

\section{Energy metabolism}

The comparable responses in post-prandial splanchnic glucose release indicate that net glucose metabolism in the splanchnic region was not influenced by the quality of the protein but that other processes in the periphery are responsible like an enhanced insulin response in the TRP supplemented meal (25).

In addition, net PDV production of lactate and alanine (products of the post-prandial glucose glycolysis) (36) was not different between the test meals, and differences in alanine content between the test meals had no effect on the net intestine production (data not shown). Therefore, we conclude that post-prandial intestinal glucose glycolysis was not changed.

The amount of lactate taken up by the liver during a normal high-quality meal was reduced, most likely due to inhibition of gluconeogenesis by the increase of insulin. However lactate (substrate for the gluconeogenesis) uptake in the low quality meal was much higher and reached comparable levels as in the post-absorptive state. This observation and the fact that a higher systemic concentration of lactate was found indicates that more lactate is produced in the periphery after intake of the low quality meal, resulting in enhanced turnover in the Cori-cycle. Elevated systemic lactate levels can stimulate gluconeogenesis (an energy consuming process) despite the portal net glucose appearance. It also indicates that a low quality meal can compromise metabolism in the periphery (muscle) and result in higher postprandial energy expenditure. Metabolic studies on a muscle compartment are necessary to confirm this hypothesis. Interesting, adding the deficient amino acid TRP to this meal resulted in normalization of lactate metabolism. 


\section{Gut health after a single low quality protein meal}

CIT production of the intestine can be a marker for gut function/health $(30,31)$. In the present study, PDV CIT production was not influenced by either the quality of the meal or the available amount of potential precursors in the meal (GLN, ARG or PRO), which suggests that the healthy gut is able to compensate to keep its major functions like CIT production when a low quality meal is given. It could well be that in this situation the intestine is breaking down endogenous proteins to secure continuity of protein synthesis of other endogenous proteins which is necessary for the most important intestinal function; absorption and barrier function. It is also possible that the intestine is compensating for the higher demand for AA's for peripheral protein synthesis that is stimulated by the meal itself (insulin). This supports the hypothesis that the intestine can act as buffer/interface for systemic AA bio-availability for the rest of the body in non-optimum food situations (11). Further studies with stable isotopes of AA are necessary to confirm this.

These data also support the hypothesis that the total amino acid profile of a protein meal determines the biological value of the meal for the intestine and is independent of the sources of the proteins $(20,37)$. Therefore, it is important particularly in vegetarian diets that a meal contains different protein sources to prevent that a complete meal becomes of very low of quality because of one deficient EAA_N.

\section{Conclusions}

After a low quality protein meal, the healthy gut is not able to generate an anabolic response. Our data suggest that in this situation, the intestine breaks down its own labile protein pool to fulfill the increased systemic amino acid demand. Supplementation of the deficient essential amino acid does improve intestinal metabolism, but the next limiting amino acid will determine the quality of the ingested protein for the remainder of the body. It is likely that chronic intake of low quality proteins can lead to exhaustion of the labile intestinal protein pool, possibly resulting in reduced function of the gut and susceptibility to intestinal and other disease states.

\section{Acknowledgements}

The authors wish to express many thanks to Hans M.H. van Eijk, PhD for analytical help and Mrs. Joyce Suyk and Mr. May C.F. Bost for help in the operative procedure

\section{References}

1. Dudley MA, Wykes LJ, Dudley AW, Jr., Burrin DG, Nichols BL, Rosenberger J, et al. Parenteral nutrition selectively decreases protein synthesis in the small intestine. Am J Physiol. 1998;274(1 Pt 1):G131-7.

2. Sax HC, Illig KA, Ryan CK, Hardy DJ. Low-dose enteral feeding is beneficial during total parenteral nutrition. Am J Surg. 1996;171(6):587-90. 

effects of blood on amino acid metabolism by intravenous isoleucine. Gastroenterology. 1991;101(6):1613-20.

4. Deutz NE, Ten Have GA, Soeters PB, Moughan PJ. Increased intestinal amino-acid retention from the addition of carbohydrates to a meal. Clin Nutr. 1995;14(6):354-64.

5. Wu G. Amino acids: metabolism, functions, and nutrition. Amino Acids. 2009;37(1):117. Epub 2009/03/21.

6. Van Der Schoor SR, Reeds PJ, Stoll B, Henry JF, Rosenberger JR, Burrin DG, et al. The high metabolic cost of a functional gut. Gastroenterology. 2002;123(6):1931-40. Epub 2002/11/28.

7. Reeds PJ, Burrin DG, Stoll B, van Goudoever JB. Role of the gut in the amino acid economy of the host. Nestle Nutr Workshop Ser Clin Perform Programme. 2000;3:25-40; discussion -6 .

8. Stein HH, Seve B, Fuller MF, Moughan PJ, de Lange CF. Invited review: Amino acid bioavailability and digestibility in pig feed ingredients: terminology and application. J Anim Sci. 2007;85(1):172-80. Epub 2006/12/21.

9. Ten Have GA, Engelen MP, Luiking YC, Deutz NE. Absorption kinetics of Amino Acids, Peptides, and Intact Proteins. International journal of sport nutrition and exercise metabolism. 2007;17(Supplement):S23-S36.

10. Deutz NE, Bruins MJ, Soeters PB. Infusion of soy and casein protein meals affects interorgan amino acid metabolism and urea kinetics differently in pigs. I Nutr. 1998;128(12):2435-45.

11. Soeters PB, de Jong CH, Deutz NE. The protein sparing function of the gut and the quality of food protein. Clin Nutr. 2001;20(2):97-9.

12. De Blaauw I, Deutz NE, van der Hulst RR, von Meyenfeldt MF. Glutamine depletion and increased gut permeability in nonanorectic, non-weight-losing tumor-bearing rats. Gastroenterology. 1997;112(1):118-26.

13. Bruins MJ, Luiking YC, Soeters PB, Lamers WH, Akkermans LM, Deutz NE. Effects of long-term intravenous and intragastric L-arginine intervention on jejunal motility and visceral nitric oxide production in the hyperdynamic compensated endotoxaemic pig. Neurogastroenterol Motil. 2004;16(6):819-28.

14. Campbell DI, Elia M, Lunn PG. Growth faltering in rural Gambian infants is associated with impaired small intestinal barrier function, leading to endotoxemia and systemic inflammation. J Nutr. 2003;133(5):1332-8. Epub 2003/05/06.

15. Campbell DI, Murch SH, Elia M, Sullivan PB, Sanyang MS, Jobarteh B, et al. Chronic T cell-mediated enteropathy in rural west African children: relationship with nutritional status and small bowel function. Pediatr Res. 2003;54(3):306-11. Epub 2003/06/06.

16. Kelly P, Menzies I, Crane R, Zulu I, Nickols C, Feakins R, et al. Responses of small intestinal architecture and function over time to environmental factors in a tropical population. Am J Trop Med Hyg. 2004;70(4):412-9. Epub 2004/04/22.

17. Guerrant RL, Oria RB, Moore SR, Oria MO, Lima AA. Malnutrition as an enteric infectious disease with long-term effects on child development. Nutr Rev. 2008;66(9):487-505. Epub 2008/08/30.

18. Ziegler TR, Evans ME, Fernandez-Estivariz C, Jones DP. Trophic and cytoprotective nutrition for intestinal adaptation, mucosal repair, and barrier function. Annu Rev Nutr. 2003;23:229-61.

19. Vidueiros SM, Fernandez I, Slobodianik N, Roux ME, Pallaro A. Nutrition disorder and immunologic parameters: study of the intestinal villi in growing rats. Nutrition. 2008;24(6):57581. Epub 2008/04/11. 
20. Reeds PJ. Dispensable and indispensable amino acids for humans. J Nutr. 2000;130(7):1835S-40S.

21. Luiking YC, Deutz NE, Jakel M, Soeters PB. Casein and soy protein meals differentially affect whole-body and splanchnic protein metabolism in healthy humans. J Nutr. 2005;135(5):1080-7.

22. Ten Have GA, Bost MC, Suyk-Wierts JC, van den Bogaard AE, Deutz NE. Simultaneous measurement of metabolic flux in portally-drained viscera, liver, spleen, kidney and hindquarter in the conscious pig. Lab Anim. 1996;30(4):347-58. Epub 1996/10/01.

23. Bruins MJ, Soeters PB, Lamers WH, Meijer AJ, Deutz NEP. L-Arginine supplementation in hyperdynamic endotoxemic pigs: Effect on nitric oxide synthesis by the different organs. Crit Care Med. 2002;30(3):508-17.

24. Stoll B, Burrin DG. Measuring splanchnic amino acid metabolism in vivo using stable isotopic tracers. Journal of animal science. 2006;84 Suppl:E60-72.

25. Cortamira NO, Seve B, Lebreton Y, Ganier P. Effect of dietary tryptophan on muscle, liver and whole-body protein synthesis in weaned piglets: relationship to plasma insulin. Br J Nutr. 1991;66(3):423-35. Epub 1991/11/01.

26. Bruins MJ, Deutz NE, Soeters PB. Aspects of organ protein, amino acid and glucose metabolism in a porcine model of hypermetabolic sepsis. Clin Sci (Lond). 2003;104(2):127-41.

27. Olde Damink SW, Deutz NE, Dejong CH, Soeters PB, Jalan R. Interorgan ammonia metabolism in liver failure. Neurochem Int. 2002;41(2-3):177-88.

28. van Eijk HM, Rooyakkers DR, Deutz NE. Rapid routine determination of amino acids in plasma by high-performance liquid chromatography with a 2-3 microns Spherisorb ODS II column. J Chromatogr. 1993;620(1):143-8.

29. De Backer D, Marx G, Tan A, Junker C, Van Nuffelen M, Huter L, et al. Arterial pressure-based cardiac output monitoring: a multicenter validation of the third-generation software in septic patients. Intensive Care Med. 2010. Epub 2010/12/15.

30. Lutgens LC, Blijlevens NM, Deutz NE, Donnelly JP, Lambin P, de Pauw BE. Monitoring myeloablative therapy-induced small bowel toxicity by serum citrulline concentration: a comparison with sugar permeability tests. Cancer. 2005;103(1):191-9.

31. Berkeveld M, Langendijk P, Verheijden JH, Taverne MA, van Nes A, van Haard P, et al. Citrulline and intestinal fatty acid-binding protein: longitudinal markers of postweaning small intestinal function in pigs? J Anim Sci. 2008;86(12):3440-9. Epub 2008/08/19.

32. Bos C, Stoll B, Fouillet H, Gaudichon C, Guan X, Grusak MA, et al. Postprandial intestinal and whole body nitrogen kinetics and distribution in piglets fed a single meal. Am J Physiol Endocrinol Metab. 2005;288(2):E436-46. Epub 2004/10/28.

33. Konturek SJ, Konturek PC, Brzozowski T, Bubenik GA. Role of melatonin in upper gastrointestinal tract. J Physiol Pharmacol. 2007;58 Suppl 6:23-52. Epub 2008/03/28.

34. Wapnir RA, Teichberg S. Regulation mechanisms of intestinal secretion: implications in nutrient absorption. J Nutr Biochem. 2002;13(4):190-9.

35. Fukagawa NK, Minaker KL, Rowe JW, Goodman MN, Matthews DE, Bier DM, et al. Insulin-mediated reduction of whole body protein breakdown. Dose-response effects on leucine metabolism in postabsorptive men. J Clin Invest. 1985;76(6):2306-11. Epub 1985/12/01.

36. Mithieux G, Rajas F, Gautier-Stein A. A novel role for glucose 6-phosphatase in the small intestine in the control of glucose homeostasis. J Biol Chem. 2004;279(43):44231-4. Epub 2004/08/11.

37. Reeds PJ, Burrin DG. The gut and amino acid homeostasis. Nutrition. 2000;16(7-8):666-8. 


\section{CHAPTER 5}




\title{
Enhanced lacto-tri-peptide bio- availability by co-ingestion of macronutrients
}

\author{
Gabriella A.M. Ten Have, Pieter C. van der Pijl, Arie K. \\ Kies and Nicolaas E.P. Deutz
}

PLoS One. 2015 Jun 22;10(6):e0130638

\section{Abstract}

Some food-derived peptides possess bioactive properties, and may affect health positively. For example, the C-terminal lacto-tri-peptides Ile-Pro-Pro (IPP), Leu-ProPro (LPP) and Val-Pro-Pro (VPP) (together named here XPP) are described to lower blood pressure. The bioactivity depends on their availability at the site of action. Quantitative trans-organ availability/kinetic measurements will provide more insight in C-terminal tri-peptides behavior in the body. We hypothesize that the composition of the meal will modify their systemic availability.

We studied trans-organ XPP fluxes in catheterized pigs ( $25 \mathrm{~kg} ; \mathrm{n}=10$ ) to determine systemic and portal availability, as well as renal and hepatic uptake of a waterbased single dose of synthetic XPP and a XPP containing protein matrix (casein hydrolyte, $\mathrm{CasH}$ ). In a second experiment ( $\mathrm{n=10)}$, we compared the CasH-containing protein matrix with a CasH-containing meal matrix and the modifying effects of macronutrients in a meal on the availability (high carbohydrates, low quality protein, high fat, and fiber).

Portal availability of synthetic XPP was $0.08 \pm 0.01 \%$ of intake and increased when a protein matrix was present (respectively 3.1, 1.8 and 83 times for IPP, LPP and VPP). Difference between individual XPP was probably due to release from longer peptides. CasH prolonged portal bioavailability with $18 \mathrm{~min}$ (absorption half-life, synthetic XPP: $15 \pm 2$ min, CasH: $33 \pm 3$ min, $p<0.0001)$ and increased systemic elimination with $20 \mathrm{~min}$ (synthetic XPP: $12 \pm 2 \mathrm{~min}$; CasH: $32 \pm 3 \mathrm{~min}, \mathrm{p}<0.0001$ ). Subsequent renal and hepatic uptake is about $75 \%$ of the portal release. A meal containing $\mathrm{CasH}$, increased portal 1.8 and systemic bioavailability 1.2 times. Low protein quality and fiber increased XPP systemic bioavailability further (respectively 1.5 and 1.4 times).

We conclude that the amount and quality of the protein, and the presence of fiber in a meal, are the main factors that increase the systemic bioavailability of foodderived XPP. 


\section{Introduction}

In the recent years our understanding of potential food-derived bioactive peptides and their potential health benefits advanced $(1,2)$, mainly on the role of bioactive peptides from milk. For instance, the effects on blood pressure of the lacto-tri-peptides Isoleucine-Proline-Proline (IPP) and Valine-Proline-Proline (VPP) is described in animal (3) and human studies (4-12). The hypothesized working mechanism of these peptides is inhibition of the angiotensin I converting (ACE) enzyme (4) and is related to the presence of a proline residue on the C-terminal of the lacto-tri-peptides (13). Animal and human studies also suggest that IPP and VPP reduce arterial stiffness and improve endothelial activity $(14,15)$.

The beneficial effects of food-derived bioactive peptides depend on availability on the site of action. For instance, to exert a potential ACE inhibition effect after oral intake of the lacto-tri-peptides, the peptides have to reach the cardiovascular system in an active form (16). Metabolic active organs like the intestine, liver, and kidney play a major role in the availability. Fluxes of these peptides through those organs are likely to be dependent on the fluxes of other macronutrients, especially proteins and amino acids (17-22). No fundamental knowledge of quantitative organ flux data are available for food-derived bioactive lacto-tri-peptides to get more insight in the behavior of those peptides in the body (23). Therefore, the main aim of the present study is to collect quantitative data about how much food-derived bioactive lacto-tri-peptides is absorbed and released to the portal system, the role of kidney and liver and their effect on the systemic availability under different nutritional conditions. We studied IPP, VPP and LPP (Leucine-Proline-Proline), named together as XPP as model for C-terminal proline containing food-derived peptides in general, because they are relatively resistant against breakdown in the gastrointestinal tract and therefore expected to be similar in their kinetics $(3,24)$.

Previously, we found that the systemic bioavailability of a single dose of synthetic XPP, when given orally as a single dose, was less than $0.1 \%$ (25) with a half-life maximum at $15 \mathrm{~min}$. In the present study, we hypothesize that dietary composition modifications improve absorption kinetics of XPPs and thus their bioavailability.

Therefore, we studied trans-organ XPP fluxes in catheterized pigs to determine the effect of a protein matrix on the XPP systemic and portal availability, renal and hepatic uptake using a XPP containing casein hydrolyte (CasH). Casein is the main lacto-protein that is rich of encrypted XPP's (www.genome.jp). This, in contrast with the lacto-protein whey or a non-milk protein soy with no encrypted XPP's. The studied $\mathrm{CasH}$ is rich of liberated IPP and LPP, produced by using enzymes including a specific prolyl-endoprotease (26). This CasH is proven to have ACE inhibitory potential (12) and has a faster digestion and absorption rate (27) than casein, which potentially relates to the maximally available systemic XPP concentrations. 
In a second experiment, we studied the potential effect on XPP availability when the XPP containing CasH was added to a meal and studied the potential modifying effects of macro-nutrients (high carbohydrates, low quality protein, high fat, and fiber) in the meal. Quantification of intestinal absorption kinetics of XPP and the role of liver and kidney is not possible in humans, therefore we measured the transorgan XPP fluxes in conscious multi-catheterized pigs $(28,29)$ as the gastro-intestinal tract of the pig is comparable with that of humans (30-32).

The quantitative results of the present study are important as crucial fundamental information for the development of therapies with bioactive peptides.

\section{Materials and Methods}

\section{Animals}

For each study, we used 10 pathogen-free, female piglets (Dutch landrace $x$ Yorkshire; 8-12 weeks of age; $25.2 \pm 1.1 \mathrm{~kg}$ body weight) that were adapted to individual housing in straw containing pens $(2 \times 3 \mathrm{~m})$ with $12 \mathrm{~h}$ day-night cycle and an environmental temperature of $23^{\circ} \mathrm{C}$ one week before surgery. Catheters for blood sampling were placed during surgery (28) under isoflurane ( $2 \%$ mixture with oxygen) anesthesia and flunixin meglumine (50 mg/25 kg bw) analgesia, in the abdominal aorta, the portal vein, the hepatic vein, and the renal vein. Catheters for infusions of post-operative medication or para-aminohippuric acid solution (PAH; flow measurements) were placed in the central vein and splenic vein and for gastric administration a gastric catheter. We checked the position of the catheter tips with X-ray during surgery using fluoroscopy and an iodine containing contrast solution.

During the recovery period (7-10 days), twice daily, we and the assigned veterinarian of the animal care facility monitored the animals for general behavior, body temperature, food intake, body weight and catheter patency and administered buprenorphine $(0.03 \mathrm{mg} / \mathrm{kg})$ analgesia, intra-venous, twice daily on day $1-4$ and when needed. Animals were accustomed to a small movable cage $(0.9 \times 0.5 \times 0.3 \mathrm{~m})$ to perform the experiments. At all times, animals would be euthanized with $0.5 \mathrm{ml} / \mathrm{kg}$ bw Euthanasol ${ }^{\circledR}$ (390 mg pentobarbital sodium +50 mg phenytoin sulfate $/ \mathrm{ml}$ ), intravenous, if imminent death is expected.

\section{Ethics Statement}

The animal ethics committee of Maastricht University, The Netherlands approved the studies: 2004-101. We performed the surgery under isoflurane anesthesia, and all efforts were made to minimize suffering. The researchers and the assigned veterinarian of the animal care facility monitored the anesthesia and recovery closely. 


\section{Experimental protocol}

\section{Study 1}

After a postoperative recovery period of 7-10 days, we conducted five test days with XPP mixtures according to a Williams cross-over design (33) with a minimum of 2 days (washout) between the test days. One intra-venous administered IPP, LPP and VPP mixture (data previously published (25)) and four intra-gastric administered XPP mixtures were studied (Table 1). We studied a control solution (Control-group) without XPP, but iso-ionogenic to the other mixtures for sodium, potassium and calcium ions, to determine potential occurring endogenous XPP fluxes. We gave a water-based matrix with comparable amounts of IPP, LPP and VPP (XPP-group) for comparison with a protein based XPP mixture (casein hydrolyte, CasH-group). The CasH contained known amounts of free XPP with a C-terminal proline. We gave a XPP spiked protein matrix (CasH+XPP group) to evaluate the influence of a protein matrix on individual XPP's. In the present study, we only will discuss the data of the four intra-gastric administered XPP. We administered these mixtures as a single bolus with an end volume of $14 \mathrm{ml} / \mathrm{kg}$ body weight and temperature of $37^{\circ} \mathrm{C}$.

Table 1. Composition of test mixtures - Study 1

\begin{tabular}{llllll}
\hline Substance & Unit & Control & XPP & CasH $^{1}$ & CasH+XPP \\
\hline IPP $^{2}$ & $\mu \mathrm{mol} / \mathrm{kg} \mathrm{bw}$ & 0.0 & 12.3 & 10.7 & 23.0 \\
$\mathrm{LPP}^{2}$ & $\mu \mathrm{mol} / \mathrm{kg} \mathrm{bw}$ & 0.0 & 12.3 & 32.9 & 45.2 \\
$\mathrm{VPP}^{2}$ & $\mu \mathrm{mol} / \mathrm{kg} \mathrm{bw}$ & 0.0 & 12.8 & 0.62 & 13.4 \\
Total XPP $^{3}$ & $\mu \mathrm{mol} / \mathrm{kg} \mathrm{bw}$ & 0.0 & 37.4 & 44.2 & 81.6 \\
Protein & $\mathrm{mg} / \mathrm{kg} \mathrm{bw}$ & 0.0 & 0.0 & 648 & 648 \\
Sodium & $\mathrm{mM}$ & 3.01 & 3.01 & 3.01 & 3.01 \\
Potassium & $\mathrm{mM}$ & 71.14 & 71.14 & 71.14 & 71.14 \\
Calcium & $\mathrm{mM}$ & 1.96 & 1.96 & 1.96 & 1.96 \\
Phosphate & $\mathrm{mM}$ & 71.1 & 71.1 & 102.9 & 102.9 \\
\hline
\end{tabular}

1 Chemical purities of the IPP, LPP, and VPP synthetic products were 93.4, 95.0 and 98.7\%, respectively (Bachem, Weil am Rhein, Switzerland).

2 The given amounts of tri-peptides isoleucine-proline-proline ( IPP), leucine-proline-proline (LPP), valine-proline-proline (VPP) in the casein hydrolysate (CasH, Casimax ${ }^{\circledR}$, DSM Food Specialties, Delft, The Netherlands). The casein hydrolysate contained 57\% protein with 5.4 , 16.5 and $0.3 \mathrm{mg} / \mathrm{g}$ protein of LPP, LPP and VPP, respectively.

3 Total XPP = total amount of IPP, LPP and VPP.

\section{Study 2}

After a postoperative recovery period of 7-10 days, we conducted six test days with CasH interventions according to a Williams cross-over design (33) with a minimum of 2 days (washout) between the test days. We compared a CasH supplemented meal matrix (Basal-group) to a CasH only protein matrix (CasH2-group). We also compared four other CasH supplemented meals with low protein quality (LQprot-group), 
high carbohydrate (hCHO-group), high fat (hFat-group) and fiber containing Basal meal (Fiber-group) to Basal to evaluate the influence of macronutrients (Table 2). The Basal meal contains a ratio of 25:50:25 En\% of the macronutrients protein, carbohydrates and fat. All meals were iso-caloric (30\% of daily intake: $37.2 \mathrm{kcal} / \mathrm{kg}$ bodyweight) and composed by Research Diet Services (Wijk bij Duurstede, The Netherlands). The protein fraction in the meals was a high quality whey protein isolate, while in the low quality protein diet a soy protein isolate that had $24 \%$ less essential amino acids. We gave all meals intra-gastric as a single bolus with an end volume of $24 \mathrm{ml} / \mathrm{kg}$ bodyweight and $37^{\circ} \mathrm{C}$ and observed for $360 \mathrm{~min}$.

\section{Table 2. Composition of test meals - Study 2}

\begin{tabular}{|c|c|c|c|c|c|c|c|c|}
\hline & Nutrient & Units & CasH2 & Basal & LQprot & $\mathrm{hCHO}$ & hFat & Fiber \\
\hline \multirow[t]{9}{*}{ Meal } & Whey protein & Energy \% & 0 & 25 & 0 & 25 & 25 & 25 \\
\hline & Soy protein & Energy \% & 0 & 0 & 25 & 0 & 0 & 0 \\
\hline & $\mathrm{CHO}$ & Energy \% & 0 & 50 & 50 & 65 & 25 & 50 \\
\hline & Fat & Energy \% & 0 & 25 & 25 & 10 & 50 & 25 \\
\hline & Total energy intake & $\mathrm{kcal} / \mathrm{kg}$ bw & 0 & 37.2 & 37.2 & 37.2 & 37.2 & 37.2 \\
\hline & $\begin{array}{l}\text { Whey protein } \\
\text { isolate }^{2}\end{array}$ & $\mathrm{~g} / \mathrm{kg} \mathrm{bw}$ & 0 & 2.32 & 0 & 2.32 & 2.32 & 2.32 \\
\hline & Soy protein isolate ${ }^{2}$ & $\mathrm{~g} / \mathrm{kg} \mathrm{bw}$ & 0 & 0 & 2.32 & 0 & 0 & 0 \\
\hline & $\mathrm{CHO}^{\S}$ & $\mathrm{g} / \mathrm{kg}$ bw & 0 & 4.64 & 4.64 & 6.04 & 2.32 & 4.64 \\
\hline & $\mathrm{Fat}^{\S \S}$ & $\mathrm{g} / \mathrm{kg} \mathrm{bw}$ & 0 & 1.04 & 1.04 & 0.42 & 2.07 & 1.04 \\
\hline \multirow[t]{2}{*}{ Supplement } & Casein hydrolysate $^{+}$ & $\mathrm{g} / \mathrm{kg}$ bw & 0.73 & 0.73 & 0.73 & 0.73 & 0.73 & 0.73 \\
\hline & Fiber $^{t+}$ & $\mathrm{g} / \mathrm{kg}$ bw & 0 & 0 & 0 & 0 & 0 & 0.18 \\
\hline \multirow[t]{3}{*}{ Tri-peptides ${ }^{1}$ ) } & IPP & $\mu \mathrm{mol} / \mathrm{kg} \mathrm{bw}$ & 12.1 & 12.1 & 12.1 & 12.1 & 12.1 & 12.1 \\
\hline & LPP & $\mu \mathrm{mol} / \mathrm{kg} \mathrm{bw}$ & 37 & 37 & 37 & 37 & 37 & 37 \\
\hline & VPP & $\mu \mathrm{mol} / \mathrm{kg} \mathrm{bw}$ & 0.7 & 0.7 & 0.7 & 0.7 & 0.7 & 0.7 \\
\hline Gross weight & & $\mathrm{g} / \mathrm{kg} \mathrm{bw}$ & 1.28 & 9.80 & 9.94 & 10.7 & 8.32 & 9.99 \\
\hline End Volume & & $\mathrm{ml} / \mathrm{kg}$ bw & 24 & 24 & 24 & 24 & 24 & 24 \\
\hline
\end{tabular}

1 Indicated is the amount of protein supplied by the isolates.

$2 \mathrm{CHO}$ : maize starch, sucrose and glucose in the weight ratio 2:1:1.

3 Fat: soybean oil and sunflower oil in the weight ratio 4:1.

4 The casein hydrolysate product (Casimax ${ }^{\circledR}, \mathrm{DSM}$ Food Specialties, Delft, The Netherlands) contained $57 \%$ protein with $5.4,16.5$ and $0.3 \mathrm{mg} / \mathrm{g}$ protein LPP, LPP and VPP, respectively.

5 Fiber: modified citrus pectin.

Sixteen hours before each test day, we removed food. Plasma flow determination started $1 \mathrm{~h}$ before feeding ( $\mathrm{t}=0$ ) and continued throughout the experiment by a primed-continuous infusion of para aminohippuric acid through the splenic infusion 
catheter. At steady state concentrations of PAH (1 hour (28)), we took blood samples from the arterial, portal, hepatic, and renal vein. We gave the liquid test mixture or meal via the gastric catheter within 5 min. Subsequently, we took blood samples (1.5 $\mathrm{ml} / \mathrm{sample}$ ) in study 1 from the arterial, portal, hepatic and renal catheter at $\mathrm{T}=1,3,5$, $7,9,12.5,17.5,25,30,40,50,60$ and 90 min; in study 2 from the arterial and portal catheter at $T=10,20,40,60,90,120,150,180,210,240,270,300,330$ and 360 min.

\section{Sample processing}

Directly after collection in Lithium-Heparin tubes, we placed the blood samples on ice. We processed all blood samples within 1 hour after collection to ensure stability of the substances. We centrifuged at $4^{\circ} \mathrm{C}$ for $15 \mathrm{~min}$ at $3000 \mathrm{~g}$ to obtain plasma. For PAH concentration determinations we added $40 \mu \mu \mathrm{l}$ of $50 \% \mathrm{w} / \mathrm{v}$ Tri-chloroacetic acid solution (TCA) to $400 \mu \mu$ of plasma and mixed. For XPP analyses, we added $10 \mu \mu \mathrm{l}$ of $10 \% \mathrm{w} / \mathrm{v}$ tri-fluoroacetic acid (TFA) to $500 \mu \mu \mathrm{l}$ plasma and mixed. We froze the samples in liquid nitrogen and stored at $80^{\circ} \mathrm{C}$ until further analysis. We took samples from the PAH infusion solutions and the test mixtures.

\section{Biochemical analyses}

We analyzed the plasma samples and test mixtures for the peptides IPP, LPP and VPP (XPP) content $(25,34)$. We added $50 \mu$ internal standard solution, containing U-13C-IPP, U-13C-VPP, and U-13C-LPP to homogenized plasma (20 $\mu \mathrm{l})$ and $480 \mu \mathrm{l}$ water. After mixing, we acidified this aliquot with tri-fluoroacetic acid to $\mathrm{pH}<3$. We removed proteins by heating the aliquot at $95^{\circ} \mathrm{C}$ for $2 \mathrm{~min}$, followed by centrifugation at $22,000 \mathrm{~g}$ for $30 \mathrm{~min}$ at $15^{\circ} \mathrm{C}$. We quantified the XPP's in the supernatant with LC-MS (Quattro Ultima, Waters, Milford, MA, USA). The detection limit of quantification of this procedure was $0.28,0.28$, and $0.71 \mathrm{nM}$ for IPP, LPP, and VPP respectively.

We tested samples of test mixtures by mixing $100 \mu \mathrm{l}$ of the sample with $100 \mu \mathrm{l}$ of a standard solution of universally 13C- labeled IPP [U-13C-IPP] and VPP [U-13CVPP] (Biopeptide Co., San Diego, CA, USA). Then we mixed for $1 \mathrm{~min}$, followed by centrifugation for $20 \mathrm{~min}$ at $16,000 \times \mathrm{g}$ at room temperature. This procedure allows determination of XPPs in the test mixtures between 1.9 and $123 \mu \mathrm{M}$.

We analyzed in a pilot set of plasma samples from Study 1, the occurrence of dipeptides with proline (Proline-Proline (PP), leucine (LP), valine (VP), isoleucine (IP)). We determined di-peptide concentrations with an automated LC-ESI-MS system (QTrap 5500 MS (AB Sciex, Foster City, CA, USA) with ExpressHT Ultra LC (Eksigent Div., AB Sciex, Foster City, CA, USA). We added supernatant (20 $\mu$ l) of centrifuged TCA deproteinized plasma to a $0.1 \mathrm{~N}$ hydrochloric acid containing internal standard $(20 \mu \mathrm{l})$ of a stable isotopomer [D8-valine, D5-proline]. We derivatized internal standard containing samples and di-peptide external standards with 9-Fluorenylmethoxycarbonyl (Fmoc), neutralized and injected $160 \mathrm{~nL}$ of the solution 
on a micro LC column $0.5 \times 100 \mathrm{~mm}$ HALO C18, $2.7 \mathrm{um}, 90 \mathrm{~A}$ pores at $35^{\circ} \mathrm{C}$. We eluted the analytes with a segmentally linear gradient from 35\% to $85 \%$ acetonitrile in water supplemented with ammonium acetate to $10 \mu \mathrm{M}$ and $5 \%$ isopropanol. We detected by electrospray triple quadrupole tandem mass spectrometry in multiple reactions monitoring mode. For concentration calculations of di-peptides, we normalized MS signals of the samples and the external standards with their internal standard. We determined di-peptide concentrations with the calibration curve of the external standards.

We determined plasma PAH concentrations with a standard enzymatic method on an automatic spectrophotometric analyzer (Cobas Mira, Hoffmann-La Roche, Basel, Switzerland) as described (22, 30-32). In brief, we measured plasma PAH concentration at 465 nm using Ehrlich's reagent: $1 \mathrm{~g}$ p-dimethylaminobenzaldehyde (Merck, Darmstadt, Germany), $35 \mathrm{ml}$ alcohol 96\%, 4 ml TCA 2N, $61 \mathrm{ml}$ water (bi-distilled) and a solution of $35 \%$ alcohol.

\section{Calculations}

\section{Systemic pharmacokinetics}

Using a 1-compartment model (25) we determined in study 1, absorption half-life (t t12,a;min), time to maximum plasma concentration (tmax;min), maximum plasma concentration (Cmax;nM), elimination half-life (t $1 / 2, e ; \mathrm{min})$ and systemic absolute bioavailability (fAbs;\%). For study 2 , we used a non-compartmental analysis calculate AUCs of the arterial XPP time curves from 0 to $360 \mathrm{~min}$ (nmol//*min). We calculated the systemic bioavailability relative to regular, basal meal using the following equation:

$f_{\text {Rel }}=\left(\right.$ AUC $\left._{\text {Basal }}{ }^{*} D_{\text {test }}\right) /\left(\right.$ AUC $_{\text {Test }}{ }^{*}$ DBasal $^{*}{ }^{* 100 \%}$

where $\mathrm{fRel}_{\mathrm{R}}=$ the bioavailability for a administered $\mathrm{XPP}$ from a meal relative to Basal (\%), $A \cup C_{\text {Basal }}=$ the $A \cup C$ for Basal (nmol//*min), DTest $=$ the dose of an XPP in the meal (nmol), $A \cup C_{\text {Test }}=$ the $A \cup C$ for the meal (nmol//*min), DBasal $=$ the XPP dose of Basal (nmol).

\section{XPP organ fluxes}

We measured plasma flow, necessary to calculate amino acid fluxes across organs. We calculated PDV fluxes of IPP, LPP and VPP by portal arterial XPP concentration difference times portal plasma flow, liver fluxes by subtracting PDV flux from splanchnic flux (hepatic arterial difference* liver plasma flow) and renal flux by renal plasma flow times renal-arterial XPP concentration difference $(22,28,31,32,35)$. We expressed organ flux as pmol/kg body weight/min and a positive flux is net release of XPP and negative flux net uptake. 
We calculated total net release or uptake (=organ net balance) of XPP across an organ from the area under curve of the respective organ flux time course. We expressed total net balance as pmol/kg bodyweight. For the PDV, the portal bioavailability is the total net balance as fraction dose of IPP, LPP or VPP in the test mixtures/ meals. We calculated the free available XPP in the test mixtures of study 1 or test meals in study 2 from information, made available by the manufacturer.

\section{Statistical procedures}

We used Prism 6 (Graphpad Software, La Jolla, CA, USA) for statistics, expressed the results as means \pm SEM and set the level of significance on $p<0.05$ and tendencies are defined as $p<0.01$. We tested the data for normality and used the Wilcoxon test to determine if means are different from zero.

Comparisons between matrixes and mean XPP responses where done with two-way ANOVA. When appropriate, followed by post-hoc test (Sidak's, Dunnett's, Fisher's LSD or Wilcoxon Signed Rank test as indicated in tables and figures) for planned physiological relevant multiple comparison.

We compared the means of two groups with unpaired t-test or Wilcoxon matched pairs signed rank test, means of more than three groups with One-way repeated measures ANOVA. When appropriate, followed by a post-hoc test (Dunnett's) for planned physiological relevant multiple comparison.

We compared means of groups in the time courses with two-way ANOVA test, mixed model: repeated measurements for time (fixed effect) but not for test mixtures, for planned physiological relevant comparisons.

\section{Results}

\section{Effect of a protein matrix on systemic bioavailability and pharmacokinetics of XPP peptides.}

Systemic (=arterial) concentrations of XPP in time (Figure 1) showed no measurable baseline/post-absorptive endogenous XPP (Control group, tested with Wilcoxon). The XPP concentrations in the CasH groups were not back to baseline at 90 min post-prandial (one-way ANOVA with Dunnett's post-hoc test: IPP $p<0.002$; LPP $p<0.001$; VPP $p<0.05)$. The calculated systemic absolute bioavailability (fabs $(\%)=$ fraction dose absorbed, Table 3) was increased in the protein based $\mathrm{CasH}$ group (IPP: 4.7 times, $p<0.0004$; LPP: 2.5 times, $p<0.002$; VPP: 121 times, $p<0.0001$ ). The spiked CasH group (CasH+XPP) showed lower systemic bioavailability for VPP (65 times, $\mathrm{p}<0.0001)$ in comparison to $\mathrm{CasH}$. There was a delay in the time to maximum 


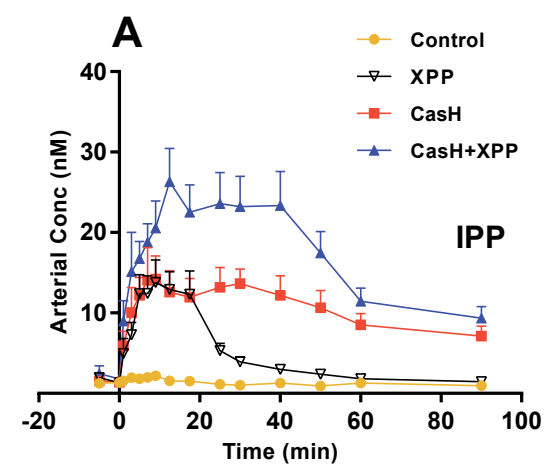

B
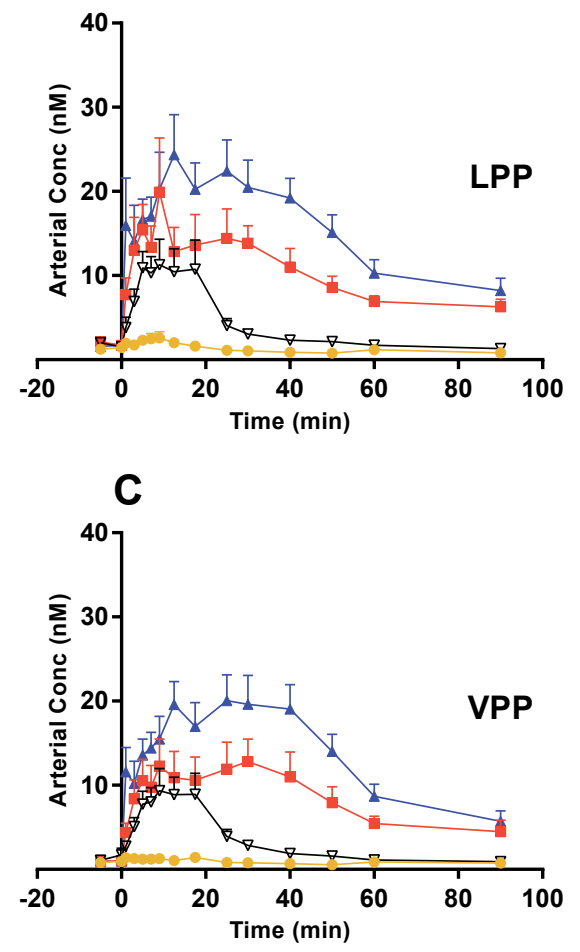

Figure 1. Systemic levels of XPP peptides - Effect of protein matrix

Post-prandial arterial concentrations after intra-gastric administration of control salt solution (Control), synthetic XPP's (XPP), casein hydrolysate rich in XPP (CasH) and spiked CasH (CasH + XPP). A: Isoleucine-proline-proline (IPP). B: Leucine-proline-proline (LPP). C: Valine-prolineproline (VPP). Respective number of observations for Control, XPP, CasH and CasH +XPP are for graph $A: n=9,10,9$ and 10; graph $B: n=10,10,9$ and 10; graph $C: n=8,10,9$ and 10. Values are mean \pm SEM. Statistics: repeated measures two-way ANOVA, mixed model, planned comparisons. All curves are significantly different from the XPP mixture: effect test mixture $p<0.001$; effect time $p<0.001$; interaction $p<0.001$. 


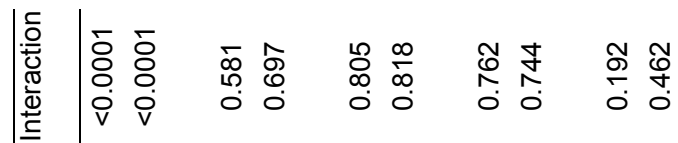

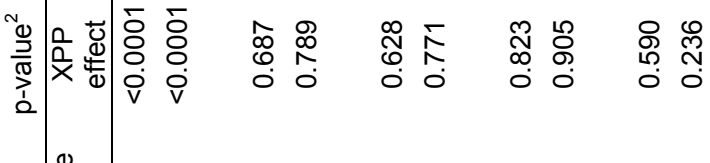

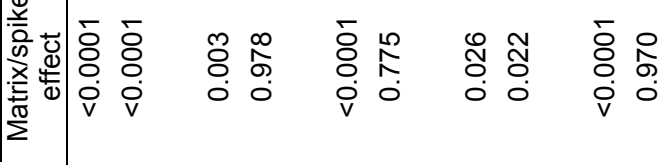

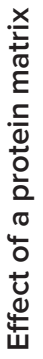

ชั

虽

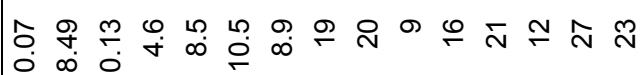

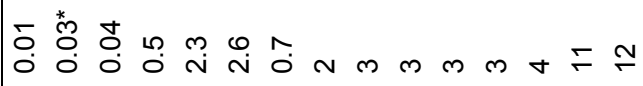

모

$+1+1+1+1+1+1+1+1+1+1+1+1+1+1$
$+1+2$

舟

б

呈

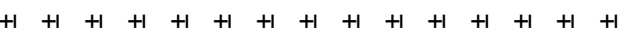

号

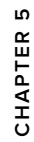

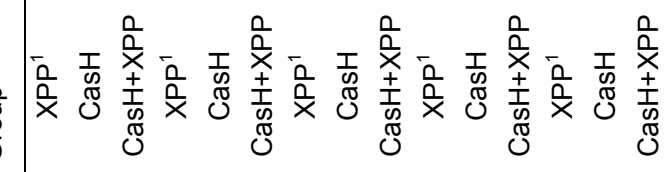

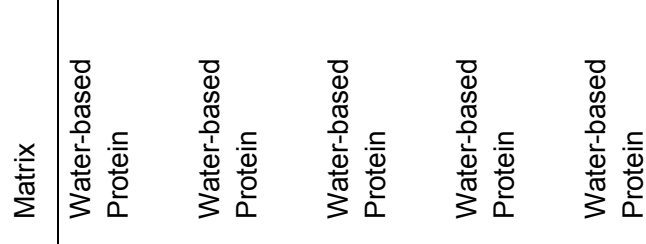

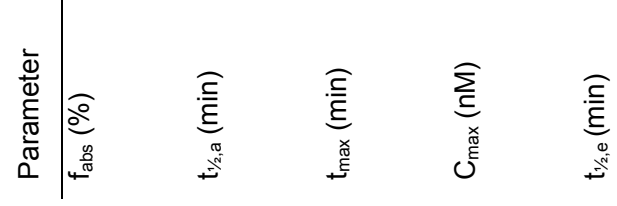


Table 3 (left). Systemic bioavailability and pharmacokinetics of XPP peptides - Effect of a protein matrix

Pharmacokinetic parameters derived from the systemic response of an intra gastric bolus administrated of the tri-peptides isoleucine-proline-proline (IPP), leucine-proline-proline (LPP), valine-proline-proline (VPP) in water-based matrix (synthetic XPP) or in a protein matrix (casein hydrolysate rich in XPP: CasH). fabs (\%): systemic absolute bioavailability, t1/2,a: absorption half-life, tmax: time to maximum plasma concentration, $\mathrm{C}_{\text {max: }}$ maximum plasma concentration, $t \frac{1}{2}$,e: elimination half-life. Parameters calculated with a 1-compartment model. Values are means \pm SEM. For fabs $n=8$. For other parameters XPP: $n=10$; CasH: $n=10 ;$ CasH+XPP: $n=9$. Significance: $p<0.05$.

1): Data XPP group from the present study 1, are published in van der Pijl et al. (25).

2): Significance for comparison of water-based matrix (XPP) with protein matrix (CasH). Or significance for comparison between XPP spiked (CasH+XPP) and non-spiked protein matrix (CasH): Two-way ANOVA. When appropriate, a post-hoc unpaired t-test is done:

*): $\mathrm{p}<0.05$ significance for comparison IPP, LPP or VPP of XPP relative to CasH.

\#): $p<0.05$ significance for comparison IPP, LPP or VPP of CasH relative to CasH+ XPP.

XPP plasma concentration ( $\mathrm{t}_{\max }$ ) with the CasH groups compared to the XPP test mixture (12 min, $p<0.0001$, Wilcoxon matched pairs signed rank test). The calculated absorption half-life (t $1 / 2, a)$ and elimination half-life $\left(t \frac{1}{2}, \mathrm{e}\right)$ was delayed significantly in CasH groups (resp. 6 min and 20 min, p<0.0001, Wilcoxon matched pairs signed rank test). Plasma concentrations of the dipeptides LP, IP, VP and PP in the postprandial period, measured in a limited set of samples, were in the range of 1-7 $\mu \mathrm{M}$, compared to 1-30 nM for XPP's in this sample set (Supplemental Table 1. Dipeptide/ tripeptide ratio - Study 1).

\section{Effect of meal matrix on portal availability of XPP peptides}

PDV fluxes showed a net release of all XPP after intra gastric administration of XPP in different matrixes (Figure 2). We observed no endogenous production of XPP by the PDV. The patterns of the curves were comparable for IPP, LPP and VPP. No differences in PDV plasma flows were observed (Supplemental Table 2. Plasma flows - Study 1.). The single dose water-based synthetic XPP matrix, showed comparable portal availability for IPP, LPP and VPP, with an average of $0.08 \pm 0.01 \%$ of the intake (Table 4). The portal availability was higher in a protein (CasH) matrix than in waterbased XPP matrix, but different between IPP, LPP and VPP (IPP 3.1 times, $p=0.026$; LPP 1.8 times, $p=0.23$; VPP 83 times, $p<0.0001$ ). For VPP, the spiked CasH showed a different portal availability in comparing to the non-spiked CasH matrix (0.05 times of $\mathrm{CasH}, \mathrm{p}<0.0001)$. To understand the significant XPP effect and spike effect, data were further analyzed, by expressing the data as \% of theoretically intake of XPP (Supplemental Table 3. Theoretical XPP intake and their portal availability - Study 1.), considering the tri-peptide sequences in the source of the $\mathrm{CasH}$ : amino acid sequence of bovine $k$ and $\beta$ casein (www.genome.jp; CASB-BOVIN, CASK-BOVIN). No spike effect of VPP was observed when corrected of potential non free available 

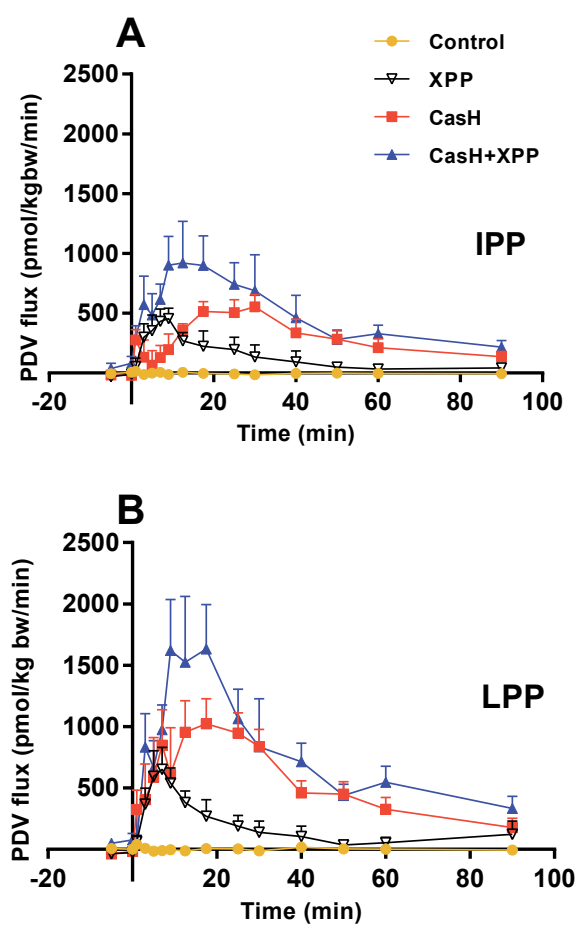

88

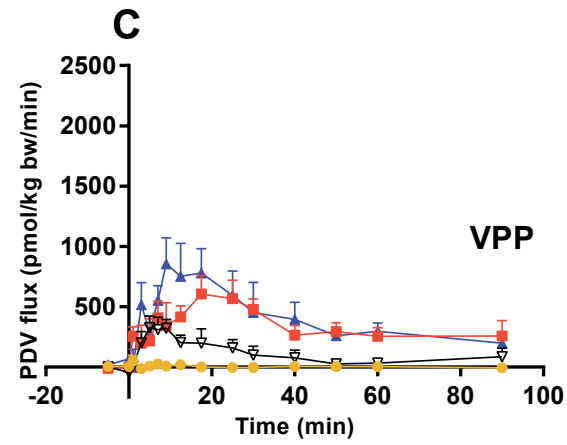

Figure 2. Portal Drained Viscera fluxes of XPP - Effect of a protein matrix

Post-prandial portal drained viscera (PDV) fluxes after intra-gastric administration of tri-peptide (XPP) mixtures: control salt solution (Control), synthetic XPP's (XPP), casein hydrolyte rich in XPP (CasH) or spiked CasH (CasH + XPP). A: Isoleucine-proline-proline (IPP). B: Leucineproline-proline (LPP). C: Valine-proline-proline (VPP). Respective number of observations for Control, XPP, CasH and CasH +XPP are for graph A: $n=6,9,8$ and 9; graph B: $n=6,10,9$ and 9; graph $C: n=5,9,9$ and 9 . Values are mean \pm SEM. Positive values is net release, negative values is net uptake. Statistics: repeated measures two-way ANOVA, mixed model, planned comparisons. All curves are significantly different from the XPP mixture: effect test mixture $p<0.01$; effect time $p<0.01$; interaction $p<0.01$. 


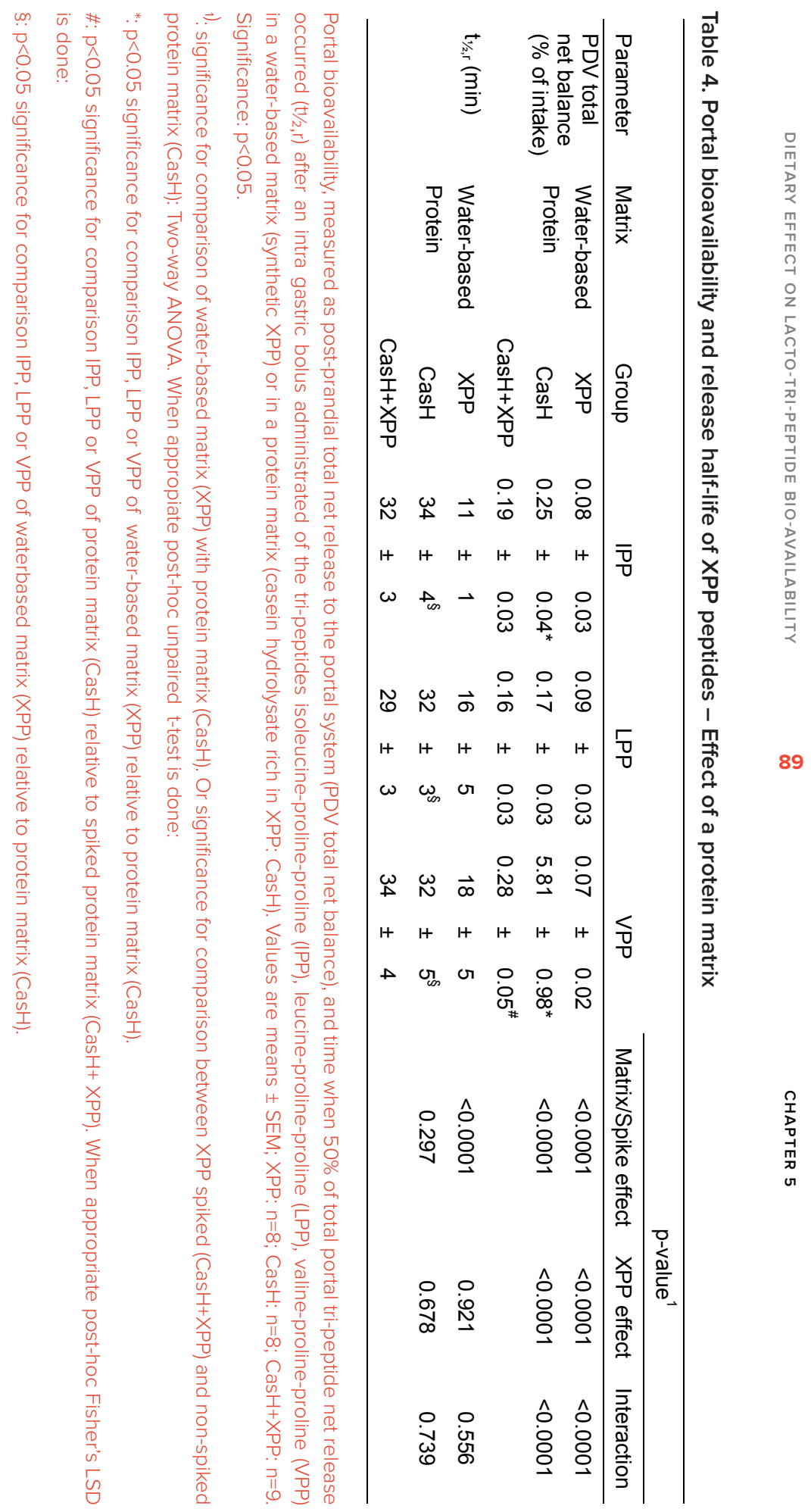


VPP sequences in the $\mathrm{CasH}$. With this correction we determined that on average the portal availability was 1.8 times higher in the protein matrix (XPP $0.08 \pm 0.01$ vs CasH $0.14 \pm 0.02$, two-way ANOVA, matrix effect, $p=0.004$ ). Further analyses of the PDV flux curves showed an increase of 2.1 times of the portal release half-life (Table 4 ) in the protein matrix in comparison to the water-based matrix (XPP: $15 \pm 2$ min vs CasH: $33 \pm 3$ min, two-way ANOVA, matrix effect $<0.0001$ ).

\section{Multiple organ interactions of XPP peptides}

In Study 1, we determined the XPP fluxes across multiple organs, to understand more about the influence of different organs on the systemic availability. We found no effect of the matrixes on the plasma flows of PDV, liver and kidneys (Supplemental Table 4). In the post-absorptive Control group, total net balances of XPP were not different from zero (Figure 3). Post-prandial balances showed a net release of XPP by the PDV, followed by a partial uptake by the liver, resulting in net release of XPP by the splanchnic area. Systemic available XPP are in part, taken up by the kidneys.

\section{Effect of meal matrix on the systemic availability of XPP peptides}

In study 2, we compared XPP responses between a protein and a meal matrix and the potential effects of different macro-nutrients in a meal matrix. With the Basal meal matrix, the systemic availabilities of XPP's were 1.2 times ( $p=0.019$, Wilcoxon rank test) increased over a post-prandial period of six hours in comparison to the protein matrix CasH2 (Table 5). The low quality protein and fiber were the macronutrients that increased the XPP systemic availability in a meal further with resp. 1.5 and 1.4 times in comparison to the Basal meal. (Two-way ANOVA with post-hoc Dunnett's test; macronutrient effect: resp. $p=0.0003$ and $p=0.0009$ ).

\section{Effect of a meal matrix on portal availability of XPP peptides}

Expressed as the fraction dose of total PDV net balance over the experimental period of six hours, the portal availability was increased with an average 1.8 times (56 $\pm 7 \%$ of Basal, two-way ANOVA, matrix effect, <0.0001) if the XPP containing CasH was given with a complete meal (Table 6). In contrast with the systemic bioavailability, we did not observe differences between the basal and the other meals and in PDV plasma flows (Supplemental Table 4. PDV Plasma flows - Study 2.). Further analysis of the PDV flux curves showed an increase of 2.6 times of the portal release half-life (Table 7) in the meal matrix in comparison to the protein matrix (CasH2: $47 \pm 5$ min; Basal: $122 \pm 11$ min; two-way ANOVA, matrix effect <0.0001). Also, the portal release half-life was reduced in a low quality protein meal 1.5 times, and increased 1.3 times in a high fat containing meal (LQprot: $80 \pm 7$; hFat: 163 \pm 10 ; two-way ANOVA with post-hoc Dunnett's multiple comparison, macronutrient effect resp. $p=0.009$ and $p=0.014)$. 

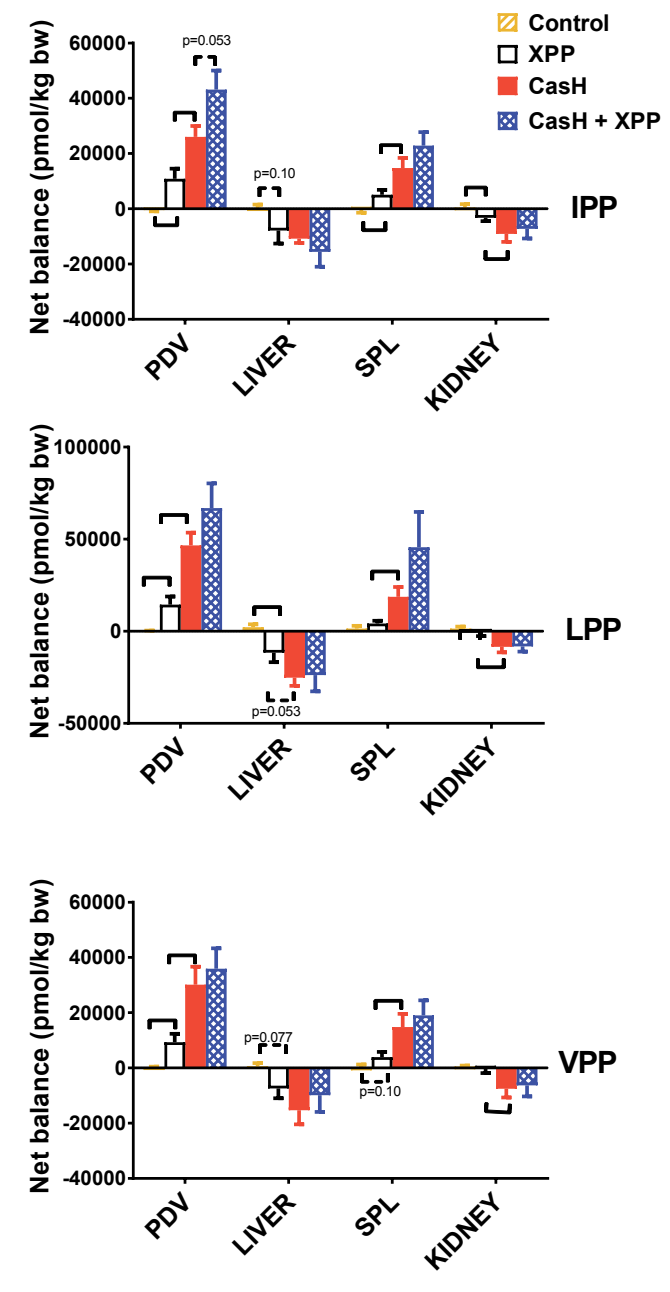

Figure 3. Organ Total Net balances of XPP peptides

Study 1. Post-prandial total net balances across organs over 90 min experimental period after intra-gastric administration of tri-peptide (XPP) mixtures: a synthetic dose of XPP (XPP), XPP containing casein hydrolyte $(\mathrm{CasH})$ and spiked $\mathrm{CasH}(\mathrm{CasH}+\mathrm{XPP})$. Organs: portal drained viscera (PDV), Liver, Splanchnic area (SPL), Kidneys. Positive balance is net release by an organ. Negative balance is net uptake by an organ. Values are mean \pm SEM. Statistics: unpaired t-test for comparison of net balances of natural occurring XPP vs administrated XPP (Control vs XPP), water-based vs protein matrix (XPP vs CasH), and for comparison protein matrixes with vs without XPP spike (CasH vs $\mathrm{CasH}+\mathrm{XPP}$ ). Hooks: significance $\mathrm{p}<0.05$. dotted hooks: tendency $p<0.10$. 


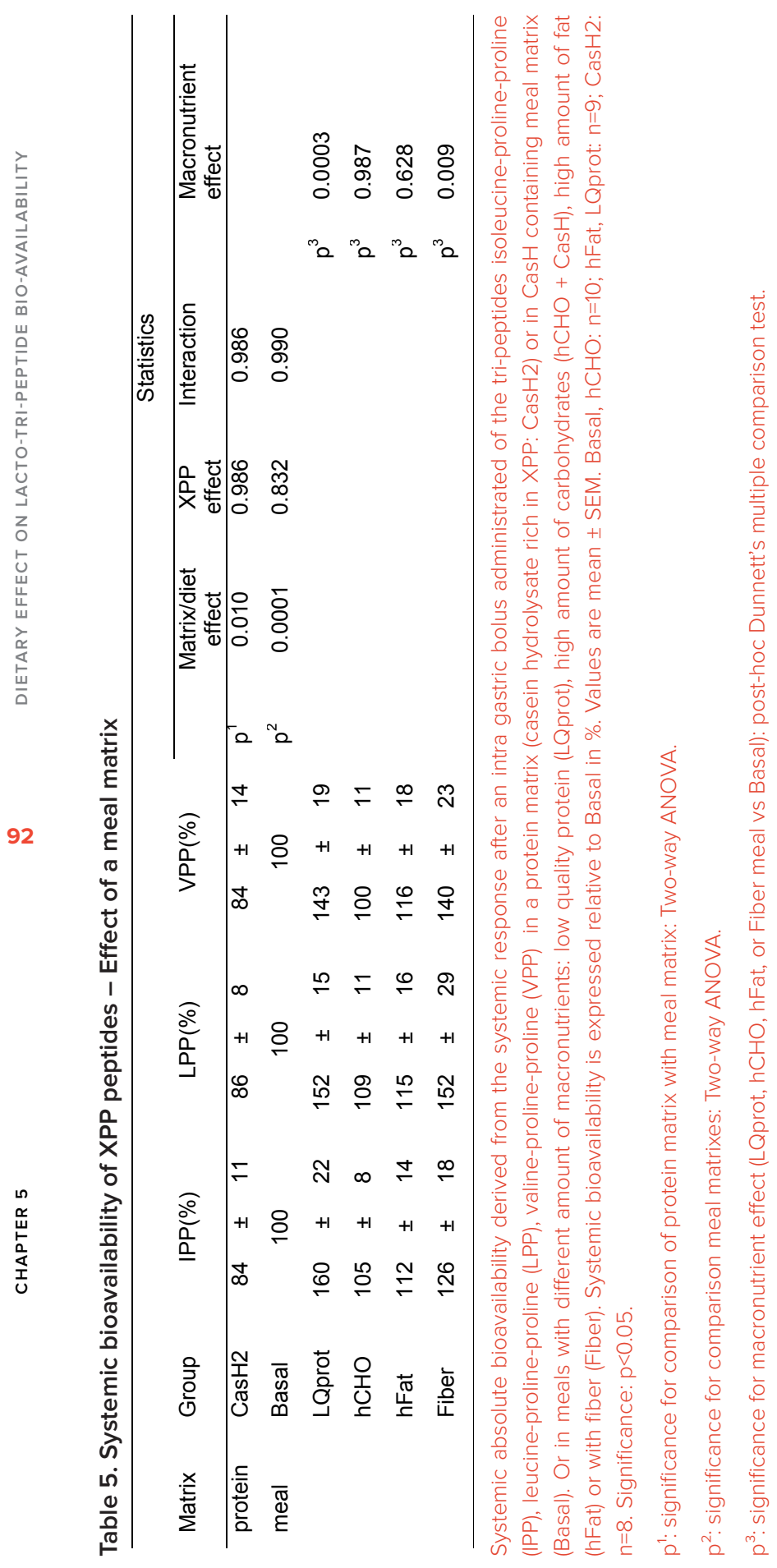




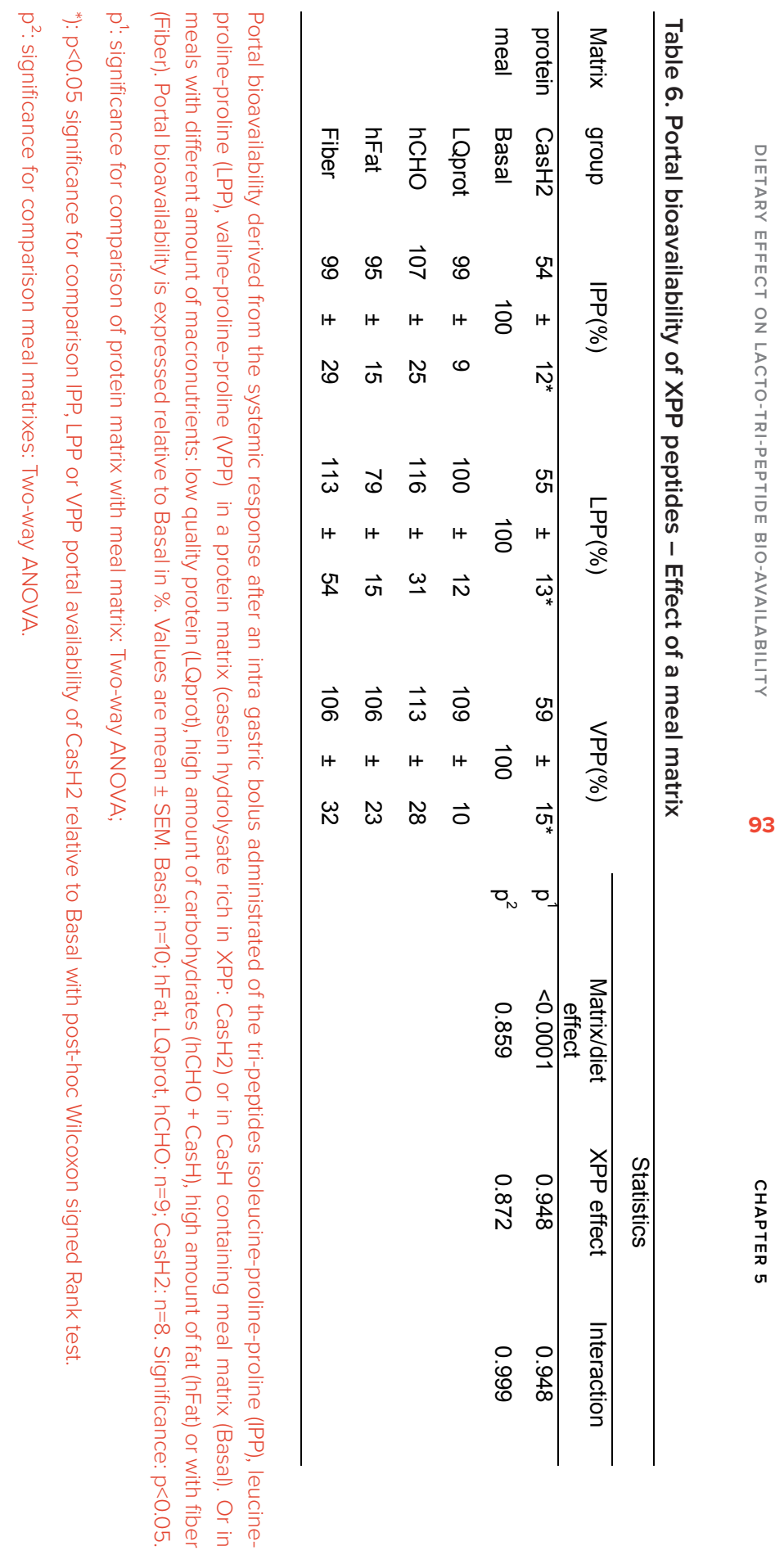




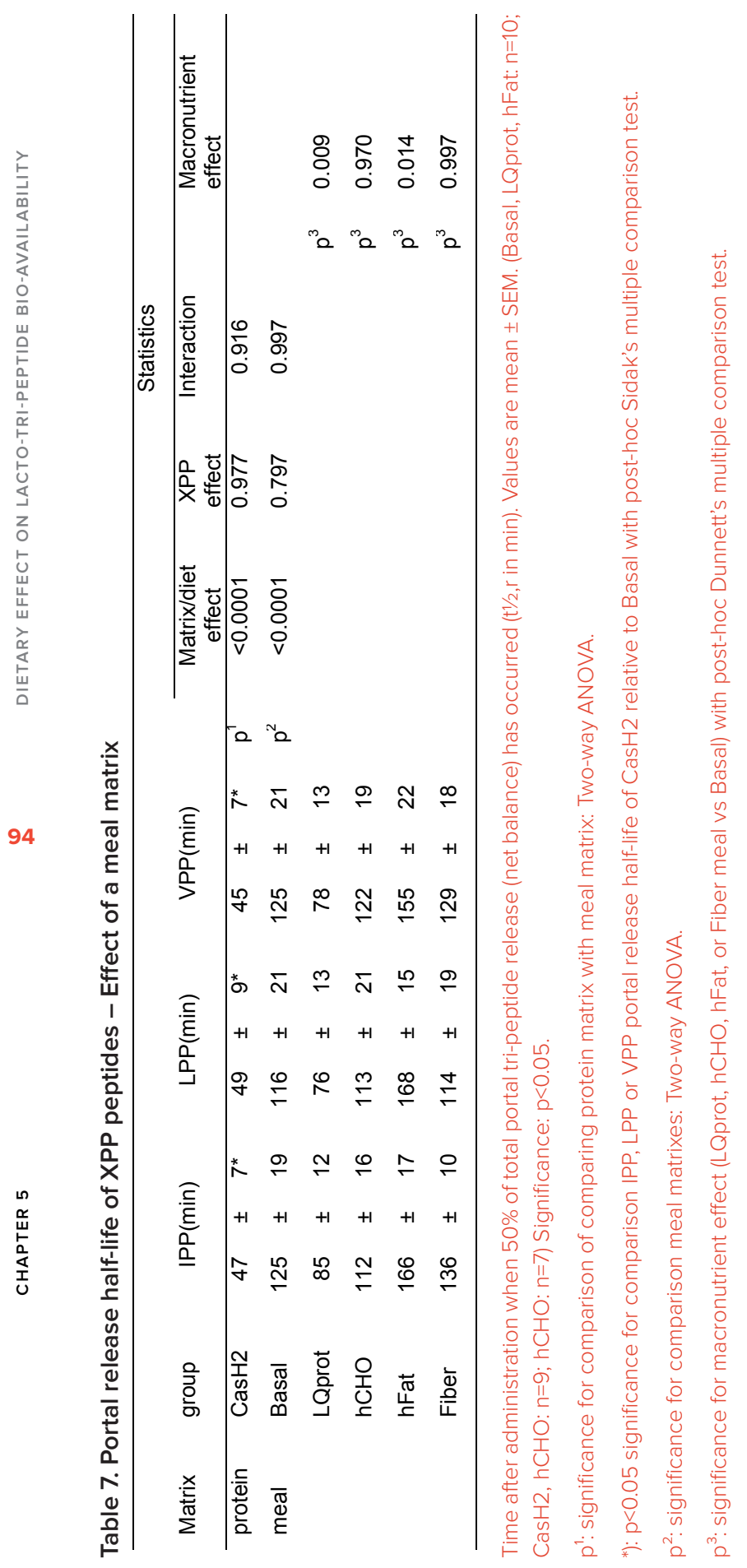




\section{Discussion}

Systemic bioavailability of orally ingested lacto-tri-peptides (XPP) may affect their potential bioactive impact. We hypothesized that macronutrients, especially protein, are able to change systemic availability and that differences in absorption kinetics of the XPP in the splanchnic region cause these changes.

Does a protein matrix improve bioavailability? In a post-absorptive state, we do not detect XPP in plasma above the limit of detection and thus any measurable XPP most likely originates from the meal (Figure 1). The calculated systemic bioavailability of XPP in the presence of a protein matrix (casein hydrolysate) does improve for all XPP, but not in the same proportion (Table 3, IPP: 4.7 times; LPP: 2.5 times; VPP: 121 times). The calculated systemic bioavailability pattern reflects the pattern of the measured portal bioavailability (Table 4), albeit underestimated because of delayed and prolonged systemic bioavailability after $90 \mathrm{~min}$. This indicates that the systemic availability mainly relates to the portal availability of XPP. A protein matrix improves the portal bioavailability of all XPP by prolonging gut absorption (Figure 2, Table 4), but also not in the same proportion. The spiked protein matrix indicates that the relative large improvement of VPP bioavailability was not matrix related.

A point to consider is whether the presence of not-freely- available XPP's (XPP's bound in longer peptides ("encrypted") in the casein hydrolyte affected our results. The portal bioavailability of XPP in a water-based matrix is comparable between the XPP. This in contrast with XPP in the protein matrix. We assume that this is related to encrypted VPP in the protein source of $\mathrm{CasH}$. The source of $\mathrm{CasH}$ is [- and [-casein and these proteins have lacto-tri-peptides encrypted (36), that are liberated during an enzymatic hydrolysis process by the manufacturer. It is likely that still encrypted XPP's in the larger peptides of the hydrolyte are liberated during the digestive process in the pig (1). These XPP also will contribute to the intestinal net balance. More in detail, the current enzymatic hydrolysis process (information of the manufacturer) liberates mainly VVVPP-peptides and only a small amount of VPP. Therefore, we conclude by theoretically calculations (Supplemental Table 3. Theoretical XPP intake and their portal availability - Study 1.) that the high fraction dose of VPP release in comparison to IPP and LPP relates to this mechanism. We found that the average improvement of XPP portal bioavailability by a protein matrix is 1.8 times, albeit underestimated because of prolonged portal bioavailability after 90 min.

The prolonged XPP net absorption from the protein meal could be the result of processes such as stomach emptying, digestion and competition between the absorption of XPP and amino acids on the lumen side. Some of the digested peptides in the dietary proteins are resistant to further digestion because of the type of amino acids bonds to other amino acids like for proline-containing peptides ( 2 , 
37). Trans-epithelial transport in the intestinal tract to the portal system of XPP can occur in three different ways: para-cellular, trans-cytotic or via peptide transport system via the proton-dependent transporter, PepT1 $(24,38)$. In vitro measurements of trans-epithelial flux of VPP across a Caco-2 cell monolayer (38) indicated that the para-cellular route is the most likely route for intact VPP. Because absorption of XPP in hydrolysates in healthy pigs is not more limited than the same amount of single amino acids $(39,40)$, we think that delayed stomach emptying caused the prolonged absorption.

The systemic pharmacokinetics suggests that the improved bioavailability mainly is caused by delay in absorption and prolonged elimination. Systemic bioavailability is not only dependent on the portal availability but also depending on the elimination from systemic plasma to other organs. We found hepatic and renal XPP uptake (Figure 3), but uptake is approx. 75\% of total PDV release. This means that other, not measured organs, also take up XPP (3). Uptake means that XPP is available for potential ACE-inhibition targets in organs like the vascular endothelial cells or absorptive epithelial cells (kidney) $(3,4)$, but also that XPP peptides are eliminated from the systemic by intracellular breakdown to amino acids by peptidases, or excretion to urine in the kidney. Further research in intra-organ kinetics is needed to quantify those processes.

Foltz et al (41) determined in vitro that also di-peptides with a C-terminal proline residue has ACE inhibitory effects. In a pilot analysis, we found that the plasma concentration of related di-peptides is 20-400 times higher than that of XPP (Supplemental Table 1. Dipeptide/tripeptide ratio - Study 1). This suggest that foodderived lacto-di-peptides have a potential synergetic role in the bioactivity, but that need to be determined in another experimental design.

Does a meal improve XPP systemic availability by improved portal availability? Systemic availability increased by a meal matrix but only by $20 \%$ (Table 5 ) in comparison to a protein matrix. Portal availability increased $80 \%$ by a meal matrix (Table 6). Higher portal release half-life (Table 7) indicates prolonged absorption and release to the portal system by the gut in a meal matrix.

Why does a complete meal matrix improve portal XPP availability? First, it is likely that the speed of absorption of regular meals is slower than a single dose of $\mathrm{CasH}$ and that this prolongs absorption of peptides. Fat is known to delays gastric emptying (42). In contrast, we did not observe any improvement of the portal bioavailability with the meals that contain a high amount of fat. Secondly, the small intestine is the interface between the gut lumen and the rest of the body and therefore controls the degree and rate of transport of amino acids coming from the meal via the portal vein (29). It could be that competition of amino acids trans-epithelial transport plays 
a role, because the amount of amino acids from digested proteins $(3.05 \mathrm{~g} / \mathrm{kg}$ bodyweight, Whey and Soy isolate together with $\mathrm{CasH}$ ) is higher in the complete meals as compared to a single dose of $\mathrm{CasH}(0.73 \mathrm{~g} / \mathrm{kg}$ bodyweight). We think however that this is also unlikely as the absolute amount of protein given in the test is still relative low (30\% of restricted daily intake), considering the potential absorption capacity of the gut of a pig (43). Therefore, we need to consider stimulated gut metabolism after the meal in comparison to a protein mixture alone (19). We found that adding carbohydrates leads to higher insulin levels, it stimulates increased protein synthesis and reduced protein breakdown, leading to more gut tissue anabolism $(44,45)$. Therefore, we think that reduced intracellular intestinal protein breakdown is related to the reduced XPP breakdown and thus higher transport to the portal vein. We therefore think that the gut anabolic response in the meals is the most likely mechanism to a higher portal bioavailability. Further research is needed to confirm our hypothesis.

What is the influence of the macronutrients on the systemic availability? Although total portal release of XPP is comparable during the experimental period (Table 6), systemic availability is higher with the low quality protein meal and high fiber meal (Table 5). Additionally in contrast, low protein decreases and high fat increases the portal release half-life of XPP (Table 7), suggesting that increased systemic availability can not be related to differences in intestinal XPP absorption, but suggests that other organs/metabolism are involved. For instance, we observed that soy protein leads to less anabolism $(17,46)$. This means that organs take up less amino acids and probably also less XPP from the systemic circulation for protein anabolism, resulting in higher systemic availability of XPP. We have no good explanation for the observation of the improved XPP systemic availability by high fiber meal. Fiber has multiple health benefits but limited info is available about a direct relation between fiber and peptide/protein metabolism (47).

\section{Conclusion}

Quantitative measurements of trans-organ availability/kinetics provide more insight of the behavior of food-derived C-terminal lacto-tri-peptides like XPP. The present study showed that XPP in a protein matrix have a prolonged portal bioavailability. In a meal with all macronutrients present, XPP are more available albeit at a low percentage (0.2-0.3\%). The digestion rate of the meal, the quality of protein, and fiber contents, mainly determine systemic XPP bioavailability after a meal. 


\section{Acknowledgements}

We thank Ayhan Şik, MSc and Ruud M Ramakers, BSc for skilled technical assistance in carrying out the animal experiments and Ed Rosing, PhD and Martijn Brandt, BSc for accurate XPP analyses and John J. Thaden, PhD for help with the measurements of the XPP. Victor Wilson, PhD, statistician for help with the statistical analysis and Guus S.M.J.E. Duchateau, PhD for continuous support.

\section{References}

1. Moller NP, Scholz-Ahrens KE, Roos N, Schrezenmeir J. Bioactive peptides and proteins from foods: indication for health effects. Eur J Nutr. 2008;47(4):171-82.

2. Picariello G, Ferranti P, Fierro O, Mamone G, Caira S, Di Luccia A, et al. Peptides surviving the simulated gastrointestinal digestion of milk proteins: biological and toxicological implications. J Chromatogr B Analyt Technol Biomed Life Sci. 2010;878(3-4):295-308.

3. Masuda O, Nakamura Y, Takano T. Antihypertensive peptides are present in aorta after oral administration of sour milk containing these peptides to spontaneously hypertensive rats. J Nutr. 1996;126(12):3063-8.

4. Yamamoto N. Antihypertensive peptides derived from food proteins. Biopolymers. 1997;43(2):129-34.

5. Nakamura T, Mizutani J, Sasaki K, Yamamoto N, Takazawa K. Beneficial potential of casein hydrolysate containing Val-Pro-Pro and Ile-Pro-Pro on central blood pressure and hemodynamic index: a preliminary study. J Med Food. 2009;12(6):1221-6.

6. de Leeuw PW, van der Zander K, Kroon AA, Rennenberg RM, Koning MM. Dosedependent lowering of blood pressure by dairy peptides in mildly hypertensive subjects. Blood Press. 2009;18(1-2):44-50.

7. van der Zander K, Jakel M, Bianco V, Koning MM. Fermented lactotripeptidescontaining milk lowers daytime blood pressure in high normal-to-mild hypertensive subjects. J Hum Hypertens. 2008;22(11):804-6.

8. van Mierlo LA, Koning MM, van der Zander K, Draijer R. Lactotripeptides do not lower ambulatory blood pressure in untreated whites: results from 2 controlled multicenter crossover studies. Am J Clin Nutr. 2009;89(2):617-23.

9. Engberink MF, Schouten EG, Kok FJ, van Mierlo LA, Brouwer IA, Geleijnse JM. Lactotripeptides show no effect on human blood pressure: results from a double-blind randomized controlled trial. Hypertension. 2008;51(2):399-405.

10. Wuerzner G, Peyrard S, Blanchard A, Lalanne F, Azizi M. The lactotripeptides isoleucine-proline-proline and valine-proline-proline do not inhibit the $\mathrm{N}$-terminal or C-terminal angiotensin converting enzyme active sites in humans. J Hypertens. 2009;27(7):1404-9.

11. van der Zander K, Bots ML, Bak AA, Koning MM, de Leeuw PW. Enzymatically hydrolyzed lactotripeptides do not lower blood pressure in mildly hypertensive subjects. Am J Clin Nutr. 2008;88(6):1697-702.

12. Boelsma E, Kloek J. IPP-rich milk protein hydrolysate lowers blood pressure in subjects with stage 1 hypertension, a randomized controlled trial. Nutrition journal. 2010;9:52.

13. Terashima M, Oe M, Ogura K, Matsumura S. Inhibition strength of short peptides derived from an ACE inhibitory peptide. J Agric Food Chem. 2011;59(20):11234-7.

14. Hirota T, Ohki K, Kawagishi R, Kajimoto Y, Mizuno S, Nakamura Y, et al. Casein 
hydrolysate containing the antihypertensive tripeptides Val-Pro-Pro and Ile-Pro-Pro improves vascular endothelial function independent of blood pressure-lowering effects: contribution of the inhibitory action of angiotensin-converting enzyme. Hypertension research : official journal of the Japanese Society of Hypertension. 2007;30(6):489-96.

15. Jakala P, Pere E, Lehtinen R, Turpeinen A, Korpela R, Vapaatalo H. Cardiovascular activity of milk casein-derived tripeptides and plant sterols in spontaneously hypertensive rats. Journal of physiology and pharmacology : an official journal of the Polish Physiological Society. 2009;60(4):11-20.

16. Vermeirssen V, Van Camp J, Verstraete W. Bioavailability of angiotensin I converting enzyme inhibitory peptides. Br J Nutr. 2004;92(3):357-66.

17. Deutz NE, Bruins MJ, Soeters PB. Infusion of soy and casein protein meals affects interorgan amino acid metabolism and urea kinetics differently in pigs. J Nutr. 1998;128(12):2435-45.

18. Deutz NE, Reijven PL, Athanasas G, Soeters PB. Post-operative changes in hepatic, intestinal, splenic and muscle fluxes of amino acids and ammonia in pigs. Clin Sci (Lond). 1992;83(5):607-14.

19. Deutz NE, Ten Have GA, Soeters PB, Moughan PJ. Increased intestinal amino-acid retention from the addition of carbohydrates to a meal. Clin Nutr. 1995;14(6):354-64.

20. Deutz NE, Welters CF, Soeters PB. Intragastric bolus feeding of meals containing elementary, partially hydrolyzed or intact protein causes comparable changes in interorgan substrate flux in the pig. Clin Nutr. 1996;15(3):119-28.

21. Bruins MJ, Soeters PB, Deutz NE. Endotoxemia affects organ protein metabolism differently during prolonged feeding in pigs. J Nutr. 2000;130(12):3003-13.

22. Ten Have GA, Engelen MP, Soeters PB, Deutz NE. Absence of post-prandial gut anabolism after intake of a low quality protein meal. Clin Nutr. 2011.

23. Foltz M, van der Pijl PC, Duchateau GS. Current in vitro testing of bioactive peptides is not valuable. J Nutr. 2010;140(1):117-8.

24. Rubio-Aliaga I, Daniel H. Peptide transporters and their roles in physiological processes and drug disposition. Xenobiotica. 2008;38(7-8):1022-42.

25. van der Pijl PC, Kies AK, Ten Have GA, Duchateau GS, Deutz NE. Pharmacokinetics of proline-rich tripeptides in the pig. Peptides. 2008;29(12):2196-202.

26. Edens L, Dekker P, van der Hoeven R, Deen F, de Roos A, Floris R. Extracellular prolyl endoprotease from Aspergillus niger and its use in the debittering of protein hydrolysates. J Agric Food Chem. 2005;53(20):7950-7.

27. Koopman R, Crombach N, Gijsen AP, Walrand S, Fauquant J, Kies AK, et al. Ingestion of a protein hydrolysate is accompanied by an accelerated in vivo digestion and absorption rate when compared with its intact protein. Am J Clin Nutr. 2009;90(1):106-15.

28. Ten Have GA, Bost MC, Suyk-Wierts JC, van den Bogaard AE, Deutz NE. Simultaneous measurement of metabolic flux in portally-drained viscera, liver, spleen, kidney and hindquarter in the conscious pig. Lab Anim. 1996;30(4):347-58.

29. Ten Have GA, Engelen MP, Luiking YC, Deutz NE. Absorption kinetics of Amino Acids, Peptides, and Intact Proteins. Int J Sport Nutr Exerc Metab. 2007;17(Supplement):S23-S36.

30. Bruins MJ, Deutz NE, Soeters PB. Aspects of organ protein, amino acid and glucose metabolism in a porcine model of hypermetabolic sepsis. Clin Sci (Lond). 2003;104(2):127-41.

31. Ytrebo LM, Sen S, Rose C, Davies NA, Nedredal GI, Fuskevaag OM, et al. Systemic and regional haemodynamics in pigs with acute liver failure and the effect of albumin dialysis. Scand J Gastroenterol. 2006;41(11):1350-60.

32. Poeze M, Bruins MJ, Kessels F, Luiking YC, Lamers WH, Deutz NE. Effects of 
L-arginine pretreatment on nitric oxide metabolism and hepatosplanchnic perfusion during porcine endotoxemia. The American journal of clinical nutrition. 2011;93(6):1237-47.

33. Williams EJ. Experimental designs balanced for the estimation of residual effects of treatments. Australian Journal of Scientific Research. 1949;Ser. A 2:149-68.

34. van Platerink CJ, Janssen HG, Haverkamp J. Application of at-line two-dimensional liquid chromatography-mass spectrometry for identification of small hydrophilic angiotensin I-inhibiting peptides in milk hydrolysates. Anal Bioanal Chem. 2008;391(1):299-307.

35. Bruins MJ, Luiking YC, Soeters PB, Lamers WH, Akkermans LM, Deutz NE. Effects of long-term intravenous and intragastric L-arginine intervention on jejunal motility and visceral nitric oxide production in the hyperdynamic compensated endotoxaemic pig. Neurogastroenterol Motil. 2004;16(6):819-28.

36. Bostaurus (cow):281099 [Internet]. DBGET integrated database retrieval system. 2013 [cited 10/22/2013]. Available from: http://www.genome.jp/dbget-bin/www_bget?bta:281099.

37. Boutrou R, Gaudichon C, Dupont D, Jardin J, Airinei G, Marsset-Baglieri A, et al. Sequential release of milk protein-derived bioactive peptides in the jejunum in healthy humans. Am J Clin Nutr. 2013;97(6):1314-23.

38. Satake M, Enjoh M, Nakamura Y, Takano T, Kawamura Y, Arai S, et al. Transepithelial transport of the bioactive tripeptide, Val-Pro-Pro, in human intestinal Caco-2 cell monolayers. Biosci Biotechnol Biochem. 2002;66(2):378-84.

39. Alpers $\mathrm{DH}$. Enteral feeding and gut atrophy. Curr Opin Clin Nutr Metab Care. 2002;5(6):679-83

40. Hammarqvist F, Luo JL, Cotgreave IA, Andersson K, Wernerman J. Skeletal muscle glutathione is depleted in critically ill patients. Crit Care Med. 1997;25(1):78-84.

41. Foltz M, van Buren L, Klaffke W, Duchateau GS. Modeling of the relationship between dipeptide structure and dipeptide stability, permeability, and ACE inhibitory activity. Journal of food science. 2009;74(7):H243-51.

42. Mourad FH, Saade NE. Neural regulation of intestinal nutrient absorption. Progress in neurobiology. 2011;95(2):149-62.

43. Hernandez F, Martinez S, Lopez C, Megias MD, Lopez M, Madrid J. Effect of dietary crude protein levels in a commercial range, on the nitrogen balance, ammonia emission and pollutant characteristics of slurry in fattening pigs. Animal : an international journal of animal bioscience. 2011;5(8):1290-8.

44. Adegoke OA, McBurney MI, Samuels SE, Baracos VE. Modulation of intestinal protein synthesis and protease mRNA by luminal and systemic nutrients. Am J Physiol Gastrointest Liver Physiol. 2003;284(6):G1017-26.

45. Liu Z, Barrett EJ. Human protein metabolism: its measurement and regulation. Am J Physiol Endocrinol Metab. 2002;283(6):E1105-12.

46. Luiking YC, Deutz NE, Jakel M, Soeters PB. Casein and soy protein meals differentially affect whole-body and splanchnic protein metabolism in healthy humans. J Nutr. 2005;135(5):1080-7.

47. Ross AB, Pere-Trepat E, Montoliu I, Martin FP, Collino S, Moco S, et al. A wholegrain-rich diet reduces urinary excretion of markers of protein catabolism and gut microbiota metabolism in healthy men after one week. J Nutr. 2013;143(6):766-73. 


\section{Supplemental Information}

\section{Supplemental Table 1. Dipeptide/tripeptide ratio - Study 1}

\begin{tabular}{lrll}
\hline Dipeptide/tripeptide & \multicolumn{3}{c}{ ratio } \\
\hline IP/IPP & 204 & \pm & 114 \\
LP/LPP & 36 & \pm & 15 \\
VP/VPP & 354 & \pm & 153 \\
PP/XPP & 28 & \pm & 12 \\
XP/XPP & 193 & \pm & 119 \\
\hline
\end{tabular}

Ratio's between the dipeptide and tripeptide plasma concentrations. Pilot data from 2 pigs with tripeptide concentrations above baseline in the range of 1-30 nM. Corresponding dipeptide concentration range: $0.5-7 \mu \mathrm{M}(\mathrm{n}=11)$. Values are means \pm SEM. Ratio's were higher than 1 (Wilcoxon Rank test, $p<0.01$ ). The VP/VPP ratio was significant different from LP/LPP and PP/ XPP (Mann-Whitney, $\mathrm{p}<0.05)$.

\section{Supplemental Table 2. Plasma flows - Study 1}

\begin{tabular}{|c|c|c|c|c|}
\hline Matrix & Group & PDV & Liver & Kidneys \\
\hline \multirow[t]{2}{*}{ water-based } & Control & $47.5 \pm 7.5$ & $71.5 \pm 10.0$ & $43.6 \pm 6.0$ \\
\hline & XPP & $45.9 \pm 4.2$ & $68.6 \pm 6.4$ & $35.6 \pm 4.0$ \\
\hline \multirow[t]{2}{*}{ protein } & $\mathrm{CasH}$ & $54.4 \pm 5.9$ & $77.6 \pm 8.2$ & $31.9 \pm 4.0$ \\
\hline & CasH + XPP & $51.7 \pm 2.4$ & $74.9 \pm 5.8$ & $33.3 \pm 5.5$ \\
\hline
\end{tabular}

Mean plasma flows in different organs of pigs after intra-gastric administration of control salt solution (Control), a synthetic dose of XPP (XPP), casein hydrolysate rich in XPP (CasH) or spiked CasH (CasH + XPP). Data are expressed as mean \pm SEM in $\mathrm{ml} / \mathrm{kg}$ bodyweight/min. No differences were observed between means of the XPP containing groups in each organ and Control (one-way ANOVA).

\section{Supplemental Table 4. PDV Plasma flows - Study 2}

\begin{tabular}{llc}
\hline Matrix & Group & PDV plasma flow \\
\hline protein & CasH2 & $57.3 \pm 8.6$ \\
& Basal & $52.2 \pm 7.2$ \\
& LQprot & $58.9 \pm 8.9$ \\
& hCHO & $57.1 \pm 6.6$ \\
& hFat & $58.1 \pm 6.7$ \\
meal & Fiber & $50.7 \pm 4.3$ \\
\hline
\end{tabular}

Mean plasma flows of portal drained viscera (PDV) in pigs after administration of casein hydroIysate (CasH2) supplement, casein hydrolysate suppleted iso-caloric meal (Basal). Or meals with different amount of macronutrients: Iow quality protein (LQprot), high amount of carbohydrates $(\mathrm{hCHO}+\mathrm{CasH})$, high amount of fat (hFat) or with fiber (Fiber). Data are expressed as mean $\pm \mathrm{SEM}$ in $\mathrm{ml} / \mathrm{kg}$ bodyweight/min. No differences were observed between means of different matrixes: $\mathrm{CasH} 2$ vs Basal, unpaired t-test. Or between means of different meals (one-way ANOVA). 


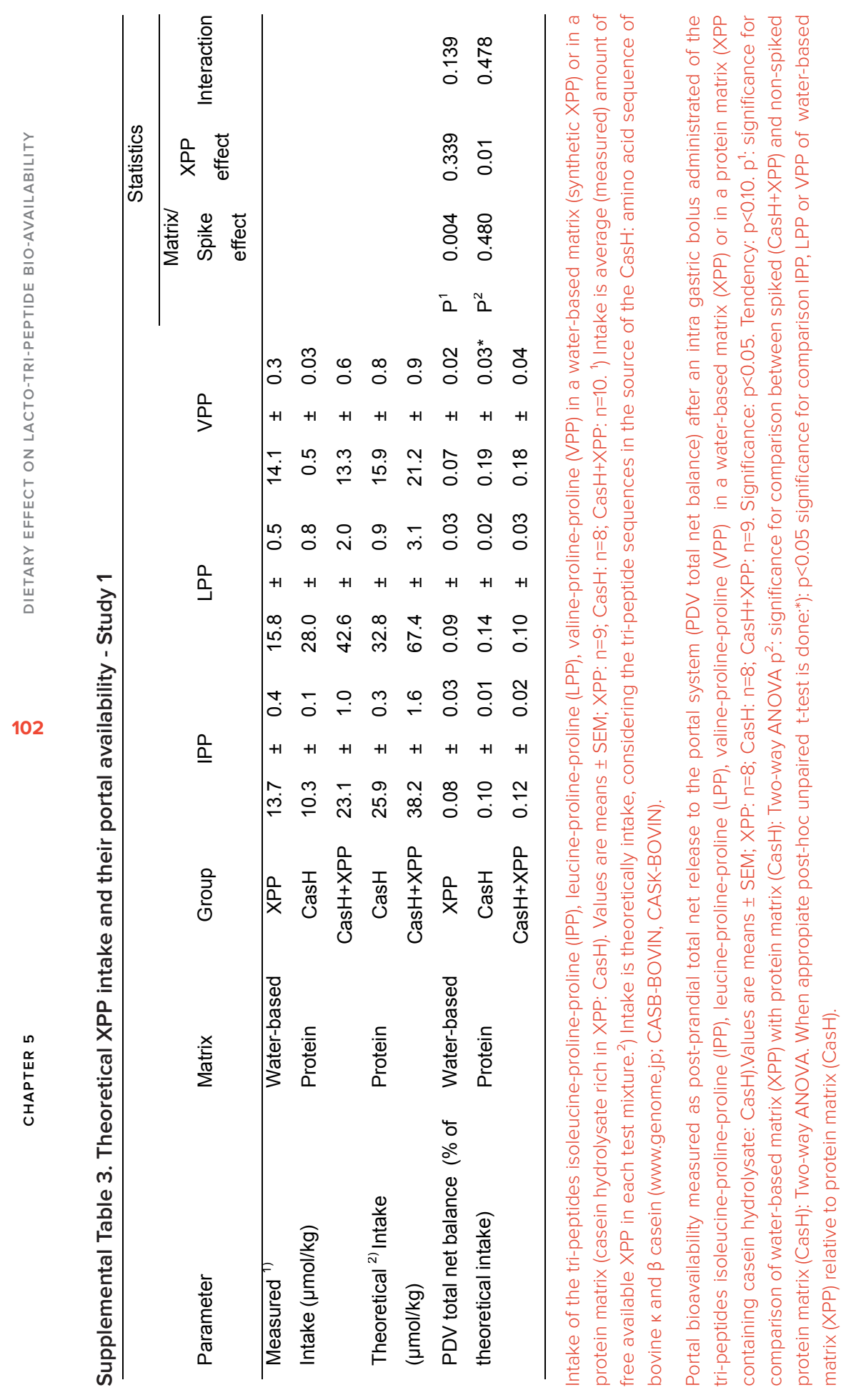




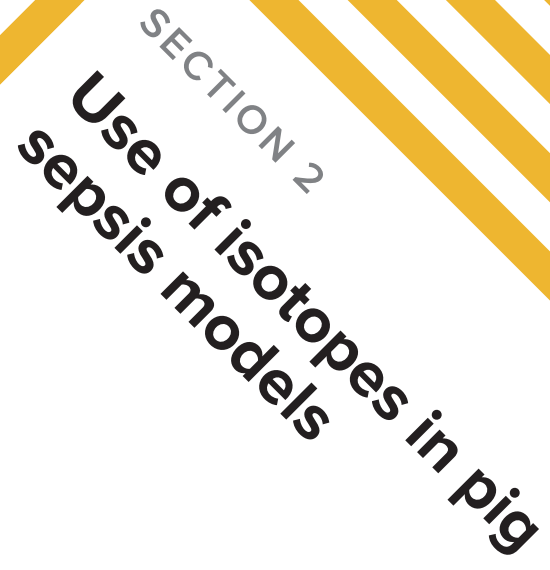


CHAPTER 6 


\title{
Characteristics of a Pseudomonas aeruginosa induced porcine sepsis model for multi-organ metabolic flux measurements
}

\author{
Gabriella A.M. ten Have, Renske C.I. Deutz, Mariëlle
}

P.K.J. Engelen, Robert R. Wolfe, Nicolaas E.P. Deutz

Lab Anim. 2017 Jan 1:23677217718003. doi:

10.1177/0023677217718003. [Epub ahead of print]

\section{Abstract}

Survival in sepsis is related to loss of muscle mass. Therefore, it is imperative to further define and understand the basic alterations in nutrient metabolism to improve targeted sepsis nutritional therapies. We developed and evaluated a controlled hyperdynamic severe sepsis pig model that can be used for in vivo multi-organ metabolic studies in a conscious state. In this catheterized pig model, bacteremia was induced IV with $10^{9} \mathrm{CFU} /$ hour Pseudomonas aeruginosa (PA) in 13 pigs for 18 hours. Both the PA and control (nine) animals received fluid resuscitation and were continuously monitored. We examined in detail the hemodynamics, blood gases, clinical chemistry, inflammation, histopathology and organ plasma flows. The systemic inflammatory response (SIRS) diagnostic scoring system was used to determine the clinical septic state. Within six hours from the start of PA infusion, a septic state developed as reflected by hyperthermia and cardiovascular changes. After 12 hours of PA infusion, severe sepsis was diagnosed. A disturbed cardiovascular function, a decreased portal drained viscera plasma flow (Control: $37.6 \pm 4.6 \mathrm{ml} /$ $\mathrm{kg}$ bw/min; PA $20.3 \pm 2.6 \mathrm{ml} / \mathrm{kg}$ bw/min, $\mathrm{p}<0.001$ ) as well moderate villous injury in the small intestines were observed. No lung, kidney or liver failure was observed. Acute phase protein CRP and II-6 levels did not change in the PA group. However, significant metabolic changes such as enhanced protein breakdown, hypocalcemia and hypocholesterolemia were found. In conclusion, the PA induced bacteremia in the catheterized pig is a clinically relevant model for acute severe sepsis and enables study of complex multi-organ metabolism. 
Sepsis is a complex bacteremia induced pathophysiological state characterized by both cardiovascular and metabolic responses (1-4). Its dynamic character, the variability in responses, and the complicated clinical logistics make it difficult to study the metabolic interaction between different organs in septic patients in a controlled setting $(5,6)$.

One characteristic metabolic alteration in sepsis is loss of muscle mass. Loss of more than $25 \%$ of muscle mass is incompatible with survival $(7,8)$. Less severe muscle loss contributes to morbidity and mortality (9), and hinders rehabilitation to normal health and function after recovery. Moreover, intestinal mucosal atrophy mainly due to reduced protein synthesis, is often present in sepsis, and contributes to increased translocation of bacteria from the gut into the bloodstream (10, 11). Increased translocation of gut bacteria perpetuates the septic state. Also, a severe decrease of plasma albumin is associated with higher mortality in sepsis (12). Albumin is the principal oncotic protein in plasma, and a decrease in concentration leads to loss of fluid from the vascular space. Finally, the physiological significance of the acute phase response has been debated (13), but clearly represents a metabolic priority in sepsis. More knowledge about the metabolic changes in sepsis should contribute to more effective potential nutritional support $(9,14)$.

Although the debate over the relevance and validity of various animal models of sepsis is still ongoing $(3,15,16)$, the high morbidity and mortality resulting from sepsis illustrate the scientific and clinical need to further define and understand the basic nutrient metabolism alterations to improve existing targeted sepsis nutritional therapies. For nutritional therapies in sepsis, an optimal animal model to study metabolic responses in the hyperdynamic septic state should be clinically relevant, controlled and performed in the conscious state. It should also have a potential therapeutic window, with the possibility to study multi-organ metabolism. For this model, the first consideration is that the septic state in the animal model should not exceed severe sepsis. Therefore, the septic shock models without potential therapeutic windows would not be valid. Secondly, induced sepsis should start with invasion of the blood stream by micro-organism, usually bacteria. It has been indicated that animal models using live bacteria are the most clinically relevant models $(15,17)$. Thirdly, pig models are preferable as they are omnivores like humans and the pig has a similar renal, cardiovascular, and gastrointestinal anatomy, physiology and metabolism. Therefore, studying therapeutic nutritional support in a pig model is viewed as a highly translational research model $(18,19)$. Fourthly, the metabolic measurements should preferably be done in a conscious state, because it is more clinical relevant/translational. In addition anesthetic management in sepsis is challenging (20), and interferes with metabolism, especially splanchnic metabolism (e.g. down regulation of the gut movements, detoxification activity of anesthetics by the 
liver). Sepsis is associated with clinical symptoms of systemic (body wide) illness such as fever, chills, malaise and general feeling of sickness. However as we know from clinical situations, pain is not a primary symptom. Therefore, it is acceptable to measure metabolism in conscious state as long as the animal is not in shock, which is characterized by multiple organ failures and the necessity of ventilation with sedation $(21,22)$. Finally, the animal should be instrumented to accommodate painless blood sampling and infusions during multi-organ metabolism study (23).

Currently, there are no animal models available with these characteristics for relevant metabolic and future nutritional therapies in sepsis. Previously, we studied multi-organ metabolism in a $24 \mathrm{~h}$ endotoxin induced catheterized sepsis pig model (24-26). Although endotoxin is the major virulent factor from a gram-negative bacterium in sepsis, it is possible that important components of the metabolic dynamics are missing in this which are present when sepsis is induced by a live bacterium $(15,17)$.

The aim of the present study was to characterize in detail the response in hemodynamics, blood gases, clinical chemistry, inflammation and organ plasma flows in a new Pseudomonas aeruginosa (PA)-induced septic instrumented porcine model. The model will be used for studying clinical relevant, multi-organ metabolism in sepsis. We modified a widely used, high dose bacteremia porcine septic shock model $(16,27-30)$ to obtain a controlled hyperdynamic conscious sepsis model by administrating the bacteria intravenously (IV) at a low dose via continuous infusion, and simultaneously preventing low blood pressure by fluid resuscitation. PA was used as it is one of the most frequently isolated bacteria in sepsis in humans in hospital intensive care units, apart from lipopolysaccharide, and other virulence factors like flagella and pili, which inject a set of toxins directly into host cells (31). The pigs were surgically catheterized and subsequently acclimatized 10 days before the induction of sepsis. This was done to better control the sepsis induction and subsequent hyperdynamic state, and to perform multi-organ metabolic flux measurements over time in the conscious state $(23,24,32)$.

\section{Materials and Methods}

\section{Animals}

Twenty seven female Yorkshire Crossbred pigs (from multiple litters, Metz Farms, Russellville, AR, USA) with an average body weight of $20-25 \mathrm{~kg}$ were used in the present study. General health status was determined by assigned veterinarian of the animal research facility (Arkansas Children's Hospital Research Institute, Little Rock, AR, USA, AALAC certified). The pigs were individually housed in steel pens 
$(2 \times 3 \mathrm{~m})$ in a controlled housing, biosafety level two facility: large animal cubicle, plastic coated mesh-style grid floor, room temperature $22-24^{\circ} \mathrm{C}$, 12 -hour light-dark cycle, and standardized food intake (1kg/day) (Harlan Teklad Vegetarian Pig/Sow Grower). The pens were enriched with toys to stimulate natural pig rooting behavior and to prevent stereotypic behavior. A heating pad was added to the pen to ensure natural body temperature control post-surgery. Water was provided ad libitum. Clinical examination was carried out at arrival, before surgery (7-10 days after arrival) and frequently post-surgery (twice daily on the first three/four days post-surgery and then every two/three days until the sepsis study). The present animal study including its justification for number, breed and single sex was approved by the animal experiment ethics committee (IACUC) of University of Arkansas Medical Sciences (Little Rock, AR, USA).

\section{Surgical procedure}

To order to perform multi-organ metabolism studies in the septic pig, we implanted catheters in blood vessels with a surgical procedure as described in detail previously $(23,32)$. After an acclimatization period of seven to ten days, a pre-surgery clinical exam and anesthesia was performed by assigned veterinarian. Anesthesia was induced with intramuscular (I.M.) injection of mixture of telazol, ketamine and xylazine $\left(\right.$ PigMix $^{\circledR}$, dose $0.4 \mathrm{ml} / \mathrm{kg}$ bw). With the subsequently intubation of the animal, general anesthesia was started and maintained with isoflurane (2\% mixture with oxygen). Via an ear vein cannula flunixin meglumine (Flunixamine ${ }^{\circledR}, 2 \mathrm{mg} / \mathrm{kg}$ bw) was administered as analgeticum and as an antibioticum, lincomycin-spectinomycin mixture (Linco-Spectin ${ }^{\circledR}$, lincomycin 5 mg/kg bw, spectinomycin 10 mg/kg bw) was administered as prophylaxis. The animal received 500-1000 ml RingersLactate during the surgery to keep up the blood pressure and fluid balance. ECG, CO2 and oxygen saturation were monitored continuously. In brief, during midline laparotomy, a catheter for blood sampling over time and monitor mean arterial pressure (MAP) was placed in the abdominal aorta. An inferior caval vein catheter was placed, allowing administration of post-surgery medication and experiment related infusions. A second arterial catheter, just before the bifurcation, and a splenic vein catheter were placed allowing infusion of para-aminohippuric acid (PAH) for plasma flow measurements. A swan ganz catheter (5 Fr, \#132F5, Edwards life sciences, Irvine, CA, USA) was inserted via the right jugular vein to monitor mean pulmonary arterial pressure (MPAP). The position of the tip of the Swan Ganz catheter was determined following the pressure readings when the catheter was pushed through the right atrium and ventricle. Additionally, an extra caval vein, a portal vein and hepatic vein catheter were placed for multi-organ blood sampling. The duration of the surgery was approximately five hours. Postoperative care was standardized as described in detail previously $(23,32)$ and monitored by assigned veterinarian. After surgery, we kept the animal hooked up to the ECG, pulse ox, and ventilator until the animal responded with multiple swallow reflexes, eye blinking, fighting the 
ventilator, and trying to rise. A second dose of lincomycin-spectinomycin was given I.M. The animal was observed (breathing and heart rate) further until it sits sternally. During the first four days post-surgery twice daily, the animals were clinically examined (body temperature, appearance and behavior), buprenorphine $(0.03 \mathrm{mg} /$ $\mathrm{kg} \mathrm{bw)} \mathrm{and} \mathrm{lincomycin-spectinomycin} \mathrm{were} \mathrm{administered} \mathrm{via} \mathrm{the} \mathrm{implanted} \mathrm{central}$ vein catheter. We fed the animals half of normal ration of food during the first two days. Catheters were kept patent by checking daily during the first three days, and by using antimicrobial and anticoagulant catheter filling $(20 \mathrm{mg} / \mathrm{ml}$ gentamycin and $225 \mathrm{U} / \mathrm{ml}$ alfa-chymotrypsin) as described previously (23). Subsequently, every two/ three days catheters were checked for patency and the animals were clinically examined. Before and after surgery, during the recovery period (seven to ten days) animals were accustomed to a small movable cage $(0.9 \times 0.5 \times 0.3 \mathrm{~m})$ to ensure that the PA experiments could be performed in the cage in conscious animals in free standing, sitting or lying positions. Until the day of the sepsis experiment, the pig spent at least ten times in this movable cage.

\section{Experimental setup of the sepsis model}

The design to characterize the septic response of the PA bacteremia is shown in Figure 1. The experiment started after a recovery period 7-10 days post-surgery. These animals were allocated for the PA group or the Control group in a randomized fashion. Basal blood pressures, plasmaflow, blood gas, clinical chemistry and inflammatory data (pre-septic period; $\mathrm{t}=-2 \mathrm{~h}$ to $\mathrm{Oh}$ ) measurements were started 4-6h after the last food intake (half of the daily amount: $0.5 \mathrm{~kg}$ ). No food was administered during the rest of the experimental period (18h). At $\mathrm{T}=0 \mathrm{~h}$ bacteremia was induced by the start of IV continuous infusion of PA (IRS 12-4-4, Shriners burns institute, University of Texas Medical Branch, Galveston; original from a burn patient at Brook

Hemodynamics monitoring: MAP, MPAP, Heart Rate, Body Temp, Bloodgas

IV Fluid resuscitation $30 \mathrm{ml} / \mathrm{kg}$ bw/hour

Primed continuous IV infusion of PAH for plasma flow measurements

Bacteremia: IV Pseudomonas aeruginosa, $10^{9} \mathrm{CFU} / \mathrm{ml} / \mathrm{hour}$

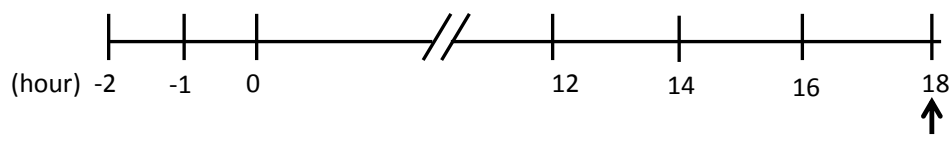

multiple blood samples

Necropsy

Figure 1. Design induction of sepsis with Pseudomonas aeruginosa bacteremia MAP: mean arterial pressure; MPAP: mean pulmonary arterial pressure; PAH: para-aminohippuric acid infusion for plasma flow measurements. IV: intravenous; bw: bodyweight. 
Army Medical Center in San Antonio, TX.; $10^{9} \mathrm{CFU} /$ hour in $1 \mathrm{ml} 0.9 \% \mathrm{NaCl}$ solution). PA dose initially obtained from the method of Rimmele et al (16) was tested for virulence and adjusted in a pilot (three animals). The Control group received 0.9\% $\mathrm{NaCl}$ solution in the same volume. Fluid resuscitation $(30 \mathrm{ml} / \mathrm{kg}$ bw/hour $0.9 \% \mathrm{NaCl}$, IV) was started at the same time as the PA infusion. General appearance (alertness, breathing) was monitored and recorded continuously. Multiple blood samples were taken over time (see details following section). At $\mathrm{T}=18$ hours animals were euthanized with $125 \mathrm{mg} / \mathrm{kg}$ pentobarbital sodium and $16 \mathrm{mg} / \mathrm{kg}$ phenytoin sodium (Euthanasol ${ }^{\circledR}$ ) administered via the central vein catheter.

\section{Hemodynamics}

Hemodynamics were monitored continuously to ensure that the hyperdynamic state was kept in the expected ranges for severe sepsis (not shock): maximal body temperature (normal range: $37-39.6{ }^{\circ} \mathrm{C}(33)$ ) increase or decrease of two to three degrees Celsius, maximal MPAP (normal range: $11-24 \mathrm{mmHg}$ (33)) of $35 \mathrm{mmHg}$, heart rate (normal range: 90-107 BPM (33)) of 200 beats per minute (BPM) and MAP (normal range: $86-123 \mathrm{mmHg}(33)$ ) higher than $60 \mathrm{mmHg}$, using the arterial and Swan Ganz catheter for invasive blood pressure measurement (monitor: Propaq Encore, Welch Allyn, Skaneateles Falls, NY, USA; transducer: TruWave, Edwards Lifesciences, Irvine CA, USA). If the heart rate became >200 bpm and/or MPAP >35 $\mathrm{mmHG}$, the PA infusion was stopped temporarily to limit the increase of the right ventricular afterload (16) until heart rate and MPAP were below 200 bpm and $35 \mathrm{~mm}$ $\mathrm{Hg}$, respectively.

\section{Clinical chemistry}

At $\mathrm{T}=0,12$ and $18 \mathrm{~h}, 500 \mu \mathrm{l}$ serum was collected and stored at $-80^{\circ} \mathrm{C}$ for a standard clinical chemistry diagnostic panel (Supplemental Table 2, Veterinary Diagnostic Laboratory, Little Rock AR, USA).

\section{Blood gas}

Arterial and central venous blood samples were collected for blood gas levels, every 6 hours, using an on-site blood gas analyzer (i-STAT, cartridge CD4+, Abbott Laboratories Inc., Abbott Park, IL). We compared and interpreted blood gas data using the alpha-stat scientific model with no temperature correction (34). Fractional oxygen extraction rate from the capillary bed were calculated: $\mathrm{SaO}_{2}-\mathrm{SvO}_{2} / \mathrm{SaO}_{2}$ (Normal range: 22-32\%, $\mathrm{SaO}_{2}=$ arterial oxygen saturation, $\mathrm{SvO}_{2}=$ venous oxygen saturation). Pulmonary oxygen exchange efficiency was calculated by dividing arterial oxygen pressure $\left(\mathrm{PaO}_{2}\right)$ with the fraction of inspired oxygen $\left(\mathrm{FiO}_{2}=0.21\right.$; Normal range: $>300$ ). 


\section{Plasma flow measurements}

We measured organ plasma flow by using PAH dilution model as described previously (35). In brief: plasmaflow was measured with a primed-continuous infusion of PAH (25mM), via a splenic and one of the arterial catheters $(75 \mathrm{ml} / \mathrm{h})$. Arterial, portal, hepatic and caval vein lithium-heparinized blood samples $(0.5 \mathrm{ml} /$ port) were collected at $\mathrm{T}=-1,-0.5,0,12,13,14,15,16,17$ and 18h for portal drained viscera (PDV), splanchnic (SPL) and hindquarter (HQ) plasma flow determination. These samples were directly cooled (four degrees Celsius), centrifuged $(8000 \times \mathrm{g}$, five min, four degrees Celsius) and plasma was deproteinized with trichloroacetic acid (50 mg per one $\mathrm{ml}$ plasma) within one hour after collection. Samples were stored at $-80^{\circ} \mathrm{C}$ until further analysis. For the measurement of the plasma flow, PAH concentrations in trichloroacetic acid deproteinized plasma samples were compared with $\mathrm{PAH}$ standards and read out with a Microplate spectrophotometer (ELx 808 Ultra microplate reader, Bio-Tek Instruments Inc, Winooski, VT, USA) (36). Plasma flow was calculated using the dilution of $\mathrm{PAH}$ across the organs (23).

\section{Histology}

At $\mathrm{T}=18 \mathrm{~h}$, immediately after euthanasia, small fragments (approximately $200 \mathrm{mg}$ ) of muscle (M. gastrocnemius, right hind leg, middle part), jejunum, ileum, liver, and lung were obtained from seven control and six PA animals. Tissues were rinsed with saline and fixation was done in $10 \%$ buffered formalin for $24 \mathrm{~h}$. Where after the tissues were transferred to $70 \%$ alcohol and stored at room temperature until further routinely processed and stained with hematoxylin and eosin (Texas A\&M University, Department of Veterinary Pathobiology, TX, USA). We examined the tissues blinded for sepsis related non-specific inflammation and/or organ specific histopathological lesions (37): in general the influx of inflammatory cells; in muscle the initial signs of atrophy (38); in jejunum/ileum mucosal villous injury using a previously described scoring system (39) and subepithelial spacing in the crypt regions. In addition, in the liver we looked for signs of the fatty change and finally in the lung the signs of diffuse alveolar damage (DAD).

\section{Inflammation parameters}

Acute phase cytokine Interleukin-6 (IL-6) and C-reactive protein (CRP) in arterial plasma were measured at time points -2,-1, 0, 12, 14, 16, 17, 17.5, and 18 hour and analyzed using commercially available ELISA following manufacturers instruction (Porcine IL-6 DuoSet, Porcine CRP Duoset ELISA, RnD systems, Minneapolis, MN, USA). Plasma IL-6 and CRP amounts will be expressed per liter plasma and mg plasma protein (30). The dynamics of IL-6 and CRP plasma concentrations over time (Supplemental Figure 1), showed non-physiological relevant changes between 12 and 18h in a pilot amount of animals (II-6: Control $\mathrm{N}=2$; PA N=4; CRP: Control $\mathrm{N}=2$; $P A N=2)$. Therefore, only the pre $(T=-2 h)$ and post PA ( $T=17 h)$ data were presented. 


\section{Statistical analyses}

Data are expressed as mean \pm SE. Graphpad Prism (version 6) was used for statistics. Levels of significance was set on $p<0.05$. Hemodynamics, organ plasma flows, histological villous scoring, blood gas data, clinical chemistry data and acute phase protein were compared with Two-way ANOVA, when appropriated with repeated measures. Also when appropriate a post-hoc Bonferroni, Tukey or uncorrected Fisher's LSD multiple comparison test was done as indicated in figures and table legend.

\section{Results}

From the 27 animals, five animals were lost due to intestinal complications (ileus, ascites or peritonitis) in the recovery phase of the catheter implantation surgery. However, no animals were lost during surgery and the PA experiment. General appearance was monitored: PA animals started shivering between one and two hours after start of PA infusion, deeper breathing (less frequent) started between four and six hours, animals were in general calm but still alert. When needed, PA infusion was stopped between seven and $18 \mathrm{~h}$ (average 14.5 hours) after start of PA to prevent the septic response from developing further into an acute septic shock. Thirteen animals with PA (PA group) and nine animals without PA (Control group) completed the study.

\section{Hemodynamics and body temperature}

The initial increased peak in heart rate was parallel to that of the MPAP up to six hours (Figure 2). The average temporary stop of PA to limit the increase of the right ventricular afterload (16) infusion lasted 18.5 min. MPAP went slowly down between 2 and 3 h after start of the PA infusion. The heart rate showed a biphasic pattern: an initial peak in the first five hours and subsequently increase that showed a stable tachycardia after ten hours. The MAP data revealed an initial hypertension phase in the first few hours, followed by a decrease after five hours. We managed to keep the MAP data stable after ten hours between 75 and $82 \mathrm{mmHg}$ (hypotension) compared with between 102 and $113 \mathrm{mmHg}$ for the controls. Body temperature increased within a few hours after start of the PA infusion and reached a maximum after 4h, followed by a gradual decrease. No hypothermia was observed throughout the study.

\section{Blood gas characteristics}

Arterial and venous oxygen saturations were kept within normal ranges (Supplemental Table 1). The fractional uptake of oxygen from the capillary bed, did not change $\left(\mathrm{O}_{2} \mathrm{ER}\right)$. The efficiency of pulmonary gas exchange (average $\mathrm{PaO}_{2} / \mathrm{FiO}_{2}$ $>300$ ) was comparable between the groups. The tissue perfusion variable lactate 

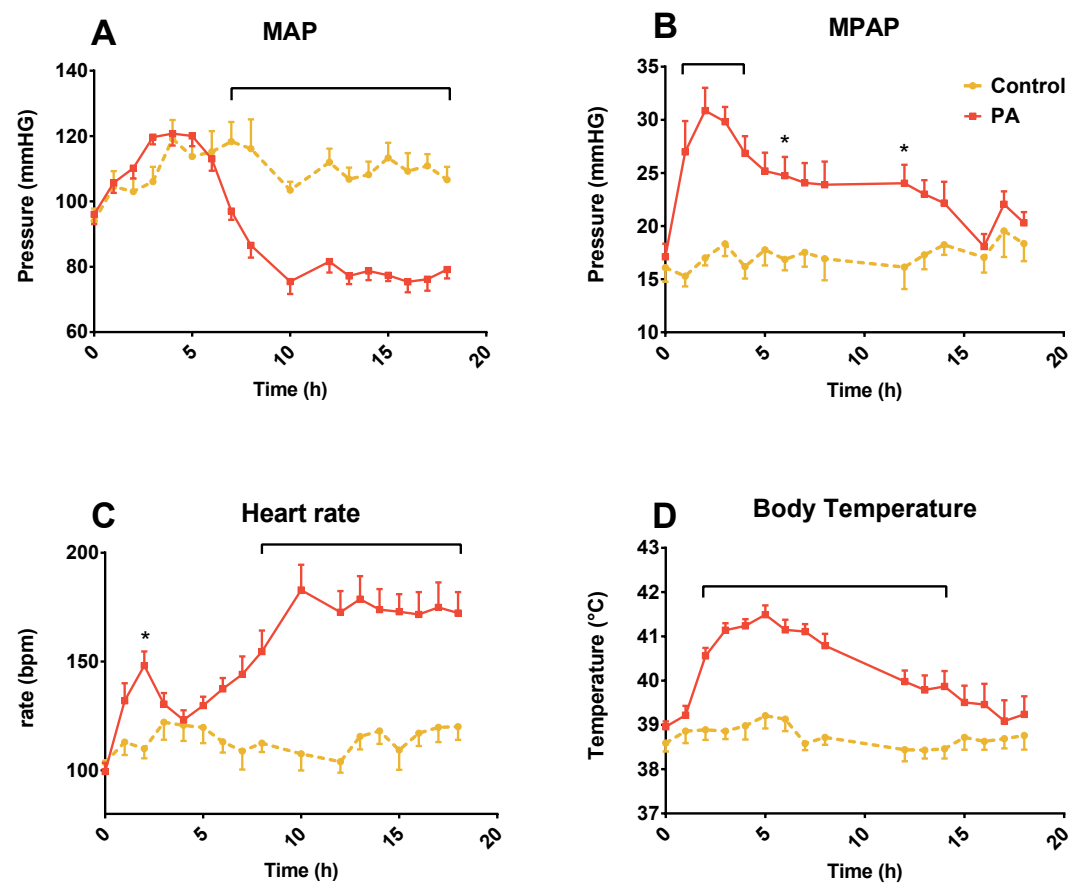

Figure 2. Hemodynamics and body temperature

Hemodynamic response on the Pseudomonas aeruginosa (PA) induced bacteremia in the pig. A: MAP is mean arterial pressure. B: MPAP is mean pulmonary arterial pressure. C: Heart rate. D: Body temperature. Control N=9; PA N=13. Values are mean \pm S.E.M. Statistics: Two-Way ANOVA comparison; when appropriate a post hoc Bonferroni multiple comparison test was done; hook or *: significance $p<0.05$.
A. $\quad$ MAP: Interaction $p<0.0001$, Time effect $p<0.0001$, PA effect $p<0.0001$.
B. MPAP: Interaction $p=0.0021$, Time effect $p=0.0053$, PA effect $p<0.0001$.
C. HR: Interaction $p<0.0001$, Time effect $p<0.0001$, PA effect $p<0.0001$.
D. Body Temp: Interaction $p<0.0001$, Time effect $p<0.0001$, PA effect $p<0.0001$.

increased over time (>1 mM); however, it does not exceed the upper limit of the normal range. The venous $\mathrm{pCO}_{2}$ and $\mathrm{HCO}_{3}$ was lower than normal in the sepsis group, the resulting $\mathrm{pH}$ was expectedly higher; however, both still within the normal range at $\mathrm{T}=18 \mathrm{~h}$.

\section{Blood flow characteristics}

Baseline plasma flow was not different between the Control and PA groups (Figure 3). PA infusion induced a decrease in plasma flow in the PDV area $(p<0.001)$, but not in the whole splanchnic area. In the $\mathrm{HQ}$, an increase of plasma flow was observed in both the PA and control groups. 


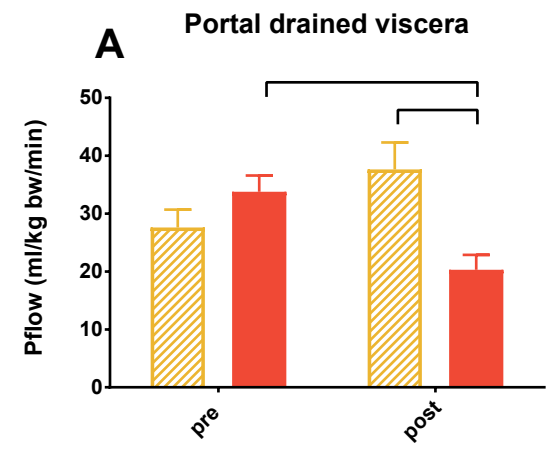

Control

PA

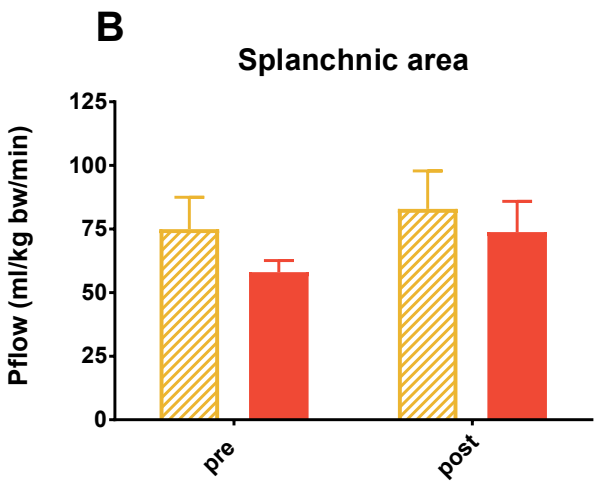

116

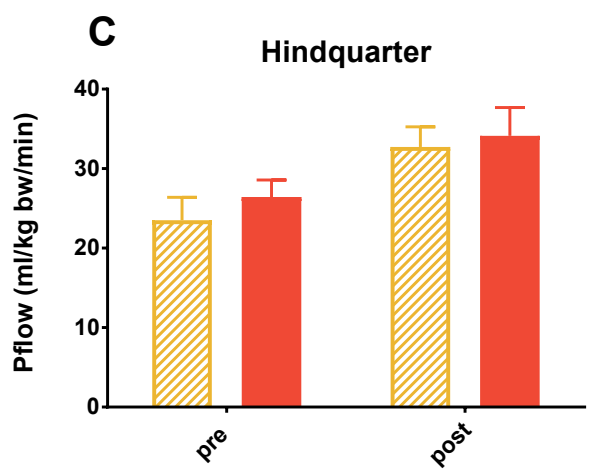

Figure 3. Organ Plasma flow

Organ plasma flows changes before and after Pseudomonas aeruginosa (PA) induced bacteremia in the pig. "Pre" is average plasma flow one hour before induction bacteremia. "Post" is average plasma flow between 14 and 18 hours after start induction bacteremia. Control $\mathrm{N}=9$; Sepsis $N=12$. Values are mean \pm S.E.M.. Statistics: Two-Way ANOVA repeated measures for time, when appropriate a post-hoc Tukey multiple comparison test; hook: significance $p<0.05$.
A. Portal drained viscera: Interaction $p=0.0008$, Time effect $p=0.5682$, PA effect $p=0.1297$
B. Splanchnic area: Interaction $p=0.7225$, Time effect $p=0.2849$, PA effect $p=0.2992$
C. Hindquarter: Interaction $p=0.6024$, Time effect $p<0.0001$, PA effect $p=0.2992$ 


\section{Clinical chemistry}

Metabolic parameters i.e. total protein, albumin, and globulin concentrations, cholesterol, as well as calcium (important for ion-homeostasis) decreased in the PA group (Supplemental Table 2.) Creatinine phosphokinase (CPK), indirect marker of muscle degradation, and lactate concentrations (Supplemental Table 1) were increased. The energy parameter glucose reached its lowest value at $T=12 \mathrm{~h}$. Urea (marker of protein metabolism and kidney function) and creatinine levels as well as urea/creatinine ratio were elevated. The liver degradation parameters alanine aminotransferase(ALT) and gamma-glutamyltranferase(GGT) were not altered but aspartate aminotransferase(AST) and alkaline phosphatase were increased.

\section{Histology}

The muscle ( $M$. gastrocnemius) showed a mild influx of inflammatory cells between the muscle fibers in all sepsis animals, but also in two of the control animals. No initial signs of muscle atrophy were observed. No increase in inflammatory cells were found in the jejunum and ileum, although in the jejunum, villous damage was found in both the control and in the PA animals. The vacuolization and irregular/ blunted villi in jejunum were more commonly present and severe in the PA animals (Figure 4). Subepithelial space in jejunal mucosa crypts was observed in five PA and two Control animals. Moderate overall flattening of ileum villi occurred in the PA animals. In the liver, we observed fatty change and mild influx of inflammatory cells around the central vein in PM animals. The lung showed no indication of DAD.

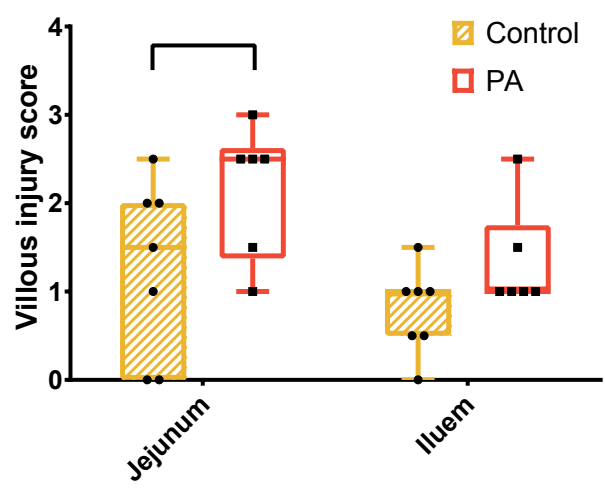




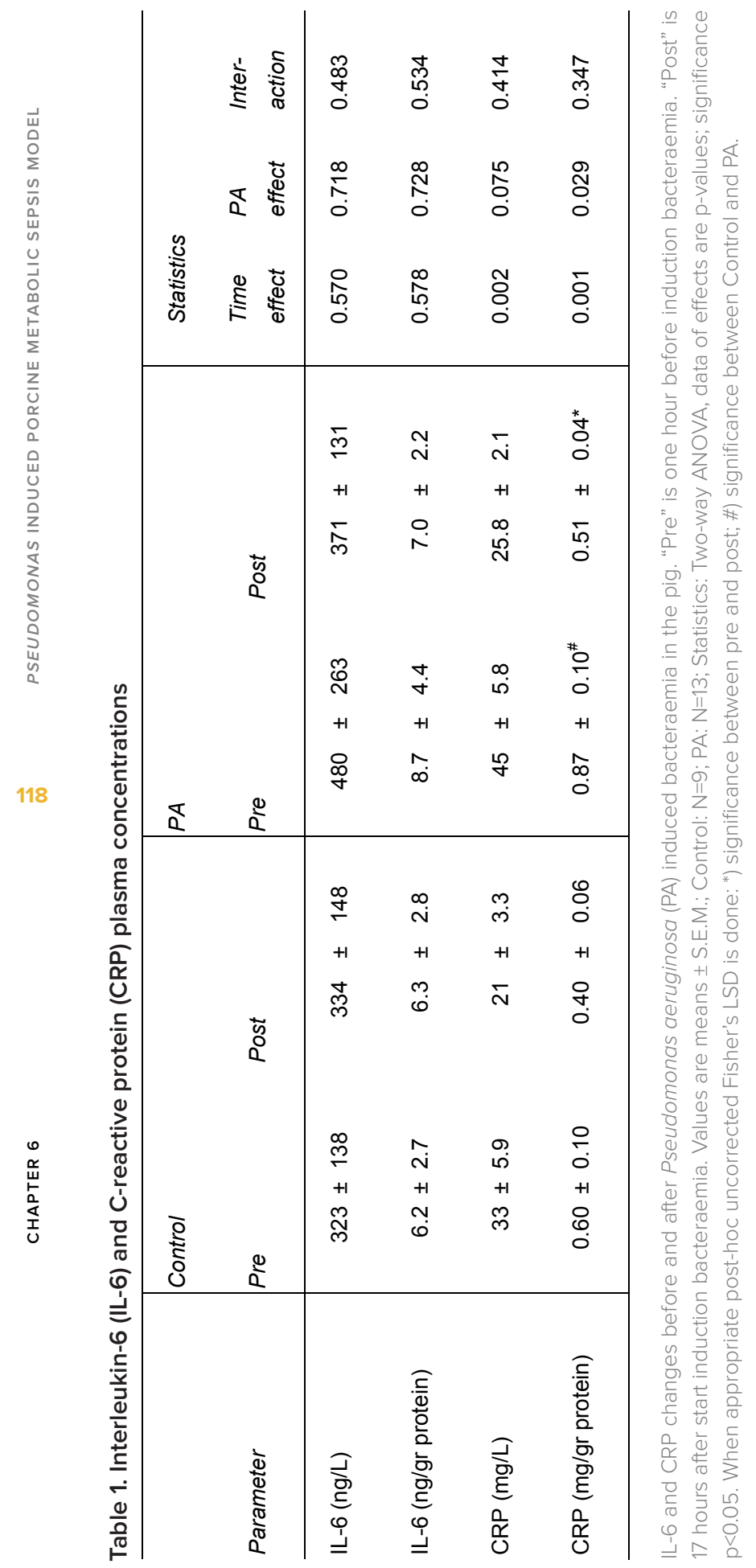




\section{Cytokines and acute phase proteins}

IL-6 data did not change during PA administration (Table 1). CRP values were lower after 17h of PA administration even after compensation for the decrease in total protein in plasma (expressed as mg/mg protein). Both IL-6 and CRP initial concentration data (resp. $419 \pm 163 \mathrm{ng} / \mathrm{L}$ and $40.0 \pm 4.3 \mathrm{mg} / \mathrm{L}$ ) showed a high coefficient of variability (CV): 183 and $50.0 \% \mathrm{CV}$, respectively.

\section{Discussion}

We developed a controlled hyperdynamic porcine model of sepsis with cardiovascular, inflammatory, metabolic characteristics as present in the human septic state. This model enables us to conduct studies unraveling the disturbances in organ metabolic pathophysiology in the early hyperdynamic septic state.

\section{Diagnose of the septic state}

Sepsis is the clinical syndrome of systemic inflammatory response (SIRS) that complicates severe infection. Diagnostic criteria include a documented pathogen plus two or more SIRS abnormalities $(1,3,4)$. The absolute values of clinical parameters can be slightly different in the pig in comparison with humans. Therefore in the present study, we compared the changes of these parameters with normal pig values. Physiological and pathophysiological responses of the pig are highly comparable with humans $(18,19)$, therefore described responses (equating to change in absolute values) of human SIRS criteria can also be used for pigs. With these main diagnostic criteria of SIRS (body temperature: increase of more than two degrees Celcius; heart rate: $>90$ beats/min; $\mathrm{PaCO}_{2}:<32 \mathrm{mmHg}$ ), we concluded that the present infection has successfully induced sepsis in pigs (Figure 2, Supplemental Table 1). This means that the acute septic response of the chosen rate of PA infusion could be controlled by monitoring the hemodynamics and general appearance (alertness, breathing) of the animal during the PA infusion. Sepsis was induced within 6 hours after the start of PA infusion. In this initial phase of sepsis, we observed that it is necessary to carefully monitor MPAP and heart rate in the first two hours to prevent a deep, unrecoverable septic shock. We found that stopping the PA infusion for a few minutes was sufficient to stabilize the animal. The increase in the right ventricular afterload is well described in the medical literature and is likely related to the release of proinflammatory mediators such as TNF $\alpha$ and immunoreactive endothelin-1 which are strong vasoconstrictors on pulmonary vessels (16).

\section{Organ dysfunction}

Severe sepsis state is characterized by one or more organ dysfunctions $(1,3,4)$. We found that despite fluid resuscitation pronounced hypotension, tachycardia and a decreased PDV plasma flow were present, indicating a disturbed cardiovascular 
function (Figure 1 and 2). The decreased PDV flow could play a role in the occurrence of the injured jejunal and ileum mucosa tissues (40). A potentially compromised PDV microcirculation (41) can especially result in a decreased absorption capacity/gut metabolism due to mild/moderate damaged villi (Figure 4). In jejunum of the PA group, it is unclear why subepithelial spacing was not only observed in the villi but also in the crypts. It is likely that the combination of the abnormal translocation of principal oncotic protein in plasma albumin to the intestines that leads to loss of fluid from the vascular space and the fluid resuscitation, could play a role(12) (42). The respiratory system showed no arterial hypoxemia (Supplemental Table 1); therefore, no mechanical ventilation was needed as confirmed by the absence of DAD occurrence in lung tissue. The tissue perfusion parameter lactate revealed hyperlactatemia ( $>1 \mathrm{mM}$ ) according to human criteria (1), but for a pig it was still in the normal physiological range (33). Therefore, we conclude that tissue perfusion is changing in the PA group, however the change is not physiological meaningful.

Acute kidney failure, as reflected by a substantial increase in the creatinine systemic plasma level, was not observed in the present model (Supplemental Table 2) (1, 4). Doi et al (43) indicated that creatinine levels can be inconclusive due to a potential decrease in the creatinine production in sepsis. The fact that we did not find any change in the arterial PAH concentrations, used for organ plasma flow calculations, confirms that renal function was not compromised in the present sepsis model. Although ALT and AST are both biomarkers for liver injury, ALT is more liver specific. Increased AST alone, indicates that there is extrahepatic injury (for instance in skeletal muscle) and fits with the increase of CPK (muscle degradation marker). We conclude that there are no indications of acute hepatic dysfunction (Supplemental Table 2), although liver histology showed mild infection related abnormalities (37). Therefore, we conclude we have developed a sepsis model characterized by severely compromised function of the cardiovascular system, and a mildly compromised function of the PDV by infusing PA. Severe sepsis was established between 12-18 h after starting the PA infusion. Furthermore, we observed several changes in clinical parameters independent of the presence of sepsis, potentially induced by fluid resuscitation (37), indicating that the control group was crucial to discriminate between sepsis and non-sepsis related changes.

\section{Systemic Inflammation symptoms}

The steep increase in body temperature indicated the onset of a PA induced inflammatory response (Figure 2). Additionally, in a follow-up study we conducted a white blood cell (WBC) count (total WBC, lymphocytes, granulocytes and platelets) in a pilot amount of animals (four) in the first six hours after start of PA (Supplemental Figure 2). The observed disappearance of lymphocytes from the systemic blood to the peripheral tissues is expected as early response on the trapped PA bacteria in tissue $(44,45)$. This results in an initial decrease of total WBC, but is quickly 
attenuated by stimulated increase in availability of granulocytes(46). Therefore, we concluded that we observed a clinical expected systemic WBC response on PA. However, this was not accompanied by a clear cytokine (IL-6) and acute phase protein (CRP) response between 12-18hours (Table 1).

Although, a general cytokine response is expected, many factors could be involved, which may explain why we did not see a response. IL-6 and CRP response on PA in the pig over time is a result of amount/strain/route of administration of PA and subsequent complex interaction of multiple host and microbial factors. Therefore, can be highly variable between different studies $(47,48)$ and between animals $(49)$.

The following explanations need to be considered. First, the uncontrolled microbial status of the farm pigs may likely be the cause of high variability of the II-6 and CRP values before the bacteria were infused (50). However, supplemental Figure 1 illustrates that the initial IL-6 or CRP variability is not related to the non-response in the period between 12 and 18h. Therefore, we conclude that it is highly unlikely that the differences in initial cytokine status has an influence on PA cytokine response. Secondly, serum amyloid A (SAA) could potentially be a more sensitive alternative for CRP, but was not measured in the present study (51). However acute phase protein response is considered to be initiated by interleukins like IL-6, also SAA. Therefore, the absence of the CRP response is potentially caused by an absence of an IL- 6 response. Thirdly, we have to consider that the absence of a clear IL-6 and CRP response is a reflection of an inefficient or delayed inflammatory response. This phenomenon in humans is associated with higher mortality in sepsis $(50,52)$. Finally, we need to consider time of measurement. Rimmele et al presented a very early (in the first six hours after administration of PA) but mild response on PA infusion in the pig (16). And is in line with observations in humans with experimental endotoxemia (46). We didn't measure IL-6 in this time window in the present study. Therefore, in a follow-up pilot study using this model (four animals), we could confirm a small IL-6 response in the first six hours (Supplemental Figure 3). Therefore, we can conclude that the cytokine response of IL- 6 in the current model occurred in a very early stage, but didn't continue in the severe septic stage. On the other hand, measurement was maybe too early. For instance, Benes et al found in a ventilated pig model an IL-6 response on comparable PA infusion was started after 18 hours (30). However, the IL-6 response was still mild in comparison with a peritonitis induced sepsis model in the pig. Overall, we can conclude that the model described in the present study showed a clinically relevant inflammatory response in the first six hours.

\section{Potential value for nutritional research in septic state}

Nutritional intervention can be supportive and of high clinical value in severe sepsis (9), in contrast to the septic shock state (53). Sepsis related metabolic changes 
like protein breakdown $(5,24)$, hypocalcemia (54) and hypocholesterolemia (55) were observed in the current model (Supplemental Table 2). Therefore, we concluded that with these metabolic characteristics, the presented PA model with implanted catheters to ensure the possibility of measuring metabolism across organs $(23,24,26)$, could be of high value for preclinical nutritional research in severe sepsis. Though other PA infused pig models are available, but they are septic shock models in an unconscious ventilated pig $(16,30)$, and therefore not suitable for nutritional research.

\section{Limitation of the model}

First, though PA is a clinically relevant bacteria in sepsis, it is not the only bacteria that can cause sepsis. When comparing other live bacteria used to induce severe sepsis in pigs, the pathophysiology of a single dose of gram positive Staphylococcus aureus (22) in a conscious pig model showed slightly different patterns in the hyperdynamics, and a late (after 18 hour) cytokine response. A similar late cytokine response was observed in a mixed bacterium model (peritonitis created by intraperitoneal autologous fecal inoculum) (19). Therefore, we conclude that although metabolic data are lacking from these models, it is highly likely that metabolic results coming from the present PA severe sepsis model can be translational to general severe sepsis, independent of the bacteria that caused the septic state. Secondly, we need to consider that the relative young age of the studied pigs cannot represent metabolic implications seen in adults. We made these concessions for practical reasons (handling, catheter protection with the used harness), but with the knowledge that the development stage of gastro intestinal tract in these animals are already mature (56). Therefore, we can still make the translation of metabolic findings to broader age range. Finally, only the initial phase of severe sepsis has been represented. This means that results cannot be easily translated to a recovery phase in sepsis (for instance after antibiotic treatment). Therefore further development of the sepsis model is needed for studying metabolism in the recovery phase.

\section{Conclusion}

The present acute sepsis model has the characteristics to be hyperdynamic and bi-phasic with a severe septic phase present between 12-18h after PA infusion. The present PA induced porcine sepsis model is a clinically relevant model for acute severe sepsis. Implanted catheters can accommodate an in-depth study of multiorgan metabolism and nutrient metabolism in sepsis. 
We thank the assigned veterinarians and animal caretakers of the Arkansas Children's Hospital Research Institute for their dedication in providing optimal care for the pigs in our experiment. We thank Joshua Spore, Bea Zoer, Eva M.C. Oosterlaken and Sonya R. Mehta for skilled technical assistance in carrying out the animal experiments and/or sample processing.

The project described was supported by Award Number R01GM084447 from the National Institute Of General Medical Sciences and by Award Number S10RR027047 from the National Center For Research Resources. The content is solely the responsibility of the authors and does not necessarily represent the official views of the National Institute Of General Medical Sciences or National Center For Research Resources or the National Institutes of Health.

\section{References}

1. Levy MM, Fink MP, Marshall JC, Abraham E, Angus D, Cook D, et al. 2001 SCCM/ESICM/ACCP/ATS/SIS International Sepsis Definitions Conference. Crit Care Med. 2003;31(4):1250-6.

2. Weycker D, Akhras KS, Edelsberg J, Angus DC, Oster G. Long-term mortality and medical care charges in patients with severe sepsis. Crit Care Med. 2003;31(9):2316-23.

3. Iskander KN, Osuchowski MF, Stearns-Kurosawa DJ, Kurosawa S, Stepien D, Valentine C, et al. Sepsis: multiple abnormalities, heterogeneous responses, and evolving understanding. Physiological reviews. 2013;93(3):1247-88.

4. Goldstein B, Giroir B, Randolph A, International Consensus Conference on Pediatric S. International pediatric sepsis consensus conference: definitions for sepsis and organ dysfunction in pediatrics. Pediatric critical care medicine : a journal of the Society of Critical Care Medicine and the World Federation of Pediatric Intensive and Critical Care Societies. 2005;6(1):2-8.

5. Rooyackers O, Kouchek-Zadeh R, Tjader I, Norberg A, Klaude M, Wernerman J. Whole body protein turnover in critically ill patients with multiple organ failure. Clin Nutr. 2015;34(1):95-100.

6. Liebau F, Sundstrom M, van Loon LJ, Wernerman J, Rooyackers O. Short-term amino acid infusion improves protein balance in critically ill patients. Crit Care. 2015;19:106.

7. Kopple JD. Pathophysiology of protein-energy wasting in chronic renal failure. J Nutr. 1999;129(1S Suppl):247S-51S

8. Kopple JD, Zhu X, Lew NL, Lowrie EG. Body weight-for-height relationships predict mortality in maintenance hemodialysis patients. Kidney Int. 1999;56(3):1136-48.

9. Deutz NE, Matheson EM, Matarese LE, Luo M, Baggs GE, Nelson JL, et al. Readmission and mortality in malnourished, older, hospitalized adults treated with a specialized oral nutritional supplement: A randomized clinical trial. Clin Nutr. 2016;35(1):18-26.

10. Piton G, Capellier G. Biomarkers of gut barrier failure in the ICU. Curr Opin Crit Care. 2016;22(2):152-60.

11. Baron P, Traber LD, Traber DL, Nguyen T, Hollyoak M, Heggers JP, et al. Gut failure 
and translocation following burn and sepsis. J Surg Res. 1994;57(1):197-204.

12. Kim MH, Ahn JY, Song JE, Choi H, Ann HW, Kim JK, et al. The C-Reactive Protein/ Albumin Ratio as an Independent Predictor of Mortality in Patients with Severe Sepsis or Septic Shock Treated with Early Goal-Directed Therapy. PLoS One. 2015;10(7):e0132109.

13. Suffredini AF, Fantuzzi G, Badolato R, Oppenheim JJ, O'Grady NP. New insights into the biology of the acute phase response. Journal of clinical immunology. 1999;19(4):203-14.

14. Bengmark S. Nutrition of the critically ill \&\#8212; a 21st-century perspective. Nutrients. 2013;5(1):162-207.

15. Deitch EA. Animal models of sepsis and shock: a review and lessons learned. Shock. 1998;9(1):1-11.

16. Rimmele T, Assadi A, Benatir F, Boselli E, Kaminski C, Arnal F, et al. Validation of a Pseudomonas aeruginosa porcine model of septic shock. J Infect. 2006;53(3):199-205.

17. Doi K, Leelahavanichkul A, Yuen PS, Star RA. Animal models of sepsis and sepsisinduced kidney injury. J Clin Invest. 2009;119(10):2868-78.

18. Soerensen KE, Olsen HG, Skovgaard K, Wiinberg B, Nielsen OL, Leifsson PS, et al. Disseminated intravascular coagulation in a novel porcine model of severe Staphylococcus aureus sepsis fulfills human clinical criteria. J Comp Pathol. 2013;149(4):463-74.

19. Clary EM, Bruch SM, Lau CL, Ali A, Chekan EG, Garcia-Oria MJ, et al. Effects of pneumoperitoneum on hemodynamic and systemic immunologic responses to peritonitis in pigs. J Surg Res. 2002;108(1):32-8.

20. Abubaih A, Weissman C. Anesthesia for Patients with Concomitant Sepsis and Cardiac Dysfunction. Anesthesiol Clin. 2016;34(4):761-74.

21. Reith S, Ortlepp JR. [State of the art - intensive care therapy of septic patients]. Dtsch Med Wochenschr. 2016;141(15):1082-90.

22. Soerensen KE, Nielsen OL, Birck MM, Soerensen DB, Leifsson PS, Jensen HE, et al. The use of sequential organ failure assessment parameters in an awake porcine model of severe Staphylococcus aureus sepsis. APMIS. 2012;120(11):909-21.

23. Ten Have GA, Bost MC, Suyk-Wierts JC, van den Bogaard AE, Deutz NE. Simultaneous measurement of metabolic flux in portally-drained viscera, liver, spleen, kidney and hindquarter in the conscious pig. Lab Anim. 1996;30(4):347-58.

24. Bruins MJ, Deutz NE, Soeters PB. Aspects of organ protein, amino acid and glucose metabolism in a porcine model of hypermetabolic sepsis. Clin Sci (Lond). 2003;104(2):127-41.

25. Bruins MJ, Luiking YC, Soeters PB, Akkermans LM, Deutz NE. Effect of prolonged hyperdynamic endotoxemia on jejunal motility in fasted and enterally fed pigs. Ann Surg. 2003;237(1):44-51.

26. Bruins MJ, Soeters PB, Deutz NE. Endotoxemia affects organ protein metabolism differently during prolonged feeding in pigs. J Nutr. 2000;130(12):3003-13.

27. Kadletz M, Dignan RJ, Mullen PG, Windsor AC, Sugerman HJ, Wechsler AS. Pulmonary artery endothelial cell function in swine pseudomonas sepsis. J Surg Res. 1996;60(1):186-92.

28. Han JJ, Windsor A, Drenning DH, Leeper-Woodford S, Mullen PG, Bechard DE, et al. Release of endothelin in relation to tumor necrosis factor-alpha in porcine Pseudomonas aeruginosa-induced septic shock. Shock. 1994;1(5):343-6.

29. Assadi A, Desebbe O, Kaminski C, Rimmele T, Benatir F, Goudable J, et al. Effects of sodium nitroprusside on splanchnic microcirculation in a resuscitated porcine model of septic shock. Br J Anaesth. 2008;100(1):55-65.

30. Benes J, Chvojka J, Sykora R, Radej J, Krouzecky A, Novak I, et al. Searching for 
mechanisms that matter in early septic acute kidney injury: an experimental study. Crit Care. 2011;15(5):R256.

31. Ramachandran G. Gram-positive and gram-negative bacterial toxins in sepsis: a brief review. Virulence. 2014;5(1):213-8.

32. Poeze M, Bruins MJ, Kessels F, Luiking YC, Lamers WH, Deutz NE. Effects of L-arginine pretreatment on nitric oxide metabolism and hepatosplanchnic perfusion during porcine endotoxemia. The American journal of clinical nutrition. 2011;93(6):1237-47.

33. Hannon JP, Bossone CA, Wade CE. Normal physiological values for conscious pigs used in biomedical research. In: research DoMt, editor. San Francisco, CA, USA: Letterman Army Institute of Reserach; 1989. p. 1-20.

34. Bisson J, Younker J. Correcting arterial blood gases for temperature: (when) is it clinically significant? Nursing in critical care. 2006;11(5):232-8.

35. Schooneman MG, Ten Have GA, van Vlies N, Houten SM, Deutz NE, Soeters MR. Transorgan fluxes in a porcine model reveal a central role for liver in acylcarnitine metabolism. Am J Physiol Endocrinol Metab. 2015;309(3):E256-64.

36. Agarwal R. Rapid microplate method for PAH estimation. American journal of physiology Renal physiology. 2002;283(2):F236-41.

37. Lucas S. The autopsy pathology of sepsis-related death. Current Diagnostic Pathology. 2007:13:375-88.

38. Morales MG, Olguin H, Di Capua G, Brandan E, Simon F, Cabello-Verrugio C. Endotoxin-induced skeletal muscle wasting is prevented by angiotensin-(1-7) through a p38 MAPK-dependent mechanism. Clin Sci (Lond). 2015;129(6):461-76.

39. Feinman R, Deitch EA, Watkins AC, Abungu B, Colorado I, Kannan KB, et al. HIF-1 mediates pathogenic inflammatory responses to intestinal ischemia-reperfusion injury. Am J Physiol Gastrointest Liver Physiol. 2010;299(4):G833-43.

40. Chiu CJ, McArdle AH, Brown R, Scott HJ, Gurd FN. Intestinal mucosal lesion in low-flow states. I. A morphological, hemodynamic, and metabolic reappraisal. Arch Surg. 1970;101(4):478-83.

41. Dyson A, Cone S, Singer M, Ackland GL. Microvascular and macrovascular flow are uncoupled in early polymicrobial sepsis. Br J Anaesth. 2012;108(6):973-8.

42. Levitt DG, Levitt MD. Human serum albumin homeostasis: a new look at the roles of synthesis, catabolism, renal and gastrointestinal excretion, and the clinical value of serum albumin measurements. Int J Gen Med. 2016;9:229-55.

43. Doi K, Yuen PS, Eisner C, Hu X, Leelahavanichkul A, Schnermann J, et al. Reduced production of creatinine limits its use as marker of kidney injury in sepsis. J Am Soc Nephrol. 2009;20(6):1217-21.

44. Venet F, Davin F, Guignant C, Larue A, Cazalis MA, Darbon R, et al. Early assessment of leukocyte alterations at diagnosis of septic shock. Shock. 2010;34(4):358-63.

45. Bijli KM, Kanter BG, Minhajuddin M, Leonard A, Xu L, Fazal F, et al. Regulation of endothelial cell inflammation and lung polymorphonuclear lymphocyte infiltration by transglutaminase 2. Shock. 2014;42(6):562-9.

46. Andreasen AS, Pedersen-Skovsgaard T, Mortensen OH, van Hall G, Moseley PL, Pedersen BK. The effect of glutamine infusion on the inflammatory response and HSP70 during human experimental endotoxaemia. Crit Care. 2009;13(1):R7.

47. Correa TD, Vuda M, Blaser AR, Takala J, Djafarzadeh S, Dunser MW, et al. Effect of treatment delay on disease severity and need for resuscitation in porcine fecal peritonitis. Crit Care Med. 2012;40(10):2841-9. 
Assays for Determining Pathogenic Potential of Pseudomonas Strains Used in Biotechnology. PLoS One. 2015;10(11):e0143604.

49. Tambuyzer T, De Waele T, Chiers K, Berckmans D, Goddeeris BM, Aerts JM. Interleukin- 6 dynamics as a basis for an early-warning monitor for sepsis and inflammation in individual pigs. Research in veterinary science. 2014;96(3):460-3.

50. Moore SA, Levy BH, Prematilake C, Dissanaike S. The Prediction Predicament: Rethinking Necrotizing Soft Tissue Infections Mortality. Surg Infect (Larchmt). 2015;16(6):813-21.

51. Pomorska-Mol M, Markowska-Daniel I, Kwit K, Stepniewska K, Pejsak Z. C-reactive protein, haptoglobin, serum amyloid $A$ and pig major acute phase protein response in pigs simultaneously infected with H1N1 swine influenza virus and Pasteurella multocida. BMC Vet Res. 2013;9:14.

52. Raju MS, V J, Kamaraju RS, Sritharan V, Rajkumar K, Natarajan S, et al. Continuous evaluation of changes in the serum proteome from early to late stages of sepsis caused by Klebsiella pneumoniae. Mol Med Rep. 2016;13(6):4835-44.

53. Vassal O, Bonnet JM, Barthelemy A, Allaouchiche B, Goy-Thollot I, Louzier V, et al. Renal haemodynamic response to amino acids infusion in an experimental porcine model of septic shock. Acta Anaesthesiol Scand. 2015;59(5):598-608.

54. Muller B, Becker KL, Kranzlin M, Schachinger H, Huber PR, Nylen ES, et al. Disordered calcium homeostasis of sepsis: association with calcitonin precursors. European journal of clinical investigation. 2000;30(9):823-31.

55. Green P, Theilla M, Singer P. Lipid metabolism in critical illness. Curr Opin Clin Nutr Metab Care. 2016;19(2):111-5.

56. Guilloteau P, Zabielski R, Hammon HM, Metges CC. Nutritional programming of gastrointestinal tract development. Is the pig a good model for man? Nutr Res Rev. 2010;23(1):4-22.

57. Harris WH. Hemoglobin, blood gases and serum electrolyte values in swine. Can Vet J. 1974;15(10):282-5.

58. Fraser CM, Bergeron JA, Mays A, Aiello SE, editors. The Merck Veterinary manual. seventh edition ed. Rahway, NJ, USA: Merck \& Co., Inc.; 1991.

59. Fujii T, Phillips B. Quick Review: Oxygen Transport. The internet Journal of Academic Physician Assistants. 2002;3(1):1-4.

\section{Supplemental information}

Supplemental Table 1 (right). Arterial and Venous blood gas values before and after 6, 12 and 18 hours of Pseudomonas aeruginosa (PA) infusion

Data are means \pm S.E.M.; Control $\mathrm{N}=8$; Sepsis $\mathrm{N}=11 . \mathrm{CaO}_{2}$ : content of oxygen in arterial blood; $\mathrm{ERO}_{2}$ : extraction ratio of oxygen; $\mathrm{FiO}_{2}$ : fraction of inspired oxygen; $\mathrm{PaO}_{2} / \mathrm{FiO}_{2}$ : oxygenation index. Statistics: Two-way ANOVA; data of effects are p-values; significance $p<0.05$. References normal ranges: *) (57); $\left.\left.\left.{ }^{* *}\right)(33) ;{ }^{* * *}\right)(58) ;{ }^{* * *}\right)(59)$. 


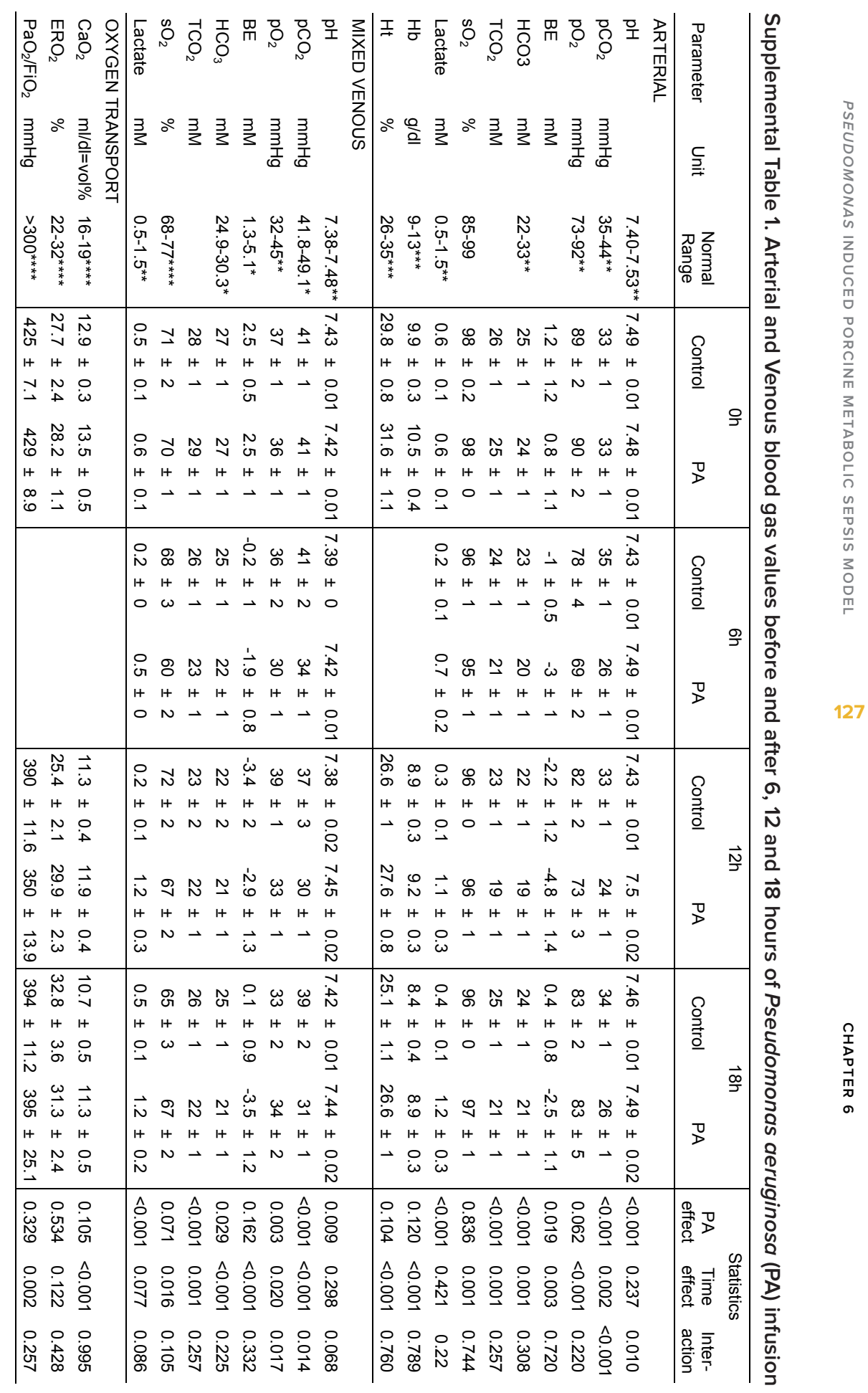




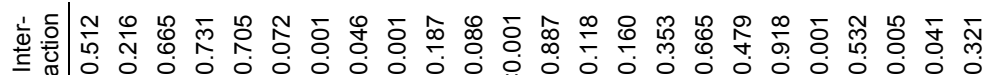

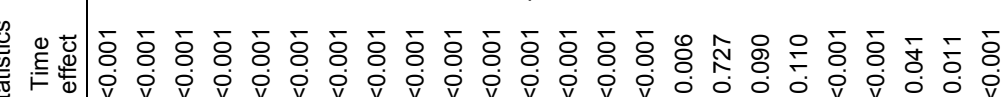

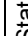

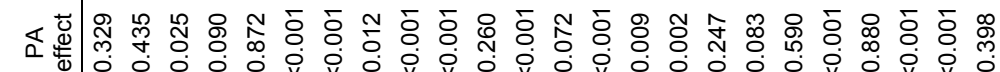

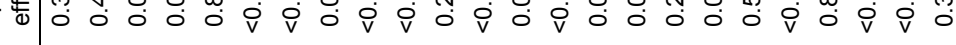

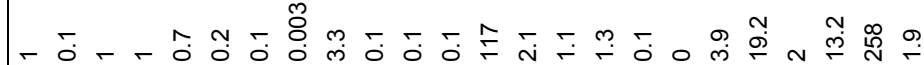

$\begin{array}{lllllllllllllllllllllllllllllllll}0 & +1 & +1 & +1 & +1 & +1 & +1 & +1 & +1 & +1 & +1 & +1 & +1 & +1 & +1 & +1 & +1 & +1 & +1 & +1 & +1 & +1 & +1 & +1 & +1\end{array}$ 拈

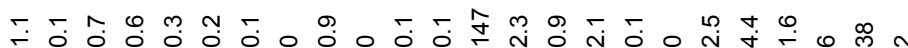

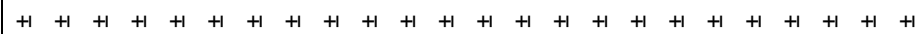

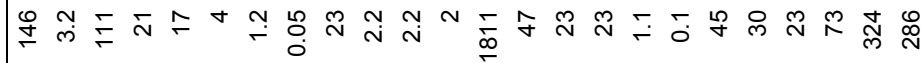

占

$\begin{array}{llllllllllllllllllllllllll}\mathbb{a} & +1 & +1 & +1 & +1 & +1 & +1 & +1 & +1 & +1 & +1 & +1 & +1 & +1 & +1 & +1 & +1 & +1 & +1 & +1 & +1 & +1 & +1 & +1 & +1\end{array}$

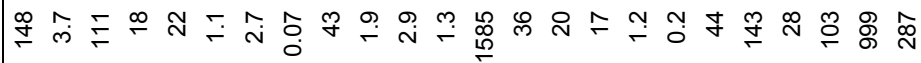

$\frac{\mathfrak{c}}{\mathfrak{2}}$

寊

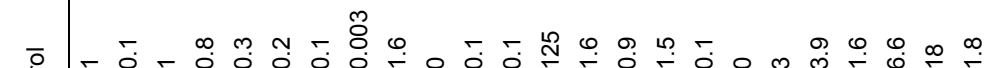

0
0

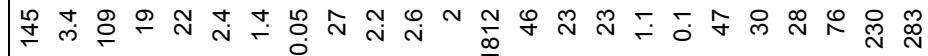

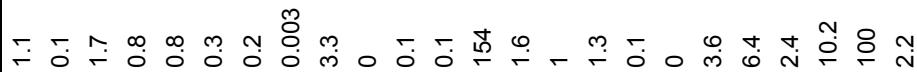

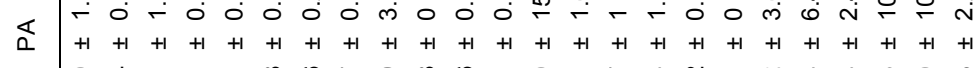

乎

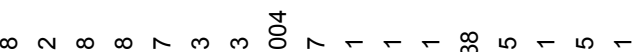

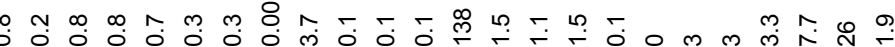

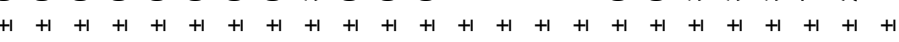

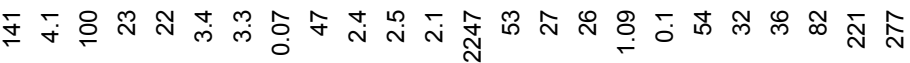

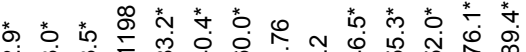

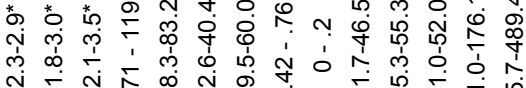

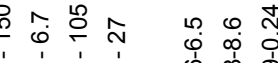
它

认ั

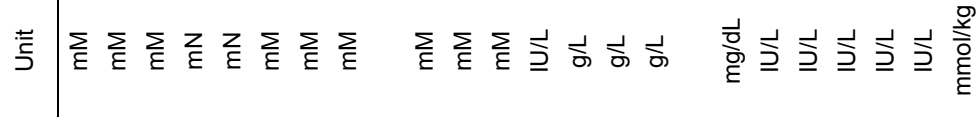

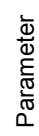


Supplemental table 2 (left). Arterial clinical chemistry values before and after 12 and $18 \mathrm{~h}$ of Pseudomonas aeruginosa (PA) infusion

Data are means \pm S.E.M; Control N=9; Sepsis $N=13$; ALT: alanine aminotransferase; AST: aspartate aminotransferase; GGT: gamma-glutamyltransferase; CPK: creatinine phosphokinase. Statistics: Two-way ANOVA; data of effects are $p$-values; significance $p<0.05$. References normal ranges: *) (58).
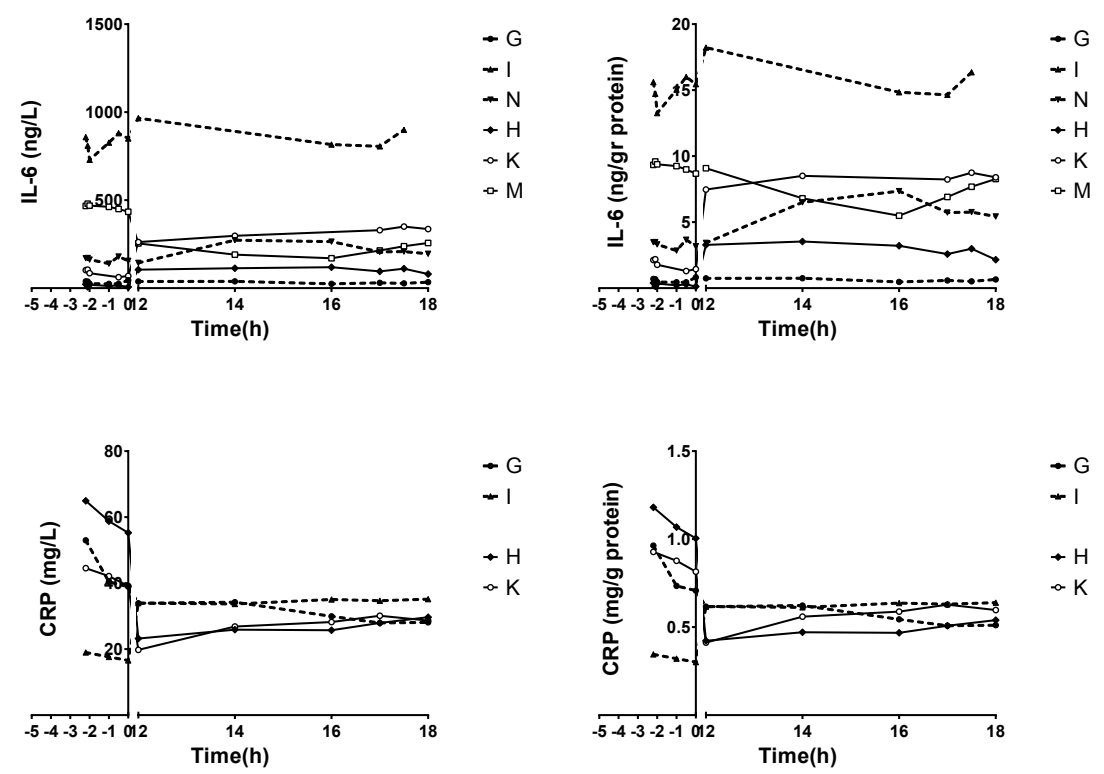

Supplemental Figure 1. Individual time courses of arterial Interleukin-6 (II-6) and C-reactive protein (CRP) values, pilot

Dash line are control animals; Straight line are Pseudomonas aeruginosa (PA) animals. II-6: Control N=2; PA N=4; CRP: Control N=2; PA N=2. Left graphs expressed per liter; Right graphs expressed per gram plasma protein. Regression slope of time courses (-2-0; 12-18h) not significant from zero for IL-6 and minor positive and negative slopes in the CRP time courses. 

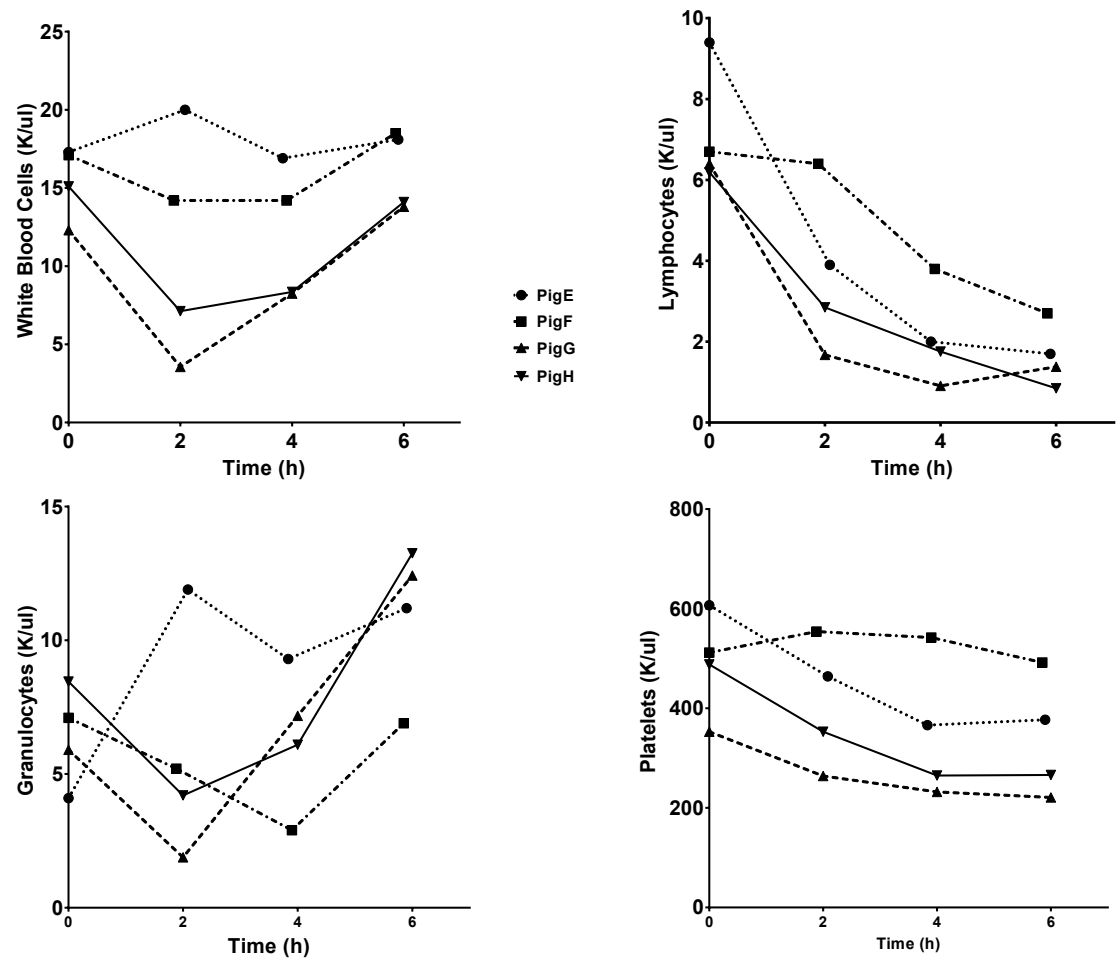

In pilot amount of pigs (four) from a follow-up study. During the first six hours of Pseudomonas aeruginosa induced development of sepsis.

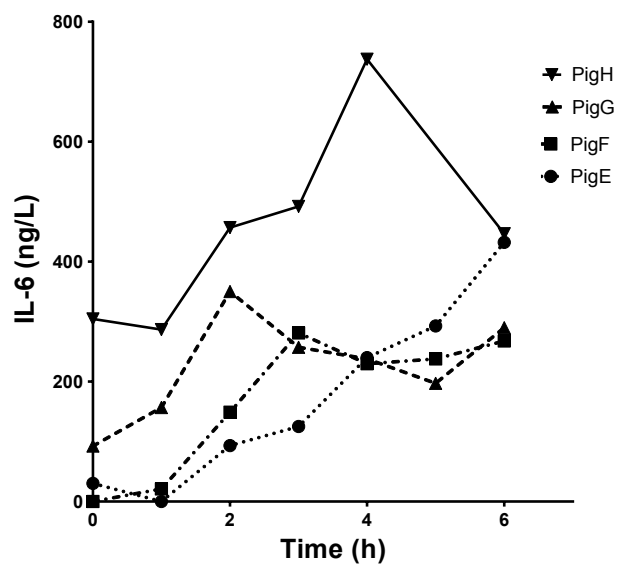

\section{Supplemental Figure 3. Interleukin (IL-6)}

In pilot amount of pigs (four) from a follow-up study. During the first six hours of Pseudomonas aeruginosa induced development of sepsis. 
CHAPTER 7 


\title{
Phenylalanine isotope pulse method to measure effect of sepsis on protein breakdown and membrane transport in the pig.
}

\author{
Gabriella A.M. ten Have, Mariëlle P.K.J. Engelen, Robert \\ R. Wolfe, Nicolaas E.P. Deutz.
}

Am J Physiol Endocrinol Metab. 2017 Mar 14:ajpendo.00351.2016.

\section{Abstract}

The primed-continuous ( $\mathrm{PC}$ ) phenylalanine (Phe) stable isotope infusion methodology is often used as a proxy for measuring whole body protein breakdown (WbPB) in sepsis. It is unclear, if WbPB data obtained by an easy-to-use single IV Phe isotope pulse administration (PULSE) are comparable to those by PC. Compartmental modeling with PULSE could provide us more insight in WbPB in sepsis. Therefore, in the present study, we compared PULSE with PC as proxy for WbPB in an instrumented pig model with Pseudomonas aeruginosa induced severe sepsis (Healthy: $n=9$; Sepsis: $n=13)$. Seventeen hours after sepsis induction, we compared the Wb rate of appearance $\left(\mathrm{WbR}_{\mathrm{a}}\right)$ of Phe obtained by PC(L-[ring- $\left.{ }^{13} \mathrm{C}_{6}\right]$-Phe) and PULSE(L-[15N]Phe) in arterial plasma using LC-MS/MS and (non)compartmental modeling.

PULSE-WbRa was highly correlated with PC-WbRa $(r=0.732, p<0.0001)$ and WbPB $(r=0.897, p<0.0001)$ independent of the septic state. PULSE-WbRa was 1.6 times higher than PC-WbRa $(p<0.001)$. Compartmental and non-compartmental PULSE modeling provides comparable WbRa values, although compartmental modeling was more sensitive. WbPB was elevated in sepsis (Healthy: 3378 \pm 103 ; Sepsis: 4333 $\pm 160 \mathrm{nmol} / \mathrm{kg} \mathrm{BW} / \mathrm{min}, \mathrm{p}=0.0002)$. With PULSE, sepsis was characterized by an increase of the metabolic shunting (Healthy: $3021 \pm 347$; Sepsis: $4233 \pm 344 \mathrm{nmol} / \mathrm{kg}$ $\mathrm{BW} / \mathrm{min}, \mathrm{p}=0.026)$. Membrane transport capacity was the same.

Both PC and PULSE-methods are able to assess changes in WbRa of plasma Phe reflecting WbPB changes with high sensitivity, independent of the (patho-)physiological state. The easy-to-use (non-)compartmental PULSE reflects better the real WbPB than PC. With PULSE compartmental analysis, we conclude that the membrane transport capacity for amino acids is not compromised in severe sepsis. 
The primed-constant and continuous infusion methodology $(P C)$ is often used for measuring whole body rate of appearance $\left(\mathrm{WbR}_{\mathrm{a}}\right)$ of amino acids in humans and animals (1-3). The principle is, that a single pool model (Figure 1) in which amino acids (e.g. from whole body protein breakdown, $\mathrm{WbPB}$ ) are appearing, is the same pool in which the stable amino acid isotopes are infused. It is assumed that the pool represents mainly the extracellular compartment. In this model, $\mathrm{WbR}_{\mathrm{a}}$ is calculated by dividing the tracer (stable isotope) infusion rate by the tracer/tracee ratio (TTR) in the extracellular pool at steady state, and in which the tracee is the natural occurring amino acid. Arterial blood is mostly used to represent the extracellular pool. The main advantage of measuring WbRa using this method is that when the TTR is low, the effects on tracee metabolism are minimal. Furthermore, when steady state is obtained, only two blood samples are needed (background and at steady state). The main disadvantage is that it might take several hours to reach a tracer steady state, although this can be overcome by correctly priming the tracer pool. The estimation of the pool size for stable tracer studies depends on the size of the plasma and tissue pool of the tracee. In studies in diseased animals or humans, the individual variation of the pool size affects the probability to correctly prime the pool. In sepsis for example, plasma phenylalanine (Phe) concentrations can be increased 2 fold, while other amino acid concentrations also can be increased many fold up or down (4). Furthermore, it is very difficult to correctly prime the pools of urea, glutamine, taurine, and 3-methylhistidine $(5,6)$. Moreover, the PC method is highly dependent on the accuracy of the infusion pump. Although the calculation is very simple, $\mathrm{WbR}_{\mathrm{a}}$ is the only information that can be obtained from the PC method as no further metabolic modeling is possible.

To overcome these disadvantages, we explored the use of a single pulse of tracer (PULSE) into the extracellular pool (Figure 1) to measure WbRa (1). In this approach, the shape of the TTR decay curve depends on how many pools are connected to the extracellular pool. For most substrates like amino acids, the intracellular pool is the main secondary pool and the amino acid decay curve therefore reflects two compartments. This TTR decay curve can be used to perform both non-compartmental and compartmental modeling (7-9). Compartmental modeling enables the calculation of the Ra into the extracellular and the intracellular pools, as well as the fluxes between the pools. The appearance of an essential amino acid (e.g. Phe) into the intracellular pool represents protein breakdown (PB). Advantages of the PULSE method are that only one single tracer dose needs to be administered, no pumps are required, low pharmacy costs, and there is no need to prime. A major disadvantage however is that multiple blood samples are needed to describe the enrichment decay curve accurately. Although the calculations are not as simple as with the PC model, modern modeling/fitting programs are more available and userfriendly. For routine Ra measurements, the non-compartmental WbRa calculation 
PC

(non-compartmental)

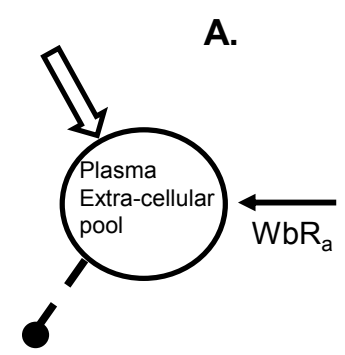

PULSE

(compartmental)

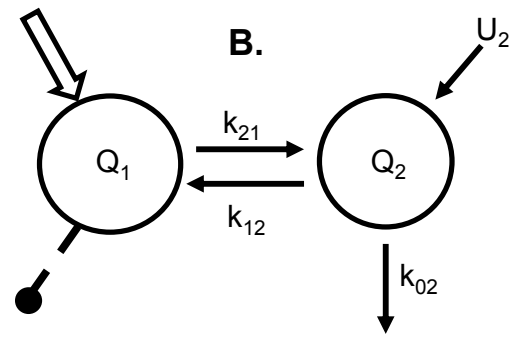

C.

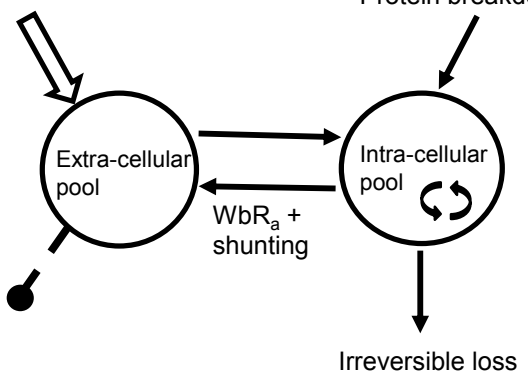

Figure 1: Calculation models for whole body protein breakdown

Two calculation models for whole body protein breakdown (WbPB) by determination of whole body rate of appearance (WbRa) of phenylalanine (Phe). (A) Non-compartmental model using a primed-continuous infusion of $L-\left[\right.$ ring $\left.-{ }^{13} \mathrm{C}_{6}\right]$-Phe $(\mathrm{PC})$. WbRa is the tracer infusion rate divided by the fraction of tracer found in plasma/extra cellular pool (TTR=tracer/tracee ratio) in a tracer steady state (B) Compartmental model using bolus infusion, L-[ $\left[{ }^{15} \mathrm{~N}\right]-\mathrm{Phe}$ (PULSE) and SAAMII computer modeling. Computer parameters: $Q=$ pool sizes of the compartments; $k_{21}=$ rate parameter to pool 2, from pool 1; $k_{02}$ rate parameter of irreversible loss from pool 2; $U_{2}$ : rate of appearance of Phe in pool 2. (C) Physiological assignment of SAAMII computer model: Flux $\left(F_{12}\right)$ of Phe from the intra-cellular pool to the extra cellular pool represents the $k_{21}$ times $Q_{1}$, and is equal to $F_{21}$ in physiological steady state; $U_{2}\left(=F_{20}\right)$ represents $\mathrm{WbPB}$; Flux of Phe from the intra-cellular pool to hydroxylation and protein synthesis represents the irreversible loss $\left(F_{02}\right.$ $\left.=k_{02} Q_{2}\right) ;$ WbRa of Phe is the fraction of Phe from protein breakdown that appears in $Q_{1}$ that is not irreversible lost; The amount of Phe flux between $Q_{1}$ and $Q_{2}$ that is not irreversible lost is shunt back to $Q_{1}\left(F_{21}-W b R a\right)$.

in the PULSE method using the fitting of the decay curve with e.g. the software program GraphPad Prism or add-ins of EXCEL, and additional relative simple calculations of $\mathrm{WbR}_{\mathrm{a}}$ can be attractive (1). Previously, the computer modeling program SAAM has been used in the compartmental analysis to measure 3-methylhistidine appearance (as indirect marker of myofibrillar muscle PB) (10) or citrulline production 
in humans (11). Compartmental modeling can be extended to measure the flux between the different metabolic routes $(8,12)$. PULSE methods with a small amount of tracer are also used to measure tissue specific fractional protein synthesis (FSR) and breakdown (FBR) (13-16), but these methods require additional sampling from the tissue compartment.

In the past, comparisons of the tracer PULSE and PC infusion methods were done with theoretically modeling (17), in healthy subjects and animals (9, 10, 13), but not in disease states. In disease conditions with large changes in the tracee pools and in situation in which there is a large effect of the disease on e.g. whole body protein breakdown, it is unclear if the PULSE method is providing the same clinical information as the more generally used PC method. Specifically in disease states, the additional information coming from the PULSE compartmental modeling can increase the understanding of the clinical situation. Therefore, in the present study, we measured whole body rate of appearance $\left(\mathrm{WbR}_{\mathrm{a}}\right)$ of Phe as proxy for WbPB using the PC protocol in comparison to a single-dose PULSE tracer protocol using Phe stable isotopes during Pseudomonas aeruginosa (PM) induced severe sepsis in the pig. The severe septic state is used as a disease model in which large changes in tracee pools and substantial effects on WbPB can be expected (18). We hypothesize that both the PC and the PULSE method are able to provide the expected enhanced WbPB information in severe sepsis. We compared the more practical non-compartmental modeling with the PULSE method with the compartmental modeling to obtain this information for a routine setting. Finally, we explored with the compartmental modeling the extra intracellular information (eg membrane transport capacity).

\section{Material and Methods}

\section{Animals}

We used Female Yorkshire cross/domestic pigs (20-25 kg BW) in our experimental studies. We housed the pigs in steel pens $(2 \mathrm{~m} \times 3 \mathrm{~m})$ in a controlled housing facility (large animal cubicle, room temperature $22-24^{\circ} \mathrm{C}, 12$ hours light-dark cycle, standardized food 1kg/day (Harlan Teklad Vegetarian Pig/Sow Grower) and provided water ad libitum.

\section{Surgical procedure}

Animals received catheters and a jejunal stoma during a surgical procedure as described in detail previously $(19,20)$. In brief, during midline laparotomy, we placed catheters for blood sampling into the abdominal aorta and in the caval vein for administering post-surgery medication and experiment related tracer infusions. We 
placed a second arterial catheter to monitor mean arterial blood pressure (MAP) and a Swan ganz catheter (5 Fr, \#132F5,Edwards life sciences, Irvine, CA, USA) via the right jugular vein to monitor mean pulmonary blood pressure (MPAP). We standardized preoperative and postoperative care $(19,20)$. During the recovery period (7-10 days) animals were accustomed to a small movable cage $(0.9 \times 0.5 \times 0.3 \mathrm{~m})$ and performed the experiments in this cage in awake animals. This study was approved by the animal experiment ethics committee of University of Arkansas Medical Sciences.

\section{Experimental Design (Figure 2)}

The experiment started after a recovery period of 7-10 days. Four hours after the last food intake (half of the daily amount: $0.5 \mathrm{~kg}$ ), we selected animals for the Sepsis group and the Healthy group in a randomized fashion (Healthy $n=9$, Sepsis $n=13$ ). We monitored basal monitoring blood pressures in the pre-septic period ( $T=-2 \mathrm{~h}-0$ h). At $\mathrm{T}=\mathrm{Oh}$, we induced sepsis by continuous infusion of Pseudomonas aeruginosa (PM, $10^{9} \mathrm{CFU} / \mathrm{ml} /$ hour), while the Healthy group received an equal volume of $0.9 \%$ $\mathrm{NaCl}$ solution. We started fluid resuscitation (30 ml/kg bw/hour) also at T=0h and monitored hemodynamics continuously. For 1 hour, between 17 and 18 hours after the start of PM, we compared the PC tracer protocol with the PULSE tracer protocol and at $\mathrm{t}=18 \mathrm{~h}$, we euthanized the pigs with $125 \mathrm{mg} / \mathrm{kg}$ pentobarbital sodium and 16 $\mathrm{mg} / \mathrm{kg}$ phenytoin sodium (Euthanasol ${ }^{\circledR}$ ) administered via the central vein catheter.

\section{Pseudomonas aeruginosa}

For the induction of sepsis, we used a live PM human strain (IRS 12-4-4, Shriners burns institute, University of Texas Medical Branch, Galveston). Originally this PM

\section{Hyper dynamics monitoring: MAP, MPAP, Body Temp}

Fluid resuscitation $(30 \mathrm{ml} / \mathrm{kg}$ bw/hour)

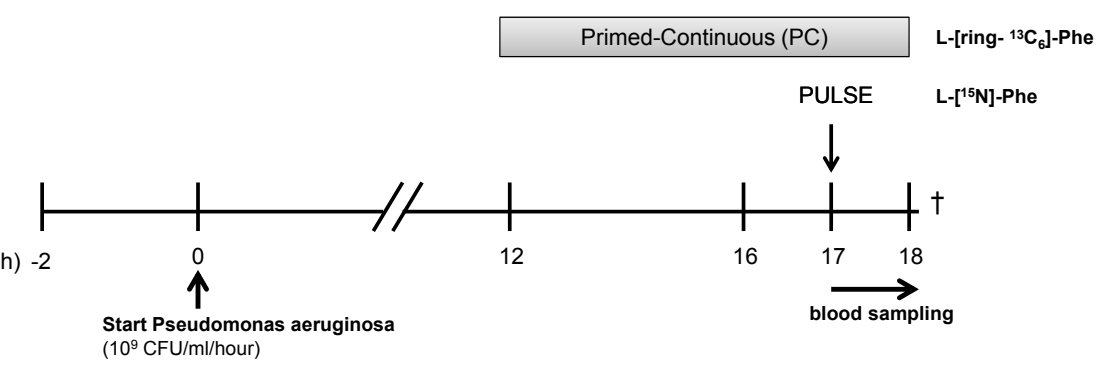

Figure 2: Protocol

PC: primed-continuous infusion: L-[ring- $\left.{ }^{13} \mathrm{C}_{6}\right]$-Phenylalanine (Phe). PULSE: bolus infusion: L- $\left[{ }^{15} \mathrm{~N}\right]-$ Phe. Abbreviations: MPAP = mean pulmonary artery pressure, MAP = mean arterial pressure. 
strain was isolated from a burn patient at Brook Army Medical Center in San Antonio, TX. Based on pilot virulence experiment, $10^{9}$ Colony Forming Units per hour (CFU/ hour) in a volume of $1 \mathrm{ml} 0.9 \% \mathrm{NaCl}$ solution was needed to obtain similar cardiovascular responses and hemodynamic variables with characteristics of severe hyperdynamic sepsis. We monitored hemodynamics continuously to ensure that the hyperdynamic state was kept in the expected ranges for severe sepsis (body temp increase of $2-3^{\circ} \mathrm{C}$, respiratory rate increased, MPAP increased but $<35 \mathrm{mmHg}$, heart rate increased but $<200$ BPM).

\section{Infusion and sampling protocol}

\section{Stable isotopes}

We used two stable isotopes of Phe: L-[ring $\left.{ }^{13} \mathrm{C}_{6}\right]-$ Phe and $\mathrm{L}-\left[{ }^{[5} \mathrm{N}\right]-$ Phe (Cambridge Isotopes, Andover, MA) as tracers to study WbRa of Phe with two different tracer infusion protocols. We previously used Phe to determine WbPB (1, 20, 21). We based the prime amount and tracer infusion rates on our previous studies (21). For the PC infusion protocol, we used L-[ring- ${ }^{13} \mathrm{C}_{6}$ ]-Phe. The prime $(1.58 \mu \mathrm{mol} / \mathrm{kg}$ bw) and infusion (4.32 $\mu \mathrm{mol} / \mathrm{kg}$ bw/hour) was given respectively in a volume of $2 \mathrm{ml} / \mathrm{kg}$ bw and 2 $\mathrm{ml} / \mathrm{kg} \mathrm{bw} /$ hour, and started 12 hours after the start of PM, 5 hours before the PULSE protocol in which we used L-[15 $\mathrm{N}]-$ Phe $(26.3 \mu \mathrm{mol} / \mathrm{kg}$ bw in a volume $0.5 \mathrm{ml} / \mathrm{kg} \mathrm{bw})$. We gave all tracers via the central caval vein catheter.

\section{Blood sampling and sample processing}

We took blood samples and directly cooled them on ice. We processed the blood samples within one hour $(19,21,22)$. In brief: for amino acid concentration and enrichment analysis, we centrifuged heparinized blood at $4^{\circ} \mathrm{C}, 5$ minutes, $8000 \mathrm{G}$ and deproteinized $250 \mu \mathrm{L}$ plasma with $25 \mu \mathrm{L}$ tri-chloroacetic acid solution (TCA, 50\% $\mathrm{w} / \mathrm{v}$ ) and finally snap freeze in liquid nitrogen and store at $-80 \mathrm{C}$.

\section{Amino acid concentration and enrichments}

We determined amino acid isotope concentrations and amino acid enrichments (tracer-to-tracee ratios, TTR) on a fully automated LC-ESI-MS system (QTrap 5500 MS (AB Sciex, Foster City, CA, USA) with ExpressHT Ultra LC (Eksigent Div., AB Sciex, Foster City, CA, USA). For concentration and TTR measurements we added $20 \mu \mathrm{l}$ of TCA deproteinized plasma supernatant to $20 \mu \mathrm{L} L-\left[U_{-}{ }^{13} C_{9}\right]$-Phe internal standard. Within 3 days before the LC-ESI-MS analysis, we derivatized the samples together with external standards at concentrations within the physiological range (calibration curve of concentration) and enriched external standards in the range of expected TTRs (calibration curve for TTRs) with 9-fluorenylmethoxycarbonyl (Fmoc). After neutralization, we injected $160 \mathrm{~nL}$ of the solution onto a micro LC column $0.5 \mathrm{x}$ 100 mm HALO C18, 2.7 um, 90A pores (ABsciex, Foster City, CA, USA), kept at $35^{\circ} \mathrm{C}$. We eluted analytes with a segmentally linear gradient from 35\% to $85 \%$ acetonitrile in water supplemented with ammonium acetate to $10 \mu \mathrm{M}$ and $5 \%$ isopropanol. We 
used electrospray triple quadrupole tandem mass spectrometry in multiple reactions monitoring mode for detection. We fragmented the Fmoc amino acid derivatives in the collision cell for detection of either free aminoacyl anions or a fragment larger by 26 atom mass units (coming from the Fmoc derivative) to have the highest sensitivity. We simultaneously did mass analyses for Phe, its tracers and internal standards. We calculated the mass signal areas to enable TTR or tracee concentrations calculations.

\section{Calculations}

\section{Calculation of Phe tracee concentration and TTR from LCMS obtained signals}

For concentration calculations, we normalized the tracee signals of the samples and the external standards with their internal $L-\left[{ }^{13} \mathrm{C}_{9}\right]$-Phe standard. We determined the plasma tracee concentration with the calibration curve external Phe standards. Plasma enrichment (TTR) was determined using the peak area ratio of the tracee and tracer signal and a calibration curve of enriched external standards. For the tracer concentration in the infusions, we used a calibration curve of tracer containing external standards.

\section{Calculation WbRa with PC and PULSE method (Figure 1)}

In the present study we compare the calculations of $\mathrm{WbR}$ a of Phe into the circulation in a post-absorptive (patho-)physiological state, using non-compartmental model in the PC group versus a compartmental model made up by two compartments in the PULSE group (1).

$P C$ : We derived $\mathrm{WbR}_{a}$ from equation 1 by using the $\mathrm{L}-\left[\right.$ ring $\left.-{ }^{13} \mathrm{C}_{6}\right]$-Phe isotope. The tracer infusion rate (I) is divided by TTRA. TTRA is the tracer to tracee ratio in arterial plasma.

\section{Equation (1) $\quad \mathrm{WbR}_{\mathrm{a}}=\mathrm{I} / \mathrm{TTR}$}

PULSE: We used non-compartmental analysis in GraphPad Prism (version 6) to perform curve fitting of the exponential decay curve. We found that all data fitted best ( $\mathrm{R}^{2}$ and observation) with a two-exponential fit:

Equation (2) Enrichment $(t)=A_{1} e^{-B_{1} t}+A_{2} e^{-B_{2} t}$

The non-compartmental WbRa equation 1 is generally applicable to multiple exponential decays. This equation can be written in terms of the parameters of the curve fits as follows:

Equation (3) $\quad \mathrm{WBR}_{\mathrm{a}}=\mathrm{I} /\left(\mathrm{A}_{1} / \mathrm{B}_{1}+\mathrm{A}_{2} / \mathrm{B}_{2}\right)$ 
The two-exponential fit is related to a two compartmental model in line with what is expected for essential amino acids (1). The two compartmental analysis of the tracer decay curve follows the Michaelis-Menten elimination kinetics (23). The assumption that the rate of change of the amount of tracer in a pool is equal to the rate that tracer enters the pool minus the rate exits the pool can be stated mathematically, as we described previously (1):

$$
\begin{array}{ll}
\text { Equation (4) } & \mathrm{dq}_{1}(\mathrm{t}) / \mathrm{dt}=\mathrm{k}_{12} * \mathrm{q}_{2}(\mathrm{t})-\mathrm{k}_{21} * \mathrm{q}_{1}(\mathrm{t}) \\
\text { Equation (5) } & \mathrm{dq}_{2}(\mathrm{t}) / \mathrm{dt}=\mathrm{k}_{21} * \mathrm{q}_{1}(\mathrm{t})-\mathrm{k}_{12} * \mathrm{q}_{2}(\mathrm{t})-\mathrm{k}_{02} * \mathrm{q}_{2}(\mathrm{t}) \\
\text { Equation (6) } & \mathrm{TTR}(\mathrm{t})=\mathrm{q}_{1}(\mathrm{t}) / \mathrm{Q}_{1}
\end{array}
$$

Where $d_{q_{1}}(t) / d t$ and $d_{q_{2}}(t) / d t$ are the rates of change of the tracer pool size in pools 1 and 2 respectively, and $Q_{1}$ the tracee pool size in pool 1. These equations/model (Figure 1), the measured enrichment data, and the size of the administered tracer pulse are entered into the SAAM II software that provides the calculated values of $k_{21}, k_{21}, k_{02}$ and $Q_{1}$. We converted the $k$ values to whole-body rate of appearance $\left(\mathrm{WbR}_{\mathrm{a}}\right)$ or intracellular production as described previously $(1,11)$. We estimated the transfer rates as fractional rates between pools $\left(k_{12}, k_{21}, k_{02}\right)$ and the extracellular pool size $\left(Q_{1}\right)$ in all subjects. Assuming that the measurements were done in a physiological steady state, meaning no net loss or production of Phe tracee in $Q_{1}$ during the experimental period, we calculated the flux $(F)$ from $Q_{2}$ to $Q_{1}\left(F_{12}\right)$ with the fractional rate of intracellular uptake $\left(k_{21}\right)$ from $Q_{1}$ :

$$
\text { Equation (7) } \quad \text { Flux }=F_{12}=F_{21}=k_{21} Q_{1}
$$

The size of the intracellular pool $\left(Q_{2}\right)$ can be calculated with the fractional rate of intracellular release $\left(\mathrm{k}_{12}\right)$ :

$$
\text { Equation (8) Intracellular pool }=Q_{2}=F_{12} / k_{12}
$$

The irreversible loss from the intracellular pool $\left(F_{02}\right)$ calculated with the fractional rate of irreversible loss $\left(\mathrm{k}_{02}\right)$ :

$$
\text { Equation (9) Irreversible loss }=F_{02}=k_{02} Q_{2}
$$

In a physiological steady state, irreversible loss is equal to appearance of Phe in the intracellular pool. Assuming that the appearance of Phe in the intracellular pool is coming from $\mathrm{PB}$, and $\mathrm{PB}$ is occurring intracellular in post-absorptive state, we thus determine WbPB: 
The fraction of the amount of Phe coming from PB that will appear in the extracellular pool $\left(Q_{1}\right)$ is the amount that is not irreversibly lost:

Equation (11) $\quad \mathrm{WbR}_{\mathrm{a}}=\mathrm{F}_{20} *\left(1-\mathrm{F}_{02} /\left(\mathrm{F}_{20}+\mathrm{F}_{21}\right)\right)$

The amount of Phe flux between $Q_{1}$ and $Q_{2}$ that is not irreversibly lost is shunt back to $Q_{1}$ :

Equation (12) Shunting $=F_{12}-$ WbRa

\section{Statistical analyses}

Results are presented as means \pm SEM. Graphpad Prism (version 6) was used for statistics. Level of significance was set on $p<0.05$. To determine physiological (tracee) or tracer Phe steady state during the experimental period, linear regression was used to determine if the slope of the best fitted line was different from zero. No difference from zero is steady state. To compare data between the Healthy and Sepsis group an unpaired t-test was used. To compare physiologically relevant models/parameters a Pearson correlation test was used. Best-fitted line that describes the relation between both models/parameters was done with linear regression. A shared fitted line was determined when no significant differences were observed between the Healthy and the Sepsis fitted line. Additionally, a Bland-Altman plot was made to determine potential discrepancy between methods (24). For the limits of agreement 2 times the standard deviation of the average discrepancy was used. Post-hoc Wilcoxon Signed Rank test and D’Agostino\&Pearson normality test was used to characterize a discrepancy.

\section{Results}

\section{Characteristics/Validation of tracer models}

Plasma tracer/tracee ratios of both tracer methods.

Figure 3 characterizes the shape of the enrichment (TTR) curve after a PULSE of $\mathrm{L}_{-}\left[{ }^{15} \mathrm{~N}\right]-\mathrm{Ph}$ and the PC infusion enrichment of $\mathrm{L}^{-\left[{ }^{13} \mathrm{C}_{6}\right]-P h e}$ in a septic and healthy animal. The exponential decay curves of all individual animals are shown in Supplemental Figures 1 and 2. We evaluated the residual errors by visual inspection. We determined goodness of fit with the coefficient of determination (R square): $0.9991 \pm 0.0002$ (Healthy) and 0.9991 \pm 0.002 (Sepsis). We determined, using linear regression, that $L-\left[{ }^{13} C_{6}\right]$-Phe tracer steady state (horizontal lines in time during the experimental period) was present. In all animals the TTR was in steady state. 


\section{Tracee concentrations}

We found that the arterial tracee Phe concentrations during the experimental period (Figure 4) were stable in the Healthy group but slightly increasing in the Sepsis group (regression slope was different from zero: $p=0.0211$ ). The plasma Phe concentration was increased in the sepsis animals (median over the experimental period, Healthy: $64.6 \pm 4.0 \mu \mathrm{M}$; Sepsis $113 \pm 8.3 \mu \mathrm{M}$; $\mathrm{p}=0.0002$ ).

\section{Precision of model parameters with PULSE method}

In Table 1 we show the results of the fitting of the decay curves of Phe enrichments performed with Graphpad Prism. For non-compartmental analysis with the PULSE method, the correlation between the fit and the data $\left(R^{2}\right)$ should be greater than 0.95 (1). We found that for all fitted curves, the $R^{2}$ was higher than 0.95 . The correlation between the various parameters (dependency) indicates that the use of a two-exponential equation is appropriate.

The coefficient of variation of each compartmental model parameter should be reasonable and models with coefficients above $100 \%$ should be rejected (1). We found for all animals that the parameter estimations performed with SAAM II (Table 2) had all coefficients of variation below $100 \%$.

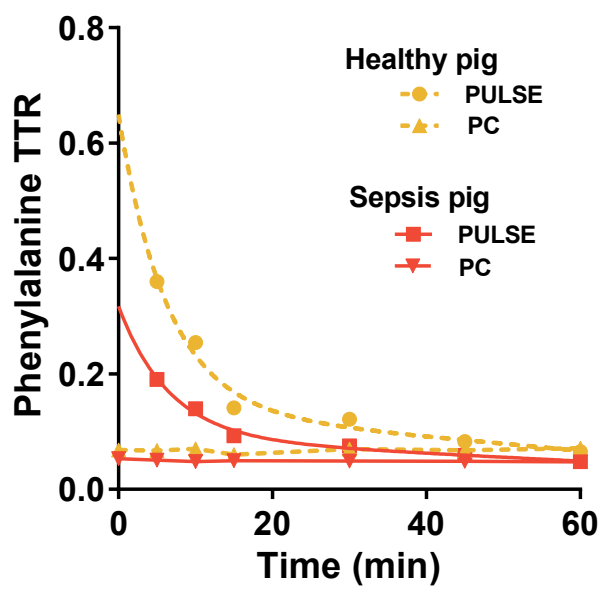

Figure 3: Example plasma tracer/tracee ratio's

Example of plasma tracer/tracee time courses of arterial phenylalanine (Phe) in a Healthy and a Sepsis animal. PC: Steady state curve, $5 \mathrm{~h}$ after the start of a primed-continuous infusion of L-[ring- ${ }^{13} \mathrm{C}_{6}$ ]-Phe tracer; PULSE: A two-exponential decay curve after a bolus infusion tracer is $\mathrm{L}-\left[{ }^{15} \mathrm{~N}\right]-\mathrm{Phe}$. TTR is tracer/tracee ratio. 
Table 1 Precision of decay curves of Phe enrichment

\begin{tabular}{|c|c|c|c|c|c|c|c|c|}
\hline \multirow[b]{2}{*}{$\begin{array}{l}\text { Para- } \\
\text { meter }\end{array}$} & \multicolumn{3}{|c|}{ Healthy } & \multirow[b]{2}{*}{$\begin{array}{l}\text { Mean } \\
\text { Depen- } \\
\text { dency }\end{array}$} & \multicolumn{3}{|l|}{ Sepsis } & \multirow[b]{2}{*}{$\begin{array}{l}\text { Mean } \\
\text { Depen- } \\
\text { dency }\end{array}$} \\
\hline & $\begin{array}{l}\text { Mean } \\
\text { Value }\end{array}$ & $\begin{array}{l}\text { Mean } \\
\text { SD }\end{array}$ & $\begin{array}{l}\text { Mean } \\
\text { CV\% }\end{array}$ & & $\begin{array}{l}\text { Mean } \\
\text { Value }\end{array}$ & $\begin{array}{l}\text { Mean } \\
\text { SD }\end{array}$ & $\begin{array}{l}\text { Mean } \\
\text { CV\% }\end{array}$ & \\
\hline$A_{1}$ & 0.371 & 0.025 & 6.7 & 0.932 & 0.319 & 0.084 & 15 & 0.910 \\
\hline$A_{2}$ & 0.231 & 0.020 & 8.6 & 0.988 & 0.173 & 0.016 & 8.4 & 0.982 \\
\hline $\mathrm{B}_{1}$ & 0.153 & 0.018 & 11 & 0.984 & 0.188 & 0.042 & 18 & 0.981 \\
\hline $\mathrm{B}_{2}$ & 0.021 & 0.002 & 8.5 & 0.956 & 0.020 & 0.002 & 10 & 0.940 \\
\hline$R^{2}$ & 0.9998 & & & & 0.9991 & & & \\
\hline
\end{tabular}

Mean variations of data of two-exponential fit of individual decay curves of Phe enrichment Equation: Enrichment $(t)=A_{1} e^{-B_{1} t}+A_{2} e^{-B_{2} t}$. Fitted with Graphpad Prism. Used fitting constrains: $B_{1}<0.4, B_{2}>0$, plateau=0. $R^{2}$ is the correlation between the fit and the data. Healthy $n=9$; Sepsis $n=13$.

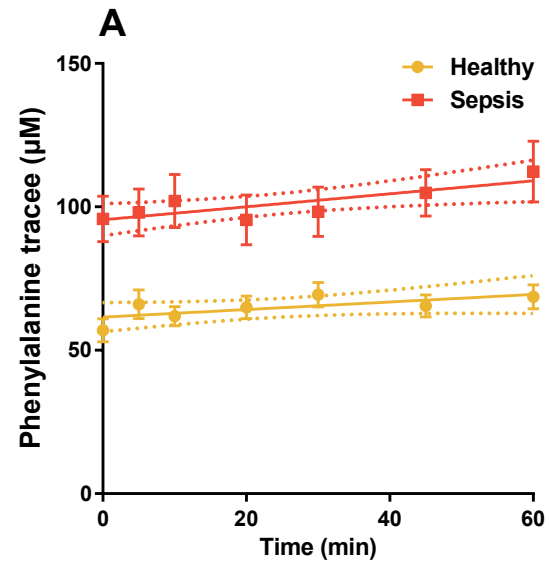

PLASMA
B

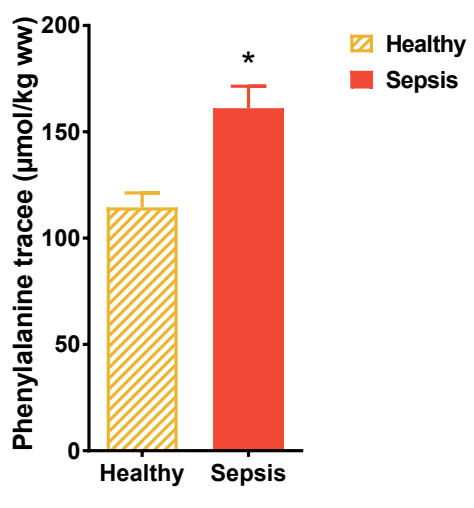

TISSUE

Figure 4. Phenylalanine (Phe,tracee) concentrations

A: Arterial plasma Phe tracee (non-tracer) concentrations after administration of L-[ $\left.{ }^{15} \mathrm{~N}\right]-\mathrm{Phe}$ pulse. Between 17-18h after start administration of Pseudomonas aeruginosa. Data expressed as mean \pm SD. Healthy $n=9$, Sepsis $n=13$. Statistics for physiological steady state: linear regression, significance $p<0.05$ if slope was different from zero. Slope in Sepsis group was different from zero ( $p=0.0211)$. B: Average tissue Phe concentration from muscle, jejunum, ileum, liver, lung in $\mu \mathrm{mol} / \mathrm{kg}$ ww. At necropsy, $18 \mathrm{~h}$ after start administration of Pseudomonas aeruginosa. Data expressed as mean \pm SEM. Healthy $n=9$, Sepsis $n=13$. Statistics: sepsis compared to Healthy, unpaired t-test. *) significance $p<0.05$. 
Table 2 Precision of compartmental model

\begin{tabular}{|c|c|c|c|c|c|c|}
\hline & Healthy & & & Sepsis & & \\
\hline Parameter & Mean Value & Mean SD & $\begin{array}{l}\text { Mean } \\
\text { CV\% }\end{array}$ & $\begin{array}{l}\text { Mean } \\
\text { Value }\end{array}$ & Mean SD & $\begin{array}{l}\text { Mean } \\
\text { CV\% }\end{array}$ \\
\hline $\mathrm{Q}_{1}(\mu \mathrm{mol})$ & 1121 & 49 & 4.1 & 1655 & 37 & 2.5 \\
\hline $\mathrm{k}_{02}\left(\min ^{-1}\right)$ & 0.033 & 0.005 & 14 & 0.028 & 0.003 & 11 \\
\hline$k_{12}\left(\min ^{1}\right)$ & 0.051 & 0.020 & 26 & 0.044 & 0.006 & 14 \\
\hline $\mathrm{k}_{21}\left(\min ^{-1}\right)$ & 0.115 & 0.019 & 14 & 0.105 & 0.008 & 7.0 \\
\hline
\end{tabular}

Mean variations of parameters generated from the two compartmental analysis of individual decay curves of Phe enrichment with computer software SAAM II

$Q_{1}$ is plasma pool size; $k_{02}, k_{12}, k_{21}$ are proportionality constants. Healthy $n=9$; Sepsis $n=13$.

Formula's used:

$\mathrm{dq}_{1}(\mathrm{t}) / \mathrm{dt}=\mathrm{k}_{12} * \mathrm{q}_{2}(\mathrm{t})-\mathrm{k}_{21} * \mathrm{q}_{1}(\mathrm{t})$

$\mathrm{dq}_{2}(\mathrm{t}) / \mathrm{dt}=\mathrm{k}_{21} * \mathrm{q}_{1}(\mathrm{t})-\mathrm{k}_{12} * \mathrm{q}_{2}(\mathrm{t})-\mathrm{k}_{02} * \mathrm{q}_{2}(\mathrm{t})$

$\operatorname{TTR}(\mathrm{t})=\mathrm{q}_{1}(\mathrm{t}) / \mathrm{Q}_{1}$

Where $d q_{1}(t) / d t$ and $d q_{2}(t) / d t$ are the rates of change of the tracer pool size in pools 1 and 2 respectively.

\section{Whole body rate of appearance (WbR $\mathbf{G}$ ) comparisons}

We compared healthy and septic animals to determine if both PC and PULSE can be used to observe changes in WbPB (Figure 5). We found that WbRa of Phe was higher in the sepsis group with both methods (PC: $p=0.003$; PULSE: $p=0.0001$ ), albeit that

$W_{b} R_{a}$ was higher with the PULSE method in both groups (Figure 5A, 5B). Data from the non-compartmental WbRa-PULSE analysis were not different (Healthy: $p=0.788$; Sepsis: $p=0.841$ ) from those from the compartmental WbRa-PULSE analysis (Figure 5B). Although the PC and PULSE WbRa data correlated very well $(r=0.732, p<0.0001)$ (Figure $5 \mathrm{C}$ ), a systemic difference between the methods was present as reflected by the 1.6 times higher WbRa with the PULSE method, independent of the septic state (Figure 5D). Although the WbRa compartmental versus non-compartmental data with the PULSE method correlated highly ( $r=0.969, p<0.0001$ ) (Figure $5 E$ ), the discrepancy didn't pass the D'Agostino\&Pearson normality test due to higher variability of the data obtained by the non-compartmental PULSE analysis.

\section{Additional metabolic information with the PULSE tracer model}

\section{$\mathrm{WbR}_{\mathrm{a}}$ in comparison with WbPB}

To determine if the WbRa of Phe in the extra cellular pool is representing WbPB, we compared it with the WbPB (Figure 6). While the WbPB was higher $(p=0.002)$ in the sepsis group, the correlation with WbRa-PULSE was excellent $(r=0.897: p<0.0001)$ and was 1.7 times higher than the WbRa-PULSE independent of the septic state.

\section{Comparison whole body Phe pools}

Both extracellular and intracellular Phe pool are increased in sepsis (Table 3) and are highly correlated $(r=0.802$ : $p<0.001)$ and the intracellular pool is 2.2 times larger 
A

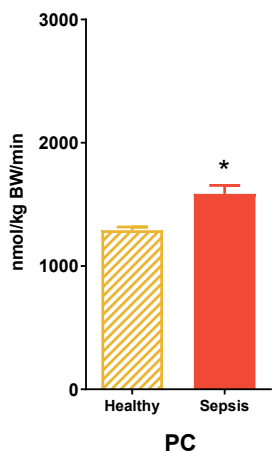

C Non-compartmental

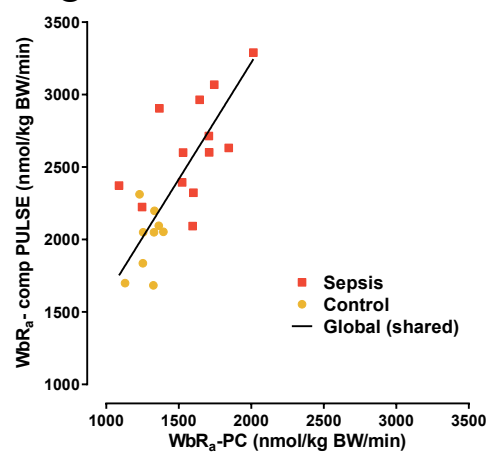

$E$

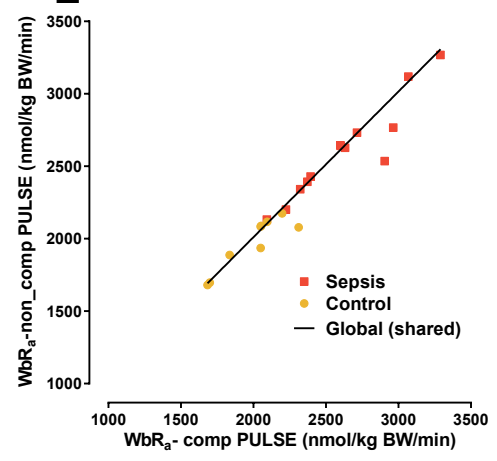

B

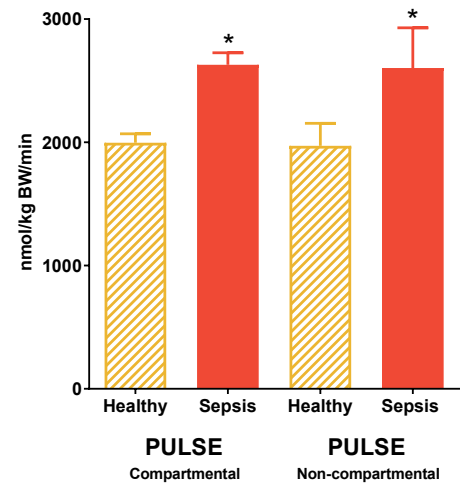

D

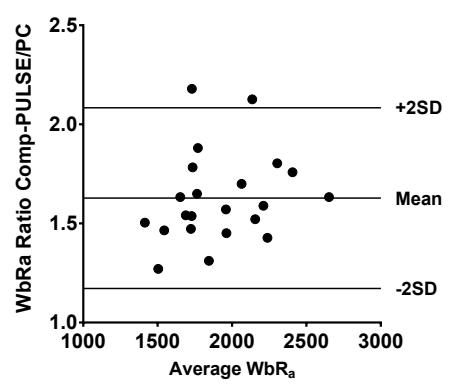

$\mathbf{F}$

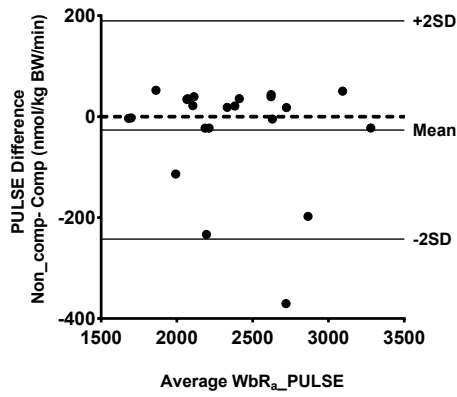

Figure 5. Effect of sepsis on whole body rate of appearance (WbRa) of phenylalanine (Phe) using two different tracer methods

WbRa as proxy for whole body protein breakdown in sepsis and healthy pigs. Calculated with A. PC tracer method: primed-continuous infusion of L-[ring $\left.{ }^{13} \mathrm{C}_{6}\right]$-Phe. Or B. with the PULSE tracer method: bolus infusion of $\mathrm{L}-\left[{ }^{15} \mathrm{~N}\right]-\mathrm{Ph}$. Compartmental and non-compartmental data analyses. Data expressed as mean \pm SEM. Healthy $n=9$, Sepsis $n=13$. Statistics: sepsis compared to Healthy, unpaired t-test. *) significance $p<0.05$. C: Correlation between WbRa-PULSE (compartmental) vs WbRa-PC. Statistics for correlation: Pearson correlation ( $r=0.732)$, with a likelihood for real correlation $(p<0.0001)$. Linear regression is used for the prediction of best line $\left(r^{2}=0.507\right.$, slope 1.61). D. Bland-Altman plot. Ratio vs the average of the two different tracer 
methods. The discrepancy (mean \pm SD) is $1.628 \pm 0.2279$. Wilcoxon Signed Rank test was used to determine the discrepancy was different from one: $p<0.0001$. Discrepancy passed D’Agostino\&Pearson normality test. E. Correlation between WbRa-PULSE (compartmental) vs WbRa-PULSE (non-compartmental). Statistics for correlation: Pearson correlation ( $r=0.969)$, with a likelihood for real correlation $(p<0.0001)$. Linear regression is used for the prediction of best line $\left(r^{2}=0.9386\right.$, slope $\left.=1.005\right)$. F. Bland-Altman plot. Difference vs the average of the two different PULSE data analysis methods. The discrepancy (mean \pm SD) is $-26.51 \pm 108.1$. Wilcoxon Signed Rank test was used to determine the discrepancy was different from zero: No significance. Discrepancy didn't passed D’Agostino\&Pearson normality test.

A

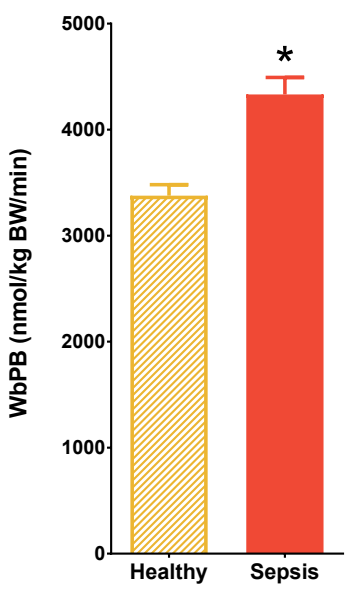

B

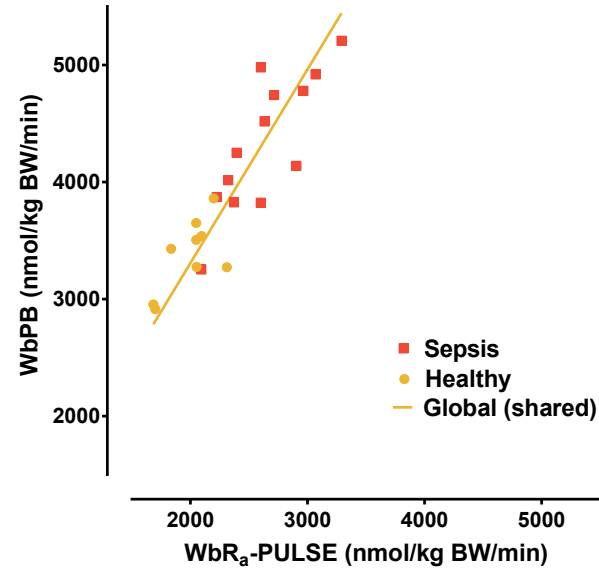

Figure 6. Effect of sepsis on whole body protein breakdown (WbPB)

WbPB data obtained with compartmental modeling (PULSE method). A: WbPB. Data expressed as mean \pm SEM. Healthy $n=9$, Sepsis $n=13$. Statistics: Sepsis compared to Healthy, unpaired t-test, *) significance $p<0.0002$. B: correlation between WbPB and whole body rate of appearance (WbRa). Statistics: Pearson correlation ( $r=0.897)$, with a likelihood for real correlation $(p<0.0001)$. Linear regression is used for the prediction of best line $\left(r^{2}=0.803\right.$, slope $\left.=0.60\right)$.

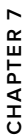

than the extracellular pool. A significant relationship is also present between the extracellular Phe pool size and plasma Phe concentration ( $r=0.613 ; p=0.002)$.

\section{Other metabolic parameters}

Beside the increase in Phe pools, we also observed an increase in Phe flux between the extra- and intracellular pools, an increase in the amount of shunting (Table 3), and an increase in irreversible loss. No changes were found in the fractional release, uptake and irreversible loss of Phe in the intracellular pool. 
Table 3. Whole body metabolic information obtained with PULSE model

\begin{tabular}{|c|c|c|c|c|}
\hline Parameter & Unit & Healthy & Sepsis & P-value \\
\hline Extracellular pool $\left(Q_{1}\right)$ & $\mu \mathrm{mol} / \mathrm{kg} \mathrm{BW}$ & $44(2.2)$ & $68(5.5)$ & 0.002 \\
\hline Intracellular pool $\left(Q_{2}\right)$ & $\mu \mathrm{mol} / \mathrm{kg} \mathrm{BW}$ & $105(8.0)$ & $157(8.2)$ & 0.0003 \\
\hline Flux $\left(F_{12}=F_{21}\right)$ & $\mu \mathrm{mol} / \mathrm{kg} \mathrm{BW} / \mathrm{min}$ & $5.02(0.40)$ & $6.86(0.42)$ & 0.006 \\
\hline Shunting ( $\left.F_{12}-W b R_{a}\right)$ & $\mu \mathrm{mol} / \mathrm{kg} \mathrm{BW} / \mathrm{min}$ & $3.02(0.35)$ & $4.23(0.34)$ & 0.026 \\
\hline $\begin{array}{l}\text { Irreversible loss }\left(\mathrm{F}_{02}\right) \\
\text { Fractional rate of irrevers- }\end{array}$ & $\mu \mathrm{mol} / \mathrm{kg} \mathrm{BW} / \mathrm{min}$ & $3.38(0.10)$ & $4.33(0.16)$ & 0.0002 \\
\hline ible loss $\left(k_{02}\right)$ & $\% / \min$ & $3.33(0.21)$ & $2.83(0.15)$ & 0.065 \\
\hline $\begin{array}{l}\text { Fractional rate of intracel- } \\
\text { lular release }\left(k_{12}\right) \\
\text { Fractional rate of intracel- }\end{array}$ & $\% / \min$ & $5.09(0.69)$ & $4.43(0.25)$ & 0.316 \\
\hline $\begin{array}{l}\text { Fractional rate of intracel- } \\
\text { lular uptake }\left(\mathrm{k}_{21}\right)\end{array}$ & $\% / \min$ & $11.5(0.71)$ & $10.6(0.76)$ & 0.423 \\
\hline
\end{tabular}

Mean values of parameters obtained and calculated from the fitting of individual decay curves of Phe enrichment. Expressed as mean \pm SEM. Healthy $n=9$; Sepsis $n=13$. Statistics: unpaired t-test.

\section{Discussion}

The present study shows that the WbRa obtained by the PULSE method is highly correlated with that of the PC method, albeit the absolute values were higher for the PULSE method but independent of the presence of sepsis. Furthermore, the PULSE method is able to measure protein breakdown using a compartmental model made up of two compartments, and provides additional valuable information i.e. membrane transport characteristics (fractional release, uptake, irreversible loss). The Phe pools and fluxes between pools are increased in the sepsis animals, however the transport characteristics between the pools are not changed.

\section{Validation models}

\section{Choice of tracers}

The stable isotope tracers of Phe that can be used for both the PULSE as the PC method are dependent on the choice of analytical method (e.g. LCMS, GCMS or IRMS), precision of this method (e.g. higher masses can give more sensitivity, possible use of neutral loss approach). Additionally, in the present study we considered potential chemical interference during the analytical procedures. For instance, if masses of two tracers are too close to each other, contribution of the lower mass (natural abundance) to the higher mass can be expected. Although biological interference (unwanted modifications of tracers in the body) like the potential disappearance of ${ }^{15} \mathrm{~N}$-tracer by transamination can occur (25), for the PULSE method it will not interfere with the $\mathrm{WbR}_{\mathrm{a}}$ (part of irreversible loss). Also, the transamination of phenylalanine in the body and back can be considered very low, because for instance the use of ${ }^{15} \mathrm{~N}$-Phe and $1-{ }^{13} \mathrm{C}$-Phe gives comparable TTR's (26). This is another reason why phenylalanine is a preferred amino acid in our type of measurements. Any 
stable isotope tracer of phenylalanine can be used for $\mathrm{WbR}_{\mathrm{a}}$, as long as it is possible to measure both high and low enrichments with sufficient precision.

\section{Physiological steady state}

During the $1 \mathrm{~h}$ testing period, our animals were all in a physiological steady state and tracer steady state for the PC method. We found that the Phe tracee curves were not significantly affected by the amount of the Phe isotope in the PULSE, although an increasing tracee concentration was found in the septic animals, likely related to the septic condition. Because the increase was minor and the tracer enrichment was in a steady state for the PC method, we didn't adjust the PC-WbRa formula with e.g. Steele equation correction for non-steady state measurements (27), which for Phe only has a minor effect on the final results. We suggest that future studies should use small amounts of tracers to reduce the effect on metabolism further.

\section{Fitting}

Due to time limitations and experimental design constraints, we did not take more than 6 samples to describe the PULSE decay curve in the present study. However, the Goodness of fit and CV\% of each parameter with as well Graphpad Prism (for non-compartmental data analysis) as SAAM II software (for compartmental data analysis) shows high reliable decay curves thanks to very accurate LCMS TTR measurements (CV\%<2\%). For the chosen compartmental model (two compartments) $(11,13,23,28)$ and to determine changes in sepsis, this number of samples was sufficient in the present study. In case of more complex compartmental models (three or more compartments) or less accurate analytical methods, we think that more sample points are necessary.

\section{Identifiability}

The general idea is to use compartments to model various components of the physiological metabolic system. The structure of the model is developed from evaluation of the relationships between the exponential functions of the decay curve and an understanding of the metabolic system to be modeled. Usually the number of exponential terms is directly related to the number of compartments in the model. The minimal number of detectable compartments in the current study is two based on the goodness of fit. The physiological identifiability of the two compartments PULSE model is not necessary univocal, but this interpretation fits with a general used two-pool model for amino acid metabolism (1) where Q1 is considered the extracellular pool and $Q_{2}$ the intracellular pool. If we compare $Q_{1}$ Phe pool with the plasma Phe pool (plasma Phe concentration times estimated plasma volume per $\mathrm{kg}: 80 \mathrm{ml} / \mathrm{kg}$ blood * plasma fraction) we concluded that the plasma pool is only $9 \%$ of the $Q_{1}$ Phe pool (healthy: $9.0 \pm 2.0 \%$; sepsis $9.4 \pm 2.5 \%$ ). Assuming that the extracellular concentration is $20 \%$ higher in healthy subjects then the arterial plasma concentration (29), we determined that the extra vascular Phe pool is about 
$58 \%$ of the Q1 Phe pool (healthy: $53 \pm 4$; sepsis $61 \pm 8$, weight gain by resuscitation included). Although we assume that $Q_{1}$ is the extracellular Phe compartment, these calculated pools combined represents only $70 \%$ of $Q_{1}$ Phe pool. $Q_{2}$ is the space were Phe is entering in a post-absorptive state from $\mathrm{PB}$ and can be used directly for protein synthesis (=recycling) or hydroxylation that takes place intracellular. If we compare the $Q_{2}$ Phe pool with the intracellular pool (calculated with an average Phe concentration in tissue, data in Figure 6), the intracellular pool represents $61 \%$ of the $Q_{2}$ pool (healthy: $66 \pm 4 \%$; sepsis $58 \pm 3 \%$ ). At the moment, we do not have a good explanation for these discrepancies $(1,23,28)$. It could well be that the above general assumptions (not observations) for the physiological pools are not realistic enough to compare it with the numeric $Q_{1}$ and $Q_{2}$ pools in animals that are in resuscitated physiological condition. It could well be that the numeric fast exchanging $Q_{1}$ Phe pool is partly also intracellular and the slow exchanging $Q_{2}$ pool is partly also located extracellular. So the numerical $Q_{1}$ and $Q_{2}$ pools are likely not strict physiological separated. Further research is needed to determine the exact translation of $\mathrm{Q} 1$ and $\mathrm{Q} 2$ pools to physiological pools.

\section{$\mathrm{WbR}_{\mathrm{a}}$ PULSE in relation to $\mathrm{WbR}_{\mathbf{a}} \mathbf{P C}$}

In the present study, we measured WbRa of Phe as an index/proxy for WbPB. We showed that both WbRa-PULSE and PC are related to total intracellular rate of appearance of Phe from PB in the healthy and disease state. However, the absolute value of $\mathrm{WbR}_{\mathrm{a}}$ was 1.6 times lower with the PC method.

For both methods, sampling was done in the same arterial plasma pool and TTR was analyzed in the same blood sample on the LC-MS/MS and calibrated with enriched standard curves. Although, the exact amount of the tracer administered in the PC model, is more error sensitive (e.g. prime is estimated, performance infusion pump, temporary pump errors) than with the PULSE model (single dose with syringe), we think it is unlikely that this can explain the discrepancy in WbRa between the models.

Rakotoambinina et al (9) also compared the PULSE approach with the PC for the non-essential amino acid taurine and found also 1.8 times higher $\mathrm{WbR}_{\mathrm{a}}$ with the PULSE approach. One of their explanations was that the area under the curve (AUC) under the decay curve could not well be determined because of the detection level of the used GC-MS. The AUC is related to the final absolute WbRa. In the present study we described the decay curve with 6 time points in a one-hour time window. The reliability of the curves was very high due to accurate LC-MS/MS measurements (CV\% <2\%). In practice, the calculation of non-compartmental WbRa with the PULSE method is strongly influenced by the tail of the decay curve because it can influence the area under the curve substantially. The calculation of the pool size $Q_{1}$ is strongly influenced by the enrichment close to zero. $Q_{1}$ is used to calculate the compartmental WbRa. Since the non-compartmental and compartmental PULSE 
approaches give comparable WbRa's, we are able to conclude that although the decay curves were fitted with only six sample points, these samples were collected on the right time and provided valid data. Therefore, in the present study a potential inaccuracy of the decay curve fitting cannot explain the WbRa difference between the PULSE and PC method.

The major difference between the PC and PULSE approach is the level of simplicity that is chosen to describe PB by determining WbRa of Phe. The WbRa-PC provides an underestimation of the intracellular appearance $(1,30)$. This underestimation is related to the fact that the $\mathrm{WbRa}-\mathrm{PC}$ is greatly limited by the necessary assumption that production appears in the sample compartment (i.e. plasma) in which the tracer is also administered. Physiologically only a few substrates appear directly in plasma, such as glucose and urea. In post-absorptive state that we studied, the essential amino acid Phe appears intracellularly. The WbRa-PULSE will better represent the dilution both in plasma as well as in tissue. This is related to the fact that the decay curve of the PULSE method has two exponentials that in the compartmental analysis represents most likely the extracellular and intracellular pools. However when we measure changes in PB both WbRa methods will identify these changes, albeit at different turnover rates. We therefore conclude that WbRa-PULSE better reflects the actual absolute PB rate. In case the total amount of PB is of interest in a scientific question, the compartmental PB analysis with the PULSE method will be the only option that is appropriate, because $\mathrm{WbR}_{\mathrm{a}}-\mathrm{PC}$ will not account for the intracellular irreversible loss.

\section{Protein kinetic changes in severe sepsis animals obtained with PULSE method}

WbRa of Phe is related to $\mathrm{WbPB}$, and is considered as important information to compare different physiological conditions. In the present study, both PC as PULSE WbRa provide us the same information: WbPB is enhanced in sepsis. The PULSE method provides us more additional information. For instance, how Phe compartments relate to each other $(1,7,13)$, how $\mathrm{WbR}_{\text {a }}$ relates to WbPB (ratio). A fraction of the difference of those two is the total amount of Phe that is directly reused (= recycling) for protein synthesis. The other fraction is hydroxylated to tyrosine. An extra tyrosine pulse tracer and the enrichment of the tyrosine metabolite, coming from the chosen Phe-tracer- PULSE is needed to determine the exact fraction of hydroxylation and subsequently the fraction of Phe recycling and whole body protein synthesis. In this case, it is not preferred to use a ${ }^{15} \mathrm{~N}$-Phe tracer due to potential (but low) interference of transamination $(25,26)$.

Also with the PULSE method we could determine that the change in $\mathrm{WbR}_{\mathrm{a}}$ in the current sepsis model, is caused by differences in Phe intracellular appearance and not the membrane transport capacity between the pools. We found that membrane 
transport of Phe in severe sepsis is not compromised, but that only WbPB in sepsis is increased $(18,31)$ and supports the notion that PULSE isotope methods provide us with additional physiologically important information about membrane transport.

\section{Conclusion}

The increased $\mathrm{WbR}_{\mathrm{a}}$ found by both the PC method and PULSE method reflects an enhanced WbPB in sepsis. In contrast to the PC method, the easy-to-use PULSE method provides additional information about intra- and extracellular fluxes, cell membrane transport capacity and WbPB. WbRa-PULSE therefore better reflects the actual absolute PB. Additionally, the non-compartmental PULSE data analysis can be very practical in routine measurements and circumvent the problems of pool priming seen with PC methods but is a little less sensitive than the compartmental PULSE data analysis. In severe sepsis, on whole body level, membrane transport capacity of amino acids is not compromised and is therefore not a rate limited factor for protein synthesis.

\section{References}

1. Wolfe RR, Chinkes DL. Isotope Tracers in Metabolic Research: Principles and Practise of Kinetic Analysis. New York: Wiley; 2005. 274 p.

2. Engelen MP, Com G, Wolfe RR, Deutz NE. Dietary essential amino acids are highly anabolic in pediatric patients with cystic fibrosis. Journal of cystic fibrosis : official journal of the European Cystic Fibrosis Society. 2013.

3. de Blaauw I, Heeneman S, Deutz NE, von Meyenfeldt MF. Increased whole-body protein and glutamine turnover in advanced cancer is not matched by an increased muscle protein and glutamine turnover. J Surg Res. 1997;68(1):44-55.

4. Luiking YC, Poeze M, Ramsay G, Deutz NE. Reduced citrulline production in sepsis is related to diminished de novo arginine and nitric oxide production. Am J Clin Nutr. 2009;89(1):142-52.

5. Matthews DE, Downey RS. Measurement of urea kinetics in humans: a validation of stable isotope tracer methods. Am J Physiol. 1984;246(6 Pt 1):E519-27.

6. Van Acker BA, Hulsewe KW, Wagenmakers AJ, Deutz NE, Van Kreel BK, Halliday D, et al. Absence of glutamine isotopic steady state: implications for the assessment of wholebody glutamine production rate. Clin Sci (Lond). 1998;95(3):339-46.

7. Rathmacher JA, Nissen SL. Development and application of a compartmental model of 3-methylhistidine metabolism in humans and domestic animals. Adv Exp Med Biol. 1998;445:303-24.

8. Fouillet H, Gaudichon C, Mariotti F, Mahe S, Lescoat P, Huneau JF, et al. Compartmental modeling of postprandial dietary nitrogen distribution in humans. Am J Physiol Endocrinol Metab. 2000;279(1):E161-75.

9. Rakotoambinina B, Marks L, Badran AM, Igliki F, Thuillier F, Crenn P, et al. Taurine kinetics assessed using [1,2-13C2]taurine in healthy adult humans. American Journal of Physiology - Endocrinology And Metabolism. 2004;287(2):E255-E62. 

production in pigs by compartmental analysis. J Anim Sci. 1996;74(1):46-56.

11. Engelen MP, Com G, Anderson PJ, Deutz NE. New stable isotope method to measure protein digestibility and response to pancreatic enzyme intake in cystic fibrosis. Clin Nutr. 2014;33(6):1024-32.

12. Mariotti F, Petzke KJ, Bonnet D, Szezepanski I, Bos C, Huneau JF, et al. Kinetics of the utilization of dietary arginine for nitric oxide and urea synthesis: insight into the argininenitric oxide metabolic system in humans. Am J Clin Nutr. 2013;97(5):972-9.

13. Zhang XJ, Chinkes DL, Wolfe RR. Measurement of muscle protein fractional synthesis and breakdown rates from a pulse tracer injection. Am J Physiol Endocrinol Metab. 2002;283(4):E753-64.

14. Zhang XJ, Chinkes DL, Herndon DN, Wolfe RR. Measurement of protein fractional synthesis and breakdown rates in the skin of rabbits using a subflooding dose method. Metabolism. 2009;58(9):1239-47.

15. Bregendahl K, Yang X, Liu L, Yen JT, Rideout TC, Shen Y, et al. Fractional protein synthesis rates are similar when measured by intraperitoneal or intravenous flooding doses of L-[ring-2H5]phenylalanine in combination with a rapid regimen of sampling in piglets. J Nutr. 2008;138(10):1976-81.

16. Symons TB, Sheffield-Moore M, Chinkes DL, Ferrando AA, Paddon-Jones D. Artificial gravity maintains skeletal muscle protein synthesis during 21 days of simulated microgravity. Journal of applied physiology. 2009;107(1):34-8.

17. Ramakrishnan R. Studying apolipoprotein turnover with stable isotope tracers: correct analysis is by modeling enrichments. Journal of lipid research. 2006;47(12):2738-53.

18. Rooyackers $\mathrm{O}$, Kouchek-Zadeh R, Tjader I, Norberg A, Klaude M, Wernerman J. Whole body protein turnover in critically ill patients with multiple organ failure. Clin Nutr. 2015;34(1):95-100.

19. Carneiro-Filho BA, Bushen OY, Brito GA, Lima AA, Guerrant RL. Glutamine Analogues As Adjunctive Therapy for Infectious Diarrhea. Curr Infect Dis Rep. 2003;5(2):114-9.

20. Poeze M, Bruins MJ, Kessels F, Luiking YC, Lamers WH, Deutz NE. Effects of L-arginine pretreatment on nitric oxide metabolism and hepatosplanchnic perfusion during porcine endotoxemia. The American journal of clinical nutrition. 2011;93(6):1237-47.

21. Bruins MJ, Deutz NE, Soeters PB. Aspects of organ protein, amino acid and glucose metabolism in a porcine model of hypermetabolic sepsis. Clin Sci (Lond). 2003;104(2):127-41.

22. Bruins MJ, Lamers WH, Meijer AJ, Soeters PB, Deutz NE. In vivo measurement of nitric oxide production in porcine gut, liver and muscle during hyperdynamic endotoxaemia. Br J Pharmacol. 2002;137(8):1225-36.

23. Godfrey K. Compartmental Models and their Application. London, UK: Academic Press Inc. LTD; 1983. 293 p.

24. Bland JM, Altman DG. Measuring agreement in method comparison studies. Stat Methods Med Res. 1999;8(2):135-60.

25. Shrawder E, Martinez-Carrion M. Evidence of phenylalanine transaminase activity in the isoenzymes of aspartate transaminase. J Biol Chem. 1972;247(8):2486-92.

26. Wachter U, Tugtekin I, Georgieff M, Radermacher P, Vogt J. Simultaneous determination of plasma enrichments of 1-13C- and 15N-labelled phenylalanine and tyrosine. Isotopes Environ Health Stud. 1998;34(3):311-6.

27. Steele R. Influences of glucose loading and of injected insulin on hepatic glucose output. Annals of the New York Academy of Sciences. 1959;82:420-30.

28. Carson ERC, C. ; Finkelstein, L. The mathematical modeling of metabolic and 
endocrine systems. Canada: John Wiley \& Sons, Inc; 1983. 394 p.

29. Miller S, Chinkes D, MacLean DA, Gore D, Wolfe RR. In vivo muscle amino acid transport involves two distinct processes. Am J Physiol Endocrinol Metab. 2004;287(1):E136-41.

30. DiStefano JJ, 3rd, Landaw EM. Multiexponential, multicompartmental, and noncompartmental modeling. I. Methodological limitations and physiological interpretations. Am J Physiol. 1984;246(5 Pt 2):R651-64.

31. Bruins MJ, Soeters PB, Deutz NE. Endotoxemia affects organ protein metabolism differently during prolonged feeding in pigs. J Nutr. 2000;130(12):3003-13.

\section{Supplemental Information}
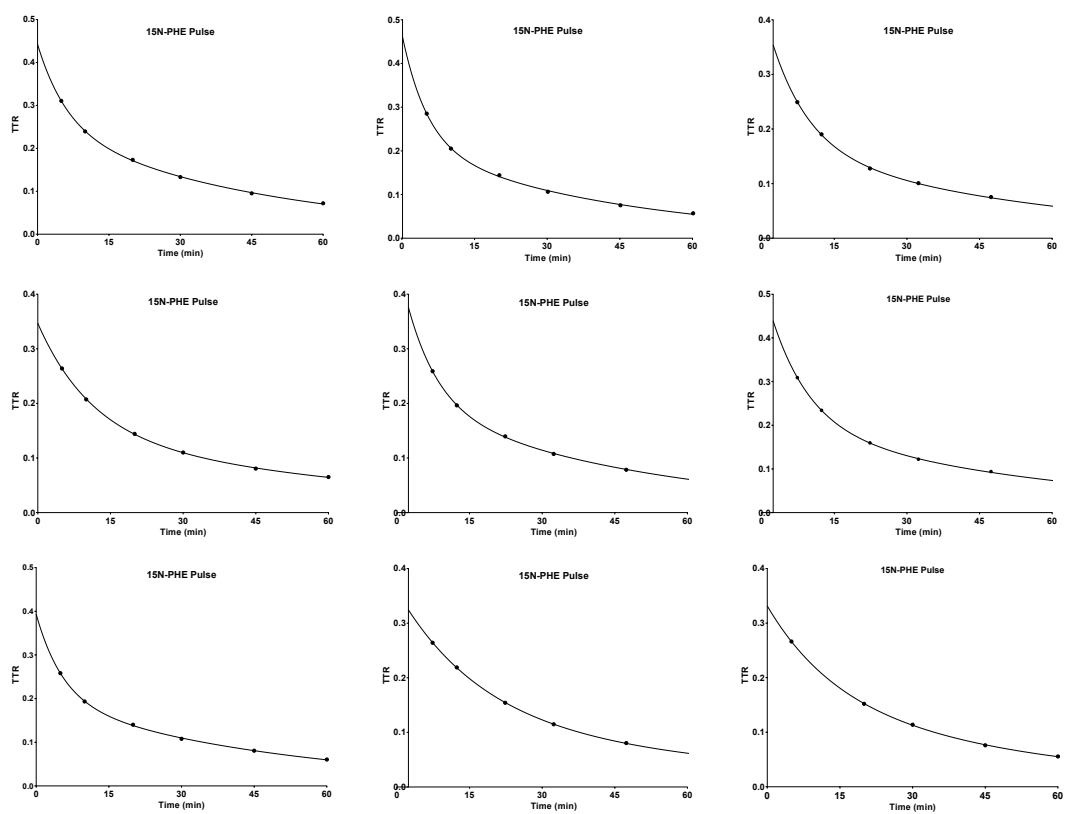

Supplemental Figure 1. Individual decay curves of Healthy Animals (PULSE)

Plasma tracer/tracee (TTR) time courses of arterial Phe in healthy animals. PULSE: A twoexponential decay curve after a bolus infusion tracer with L-[ $\left.{ }^{15} \mathrm{~N}\right]-\mathrm{Phe}$. 

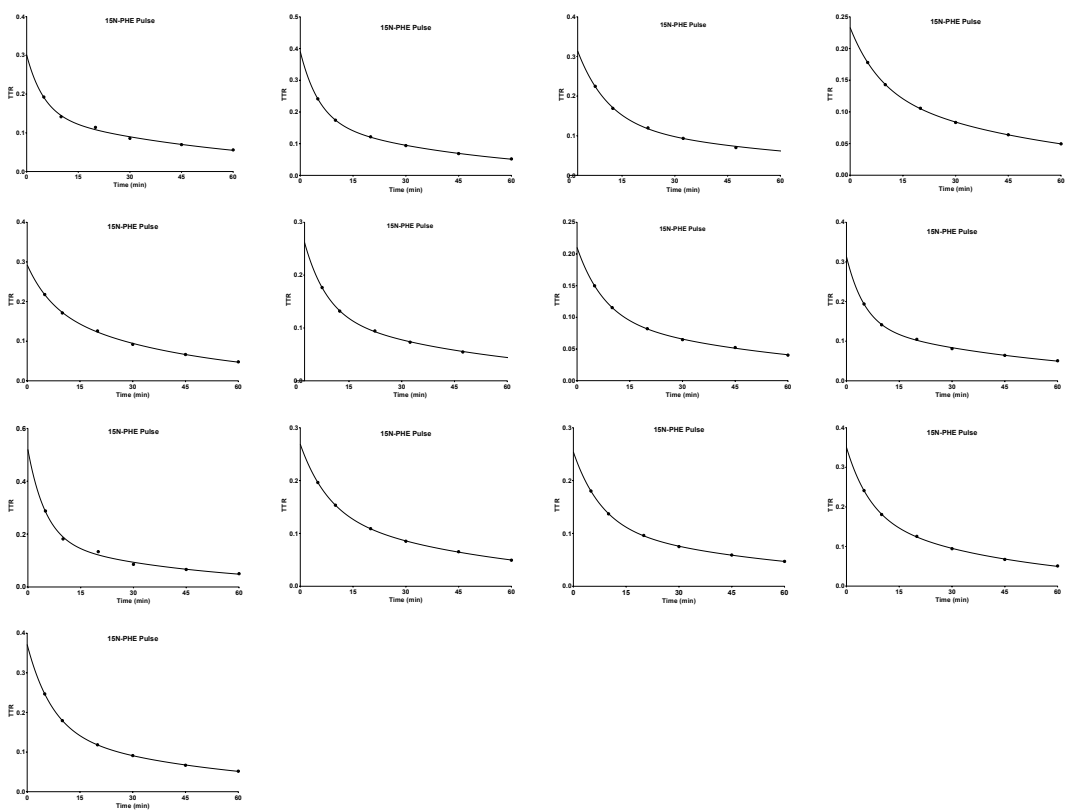

Supplemental Figure 2. Individual decay curves of Sepsis Animals (PULSE)

Plasma tracer/tracee (TTR) time courses of arterial Phe in sepsis animals. PULSE: A twoexponential decay curve after a bolus infusion tracer with L-[15N]-Phe.

\section{Supplemental Table 1: Phenylalanine (Phe) concentration in tissues}

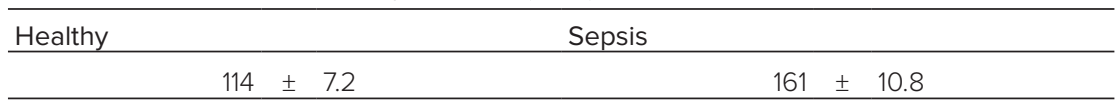

Average tissue Phe concentration from muscle, jejunum, ileum, liver, lung in $\mu \mathrm{mol} / \mathrm{kg}$ wet weight 



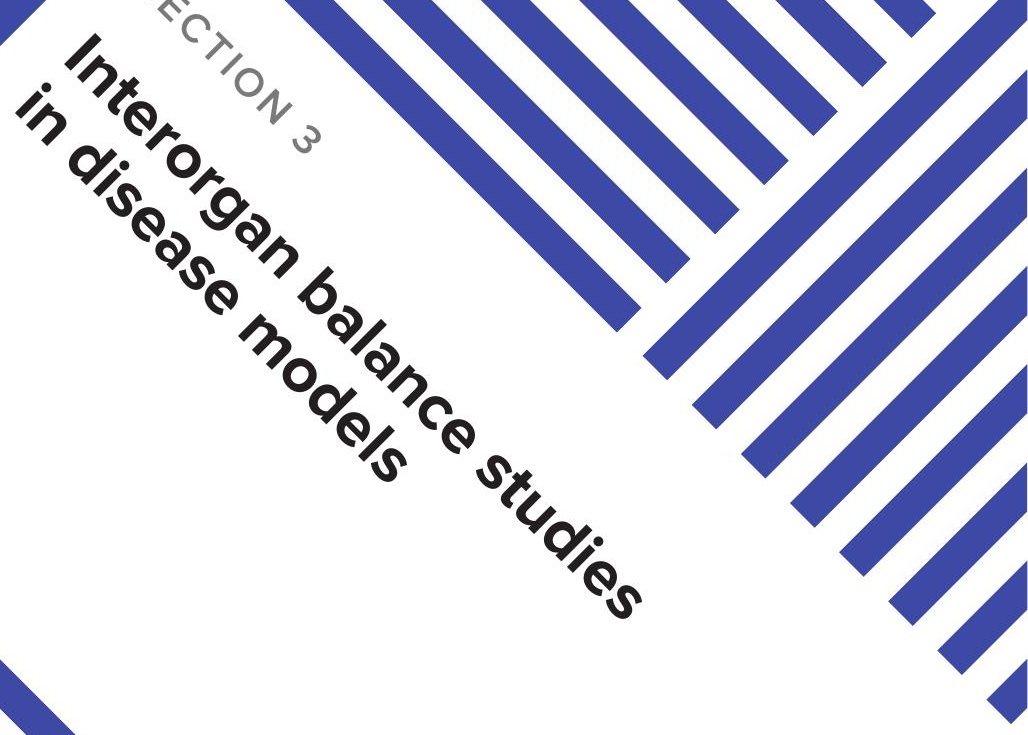


CHAPTER 8 


\title{
Arginine de novo and nitric oxide production in disease states
}

\author{
Yvette C. Luiking, Gabriella A.M. Ten Have, Robert R. \\ Wolfe, Nicolaas E.P. Deutz
}

Am J Physiol Endocrinol Metab. 2012 Nov 15;303(10):E1177-89.

\section{Abstract}

Arginine is derived from dietary protein intake, body protein breakdown or endogenous de novo arginine production. The latter may be linked to the availability of citrulline, which is the immediate precursor of arginine and limiting factor for de novo arginine production. Arginine metabolism is highly compartmentalized due to the expression of the enzymes involved in arginine metabolism in various organs. A small fraction of arginine enters the NO synthase (NOS) pathway. Tetrahydrobiopterin $(\mathrm{BH} 4)$ is an essential and rate-limiting cofactor for the production of NO. Depletion of $\mathrm{BH} 4$ in oxidative stressed endothelial cells can result in so-called NOS3 'uncoupling', resulting in production of superoxide instead of NO. Moreover, distribution of arginine between intracellular transporters and arginine-converting enzymes, as well as between the arginine-converting and arginine-synthesizing enzymes, determines the metabolic fate of arginine. Alternatively, NO can be derived from conversion of nitrite.

Reduced arginine availability stemming from reduced de novo production and elevated arginase activity have been reported in various conditions of acute and chronic stress, which are often characterized by increased NOS2 and reduced NOS3 activity. Cardiovascular and pulmonary disorders such as atherosclerosis, diabetes, hypercholesterolemia, ischemic heart disease and hypertension are characterized by NOS3 uncoupling. Therapeutic applications to influence (de novo) arginine and NO metabolism aim at increasing substrate availability or at influencing the metabolic fate of specific pathways related to NO bioavailability and prevention of NOS3 uncoupling. These include supplementation of arginine or citrulline, provision of NO donors including inhaled NO and nitrite (sources), NOS3 modulating agents, or the targeting of endogenous NOS inhibitors like asymmetric dimethylarginine. 


\section{Introduction}

The purpose of this review is to give an overview of and highlight recent developments on arginine metabolism and nitric oxide (NO) production in health and disease. The endogenous de novo production of arginine will be a special focus, and alterations in this pathway in disease and its relevance will be discussed. Finally, therapeutic applications to influence (de novo) arginine and NO metabolism will be summarized.

\section{Physiology of arginine, and arginine de novo and NO metabolism}

Arginine is a conditionally essential amino acid in healthy adults and therefore there is no specific nutritional requirement (1). However, dietary arginine is required in neonates, infants and in certain conditions and diseases (2). In the 1980s, L-arginine was identified as the precursor for $\mathrm{NO}$ (3). While first discovered in endothelial cells (4), NO appeared to be an ubiquitous molecule present in a variety of cells, including cells from the cardiovascular and nervous system and also inflammatory cells. As such, NO has many physiological functions, and the relationship between arginine availability and NO production emphasizes the functional relevance of arginine.

\section{Metabolic pathways}

Arginine is derived from dietary intake, body protein breakdown or endogenous de novo arginine production (Figure 1). In the post-absorptive state, whole body arginine flux in healthy adults is about 70-90 $\mu \mathrm{mol} / \mathrm{kg} . \mathrm{h}$, which equals $15-20 \mathrm{~g} /$ day $(5,6)((7)$ for review), while daily dietary arginine intake is about 4-6 grams $(8,9)$. De novo arginine production, which contributes about $10-15 \%$ to whole body arginine production under normal conditions $(6,10)$, involves the conversion of citrulline to arginine and is catalyzed by the enzymes argininosuccinate synthase (ASS) and argininosuccinate lyase (ASL) $(11,12)$. This conversion is part of the so-called intestinal-renal axis, with intestinal production of citrulline and renal synthesis of arginine (13-17). Citrulline availability is a limiting factor in this conversion (12).

Arginine is a constituent for body protein synthesis, $80 \%$ of which is derived from recycling amino acids originated from protein breakdown. Moreover, arginine plays a key role in several other metabolic pathways catalyzed by various enzyme systems (see Refs $(2,18,19)$ for recent reviews) (Figure 2). Arginine metabolism is highly compartmentalized within the body since the enzymes involved in arginine metabolism are expressed in various organs, although to a different extent. The only exceptional organ is the liver, which contains the complete urea cycle and its related enzymes. However in healthy conditions, arginine produced in the liver urea cycle is not exchanged to plasma (20). Due to compartmentalization, arginine metabolism and recycling is only partly in balance with plasma arginine concentration. This so-called 'arginine paradox' explains that acute exogenous arginine provision 


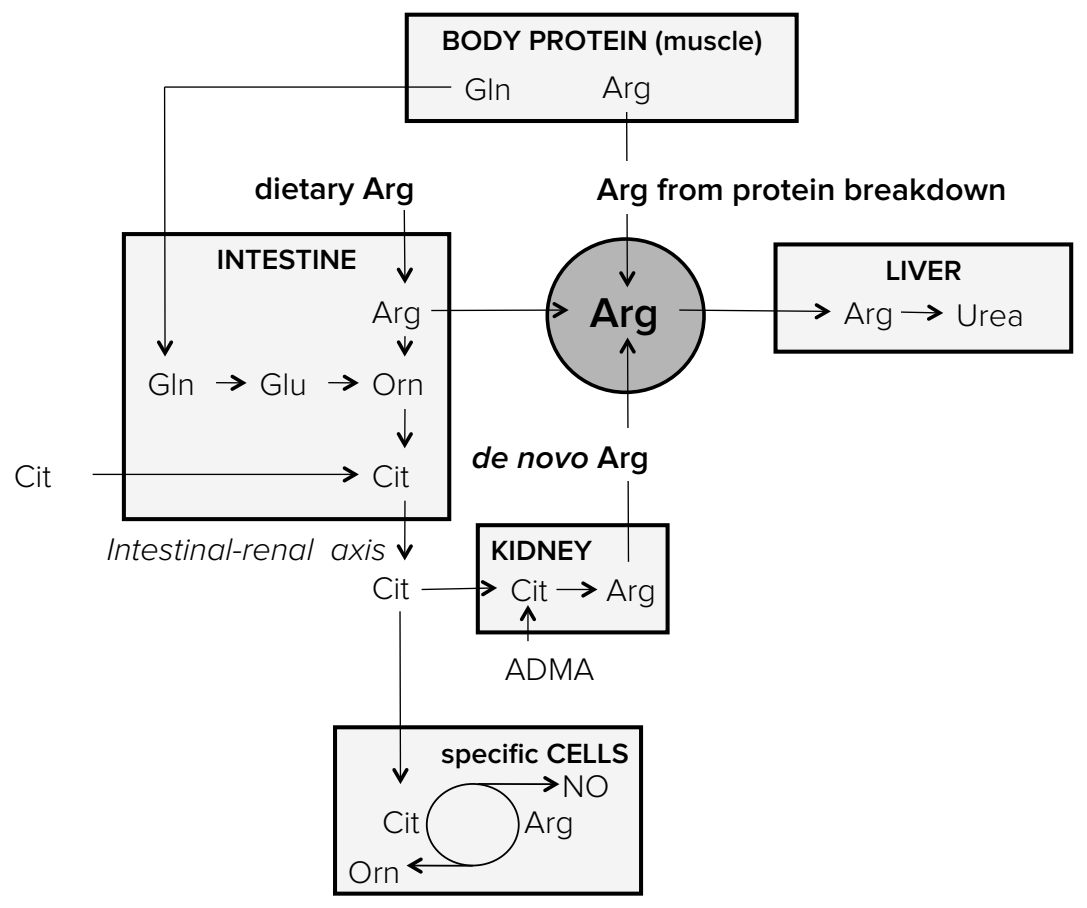

Figure 1. Extra-hepatic systemic arginine availability in healthy humans

$A R G$, arginine; $C I T$, citrulline; $A S S$, argininosuccinate synthase; $A S L$, argininosuccinate lyase.

can still increase NO production even though the intracellular arginine concentration far exceeds the $\mathrm{Km}_{\mathrm{m}}$ of eNOS (21). It is also likely that, once transported into the cell, arginine can no longer gain access to the membrane-bound eNOS. This makes intracellular arginine less useful as a reference point (22).

Arginine flux as measured with an intravenously-infused stable isotope and subsequent dilution of this isotope in the plasma compartment reflects the whole body appearance of arginine in plasma. This plasma arginine flux does not account for hidden compartments (such as liver cells) in which arginine is produced without first being released into plasma. Of the plasma arginine flux, 15\% enters the (extrahepatic) arginase pathway (6) that degrades arginine to ornithine and urea.

There are two isoforms of the enzyme arginase. Type I (cytosolic) arginase is predominantly expressed in the liver, as part of the urea cycle, but was also demonstrated at lower levels in various extrahepatic organs in rodents with a main role in production of ornithine for polyamines biosynthesis (23). Type II (mitochondrial) arginase is expressed in low levels in extra-hepatic tissues and cells (such as brain, kidney, small intestine, red blood cells, and immune cells) and is mainly involved in the synthesis of ornithine, proline and glutamate $(24,25)$. Based on the variability of 


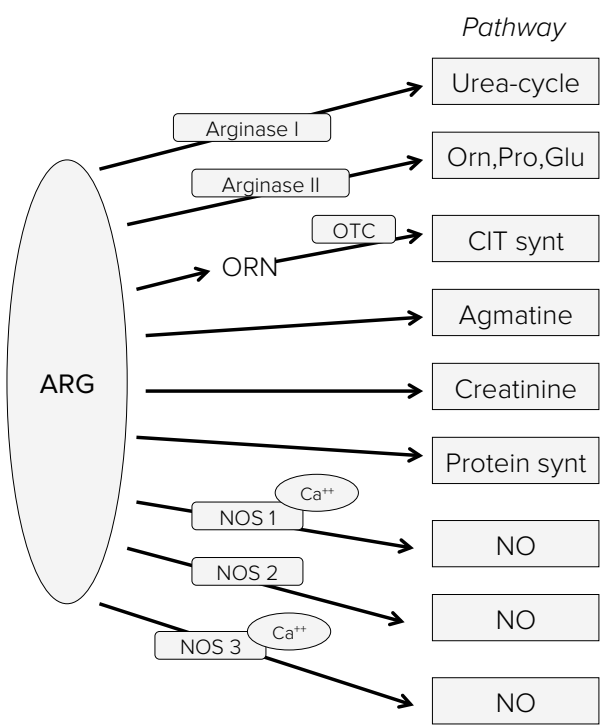

Figure 2 Compartments of arginine metabolism in healthy humans

ARG, arginine; ORN, ornithine; PRO, proline; GLU, glutamate; CIT, citrulline; NO, nitric oxide; NOS, nitric oxide synthase; Ca, calcium.

arginase I and II among organs in rodents, organ-specific roles of arginase isoforms have been suggested (26). Under normal conditions, about $40 \%$ of dietary arginine is extracted in the splanchnic area (27), which is likely due to the relatively high arginase activity in the intestinal mucosa. Arginine is a substrate for citrulline synthesis in the intestine through conversion by arginase II and ornithine transcarbamylase (OTC) metabolic pathways with interorgan exchange of ornithine $(28,29)$.

Arginine is substrate for creatine synthesis, which also requires glycine and methionine. Creatine synthesis consumes some $20-30 \%$ of arginine's amidino groups, whether provided in the diet or synthesized within the body, and therefore imposes an appreciable burden on the metabolism of arginine. Creatine is excreted from the body as urinary creatinine. This is a non-enzymatic and unregulated breakdown process, that occurs at a rate of approximately $1.7 \%$ of total body creatine and creatine phosphate per day ((30) for review).

About 1.5\% of arginine flux enters the NO synthase (NOS) pathway (6) that converts arginine to $\mathrm{NO}$ and citrulline by either of three isoforms of the NOS enzyme (31, 32). NOS1 (neuronal NOS) and NOS3 (endothelial NOS) are constitutive enzymes that are controlled by intracellular $\mathrm{Ca}^{2+}$ /calmodulin. NOS2 is inducible at the level of gene transcription, $\mathrm{Ca}^{2+}$ independent, and expressed by macrophages and other tissues in response to (pro)-inflammatory mediators. A mitochondrial NOS isoform (mtNOS) for production of $\mathrm{NO}$ in mitochondria has been proposed but several studies have challenged the existence of a mitochondrial isoform (33). Several 
co-factors are known for NOS of which tetrahydrobiopterin (BH4) is essential and rate-limiting, and is synthesized from guanosine triphosphate (GTP) via the GTPcyclohydrolase-I (GTP-CH) pathway ((34) for recent review). Other known cofactors are flavin adenine dinucleotide (FAD), flavin mononucleotide (FMN) and heme (reviewed by (35)). Finally, arginine can be decarboxylated to agmatine, which acts as a cell-signaling molecule (18).

NO can also be derived from conversion of nitrite. The conversion of nitrite to $\mathrm{NO}$ can occur via simple non-enzymatic nitrite reduction under acidic conditions $\left(e^{-}+2 \mathrm{H}^{+}+\mathrm{NO}_{2}{ }^{-} \rightarrow \mathrm{NO}^{-}+\mathrm{H}_{2} \mathrm{O}\right)$, resulting in $\mathrm{NO}$ production in the stomach (36). This NOS-independent $\mathrm{NO}$ production is controlled by oxygen tension, $\mathrm{pH}$, reducing substrates and nitrite levels (37). Production of NO from nitrite was first observed in heart tissue under conditions of ischemia with intracellular acidosis (38) and occurs primarily in tissues and not in blood (39). The level of nitrite-derived NO under ischemic conditions with acidosis is comparable to maximum constitutive NOS production, which makes this NOS-independent route a practical alternative pathway under ischemic conditions where NO production from NOS is compromised (37). Dietary nitrate, mainly from vegetables, is reduced to bioactive $\mathrm{NO}_{2}{ }^{-}$by facultative anaerobic bacteria in the saliva, and as such can serve as NO source. Arginine metabolic pathways and enzymes are summarized in Figure 3.

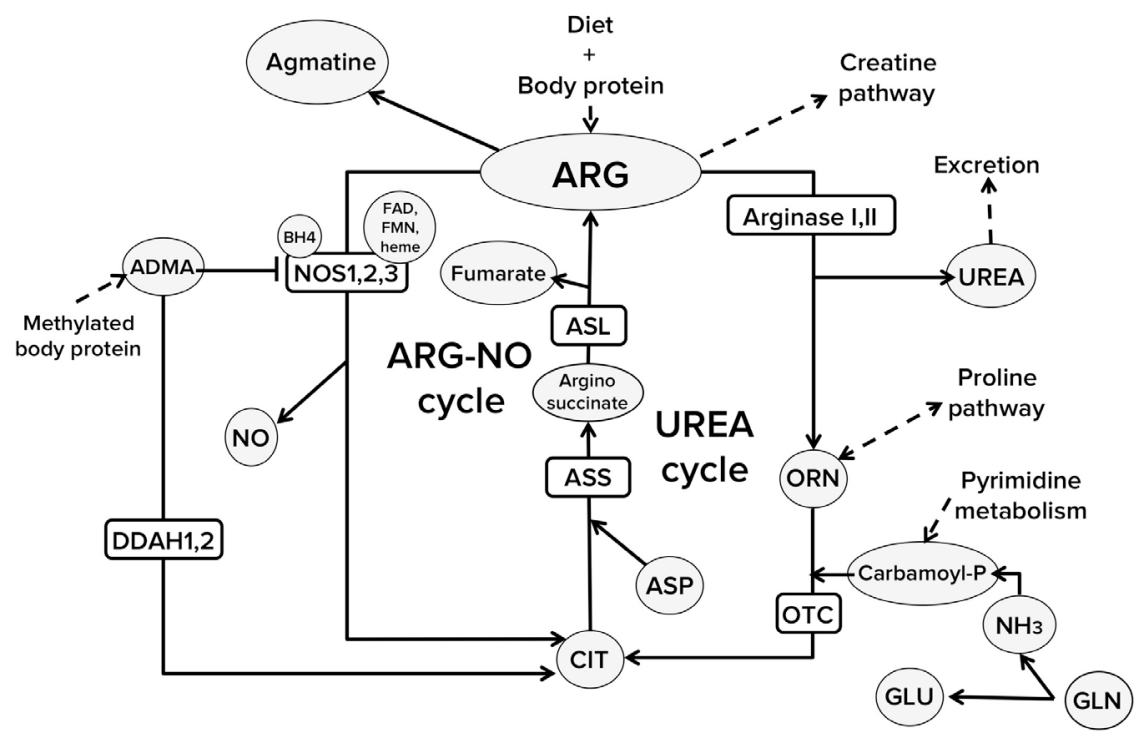

Figure 3. Arginine metabolic pathways in healthy humans

ARG, arginine; ORN, ornithine; PRO, proline; GLU, glutamate; CIT, citrulline; ASP, aspartate; GLN, glutamine; NO, nitric oxide; NOS, nitric oxide synthase; ASS, argininosuccinate synthase; ASL, argininosuccinate lyase; ; ADMA, asymmetric dimethylarginine; L-NMMA, NGmethyl- L-arginine; $\mathrm{BH} 4$, tetrahydrobiopterin; FAD, flavin adenine dinucleotide; FMN, flavin mononucleotide. 


\section{Role of arginine and NO in normal physiology}

Arginine is a constituent of body proteins and is an intermediate in the urea cycle in the liver. The urea cycle is a series of five reactions in which urea synthesis is the final step in the detoxification of ammonia. Moreover, the urea cycle has been considered as a major pathway for the removal of metabolically generated bicarbonate, and as such a role for the liver in $\mathrm{pH}$ homeostasis (40). Other roles of arginine are related to arginine-derived metabolites. These include, among others that we will not further specify here, ornithine and derived polyamines (putrescine, spermine, and spermidine), which are important for cell growth and differentiation. Proline, which is hydroxylated to hydroxyproline post-translationally, can also be derived from arginine and has a role in collagen formation, tissue repair and wound healing. Creatine is also derived from arginine and plays a role in energy metabolism in muscle and neurons (see Ref. (20) for review). Apart from actions via its metabolites, arginine directly activates p70 S6 kinase and phosphorylation of 4E-BP1 through the mTOR signaling pathway (41) with stimulation of protein synthesis in a NO-independent way (42).NO has various roles in normal physiology, but we will not review all roles in full detail. NO derived from NOS1 and NOS3 acts as a neurotransmitter and a vasodilator, respectively (32). NO in the brain regulates many physiological processes affecting behavior and cognitive function, including synaptic plasticity. In addition, NO controls brain blood flow, promotes angiogenesis, maintains cellular redox state, cell immunity and neuronal survival. However, despite the many diverse roles of $\mathrm{NO}$, regulation of the amount produced is important, as over-production of NO may lead to neurodegeneration (43). NO is synthesized at high levels by NOS2 when activated during inflammatory processes by elevated circulating cytokine concentrations (mainly TNF- $\alpha$, and IL-1, IL-6, and IL-8) and/or microbial products like LPS $(31,32,44,45)$. This NO has immune regulatory functions, such as control or killing of infectious pathogens, modulation of cytokine production and T-helper cell development. Moreover, NO can act as a free radical scavenger (46). Local NO responses are concentration and exposure time dependent (47). In general at low concentrations, NO promotes cell survival and proliferation and at high concentrations promotes cell cycle arrest, apoptosis and senescence. As such, arginine has an indirect role in NO-mediated functions, including immune modulation $(48,49)$ or acts immune response-enhancing during immunological challenge (50).

\section{Factors that mediate arginine and NO metabolism and availability}

The level of dietary arginine intake and endogenous production on the one hand, and the extent of utilization or clearance on the other hand influence arginine metabolism and availability (Figure 4). 


\section{Systemic and intracellular arginine availability}

Transporters for arginine uptake in the cell are often co-localized with arginineconverting enzymes and as such can modulate cellular arginine metabolism (20). For example, cationic amino acid transporter (CAT)-1 and NOS3 are co-localized in plasma membrane caveolae (51). This facilitates specific channelling of arginine to endothelial NO production without mixing with the total intracellular pool. This is in line with observations in vitro of extracellular rather than intracellular arginine being the major determinant for NO production in endothelial cells (22). Another example of relation between arginine metabolism and its transporter is the upregulation by inflammatory cytokines of CAT-2 and down-regulation of CAT-1 arginine transporters $(52,53)$, that results in increased availability of arginine to NOS2 and decreased availability to NOS3. Competition with lysine, ornithine, glutamine and certain endogenous NOS-inhibitors that use the same transporter as arginine may compromise intracellular arginine transport in conditions of low arginine (54). In addition to the link between arginine transport and intracellular arginine availability, the coupling between arginine-synthesizing and converting enzymes or the competition between enzymes for arginine as a substrate determine its metabolic fate. For example, coupling between de novo arginine synthesis and NO production is supported by co-localization in endothelial cells of NOS3 and ASS/ASL (21). On the other hand, substrate competition between arginase and NOS reciprocally regulates NO levels in endothelial cells $(20,55,56)$.

De novo arginine production from citrulline can be impaired by renal failure (57-59), but citrulline delivery to the kidney is the rate limiting determinant of renal arginine production $(12,59)$. Impaired intestinal function is a major underlying reason for reduced citrulline availability (see Ref. (60) for review)(61), supported by the observations that 80 to $90 \%$ of the citrulline is derived from conversion of glutamine to citrulline in the intestine $(16,62-65)$.

Dietary arginine availability influences its own catabolism and that of other amino acids by controlling ureagenesis. Arginine is not only a substrate for ureagenesis, but also an activator of $\mathrm{N}$-acetyl glutamate synthetase, which is a key ureagenic enzyme (66). On a low-arginine diet, arginine catabolism (i.e. arginine hydroxylation with conversion to ornithine) is reduced with maintained de novo arginine production and reduced plasma arginine (67-69). This also applies when a low protein (nitrogen) diet is fed and amino acids are more efficiently used for other processes than oxidation, and subsequently less ammonia is present for detoxification in the liver urea cycle. However, a concept presented by the Cynober group states that, under the condition of low dietary protein intake, the intestinal conversion of arginine into citrulline by intestinal arginase and OTC is activated, . The newly formed citrulline then bypasses liver metabolism and is converted back to arginine in the 


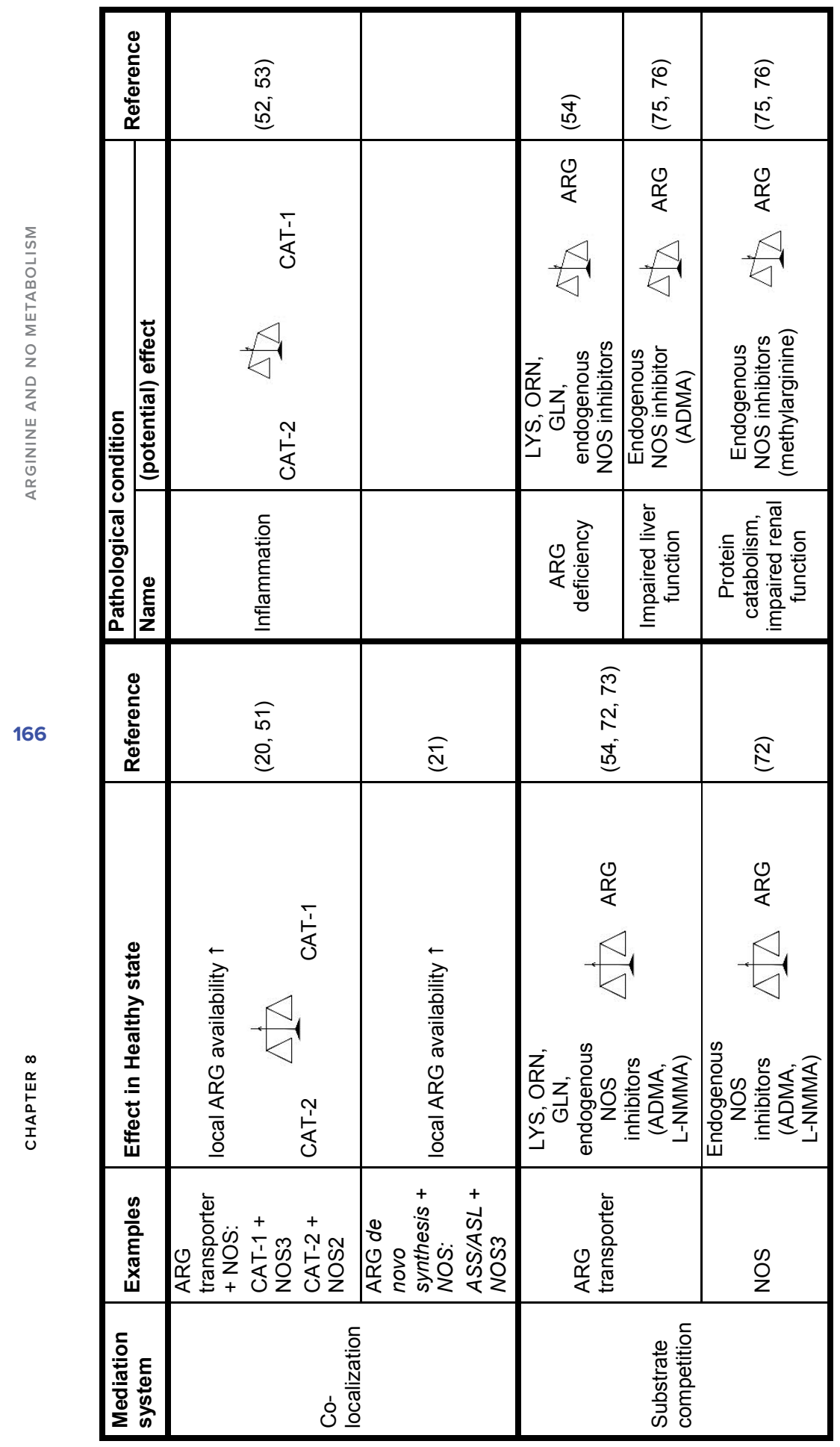




\begin{tabular}{|c|c|c|c|}
\hline \multicolumn{2}{|c|}{$\widehat{\bar{\theta}}$} & \multicolumn{2}{|l|}{ త్ర్ర } \\
\hline & 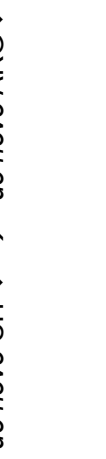 & 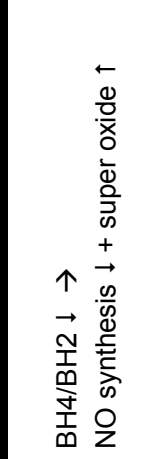 & 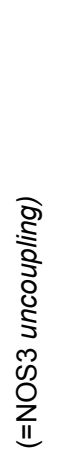 \\
\hline \multicolumn{2}{|c|}{ 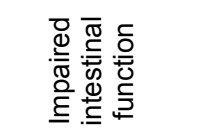 } & \multicolumn{2}{|l|}{ 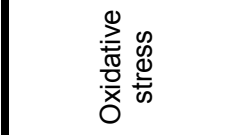 } \\
\hline ठิ) & $\begin{array}{l}\widehat{9} \\
0 \\
0 \\
0 \\
0\end{array}$ & 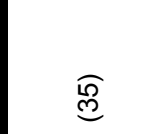 & $\stackrel{\widehat{\infty}}{=}$ \\
\hline 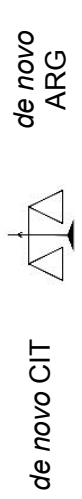 & $\begin{array}{l}5 \overline{5} \\
0 \\
0 \\
0 \\
8 \\
8 \\
\square \\
\square\end{array}$ & 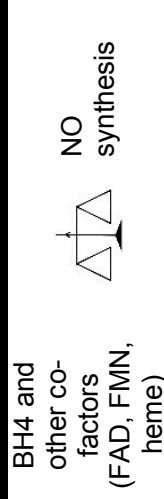 & 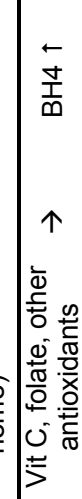 \\
\hline $\begin{array}{l}0 \\
0 \\
0 \\
0 \\
0 \\
0 \\
\frac{1}{\alpha}\end{array}$ & $\begin{array}{l}\frac{5}{0} \\
0 \\
0 \\
0 \\
8\end{array}$ & $\underset{\infty}{\stackrel{\nabla}{\mathbf{T}}}$ & \\
\hline & & 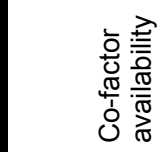 & \\
\hline
\end{tabular}


kidney (de novo arginine production), while at the same time ureagenesis is limited and arginine and other amino acids are spared for protein synthesis $(70,71)$.

\section{Endogenous NOS inhibitors and enzymatic cofactors.}

Methylarginines, such as a-symmetric dimethylarginine (ADMA) and $N^{G}$-methylL-arginine (L-NMMA), are the most powerful endogenous and competitive nonspecific NOS inhibitors that compete with L-arginine for the active site of NOS and for intracellular transport ((72) for review)(73). These methylarginines are derived from the catabolism of post-translational modified proteins that contain methylated arginine residues. Methylarginines are eliminated from the body by a combination of renal excretion and metabolism through enzymatic degradation by dimethylaminohydrolase (DDAH) to citrulline and methylamines (74). Increased protein catabolism and impaired renal function can thus contribute to elevated levels of methylarginine. High hepatic expression of DDAH and uptake of ADMA makes the liver important in the metabolism of ADMA, and hepatic dysfunction a prominent determinant of ADMA concentration $(75,76)$. Reference values $\left(2.5^{\text {th }}-97.5^{\text {th }}\right.$ percentile $)$ for the L-arginine/ADMA ratio are between 74.3 and 225 (77).

Enzymatic cofactors for $\mathrm{NO}$ production, such as tetrahydrobiopterin (BH4), can be affected by several factors and can subsequently influence NO production and endothelial function. Factors that regulate $\mathrm{BH} 4$ activity are nutritional, therapeutic, and endothelium-derived factors. Vitamin C, folate, and other antioxidants enhance endothelial $\mathrm{BH} 4$ bioavailability through chemical stabilization or scavenging of reactive oxygen species (78). Depletion of $\mathrm{BH} 4$ by oxidation into 7,8-dihydrobiopterin $(\mathrm{BH} 2)$ in oxidative stressed endothelial cells can result in so-called NOS3 'uncoupling', with production of superoxide instead of NO. It is now believed that the intracellular $\mathrm{BH} 4$ to $\mathrm{BH} 2$ ratio, rather than absolute concentrations of $\mathrm{BH} 4$, is the key determinant of this NOS3 uncoupling (34).

\section{Metabolic alterations related to age, sex, animal species and strain differences}

In neonates, de novo arginine production via the interorgan intestinal-renal axis is not yet developed (2), but conversion of citrulline to arginine occurs in the intestine. The precursor of intestinal citrulline in this condition is proline. In addition, the absence of arginase in the placenta of the mother and in the neonatal intestines suggests a metabolic strategy to maximize the availability of arginine in the systemic circulation from mother to fetus and from maternal milk to neonate. In addition, polyamines and NO are essential for growth and angiogenesis. Therefore, limited de novo arginine production capacity makes arginine an essential amino acid in early life. In neonatal piglets, dietary arginine is conserved in times of deficiency by decreasing arginine hydroxylation and increasing recycling (79). Also in a wide range of livestock reproduction processes, non-optimum availability of arginine results in suppression or 
inhibition of arginine metabolism (2). NO bioavailability diminishes with aging and as such adds to the pathogenesis of disturbances in endothelium-dependent vasodilatation related to aging (80). This diminished $\mathrm{NO}$ bioavailability has been related to a decrease in $\mathrm{BH} 4$ and uncoupling of NOS3 with increased oxidative stress in aging $(81,82)$, but also to increased arginase activity with subsequently reduced arginine availability for NOS ((83) for review). In the brain, the excitatory glutamate-NO-cGMP neurotransmission is normally in balance with the inhibitory GABA neurotransmission. In rats, an age-related regional imbalance of the glutamate/GABA balance was observed, caused by decreased glutamate levels. This is correlated with changes in levels of L-arginine and its metabolites in many brain regions (84).

In mice, differences in arginine metabolism are described between sex and strains, such as between C57BL6/J and FVB mice, as well as between B6 and ICR mice (85, 86). In female mice, plasma arginine was reported to be higher, and strain differences with regard to whole body de novo arginine production and portal-drained visceral (PDV) arginine metabolism were found (85).

\section{Alterations of arginine and NO metabolism in disease}

Arginine metabolism is altered in disease states both with regard to its synthesis and catabolism. This can result in a disruption of the normal homeostasis between metabolic pathways and the fasted blood arginine level. Tang et al (87) proposed the global arginine bioavailability ratio (GABR), defined as plasma arginine divided by the sum of ornithine and citrulline, to account for arginine catabolic metabolites. This ratio was derived as a better index of reduced NO synthetic capacity than systemic arginine levels alone. Impaired intestinal absorption (88), impaired organ function such as intestinal dysfunction (89) or renal dysfunction (90), enzyme competition and impaired cellular uptake may further compromise (de novo) arginine metabolism and (cellular) arginine availability. Subsequent functional consequences of altered arginine metabolism, such as altered endothelial function with hemodynamic changes on systemic (especially hypertension) and (micro) circulatory level, as well as immune alterations, are well known.

\section{Metabolic alterations of arginine, arginine de novo and NO under stress conditions}

Normal fasted plasma arginine ranges between 80-100 $\mu \mathrm{mol} / \mathrm{L}$ (162) while recent reference values $\left(2.5^{\text {th }}-97.5^{\text {th }}\right.$ percentiles) from relatively healthy subjects from the Framingham Offspring Cohort were reported to be lower between 41-114 $\mu \mathrm{mol} / \mathrm{L}$ (77). This could well be related to the fact that in this study, the special treatment of plasma samples for arginine (immediately cooling and cooled spinning down 
within $30 \mathrm{~min}$ ) was not done (91). When taking care of proper treatment of blood samples, under stressed conditions plasma arginine is decreased (92), to levels as low as $50 \mu \mathrm{mol} / \mathrm{L}$ in patients with sepsis (93-95). The intestinal-renal pathway resulting in de novo arginine synthesis from citrulline is impaired in sepsis (95), which may be the results of limited citrulline availability due to intestinal failure $(95$, 96) and impaired glutamine to citrulline conversion (97), or limited arginine production due to renal failure $(12,98)$. Higher level proteolysis can mask the decline in de novo synthesis of arginine, and as a consequence total arginine availability may be maintained $(95,99)$.

Increased utilization of arginine for the synthesis of proteins associated with the stress response, such as the acute phase proteins, will reduce arginine availability in these conditions and may result in alterations in (isoform-specific) NOS enzyme activity. Increased NOS2 activity during sepsis coincides with the down-regulation of the activity of other NOS isoforms (100-102). This reduces NO production enzyme-specifically in conditions of overall $\mathrm{NO}$ production being either lowered or not different from healthy controls $(95,103)$. Increased plasma arginine clearance (103) due to enhanced arginase activity also reduces arginine availability for other catabolic pathways. Moreover, increased arginine oxidation is observed during sepsis in paediatric patients (99). The nutritional status also affects the metabolic response to endotoxemia, as demonstrated in a pig model of sepsis. A well-nourished condition before prolonged endotoxemia in this model resulted in a better ability to adapt to endotoxin-induced metabolic deterioration of arginine-NO metabolism compared with reduced caloric intake before endotoxemia (104).

\section{Acute conditions, such as trauma, sepsis and acute liver failure}

In sepsis, a reduced plasma arginine concentration was related to worse survival (93). Increased mortality in critically ill patients has also been related to elevated ADMA levels, which by substrate competition may be a causative factor in the development of multiple organ dysfunction (105). Sepsis, in particular septic shock, is characterized by elevated cardiac output and hypotension caused by vasodilatation that are associated with misdistribution of blood flow and low peripheral vascular resistance. These characteristics of sepsis have been attributed to an increased NO production by NOS2 $(44,106)$. However, simultaneously decreased NOS3 expression may be related to microcirculatory shutdown and shunting, contributing to the reduced microvascular blood flow and impaired tissue oxygenation (107-111). Others suggested that an elevated NO production in critically ill patients impairs substrate and oxygen utilization by enhanced protein nitrosylation and inhibition of mitochondrial respiration $(112,113)$.

Increased NO production is likely also responsible for the hyperdynamic circulation found in patients with liver cirrhosis, and may be an important mediator of 
the exaggerated circulatory abnormalities during acute systematic inflammation in so-called acute-on-chronic liver failure with increased cerebral blood flow (114). Up-regulation of the NOS2 pathway in the endothelium with simultaneous downregulation of NOS3 mediated NO production, was observed in critically ill cirrhotic patients after transjugular intrahepatic stent-shunt (TIPPS) placement through exacerbation of existing endotoxemia (115). Recently, in a pig model with early phase of acute liver failure, arginine deficiency and increased ADMA did not limit whole body NO production. Arginine deficiency was caused by arginase-related arginine clearance. The stimulated intestinal-renal axis was insufficient to compensate the arginine deficiency (116).

\section{Chronic conditions, such as obesity, diabetes and cardiovascular diseases}

Abnormalities in NO production and transport in vascular systems result in various cardiovascular pathologies including hypertension, atherosclerosis and angiogenesis-associated disorders (for recent review on the role of $\mathrm{NO}$ in the vasculature see (117)). Reduced basal, NOS3-mediated, NO synthesis, or action leads to vasoconstriction, elevated blood pressure or thrombus formation. On the other hand, NO overproduction by NOS2 leads to vasodilation, hypotension, vascular leakage, disruption of cell metabolism and atherosclerosis, either directly or indirect via the formation of $\mathrm{NO}$ adducts such as peroxynitrite (43). Cardiovascular and pulmonary disorders such as atherosclerosis, diabetes, hypercholesterolaemia, ischemic heart disease and hypertension are characterized by NOS3 uncoupling with formation of superoxide instead of $\mathrm{NO}((34,118)$ for recent reviews). The lower $\mathrm{NO}$ production in these conditions due to a deficiency of $\mathrm{BH} 4$ also underlies the impaired action of insulin in the vasculature of obese and diabetic subjects (119). Diet-induced oxidative scavenging of $\mathrm{NO}$ and reduced $\mathrm{NO}$ bioavailability was also shown to accompany early diet-induced insulin resistance (120). Oxidative stress causing S-glutathionylation of NOS3 in endothelial cells with loss of NO and gain of superoxide is increased in hypertensive vessels (121). By inhibiting NO production, elevated cellular levels of endogenous methylarginines can impair vascular relaxation and are mediators of vascular dysfunction in disease. Elevated ADMA levels have been reported in hypercholesterolemia, atherosclerosis, hypertension, chronic heart failure, diabetes mellitus and chronic renal failure $(73,122)$. L-arginine: ADMA ratio was positively associated with the estimated glomerular filtration rate and diastolic blood pressure in a large cohort study (77), and may act as a clinical diagnostic tool for improved cardiovascular risk assessment (122). ADMA was also identified as an independent risk marker for mortality in ambulatory patients with peripheral arterial disease (123). 


\section{Neurological diseases}

In the brain, arginine as a precursor for $\mathrm{NO}$ is necessary for cerebrovascular homeostasis (NOS3) and is involved in learning and memory capacities via the glutamateNO-cGMP pathway (NOS1). In neurological diseases, one or both of these routes are impaired. Also, high amounts of induced $\mathrm{NO}$ production by inflammatory factors (NOS2) contribute to oxygen stress and therefore can play a role in the severity of the diseases. In the onset of brain stroke, cerebrovascular disease and Alzheimer disease, hypoperfusion as underlying cause of oxygen stress is one of the present avenues to understand the initiation of these diseases ((124) for review). A chronic imbalance of NOS in the brain is believed to be a key element.

In hepatic encephalopathy $(\mathrm{HE})$, a neurocognitive disorder in which brain function is impaired and is associated with both acute and chronic liver dysfunction, hyperammonemia plays an important role in the pathophysiology. Alterations in glutamate-NO-cGMP pathway are described, especially in acute HE and in relation with excessive glutamine production (ammonia detoxification by conversion of glutamate into glutamine) in the brain (125). Besides a direct effect on the glutamate neurotransmission cycle, glutamine can also limit the transport of arginine into the neurons and astrocytes, because it competes with the glutamine transport (126). The implications are not clear yet and may differ in different stages of the disease.

\section{Cancer}

Humans with cancer have a decreased systemic availability of arginine independent of the type of cancer, age, sex or cachectic state (127). In mice, cancer affects de novo arginine production probably through diminished intestinal citrulline production (128). In addition, high arginase activity is observed via the myeloid suppressor cells in the microenvironment of tumors (129). Other research is focused on tumor growth and the arginine dependence of certain tumors that do not express ASS, Such tumors, such as melanoma and hepatocellular carcinoma are sensitive to arginine depletion by arginine degrading enzymes such as arginine deiminase (see Ref. (130) for review) or a recombinant form of human arginase I (131). A disturbed arginine metabolism could be a factor that is causing relative poor clinical outcome. $\mathrm{NO}$ is not only required for an adequate immune reaction during a surgical trauma after a tumor extraction, but also contributes to cytotoxic induced antitumor processes $(132,133)$.

\section{Therapeutic approaches to influence arginine and NO metabolism}

Reduced arginine intake in disease or malnutrition as well as increased metabolic needs can result in arginine deficiency or its increased requirement. Therapeutic 
approaches can aim at increasing substrate availability by supplementation, or at influencing the metabolic fate of specific pathways related to NO bioavailability and prevention of NOS3 uncoupling.

\section{Arginine supplementation}

Arginine supplementation varying between $3 \mathrm{~g} /$ day up to over $100 \mathrm{~g} /$ day has been used in clinical studies. Single doses of 3-8g appear to be safe and rarely provoke adverse events (134), but single doses exceeding $9 \mathrm{~g}$ and especially when part of a dosing regimen of over $30 \mathrm{~g} /$ day have been associated with gastrointestinal discomfort, nausea and (osmotic) diarrhea (135). Arginine has been used in supplemental nutrition for surgical patients, burn patients, and patients with sepsis and cancer to benefit regulation of blood pressure, wound healing, immunomodulation, or to serve as an anabolic stimulus. However, the benefits of arginine in these conditions are not uniformly proven and accepted.

Arginine supplementation in sepsis patients has been combined with a mixture of amino acids and other nutrients, referred to as immunonutrition (136-139). Several reviews and opinion papers on its use have been published (140-147), but conclusions regarding the benefits and potential use in sepsis are not uniform. Arginine treatment starting before endotoxemia in a pig model appeared beneficial by improved hepatosplanchnic perfusion and oxygenation during prolonged endotoxemia, without causing deleterious systemic side effects. This is probably mediated through enhanced NO synthesis (148). Arginine supplementation has inconsistent effects on intestinal ischemia-reperfusion injury, and a recent publication with longterm intra-duodenal arginine supplementation in a rat model of intestinal ischemia and reperfusion injury did not show observable benefits on intestinal morphology or on the inflammatory response (149). Remarkably, serum NOx was even decreased with arginine supplementation in this study, which suggests that arginine was probably not available for $\mathrm{NO}$ production due to substrate competition for arginine. Supplementing arginine in partial enteral feeding in neonatal pigs modestly increased intestinal mucosal growth through a NO-independent mechanism (150).

Beneficial effects of arginine supplementation were observed in patients with sickle cell disease suffering from pulmonary hypertension, as well as in the prevention of age-related glomerular injury, in reversing impaired vasodilatation in clinically asymptomatic hypercholesterolemic adults, and improving wound healing (151-155). A growing body of evidence indicates that arginine supplementation is beneficial in growth, health and disease and may provide novel and effective therapies for obesity, diabetes and metabolic syndrome (2). Malnourished head and neck cancer patients who received arginine-enriched nutrition peri-operatively demonstrated better 10-year survival (156). Moreover, arginine was proposed as a therapy in hypertension, to interrupt the vicious cycle that initiates and maintains low NO (157). 
A recent meta-analysis concluded that short-term oral L-arginine supplementation is effective in improving the fasting vascular endothelial function, i.e. flow-mediated vasodilation (FMD), when the baseline FMD is low and thus endothelial dysfunction can be restored (158). FMD is an early pathophysiological feature of cardiovascular disease and reflects local bioavailability of NO under physiological stimulation. Whether long-term arginine supplementation is beneficial is debated, since exogenous arginine also increases arginase with subsequent diversion of arginine from NOS and subsequent NO production (159).

\section{Citrulline supplementation}

Citrulline supplementation as a single oral dose of 2, 5, 10 or $15 \mathrm{~g}$ is safe and well tolerated in healthy adults with no effect on plasma levels of insulin and growth hormone and urinary excretion of citrulline remaining low $(<5 \%)$ even at high doses. Citrulline supplementation has proven to be an effective precursor for arginine and ornithine, but saturation of the renal conversion of citrulline into arginine probably occurred at the highest citrulline dose (15g) (160). Citrulline-malate is an alternative citrulline source that is also applied as anti-asthenia treatment and quickly lowers ammonia levels in hyperammonaemia (89).

Citrulline supplementation likely restores the optimal balance between arginine production and metabolism, as well as improving NO production and related functions. In an arginine-deprived in vitro model of macrophages, addition of citrulline restored NO production, while glutamine interfered with citrulline-mediated NO production (161). Therefore, in conditions of acute or chronic inflammation with arginine deficiency, citrulline supplementation is a potentially powerful approach to restoring NO production (71). In sickle cell disease, oral citrulline supplementation maintained elevated arginine levels and maintained nearly normal total leukocyte and neutrophil counts, and has therefore been suggested as a useful palliative therapy in this condition (162). Citrulline supplementation ameliorated the development of pulmonary hypertension and increased $\mathrm{NO}$ production in piglets exposed to chronic hypoxia (163); this suggests that neonates exposed to prolonged periods of hypoxia from cardiac or pulmonary causes may potentially benefit from citrulline supplementation. In middle-aged men, citrulline supplementation improved arterial stiffness, which is considered a powerful predictor of cardiovascular disease (164). Citrulline supplementation restored nitrogen balance and generated large amounts of arginine in rats with short bowel syndrome (165).

\section{NO donors and NOS3 modulating agents}

NO can also be derived from so-called NO donors, inhaled NO, and nitrite (sources). NO donors, such as nitroglycerin are well known and used as vasodilators to treat heart conditions such as angina and chronic heart failure. In septic patients, nitroglycerin increased sublingual microvascular flow, even though arterial and central 
venous pressure dropped temporarily (166). The use of inhaled NO in the perioperative setting for the treatment of pulmonary hypertension in children is recommended (167). Short-term nitrite therapy reversed age-associated vascular endothelial dysfunction, large elastic artery stiffness, oxidative stress, and inflammation in old mice, by restoring NO bioavailability through a NOS-independent conversion. The authors therefore suggested that sodium nitrite may be a novel therapy for treating arterial aging in humans (168). Nitrite is also currently undergoing or planned for clinical trials as a vasodilator drug in patients with cardiovascular diseases such as ischemic stress, sickle cell disease, coronary artery disease, and pulmonary hypertension (37). Nitrate-rich vegetable juice acutely increased nitrite (within 2.5h), and reduced blood pressure as well as oxygen costs of moderate intensity exercise in normotensive subjects. These effects were sustained during continuous juice intake over 15 days (169).

Other novel pharmacological approaches under development to increase NO bioavailability are targeted at preventing NOS3 uncoupling or enhancing NOS3 expression $((34,118,170,171)$ for recent reviews). Regarding the latter, $\mathrm{BH} 4$ or its synthetic versions may be a new therapeutic strategy to tackle myocardial and endothelial dysfunction (172). Other agents or therapies e.g. statins, intravenous ascorbic acid administration or exercise, act on preventing $\mathrm{BH} 4$ loss, on improving $\mathrm{BH} 4$ availability or on $\mathrm{BH} 4$ stability by scavenging superoxide; this functions by improved endothelial NOS coupling and vascular NO bioavailability $(82,171,173)$. Local ascorbic acid infusion was demonstrated to improve NO-mediated muscle blood flow during exercise in elderly (174). While $\mathrm{BH} 4$ repletion only partly restored NOS activity and NO-dependent vasodilation, reversion of another redox-regulated mechanism controlling NOS function by thiol-specific reducing agents can restore vasodilation when NOS3 S-glutathionylation is increased (121). A pharmacological NOS3 enhancer (AVE3085) ameliorated endothelial dysfunction in $\mathrm{db} / \mathrm{db}$ mice through increased NO bioavailability, which makes targeting NOS3 and NO a promising approach to combat diabetic vasculopathy (175).

\section{Targeting endogenous NO inhibitors}

An alternative approach to increase $\mathrm{NO}$ bioavailability is via targeting endogenous inhibitors of nitric oxide synthesis such as ADMA or arginase. Pharmacological modification of dimethylarginine dimethylaminohydrolase (DDAH) enzymes that metabolize ADMA (176) or treatment with the arginase inhibitor $\mathrm{N}(\omega)$-hydroxy-norL-arginine (nor-NOHA) are options $(177,178)$. Nor-NOHA restored the microvascular coronary artery function in type 2 diabetic rats and caused cardioprotection against myocardial ischemia-reperfusion injury in rats by a mechanism with increased utilization of arginine by NOS and increased NO availability $(177,178)$. Recently published reference levels for L-arginine:ADMA ratio may be helpful for evaluation of 
the effects of L-arginine supplementation in participants with an impaired L-arginine/ NO pathway (77).

\section{Therapies that influence NO-mediated effects}

Patients receiving IL-2 cytokine treatment for advanced malignancy demonstrate increase endogenous nitrate synthesis (132), while NOS3 knockout mice were resistant to IL-2 induced hypotension and vascular leak. Methylene blue, by inhibiting guanylate cyclase and cGMP, could inhibit this NOS3 mediated vascular leak (179). Selective inhibition of p38 mitogen-activated protein kinase (MAPK), a mediator of vascular inflammation and activated by oxidized low-density lipoproteins, improved NO-mediated vasodilation in patients with hypercholesterolemia (180). The authors suggested that p38 MAPK could therefore be a novel target for patients with cardiovascular disease.

\section{Summary, conclusion and future research}

While NO production is dependent on arginine availability as its precursor, the odd circumstance is that only a small percentage of arginine is used for NO synthesis and that either too much or too little NO is detrimental. This suggests that the relation between arginine availability and $\mathrm{NO}$ production is not simply a case of precursor availability. Rather, it is more likely the combination of the availability of arginine, along with cofactors and rate-limiting enzymes that determine the rate of production of NO. The compartmentalization of arginine metabolism plays a role here, in what is also referred to as the 'arginine paradox'.

During the past few years, it has become recognized that endothelial NOS uncoupling and NOS3 dependent superoxide generation, induced by stress, are key mediators in the pathogenesis of cardiovascular and pulmonary diseases. Local arginine deficiency, which can be the result of arginine catabolism via arginase or competition with methylarginines, results in endothelial NOS uncoupling. Modulating NOS uncoupling and targeting NOS3-dependent ROS formation are recent developments that require further clinical testing. Arginine seems to have a critical and dual role here, both as a substrate for NOS and as a radical scavenger. Since arginine released from local protein breakdown may not be available for NOS, coupling of the enzymes for de novo arginine and NOS3 could make citrulline a good and maybe even better source for NO. The antioxidant action of citrulline could further contribute to prevent NOS uncoupling, but this is not yet known. Specific drugs that act on increasing local arginine availability for $\mathrm{NO}$ production, or those that mediate or provide co-factors for NO production are also considered useful.

In conclusion, the complex regulation of $\mathrm{NO}$ synthesis and intracellular availability of arginine as its precursor probably requires an approach beyond the primary provision of extra arginine. A multi-target approach addressing substrate competition, 
precursor availability and cofactor availability may be useful, and future research could focus on developing such strategies that can optimize NO bioavailability. This can be applied to conditions of compromised or unbalanced NO production, such as those of endothelial dysfunction in various acute and chronic diseases.

\section{Acknowledgement}

YC Luiking is an employee of Danone Research, Centre for Specialised Nutrition, The Netherlands.

\section{References}

1. Barbul A. Arginine: biochemistry, physiology, and therapeutic implications. JPEN J Parenter Enteral Nutr. 1986;10(2):227-38.

2. Wu G, Bazer FW, Davis TA, Kim SW, Li P, Marc Rhoads J, et al. Arginine metabolism and nutrition in growth, health and disease. Amino Acids. 2009;37(1):153-68.

3. Palmer RM, Ashton DS, Moncada S. Vascular endothelial cells synthesize nitric oxide from L-arginine. Nature. 1988;333(6174):664-6.

4. Moncada S, Radomski MW, Palmer RM. Endothelium-derived relaxing factor. Identification as nitric oxide and role in the control of vascular tone and platelet function. Biochem Pharmacol. 1988;37(13):2495-501.

5. Castillo L, Sanchez M, Vogt J, Chapman TE, DeRojas-Walker TC, Tannenbaum SR, et al. Plasma arginine, citrulline, and ornithine kinetics in adults, with observations on nitric oxide synthesis. Am J Physiol. 1995;268(2 Pt 1):E360-7.

6. Castillo L, Beaumier L, Ajami AM, Young VR. Whole body nitric oxide synthesis in healthy men determined from [15N] arginine-to-[15N]citrulline labeling. Proc Natl Acad Sci U S A. 1996;93(21):11460-5.

7. Luiking YC, Deutz NE. Isotopic investigation of nitric oxide metabolism in disease. Curr Opin Clin Nutr Metab Care. 2003;6(1):103-8.

8. Heys SD, Gardner E. Nutrients and the surgical patient: current and potential therapeutic applications to clinical practice. J R Coll Surg Edinb. 1999;44(5):283-93.

9. Visek WJ. Arginine needs, physiological state and usual diets. A reevaluation. J Nutr. 1986;116(1):36-46.

10. Dejong $\mathrm{CH}$, Welters CF, Deutz NE, Heineman E, Soeters PB. Renal arginine metabolism in fasted rats with subacute short bowel syndrome. Clin Sci (Colch). 1998;95(4):409-18.

11. Featherston WR, Rogers QR, Freedland RA. Relative importance of kidney and liver in synthesis of arginine by the rat. Am J Physiol. 1973;224(1):127-9.

12. Dhanakoti SN, Brosnan JT, Herzberg GR, Brosnan ME. Renal arginine synthesis: studies in vitro and in vivo. Am J Physiol. 1990;259(3 Pt 1):E437-42.

13. Yu YM, Burke JF, Tompkins RG, Martin R, Young VR. Quantitative aspects of interorgan relationships among arginine and citrulline metabolism. Am J Physiol. 1996;271(6 Pt 1):E1098-109.

14. Windmueller HG, Spaeth AE. Source and fate of circulating citrulline. Am J Physiol. 
1981;241(6):E473-80.

15. van de Poll MC, Siroen MP, van Leeuwen PA, Soeters PB, Melis GC, Boelens PG, et al. Interorgan amino acid exchange in humans: consequences for arginine and citrulline metabolism. Am J Clin Nutr. 2007;85(1):167-72.

16. Ligthart-Melis $\mathrm{GC}$, van de Poll MC, Boelens PG, Dejong $\mathrm{CH}$, Deutz NE, van Leeuwen PA. Glutamine is an important precursor for de novo synthesis of arginine in humans. Am J Clin Nutr. 2008;87(5):1282-9.

17. van de Poll MCG, Soeters PB, Deutz NEP, Fearon KCH, Dejong CHC. Renal metabolism of amino acids: its role in interorgan amino acid exchange. Am J Clin Nutr. 2004;79(2):185-97.

18. Morris SM, Jr. Arginine metabolism: boundaries of our knowledge. J Nutr. 2007;137(6 Suppl 2):1602S-9S.

19. Cynober L. Pharmacokinetics of arginine and related amino acids. J Nutr. 2007;137(6 Suppl 2):1646S-9S.

20. Wu G, Morris SM, Jr. Arginine metabolism: nitric oxide and beyond. Biochem J. 1998;336 ( Pt 1):1-17.

21. Flam BR, Eichler DC, Solomonson LP. Endothelial nitric oxide production is tightly coupled to the citrulline-NO cycle. Nitric Oxide. 2007;17(3-4):115-21.

22. Shin S, Mohan S, Fung HL. Intracellular L-arginine concentration does not determine NO production in endothelial cells: implications on the "L-arginine paradox". Biochem Biophys Res Commun. 2011;414(4):660-3.

23. Yu H, Yoo PK, Aguirre CC, Tsoa RW, Kern RM, Grody WW, et al. Widespread expression of arginase I in mouse tissues. Biochemical and physiological implications. J Histochem Cytochem. 2003;51(9):1151-60.

24. Jenkinson CP, Grody WW, Cederbaum SD. Comparative properties of arginases. Comp Biochem Physiol B Biochem Mol Biol. 1996;114(1):107-32.

25. Morris SM, Jr. Recent advances in arginine metabolism: roles and regulation of the arginases. Br J Pharmacol. 2009;157(6):922-30.

26. Choi S, Park C, Ahn M, Lee JH, Shin T. Immunohistochemical study of arginase 1 and 2 in various tissues of rats. Acta Histochem. 2012;114(5):487-94.

27. Castillo L, Chapman TE, Yu YM, Ajami A, Burke JF, Young VR. Dietary arginine uptake by the splanchnic region in adult humans. Am J Physiol. 1993;265(4 Pt 1):E532-9.

28. Marini JC, Didelija IC, Castillo L, Lee B. Glutamine: precursor or nitrogen donor for citrulline synthesis? Am J Physiol Endocrinol Metab. 2010;299(1):E69-79.

29. Marini JC, Keller B, Didelija IC, Castillo L, Lee B. Enteral arginase II provides ornithine for citrulline synthesis. Am J Physiol Endocrinol Metab. 2011;300(1):E188-94.

30. Brosnan JT, Brosnan ME. Creatine metabolism and the urea cycle. Mol Genet Metab. 2010;100 Suppl 1:S49-52.

31. Knowles RG, Moncada S. Nitric oxide synthases in mammals. Biochem J. 1994;298 (Pt 2):249-58.

32. Moncada S, Higgs A. The L-arginine-nitric oxide pathway. N Engl J Med. 1993;329(27):2002-12.

33. Lacza Z, Pankotai E, Csordas A, Gero D, Kiss L, Horvath EM, et al. Mitochondrial $\mathrm{NO}$ and reactive nitrogen species production: does mtNOS exist? Nitric Oxide. 2006;14(2):162-8.

34. Crabtree MJ, Channon KM. Synthesis and recycling of tetrahydrobiopterin in endothelial function and vascular disease. Nitric Oxide. 2011;25(2):81-8. 
36. Benjamin N, O’Driscoll F, Dougall H, Duncan C, Smith L, Golden M, et al. Stomach NO synthesis. Nature. 1994;368(6471):502.

37. Zweier JL, Li H, Samouilov A, Liu X. Mechanisms of nitrite reduction to nitric oxide in the heart and vessel wall. Nitric Oxide. 2010;22(2):83-90.

38. Zweier JL, Wang P, Samouilov A, Kuppusamy P. Enzyme-independent formation of nitric oxide in biological tissues. Nat Med. 1995;1(8):804-9.

39. Li H, Cui H, Kundu TK, Alzawahra W, Zweier JL. Nitric oxide production from nitrite occurs primarily in tissues not in the blood: critical role of xanthine oxidase and aldehyde oxidase. J Biol Chem. 2008;283(26):17855-63.

40. Haussinger D. Liver regulation of acid-base balance. Miner Electrolyte Metab. 1997;23(3-6):249-52.

41. Ban H, Shigemitsu K, Yamatsuji T, Haisa M, Nakajo T, Takaoka M, et al. Arginine and Leucine regulate p70 S6 kinase and 4E-BP1 in intestinal epithelial cells. Int J Mol Med. 2004;13(4):537-43.

42. Bauchart-Thevret C, Cui L, Wu G, Burrin DG. Arginine-induced stimulation of protein synthesis and survival in IPEC-J2 cells is mediated by mTOR but not nitric oxide. Am J Physiol Endocrinol Metab. 2010;299(6):E899-909.

43. Moncada S, Higgs EA. The discovery of nitric oxide and its role in vascular biology. Br J Pharmacol. 2006;147 Suppl 1:S193-201.

44. Nakae H, Endo S, Kikuchi M, Yamada Y, Shibata M, Ishikura H, et al. Nitrite/nitrate (NOx) levels and hemodynamics during septic shock. Surg Today. 2000;30(8):683-8.

45. Groeneveld PH, Kwappenberg KM, Langermans JA, Nibbering PH, Curtis L. Relation between pro- and anti-inflammatory cytokines and the production of nitric oxide (NO) in severe sepsis. Cytokine. 1997;9(2):138-42.

46. Titheradge MA. Nitric oxide in septic shock. Biochim Biophys Acta. 1999;1411(2-3):437-55.

47. Thomas DD, Ridnour LA, Isenberg JS, Flores-Santana W, Switzer CH, Donzelli S, et al. The chemical biology of nitric oxide: implications in cellular signaling. Free Radic Biol Med. 2008;45(1):18-31.

48. Reynolds JV, Daly JM, Zhang S, Evantash E, Shou J, Sigal R, et al. Immunomodulatory mechanisms of arginine. Surgery. 1988;104(2):142-51.

49. Daly JM, Reynolds J, Sigal RK, Shou J, Liberman MD. Effect of dietary protein and amino acids on immune function. Crit Care Med. 1990;18(2 Suppl):S86-93.

50. Li P, Yin YL, Li D, Kim SW, Wu G. Amino acids and immune function. Br J Nutr. 2007;98(2):237-52.

51. McDonald KK, Zharikov S, Block ER, Kilberg MS. A caveolar complex between the cationic amino acid transporter 1 and endothelial nitric-oxide synthase may explain the “arginine paradox". J Biol Chem. 1997;272(50):31213-6.

52. Reade MC, Clark MF, Young JD, Boyd CA. Increased cationic amino acid flux through a newly expressed transporter in cells overproducing nitric oxide from patients with septic shock. Clin Sci (Lond). 2002;102(6):645-50.

53. Schwartz D, Schwartz IF, Gnessin E, Wollman Y, Chernichovsky T, Blum M, et al. Differential regulation of glomerular arginine transporters (CAT-1 and CAT-2) in lipopolysaccharide-treated rats. Am J Physiol Renal Physiol. 2003;284(4):F788-95.

54. Luiking YC, Deutz NE. Biomarkers of arginine and lysine excess. J Nutr. 2007;137(6 Suppl 2):1662S-8S. 
55.

Bansal V, Ochoa JB. Arginine availability, arginase, and the immune response. Curr Opin Clin Nutr Metab Care. 2003;6(2):223-8.

56. Li H, Meininger CJ, Hawker JR, Jr., Haynes TE, Kepka-Lenhart D, Mistry SK, et al. Regulatory role of arginase I and II in nitric oxide, polyamine, and proline syntheses in endothelial cells. Am J Physiol Endocrinol Metab. 2001;280(1):E75-82.

57. Bouby N, Hassler C, Parvy P, Bankir L. Renal synthesis of arginine in chronic renal failure: in vivo and in vitro studies in rats with 5/6 nephrectomy. Kidney Int. 1993;44(4):676-83.

58. Bouby N, Coutaud C, Bankir L. Arginine synthesis by the proximal convoluted tubule in rats with chronic renal failure. Miner Electrolyte Metab. 1992;18(2-5):101-3.

59. Chen GF, Baylis C. In vivo renal arginine release is impaired throughout development of chronic kidney disease. Am J Physiol Renal Physiol. 2010;298(1):F95-102.

60. Crenn P, Messing B, Cynober L. Citrulline as a biomarker of intestinal failure due to enterocyte mass reduction. Clin Nutr. 2008;27(3):328-39.

61. Crenn P, Coudray-Lucas C, Thuillier F, Cynober L, Messing B. Postabsorptive plasma citrulline concentration is a marker of absorptive enterocyte mass and intestinal failure in humans. Gastroenterology. 2000;119(6):1496-505.

62. Boelens PG, Melis GC, van Leeuwen PA, ten Have GA, Deutz NE. Route of administration (enteral or parenteral) affects the contribution of L-glutamine to de novo L-arginine synthesis in mice: a stable-isotope study. Am J Physiol Endocrinol Metab. 2006;291(4):E683-90.

63. Boelens PG, van Leeuwen PA, Dejong CH, Deutz NE. Intestinal renal metabolism of $\mathrm{L}$-citrulline and L-arginine following enteral or parenteral infusion of L-alanyl-L-[2,15N] glutamine or L-[2,15N]glutamine in mice. Am J Physiol Gastrointest Liver Physiol. 2005;289(4):G679-85.

64. Ligthart-Melis GC, van de Poll MC, Dejong CH, Boelens PG, Deutz NE, van Leeuwen PA. The route of administration (enteral or parenteral) affects the conversion of isotopically labeled L-[2-15N]glutamine into citrulline and arginine in humans. JPEN J Parenter Enteral Nutr. 2007;31(5):343-48; discussion 9-50.

65. van de Poll MC, Ligthart-Melis GC, Boelens PG, Deutz NE, van Leeuwen PA, Dejong $\mathrm{CH}$. Intestinal and hepatic metabolism of glutamine and citrulline in humans. J Physiol. 2007;581(Pt 2):819-27.

66. Cynober L, Le Boucher J, Vasson M-P. Arginine metabolism in mammals. Nutritional Biochemistry. 1995;6:402-13.

67. Castillo L, Chapman TE, Sanchez M, Yu YM, Burke JF, Ajami AM, et al. Plasma arginine and citrulline kinetics in adults given adequate and arginine-free diets. Proc Natl Acad Sci U S A. 1993;90(16):7749-53.

68. Castillo L, Sanchez M, Chapman TE, Ajami A, Burke JF, Young VR. The plasma flux and oxidation rate of ornithine adaptively decline with restricted arginine intake. Proc Natl Acad Sci U S A. 1994;91(14):6393-7.

69. Tharakan JF, Yu YM, Zurakowski D, Roth RM, Young VR, Castillo L. Adaptation to a long term (4 weeks) arginine- and precursor (glutamate, proline and aspartate)-free diet. Clin Nutr. 2008;27(4):513-22.

70. Moinard C, Cynober L. Citrulline: a new player in the control of nitrogen homeostasis. J Nutr. 2007;137(6 Suppl 2):1621S-5S.

71. Cynober L, Moinard C, De Bandt JP. The 2009 ESPEN Sir David Cuthbertson. Citrulline: A new major signaling molecule or just another player in the pharmaconutrition game? Clin Nutr. 2010. 
inhibit nitric oxide synthases. Cardiovasc Res. 1999;43(3):542-8.

73. Cardounel AJ, Cui H, Samouilov A, Johnson W, Kearns P, Tsai AL, et al. Evidence for the pathophysiological role of endogenous methylarginines in regulation of endothelial NO production and vascular function. J Biol Chem. 2007;282(2):879-87.

74. Palm F, Onozato ML, Luo Z, Wilcox CS. Dimethylarginine dimethylaminohydrolase (DDAH): expression, regulation, and function in the cardiovascular and renal systems. Am J Physiol Heart Circ Physiol. 2007;293(6):H3227-45.

75. Nijveldt RJ, Teerlink T, Van Der Hoven B, Siroen MP, Kuik DJ, Rauwerda JA, et al. Asymmetrical dimethylarginine (ADMA) in critically ill patients: high plasma ADMA concentration is an independent risk factor of ICU mortality. Clin Nutr. 2003;22(1):23-30.

76. Nijveldt RJ, Teerlink T, Siroen MP, van Lambalgen AA, Rauwerda JA, van Leeuwen $P A$. The liver is an important organ in the metabolism of asymmetrical dimethylarginine (ADMA). Clin Nutr. 2003;22(1):17-22.

77. Luneburg N, Xanthakis V, Schwedhelm E, Sullivan LM, Maas R, Anderssohn M, et al. Reference intervals for plasma L-arginine and the L-arginine:asymmetric dimethylarginine ratio in the Framingham Offspring Cohort. J Nutr. 2011;141(12):2186-90.

78. Shi W, Meininger CJ, Haynes TE, Hatakeyama K, Wu G. Regulation of tetrahydrobiopterin synthesis and bioavailability in endothelial cells. Cell Biochem Biophys. 2004;41(3):415-34.

79. Urschel KL, Shoveller AK, Pencharz PB, Ball RO. Arginine synthesis does not occur during first-pass hepatic metabolism in the neonatal piglet. Am J Physiol Endocrinol Metab. 2005;288(6):E1244-51.

80. Taddei S, Virdis A, Ghiadoni L, Salvetti G, Bernini G, Magagna A, et al. Age-related reduction of $\mathrm{NO}$ availability and oxidative stress in humans. Hypertension. 2001;38(2):274-9.

81. Higashi Y, Sasaki S, Nakagawa K, Kimura M, Noma K, Hara K, et al.

Tetrahydrobiopterin improves aging-related impairment of endothelium-dependent vasodilation through increase in nitric oxide production. Atherosclerosis. 2006;186(2):390-5.

82. Sindler AL, Delp MD, Reyes R, Wu G, Muller-Delp JM. Effects of ageing and exercise training on eNOS uncoupling in skeletal muscle resistance arterioles. J Physiol. 2009;587(Pt 15):3885-97.

83. Santhanam L, Christianson DW, Nyhan D, Berkowitz DE. Arginase and vascular aging. J Appl Physiol. 2008;105(5):1632-42.

84. Liu $\mathrm{P}$, Jing $\mathrm{Y}$, Zhang $\mathrm{H}$. Age-related changes in arginine and its metabolites in memory-associated brain structures. Neuroscience. 2009;164(2):611-28.

85. Luiking YC, Hallemeesch MM, Vissers YL, Lamers WH, Deutz NE. In vivo whole body and organ arginine metabolism during endotoxemia (sepsis) is dependent on mouse strain and gender. J Nutr. 2004;134(10 Suppl):2768S-74S; discussion 96S-97S.

86. Marini JC, Erez A, Castillo L, Lee B. Interaction between murine spf-ash mutation and genetic background yields different metabolic phenotypes. Am J Physiol Endocrinol Metab. 2007;293(6):E1764-71.

87. Tang WH, Wang Z, Cho L, Brennan DM, Hazen SL. Diminished global arginine bioavailability and increased arginine catabolism as metabolic profile of increased cardiovascular risk. J Am Coll Cardiol. 2009;53(22):2061-7.

88. Gardiner KR, Gardiner RE, Barbul A. Reduced intestinal absorption of arginine during sepsis. Crit Care Med. 1995;23(7):1227-32.

89. Curis E, Nicolis I, Moinard C, Osowska S, Zerrouk N, Benazeth S, et al. Almost all about citrulline in mammals. Amino Acids. 2005;29(3):177-205.

90. Ceballos I, Chauveau P, Guerin V, Bardet J, Parvy P, Kamoun P, et al. 
Early alterations of plasma free amino acids in chronic renal failure. Clin Chim Acta. 1990;188(2):101-8.

91. Van Eijk HM, Dejong CH, Deutz NE, Soeters PB. Influence of storage conditions on normal plasma amino-acid concentrations. Clin Nutr. 1994;13(6):374-80.

92. Vente JP, von Meyenfeldt MF, van Eijk HM, van Berlo CL, Gouma DJ, van der Linden CJ, et al. Plasma-amino acid profiles in sepsis and stress. Ann Surg. 1989;209(1):57-62.

93. Freund $\mathrm{H}$, Atamian S, Holroyde J, Fischer JE. Plasma amino acids as predictors of the severity and outcome of sepsis. Ann Surg. 1979;190(5):571-6.

94. Milewski PJ, Threlfall CJ, Heath DF, Holbrook IB, Wilford K, Irving MH. Intracellular free amino acids in undernourished patients with or without sepsis. Clin Sci (Lond). 1982;62(1):83-91.

95. Luiking YC, Poeze M, Ramsay G, Deutz NE. Reduced citrulline production in sepsis is related to diminished de novo arginine and nitric oxide production. Am J Clin Nutr. 2009;89(1):142-52.

96. Piton G, Manzon C, Cypriani B, Carbonnel F, Capellier G. Acute intestinal failure in critically ill patients: is plasma citrulline the right marker? Intensive Care Med. 2011.

97. Kao C, Hsu J, Bandi V, Jahoor F. Impaired synthesis of citrulline from glutamine in sepsis. Am J Respir Crit Care Med. 2012;185(1):A1137.

98. Prins HA, Nijveldt RJ, Gasselt DV, van Kemenade F, Teerlink T, van Lambalgen $A A$, et al. The flux of arginine after ischemia-reperfusion in the rat kidney. Kidney Int. 2002;62(1):86-93.

99. Argaman Z, Young VR, Noviski N, Castillo-Rosas L, Lu XM, Zurakowski D, et al. Arginine and nitric oxide metabolism in critically ill septic pediatric patients. Crit Care Med. 2003;31(2):591-7.

100. Beach PK, Spain DA, Kawabe T, Harris PD, Garrison RN. Sepsis increases NOS-2 activity and decreases non-NOS-mediated acetylcholine-induced dilation in rat aorta. J Surg Res. 2001;96(1):17-22.

101. Hallemeesch MM, Janssen BJA, De Jonge WJ, Soeters PB, Lamers WH, Deutz NEP. NO production by cNOS and iNOS reflects blood pressure changes in LPS-challenged mice. Am J Physiol Endocrinol Metab. 2003;285:E871-5.

102. Scott JA, Mehta S, Duggan M, Bihari A, McCormack DG. Functional inhibition of constitutive nitric oxide synthase in a rat model of sepsis. Am J Respir Crit Care Med. 2002;165(10):1426-32.

103. Kao CC, Bandi V, Guntupalli KK, Wu M, Castillo L, Jahoor F. Arginine, citrulline and nitric oxide metabolism in sepsis. Clin Sci (Lond). 2009;117(1):23-30.

104. Poeze M, Bruins MJ, Luiking YC, Deutz NE. Reduced caloric intake during endotoxemia reduces arginine availability and metabolism. Am J Clin Nutr. 2010;91(4):992-1001.

105. Nijveldt RJ, Teerlink T, van Leeuwen PA. The asymmetrical dimethylarginine (ADMA)-multiple organ failure hypothesis. Clin Nutr. 2003;22(1):99-104.

106. Annane D, Sanquer S, Sebille V, Faye A, Djuranovic D, Raphael JC, et al. Compartmentalised inducible nitric-oxide synthase activity in septic shock. Lancet. 2000;355(9210):1143-8.

107. Prins HA, Houdijk AP, Wiezer MJ, Teerlink T, van Lambalgen AA, Thijs LG, et al. The effect of mild endotoxemia during low arginine plasma levels on organ blood flow in rats. Crit Care Med. 2000;28(6):1991-7.

108. Saetre T, Gundersen Y, Smiseth OA, Scholz T, Carlsen H, Nordsletten L, et al. Hepatic oxygen metabolism in porcine endotoxemia: the effect of nitric oxide synthase 
inhibition. Am J Physiol. 1998;275(6 Pt 1):G1377-85.

109. Parrillo JE. Pathogenetic mechanisms of septic shock. N Engl J Med. 1993;328(20):1471-7.

110. Ince C, Sinaasappel M. Microcirculatory oxygenation and shunting in sepsis and shock. Crit Care Med. 1999;27(7):1369-77.

111. Lehr HA, Bittinger F, Kirkpatrick CJ. Microcirculatory dysfunction in sepsis: a pathogenetic basis for therapy? J Pathol. 2000;190(3):373-86.

112. Preiser JC, De Backer D, Vincent JL. Nitroglycerin for septic shock. Lancet. 2003;361(9360):880; author reply

113. Fink MP. Cytopathic hypoxia. Mitochondrial dysfunction as mechanism contributing to organ dysfunction in sepsis. Crit Care Clin. 2001;17(1):219-37.

114. Liu H, Lee SS. Acute-on-chronic liver failure: the heart and systemic hemodynamics. Curr Opin Crit Care. 2011;17(2):190-4.

115. Jalan R, Olde Damink SW, Ter Steege JC, Redhead DN, Lee A, Hayes PC, et al. Acute endotoxemia following transjugular intrahepatic stent-shunt insertion is associated with systemic and cerebral vasodilatation with increased whole body nitric oxide production in critically ill cirrhotic patients. J Hepatol. 2011;54(2):265-71.

116. Sharma V, Ten Have G, Ytrebo L, Sen S, Rose C, Dalton N, et al. Nitric Oxide and L-Arginine metabolism in a devascularized porcine model of Acute Liver Failure. Am J Physiol. 2012:in press.

117. Chen K, Pittman RN, Popel AS. Nitric oxide in the vasculature: where does it come from and where does it go? A quantitative perspective. Antioxid Redox Signal. 2008;10(7):1185-98.

118. Gielis JF, Lin JY, Wingler K, Van Schil PE, Schmidt HH, Moens AL. Pathogenetic role of eNOS uncoupling in cardiopulmonary disorders. Free Radic Biol Med. 2011;50(7):765-76.

119. Wu G, Meininger CJ. Nitric oxide and vascular insulin resistance. Biofactors. 2009;35(1):21-7.

120. Blouet C, Mariotti F, Mathe V, Tome D, Huneau JF. Nitric oxide bioavailability and not production is first altered during the onset of insulin resistance in sucrose-fed rats. Exp Biol Med (Maywood). 2007;232(11):1458-64.

121. Chen CA, Wang TY, Varadharaj S, Reyes LA, Hemann C, Talukder MA, et al. S-glutathionylation uncouples eNOS and regulates its cellular and vascular function. Nature. 2011;468(7327):1115-8.

122. Sibal L, Agarwal SC, Home PD, Boger RH. The Role of Asymmetric Dimethylarginine (ADMA) in Endothelial Dysfunction and Cardiovascular Disease. Curr Cardiol Rev. 2010;6(2):82-90.

123. Boger RH, Endres HG, Schwedhelm E, Darius H, Atzler D, Luneburg N, et al. Asymmetric dimethylarginine as an independent risk marker for mortality in ambulatory patients with peripheral arterial disease. J Intern Med. 2011;269(3):349-61.

124. Aliev G, Palacios HH, Lipsitt AE, Fischbach K, Lamb BT, Obrenovich ME, et al. Nitric oxide as an initiator of brain lesions during the development of Alzheimer disease. Neurotox Res. 2009;16(3):293-305.

125. Albrecht J, Zielinska M, Norenberg MD. Glutamine as a mediator of ammonia neurotoxicity: A critical appraisal. Biochem Pharmacol. 2010;80(9):1303-8.

126. Zielinska M, Ruszkiewicz J, Hilgier W, Fresko I, Albrecht J. Hyperammonemia increases the expression and activity of the glutamine/arginine transporter $y+$ LAT2 in rat cerebral cortex: implications for the nitric oxide/cGMP pathway. Neurochem Int. 
2011;58(2):190-5.

127. Vissers YL, Dejong CH, Luiking YC, Fearon KC, von Meyenfeldt MF, Deutz NE. Plasma arginine concentrations are reduced in cancer patients: evidence for arginine deficiency? Am J Clin Nutr. 2005;81(5):1142-6.

128. Vissers YL, von Meyenfeldt MF, Luiking YC, Dejong CH, Deutz NE. Interorgan synthesis of arginine is down-regulated in tumor-bearing mice undergoing surgical trauma. Metabolism. 2008;57(7):896-902.

129. Zea AH, Rodriguez PC, Atkins MB, Hernandez C, Signoretti S, Zabaleta J, et al. Arginase-producing myeloid suppressor cells in renal cell carcinoma patients: a mechanism of tumor evasion. Cancer Res. 2005;65(8):3044-8.

130. Feun L, You M, Wu CJ, Kuo MT, Wangpaichitr M, Spector S, et al. Arginine deprivation as a targeted therapy for cancer. Curr Pharm Des. 2008;14(11):1049-57.

131. Lam TL, Wong GK, Chow HY, Chong HC, Chow TL, Kwok SY, et al. Recombinant human arginase inhibits the in vitro and in vivo proliferation of human melanoma by inducing cell cycle arrest and apoptosis. Pigment Cell Melanoma Res. 2011;24(2):366-76.

132. Hibbs JB, Jr., Westenfelder C, Taintor R, Vavrin Z, Kablitz C, Baranowski RL, et al. Evidence for cytokine-inducible nitric oxide synthesis from $\mathrm{L}$-arginine in patients receiving interleukin-2 therapy. J Clin Invest. 1992;89(3):867-77.

133. Yim CY, Bastian NR, Smith JC, Hibbs JB, Jr., Samlowski WE. Macrophage nitric oxide synthesis delays progression of ultraviolet light-induced murine skin cancers. Cancer Res. 1993;53(22):5507-11.

134. Boger RH. The pharmacodynamics of L-arginine. J Nutr. 2007;137(6 Suppl 2):1650S-5S.

135. Grimble GK. Adverse gastrointestinal effects of arginine and related amino acids. J Nutr. 2007:137(6 Suppl 2):1693S-701S.

136. Bower RH, Cerra FB, Bershadsky B, Licari JJ, Hoyt DB, Jensen GL, et al. Early enteral administration of a formula (Impact) supplemented with arginine, nucleotides, and fish oil in intensive care unit patients: results of a multicenter, prospective, randomized, clinical trial. Crit Care Med. 1995;23(3):436-49.

137. Atkinson S, Sieffert E, Bihari D. A prospective, randomized, double-blind, controlled clinical trial of enteral immunonutrition in the critically ill. Guy's Hospital Intensive Care Group. Crit Care Med. 1998;26(7):1164-72.

138. Galban C, Montejo JC, Mesejo A, Marco P, Celaya S, Sanchez-Segura JM, et al. An immune-enhancing enteral diet reduces mortality rate and episodes of bacteremia in septic intensive care unit patients. Crit Care Med. 2000;28(3):643-8.

139. Bertolini G, lapichino G, Radrizzani D, Facchini R, Simini B, Bruzzone P, et al. Early enteral immunonutrition in patients with severe sepsis: results of an interim analysis of a randomized multicentre clinical trial. Intensive Care Med. 2003;29(5):834-40.

140. McCowen KC, Bistrian BR. Immunonutrition: problematic or problem solving? Am J Clin Nutr. 2003;77(4):764-70.

141. Heyland DK, Novak F, Drover JW, Jain M, Su X, Suchner U. Should immunonutrition become routine in critically ill patients? A systematic review of the evidence. Jama. 2001;286(8):944-53.

142. Suchner U, Heyland DK, Peter K. Immune-modulatory actions of arginine in the critically ill. Br J Nutr. 2002;87 Suppl 1:S121-32.

143. Koretz RL. The impact of immunonutrition. Gastroenterology. 1995;109(5):1713-4.

144. Heyland DK, Samis A. Does immunonutrition in patients with sepsis do more harm than good? Intensive Care Med. 2003;29(5):669-71. 
145. Weimann A, Bastian L, Bischoff WE, Grotz M, Hansel M, Lotz J, et al. Influence of arginine, omega-3 fatty acids and nucleotide-supplemented enteral support on systemic inflammatory response syndrome and multiple organ failure in patients after severe trauma. Nutrition. 1998;14(2):165-72.

146. Georgieff M, Tugtekin IF. Positive role of immune nutrition on metabolism in sepsis and multi-organ failure. Kidney Int Suppl. 1998;64:S80-3.

147. Heyland DK, Dhaliwal R, Drover JW, Gramlich L, Dodek P. Canadian clinical practice guidelines for nutrition support in mechanically ventilated, critically ill adult patients. JPEN J Parenter Enteral Nutr. 2003;27(5):355-73.

148. Poeze M, Bruins MJ, Kessels F, Luiking YC, Lamers WH, Deutz NE. Effects of L-arginine pretreatment on nitric oxide metabolism and hepatosplanchnic perfusion during porcine endotoxemia. Am J Clin Nutr. 2011.

149. Lee CH, Hsiao CC, Hung CY, Chang YJ, Lo HC. Long-Term Enteral Arginine Supplementation in Rats with Intestinal Ischemia and Reperfusion. J Surg Res. 2011.

150. Puiman PJ, Stoll B, van Goudoever JB, Burrin DG. Enteral arginine does not increase superior mesenteric arterial blood flow but induces mucosal growth in neonatal pigs. J Nutr. 2011;141(1):63-70.

151. Reckelhoff JF, Kellum JA, Jr., Racusen LC, Hildebrandt DA. Long-term dietary supplementation with L-arginine prevents age-related reduction in renal function. Am J Physiol. 1997;272(6 Pt 2):R1768-74.

152. Morris CR, Morris SM, Jr., Hagar W, Van Warmerdam J, Claster S, Kepka-Lenhart D, et al. Arginine therapy: a new treatment for pulmonary hypertension in sickle cell disease? Am J Respir Crit Care Med. 2003;168(1):63-9.

153. Boger RH, Bode-Boger SM, Szuba A, Tsao PS, Chan JR, Tangphao O, et al. Asymmetric dimethylarginine (ADMA): a novel risk factor for endothelial dysfunction: its role in hypercholesterolemia. Circulation. 1998;98(18):1842-7.

154. Gurbuz AT, Kunzelman J, Ratzer EE. Supplemental dietary arginine accelerates intestinal mucosal regeneration and enhances bacterial clearance following radiation enteritis in rats. J Surg Res. 1998;74(2):149-54.

155. Barbul A, Lazarou SA, Efron DT, Wasserkrug HL, Efron G. Arginine enhances wound healing and lymphocyte immune responses in humans. Surgery. 1990;108(2):331-6; discussion 6-7.

156. Buijs N, van Bokhorst-de van der Schueren MA, Langius JA, Leemans CR, Kuik DJ, Vermeulen MA, et al. Perioperative arginine-supplemented nutrition in malnourished patients with head and neck cancer improves long-term survival. Am J Clin Nutr. 2010;92(5):1151-6.

157. Rajapakse NW, Mattson DL. Role of L-arginine in nitric oxide production in health and hypertension. Clin Exp Pharmacol Physiol. 2009;36(3):249-55.

158. Bai Y, Sun L, Yang T, Sun K, Chen J, Hui R. Increase in fasting vascular endothelial function after short-term oral L-arginine is effective when baseline flow-mediated dilation is low: a meta-analysis of randomized controlled trials. Am J Clin Nutr. 2009;89(1):77-84.

159. Dioguardi FS. To give or not to give? Lessons from the arginine paradox. J Nutrigenet Nutrigenomics. 2011;4(2):90-8.

160. Moinard C, Nicolis I, Neveux N, Darquy S, Benazeth S, Cynober L. Dose-ranging effects of citrulline administration on plasma amino acids and hormonal patterns in healthy subjects: the Citrudose pharmacokinetic study. Br J Nutr. 2008;99(4):855-62.

161. Bryk J, Ochoa JB, Correia MI, Munera-Seeley V, Popovic PJ. Effect of citrulline and glutamine on nitric oxide production in RAW 264.7 cells in an arginine-depleted environment. JPEN J Parenter Enteral Nutr. 2008;32(4):377-83. 
162. Waugh WH, Daeschner CW, 3rd, Files BA, McConnell ME, Strandjord SE. Oral citrulline as arginine precursor may be beneficial in sickle cell disease: early phase two results. J Natl Med Assoc. 2001;93(10):363-71.

163. Ananthakrishnan M, Barr FE, Summar ML, Smith HA, Kaplowitz M, Cunningham G, et al. L-Citrulline ameliorates chronic hypoxia-induced pulmonary hypertension in newborn piglets. Am J Physiol Lung Cell Mol Physiol. 2009;297(3):L506-11.

164. Ochiai M, Hayashi T, Morita M, Ina K, Maeda M, Watanabe F, et al. Short-term effects of I-citrulline supplementation on arterial stiffness in middle-aged men. Int J Cardiol. 2010;Epub Nov 8.

165. Osowska S, Moinard C, Neveux N, Loi C, Cynober L. Citrulline increases arginine pools and restores nitrogen balance after massive intestinal resection. Gut. 2004;53(12):1781-6.

166. Spronk PE, Ince C, Gardien MJ, Mathura KR, Oudemans-van Straaten HM, Zandstra DF. Nitroglycerin in septic shock after intravascular volume resuscitation. Lancet. 2002;360(9343):1395-6.

167. Barr FE, Macrae D. Inhaled nitric oxide and related therapies. Pediatr Crit Care Med. 2010;11(2 Suppl):S30-6.

168. Sindler AL, Fleenor BS, Calvert JW, Marshall KD, Zigler ML, Lefer DJ, et al. Nitrite supplementation reverses vascular endothelial dysfunction and large elastic artery stiffness with aging. Aging Cell. 2011;10(3):429-37.

169. Vanhatalo A, Bailey SJ, Blackwell JR, DiMenna FJ, Pavey TG, Wilkerson DP, et al. Acute and chronic effects of dietary nitrate supplementation on blood pressure and the physiological responses to moderate-intensity and incremental exercise. Am J Physiol Regul Integr Comp Physiol. 2010;299(4):R1121-31.

170. Domenico R. Pharmacology of nitric oxide: molecular mechanisms and therapeutic strategies. Curr Pharm Des. 2004;10(14):1667-76.

171. Forstermann $U$, Li H. Therapeutic effect of enhancing endothelial nitric oxide synthase (eNOS) expression and preventing eNOS uncoupling. Br J Pharmacol. 2011;164(2):213-23.

172. Moens AL, Kietadisorn R, Lin JY, Kass D. Targeting endothelial and myocardial dysfunction with tetrahydrobiopterin. J Mol Cell Cardiol. 2011:in press.

173. Antoniades C, Bakogiannis C, Leeson P, Guzik TJ, Zhang MH, Tousoulis D, et al. Rapid, direct effects of statin treatment on arterial redox state and nitric oxide bioavailability in human atherosclerosis via tetrahydrobiopterin-mediated endothelial nitric oxide synthase coupling. Circulation. 2011;124(3):335-45.

174. Crecelius AR, Kirby BS, Voyles WF, Dinenno FA. Nitric oxide, but not vasodilating prostaglandins, contributes to the improvement of exercise hyperemia via ascorbic acid in healthy older adults. Am J Physiol Heart Circ Physiol. 2010;299(5):H1633-41.

175. Cheang WS, Wong WT, Tian XY, Yang Q, Lee HK, He GW, et al. Endothelial nitric oxide synthase enhancer reduces oxidative stress and restores endothelial function in $\mathrm{db} / \mathrm{db}$ mice. Cardiovasc Res. 2011;92(2):267-75.

176. Leiper J, Nandi M. The therapeutic potential of targeting endogenous inhibitors of nitric oxide synthesis. Nat Rev Drug Discov. 2011;10(4):277-91.

177. Gronros J, Jung C, Lundberg JO, Cerrato R, Ostenson CG, Pernow J. Arginase inhibition restores in vivo coronary microvascular function in type 2 diabetic rats. Am J Physiol Heart Circ Physiol. 2011;300(4):H1174-81.

178. Jung C, Gonon AT, Sjoquist PO, Lundberg JO, Pernow J. Arginase inhibition mediates cardioprotection during ischaemia-reperfusion. Cardiovasc Res. 2010;85(1):147-54. 
179. Samlowski WE, Kondapaneni M, Tharkar S, McGregor JR, Laubach VE, Salvemini D. Endothelial nitric oxide synthase is a key mediator of interleukin-2-induced hypotension and vascular leak syndrome. J Immunother. 2011;34(5):419-27.

180. Cheriyan J, Webb AJ, Sarov-Blat L, Elkhawad M, Wallace SM, Maki-Petaja KM, et al. Inhibition of p38 mitogen-activated protein kinase improves nitric oxide-mediated vasodilatation and reduces inflammation in hypercholesterolemia. Circulation. 2011;123(5):515-23. 


\section{CHAPTER 9}




\title{
Nitric oxide and L-arginine metabolism in a devascularized porcine model of acute liver failure
}

\author{
Vikram Sharma, Gabriella A. M. Ten Have, Lars Ytrebo, \\ Sambit Sen, Christopher F. Rose, R. Neil Dalton, Charles \\ Turner, Arthur Revhaug, Hans M. H. van-Eijk, Nicolaas \\ E. P. Deutz, Rajiv Jalan, Rajeshwar P. Mookerjee, and \\ Nathan A. Davies
}

Am J Physiol Gastrointest Liver Physiol. 2012 Aug 1;303(3):G435-41.

\section{Abstract}

In acute liver failure (ALF), the hyperdynamic circulation is believed to be the result of overproduction of nitric oxide (NO) in the splanchnic circulation. However, it has been suggested that arginine concentrations (the substrate for $\mathrm{NO}$ ) are believed to be decreased, limiting substrate availability for NO production. To characterize the metabolic fate of arginine in early-phase ALF, we systematically assessed its interorgan transport and metabolism and measured the endogenous NO synthase inhibitor asymmetric dimethylarginine (ADMA) in a porcine model of ALF. Female adult pigs $(23-30 \mathrm{~kg})$ were randomized to sham $(\mathrm{N}=8)$ or hepatic devascularization $\operatorname{ALF}(\mathrm{N}=8)$ procedure for $6 \mathrm{~h}$. We measured plasma arginine, citrulline, ornithine levels; arginase activity, NO, and ADMA. Whole body metabolic rates and interorgan flux measurements were calculated using stable isotope-labeled amino acids. Plasma arginine decreased $>85 \%$ of the basal level at $t=6 \mathrm{~h}(\mathrm{p}<0.001)$, whereas citrulline and ornithine progressively increased in ALF $(p<0.001$ and $p<0.001$, vs. sham respectively). No difference was found between the groups in the whole body rate of appearance of arginine or NO. However, ALF showed a significant increase in de novo arginine synthesis $(p<0.05)$. Interorgan data showed citrulline net intestinal production and renal consumption that was related to net renal production of arginine and ornithine. Both plasma arginase activity and plasma ADMA levels significantly increased in ALF ( $p<0.001)$. In this model of early-phase ALF, arginine deficiency or higher ADMA levels do not limit whole body NO production. Arginine deficiency is caused by arginase-related arginine clearance in which arginine production is stimulated de novo. 


\section{Introduction}

Acute liver failure (ALF) is characterized by sudden and severe liver dysfunction with rapid progression to coagulopathy, encephalopathy, and multiorgan failure. Without liver transplantation, mortality from ALF is about $50 \%$ (1). A hallmark of ALF is the presentation of systemic hypotension and a hyperdynamic circulation (2). This is associated with increased guanylate cyclase (GC) activation by nitric oxide (NO) (3), which converts guanidine triphosphate to cyclic guanidine monophosphate and is thought to cause the vasodilatation seen in ALF (4). Previously, we have reported the characteristics of the same devascularized porcine liver model of ALF, which was shown to have a hyperdynamic circulation as evidenced by a high cardiac output and low mean arterial pressure and systemic vascular resistance (5). However, in this hyperacute model of ALF, no significant changes in the metabolites of NO were observed. Indeed, the kinetics of NO in this model and whether it is involved in the initiation of the vasodilation of ALF remain unknown.

L-Arginine has several important biological functions (6-8), one of which is to be the nitrogen-donating substrate for endothelial NO synthase (eNOS) to produce NO and citrulline in stoichiometric quantities (9). The plasma concentration of arginine is believed to be the rate-limiting factor in its synthesis (10). In ALF, plasma concentrations of L-arginine have been measured, but the results are contradictory with some studies suggesting that the levels remain the same (11) and others showing a reduction (12) or an increase $(13,14)$.

Other regulators that may influence the amount of $\mathrm{NO}$ generated in ALF are the concentrations of the endogenous NOS inhibitor asymmetric dimethyl arginine (ADMA) (13) and levels of plasma arginase activity (15). In a previous study, we have shown that ADMA levels were higher in patients with ALF and that the increase was associated with worse outcome (16). Similarly, an increase in the level of plasma arginase, released during hepatic stresses (17), can reduce the amount of available arginine, preventing eNOS-mediated NO synthesis. Both these observations can relate to a reduced NO production.

It is still unclear as to the exact mechanism of the vascular derangement in the initiation of vasodilation in ALF. Our group has previously described the classical hyperdynamic circulation and reduced systemic vascular resistance, along with hepatic encephalopathy, high intracranial pressure, and coagulopathy in a devascularized porcine model of ALF, which is ideal to study the initiation of metabolic disturbances in relation to hemodynamic changes (18). The aim of the study was to evaluate the evolution of disturbances in NO metabolism in relation to its regulators, L-arginine, ADMA, and arginase, in the first $6 \mathrm{~h}$ of ALF using stable isotope technology. 


\section{Materials and Methods}

\section{Study outline}

The Norwegian Experimental Animal Board approved the present study. Sixteen female Landrace pigs $(23-30 \mathrm{~kg})$ were randomly allocated into Sham-operated control or ALF groups. Study outline is shown in Figure 1. Blood and urine sampling was performed 30-45 min after creation of the portacaval shunt (PCS) or completion of Sham surgery ( $t=0 \mathrm{~h}$ ). ALF was induced by hepatic artery ligation ( $t=0 \mathrm{~h}$ ) immediately after completion of the sampling procedures. The experiments were terminated with an overdose of pentobarbital sodium and potassium chloride at $t=6 \mathrm{~h}$.

\section{Animal preparation and surgery}

The pigs were kept in the animal department for at least 2 days before the experiments. Details regarding the animal room facilities, anesthesia, and surgical preparation have been previously reported $(19,20)$. Briefly, the pigs underwent a tracheotomy, were intubated, and ventilated on a volume-controlled respirator (Servo 900; Elema-Schnander, Stockholm, Sweden). Ventilation was not altered after $t=0 \mathrm{~h}$. Core body temperature was maintained normothermic at $38.5 \pm 1^{\circ} \mathrm{C}$ with a heating pad and blankets. All animals received $500 \mathrm{ml}$ of $0.9 \% \mathrm{NaCl}$ containing $625 \mathrm{mg}$ of glucose as a preoperative load to prevent dehydration. During the experiment, $0.9 \% \mathrm{NaCl}$ was infused at a rate of $3 \mathrm{ml} / \mathrm{kg}$ per h. $0.9 \% \mathrm{NaCl}, 50 \%$ glucose, and $20 \%$ human albumin (Octapharm, Hurdal, Norway) was infused continuously at the rate of

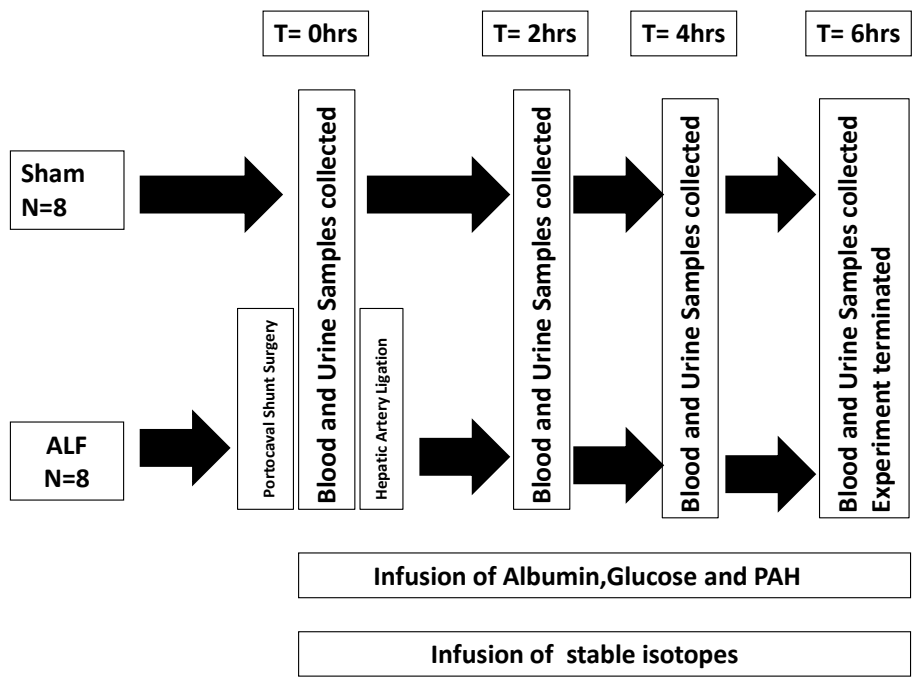

Figure 1. Study design for the porcine model of acute liver failure PAH, $p$-Amminohippuric acid; ALF, acute liver failure. 
$3 \mathrm{ml} \mathrm{kg} \mathrm{per} \mathrm{h,} 0.6048 \mathrm{ml} / \mathrm{kg}$ per h, and $0.66 \mathrm{ml} / \mathrm{kg}$ per h, respectively. Sham animals were given half the amount of glucose to make the glucose levels comparable between the groups. Anesthesia was stopped in the ALF group after the liver was devascularized although, if the degree of sedation became insufficient, small doses of fentanyl and midazolam were given as a bolus. Sham-operated animals received continuous anesthesia during the experimental period and received equal amounts of intravenous fluids. ALF was induced with an end-to-side PCS followed by ligation of the hepatic arteries. Details of the surgery, including the Sham-operation procedure, have been described elsewhere $(18,20)$.

\section{Positioning of catheters, flow probes, sampling, and analytical procedures}

Catheters, combining our previously described approach in mice (21) and pigs (22), were inserted in the abdominal aorta, renal vein, portal vein, and femoral vein for arterial and venous across-organ blood sampling. A 16-G central venous catheter (Secalon T; Ohmeda, Swindon, UK) was introduced into the left external jugular vein for administration of drugs and fluids. p-Amminohippuric acid (PAH; 25 mM; A1422; Sigma, St. Louis, MO) was infused at a rate of $30 \mathrm{ml} / \mathrm{h}$ through this catheter after an initial bolus of $6 \mathrm{ml}$ (22). Portal and femoral blood flows were measured by the use of perivascular ultrasonic transit time flow probes (CardioMed Systems; Medistim A/S, Oslo, Norway). A 5-Fr Edwards Swan-Ganz catheter (Baxter Healthcare, Irvine, CA) was floated into the pulmonary artery via the right external jugular vein. The urine bladder was drained via a cystotomy. Blood and urine samples were collected on ice at the times for measurement of blood flow and processed as described previously (23). Tissue samples were freeze clamped with Wollenberger tongs cooled in liquid nitrogen (21) and frozen at $-80^{\circ} \mathrm{C}$. Ammonia, urea, and $\mathrm{PAH}$ concentrations were determined spectrophotometrically (23). Amino acids concentrations were determined using HPLC (24).

\section{The use of stable amino acid isotopes}

A venous blood sample was drawn for the measurement of the natural abundance of plasma amino acids in each subject, before the start of the stable isotope primedcontinuous infusion protocol. Stable isotopes were administrated into the left external jugular vein, which included a priming dose followed by continuous infusion of a mixture of $L$-[guanido- ${ }^{15} \mathrm{~N}_{2}$ ] arginine $\left(\mathrm{N}_{2}\right.$-arginine prime: $1 \mathrm{mg} / \mathrm{kg}$ body wt per $\left.\mathrm{h}\right)$ and L-[ureido- ${ }^{13} \mathrm{C} ; 5,5-{ }^{2} \mathrm{H}_{2}$ ] citrulline (C1D2-citrulline, prime: $0.1 \mathrm{mg} / \mathrm{kg}$ body wt, infusion: $0.1 \mathrm{mg} / \mathrm{kg}$ body wt per h; Cambridge Isotope Laboratories, Woburn, MA) (25). Plasma enrichments of amino acid isotopes were determined by measuring the ratio of the amino acid stable isotope/ amino acid (tracer/tracee ratio) with a liquid chromatography mass spectrometry method (26). 
Plasma ADMA was measured as described previously (27) using fragmentationspecific stable isotope dilution electrospray mass spectrometry-mass spectrometry. Samples were deproteinized with acetonitrile containing ${ }^{2} \mathrm{H}_{6}$-ADMA, chromatographed (acetonitrile:water, 1:1; with $0.025 \%$ formic acid) on a Teicoplanin guard column 10-mm X $2.1 \mathrm{~mm}$ inner diameter (Chirobiotic T; ASTEC, Congleton, UK), and analyzed using a SCIEX API4000 (Applied Biosystems, Warrington, UK) in positiveion multiple-reaction-monitoring mode.

\section{Calculations}

Plasma flow rate $(\mathrm{ml} / \mathrm{kg}$ body wt per min) of the kidneys was calculated using the formulae based on the method of indicator dilution and Fick's principle $(23,28)$. The $\mathrm{PAH}$-determined blood flow and data from the perivascular blood flow probes were converted to plasma flow using the hematocrit. Substrate fluxes across organs were calculated as the venous-arterial concentration difference multiplied by the plasma flow. Positive values reflect substrate release, and negative fluxes reflect substrate uptake. Kidney and hind leg data are multiplied by two to reflect both organs. The flux of amino acid across the leg represented flux across a muscle compartment with defined arterial and venous sampling. Plasma arginase activity was assessed by colorimetric assay (17). Whole body rate of appearances of amino acids were calculated by the continuous stable amino acid isotope infusion rate divided by the arterial plasma enrichment of that isotope (29).

Whole body rate of appearances of NO was determined with the flux of plasma L-arginine to L-citrulline-stable isotopes as described previously (25). Whole body rate of appearance of de novo arginine was determined with the flux of plasma $\mathrm{L}$-citrulline to L-arginine-stable isotopes as described previously (30).

Whole body arginine clearance is defined as the amount of arginine that is cleared each minute from arginine and was calculated by whole body rate of appearance of arginine divided by the arterial arginine concentration (31).

\section{Statistics}

Data are expressed as means \pm SE. Significance of difference between groups was tested with Student's t-test or the Mann-Whitney comparisons test for nonparametric data as appropriate. Continuous data sets were compared using two-way ANOVA; $P<0.05$ was taken to be statistically significant. Software used included Microsoft Excel 2007 (Microsoft, Redmond, WA) and GraphPad Prism 4.0 (GraphPad Software, San Diego, CA). 


\section{Results}

\section{Whole body rate of appearance of NO in ALF}

The whole body rate of appearance of $\mathrm{NO}$ at the time of PCS or after ALF induction was not significantly different between the two groups. There was no significant change in the whole body rate of appearance of NO between the Sham-operated and ALF groups throughout the experimental period (Figure 2).

\section{L-arginine, citrulline, and glutamine metabolism}

Arterialarginine concentration was not significantly different from the basal level after the creation of the PCS and at induction of ALF but was reduced significantly ( $p$ $<0.001$ ) at $2 \mathrm{~h}$ and continued to decrease until end of the experimental period compared with the Sham group, in which no significant change was observed (Figure 3A). Citrulline concentration was not significantly different between Sham and ALF pigs after creation of PCS; however, the level significantly increased $(p<0.01)$ after the induction of $A L F$, and it continued to increase throughout the experimental period $(p<0.001)$ compared with the Sham group, in which no significant changes were observed (Figure 3B). Glutamine concentration was not different from the Sham-operated group after induction of PCS (Sham $547 \pm 45 \mu \mathrm{M}$, ALF $623 \pm 57$ $\mu \mathrm{M}$ at $t=0)$ but increased significantly after ALF induction $(\mathrm{p}<0.05)$ and continued to increase significantly till the end of the experiment $(p<0.001)$, as shown and discussed in a previous paper by our group (32).

\section{Interorgan metabolism of L-arginine and citrulline}

The effect of PCS and ALF on muscle metabolism is shown in Table 1, which shows the net flux across the muscle. Glutamine was released from the leg muscle throughout the experimental period, increasing non-significantly at $2 \mathrm{~h}$ after the induction of ALF, which was not observed in the Sham group (32). There is no significant uptake or release of citrulline or arginine from the muscle.

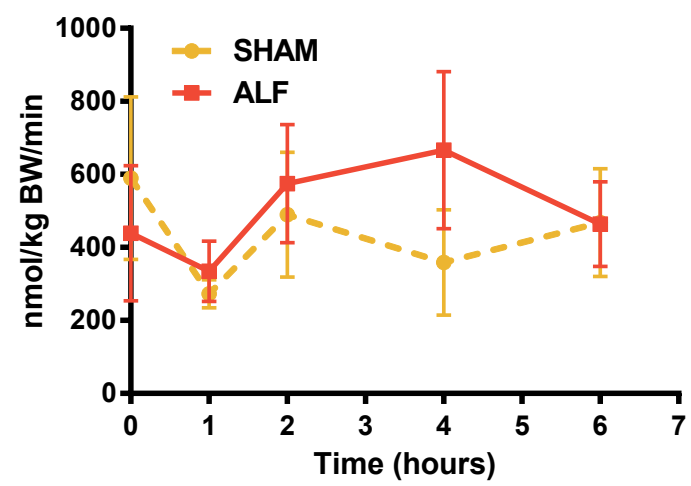

Figure 2. Whole body rate of appearance of nitric oxide (NO) in Sham-operated and ALF pigs Data shown are means \pm SE. 

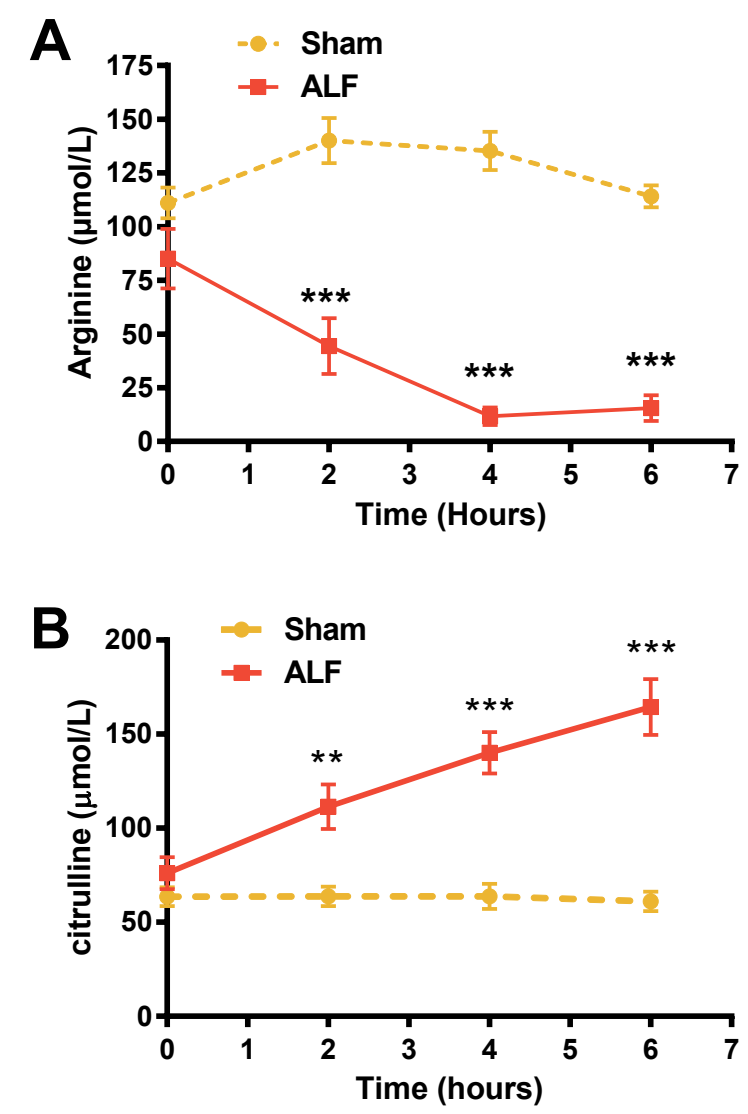

Figure 3. Plasma arginine (A), citrulline (B) concentration in Sham-operated and ALF pigs with time

$\left({ }^{* *} P<0.01,{ }^{* * *} P<0.001,2\right.$-way ANOVA). Data shown are means $\pm \mathrm{SE}$.

Table 1. Net flux of L-arginine and citrulline across muscle

\begin{tabular}{|c|c|c|c|}
\hline & Time & Sham & ALF \\
\hline \multirow{4}{*}{$\begin{array}{l}\text { Arginine } \\
(\mu \mathrm{mol} / \mathrm{kg} \mathrm{bw} / \mathrm{min})\end{array}$} & $\mathrm{T}=0 \mathrm{hrs}$ & $-0.0072 \pm 0.018$ & $-0.0048 \pm 0.017$ \\
\hline & $\mathrm{T}=2 \mathrm{hrs}$ & $0.0702 \pm 0.024$ & $0.0361 \pm 0.0358$ \\
\hline & $\mathrm{T}=4 \mathrm{hrs}$ & $0.0721 \pm 0.019$ & $-0.0082 \pm 0.030$ \\
\hline & $\mathrm{T}=6 \mathrm{hrs}$ & $0.0432 \pm 0.009$ & $0.0033 \pm 0.025$ \\
\hline \multirow{4}{*}{$\begin{array}{l}\text { Citrulline } \\
(\mu \mathrm{mol} / \mathrm{kg} \mathrm{bw} / \mathrm{min})\end{array}$} & $\mathrm{T}=0 \mathrm{hrs}$ & $-0.0038 \pm 0.013$ & $0.0015 \pm 0.011$ \\
\hline & $\mathrm{T}=2 \mathrm{hrs}$ & $0.0166 \pm 0.006$ & $0.0037 \pm 0.017$ \\
\hline & $\mathrm{T}=4 \mathrm{hrs}$ & $-0.0118 \pm 0.008$ & $0.0163 \pm 0.034$ \\
\hline & $\mathrm{T}=6 \mathrm{hrs}$ & $-0.0106 \pm 0.010$ & $-0.0444 \pm 0.050$ \\
\hline
\end{tabular}


The effect of PCS and ALF on portal-derived viscera (PDV) metabolism was determined. Glutamine was taken up by the PDV throughout the experimental period in both the PCS and ALF groups (32). Citrulline was released from the PDV at the point of creation of the PCS, and the release significantly increased in the ALF animals compared with the Sham animals $(p<0.05)$. There were no significant differences in net fluxes of arginine in the PDV (Table 2).

Table 2. Net flux of L-arginine and citrulline across the PDV

\begin{tabular}{llll}
\hline & Time & Sham & ALF \\
\hline & $\mathrm{T}=0 \mathrm{hrs}$ & $-0.0256 \pm 0.135$ & $0.1387 \pm 0.061$ \\
Arginine & $\mathrm{T}=2 \mathrm{hrs}$ & $0.1665 \pm 0.131$ & $0.3117 \pm 0.056$ \\
$(\mu \mathrm{mol} / \mathrm{kg} \mathrm{bw} / \mathrm{min})$ & $\mathrm{T}=4 \mathrm{hrs}$ & $0.1440 \pm 0.127$ & $0.0941 \pm 0.079$ \\
& $\mathrm{~T}=6 \mathrm{hrs}$ & $-0.0444 \pm 0.084$ & $0.0491 \pm 0.118$ \\
& & & \\
Citrulline & $\mathrm{T}=0 \mathrm{hrs}$ & $0.1647 \pm 0.107$ & $0.3768 \pm 0.092$ \\
$(\mu \mathrm{mol} / \mathrm{kg} \mathrm{bw} / \mathrm{min})$ & $\mathrm{T}=2 \mathrm{hrs}$ & $0.4195 \pm 0.092$ & $0.6975 \pm 0.069$ \\
& $\mathrm{~T}=4 \mathrm{hrs}$ & $0.3421 \pm 0.068$ & $0.5882 \pm 0.094$ \\
& $\mathrm{~T}=6 \mathrm{hrs}$ & $0.2451 \pm 0.101$ & $0.8322 \pm 0.572^{*}$ \\
\hline
\end{tabular}

${ }^{*} p<0.05$ Sham vs. ALF, 2-way ANOVA. PDV, portal-derived viscera.

The effect of PCS and ALF on renal metabolism was also determined. Citrulline was taken up by the kidney after the creation of the PCS, and the uptake increased significantly compared with the Sham-operated group ( $p=0.05)$. Argininewas released from the kidney after the induction of ALF, and the release remained significantly higher compared with theSham animals ( $p<0.0001)$ for the remainder of the study period. There was no significant change in the metabolism of glutamine (32) (Table 3).

Table 3. Net flux of L-arginine and citrulline across the kidneys

\begin{tabular}{|c|c|c|c|}
\hline & Time & Sham & ALF \\
\hline \multirow{4}{*}{$\begin{array}{l}\text { Arginine } \\
(\mu \mathrm{mol} / \mathrm{kg} \text { bw/min) }\end{array}$} & $\mathrm{T}=0 \mathrm{hrs}$ & $0.5448 \pm 0.083$ & $0.6855 \pm 0.112$ \\
\hline & $\mathrm{T}=2 \mathrm{hrs}$ & $0.2924 \pm 0.123$ & $0.5887 \pm 0.101$ \\
\hline & $\mathrm{T}=4 \mathrm{hrs}$ & $0.3604 \pm 0.135$ & $0.5279 \pm 0.134$ \\
\hline & $\mathrm{T}=6 \mathrm{hrs}$ & $0.2439 \pm 0.112$ & $0.4351 \pm 0.255^{\dagger}$ \\
\hline \multirow{4}{*}{$\begin{array}{l}\text { Citrulline } \\
(\mu \mathrm{mol} / \mathrm{kg} \mathrm{bw} / \mathrm{min})\end{array}$} & $\mathrm{T}=0 \mathrm{hrs}$ & $-1.246 \pm 0.159$ & $-1.1107 \pm 0.175$ \\
\hline & $\mathrm{T}=2 \mathrm{hrs}$ & $-0.8352 \pm 0.150$ & $-1.3775 \pm 0.358$ \\
\hline & $\mathrm{T}=4 \mathrm{hrs}$ & $-1.0228 \pm 0.160$ & $-1.4413 \pm 0.305$ \\
\hline & $\mathrm{T}=6 \mathrm{hrs}$ & $-0.8241 \pm 0.072$ & $-1.4620 \pm 0.427^{*}$ \\
\hline
\end{tabular}

*) $\left.p<0.05 ;^{\dagger}\right) p<0.001$, Sham vs. ALF, 2-way ANOVA. 

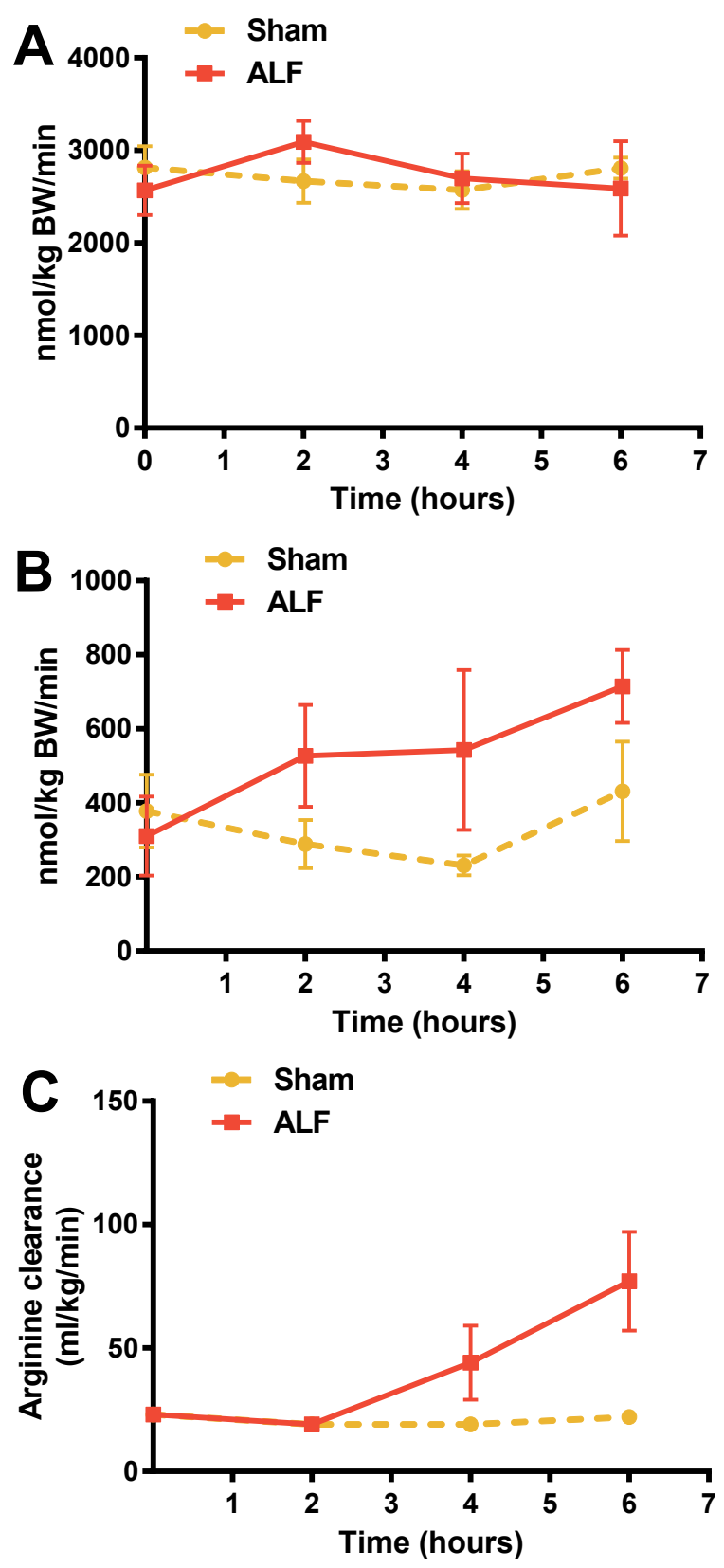

Figure 4. Whole body rate of appearance of arginine (WbRa, A), de novo arginine (B), and arginine clearance $(C)$ of Sham-operated and ALF pigs with time

*) $\left.p<0.05 ;{ }^{* * *}\right) p<0.001 ; 2$-way ANOVA. Data shown are means \pm SE. 


\section{Whole body rate of appearance of arginine and de novo arginine in ALF}

The whole body rate of appearance of arginine showed no difference between the Sham and ALF groups at the point of creation of the PCS. There was also no significant difference observed between the Sham and ALF group in the whole body appearance of arginine throughout the experimental period (Figure 4A). The whole body rate of appearance of de novo arginine was non-significantly different between the two groups at the point of creation of the PCS. The rate of appearance of arginine increased non-significantly in the ALF group compared with the Sham-operated group through the period of experimentation although it showed a significant increase in production in the ALF group at the end of the experiment compared with the Sham group ( $p<0.05$, Figure $4 B$ ). There was a significant difference in the clearance of arginine between the Sham and the ALF pigs, wherein the
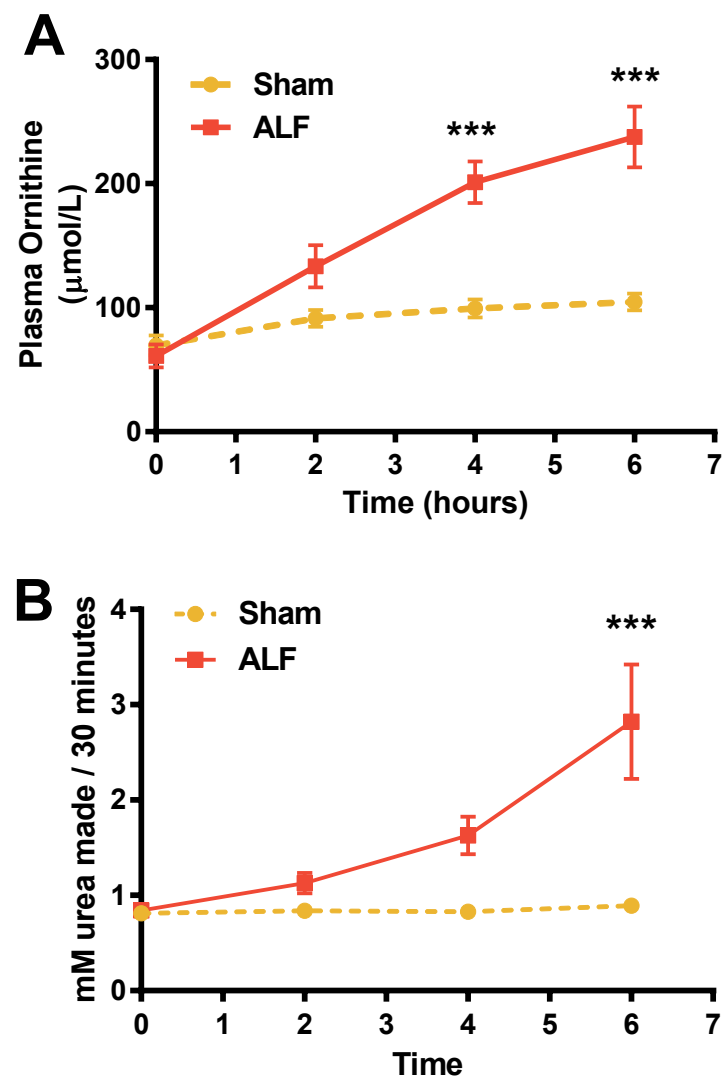

Figure 5. Plasma ornithine (A) and plasma arginase activity (B) in Sham-operated and ALF pigs throughout the experimental period

There is a significant increase in the plasma ornithine levels with a corresponding significant increase in the plasma arginase activity at the end of the experimental period. ${ }^{* * *}$ ) $p<0.001$; 2-way ANOVA. Data shown are means $\pm \mathrm{SE}$. 
ALF pigs showed a significantly increased arginine clearance at the end of the experiment compared with the Sham and the levels of clearance remained constant throughout the experimental period ( $p<0.001$, Figure 4 ).

\section{Plasma arginase activity}

Ornithine concentration was not different between Sham and ALF groups at the creation of PCS, but the arterial concentration of ornithine increased after induction of ALF and continued to increase throughout the duration of the experiment to a significant level ( $p<0.001$ at 6 h) compared with the Sham-operated animals (Figure $5 \mathrm{~A}$ ) in which no significant changes were observed. The plasma arginase activity was equal in the two groups at the point of PCS and the induction of ALF. The plasma arginase activity increased through the course of the experiment and, at the end of the experiment, was significantly increased ( $<<0.001$, Figure 5B) in the ALF group compared with the Sham-operated group.

\section{Plasma arterial ADMA}

The arterial ADMA concentration was not significantly different between the two groups after the creation of the PCS. The ADMA levels remained constant in the Sham group through the course of the experiment. However, after the induction of ALF, the arterial concentrations of ADMA were increased at $2 \mathrm{~h}$ and continued to be significantly elevated throughout the remainder of the study period ( $p<0.001$, Figure 6). The normal values of L-arginine to ADMA ratio is about 100:1 in healthy controls. However, in the setting of ALF, the L-arginine to ADMA ratio was markedly reduced ( $p<0.001$, Figure 7 ).

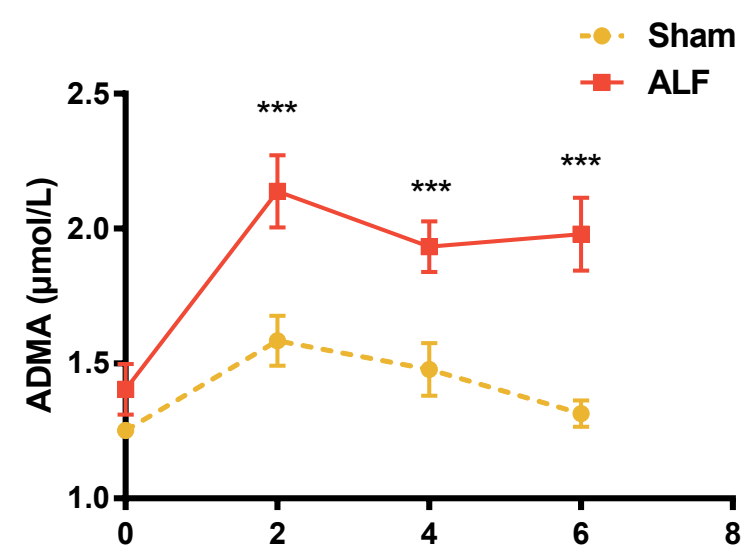

Figure 6. Plasma asymmetric dimethyl arginine (ADMA) concentration in Sham-operated and ALF pigs with time

There is a significant increase in the arterial ADMA concentration in the ALF pigs compared with the Sham-operated pigs at $2 \mathrm{~h}$ and continues to increase significantly until the end of experiment. $\left.{ }^{* * *}\right) \mathrm{p}<0.001 ; 2$-way ANOVA. Data shown are means $\pm \mathrm{SE}$ 


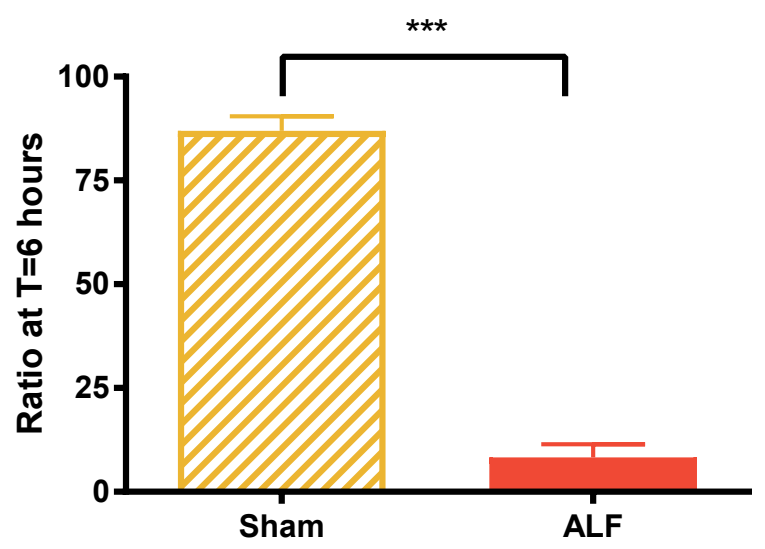

Figure 7. Ratio of arginine to ADMA in Sham-operated and the ALF pig groups at $6 \mathrm{~h}$ postinduction of ALF

There is a significant decrease in the arginine to ADMA ratio in the ALF pig compared with the Sham-operated pigs at the end of 6 h. $\left.{ }^{* * *}\right) p<0.001$, Mann-Whitney test. The data shown are means $\pm \mathrm{SE}$.

\section{Discussion}

The most important finding of this study was the observation that the rate of NO production was not significantly different between animals with ALF and Sham controls, indicating that $\mathrm{NO}$ is unlikely to be involved in initiating the vasodilation and hypotension observed in early ALF. These results are unexpected and may in part be explained by substantially reduced plasma arginine levels through the action of arginase. However, we also show that there is an increase in de novo arginine synthesis, resulting in only a relative arginine deficiency, although there appears to be insufficient capacity in this mechanism to restore normal levels. In relation to ADMA, the significant elevation seen in ALF plasma is likely to reflect the impairment of hepatic ADMA metabolism and/or its increased generation. Given the devascularized liver in this model, the significance of higher arterial ADMA values compared with sham are likely to reflect the spillover of an increased capacity to compete with arginine at tissue level, in turn limiting the generation of $\mathrm{NO}$. It is also possible that the site of $\mathrm{NO}$ production within the endothelial cells is unaffected by the reduction in plasma arginine. Furthermore, our findings of no significant change in rate of NO production are still compatible with other studies in ALF that have observed changes in activity of guanylate cyclase $(3,4)$. We and others have shown that the activation of guanylate cyclase can be independent of $\mathrm{NO}(33,34)$ and that guanylate cyclase activation itself may be altered in the context of liver injury.

This experimental model of hyperacute ALF has been well characterized (18). The ability to infuse relevant amino acids with stable isotope labels to measure the 
conversion of arginine to citrulline as a surrogate for $\mathrm{NO}$ production was employed in this model to evaluate the role of $\mathrm{NO}$ in initiating the hemodynamic disturbances, which is not possible to do in a clinical setting, as patients will invariably present late in the course of the condition. These findings lead us to speculate that there may be some other mechanism involved in the pathophysiology of the vascular abnormality seen in the onset of ALF as seen in patients (2) and also this model (5). This result is in contrast to other published articles where increases in the metabolites of NO were described (4). The human studies were performed when ALF was already manifest, and the condition is invariably associated with systemic inflammation. The present study focused on evaluating the role of NO in initiating hemodynamic disturbances and was founded on prior evaluation of this model where no clear evidence could be shown for systemic inflammation. Thus the changes observed can be considered to occur more on a background of metabolic dysfunction, as demonstrated by hyperammonemia, hyperlactatemia, and derangements in glycolysis previously described in this model (35).

The sole nitrogen-donating substrate for the synthesis of $\mathrm{NO}$ is L-arginine. Arginine is oxidized to NO and citrulline by the enzyme NOS. In the setting of ALF, plasma arginine levels have been found to be lower than those in healthy animals. This finding was described in the early description of this devascularized porcine model of acute liver failure, wherein they also found that the concentrations of citrulline and ornithine were increased (36). In septic patients, low arginine levels $(37,38)$ have also been observed, which is associated with poor outcome. Arginine clearance was found to progressively increase over the duration of the study (Figure $4 \mathrm{C}$ ). Although the whole body rate of appearance of arginine was unchanged, the reduction in the plasma levels was significant. This increased clearance rate most likely provided stimulation for the observed de novo synthesis of arginine via the intestinal-renal axis; however, the amount generated during the experimental period was insufficient to restore normal plasma levels. The mechanism of the increased de novo synthesis of L-arginine is revealed from the interorgan experiments. The increased glutamine that was released from the muscle was taken up by the PDV (gut), where it was converted to citrulline. This citrulline was converted stoichiometrically to arginine in the kidney. It is important to acknowledge that there are limitations to this hyperacute model of ALF in that the experimental duration is over $6 \mathrm{~h}$ and occurs in a devascularized liver. The findings, while questioning the exclusive role of $\mathrm{NO}$ in modulating the hyperdynamic circulation in ALF, do need further testing in other ALF model systems.

A second important observation was the finding of increased plasma levels of ornithine. The increase in ornithine and the simultaneous reduction in arginine indicate that there is an increase in arginase activity, which was found to be markedly increased in the ALF animals. Arginase I is a cytosolic enzyme that forms a part 
of the urea cycle and is predominantly present in the liver (39); arginase II is a mitochondrial protein found most abundantly in the kidney (40). Other studies have also found arginase to be present in the plasma following liver injury $(41,42)$. In this model of hepatic arterial devascularization, the hepatic vein, and hence venous drainage from the liver, remains intact. It is likely that the arginase that was found in the plasma is derived from the breakdown of and leakage from hepatocytes and enters the systemic circulation due to a small amount of residual collateral venous drainage from the liver post-PCS surgery and hepatic artery ligation. Although the major blood supply routes to the liver are removed, there still remains a small but significant contribution from collateral vessels above the section point of the portal vein (43). Although we propose that the majority of the measured arginase activity derives from the liver, the kidney and other vasculature may undertake a substantial role in the production and release of arginase. The mechanism of reduction in arginine may be related to this increase in arginase activity, which is known to regulate the bioavailability of arginine. This inference can be drawn from genetic studies wherein it has been shown that arginase I deficiency leads to hyperargininemia in both humans and mice $(44,45)$. Similarly, a deficiency of arginase II also leads to a twofold increase in the level of arginine (46)]. Apart from these mechanisms, the arginase-mediated depletion of arginine may also inhibit the expression of inducible NOS by repressing the translation and stability of the inducible NOS protein $(47,48)$.

Furthermore, the significantly increased ADMA levels also provide a possible mechanism for an impaired production of NO in this model of ALF. ADMA, an endogenous inhibitor of NOS, is synthesized by the action of protein arginine methyl transferase on arginine residues of nuclear proteins. ADMA is released following proteolysis, and most of the freely circulating ADMA is metabolized by dimethylarginine dimethylaminohydrolase, which occurs mostly in the liver (49) but can also occur in other organs such as the kidney (50). Data from this model study have previously been described and shown to have renal dysfunction and changes in vascular dynamics as part of the developing multiple organ dysfunction syndrome (5). Renal dysfunction, particularly in the context of reno-vascular hypertension, has been described as being associated with higher than normal range values of ADMA (51). Plasma ADMA levels have been shown to be markedly increased in ALF, and its levels correlate with severity of liver failure and the presence of added inflammation and are significantly higher than the levels described in renal disease, which would support the assertion that the ADMA levels in these models are primarily impacted on by hepatic impairment $(16,52)$. As ADMA is a competitive inhibitor of the NOS-arginine binding site, lowering the arginine to ADMA ratio may directly result in a reduction in NOS activity. In our study, an increase in plasma ADMA level and a reduction of the arginine to ADMA ratio were observed. This suggests that the lack of increase in NO production that was observed in the ALF group may be attributable to the reduction in eNOS activity in the presence of an elevated ADMA and Iow arginine 
plasma levels. This is further compounded by the fact that high arginase also plays its part in reducing the eNOS activity and arginine levels.

In conclusion, this study shows for the first time that, during the initiation of ALF, NO may not play a significant role in mediating the hemodynamic disturbances. This lack of augmentation in the production of $\mathrm{NO}$ may be due to a relative reduction in L-arginine concentration despite increased de novo production, through the action of arginase and a reduction in its effect on NOS by the relative increase in the levels of its competitive antagonist, ADMA.

\section{References}

1. Bernal W, Auzinger G, Dhawan A, Wendon J. Acute liver failure. Lancet. 2010;376(9736):190-201.

2. Trewby PN, Williams R. Pathophysiology of hypotension in patients with fulminant hepatic failure. Gut. 1977;18(12):1021-6.

3. Russwurm M, Koesling D. NO activation of guanylyl cyclase. EMBO J. 2004;23(22):4443-50.

4. Schneider F, Lutun P, Boudjema K, Wolf P, Tempe JD. In vivo evidence of enhanced guanylyl cyclase activation during the hyperdynamic circulation of acute liver failure. Hepatology. 1994;19(1):38-44.

5. Ytrebo LM, Sen S, Rose C, Davies NA, Nedredal GI, Fuskevaag OM, et al. Systemic and regional haemodynamics in pigs with acute liver failure and the effect of albumin dialysis. Scand J Gastroenterol. 2006;41(11):1350-60.

6. Barbul A. Arginine and immune function. Nutrition. 1990;6(1):53-8; discussion 9-62.

7. Barbul A, Lazarou SA, Efron DT, Wasserkrug HL, Efron G. Arginine enhances wound healing and lymphocyte immune responses in humans. Surgery. 1990;108(2):331-6; discussion 6-7.

8. Brusilow SW, Finkelstien J. Restoration of nitrogen homeostasis in a man with ornithine transcarbamylase deficiency. Metabolism. 1993;42(10):1336-9.

9. Palmer RM, Rees DD, Ashton DS, Moncada S. L-arginine is the physiological precursor for the formation of nitric oxide in endothelium-dependent relaxation. Biochem Biophys Res Commun. 1988;153(3):1251-6.

10. Alderton WK, Cooper CE, Knowles RG. Nitric oxide synthases: structure, function and inhibition. Biochem J. 2001;357(Pt 3):593-615.

11. Tietge UJ, Bahr MJ, Manns MP, Boker KH. Plasma amino acids in cirrhosis and after liver transplantation: influence of liver function, hepatic hemodynamics and circulating hormones. Clin Transplant. 2002;16(1):9-17.

12. Yagnik GP, Takahashi Y, Tsoulfas G, Reid K, Murase N, Geller DA. Blockade of the L-arginine/NO synthase pathway worsens hepatic apoptosis and liver transplant preservation injury. Hepatology. 2002;36(3):573-81.

13. Cardounel AJ, Cui H, Samouilov A, Johnson W, Kearns P, Tsai AL, et al. Evidence for the pathophysiological role of endogenous methylarginines in regulation of endothelial $\mathrm{NO}$ production and vascular function. J Biol Chem. 2007;282(2):879-87. 
14. Clemmesen JO, Kondrup J, Ott P. Splanchnic and leg exchange of amino acids and ammonia in acute liver failure. Gastroenterology. 2000;118(6):1131-9.

15. Durante W, Johnson FK, Johnson RA. Arginase: a critical regulator of nitric oxide synthesis and vascular function. Clin Exp Pharmacol Physiol. 2007;34(9):906-11.

16. Mookerjee RP, Malaki M, Davies NA, Hodges SJ, Dalton RN, Turner C, et al. Increasing dimethylarginine levels are associated with adverse clinical outcome in severe alcoholic hepatitis. Hepatology. 2007;45(1):62-71.

17. Das A, Hoare M, Davies N, Lopes AR, Dunn C, Kennedy PT, et al. Functional skewing of the global CD8 T cell population in chronic hepatitis B virus infection. J Exp Med. 2008;205(9):2111-24.

18. Ytrebo LM, Nedredal GI, Langbakk B, Revhaug A. An experimental large animal model for the assessment of bioartificial liver support systems in fulminant hepatic failure. Scand J Gastroenterol. 2002;37(9):1077-88.

19. Ytrebo LM, Ingebrigtsen T, Nedredal GI, Elvenes OP, Korvald C, Romner B, et al. Protein S-100beta: a biochemical marker for increased intracranial pressure in pigs with acute hepatic failure. Scand J Gastroenterol. 2000;35(5):546-51.

20. Ytrebo LM, Korvald C, Nedredal GI, Elvenes OP, Nielsen Grymyr OJ, Revhaug A. $\mathrm{N}$-acetylcysteine increases cerebral perfusion pressure in pigs with fulminant hepatic failure. Crit Care Med. 2001;29(10):1989-95.

21. Hallemeesch MM, Ten Have GA, Deutz NE. Metabolic flux measurements across portal drained viscera, liver, kidney and hindquarter in mice. Lab Anim. 2001;35(1):101-10.

22. Ten Have GA, Bost MC, Suyk-Wierts JC, van den Bogaard AE, Deutz NE. Simultaneous measurement of metabolic flux in portally-drained viscera, liver, spleen, kidney and hindquarter in the conscious pig. Lab Anim. 1996;30(4):347-58.

204 23. Dejong CH, Kampman MT, Deutz NE, Soeters PB. Altered glutamine metabolism in rat portal drained viscera and hindquarter during hyperammonemia. Gastroenterology. 1992;102(3):936-48.

24. Jalan R. Acute liver failure: current management and future prospects. J Hepatol. 2005;42 Suppl(1):S115-23.

25. van Eijk HM, Luiking YC, Deutz NE. Methods using stable isotopes to measure nitric oxide (NO) synthesis in the I-arginine/NO pathway in health and disease. J Chromatogr B Analyt Technol Biomed Life Sci. 2006.

26. van Eijk HM, Suylen DP, Dejong CH, Luiking YC, Deutz NE. Measurement of amino acid isotope enrichment by liquid chromatography mass spectroscopy after derivatization with 9-fluorenylmethylchloroformate. J Chromatogr B Analyt Technol Biomed Life Sci. 2007;856(1-2):48-56.

27. Mookerjee RP, Dalton RN, Davies NA, Hodges SJ, Turner C, Williams R, et al. Inflammation is an important determinant of levels of the endogenous nitric oxide synthase inhibitor asymmetric dimethylarginine (ADMA) in acute liver failure. Liver Transpl. 2007;13(3):400-5.

28. Owen EE, Tyor MP, Flanagan JF, Berry JN. The kidney as a source of blood ammonia in patients with liver disease: the effect of acetazolamide. J Clin Invest. 1960;39:288-94.

29. Wolfe RR, Chinkes DL. Isotope Tracers in Metabolic Research: Principles and Practise of Kinetic Analysis. New York: Wiley; 2005. 274 p.

30. Poeze M, Bruins MJ, Kessels F, Luiking YC, Lamers WH, Deutz NE. Effects of L-arginine pretreatment on nitric oxide metabolism and hepatosplanchnic perfusion during porcine endotoxemia. The American journal of clinical nutrition. 2011;93(6):1237-47. 
and organ arginine metabolism during endotoxemia (sepsis) is dependent on mouse strain and gender. J Nutr. 2004;134(10 Suppl):2768S-74S; discussion 96S-97S.

32. Ytrebo LM, Sen S, Rose C, Ten Have GA, Davies NA, Hodges S, et al. Interorgan ammonia, glutamate, and glutamine trafficking in pigs with acute liver failure. Am J Physiol Gastrointest Liver Physiol. 2006;291(3):G373-81.

33. Davies NA, Hodges SJ, Pitsillides AA, Mookerjee RP, Jalan R, Mehdizadeh S. Hepatic guanylate cyclase activity is decreased in a model of cirrhosis: a quantitative cytochemistry study. FEBS Lett. 2006;580(8):2123-8.

34. Knorr A, Hirth-Dietrich C, Alonso-Alija C, Harter M, Hahn M, Keim Y, et al. Nitric oxide-independent activation of soluble guanylate cyclase by BAY 60-2770 in experimental liver fibrosis. Arzneimittelforschung. 2008;58(2):71-80.

35. Sen S, Rose C, Ytrebo LM, Davies NA, Nedredal GI, Drevland SS, et al. Effect of albumin dialysis on intracranial pressure increase in pigs with acute liver failure: a randomized study. Crit Care Med. 2006;34(1):158-64.

36. Mazziotti A, Bernardi M, Antonini L, Dioguardi FS, Bellusci R, Papa V, et al. Plasma amino acid patterns in experimental acute hepatic failure: comparison between hepatectomy and liver devascularization in pigs. Surgery. 1981;90(3):527-34.

37. Freund H, Atamian S, Holroyde J, Fischer JE. Plasma amino acids as predictors of the severity and outcome of sepsis. Ann Surg. 1979;190(5):571-6.

38. Milewski PJ, Threlfall CJ, Heath DF, Holbrook IB, Wilford K, Irving MH. Intracellular free amino acids in undernourished patients with or without sepsis. Clin Sci (Lond). 1982;62(1):83-91.

39. Vockley JG, Jenkinson CP, Shukla H, Kern RM, Grody WW, Cederbaum SD. Cloning and characterization of the human type II arginase gene. Genomics. 1996;38(2):118-23.

40. Morris SM, Jr., Bhamidipati D, Kepka-Lenhart D. Human type II arginase: sequence analysis and tissue-specific expression. Gene. 1997;193(2):157-61.

41. Durante W, Liao L, Reyna SV, Peyton KJ, Schafer Al. Physiological cyclic stretch directs $L$-arginine transport and metabolism to collagen synthesis in vascular smooth muscle. FASEB J. 2000;14(12):1775-83.

42. Durante W, Liao L, Reyna SV, Peyton KJ, Schafer Al. Transforming growth factorbeta(1) stimulates L-arginine transport and metabolism in vascular smooth muscle cells: role in polyamine and collagen synthesis. Circulation. 2001;103(8):1121-7.

43. Iber FL, Kerr DN, Dolle W, Sherlock S. Measurement of Blood Flow in the Collateral Vessels of the Portal Vein; Preliminary Results of a New Method. J Clin Invest. 1960;39(8):1201-7.

44. Iyer RK, Yoo PK, Kern RM, Rozengurt N, Tsoa R, O’Brien WE, et al. Mouse model for human arginase deficiency. Mol Cell Biol. 2002;22(13):4491-8.

45. Zhang C, Hein TW, Wang W, Chang Cl, Kuo L. Constitutive expression of arginase in microvascular endothelial cells counteracts nitric oxide-mediated vasodilatory function. FASEB J. 2001;15(7):1264-6.

46. Shi O, Morris SM, Jr., Zoghbi H, Porter CW, O'Brien WE. Generation of a mouse model for arginase II deficiency by targeted disruption of the arginase II gene. Mol Cell Biol. 2001;21(3):811-3.

47. El-Gayar S, Thuring-Nahler H, Pfeilschifter J, Rollinghoff M, Bogdan C. Translational control of inducible nitric oxide synthase by IL-13 and arginine availability in inflammatory macrophages. J Immunol. 2003;171(9):4561-8.

48. Li H, Meininger CJ, Hawker JR, Jr., Haynes TE, Kepka-Lenhart D, Mistry SK, et al. Regulatory role of arginase I and II in nitric oxide, polyamine, and proline syntheses in endothelial cells. Am J Physiol Endocrinol Metab. 2001;280(1):E75-82. 
PA. The liver is an important organ in the metabolism of asymmetrical dimethylarginine (ADMA). Clin Nutr. 2003;22(1):17-22.

50. Yilmaz MI, Saglam M, Caglar K, Cakir E, Ozgurtas T, Sonmez A, et al. Endothelial functions improve with decrease in asymmetric dimethylarginine (ADMA) levels after renal transplantation. Transplantation. 2005;80(12):1660-6.

51. Ueda S, Yamagishi S, Okuda S. New pathways to renal damage: role of ADMA in retarding renal disease progression. J Nephrol. 2010;23(4):377-86.

52. Fliser D. Asymmetric dimethylarginine (ADMA): the silent transition from an 'uraemic toxin' to a global cardiovascular risk molecule. European journal of clinical investigation. 2005;35(2):71-9. 



\section{Inhibition of jejunal protein synthesis and breakdown in Pseudomonas aeruginosa induced severe sepsis pig model}

Gabriella A.M. ten Have, Mariëlle P.K.J. Engelen, Robert

R. Wolfe, Nicolaas E.P. Deutz

\section{Abstract}

Maintenance of gut integrity has long been recognized as crucial for survival in sepsis but alterations in protein metabolism have not previously been documented. Therefore in the present study, we measured in a Pseudomonas aeruginosa induced porcine sepsis model, fractional protein synthesis (FSR) and breakdown rates (FBR) in jejunal mucosa in a fasted conscious state. We determined FSR by the measurement of the incorporation of stable isotope labeled amino acid (primed continuous infusion, L-[ring- ${ }^{13} \mathrm{C}_{6}$ ]-phenylalanine) into tissue protein, and FBR using the relation between blood arterial enrichment and intracellular enrichment in consecutive mucosal biopsies after a pulse of $\mathrm{L}-\left[{ }^{15} \mathrm{~N}\right]$-phenylalanine. Additionally, we determined the FSR of other splanchnic tissue (jejunum, ileum, liver) and compared them with muscle and lung tissue.

We found in this sham controlled acute severe sepsis pig model (Sham-healthy $(H)$ : $\mathrm{N}=9$; Sepsis ( $\mathrm{S}$ : $\mathrm{N}=13$ ) that jejunal mucosal protein turnover is reduced with both decreased FSR $(H$ : 3.29 \pm 0.22 ; S: $2.32 \pm 0.12 \% /$ hour, $p=0.0008)$ and FBR $(H: 0.72 \pm 0.12$; $\mathrm{S}: 0.34 \pm 0.04 \% /$ hour; $p=0.006$ ). We also found that FSR was unchanged in ileum and muscle, while it was higher in the liver $(H$ : 0.87 \pm 0.05 ; S: $1.05 \pm 0.06 \% / h o u r ; p=0.041)$ and tended to be higher in the lung $(\mathrm{H}: 0.57 \pm 0.04$; S: 0.73 $\pm 0.07 ; \mathrm{p}=0.12)$.

In conclusion, our data, obtained with a highly translational acute severe sepsis model, suggest that jejunal mucosal protein metabolism is diminished in acute severe sepsis. Comparison with other tissues indicates that the most serious acute changes in severe sepsis occur in the gut rather than the muscle. Therefore, we hypothesize that preventing a compromised gut is critical to maintain gut function during sepsis. 


\section{Introduction}

Sepsis is characterized by severe whole body protein breakdown (PB) and organ dysfunction (1). In addition to enhanced muscle breakdown (14), disturbed protein metabolism in splanchnic tissue could potentially play a role in the response to sepsis. It is known that gut atrophy causes gut dysfunction in sepsis (2, 3). Gut dysfunction is responsible for increased translocation of bacteria from the gut into the bloodstream and therefore contributes to the severity of the septic state. Furthermore, altered gut function could compromise nutrient absorption. Gut mucosa is a tissue with very high protein turnover rates $(4,5)$. Therefore, gut atrophy could reflect a reduced mucosal protein synthesis and/or increased mucosal PB. In contrast with a potential enhanced PB in the gut, the liver is expected to increase its protein synthesis (PS) due to initiation of immunological acute phase response $(6,7)$.

In humans, research on protein metabolism in sepsis has mainly focused on the occurrence of severe whole body PB and related muscle PB (8-10), in part because of the difficulty in obtaining gut tissue biopsies. Although preserving muscle is important for survival and quality of life after sepsis, the role of the gut is a critical consideration when developing/improving an appropriate nutritional interventions in septic humans. Therefore, we need to gain knowledge on gut and liver tissue protein metabolism in relation to other tissue protein metabolism in sepsis.

Studying protein synthesis and breakdown rates in the gut and liver in septic humans is very difficult (10-12) (13). It is only possible to measure gut PS and PB by taking mucosal biopsies through a stoma or during an endoscopic procedure and liver PS during diagnostic liver biopsy procedure. Therefore, we and others have developed large animal models in pigs $(14,15)$ to make this type of research possible. In clinically relevant large animal models of bacteremia induced acute severe sepsis, it is unknown how PS and PB in the gut is affected in an early stage of severe sepsis. We found in arterial-venous organ balance studies that when endotoxins are continuously infused in pigs, jejunal motility is affected (16) in relation to reduced gut protein synthesis (17). Recently, we found mild gut atrophy and disturbed portal drained viscera plasma flow in a well characterized live bacteria (Pseudomonas aeruginosa, PA) induced hyperdynamic severe sepsis catheterized pig model (18).

Therefore in the present study, we have measured jejunal mucosa protein synthesis (FSR) and breakdown rates (FBR) in a pig model of PA. In order to most appropriately simulate the human condition we have performed this study in conscious pigs. We determined protein FSR by the measurement of the incorporation of stable phenylalanine (Phe) tracer into tissue protein over a period of several hours (19) and measured protein FBR using the relation between blood arterial and intracellular enrichment after a pulse of a different stable Phe tracer (19). Additionally, for 
comparison we determined the FSR of other splanchnic tissues (jejunum, ileum, liver) as well as muscle and lung tissue obtained during necropsy.

\section{Materials and Methods}

\section{Animals}

Twenty two female Yorkshire cross/domestic pigs, average bodyweight of 20-25 $\mathrm{kg}$ were used for the present study. The pigs were individually housed in steel pens $(2 \mathrm{~m} \times 3 \mathrm{~m})$ in a controlled housing facility: room temperature $22-24^{\circ} \mathrm{C}, 12$ hours light-dark cycle, standardized food 1kg/day (Harlan Teklad Vegetarian Pig/Sow Grower). Water was provided ad libitum. This study was approved by the animal experiment ethics committee (IACUC) of University of Arkansas Medical Sciences (Little Rock, AR, USA).

\section{Implantation catheters}

We implanted several catheters in blood vessels with a surgical procedure about 10 days for the induction of sepsis as described previously (18) $(20,21)$ to determine stable tracer enrichment in afferent blood of the tissues (precursor for tissue FSR) and arterial enrichment for jejunal mucosal FBR measurement. We created a jejunostomy for sampling jejunal mucosa biopsies. Preoperative and postoperative care was standardized as described in detail previously (18) (20, 21). During the recovery period (7-10 days) animals were familiarized to a small movable cage $(0.9 \times 0.5 \times 0.3 \mathrm{~m})$ to ensure that the experiments could be performed in this cage in conscious animals.

\section{Experimental design}

The experiment (Figure 1) started after a recovery period of 7-10 days. Food was withheld for 6-8 hours before the start of the experiment. Animals were selected for the Sepsis group or the Healthy group in a randomized fashion. At $\mathrm{T}=0 \mathrm{~h}$ sepsis was induced by the start of IV continuous infusion of Pseudomonas aeruginosa (PA, $10^{9} \mathrm{CFU} / \mathrm{ml} /$ hour) and monitored continuously as we described previously (18). The control group received $0.9 \% \mathrm{NaCl}$ solution in the same volume. A primed continuous infusion of the stable amino acid tracer L-[ring ${ }^{13} \mathrm{C}_{6}$ ]-Phe (prime $1.58 \mu \mathrm{mol} / \mathrm{kg}$ bw; infusion $4.32 \mu \mathrm{mol} / \mathrm{kg}$ bw/hour) was administered intravenously in the pre-septic phase (-2 to $0 h)$ and in the septic state (12-18h) via the central vein catheter. A pulse of the stable tracer L-[15 N]-Phe $(26.3 \mu \mathrm{mol} / \mathrm{kg}$ bw) was given at $\mathrm{T}=17 \mathrm{~h}$. We collected basal blood samples and mucosa samples to determine natural abundances of the Phe isotopes at $T=-2 h$. For jejunal mucosal FSR, we took biopsies at $T=12$ and 18 for protein bound $\mathrm{L}$-[ring ${ }^{13} \mathrm{C}_{6}$ ]-Phe enrichments. And at $\mathrm{T}=12,14,16,17.08,17.5,18$ hours to determine average intracellular precursor enrichment over the incorporation time. 
For determination of jejunal mucosal FBR, we measured L- $\left[{ }^{15} \mathrm{~N}\right]-$ Phe enrichment in arterial plasma at $\mathrm{T}=17.08,17.17,17.33,17.5,17.75$ and 18 hours. And intracellular jejunal mucosal enrichment at 17.08, 17.5 and 18.0 hours. For FSR in other tissues, we collected at $\mathrm{T}=18$ hours after euthanized with $125 \mathrm{mg} / \mathrm{kg}$ pentobarbital sodium and $16 \mathrm{mg} / \mathrm{kg}$ phenytoin sodium (Euthanaso ${ }^{\circledR}$ ) administered via the central vein catheter, tissue samples of jejunum, ileum, liver, muscle and lung within 10 min after the euthanization to obtain enrichments (L-[ring13C6]-Phe) in tissue bound proteins. Blood samples were taken to determine average plasma outflow enrichment of the precursor (Phe) of each tissue during incorporation time at $\mathrm{T}=-1,-0.5,0,14$, 16, 17, 17.5, 18.0: portal vein plasma was taken for jejunum and ileum; hepatic vein plasma for liver; arterial plasma for lung and abdominal vein plasma outflow from the hindquarter for muscle.

\section{Blood and tissue sample processing}

We collected blood in lithium-heparinized tubes (microtube, Sarstedt, Newton, NC, USA) and immediately placed them on ice $\left(4^{\circ} \mathrm{C}\right)$ and processed them within one hour as described previously $(20,22,23)$. In brief: for plasma flow measurements, blood was centrifuged $\left(4^{\circ} \mathrm{C}, 5\right.$ minutes, $\left.8000 \mathrm{G}\right)$, after which $250 \mu \mathrm{L}$ plasma were deproteinized with $25 \mu \mathrm{L}$ trichloroacetic acid solution (TCA, 50\% w/v) and snap frozen in liquid nitrogen. We collected mucosa biopsies with a short endoscopic flexible biopsy needle via the jejunal stoma, and rinsed and cooled them directly in cold saline, blotted them on paper towel and transferred into a tube. The tube was snap frozen in liquid nitrogen. Samples of jejunum, ileum, liver, muscle and lung, were collected during necropsy (within $10 \mathrm{~min}$ after the euthanization) were treated in the same way. All samples were stored until further analyses at $-80^{\circ} \mathrm{C}$.

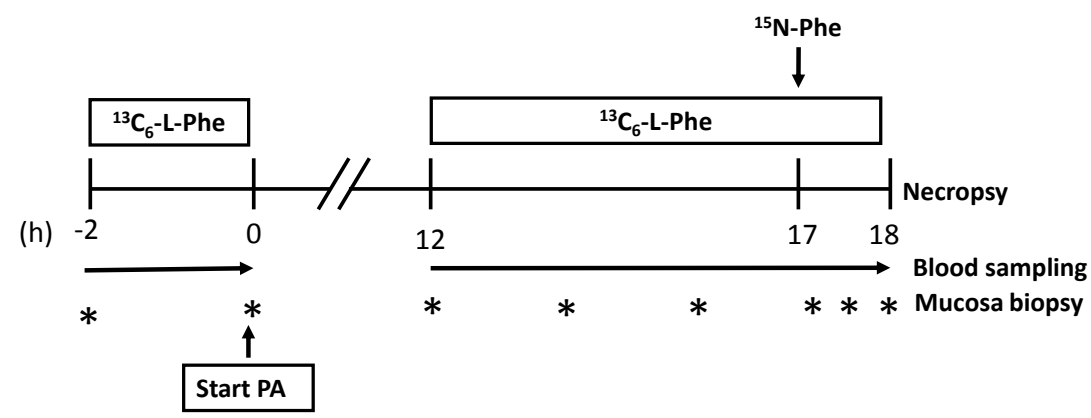

Figure 1. Experimental Design

$\mathrm{PA}=$ Pseudomonas aeruginosa $\left(1 \mathrm{e}^{9} \mathrm{CFU} / \mathrm{ml} / \mathrm{min}\right) ;{ }^{13} \mathrm{C}_{6}$-L-Phe is intravenous primed continuous infusion of stable isotope tracer ${ }^{13} \mathrm{C}_{6}$-L-phenylalanine; ${ }^{15} \mathrm{~N}$-Phe is intravenous pulse administration of ${ }^{15} \mathrm{~N}$-Phenylalanine. 
Before the analyses, tissues were crunched under freezing conditions $\left(-190^{\circ} \mathrm{C}\right)$. Tissue powder (30-80 mg) were transferred to a microtube (Sarstedt, \#72.730, Newton,NC,USA) with $250 \mu \mathrm{l} 0.098 \mathrm{M} \mathrm{TCA}\left(4^{\circ} \mathrm{C}\right)$ and $0.1 \mathrm{gr}$ glass beads and subsequently homogenized with mini-bead beater (Biospec, Bartlesville, OK, USA) for 30 seconds on low speed. After centrifugation $\left(4^{\circ} \mathrm{C}, 5\right.$ minutes, $\left.8000 \mathrm{G}\right)$, supernatant was used for intracellular amino acid concentration measurements with the LCMS. The protein pellet was washed three times with 0.3M TCA and transferred to larger volume $(8 \mathrm{ml})$ glass tube. Hydrolysation of the protein in the air-tight capped glass tube was performed with $1 \mathrm{ml} 6 \mathrm{~N} \mathrm{HCL}$ at $110^{\circ} \mathrm{C}$ for 24 hours. After hydrolysis $5 \mathrm{ml}$ MilliQ water was added to dilute the strong acid, where after the tubes were dried down. Capped samples were stored dark and at room temperature until analysis.

\section{Stable isotope tracer enrichment measurement}

We determined amino acid enrichments (tracer-to-tracee ratios, TTR) on a fully automated LC-ESI-MS/MS system (QTrap 5500 MS, AB Sciex, Foster City, CA, USA) with ExpressHT Ultra LC (Eksigent Div., AB Sciex, Foster City, CA, USA). Within 3 days before the LC-ESI-MS/MS analysis, we derivatized the samples together with enriched external standards in the range of expected TTRs (calibration curve for TTRs) with 9-fluorenylmethoxycarbonyl (Fmoc). After neutralization, we injected $160 \mathrm{~nL}$ of the solution onto a micro LC column $0.5 \times 100 \mathrm{~mm}$ HALO C18, $2.7 \mathrm{um}$, 90A pores (ABsciex, Foster City, CA, USA), kept at $35^{\circ} \mathrm{C}$. We eluted analytes with a segmentally linear gradient from $35 \%$ to $85 \%$ acetonitrile in water supplemented with ammonium acetate to $10 \mu \mathrm{M}$ and $5 \%$ isopropanol. We used electrospray triple quadrupole tandem mass spectrometry in multiple reactions monitoring mode for detection. We fragmented the Fmoc amino acid derivatives in the collision cell for detection of either free aminoacyl anions or a fragment larger by 26 atom mass units (coming from the Fmoc derivative) to have the highest sensitivity. We simultaneously did mass analyses for Phe tracee, tracers and internal standards. We calculated the mass signal areas to enable TTR.

\section{Calculations}

\section{FSR}

FSR of the tissues was determined by measurement of the incorporation of the stable isotope labeled amino acid (=precursor) over a period of time in protein (=product) as described by Wolfe et al (19):

\section{$\mathrm{FSR}=\left(\right.$ Eproduct $\left(\mathrm{T}_{2}\right)-$ Eproduct $\left.\left(\mathrm{T}_{1}\right)\right) /($ Eprecursor $\times$ Delta time $)$}

$E$ is the enrichment in unit Mole Percent Excess (MPE= TTR/(1+TTR)). Eproduct $\left(T_{1}\right)$ is the natural abundance of mucosal protein at $t=-2 h$. Eproduct $\left(T_{2}\right)=$ enrichment of tissue protein at the time of tissue collection. Eprecursor = average plasma outflow enrichment of the precursor (Phe) of each tissue during incorporation time: portal 
vein plasma for jejunum and ileum; hepatic vein plasma for liver; arterial plasma for lung and abdominal vein plasma outflow from the hindquarter for muscle. Delta time: Incorporation time (-2-Oh and 12-18h). For the FSR in mucosal biopsies we used the same formula, replaced Eproduct $\left(T_{1}\right)$ with the enrichment in mucosal protein at $\mathrm{T}=12 \mathrm{~h}$. Eproduct $\left(\mathrm{T}_{2}\right)$. Eprecursor is the average intracellular mucosa enrichment of precursor and Delta time is $12-18 \mathrm{~h}$.

\section{FBR}

The rate of decay of the intracellular enrichment (TTR), after a pulse of stable amino acid tracer ( ${ }^{15} \mathrm{~N}$-phenylalanine) in plasma, is related to FBR. Arterial and intracellular decay curves in mucosa biopsies of this tracer are used for the calculations following the method of Wolfe etal (19). FBR is measured between $\mathrm{t}=17$ and $18 \mathrm{~h}$.

\section{Net protein synthesis}

Net protein synthesis was calculated by subtracting the FBR from FSR.

\section{Statistics}

Results are presented as means \pm SEM. Graphpad Prism (version 7) was used for statistics. Levels of significance was set on $p<0.05$. Tendency on $p<0.1$. To compare data between the Healthy and Sepsis group an unpaired t-test was used.

\section{Results}

To determine changes in protein synthesis in acute severe sepsis, we measured protein turnover in the gut and of several tissues of major organs in a postabsorptive state.

\section{Jejunal mucosal protein turnover}

In relation to the FSR determination (Figure 2), the enrichments of Phe in the intracellular precursor pool and protein pool were decreased in sepsis (respectively $p=0.036, p<0.001)$ (Figure 4) $(p=0.0008)$. For FBR determination (Figure 3 ), the arterial plasma ${ }^{15} \mathrm{~N}$-Phe enrichment decay curve was lower $(p<0.0001)$ in sepsis but the intracellular enrichment curve was not changed. Both FSR ( $p=0.0008$ ) and FBR ( $p=0.0065$ ) were reduced in jejunal mucosa in septic animals (Figure 4). Mucosal FSR is on average 5.9 times higher than FBR. Net Protein synthesis and overall protein turnover (PS + PB) were decreased in sepsis (Figure 5, respectively $p=0.0247, p=0.0096)$. 
A

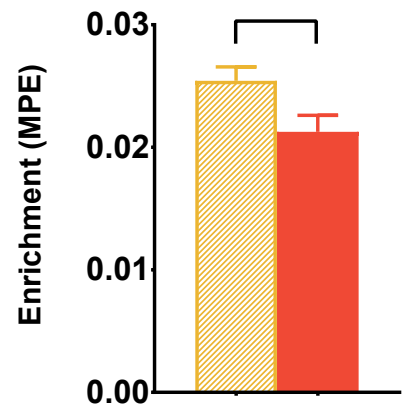

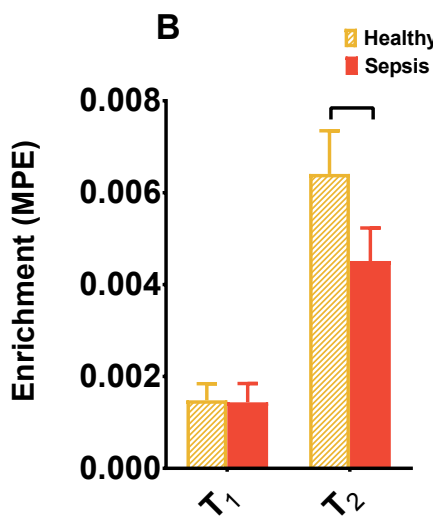

Figure 2. Enrichments of phenylalanine for mucosa FSR calculations

Expressed as mean \pm SE in MPE (= mole percent excess). A. Intracellular (precursor) enrichment (average) of phenylalanine during the incorporation time. B. Protein enrichment of phenylalanine in jejunal mucosa at the start $\left(T_{1}\right)$ and end $\left(T_{2}\right)$ of the tracer incorporation time. Healthy N=9, Sepsis N=11. Statistics: A: Student t-test; B: Two-way ANOVA with posthoc Uncorrected Fisher's LSD test: Sepsis effect $p<0.0001$, Time effect $p<0.0001$, Interaction $p<0.0001 ;$ Hook: $p<0.05$.

A

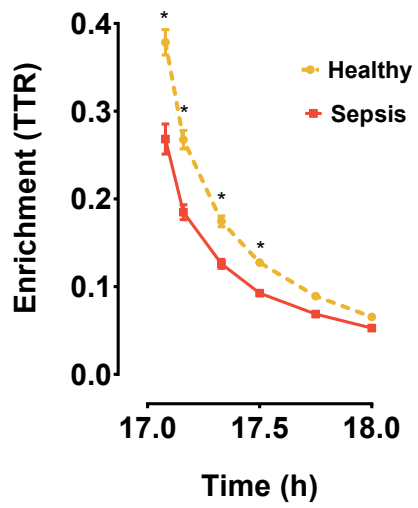

B

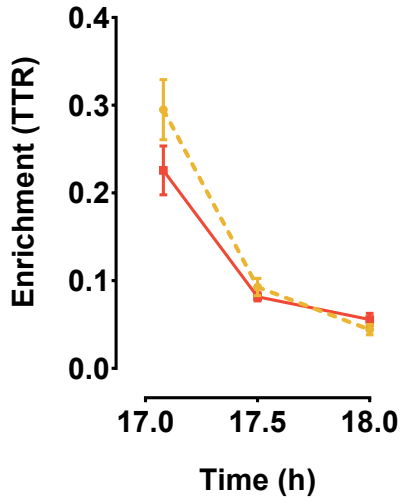

Figure 3. Enrichments of phenylalanine for mucosa FBR calculations

Expressed as mean \pm SE in TTR (= tracer/tracee ratio). A: Arterial plasma ${ }^{15} \mathrm{~N}$-phenylalanine enrichment after IV pulse administration of ${ }^{15} \mathrm{~N}$-phenylalanine 17 hours after the induction of sepsis. B. Intracellular enrichment in jejunal mucosa of ${ }^{15} \mathrm{~N}$-phenylalanine. Healthy $\mathrm{N}=9$, Sepsis $\mathrm{N}=12$. Statistics: Two way ANOVA, A: Time effect $\mathrm{p}<0.0001$; Sepsis effect $\mathrm{p}<0.0001$; Interaction $p<0.0001$. B: Time effect $p<0.0001$; Sepsis effect $p=0.134$; Interaction $p=0.089$. When appropriate a post-hoc Sidak's multiple comparisons test was done; *) $p<0.05$. 

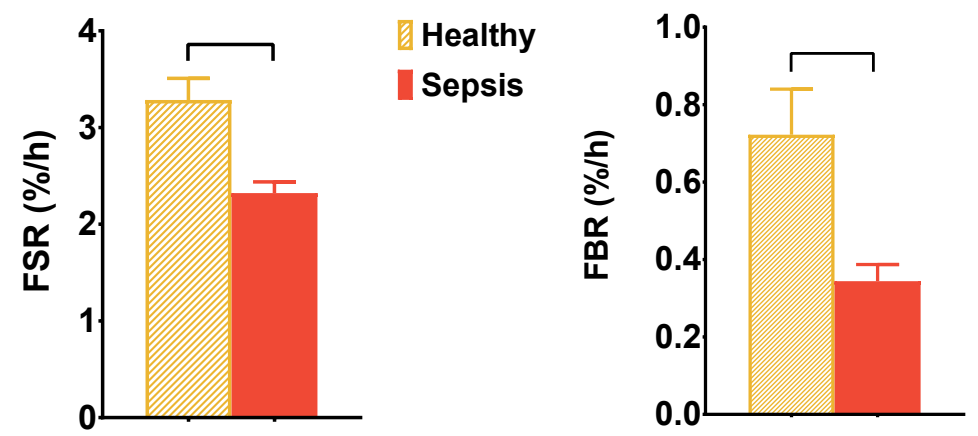

Figure 4. Fractional Synthesis Rate (FSR) and fractional breakdown rate (FBR) of protein in mucosa

Expressed as mean \pm SE in percent per hour. Post-absorptive state. FSR: Healthy $N=9$, Sepsis $\mathrm{N}=11$. FBR: Healthy $\mathrm{N}=8$, Sepsis $\mathrm{N}=9$ Statistics: Student t-test; Hook: $\mathrm{p}<0.05$.
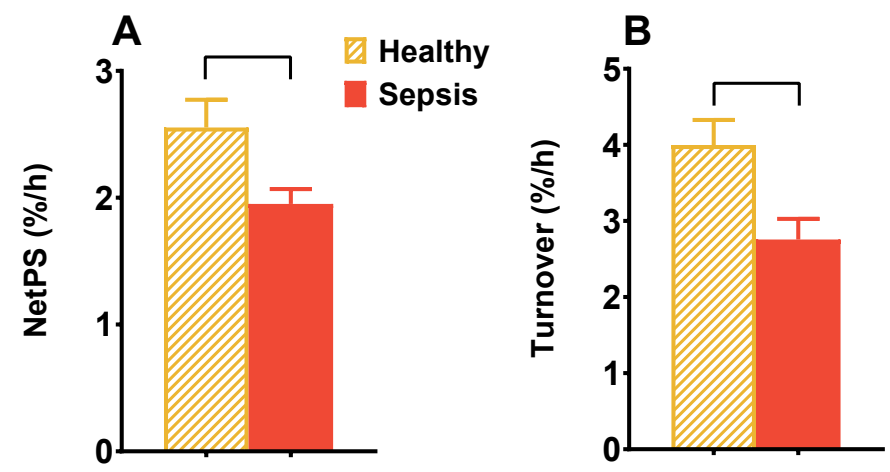

Figure 5. Mucosa Net protein Synthesis (NetPS) and protein turnover

Expressed as mean \pm SE. Post-absorptive state. Healthy: $N=8$; Sepsis: $N=10$. Statistics: Student t-test; Hook: $p<0.05$.

\section{Tissue FSR}

The average enrichment of Phe of the different possible proxies for the precursor pools (Figure 6) are decreased in sepsis animals $(p<0.0001)$. The enrichment of bound Phe in tissue protein was decreased in jejunum ( $p=0.0017)$ but not in other tissues. In a post-absorptive state (Figure 7), FSR in jejunum is about 10 times higher than in muscle. Jejunum FSR was decreased $(p=0.0328)$ in acute severe sepsis, but no effect of sepsis was seen in the ileum and muscle. Liver FSR was increased and lung FSR tended to be increased. 
A

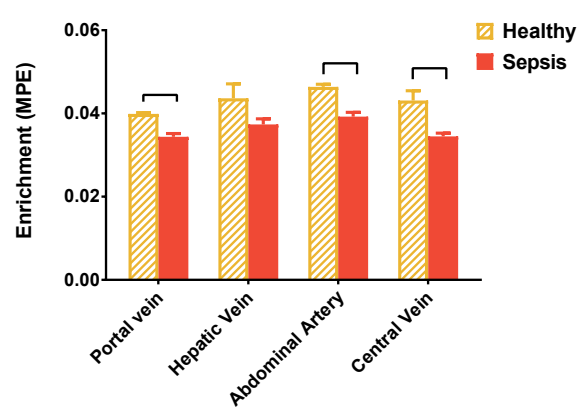

B

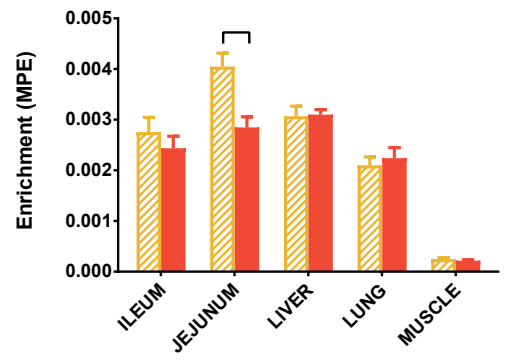

Figure 6. Enrichments of phenylalanine for tissue FSR calculations

Expressed as mean \pm SE in MPE (is mole percent excess). A: average enrichment of the phenylalanine in plasma (precursor) during the incorporation time; Healthy $N=9$; Sepsis $N=11$. B: enrichment of bound phenylalanine in tissue protein; Healthy $N=9$; Sepsis $N=13$. Statistics: Two-way ANOVA; A: sepsis effect $p<0.0001$, tissue effect $p=0.0028$, interaction $p=0.8031 ; B$ : sepsis effect $p=0.0263$, tissue effect $p<0.0001$, interaction $p=0.0052$; When appropriate with a post-hoc Holm-Sidak test; Hook: p<0.05.

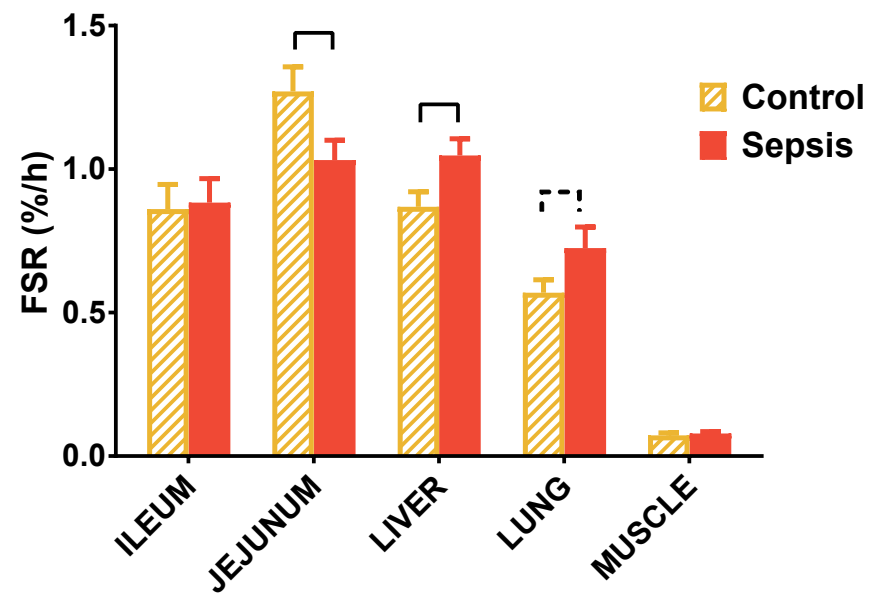

Figure 7. Fractional Synthesis Rate (FSR) of protein in tissues

Expressed as mean \pm SE in percent per hour. Post-absorptive state; Healthy $N=9$; Sepsis $N=11$. Statistics: Two-way ANOVA, sepsis effect $p=0.673$, tissue effect $p<0.0001$, interaction 0,0189; Post-hoc test Holm-Sidak: Hook: $\mathrm{p}<0.05$; Dashed hook: tendency $\mathrm{p}<0.1$.

\section{Discussion}

Disturbed net protein synthesis in the gut is the basis for gut atrophy. We studied protein synthesis and breakdown rates of gut tissue to observe how this relates with other potential PA affected tissues in the body. We found in this acute severe sepsis pig model that jejunal mucosal protein synthesis and breakdown was reduced while protein synthesis rate was higher in the liver but unchanged in ileum and muscle. 


\section{Measurement of protein synthesis}

The absolute FSR depends on the chosen precursor pool, route of administration, tracer itself (4), and the chosen incorporation time.

\section{Precursor pool}

Aminoacyl-tRNA is the true precursor of protein synthesis, but to measure its enrichment is technically very difficult, and in small tissue pieces such as gut mucosal biopsies almost impossible. The amino acids charging the tRNA must come from either the interstitial fluid compartment (being picked up at cell membrane) or from the intracellular fluid compartment. For amino acids such as phenylalanine, there is only a small difference in the enrichments in the interstitial or intracellular fluid compartment and therefore reasonably reflect the tRNA enrichment (24). The intracellular precursor enrichment during necropsy does not represent the precursor enrichment during the whole incorporation time due to the dynamics of the model. Therefore, we choose to use the average enrichment of the precursor in the plasma venous outflow of the organs during the incorporation time as the precursor enrichments to calculate the different tissue protein FSR's (19, 24).

\section{Route of administration}

In the gut, the amino acids utilized for mucosal protein synthesis originate from the luminal or basolateral side. The measured rate of mucosal protein synthesis can be affected by the route of administration of the stable tracers (25). Intravenous administered tracers will be preferentially incorporated in the crypt cells and lower villi. In post-absorptive condition, precursor amino acids are coming mainly from the basolateral side in jejunal mucosa and therefore we chose to enrich the mucosal intracellular precursor pool via the vascular side. This means that the observed altered post-absorptive mucosa FSR is mainly a change of PS in the crypt and lower villus cells.

\section{Choice of tracer}

Stable tracers of phenylalanine or leucine are chosen frequently to measure rate of incorporation in protein. In muscle, both tracers will provide the same absolute protein synthesis rate $(24,26)$. Also Bouteloup-Demange (4) found that the same absolute mucosal FSR is measured with both stable tracers when plasma enrichment is used as precursor pool, but not when intracellular enrichments are used. No explanation was provided for that observation. Therefore, absolute values of FSR should be taken with caution because these can depend on the chosen tracer and precursor pool, especially, if the absolute quantity of FSR is of interest. We used a sensitive and precise measurement of the FSR that is based on an isotope with a high mass ( 6 masses higher than tracee that has no measurable natural abundance background) and LC-MS/MS analyses. Our approach also enabled us to reduce the 
chosen total incorporation time needed to be able to pick-up tracer incorporation in the protein, especially in slow turn-over tissues like the muscle.

\section{FSR and FBR in jejunal mucosa}

In the healthy animals, the measured mucosa FSR was comparable with the human gut mucosal PS rate (4). The present mucosa PS and PB data showed that the ratio between FSR and FBR is much larger in mucosa than in other tissues. Mucosa is a net protein producing tissue. The FSR of the endogenous secreted protein mucin, which is needed for an adequate intestinal defense is very high (130\%/day) (24). Therefore, the substantial decrease of mucosal FSR in the sepsis animals will lead to compromised mucosal net protein synthesis and will affect processes including mucin production, proteins involved in intestinal protection/repair, proliferation epithelial cells and intestinal defense against bacterial translocation. The observed diminished FBR could attenuate but not prevent this effect. The observed inhibition of jejunal mucosal protein metabolism is in line with previously described histopathology of the small intestines (18), in which we observed mild jejunal mucosa injury. We believe that observed reduced portal drained viscera PDV flow (18) could play a role in the occurrence of the injured jejunal and ileum mucosa tissues (27) in relation to compromised microcirculation (28) and subsequent inhibition of jejunal mucosal protein metabolism.

\section{FSR in the liver}

In contrast to the gut, liver FSR was increased probably due to the initiation of the acute phase response (29). Although we did not found a substantial increase of IL-6 and CRP in our model (18), it remains to be established whether the increased liver FSR is related to the synthesis of other hepatic acute phase proteins like fibrinogen.

\section{FSR in other tissues.}

In the present severe sepsis model, baseline muscle FSR was not affected, potentially due to amino acid membrane transport being maintained, which ensures the availability of amino acids from plasma for charging the tRNA (30). This is in contrast to previous studies that found reduced muscle FSR in large and small animal studies, with endotoxin or bacteremia induced sepsis $(6,7,31)$. However, the results in the present paper are more in line with what is observed in critically ill humans with muscle FSR still within normal physiological ranges $(32,33)$. These observations also support the notion that our sepsis model in the pig is a reasonable model for sepsis in critically-ill patients.

Very limited data are available that describe protein synthesis dynamics in the lungs. Breuille et al (7) described an increase of lung PS in the early phase in a chronic bacteremia induced septic rat model and speculated that influx of inflammatory cells could possibly be responsible for the overall increase of PS in the lung, 
as reflected in the present model. However, in the time window we measured, lung function was maintained (18). Therefore, we conclude that with the lung function still intact, lung PS is potentially affected in early phase of severe sepsis, but it is unknown if lung PB also is affected.

\section{Nutritional implications for gut metabolism in sepsis}

We hypothesize that the decreased net PS in the jejunal mucosa will compromise the nutrient and barrier function of jejunal mucosa in severe sepsis (34). Considering the highly dynamic character of intestinal metabolism, it is unclear if this phenomenon is only temporarily present or will sustain longer in the recovery phase of severe sepsis. Although certain specific dietary components are considered to be potentially helpful for mucosal healing, clinical trials (35) or studies in clinical relevant animal models with mucosal injury are limited (36). Therefore, additional studies in sepsis recovery phase are needed to study these dynamics.

\section{Conclusion}

In conclusion, our data, obtained with a highly translational acute severe sepsis model, suggest that jejunal mucosal protein metabolism is diminished in acute severe sepsis, even if origin of the sepsis was not located in the gut. Therefore, goals of nutritional support in sepsis should not only focus on preventing muscle breakdown but also on stimulation of gut metabolism to prevent/heal the leaking/ atrophic gut.

\section{Acknowledgements}

We thank John Thaden (PhD), Renske C.I. Deutz (MD), Joshua Spore and Bea Zoer $(\mathrm{PhD})$, for skilled technical assistance in carrying out the animal experiments and/or sample processing.

The project described was supported by Award Number R01GM084447 from the National Institute Of General Medical and by Award Number S10RR027047 from the National Center For Research Resources. The content is solely the responsibility of the authors and does not necessarily represent the official views of the National Institute Of General Medical Sciences or National Center For Research Resources or the National Institutes of Health. 


\section{References}

1. Hermans $G$, Van den Berghe G. Clinical review: intensive care unit acquired weakness. Crit Care. 2015;19:274.

2. $\quad$ Song J, Wolf SE, Herndon DN, Wu XW, Jeschke MG. Second hit post burn increased proximal gut mucosa epithelial cells damage. Shock. 2008;30(2):184-8.

3. Ding LA, Li JS. Intestinal failure: pathophysiological elements and clinical diseases. World J Gastroenterol. 2004;10(7):930-3.

4. Bouteloup-Demange C, Boirie Y, Dechelotte P, Gachon P, Beaufrere B. Gut mucosal protein synthesis in fed and fasted humans. Am J Physiol. 1998;274(3 Pt 1):E541-6.

5. Montagne L, Piel C, Lalles JP. Effect of diet on mucin kinetics and composition: nutrition and health implications. Nutr Rev. 2004;62(3):105-14.

6. Orellana RA, O'Connor PM, Bush JA, Suryawan A, Thivierge MC, Nguyen HV, et al. Modulation of muscle protein synthesis by insulin is maintained during neonatal endotoxemia. Am J Physiol Endocrinol Metab. 2006;291(1):E159-66.

7. Breuille D, Arnal M, Rambourdin F, Bayle G, Levieux D, Obled C. Sustained modifications of protein metabolism in various tissues in a rat model of long-lasting sepsis. Clin Sci (Lond). 1998;94(4):413-23.

8. Cohen S, Nathan JA, Goldberg AL. Muscle wasting in disease: molecular mechanisms and promising therapies. Nat Rev Drug Discov. 2015;14(1):58-74.

9. Klaude M, Mori M, Tjader I, Gustafsson T, Wernerman J, Rooyackers O. Protein metabolism and gene expression in skeletal muscle of critically ill patients with sepsis. Clin Sci (Lond). 2012;122(3):133-42.

10. Pittiruti M, Siegel JH, Sganga G, Coleman B, Wiles CE, 3rd, Placko R. Determinants of urea nitrogen production in sepsis. Muscle catabolism, total parenteral nutrition, and hepatic clearance of amino acids. Arch Surg. 1989;124(3):362-72.

11. Rooyackers O, Kouchek-Zadeh R, Tjader I, Norberg A, Klaude M, Wernerman J. Whole body protein turnover in critically ill patients with multiple organ failure. Clin Nutr. 2015;34(1):95-100.

12. Nakshabendi IM, Obeidat W, Russell RI, Downie S, Smith K, Rennie MJ. Gut mucosal protein synthesis measured using intravenous and intragastric delivery of stable tracer amino acids. Am J Physiol. 1995;269(6 Pt 1):E996-9.

13. Decaris ML, Emson CL, Li K, Gatmaitan M, Luo F, Cattin J, et al. Turnover rates of hepatic collagen and circulating collagen-associated proteins in humans with chronic liver disease. PLoS One. 2015;10(4):e0123311.

14. Bruins MJ, Luiking YC, Soeters PB, Lamers WH, Akkermans LM, Deutz NE. Effects of long-term intravenous and intragastric L-arginine intervention on jejunal motility and visceral nitric oxide production in the hyperdynamic compensated endotoxaemic pig. Neurogastroenterol Motil. 2004;16(6):819-28.

15. Ytrebo LM, Sen S, Rose C, Davies NA, Nedredal GI, Fuskevaag OM, et al. Systemic and regional haemodynamics in pigs with acute liver failure and the effect of albumin dialysis. Scand J Gastroenterol. 2006;41(11):1350-60.

16. Bruins MJ, Luiking YC, Soeters PB, Akkermans LM, Deutz NE. Effect of prolonged hyperdynamic endotoxemia on jejunal motility in fasted and enterally fed pigs. Ann Surg. 2003;237(1):44-51.

17. Bruins MJ, Soeters PB, Deutz NE. Endotoxemia affects organ protein metabolism differently during prolonged feeding in pigs. J Nutr. 2000;130(12):3003-13.

18. Ten Have GAM, Deutz RCl, Engelen M, Wolfe RR, Deutz NEP. Characteristics of a 
Pseudomonas aeruginosa induced porcine sepsis model for multi-organ metabolic flux measurements. Lab Anim. 2017:23677217718003.

19. Wolfe RR, Chinkes DL. Isotope Tracers in Metabolic Research: Principles and Practise of Kinetic Analysis. New York: Wiley; 2005. 274 p.

20. Ten Have GA, Bost MC, Suyk-Wierts JC, van den Bogaard AE, Deutz NE. Simultaneous measurement of metabolic flux in portally-drained viscera, liver, spleen, kidney and hindquarter in the conscious pig. Lab Anim. 1996;30(4):347-58.

21. Poeze M, Bruins MJ, Kessels F, Luiking YC, Lamers WH, Deutz NE. Effects of L-arginine pretreatment on nitric oxide metabolism and hepatosplanchnic perfusion during porcine endotoxemia. The American journal of clinical nutrition. 2011;93(6):1237-47.

22. Bruins MJ, Deutz NE, Soeters PB. Aspects of organ protein, amino acid and glucose metabolism in a porcine model of hypermetabolic sepsis. Clin Sci (Lond). 2003;104(2):127-41.

23. Bruins MJ, Lamers WH, Meijer AJ, Soeters PB, Deutz NE. In vivo measurement of nitric oxide production in porcine gut, liver and muscle during hyperdynamic endotoxaemia. Br J Pharmacol. 2002;137(8):1225-36.

24. Miller S, Chinkes D, MacLean DA, Gore D, Wolfe RR. In vivo muscle amino acid transport involves two distinct processes. Am J Physiol Endocrinol Metab. 2004;287(1):E136-41.

25. Alpers DH. Protein synthesis in intestinal mucosa: the effect of route of administration of precursor amino acids. J Clin Invest. 1972;51(1):167-73.

26. Harber MP, Dickinson JM, Crane JD, Trappe SW, Trappe TA. Influence of tracer selection on protein synthesis rates at rest and postexercise in multiple human muscles. Metabolism. 2011;60(5):689-97.

27. Chiu CJ, McArdle AH, Brown R, Scott HJ, Gurd FN. Intestinal mucosal lesion in low-flow states. I. A morphological, hemodynamic, and metabolic reappraisal. Arch Surg. 1970;101(4):478-83.

28. Dyson A, Cone S, Singer M, Ackland GL. Microvascular and macrovascular flow are uncoupled in early polymicrobial sepsis. Br J Anaesth. 2012;108(6):973-8.

29. Bauer M, Press AT, Trauner M. The liver in sepsis: patterns of response and injury. Curr Opin Crit Care. 2013;19(2):123-7.

30. Ten Have GA, Engelen MP, Wolfe RR, Deutz NE. Phenylalanine isotope pulse method to measure effect of sepsis on protein breakdown and membrane transport in the pig. Am J Physiol Endocrinol Metab. 2017:ajpendo 003512016.

31. Lang $\mathrm{CH}$, Frost RA. Endotoxin disrupts the leucine-signaling pathway involving phosphorylation of mTOR, 4E-BP1, and S6K1 in skeletal muscle. J Cell Physiol. 2005;203(1):144-55.

32. Gamrin L, Essen P, Hultman E, McNurlan MA, Garlick PJ, Wernerman J. Proteinsparing effect in skeletal muscle of growth hormone treatment in critically ill patients. Ann Surg. 2000;231(4):577-86.

33. Tjader I, Rooyackers O, Forsberg AM, Vesali RF, Garlick PJ, Wernerman J. Effects on skeletal muscle of intravenous glutamine supplementation to ICU patients. Intensive Care Med. 2004;30(2):266-75.

34. Baron P, Traber LD, Traber DL, Nguyen T, Hollyoak M, Heggers JP, et al. Gut failure and translocation following burn and sepsis. J Surg Res. 1994;57(1):197-204.

35. Zhang Y, Gu F, Wang F, Zhang Y. Effects of early enteral nutrition on the gastrointestinal motility and intestinal mucosal barrier of patients with burn-induced invasive fungal infection. Pak J Med Sci. 2016;32(3):599-603.

36. Lan A, Blachier F, Benamouzig R, Beaumont M, Barrat C, Coelho D, et al. Mucosal healing in inflammatory bowel diseases: is there a place for nutritional supplementation? Inflamm Bowel Dis. 2015;21(1):198-207. 
CHAPTER 11 


\title{
Altered transorgan protein kinetics during a Pseudomonas aeruginosa induced severe sepsis in the pig
}

\author{
Gabriella A.M. ten Have, Merel P.J. Hommen, Mariëlle \\ P.K.J. Engelen, Steven W.M. Olde Damink, Robert R. \\ Wolfe, Nicolaas E.P. Deutz.
}

\section{Abstract}

Severe sepsis is characterized by multi-organ pathophysiology and tissue protein breakdown (PB). Unraveling the disturbed metabolic pathways in sepsis is therefore important to gain basic knowledge for potential improvement of clinical nutrition strategies in septic humans. Little work has been done to study inter/multi organ amino acid (AA) kinetics in sepsis.

Therefore, we studied the metabolic pathways that are involved in net catabolism in severe sepsis, by measuring AA substrate fluxes with multiple stable amino acid tracers on whole body $(\mathrm{Wb})$ level by measuring rate of appearances (Ra) of $A A$, and AA net balances (NB), PB and protein synthesis (PS) across the portal drained viscera (PDV), splanchnic area (SPL), liver and hindquarter $(\mathrm{HQ})$ in a clinically relevant Pseudomonas aeruginosa (PA) induced acute severe sepsis model in a catheterized pig in a fasted conscious state.

We measured baseline substrate metabolism (BL) and between 12 and 18 hours (POST) after start of intra venous PA infusion. Comparison between the experimental groups: Control ( $C, N=9$ ) and Sepsis (,$N=13)$. POST results are expressed as difference POST-BL \pm SE in $\mathrm{nmol} / \mathrm{kg} \mathrm{bw} / \mathrm{min}$. We could establish in this sham controlled acute severe sepsis pig model that enhanced WbPB (C:-154 \pm 31 ; S:107 \pm 39 ; $p=0.0001)$, WbPS (C:-163 $\pm 27 ; \mathrm{S}: 49 \pm 37 ; \mathrm{p}=0.0004), \mathrm{Wb}$ net catabolic state (C:2 \pm 4 ; $\mathrm{S}: 58 \pm 11 ; p=0.001$ ) and myofibrillar muscle breakdown (C:10 $\pm 1 ; \mathrm{S}: 25 \pm 1 ; p<0.0001)$ occurs in sepsis. Across PDV, both PB (C:-28 \pm 29 ; S:-274 $\pm 39 ; p=0.0002)$ and PS (C:15 \pm 49 ; S:-297 $\pm 80 ; p=0.014)$ are decreased. The liver showed substantial increased uptake of amino acids (C:44 \pm 1716 ; S:10879 $\pm 3401 ; p=0.0218$ ) and severe increase of ureagenesis (C:-3532 \pm 641 ; S:2517 \pm 661 ; $p<0.0001$ ). Overall the SPL and HQ NB could not explain the enhanced whole body PB. Interacting Glu, GIn and Ala multiorgan pathways showed that severe systemic Glu decrease in sepsis (C:-38 5 ;

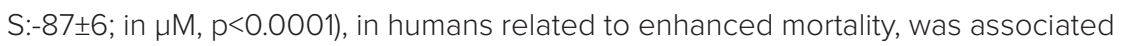




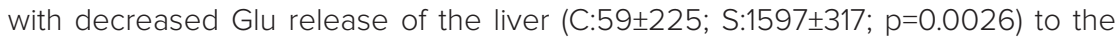
systemic plasma pool that could not be compensated by interacting Gln and Ala multiorgan pathways.

We conclude that enhanced protein turnover in the muscle cannot fully explain changes in WB protein turnover. Further, the decreased protein turnover in the PDV is in contrast to the WB changes. Consequently, protein turnover must be increased in tissues and organs that we have not measured directly. On the basis of the present results we hypothesize that in the acute phase of severe sepsis, the overall metabolic downregulation of PDV and the compromised liver are previously unappreciated metabolic factors in sepsis.

\section{Abbreviations}

AA: amino acid; Ala: alanine; Arg: arginine; Asp: asparagine; BCAA: branched chain amino acids; BL: Baseline T= -2 to Oh; EAA: essential amino acids; Cit: citrulline; FSR: fractional synthesis rate; GIn: glutamine; Glu: glutamate; His: histidine; HQ: hindquarter; Iso: isoleucine; Leu: leucine; Lys: Iysine; Met: methionine; mHis: 3-methylhistidine; MPAP: mean pulmonary arterial pressure; NB: Net balances; Orn: ornithine PA: Pseudomonas aeruginosa; PB: protein breakdown; PDV: portal drained viscera; Phe: phenylalanine; POST: period $\mathrm{T}=12$ to $18 \mathrm{~h}$ after start sepsis induction; Pro: proline; PS: protein synthesis; Ser: serine; SPL: splanchnic area; TAA: total amino acids (measured); Thr: threonine; Tryp: tryptophan; TTR: tracer-tracee ratio; Tyr: tyrosine; Val: valine; Wb: whole body; WbRa: whole body rate of appearance.

\section{Introduction}

Severe sepsis is characterized by multi-organ pathophysiology and tissue protein breakdown (PB) (1-3). Muscle tissue PB is considered to contribute substantially to whole body breakdown (WbPB) (4). As loss of muscle is related to poor survival and quality of life after sepsis, most research in sepsis is focused on muscle protein kinetics (5-8).

Altered protein kinetics in sepsis are also related to observations like gut mucosal atrophy, stimulation of liver acute phase protein synthesis \{Chapter 10\}(9-11), low plasma protein levels like albumin (12), or disturbed amino acid (AA) metabolism $(2,13)$. In addition, muscle loss, low albumin and reduced glutamate plasma levels are related to high mortality in sepsis $(13,14)$. Unraveling the disturbed metabolic pathways in sepsis is therefore important to gain basic knowledge for potential 
improvement of clinical nutrition and metabolic strategies in septic humans. Little work has been done to study interorgan protein/AA kinetics in sepsis (7) .

Although critical for the interpretation of the metabolic response during sepsis in humans, it is very difficult to study protein kinetics on an organ level $(7,15)$. Therefore, we developed a clinical relevant model in pigs with infusion of live bacteria (16). In the present study, we applied our multiorgan approach in the new sepsis model. In this model, we previously reported mild atrophy and decreased protein turnover in jejunal mucosa during sepsis (16)\{Chapter 10\}, suggesting changes in intestinal protein kinetics. Also, we observed an increased fractional protein synthesis rate (FSR) in liver tissue, indicating a stimulation of acute phase protein synthesis. The fraction of AA derived from enhanced WbPB that is derived from muscle protein breakdown that is "reused" for acute phase protein in the liver or is released from other organs like the intestines (7) is still unclear.

In the present paper, we present AA fluxes on whole body ( $\mathrm{Wb}$ ) level and AA net balances across the portal drained viscera (PDV, mainly intestines), splanchnic area (SPL, liver and PDV), liver and hindquarter ( $\mathrm{HQ}$, muscle compartment). With the use of stable isotopes we are able to obtain information on protein synthesis (PS) and (PB) in multiple organs (17) in severe sepsis. Data from the present study further unravel the disturbed metabolic pathways in sepsis and therefore provide basic knowledge for potential improvement of clinical nutrition strategies in septic humans.

\section{Materials and Methods}

\section{Animals}

We used Female Yorkshire cross/domestic pigs (20-25 kg BW) in our experimental studies. We housed the pigs in steel pens $(2 \mathrm{~m} \times 3 \mathrm{~m})$ in a controlled housing facility (large animal cubicle, room temperature $22-24^{\circ} \mathrm{C}$, 12 hours light-dark cycle, standardized food 1kg/day (Harlan Teklad Vegetarian Pig/Sow Grower) and provided water ad libitum.

\section{Surgical procedure}

Animals received catheters during a surgical procedure as described in detail previously (18) (16). In brief, during midline laparotomy, we placed catheters for blood sampling into the abdominal aorta, portal vein, hepatic vein, and in the caval vein. An extra central caval vein catheter was placed for administering post-surgery medication and experiment related tracer infusions. We placed a second arterial catheter to monitor mean arterial blood pressure and a Swan Ganz catheter (5 Fr, \#132F5, Edwards life sciences, Irvine, CA, USA) via the right jugular vein to monitor mean 
pulmonary arterial pressure (MPAP) and to obtain blood from the right ventricle. We standardized preoperative and postoperative care. During the recovery period (7-10 days) animals were accustomed to a small movable cage $(0.9 \times 0.5 \times 0.3 \mathrm{~m})$ that was subsequently used to perform the experiments in conscious animals. This study was approved by the animal experiment ethics committee of University of Arkansas Medical Sciences.

\section{Experimental Design (Figure 1)}

The experiment started after a recovery period of 7-10 days. The experiments were started at 15:00h on the experimental day, six hours after the last food intake (half of the daily amount: $0.5 \mathrm{~kg}$ ) and selection of animals for the Sepsis group and the Control group in a randomized fashion (Control $n=9$, Sepsis $n=13$ ). We obtained baseline blood pressures in the pre-septic period ( $T=-2$ to Oh, Baseline: BL). At $\mathrm{T}=\mathrm{Oh}$, sepsis was induced by continuous infusion of Pseudomonas aeruginosa (PA, $10^{9} \mathrm{CFU} / \mathrm{ml} /$ hour), while the Control group received an equal volume of $0.9 \% \mathrm{NaCl}$ solution. Fluid resuscitation was provided $(0.9 \% \mathrm{NaCl}$ solution, $30 \mathrm{ml} / \mathrm{kg} \mathrm{bw} / \mathrm{hour})$ from $\mathrm{T}=\mathrm{Oh}$ and hemodynamics was monitored continuously.

Para-aminohippuric acid was infused to determine plasma flow $(25 \mathrm{mM} ; 60 \mathrm{ml} / \mathrm{h}$ into the splenic vein for SPL and PDV plasma flow; $60 \mathrm{ml} / \mathrm{h}$ into the femoral artery for $H Q$ plasma flow) as described previously $(18,19)$. We described that in the timeframe of $12-18 \mathrm{~h}$ the pigs showed a relative stable cardiovascular period (16). Therefore, in the $\mathrm{BL}$ period and $\mathrm{T}=12$ to $18 \mathrm{~h}$ (POST) after the start of PA, we measured systemic (arterial) AA levels, Wb protein metabolism and across organ net balances (NB) of

\begin{tabular}{|c|c|}
\multicolumn{1}{c|}{ Fluid resuscitation } \\
\hline Tracer B & Tracer B \\
\hline Tracer A & Tracer A \\
\hline
\end{tabular}

(h) -2

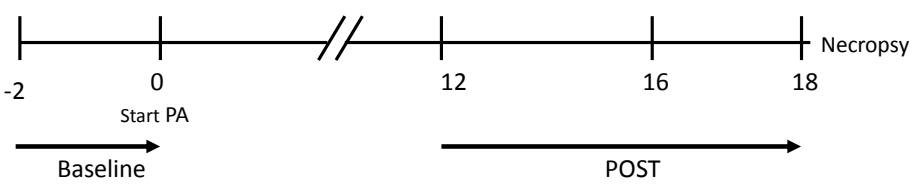

Figure 1. Experimental Design

IV infusions and blood sampling. PA is Pseudomonas aeruginosa infusion $\left(10^{9} \mathrm{CFU} / \mathrm{ml} / \mathrm{hour}\right)$. Fluid resuscitation is $0.9 \% \mathrm{NaCL}, 30 \mathrm{ml} / \mathrm{kg}$ bw/hour. Tracer A: primed-continuous infusion: $\mathrm{L}$-[ring- ${ }^{13} \mathrm{C}_{6}$ ]-phenylalanine, L-[3,3- ${ }^{2} \mathrm{H}_{2}$ ]-tyrosine, L-[1 $\left[{ }^{13} \mathrm{C}\right]-\mathrm{L}$-leucine (2 ml/kg bw/hour). Tracer $\mathrm{B}$ : primed-continuous infusion: L-[guanido- $\left.{ }^{15} \mathrm{~N}_{2}\right]$-ariginine, $\mathrm{L}-\left[{ }^{2} \mathrm{H}_{3}\right]$-methyl-histidine, $\left[{ }^{13} \mathrm{C}\right]$-urea, $(2$ $\mathrm{ml} / \mathrm{kg}$ bw/ hour). Arrow is multiple transorgan blood sampling from arterial, portal, hepatic, caval vein. 
splanchnic (SPL), Portal-Drained Viscera (PDV) and Hindquarter (HQ). With the use of AA tracers, we also measured Protein Synthesis (PS) and Protein Breakdown (PB) of SPL, PDV and HQ. At T=18, we euthanized the pigs with $125 \mathrm{mg} / \mathrm{kg}$ pentobarbital sodium and $16 \mathrm{mg} / \mathrm{kg}$ phenytoin sodium (Euthanaso ${ }^{\circledR}$ ) administered via the central vein catheter.

\section{Pseudomonas aeruginosa}

For the induction of sepsis, we used a live PA human strain (IRS 12-4-4, Shriners burns institute, University of Texas Medical Branch, Galveston). Based on pilot virulence experiment, $10^{9}$ (CFU/hour) in a volume of $1 \mathrm{ml} 0.9 \% \mathrm{NaCl}$ solution was needed to obtain similar cardiovascular responses and hemodynamic variables with characteristics of severe hyperdynamic sepsis. We monitored hemodynamics continuously to ensure that the hyperdynamic state was kept in the expected ranges for severe sepsis (body temp increase of $2-3^{\circ} \mathrm{C}$, respiratory rate increased, MPAP increased but $<35 \mathrm{mmHg}$, heart rate increased but $<200$ BPM).

\section{Infusion and sampling protocol}

\section{Stable isotopes}

Primed continuous infusions of the following stable isotopes were given via the central caval catheter: L-[ring $\left.{ }^{13} \mathrm{C}_{6}\right]-P h e, \quad L-\left[3,3-{ }^{2} \mathrm{H}\right]-\mathrm{Tyr}, \mathrm{L}-\left[1-{ }^{13} \mathrm{C}\right]-\mathrm{Leu}, \mathrm{L}-\left[{ }^{2} \mathrm{H}_{3}\right]-\mathrm{mH}$ is, $\left[{ }^{13} \mathrm{C}\right]$-Urea and L-[guanidino- $\left.{ }^{15} \mathrm{~N}_{2}\right]$-Arg (Cambridge Isotopes, Andover, MA) to determine whole body or across organ PB or PS. Additionally, myofibrillar muscle PB was determined by the whole body rate of appearance (WbRa) of methyl histidine (20, 21), and urea metabolism by studying total and extra hepatic ureagenesis (Table 1). The prime and infusion was given respectively in a volume of $2 \mathrm{ml} / \mathrm{kg}$ bw and $2 \mathrm{ml} /$ $\mathrm{kg}$ bw/hour in the time periods indicated in Figure 1.

Table 1. Stable isotope tracers

\begin{tabular}{lll}
\hline Tracer & $\begin{array}{l}\text { Prime } \\
(\mu \mathrm{mol} / \mathrm{kg} \mathrm{bw} / \mathrm{min})\end{array}$ & $\begin{array}{l}\text { Infusion rate } \\
(\mu \mathrm{mol} / \mathrm{kg} \mathrm{bw} / \mathrm{hour})\end{array}$ \\
\hline $\mathrm{L}-\left[\mathrm{ring}-{ }^{13} \mathrm{C}_{6}\right]$-phenylalanine & 1.6 & 4.3 \\
$\mathrm{~L}-\left[3,3-{ }^{2} \mathrm{H}_{2}\right]$-tyrosine & 2.5 & 5.4 \\
$\mathrm{~L}-\left[1-{ }^{13} \mathrm{C}\right]-$-leucine & 16 & 24 \\
$\mathrm{L-}\left[\right.$ guanido- $\left.{ }^{15} \mathrm{~N}_{2}\right]$-arginine & 4.6 & 8.4 \\
$\mathrm{L-}\left[{ }^{2} \mathrm{H}_{3}\right]$-methyl-histidine & 0.38 & 0.74 \\
{$\left[{ }^{13} \mathrm{C}\right]-$ urea } & 73 & 15 \\
\hline
\end{tabular}

\section{Blood sampling and Sample processing}

Blood samples were collected on ice and immediately processed within one hour as described previously $(16,19)$ In brief: for AA concentration and enrichment analysis, we centrifuged heparinized blood at $4^{\circ} \mathrm{C}, 5$ minutes, $8000 \mathrm{G}$ and deproteinized 
$250 \mu \mathrm{L}$ plasma with $25 \mu \mathrm{L}$ tri-chloroacetic acid solution (TCA, 50\% w/v) and finally snap frozen in liquid nitrogen and store at $-80 \mathrm{C}$.

\section{AA concentration and enrichments}

We determined AA isotope concentrations and AA enrichments (TTR) on a fully automated LC-MS/MS system (QTrap 5500 MS, AB Sciex, Foster City, CA, USA) with Express HT Ultra LC (Eksigent Div., AB Sciex, Foster City, CA, USA). For concentration and TTR measurements we added $20 \mu \mathrm{l}$ of TCA deproteinized plasma supernatant to $20 \mu \mathrm{AA}$ (high mass stable isotopes) internal standard. Within 3 days before the LC-MS/MS analysis, we derivatized the samples together with external standards at concentrations within the physiological range (calibration curve of concentration) and enriched external standards in the range of expected TTRs (calibration curve for TTRs) with 9-fluorenylmethoxycarbonyl (Fmoc). After neutralization, we injected $160 \mathrm{~nL}$ of the solution onto a micro LC column $0.5 \times 100 \mathrm{~mm}$ HALO C18, $2.7 \mathrm{um}$, $90 \mathrm{~A}$ pores (ABsciex, Foster City, CA, USA), kept at $35^{\circ} \mathrm{C}$. We eluted analytes with a segmentally linear gradient from 35\% to 85\% acetonitrile in water supplemented with ammonium acetate to $10 \mu \mathrm{M}$ and 5\% isopropanol. We used electrospray triple quadrupole tandem mass spectrometry in multiple reactions monitoring mode for detection. We fragmented the Fmoc AA derivatives in the collision cell for detection of either free aminoacyl anions or a fragment larger by 26 atom mass units (coming from the Fmoc derivative) to have the highest sensitivity. We simultaneously did mass analyses for AA tracee, its tracers and internal standards. We calculated the mass signal areas to enable TTR or tracee concentrations calculations.

\section{Calculations}

\section{Calculation tracee concentration and TTR from LC-MS/MS obtained signals}

For concentration calculations, we normalized the tracee signals of the samples and the external standards with their internal AA standard. We determined the plasma tracee concentration with the calibration curve of external AA standards. Plasma enrichment (TTR) was determined using the peak area ratio of the tracee and tracer signal and a calibration curve of enriched external standards. For the tracer concentration in the infusions, we used a calibration curve of tracer containing external standards.

\section{Calculation $\mathrm{Wb}$ fluxes}

Wb protein metabolism fluxes on post-absorptive state were determined by measuring WbRa of protein turnover related AA's as described previously (18, 22-24):

Equation (1) $\quad$ WbRa $=\mathrm{I} / \mathrm{TTR}$

Where "l" is infusion rate of the tracers and "TTR" the arterial steady state TTR. Proxies for WbPB are the WbRa of Phe or Leu: 
Equation (2) WbRa_Phe $=1 / T T R \_\left[\text {ring }{ }^{13} \mathrm{C}_{6}\right]-$ Phe

Or

Equation (3) WbRa_Leu $=\mathrm{I} / \mathrm{TTR} \_\left[1-{ }^{13} \mathrm{C}\right]$-Leu

The rate $(Q)$ of Phe that is hydroxylated to Tyr is:

Equation (4) Qphe>tyr $=$ WbRa_Tyr ${ }^{*}$ TTR_[ring $\left.-{ }^{13} \mathrm{C}_{6}\right]-\mathrm{Tyr} /$

TTR_[ring $-{ }^{13} \mathrm{C}_{6}$ ]-Phe

Equation (5) WbPS $=$ WbRa_Phe - QPhe $>$ Tyr

Equation (6) Urea production $=\mathrm{WbRa}$ _urea $=\mathrm{I} / \mathrm{TTR}\left[{ }^{13} \mathrm{C}\right]$-urea

Equation (7) Extra hepatic arginase activity $=$ Qarg $>$ urea $=$ WbRa_urea * TTR_ $\left[{ }^{15} \mathrm{~N}_{2}\right]$-urea/TTR_[guanido- $\left.{ }^{15} \mathrm{~N}_{2}\right]$-arginine

A proxy for skeletal muscle breakdown is the WbRa of the post-translationally modified AA mHis.

Equation (8) Myofibrillar protein breakdown $=$ WbRa_mHis $=\mathrm{l} /$

TTR_[ $\left.{ }^{2} \mathrm{H}_{3}\right]-\mathrm{mHis}$

\section{Calculation transorgan net arterial-venous balances (NB)}

Transorgan or organ NB of AA are calculated as described previously $(18,22,24)$. In principle the tracee NB across an organ is the multiplication of arterial-venous AA concentration difference with the plasma flow:

Equation (9) Transorgan net balance $=\mathrm{NB}=$ Flowplasma $*([\mathrm{~A}]-[\mathrm{V}])$

A positive value represents net uptake and a negative value represents net release. To calculated PDV, SPL and HQ AA NB's, we used the previously reported plasma flow of the present used cohort of animals (16).

\section{Calculation organ disposal and production}

Organ disposal and production of AAs which are only used for PS and come from only PB can be used as a proxy for organ PS and PB and were calculated as described previously using a two pool model $(22,24)$. Therefore for the $H Q$, we used the Phe/Leu/Tyr tracers for PB and Phe/Tyr for PS due to conversion of Leu to $\alpha$-ketoisocaproate $(\mathrm{KIC})$ in the HQ. For the total SPL area, we used Phe for PB as hydroxylation of Phe to Tyr takes place in the liver and Leu is converted to KIC in 
PDV, invalidating Phe and Leu to be used for PS. Additionally conversion of KIC to Leu in the liver invalidates the use of Leu for PB. In the PDV, Phe/Leu/Tyr tracers can be used for PB and Phe/Tyr for PS.

Equation (10) Organ tracer net balance $=\mathrm{nb}=$ Flow $*\left\{\left([\mathrm{~A}]^{*} \mathrm{TTR}_{\mathrm{art}}\right)\right.$ ([V]*TTRvenous)\}

AAs that are used for organ PS:

Equation (11) Disposal $=\mathrm{nb} / \mathrm{TTR}$ venous

AAs that comes from organ PB:

Equation (12) Production $=$ Disposal - NB

\section{Statistical analyses}

Results are presented as means \pm SEM. For the baseline period $(\mathrm{BL})$ absolute values were given. In the POST period absolute differences (difference POST-BL) are described. Graphpad Prism (version 7) was used for statistics. Levels of significance was set on $p<0.05$. To compare data between the Control and Sepsis group in the POST period an unpaired t-test was used. Wilcoxon Signed Rank Test was used to determine if fluxes, NB, disposal, production and difference POST-BL are different from zero. Average AA concentrations in BL and POST were determined with horizontal line fitting. Steady state TTR values in these same periods were estimated with plateau values obtained from one-phase association fitting of the individual TTR curves (Graphpad Prism, version 7).

\section{Results}

Clinical characteristics including hemodynamics, clinical chemistry, inflammation and plasma flow of the cohort of animals described in the present study are described extensively in Chapter 6 (16). In brief: of the 27 animals, we lost five animals in the recovery phase of the catheter implantation surgery due to intestinal complications (ileus, ascites or peritonitis). However, no animals were lost during surgery and the PA experiment. With main diagnostic criteria of SIRS (body temperature: increase of more than two degrees Celsius; heart rate: >90 beats/min; PaCO2: <32 $\mathrm{mmHg}$ ), we concluded that the present PA bacteremia has successfully induced sepsis in pigs. We found that despite fluid resuscitation pronounced hypotension, tachycardia and a decreased PDV plasma flow were present, indicating a disturbed cardiovascular function. Severe sepsis was established between 12-18 h after starting the PA infusion. 


\section{Whole body}

\section{Systemic AA concentrations (Table 2)}

Most of the AA concentrations were decreased in both experimental groups in the POST period in comparison with the baseline (Figure 2, 4, 5). Only Glu concentrations
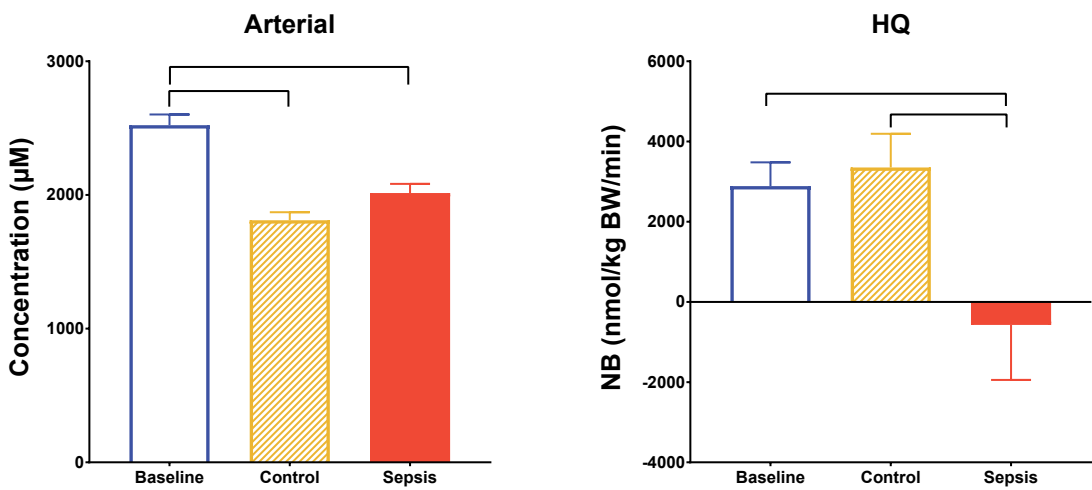

PDV
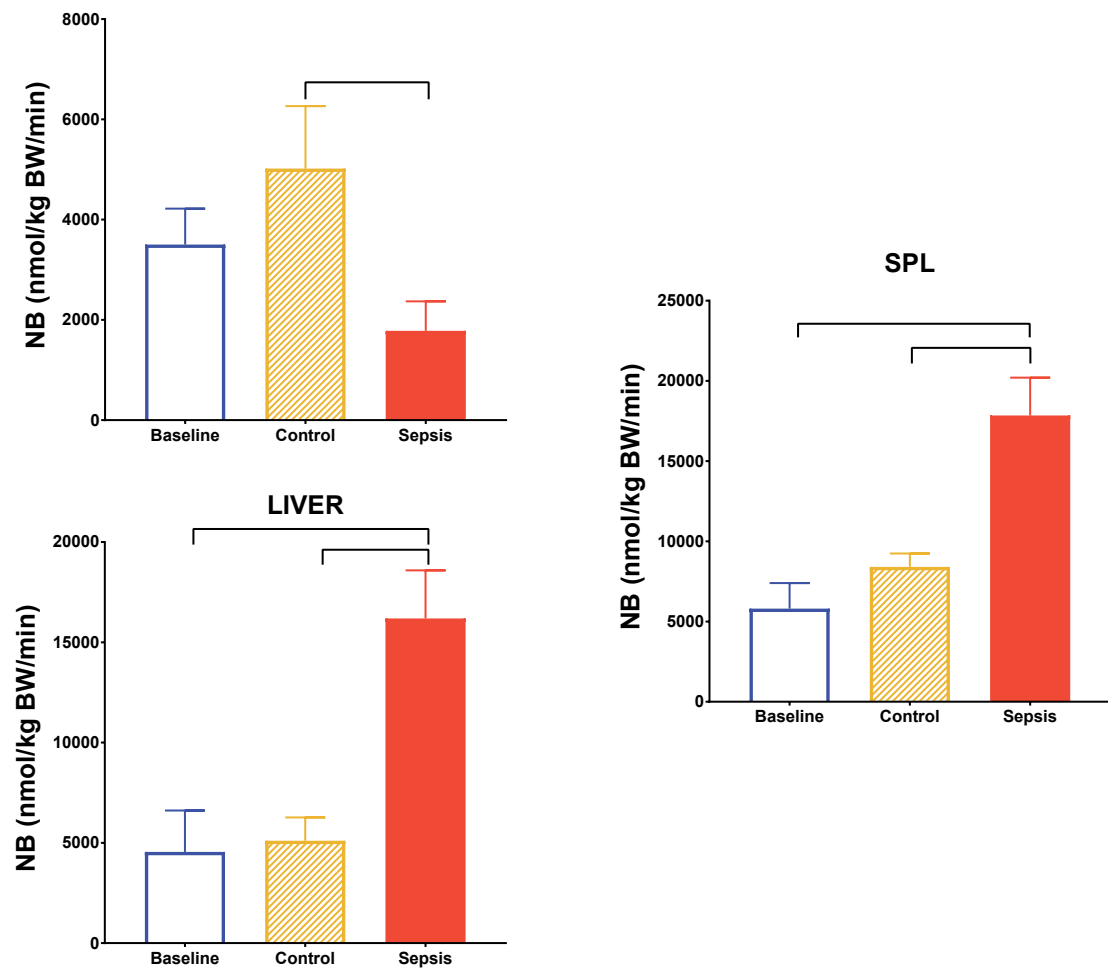

Figure 2. Total amino acid concentrations and organ net balances (NB)

Baseline and post infusion of Pseudomonas aeruginosa. HQ is hindquarter (muscle compartment), PDV is Portal drained viscera, SPL is splanchnic area. Control $n=9$, Sepsis $n=13$. Statistics: unpaired Student t-test, Significance $p<0.05$ (hook) 
decreased more severely in the Sepsis group (Figure 3). This in contrast with plasma concentrations of several EAA that were less reduced or elevated in comparison to the Control group. In addition, plasma myofibrillar protein metabolite mHis concentration was increased in the septic group. Urea concentration in the sepsis group was higher than in the Control group.

Table 2: Arterial Plasma Amino Acid Concentrations

\begin{tabular}{l|l|lll}
\hline \multirow{2}{*}{ Histidine } & Baseline & \multicolumn{3}{l}{ POST - Baseline $(\Delta)$} \\
\cline { 2 - 5 } Isoleucine & & Control & Sepsis & PT-test \\
Leucine & $78(2)$ & $-19(7)^{*}$ & $-11(4)^{*}$ & 0.3219 \\
Lysine & $67(2)$ & $1(8)$ & $23(7)^{*}$ & 0.0526 \\
Methionine & $131(35)$ & $-11(10)$ & $4(9)$ & 0.2747 \\
Phenylalanine & $81(8)$ & $49(10)^{*}$ & $61(12)^{*}$ & 0.4574 \\
Threonine & $63(9)$ & $-59(18)^{*}$ & $-20(7)^{*}$ & 0.0363 \\
Tryptophan & $76(2)$ & $5(7)$ & $54(9)^{*}$ & 0.0008 \\
Valine & $86(8)$ & $-3(18)$ & $35(15)^{*}$ & 0.1198 \\
Alanine & $27(2)$ & $-12(3)^{*}$ & $-13(3)^{*}$ & 0.7923 \\
Arginine & $207(6)$ & $-61(18)^{*}$ & $-5(15)$ & 0.0274 \\
Asparagine & $409(16)$ & $-173(41)$ & $-41(51)$ & 0.0699 \\
Citrulline & $138(9)$ & $-35(15)$ & $-35(13)^{*}$ & 0.9740 \\
Glutamate & $44(3)$ & $-22(6)^{*}$ & $-10(5)$ & 0.1191 \\
Glutamine & $62(5)$ & $-23(4)^{*}$ & $-25(6)^{*}$ & 0.7925 \\
Ornithine & $123(5)$ & $-38(5)^{*}$ & $-87(6)^{*}$ & $<0.0001$ \\
Proline & $416(19)$ & $-162(21)^{*}$ & $-141(20)^{*}$ & 0.4993 \\
Serine & $100(6)$ & $-36(9)^{*}$ & $-33(12)^{*}$ & 0.8168 \\
Tyrosine & $254(17)$ & $-162(32)^{*}$ & $-94(23)^{*}$ & 0.0946 \\
BCAA & $95(3)$ & $7(5)$ & $17(7)^{*}$ & 0.3092 \\
EAA & $54(3)$ & $-6(8)$ & $-13(4)^{*}$ & 0.4592 \\
TAA & $403(10)$ & $-71(35)$ & $22(30)$ & 0.0598 \\
3-Methyl-Histidine & $826(23)$ & $-111(82)$ & $138(64)$ & 0.0250 \\
Urea & $2522(80)$ & $-760(184)^{*}$ & $-265(183)$ & 0.0793 \\
\hline & $2992(157)$ & $-0.1(0.1)$ & $0.2(0.04)^{*}$ & 0.0211 \\
\hline & $-2063(302)^{*}$ & $165(248)$ & $<0.0001$ \\
\hline
\end{tabular}

Arterial plasma concentrations before (Baseline: $\mathrm{T}=-2$ to $\mathrm{Oh}$ ) and after (POST: $\mathrm{T}=16$ to 18h) start of Pseudomonas aeruginosa infusion. Data are expressed as mean (SEM) in $\mu \mathrm{mol} / \mathrm{l}$. "Baseline" is concentration before start PM infusion N=22. "POST-Baseline" is difference between POST and baseline period; Control: $\mathrm{N}=9$; Sepsis: $\mathrm{N}=13$; negative value is decrease, positive value is increase. BCAA: Sum of the branched-chain amino acids: Valine, Leucine and Isoleucine. EAA: histidine, isoleucine, leucine, lysine, methionine, phenylalanine, threonine, tryptophan, valine; TAA: EAA+ alanine, arginine, asparagine, citrulline, glutamate, glutamine, ornithine, proline, serine, tyrosine. Statistics POST: Different from zero tested with Wilcoxon Signed Rank test, $\left.{ }^{*}\right) \mathrm{p}<0.05$. Effect of sepsis was tested with Unpaired T-test. Significance different compared to Control, $\mathrm{P}<0.05$ (bold). Tendency: $\mathrm{P}<0.1$ 

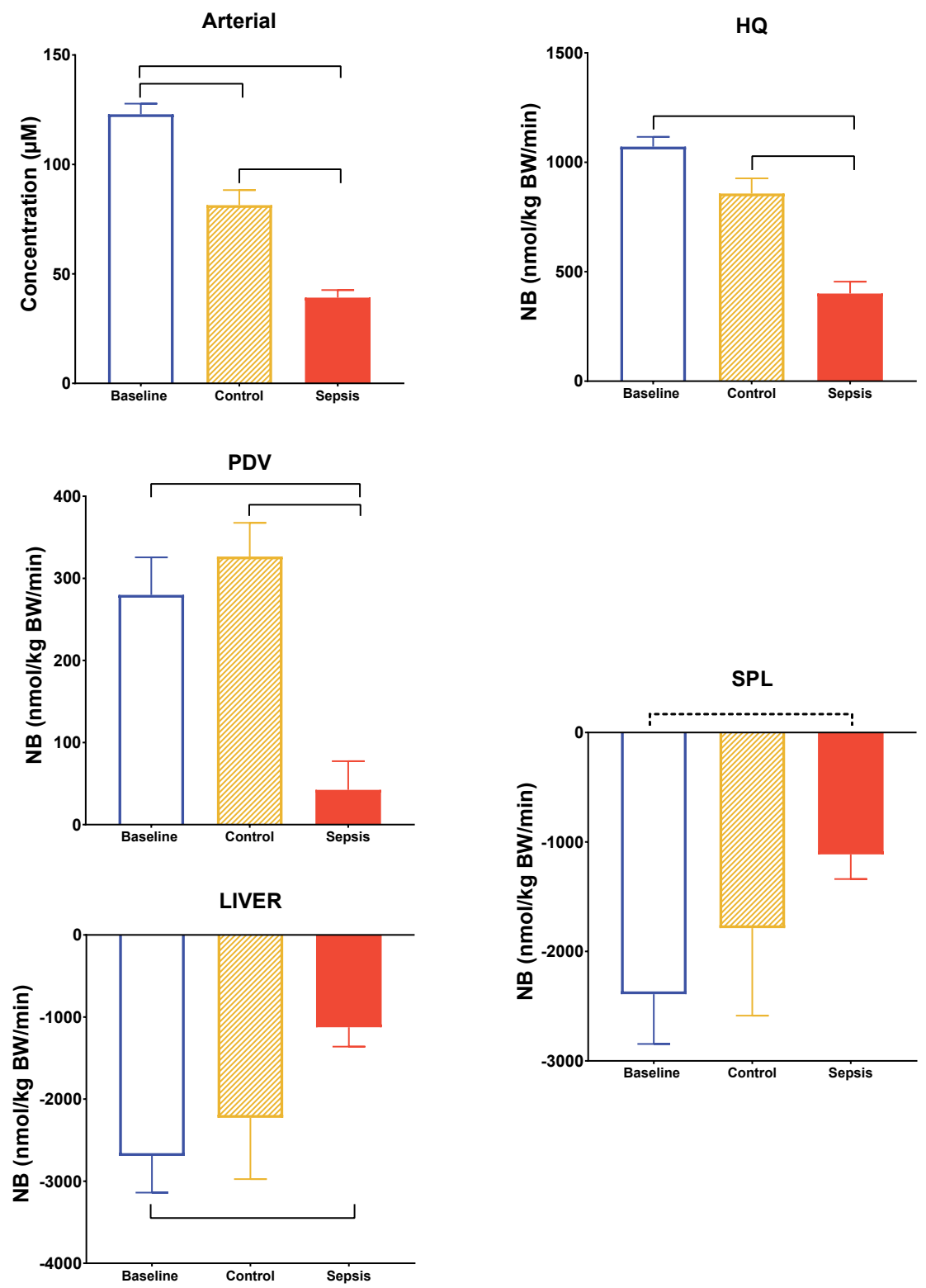

Figure 3. Glutamate arterial concentrations and organ net balances (NB)

Before (Baseline) and post infusion of Pseudomonas aeruginosa. HQ is hindquarter (muscle compartment), PDV is Portal drained viscera, SPL is splanchnic area. Control $n=9$, Sepsis $n=13$. Statistics: unpaired Student t-test, Significance $p<0.05$ (hook), Tendency $p<0.1$ (dashed hook). 


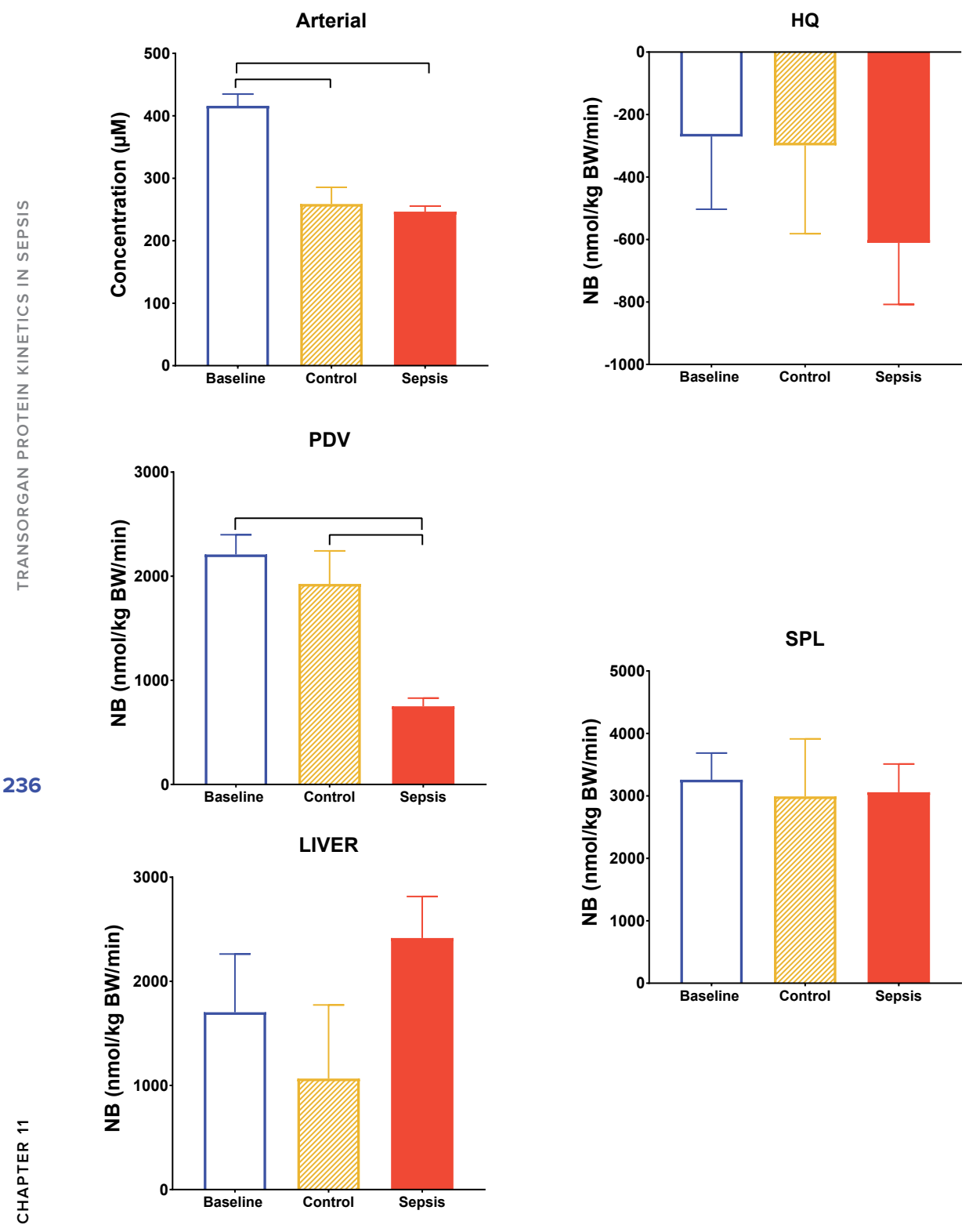

Figure 4. Glutamine arterial concentrations and organ net balances (NB)

Before (Baseline) and post infusion of Pseudomonas aeruginosa. HQ is hindquarter (muscle compartment), PDV is Portal drained viscera, SPL is splanchnic area. Control $n=9$, Sepsis $n=13$. Statistics: unpaired Student t-test, Significance $p<0.05$ (hook). 

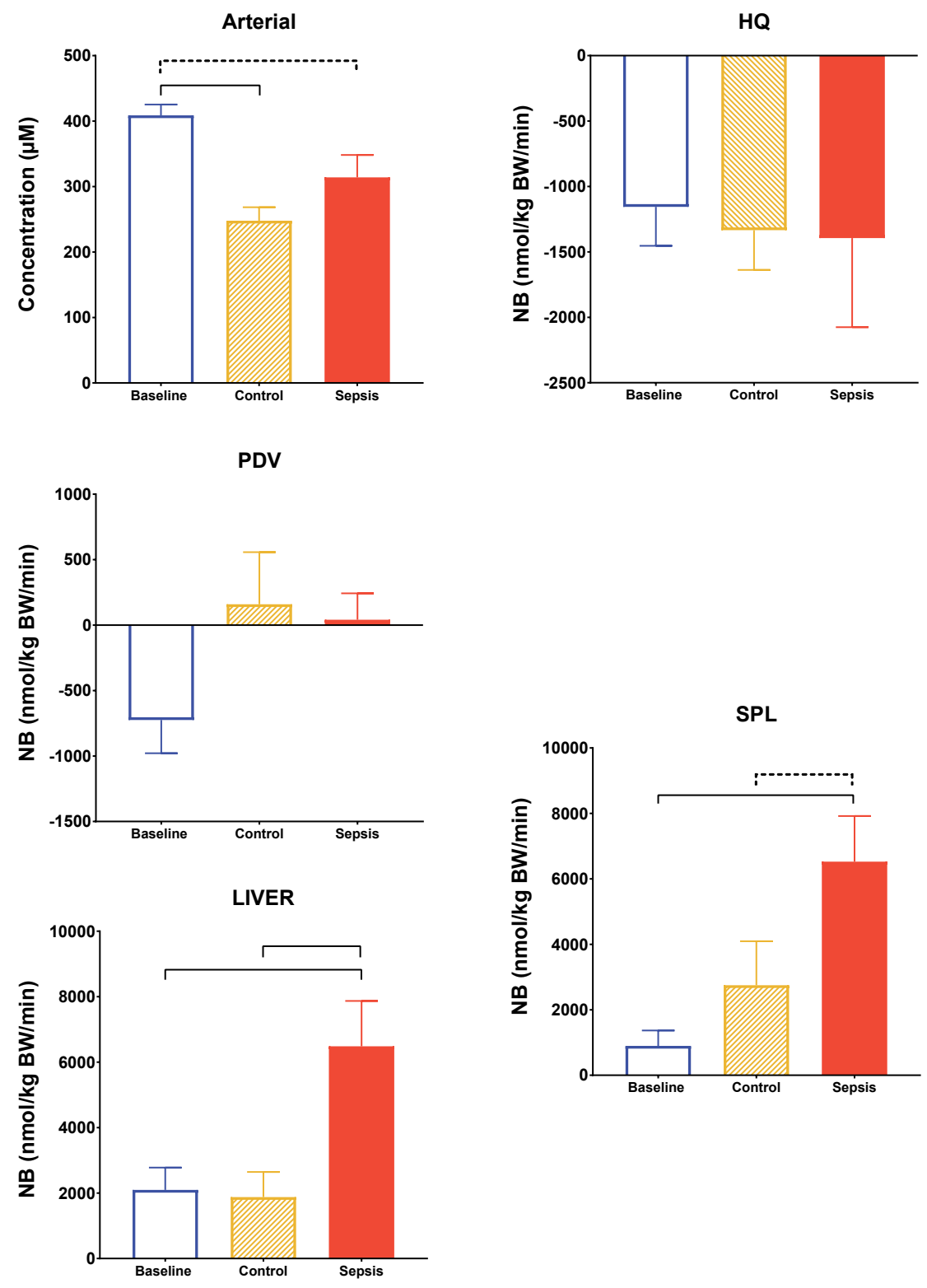

Figure 5. Alanine arterial concentrations and organ net balances (NB)

Before (Baseline) and post infusion of Pseudomonas aeruginosa. HQ is hindquarter (muscle compartment), PDV is Portal drained viscera, SPL is splanchnic area. Control $n=9$, Sepsis $n=13$. Statistics: unpaired Student t-test, Significance $p<0.05$ (hook). Tendency $p<0.1$ (dashed hook) 


\section{Rate of appearance and disappearance of Phe/Leu/Tyr as proxy for WbPS, WbPB, netPS (Table 3)}

WbPB, WbPS and net PB were higher in the Sepsis group in comparing to Control group, indicating more protein catabolism in sepsis. The increase in PB was observed both in the Ra of Phe and Leu, albeit more prominent in Phe. Sepsis also increased the fraction of WbPB derived from myofibrillar protein breakdown. In addition, total urea production and extra hepatic urea production from arginase was substantially increased.

\section{Hindquarter}

\section{Transorgan NB of AAs (Table 4)}

Except for GIn and Ala, most AA were taken up by the hindquarter in the BL period (Figure 4,5). Although most of the individual EAA were taken up less or even released in the sepsis group, this was only significant for Leu, Met and Thr. Lys was taken up substantially more in both groups, but was not different between the Sepsis and Control group. We found no significant difference between these groups for total EAA. This in contrast with TAA that showed a disappearance of the uptake (no significant net release/uptake) in the HQ in the sepsis group (Figure 2). Sepsis increased the release of $\mathrm{mHis}$ by the $\mathrm{HQ}$.

\section{Protein metabolism (Table 5)}

The HQ showed net PS during BL period. In POST period net PS is decreased significant (with the Tyr tracer) in the sepsis group. However not significant in comparing to the Control group. Also PB in the sepsis group was not significant different from the Control group Therefore, the diminished net PS (NB of Leu or Tyr) in the sepsis group is not the result of a clear enhanced PB and/or diminished PS in the $H Q$ in present severe septic state.

\section{Portal Drained Viscera}

\section{Transorgan NB of AAs (Table 6)}

In the $\mathrm{BL}$ period, the PDV is taken up almost all $\mathrm{AA}$ and is releasing Ala and Cit (Figure 2, 5). In the POST period, the Control group showed a different profile of uptake. For instance, we found an increase in uptake of individual AA like Leu, Thr, Arg, Glu and Ser and a reduced uptake of Pro. In the sepsis group the uptake of AAs is decreased in comparison to control group. And this was significant for the individual non-EAA: Arg, GIn (Figure 4) and Ser. No significant uptake of Glu was found (Figure 3). Cit production was the same between the control and sepsis group.

\section{Protein metabolism (Table 5)}

In the BL period PDV protein metabolism is in balance: no significant net PS or PB is observed. After administration of PA, Both PDV PS as PB were reduced in sepsis in comparison to control, but this decreased protein turnover did not change net PDV protein balance. 


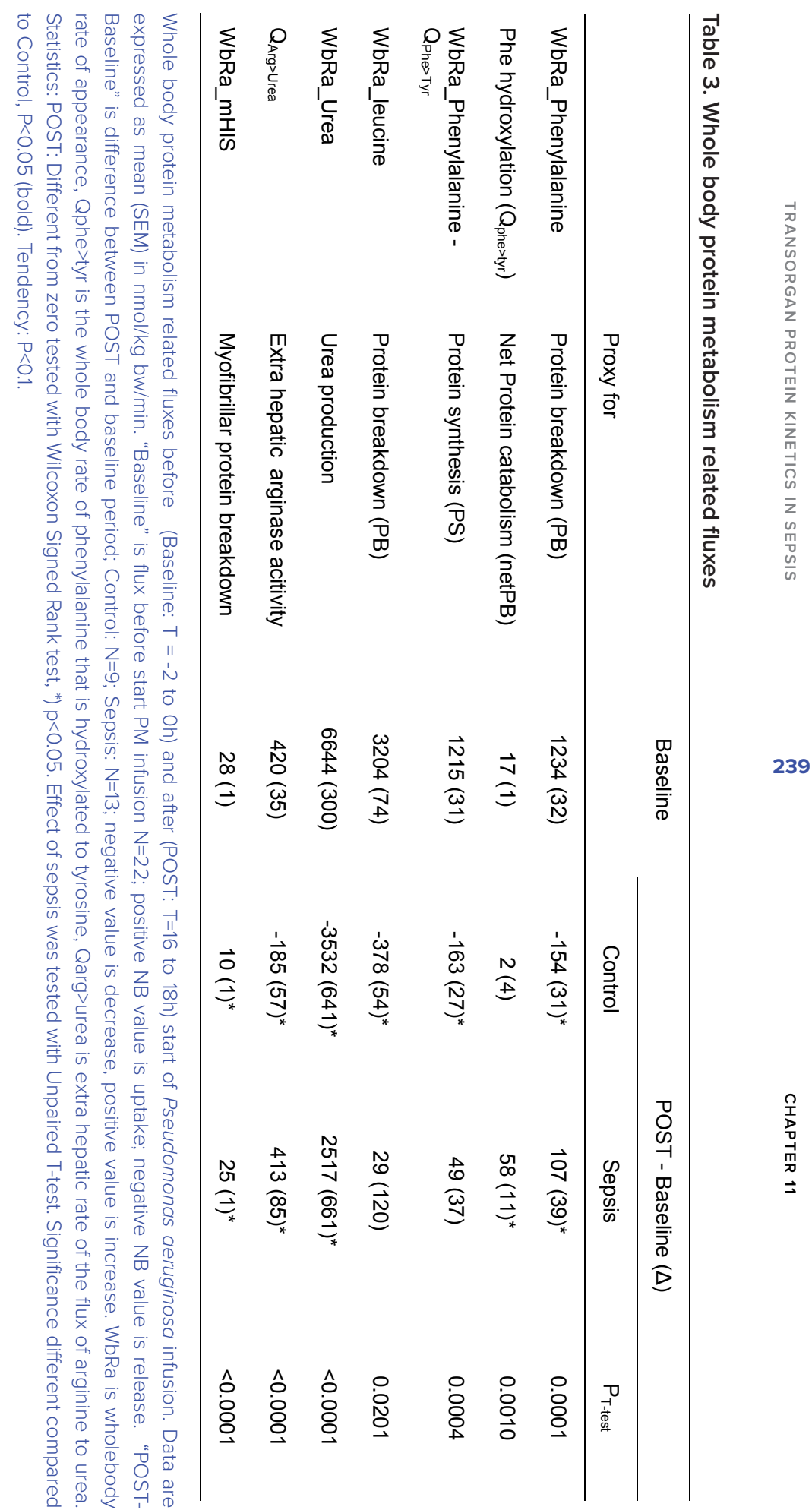


Table 4. Hindquarter amino acid net balances

\begin{tabular}{|c|c|c|c|c|c|}
\hline & \multicolumn{2}{|l|}{ Baseline } & \multicolumn{3}{|c|}{ POST - Baseline $(\Delta)$} \\
\hline & & PWilcoxon & Control & Sepsis & PT-test \\
\hline Histidine & $215(40)$ & $<0.0001$ & $-100(71)$ & $-249(77)^{*}$ & 0.1886 \\
\hline Isoleucine & $213(30)$ & $<0.0001$ & $-60(37)$ & $-163(92)$ & 0.4064 \\
\hline Leucine & $237(47)$ & $<0.0001$ & $54(42)$ & $-257(92)$ & 0.0146 \\
\hline Lysine & $235(48)$ & $<0.0001$ & $1842(293)^{*}$ & $2160(404)^{*}$ & 0.5824 \\
\hline Methionine & $120(22)$ & $<0.0001$ & $-133(50)$ & $-266(33)^{*}$ & 0.0331 \\
\hline Phenylalanine & $104(22)$ & $<0.0001$ & -119 (31) & $-188(85)$ & 0.1227 \\
\hline Threonine & $221(44)$ & $<0.0001$ & $-123(52)$ & $-484(92)^{*}$ & 0.0066 \\
\hline Tryptophan & $47(8)$ & $<0.0001$ & $-46(19)$ & $-100(17)^{*}$ & 0.0515 \\
\hline Valine & $514(67)$ & $<0.0001$ & $-206(78)^{*}$ & $-477(132)^{*}$ & 0.1316 \\
\hline Alanine & $-1156(298)$ & 0.0009 & $1114(340)^{*}$ & -1093 (669) & 0.0285 \\
\hline Arginine & $276(41)$ & $<0.0001$ & $-111(66)$ & $-520(102)^{*}$ & 0.0065 \\
\hline Asparagine & $90(24)$ & 0.0010 & $-119(64)$ & $-220(46)^{*}$ & 0.2096 \\
\hline Citrulline & $92(13)$ & $<0.0001$ & $-37(33)$ & $-63(18)^{*}$ & 0.4626 \\
\hline Glutamate & $1072(45)$ & $<0.0001$ & $-114(75)$ & $-682(95)^{*}$ & 0.0003 \\
\hline Glutamine & $-270(233)$ & 0.2572 & $151(783)$ & $-377(310)$ & 0.2283 \\
\hline Ornithine & 217 (33) & $<0.0001$ & $-129(78)$ & $-208(122)$ & 0.6297 \\
\hline Proline & $592(47)$ & $<0.0001$ & $-469(127)$ & $-621(122)$ & 0.3878 \\
\hline Serine & $453(63)$ & $<0.0001$ & $-21(92)$ & $-595(132)^{*}$ & 0.0042 \\
\hline Tyrosine & $82(24)$ & 0.0025 & $-37(29)$ & $-214(45)^{*}$ & 0.0075 \\
\hline BCAA & 964 (138) & $<0.0001$ & $-164(133)$ & $-898(290)^{*}$ & 0.0596 \\
\hline EAA & 1864 (288) & $<0.0001$ & $1090(542)$ & $-53(648)$ & 0.2204 \\
\hline TAA & 2887 (593) & 0.0002 & $1675(920)$ & $-5148(1636)^{*}$ & 0.0043 \\
\hline 3-Methyl-Histidine & $0.3(1.4)$ & 0.7854 & $-3.2(3.3)$ & $4.4(0.9)^{*}$ & 0.0137 \\
\hline
\end{tabular}

Hindquarter net balances (NB) before (Baseline: $\mathrm{T}=-2$ to Oh) and after (POST: T=16 to 18h) start of Pseudomonas aeruginosa infusion. Data are expressed as mean (SEM) in nmol/kg bw/ min. Baseline is net balance before start PM infusion $N=22$; positive NB value is uptake; negative NB value is release. "POST-Baseline" is difference between POST and baseline period; Control: N=9; Sepsis: N=13; negative value is decrease, positive value is increase. BCAA: Sum of the branched-chain amino acids: Valine, Leucine and Isoleucine. EAA: histidine, isoleucine, leucine, lysine, methionine, phenylalanine, threonine, tryptophan, valine; TAA: EAA+ alanine, arginine, asparagine, citrulline, glutamate, glutamine, ornithine, proline, serine, tyrosine. Statistics: Baseline: Different from zero tested with Wilcoxon Signed Rank test, POST: Different from zero tested with Wilcoxon Signed Rank test, $\left.{ }^{*}\right) \mathrm{p}<0.05$. Effect of sepsis was tested with Unpaired T-test. Significance different compared to Control, $\mathrm{P}<0.05$ (bold). Tendency: $\mathrm{P}<0.1$. 
Table 6. Portal drained viscera net balances

\begin{tabular}{|c|c|c|c|c|c|}
\hline & \multicolumn{2}{|l|}{ Baseline } & \multicolumn{3}{|c|}{ POST - Baseline $(\Delta)$} \\
\hline & & PWilcoxon & Healthy & Sepsis & PT-test \\
\hline Histidine & 106 (39) & 0.009 & $-21(42)$ & $-116(84)$ & 0.3832 \\
\hline Isoleucine & $75(24)$ & 0.0063 & $85(80)$ & $-32(64)$ & 0.2651 \\
\hline Leucine & $-43(44)$ & 0.157 & $201(56)^{*}$ & $139(80)$ & 0.5615 \\
\hline Lysine & $-12(52)$ & $>0.9999$ & $63(74)$ & $4(153)$ & 0.7707 \\
\hline Methionine & $133(29)$ & 0.0002 & $-135(67)$ & $-106(18)^{*}$ & 0.6571 \\
\hline Phenylalanine & $-20(39)$ & 0.7593 & $21(53)$ & $48(62)$ & 0.7627 \\
\hline Threonine & $198(33)$ & $<0.0001$ & $58(20)^{*}$ & $-42(59)$ & 0.1692 \\
\hline Tryptophan & $51(10)$ & 0.0002 & $-17(19)$ & $-34(13)^{*}$ & 0.4619 \\
\hline Valine & $344(57)$ & 0.0001 & $-136(93)$ & $-273(115)^{*}$ & 0.3917 \\
\hline Alanine & $-725(254)$ & 0.0073 & $1828(878)$ & $836(505)$ & 0.3118 \\
\hline Arginine & $162(58)$ & 0.0127 & $418(142)^{*}$ & $-119(97)$ & 0.0041 \\
\hline Asparagine & $-34(43)$ & 0.7502 & $26(60)$ & $57(49)$ & 0.6948 \\
\hline Citrulline & $-183(49)$ & 0.0329 & $-76(46)$ & $-3(52)$ & 0.3278 \\
\hline Glutamate & $280(46)$ & $<0.0001$ & $154(38)^{*}$ & $-320(90)^{*}$ & 0.0005 \\
\hline Glutamine & $2209(190)$ & $<0.0001$ & 107 (399) & $-1402(343)^{*}$ & 0.0099 \\
\hline Ornithine & $122(55)$ & 0.0425 & $-95(90)$ & $-20(96)$ & 0.7805 \\
\hline Proline & 465 (101) & 0.0002 & $-495(117)^{*}$ & $-360(88)^{*}$ & 0.3585 \\
\hline Serine & $162(58)$ & 0.0127 & $418(142)^{*}$ & $-119(97)$ & 0.0041 \\
\hline Tyrosine & 84 (31) & 0.0145 & $-55(51)$ & $-70(53)$ & 0.8549 \\
\hline BCAA & $344(104)$ & 0.0023 & $150(159)$ & $-359(308)$ & 0.2126 \\
\hline EAA & $883(1277)$ & 0.0029 & 295 (369) & $-920(700)$ & 0.1919 \\
\hline TAA & 3502 (717) & 0.0001 & 2286 (1437) & $-2445(1161)$ & 0.0181 \\
\hline Methyl-Histidine & $-2.7(1.8)$ & 0.2099 & $4.0(1.5)^{*}$ & $5.5(2.6)$ & 0.6971 \\
\hline
\end{tabular}

Portal Drained Viscera net balances (NB) before (Baseline: $T=-2$ to 0 h) and after (POST: T=16 to 18h) start of Pseudomonas aeruginosa infusion. Data are expressed as mean (SEM) in nmol/ $\mathrm{kg}$ bw/min. PRE is net balance before start PM infusion $\mathrm{N}=22$; positive NB value is uptake; negative NB value is release. "POST-Baseline" is difference between POST and baseline period; Control: $\mathrm{N}=9$; Sepsis: $\mathrm{N}=13$; negative value is decrease, positive value is increase. BCAA: Sum of the branched-chain amino acids: Valine, Leucine and Isoleucine. EAA: histidine, isoleucine, leucine, lysine, methionine, phenylalanine, threonine, tryptophan, valine; TAA: EAA+ alanine, arginine, asparagine, citrulline, glutamate, glutamine, ornithine, proline, serine, tyrosine. Statistics: Baseline: Different from zero tested with Wilcoxon Signed Rank test, POST: Different from zero tested with Wilcoxon Signed Rank test, $\left.{ }^{*}\right) p<0.05$. Effect of sepsis was tested with Unpaired T-test. Significance different compared to Control, $\mathrm{P}<0.05$ (bold). Tendency: $P<0.1$. 


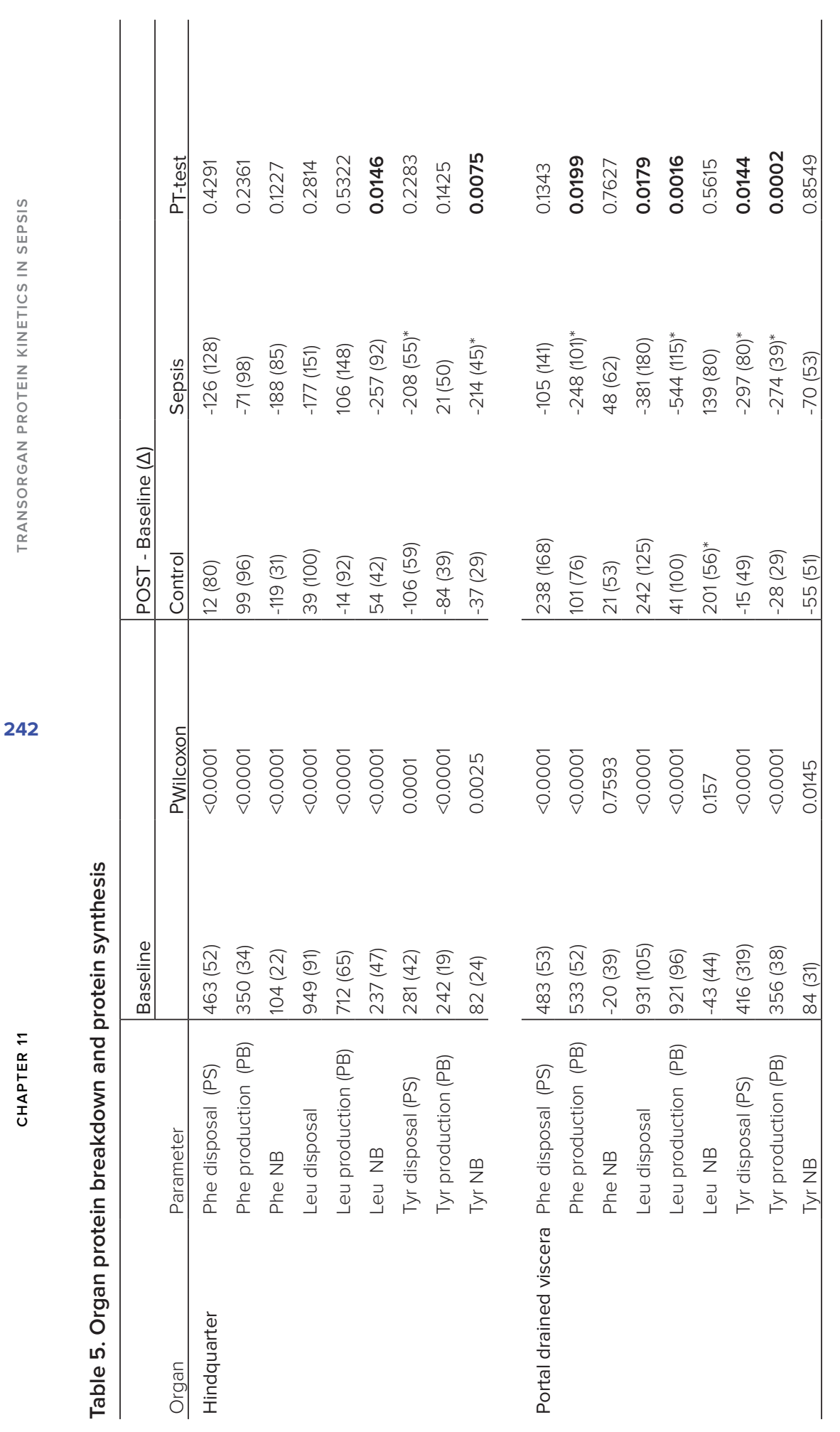




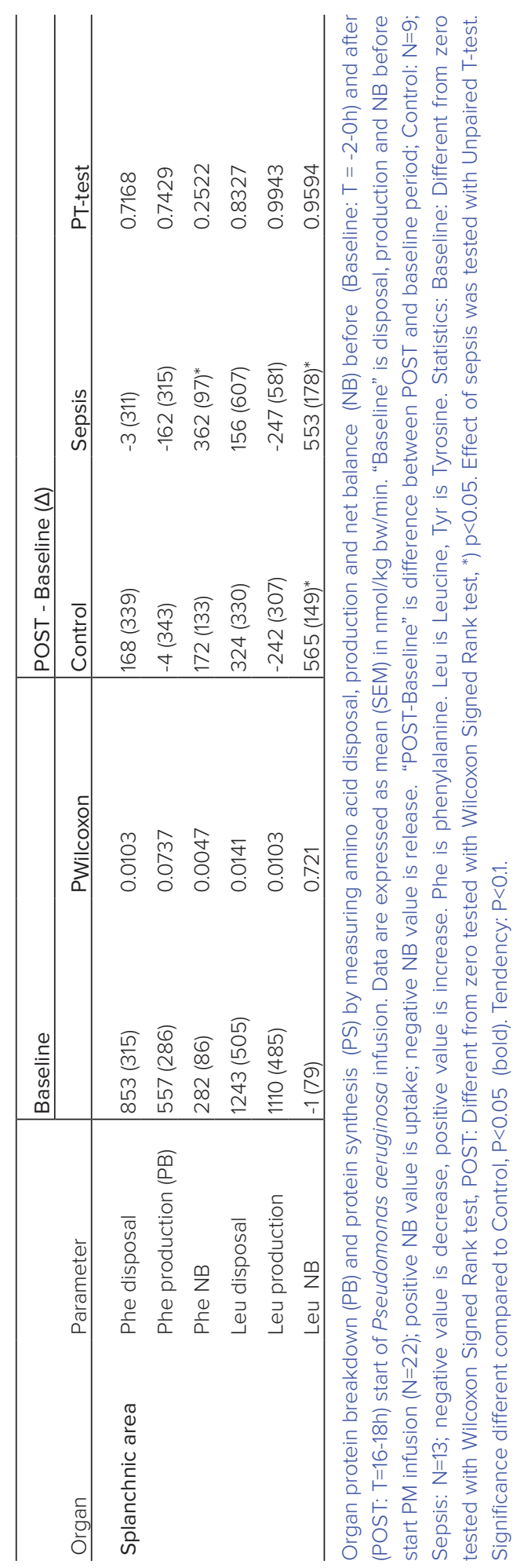




\section{Splanchnic area}

\section{Transorgan NB of AAs (Table 7)}

In the BL period the SPL area is taken up EAA except for Leu. In addition, Arg, GIn, Pro and Tyr are taken up. Glu is released from the splanchnic area. In the POST period, we observed an overall reduction of uptake of Trp, Cit, Pro and enhanced uptake of Lys in the Control group. In the sepsis group, the SPL area the uptake of AA was tripled in comparison to the control group.

\section{Table 7. Splanchnic area net balances}

\begin{tabular}{|c|c|c|c|c|c|}
\hline & \multicolumn{2}{|l|}{ Baseline } & \multicolumn{3}{|c|}{ POST - Baseline $(\Delta)$} \\
\hline & & PWilcoxon & Control & Sepsis & PT-test \\
\hline Histidine & $337(60)$ & $<0.0001$ & 69 (119) & $108(96)$ & 0.7993 \\
\hline Isoleucine & $284(75)$ & 0.0006 & 306 (162) & $123(117)$ & 0.3569 \\
\hline Leucine & $-1(79)$ & 0.721 & $565(149)^{*}$ & $553(178)^{*}$ & 0.9594 \\
\hline Lysine & $205(86)$ & 0.0390 & 347 (264) & $661(196)^{*}$ & 0.3417 \\
\hline Methionine & 595 (79) & $<0.0001$ & $-327(137)^{*}$ & $-256(67)^{*}$ & 0.6204 \\
\hline Phenylalanine & $282(86)$ & 0.0047 & $172(133)$ & $362(97)^{*}$ & 0.2522 \\
\hline Threonine & 479 (61) & $<0.0001$ & $-18(176)$ & $802(81)^{*}$ & 0.0003 \\
\hline Tryptophan & $141(17)$ & $<0.0001$ & $-94(29)^{*}$ & $-15(19)$ & 0.0273 \\
\hline Valine & $872(124)$ & $<0.0001$ & $-310(199)$ & 748 (133) & 0.1083 \\
\hline Alanine & 897 (469) & 0.0586 & $1923(567)^{*}$ & $4877(1258)^{*}$ & 0.0945 \\
\hline Arginine & 372 (112) & 0.0049 & $380(225)$ & $326(126)^{*}$ & 0.2497 \\
\hline Asparagine & $114(63)$ & 0.0841 & $-103(148)$ & $415(202)$ & 0.0728 \\
\hline Citrulline & $85(65)$ & 0.2755 & $-160(95)$ & $-323(85)^{*}$ & 0.2203 \\
\hline Glutamate & $-2390(455)$ & 0.0001 & 574 (414) & $1297(308)^{*}$ & 0.1683 \\
\hline Glutamine & $3259(428)$ & $<0.0001$ & $-1123(718)$ & $378(1066)$ & 0.2918 \\
\hline Ornithine & $100(115)$ & 0.7023 & $-12(164)$ & $-286(145)$ & 0.2276 \\
\hline Proline & $1258(134)$ & $<0.0001$ & $-732(292)^{*}$ & 89 (169) & 0.0183 \\
\hline Serine & 161 (171) & 0.4244 & 366 (292) & 64 (293) & 0.4902 \\
\hline Tyrosine & $267(58)$ & $<0.0001$ & $-169(111)$ & $204(104)$ & 0.0259 \\
\hline BCAA & 1366 (202) & 0.0001 & 561 (449) & $887(279)^{*}$ & 0.5255 \\
\hline EAA & 3072 (511) & $<0.0001$ & 710 (979) & $1994(1023)^{*}$ & 0.3886 \\
\hline TAA & 5802 (1598) & 0.0014 & 2330 (2491) & $12659(2577)^{*}$ & 0.0113 \\
\hline Methyl-Histidine & $-2.8(4.4)$ & 0.4825 & $0.4(8.1)$ & $10.8(6.3)^{*}$ & 0.3554 \\
\hline
\end{tabular}

Splanchnic area net balances (NB) before (Baseline: $T=-2$ to $0 h$ ) and after (POST: T=16 to 18h) start of Pseudomonas aeruginosa infusion. Data are expressed as mean (SEM) in nmol/kg bw/ min. Baseline is net balance before start PM infusion $N=22$; positive NB value is uptake; negative NB value is release. "POST-Baseline" is difference between POST and baseline period; Control: $N=9$; Sepsis: $N=13$; negative value is decrease, positive value is increase. BCAA: Sum of the branched-chain amino acids: Valine, Leucine and Isoleucine. EAA: histidine, isoleucine, leucine, lysine, methionine, phenylalanine, threonine, tryptophan, valine; TAA: EAA+ alanine, arginine, asparagine, citrulline, glutamate, glutamine, ornithine, proline, serine, tyrosine. Statistics: Baseline: Different from zero tested with Wilcoxon Signed Rank test, POST: Different 
from zero tested with Wilcoxon Signed Rank test, *) $p<0.05$. Effect of sepsis was tested with Unpaired T-test. Significance different compared to Control, $\mathrm{P}<0.05$ (bold). Tendency: $\mathrm{P}<0.1$.

\section{Protein metabolism (Table 5)}

In the SPL area we are only able to determine PB. PS and netPS measurement was not possible due to the disposal of the used tracers to other metabolic routes than PS. PA induced sepsis did not change PB in the SPL area.

\section{Liver}

\section{Transorgan NB of AAs (Table 8)}

Overall the liver is taken up AAs in the BL period and that is diminished in the POST period in the Control group (Figure 2). In contrast with the Control group, the sepsis group tripled the AA uptake in as well the EAA as the non-EAA AA fractions. Normal Glu release is decreased more than $50 \%$. No differences were observed in the BCAA's NB.

Table 8. Liver amino acid net balances

\begin{tabular}{|c|c|c|c|c|c|}
\hline & \multicolumn{2}{|l|}{ Baseline } & \multicolumn{3}{|c|}{ POST - Baseline $(\Delta)$} \\
\hline & Net balance & PWilcoxon & Control & Sepsis & PT-test \\
\hline Histidine & 217(59) & 0.0006 & $89(110)$ & $230(103)^{*}$ & 0.3692 \\
\hline Isoleucine & $191(62)$ & 0.0053 & 221(152) & $188(53)^{*}$ & 0.8229 \\
\hline Leucine & $42(97)$ & 0.5234 & 364(158) & $524(169)^{*}$ & 0.5166 \\
\hline Lysine & $108(75)$ & 0.1464 & $497(176)^{*}$ & $792(206)^{*}$ & 0.3239 \\
\hline Methionine & $443(67)$ & $<0.0001$ & $-192(90)$ & $-28(84)$ & 0.2061 \\
\hline Phenylalanine & $331(87)$ & 0.0006 & $34(68)$ & $248(81)^{*}$ & 0.0677 \\
\hline Threonine & $307(87)$ & 0.0004 & $-38(166)$ & $672(145)^{*}$ & 0.0046 \\
\hline Tryptophan & $106(21)$ & $<0.0001$ & $-77(26)^{*}$ & $4(22)$ & 0.0283 \\
\hline Valine & 574(151) & 0.0009 & $-174(167)$ & 235(206) & 0.1665 \\
\hline Alanine & 2095(683) & 0.0017 & $1112(535)$ & $3470(1374)^{*}$ & 0.1859 \\
\hline Arginine & 339(123) & 0.0103 & $15(116)$ & $470(142)^{*}$ & 0.0502 \\
\hline Asparagine & 204(69) & 0.0014 & $-249(124)$ & $329(105)^{*}$ & 0.0021 \\
\hline Citrulline & $202(57)$ & 0.0037 & $-152(47)^{*}$ & $-245(98)^{*}$ & 0.4889 \\
\hline Glutamate & $-2692(447)$ & $<0.0001$ & $59(225)$ & $1597(317)^{*}$ & 0.0026 \\
\hline Glutamine & 1703(559) & 0.0101 & $-1230(838)$ & 1501(888) & 0.0428 \\
\hline Ornithine & $-4(97)$ & 0.7502 & $47(164)$ & $-412(208)^{*}$ & 0.1236 \\
\hline Proline & $877(122)$ & $<0.0001$ & $-216(92)^{*}$ & $189(136)^{*}$ & 0.0463 \\
\hline Serine & $-70(155)$ & 0.6326 & $-52(328)$ & $219(244)$ & 0.5074 \\
\hline Tyrosine & $231(57)$ & 0.0007 & $-114(80)$ & $253(90)^{*}$ & 0.0087 \\
\hline BCAA & $784(276)$ & 0.0083 & $411(453)$ & $1004(202)^{*}$ & 0.2190 \\
\hline EAA & 2314(553) & 0.0003 & $415(1020)$ & $2989(704)^{*}$ & 0.0448 \\
\hline TAA & $4547(2067)$ & 0.0275 & $44(1716)$ & 10879(3401)* & 0.0218 \\
\hline Methyl-Histidine & $-0.3(3.3)$ & 0.6556 & $-3.0(6.8)$ & $5.1(4.6)$ & 0.3167 \\
\hline
\end{tabular}


Liver net balances (NB) before (Baseline: $T=-2$ to 0 h) and after (POST: $T=16$ to 18h) start of Pseudomonas aeruginosa infusion. Data are expressed as mean(SEM) in nmol/kg bw/min. "Baseline" is net balance before start PM infusion N=22; positive NB value is uptake; negative NB value is release. "POST-Baseline" is difference between POST and baseline period; Control: N=9; Sepsis: N=13; negative value is decrease, positive value is increase. BCAA: Sum of the branched-chain amino acids: Valine, Leucine and Isoleucine. EAA: histidine, isoleucine, leucine, lysine, methionine, phenylalanine, threonine, tryptophan, valine; TAA: EAA+ alanine, arginine, asparagine, citrulline, glutamate, glutamine, ornithine, proline, serine, tyrosine. Statistics: Baseline: Different from zero tested with Wilcoxon Signed Rank test, POST: Different from zero tested with Wilcoxon Signed Rank test, $\left.{ }^{*}\right) \mathrm{p}<0.05$. Effect of sepsis was tested with Unpaired T-test. Significance different compared to Control, $\mathrm{P}<0.05$ (bold). Tendency: $\mathrm{P}<0.1$

\section{Discussion}

\section{Summary data}

The presented acute severe sepsis model is characterized by increased whole body net catabolism and increased plasma EAA concentrations. We tried to find the responsible organs and tissues responsible for the increased whole body catabolism. Although the increased WbRa of $\mathrm{mHIS}$ indicates accelerated muscle myofibrillar protein breakdown, we could not detect this by an increased EAA net release and/or PB in the muscle compartment (HQ). This is likely because of increased reincorporation of EAAs from $\mathrm{PB}$ back into protein without entering plasma. Protein turnover was reduced in gut tissue, and there was no net release of AA substrates. The liver increased AA uptake substantially, in agreement with the increased urea rate of appearance. Taken together, these data indicate severe tissue protein wasting in this acute severe sepsis phase. We conclude that protein catabolism also occurred in tissues and organs we did not directly measure, as the increased net release of AA substrates from the $H Q$ and PDV cannot explain the observed net catabolism on whole body level and substantial AA substrate wasting by the liver. In addition, disturbed interorgan Glu metabolism with a key role for the liver, suggests a potential role of this AA in the initial sepsis response due to the fact that decreased systemic Glu availability is associated with higher mortality in humans (13).

\section{Changes in whole body metabolism}

The use of primed continuous tracer infusion approach, the single pool model to describe substrate kinetics (24), makes detection of changes in protein metabolism related substrates in (patho)physiological conditions possible $(2,17,25)$. Tracer steady state is an important condition to determine the whole body rate of appearance. However, it can be challenging to keep the enrichment stable during tracer steady state, due to dynamics of the sepsis condition, the rapid and unpredictable changes in the pool size that makes priming very difficult. A long period is needed to achieve a steady state in metabolites and the use of some tracers are more 
difficult to achieve a steady state, particularly urea and 3-mHis, a slow turnover rate relative to the pool size or multiple pool compartments $(21,26)$. Therefore we chose to measure the WbRa in those time ranges in which the animals were metabolically relative stable (16). In addition, instead of taking one single sample at a theoretically time point of steady state, we took multiple samples over time, so we were able to create an enrichment curve and theoretically calculate the tracer enrichment plateau. We determined the tracer steady state plateau value by fitting each individual steady state enrichment curve using one-phase association fitting (see examples in Supplemental figure 1). We believe that this approach improved the interpretation of the data.

In the Control group, whole body metabolism is characterized by reduced systemic AA concentration, WbPB, WbPS and lower muscle PB, but no change in the net catabolic state in the POST period. This is probably caused by the change in nutritional state from early post-absorptive (the BL period was 6-8 hours after the last meal) to an early starvation condition (POST period is 18-28h after the last meal). This downregulation of protein metabolism can be considered as an process of natural adaptation to a new nutritional state wherein availability of energy and protein substrate is limited (27). Additionally we cannot exclude a potential effect of the experimental conditions, such as fluid resuscitation, on metabolism (28). This supports the fact that the Control group is crucial in the study design to be able to discriminate between sepsis and non- sepsis specific changes in metabolism.

The changes we observed in systemic AA changes in sepsis agree with findings in human studies. The elevated Phe and decreased Glu concentrations are characteristic of septic, traumatized or ICU patients (2, 3, 13, 29, 30). Therefore, we conclude that our systemic Phe and Glu observations can be considered as a general phenomenon in critical ill conditions. In addition, in sepsis in the present study, higher WbPB and WbPS resulting in more severe catabolic state and enhanced AA substrate wasting (higher ureagenesis), are in line what we and others observed in septic humans $(2,3,7)$. Also the observed higher extrahepatic ureagenesis by arginase in sepsis could well affect the availability of Arg locally in organ/tissues, resulting in a different metabolic fate of Arg (31). Additionally, with the use of 3-mHis tracer we were able to quantify on a whole body level that myofibrillar muscle protein is accelerated in sepsis. Overall, we can conclude that our severe sepsis pig model mimics well the Wb protein metabolism in septic/critically ill humans.

\section{Transorgan AA and protein metabolism in the Control group}

In the Control group the $\mathrm{HQ}$ showed an increased lysine uptake and a diminished Ala release in the POST period. However, this observation had no relation with $\mathrm{HQ}$ PB/PS. Across the PDV, we did not found a difference between BL and POST in net uptake of EAA and TAA. However, the profile of the AA of this uptake changed. 
The increase of Leu uptake could indicate an increase in PDV PS, but the PS and PB data obtained from Leu tracer were inconclusive and did not confirm the disposal/production data of the Phe and Tyr tracers. Therefore we conclude that other metabolic pathways in PDV were changed in Control group in the POST period, potentially related to the difference in nutritional state between POST and BL and/or the influence of experimental factors like fluid resuscitation $(27,28)$. In the SPL area, we observed an overall reduction of uptake of some AA's, but this was not related to overall PS and PB changes in SPL area. Therefore, we conclude that protein turnover didn't change in the SPL area in the Control group. Overall we conclude that in the Control group, the whole body downregulation of protein metabolism in the post period, most likely caused by changes in nutritional status and fluid resuscitation, cannot be related to PS/PB changes in one single or more individual organs that are measured.

\section{Transorgan AA and protein during severe sepsis}

In the present study, we tried to study transorgan protein NB to unravel which organs play a key role in the enhanced WbPB and WbPS resulting in an enhanced net catabolism in acute severe sepsis. The systemic WbRa of Phe was used as proxy for WbPB. In the $\mathrm{HQ}$, we did not observe a significant net release of Phe although there was a significant net release of Tyr and Leu and TAA. HQ PS and $\mathrm{PB}$, using 3 different tracers, were inconclusive. This in contrast with the specific myofibrillar muscle WbRa mHIS data. We conclude that enhanced muscle PB is occurring in the $H Q$ but is relative low in the present acute severe sepsis phase in the pig or the reincorporation of AA into protein in the cell is enhanced. Other type of tissues, variability in protein kinetics in different muscle groups in HQ (24) and/ or relative small $\mathrm{HQ} A-\mathrm{V}$ concentration differences in early starvation state could mask the PB in the muscle. Previously described unchanged FSR in muscle in the present cohort of animals \{Chapter 10\}, confirmed the observed unchanged PS in the HQ. Therefore, we conclude that in acute PA induced severe sepsis muscle PB is enhanced, but not sufficiently to explain the observed WbPB (4). In addition, PS in muscle does not contribute to the enhanced WbPS.

The PDV showed a diminishing PB and PS resulting in diminished protein turnover, which is mainly caused by a compromised jejunal mucosa (16)\{Chapter 10\}. This in contrast with earlier observations in models of endotoxin induced sepsis wherein the PDV was preserved (17), indicating that virulent factors coming from bacteria induce a different response on PDV protein metabolism than endotoxin alone. The PDV diminished protein turnover indicates that the PDV is not contributing at all to the enhanced $\mathrm{Wb}$ protein turnover.

During sepsis, the liver consumes three times the amount of AAs compared to the Control group accompanied with a three times higher urea rate of appearance. This 
indicates severe AA wasting in the phase of acute severe sepsis. The unchanged SPL protein turnover and diminished PDV turnover indicates that liver PB and PS should be enhanced. An enhanced PS is indeed detected as increased fractional synthesis rate in liver tissue \{Chapter 10 \} and is in line with protein acute phase response in sepsis (11). However, unchanged SPL protein turnover also indicates that this part of the body is not contributing to the enhanced WbPB and WbPS. Therefore in general, we conclude that it is still unclear which tissue other than muscle is contributing to the enhanced WbPB and which other tissue than the liver is contributing to enhanced WbPS.

Figure 6 shows an estimation of the contribution of the individual organ PB to WbPB in which we compared the organ Phe production with the WbRa of Phe. We published earlier that WbRa measured with a primed continuous infusion is an underestimation of the real WbRa due to the used non-compartmental assumptions for the data analysis (19). Therefore, we corrected the measured WbRa with a factor 1.6 for this comparison. Overall with this figure and with the presented interorgan metabolism, we conclude that enhanced WbPB and WbPS measured in the systemic plasma pool cannot be explained by significant protein metabolism changes in one single or multiple measured organs, despite prominent changes in muscle and the $\mathrm{SPL}$ area protein metabolism. What can potentially explain this discrepancy? Due to influx of bacteria in all tissues and subsequently influx of inflammatory cells in tissue, a local acute phase reaction is initiated (32) resulting in a small local increased PS and PB that can contribute to WbPB and WbPS, but is too small to detect with our organ net balance measurements in post-absorptive/early starvation state where

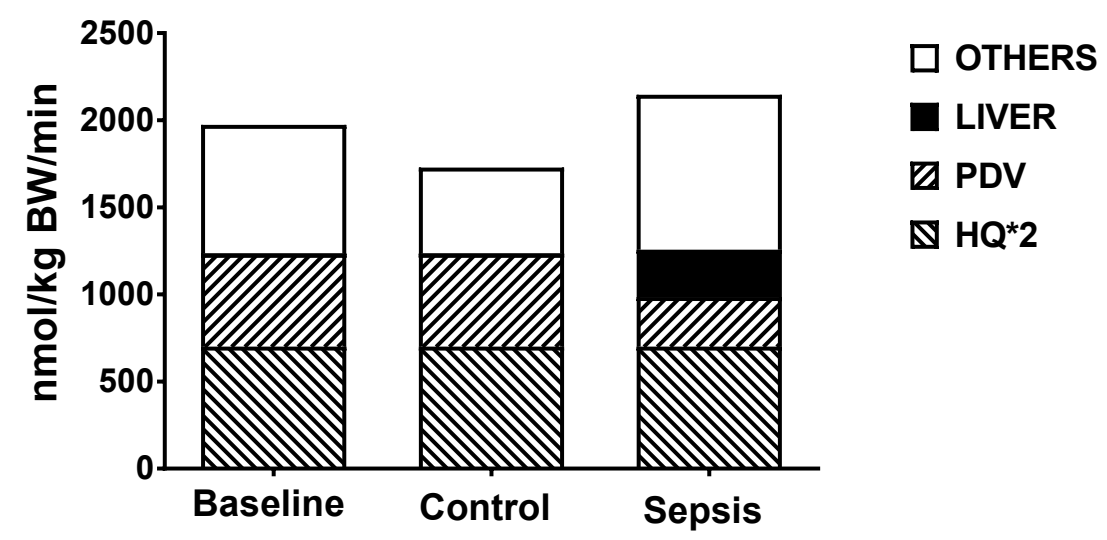

Figure 6. Contribution of organ Phe production to whole body rate of appearance of Phe (Hypothetical)

Before (Baseline) and post infusion of Pseudomonas aeruginosa. Height of the bar = whole body rate of appearance of PHE corrected with factor of 1.6 (19). HQ is hindquarter (muscle compartment), $\mathrm{HQ}^{*} 2$ = whole body muscle compartment. PDV is Portal drained viscera. LIVER = Splanchnic area - PDV. 
A-V differences are relative small. Other more advanced tracer techniques like triple pulse tracer approach (33) could be helpful in future studies to study not only fractional synthesis but also fractional breakdown rates in more different organ/tissues/ proteins to unravel the net catabolism phenomenon in severe sepsis. Finally, also changes in plasma protein synthesis and breakdown are suggested to play a role in the septic net catabolism. A severe decrease in the plasma albumin concentration in sepsis related to increased mortality in sepsis (14), are not only caused by redistribution to interstitial fluids but also potentially by diminished albumin synthesis in the liver and enhanced proteolysis or clearance (34). Data of albumin breakdown are very limited, but by measurements of disappearance of isotopic labeled plasma proteins such as albumin could be helpful to study further the contribution of plasma proteins to net catabolism (12).

\section{Glutamate/Glutamine/Alanine interorgan metabolism}

Glu, GIn and Ala metabolism are connected and characterized by high compartmentalization in different organs. In healthy situation the organs that have a net production of these AA are in balance with the organs that have a net uptake these AA. Interorgan transport of these substrates are known to be out of balance in certain pathological conditions (35). We found that severe systemic low Glu blood values are related to increased mortality in critically ill humans (13). Glu plays an intermediary role in many metabolic pathways and is therefore potentially critical for an adequate response to defeat bacteremia induced sepsis. In the present study, we observed that the systemic 3 fold decrease of plasma Glu concentration was associated with severely diminished release of Glu from the liver. Complete disappearance of the uptake by PDV preserve Glu availability for the rest of the body. The decreased uptake from the HQ is potentially caused by the decreased systemic availability.

In humans, GIn production by the $\mathrm{HQ}$ is considered to be comparable (in balance) with Glu production in the liver(35). However in the present study this is not observed. We observed decreased systemic GIn availability, but in contrast with systemic Glu levels, this was not sepsis related but probably caused by the nutrition state and/ or fluid resuscitation because the response was observed in both the controls and septic animals. The SPL area still used the same amount of GIn, however in sepsis more uptake by the liver and less by the PDV was observed. GIn is used as energy substrate in the PDV and as precursor for purine and pyrimidine synthesis, necessary for DNA synthesis in rapidly dividing cells like enterocytes (36). The decreased uptake of GIn in the PDV indicates that beside the down regulated protein turnover, also the energy producing aerobic citrate cycle is down regulated. These results indicate potential compromised intestinal function, although the production of Cit by the PDV, a general gut function parameter (37), was not affected in this septic phase. The enhanced portal availability of GIn to the liver didn't lead to more Glu 
release. It is unlikely, that glutaminase activity that is used for urea synthesis (38), is compromised considering the huge increase of ureagenesis in the present septic state. Also HQ and PDV production of Ala could influence the liver Glu release. However, the substantial enhanced uptake of Ala by the liver, indicating an enhanced Ala-Glu transamination cycle resulting in enhanced glucose production (17) and ureagenesis, didn't result in normalising the release of Glu by the liver. This indicates that Glu in the liver is routed to other metabolic routes as part/intermediary of an acute phase response to PA and therefore results in less availability for the rest of the body.

Overall we conclude, that in acute severe sepsis, systemic Glu depletion is caused by diminished release of Glu from the liver and enhanced uptake of GIn and Ala by the liver could not prevent this phenomenon. The consequences of the severely reduced systemic availability of glutamate are unclear with regard to the further progression of the pathological septic condition.

\section{Conclusion}

We have attempted to unravel the disturbed metabolic pathways that are involved in net catabolism in severe sepsis, by measuring AA substrate fluxes on $\mathrm{Wb}$ level and net balances across the PDV, SPL area, liver and HQ in a clinically relevant pig model. With the use of tracers, we established that enhanced WbPB and WbPS measured in the systemic plasma pool could not be explained by significant protein metabolism changes in one single or multiple measured organs, despite prominent changes in muscle and the SPL area protein metabolism. That the PDV is not responsible for the enhanced $\mathrm{Wb}$ protein turnover and that beside muscle multiple other organ/tissue/proteins should be involved. Further tracer studies on tissue/protein level might be helpful in future studies to determine this. Finally with the present results, we hypothesize, that in the acute phase of severe sepsis, the overall metabolic downregulated PDV and the compromised liver reflects severe AA substrate wasting, and decreased release of Glu to the whole body, that could not be compensated by interacting GIn and Ala multiorgan pathways, are potentially key metabolic factors for further progression of multi-organ failure in sepsis.

\section{Acknowledgements}

We thank Joshua Spore, BSc for skilled technical assistance of sample processing and analyses. Bea Zoer, PhD and Renske C.I. Deutz, MD for skilled technical assistance in carrying out the animal experiments. John Thaden, PhD for accurate LC-MS/MS analyses of AAs. 
The project described was supported by Award Number R01GM084447 from the National Institute of General Medical and by Award Number S10RR027047 from the National Center for Research Resources. The content is solely the responsibility of the authors and does not necessarily represent the official views of the National Institute of General Medical Sciences or National Center for Research Resources or the National Institutes of Health.

\section{References}

1. Hermans G, Van den Berghe G. Clinical review: intensive care unit acquired weakness. Crit Care. 2015;19:274.

2. Luiking YC, Poeze M, Ramsay G, Deutz NE. Reduced citrulline production in sepsis is related to diminished de novo arginine and nitric oxide production. Am J Clin Nutr. 2009;89(1):142-52.

3. Rooyackers O, Kouchek-Zadeh R, Tjader I, Norberg A, Klaude M, Wernerman J. Whole body protein turnover in critically ill patients with multiple organ failure. Clin Nutr. 2015;34(1):95-100.

4. Deutz NE, Wagenmakers AJ, Soeters PB. Discrepancy between muscle and whole body protein turnover. Curr Opin Clin Nutr Metab Care. 1999;2(1):29-32.

5. Cohen S, Nathan JA, Goldberg AL. Muscle wasting in disease: molecular mechanisms and promising therapies. Nat Rev Drug Discov. 2015;14(1):58-74.

2526 6laude M, Mori M, Tjader I, Gustafsson T, Wernerman J, Rooyackers O. Protein metabolism and gene expression in skeletal muscle of critically ill patients with sepsis. Clin Sci (Lond). 2012;122(3):133-42.

7. Pittiruti M, Siegel JH, Sganga G, Coleman B, Wiles CE, 3rd, Placko R. Determinants of urea nitrogen production in sepsis. Muscle catabolism, total parenteral nutrition, and hepatic clearance of amino acids. Arch Surg. 1989;124(3):362-72.

8. Heyland DK, Stapleton RD, Mourtzakis M, Hough CL, Morris P, Deutz NE, et al. Combining nutrition and exercise to optimize survival and recovery from critical illness: Conceptual and methodological issues. Clin Nutr. 2016;35(5):1196-206.

9. Song J, Wolf SE, Herndon DN, Wu XW, Jeschke MG. Second hit post burn increased proximal gut mucosa epithelial cells damage. Shock. 2008;30(2):184-8.

10. Ding LA, Li JS. Intestinal failure: pathophysiological elements and clinical diseases. World J Gastroenterol. 2004;10(7):930-3.

11. Strnad P, Tacke F, Koch A, Trautwein C. Liver - guardian, modifier and target of sepsis. Nat Rev Gastroenterol Hepatol. 2017;14(1):55-66.

12. Chen Y, Ren J, Qin X, Li G, Zhou B, Gu G, et al. Metabolism of albumin after continuous venovenous hemofiltration in patients with systemic inflammatory response syndrome. Biomed Res Int. 2015;2015:917674.

13. Poeze M, Luiking YC, Breedveld P, Manders S, Deutz NE. Decreased plasma glutamate in early phases of septic shock with acute liver dysfunction is an independent predictor of survival. Clin Nutr. 2008;27(4):523-30.

14. Kim MH, Ahn JY, Song JE, Choi H, Ann HW, Kim JK, et al. The C-Reactive Protein/ Albumin Ratio as an Independent Predictor of Mortality in Patients with Severe Sepsis or Septic Shock Treated with Early Goal-Directed Therapy. PLoS One. 2015;10(7):e0132109. 

et al. Interorgan amino acid exchange in humans: consequences for arginine and citrulline metabolism. Am J Clin Nutr. 2007;85(1):167-72.

16. Ten Have GAM, Deutz RCl, Engelen M, Wolfe RR, Deutz NEP. Characteristics of a Pseudomonas aeruginosa induced porcine sepsis model for multi-organ metabolic flux measurements. Lab Anim. 2017:23677217718003.

17. Bruins MJ, Deutz NE, Soeters PB. Aspects of organ protein, amino acid and glucose metabolism in a porcine model of hypermetabolic sepsis. Clin Sci (Lond). 2003;104(2):127-41.

18. Ten Have GA, Bost MC, Suyk-Wierts JC, van den Bogaard AE, Deutz NE. Simultaneous measurement of metabolic flux in portally-drained viscera, liver, spleen, kidney and hindquarter in the conscious pig. Lab Anim. 1996;30(4):347-58.

19. Ten Have GA, Engelen MP, Wolfe RR, Deutz NE. Phenylalanine isotope pulse method to measure effect of sepsis on protein breakdown and membrane transport in the pig. Am J Physiol Endocrinol Metab. 2017:ajpendo 003512016.

20. Vissers YL, von Meyenfeldt MF, Braulio VB, Luiking YC, Deutz NE. Measuring wholebody actin/myosin protein breakdown in mice using a primed constant stable isotope-infusion protocol. Clin Sci (Lond). 2003;104(6):585-90.

21. Rathmacher JA, Nissen SL, Paxton RE, Anderson DB. Estimation of 3-methylhistidine production in pigs by compartmental analysis. J Anim Sci. 1996;74(1):46-56.

22. Bruins MJ, Soeters PB, Deutz NE. Endotoxemia affects organ protein metabolism differently during prolonged feeding in pigs. J Nutr. 2000;130(12):3003-13.

23. Luiking YC, Poeze M, Deutz NE. Arginine infusion in patients with septic shock increases nitric oxide production without haemodynamic instability. Clin Sci (Lond). 2015;128(1):57-67.

24. Wolfe RR, Chinkes DL. Isotope Tracers in Metabolic Research: Principles and Practise of Kinetic Analysis. New York: Wiley; 2005. 274 p.

25. Engelen MP, Rutten EP, De Castro CL, Wouters EF, Schols AM, Deutz NE. Altered interorgan response to feeding in patients with chronic obstructive pulmonary disease. Am J Clin Nutr. 2005;82(2):366-72.

26. Jahoor F, Wolfe RR. Reassessment of primed constant-infusion tracer method to measure urea kinetics. Am J Physiol. 1987;252(4 Pt 1):E557-64.

27. Soeters PB. Macronutrient Metabolism in Starvation and Stress. Nestle Nutr Inst Workshop Ser. 2015;82:17-25.

28. Li Y, Guo M, Shen J, Zheng L, Wang J, Wang P, et al. Limited fluid resuscitation attenuates lung and intestine injury caused by hemorrhagic shock in rats. Journal of investigative surgery : the official journal of the Academy of Surgical Research. 2014;27(2):81-7.

29. Clowes GH, Jr., Randall HT, Cha CJ. Amino acid and energy metabolism in septic and traumatized patients. JPEN J Parenter Enteral Nutr. 1980;4(2):195-205.

30. Rosenblatt S, Clowes GH, Jr., George BC, Hirsch E, Lindberg B. Exchange of amino acids by muscle and liver in sepsis. Arch Surg. 1983;118(2):167-75.

31. Luiking YC, Ten Have GA, Wolfe RR, Deutz NE. Arginine de novo and nitric oxide production in disease states. Am J Physiol Endocrinol Metab. 2012;303(10):E1177-89.

32. Langhans C, Weber-Carstens S, Schmidt F, Hamati J, Kny M, Zhu X, et al. Inflammation-induced acute phase response in skeletal muscle and critical illness myopathy. PLoS One. 2014;9(3):e92048.

33. Zhang XJ, Chinkes DL, Wolfe RR. Measurement of muscle protein fractional synthesis and breakdown rates from a pulse tracer injection. Am J Physiol Endocrinol Metab. 2002;283(4):E753-64. 
35. Deutz NE. The 2007 ESPEN Sir David Cuthbertson Lecture: amino acids between and within organs. The glutamate-glutamine-citrulline-arginine pathway. Clin Nutr. 2008;27(3):321-7.

36. Ashy AA, Salleh M, Ardawi M. Glucose, glutamine, and ketone-body metabolism in human enterocytes. Metabolism. 1988;37(6):602-9.

37. Lutgens LC, Blijlevens NM, Deutz NE, Donnelly JP, Lambin P, de Pauw BE. Monitoring myeloablative therapy-induced small bowel toxicity by serum citrulline concentration: a comparison with sugar permeability tests. Cancer. 2005;103(1):191-9.

38. Watford M. Glutamine and glutamate metabolism across the liver sinusoid. J Nutr. 2000;130(4S Suppl):983S-7S.

\section{Supplemental Figure}
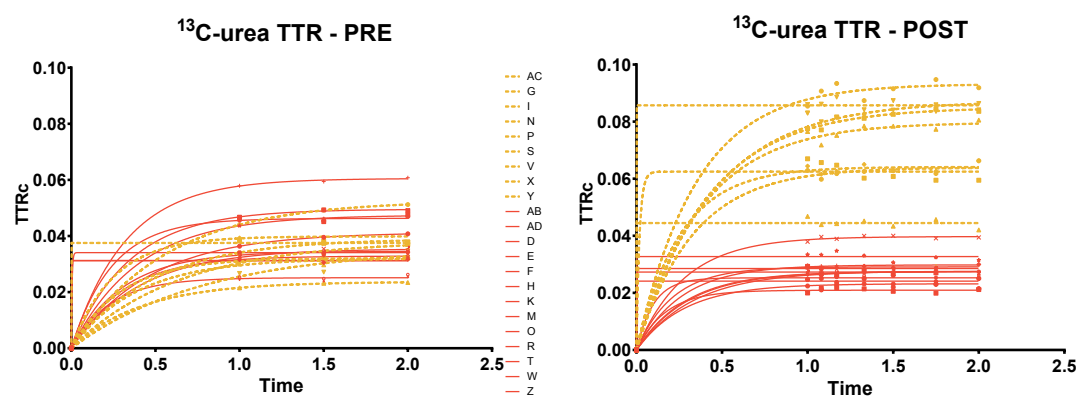

\section{${ }^{2} \mathrm{H}_{3}$-mHis TTR - PRE}

\section{${ }^{2} \mathrm{H}_{3}$-mHis TTR - POST}
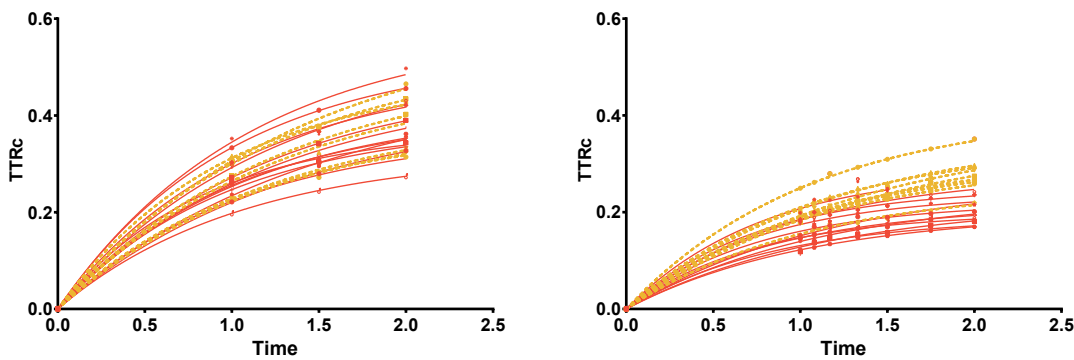

Supplemental Figure 1. Tracer/tracee ratio (TTR) curves.

Example of one-phase association fitting of Tracer/Tracee ratio (TTR) curves. Plateau values of curves will be used for steady state enrichment. Before (Baseline) and POST infusion of Pseudomonas aeruginosa. Dash line is Control, solid line is Sepsis group. Control n=9, Sepsis $\mathrm{n}=13$. 

<smiles>C=COc1ccccc1OCCOC</smiles> 
CHAPTER 12 


\section{Summary and future perspectives}

A healthy human body needs nutrients to be kept in a metabolic balance. To keep balance, complex interactions between multiple specialized organs for transportation, digestion, absorption, redistribution, utilization, conversion, breakdown of nutritional substrates during post-prandial and/or post-absorptive stage must occur. These interactions are highly dynamic and are subject to disturbances in many pathophysiological conditions (e.g. sepsis or liver failure) (1-10). To unravel (disturbed) complex nutritional substrate metabolism, quantitative and dynamic substrate flux measurements are needed to support new nutritional strategies in health and disease. To measure complex fluxes/trafficking (amounts in time) of substrates transorgan/interorgan, is extremely difficult in humans due to the multitude of ethical issues and the limitations to perform invasive procedures, especially in pathophysiological conditions (e.g. critical-ill patients). Therefore, clinically relevant animal models are essential to study (patho) physiological interorgan metabolism in a controlled, repeatable way and to be able to use invasive techniques, providing multiple measurements and mechanistic data within the same animal in several nutritional/pathological stages. The pig, with its remarkable similarity to humans, is used often in biomedical research and more generally in the field of nutrition and associated metabolic disease states.

The present dissertation is a reflection of my longstanding experience in metabolic research using a multi-catheterized pig model. I describe interorgan/ transorgan measurement of metabolism of mainly amino acids/protein, their metabolites and interaction with other macronutrients using advanced stable isotope tracer methods in healthy and disease states. The main focus is on a conscious disease model with acute severe sepsis induced by Pseudomonas aeruginosa bacteremia. In addition, we present a study in an unconscious devascularized acute liver failure model.

\section{Section 1: Interorgan balance studies during feeding}

In this section a review is given of the current knowledge of absorption kinetics of protein, peptides and amino acids (Chapter 2) (11). We stated that the small intestine acts as interface and regulator between the gut lumen and the rest of the body and controls the degree and rate of transport of amino acids derived from dietary protein via the portal vein to the liver and the systemic circulation. In a balanced-protein meal, the gut dietary amino acid utilization (30-50\%) for protein synthesis will fill a labile protein pool in the gut that can be beneficial during the postabsorptive state. 
Despite the absence of different absorption kinetics between protein, peptides, and amino acids, specific attributes can still be beneficial. For instance, when the digestibility function in the gut is compromised (critical ill patients), or in a postexercise situation in which a hydrolysate works insulinotropically. In this review, we have discussed the factors that lead to enhanced, reduced, or modified absorption kinetics. We concluded that to study these factors, a sophisticated multi-catheterized pig model and the use of isotopes to calculate absorption and gut metabolism is necessary. Data obtained from those studies in health and disease condition will support new/improve clinical nutritional strategies.

In Chapter 3 a catheterized conscious pig model is described that is developed to enable measurement of substrate fluxes across organs (12). To ensure that the model was reproducible and stable with a relative short recovery time, meticulous attention was given to optimize recovery conditions and to enhance the long term patency rate of the sampling catheters. Patency rate was probably due to the prevention of catheter-related infections using a gentamicin (20 mg/ml), a-chymotrypsin (225 D/ml) solution as catheter filling. The model enables simultaneous substrate flux measurement across the portal drained viscera, intestine, spleen, liver, kidney and hindquarter in the conscious pig and is used throughout the thesis.

In Chapter 4 and 5, the catheterized pig model is extensively used to study dietary factors that could influence the absorption rates from the lumen to the gut, metabolism of dietary component in the gut, the release of amino acids to the portal circulation from digested protein, the effect on liver metabolism and the resulting systemic availability.

In Chapter 4 we stated that gut health relates to a diet with a high digestibility and quality, but it is unknown what the acute consequences are of low quality foods on gut and liver metabolism (13). Therefore, a meal with the low quality protein gelatin (GEL; tryptophan deficient and low amount of essential amino acids) was compared to a meal with the high quality protein whey (Whey) and a tryptophan supplemented gelatin meal (GEL + TRP) (Figure 1). In the GEL group, the average net portal appearance of amino acids was $99.8 \pm 14.6 \%$ of the intake versus $61.4 \pm 9.0 \%$ in the Whey group (Figure 2). Despite the absence of tryptophan in the GEL group, a net portal appearance of tryptophan (42\% of the amount in the Whey group) was observed in the GEL group. Intestinal energy metabolism and citrulline production was not affected. Amino acid gut retention for the GEL+TRP group was comparable with the Whey group. We concluded that in a postprandial phase after intake of a low quality protein meal, net anabolism in the healthy intestine is absent. It is likely that the intestine responds with a net breakdown of endogenous (labile) proteins to secure amino acid availability for the body. The addition of the first limiting essential amino acid to this meal (TRP) improved protein anabolism in the intestine. We concluded 


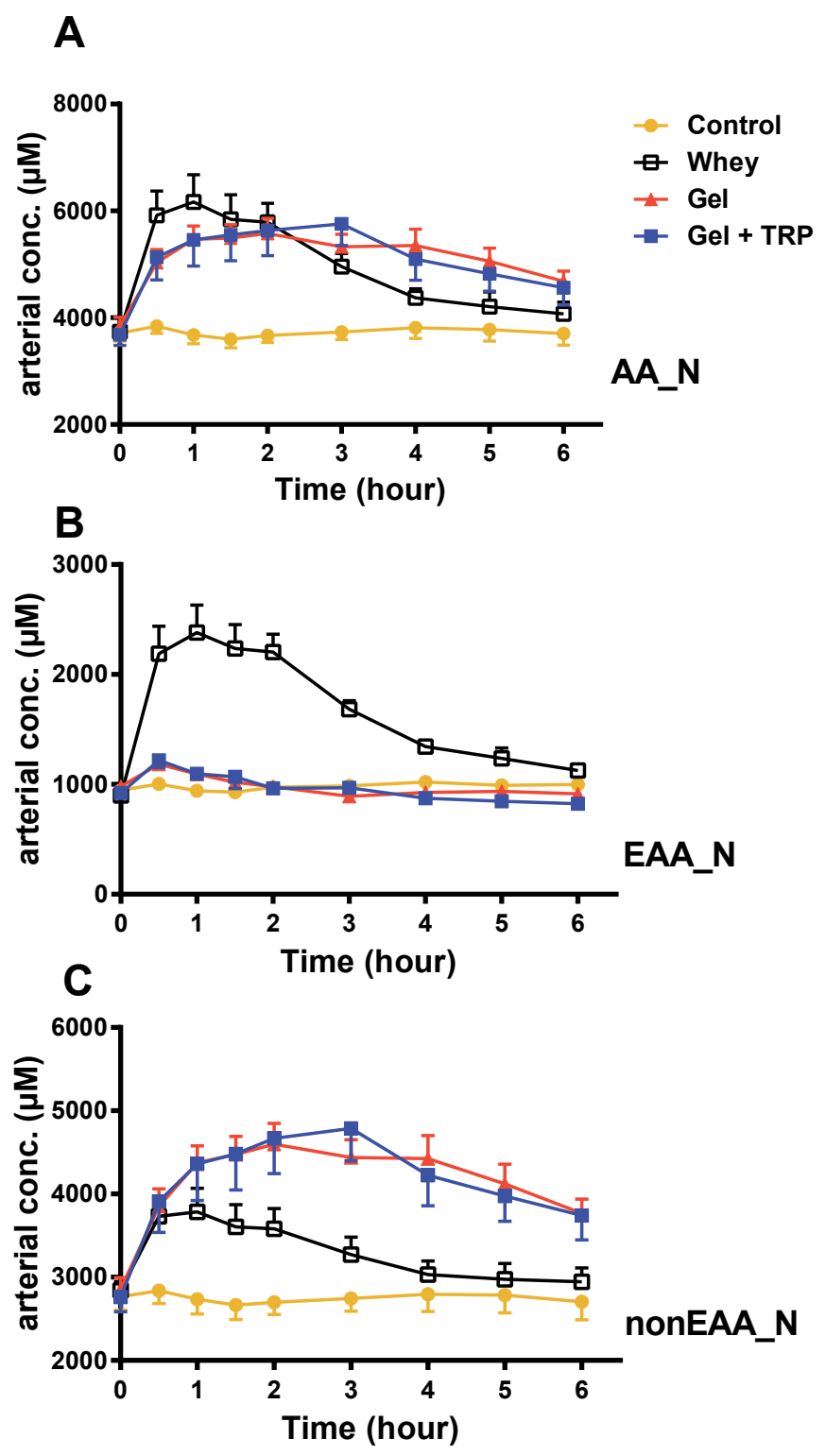

Figure 1. Arterial amino acid nitrogen

A: amino acids (AA_N) B: essential amino acid nitrogen (EAA_N); C: and non-essential amino acid nitrogen (NonEAA_N); concentrations, in $\mu \mathrm{M}$, in post-absorptive state (Control, $n=9$ ) and during 6 hours after administration of Whey protein (Whey, $n=8$ ), gelatin (Gel, $n=9$ ) or gelatin + tryptophan (Gel+TRP, $n=9$ ) meals. Values are mean \pm SEM. ANOVA tested, significant meal effect ( $p<0.05$ ): (A) protein meals vs. Control; (B) Whey vs. all other test meals; (C) proteins vs. control, Whey vs. Gel groups. Significant interaction ( $p<0.05)$ : (A) Control vs. protein meals, Gel vs. Whey; (B) Control vs. all protein meals, Whey vs. all test meals; (C) Control vs. Gel groups, Gel vs. Whey. 


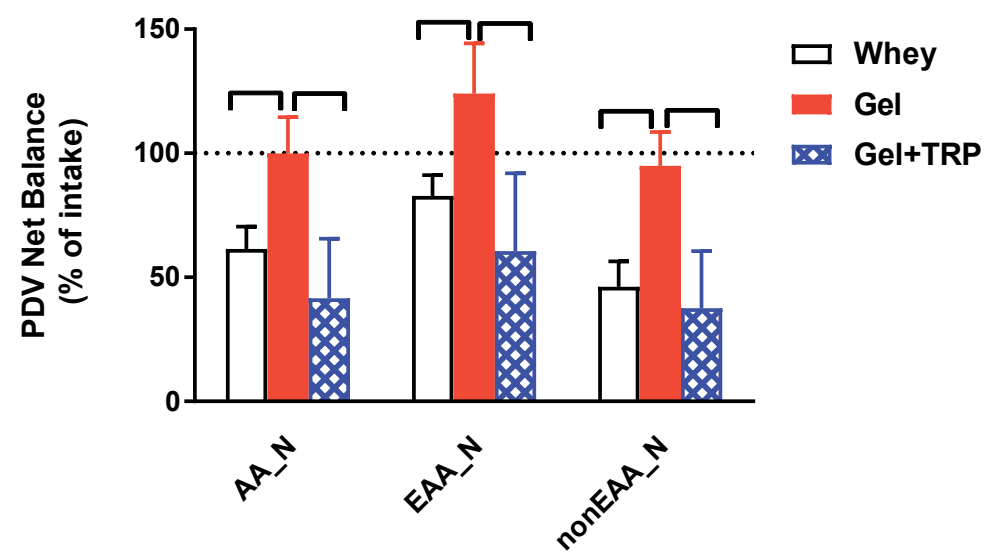

Figure 2. Total net balances across the gut

Total net balances of amino acid nitrogen (AA_N), essential amino acid nitrogen (EAA_N) and amino acid nitrogen (nonEAA_N) as percentage of the intake, over 6 hours after administration of Whey protein (Whey, $n=8$ ), gelatin (Gel, $n=9$ ) or gelatin + tryptophan (Gel+TRP, $n=9$ ) meals. Values are mean \pm SEM. Significance with Student t-test: $p<0.05$, indicated with hooks.

that protein quality of a meal is related to the anabolic response of the intestine to the meal.

In the last chapter of this section (Chapter 5) we studied the bioavailability kinetics of some food-derived peptides that possess bioactive properties, and may affect health positively (14). We stated that the potential blood pressure lowering C-terminal lacto-tri-peptides Ile-Pro-Pro (IPP), Leu-Pro-Pro (LPP) and Val-Pro-Pro (VPP) (together named here XPP) depends on their availability at the site of action. We hypothesize that the composition of the meal will modify their systemic availability. We studied trans-organ XPP fluxes to determine systemic and portal availability, as well as renal and hepatic uptake of a water-based single dose of synthetic XPP and a XPP containing protein matrix (casein hydrolyte, CasH). In a second experiment, we compared the CasH-containing protein matrix with a $\mathrm{CasH}$-containing meal matrix and the modifying effects of macronutrients (high carbohydrates, low quality protein, high fat, and fiber) in a meal on the availability. Portal availability of synthetic XPP was $0.08 \pm 0.01 \%$ of intake and increased when a protein matrix was present. Subsequent renal and hepatic uptake is about $75 \%$ of the portal release. In a meal with all macronutrients present, XPP are more portally available, albeit at a low percentage (0.2-0.3\%). We concluded that the digestion rate of the meal, the quality of protein, and fiber contents, mainly determine systemic XPP bioavailability after a meal. 


\section{Section 2: Use of isotopes in pig sepsis models}

In this section, we described a new developed Pseudomonas aeruginosa (PA) induced porcine sepsis model as a clinically relevant model for acute severe sepsis (Chapter 6) (15). Muscle wasting in sepsis, a life-threatening organ dysfunction caused by a dysregulated host response to infection in critically ill, has symptoms like gut atrophy and stimulation of liver acute phase response, indicating complex interactions between organs. To study the multi-organ metabolism, in this new sepsis model, bacteremia was induced by intravenous administration of Pseudomonas aeruginosa $\left(10^{9} \mathrm{CFU} /\right.$ hour $)$ in 13 catheterized pigs for 18 hours. Both the septic and control animals received fluid resuscitation and were continuously monitored. The systemic inflammatory response (SIRS) diagnostic scoring system
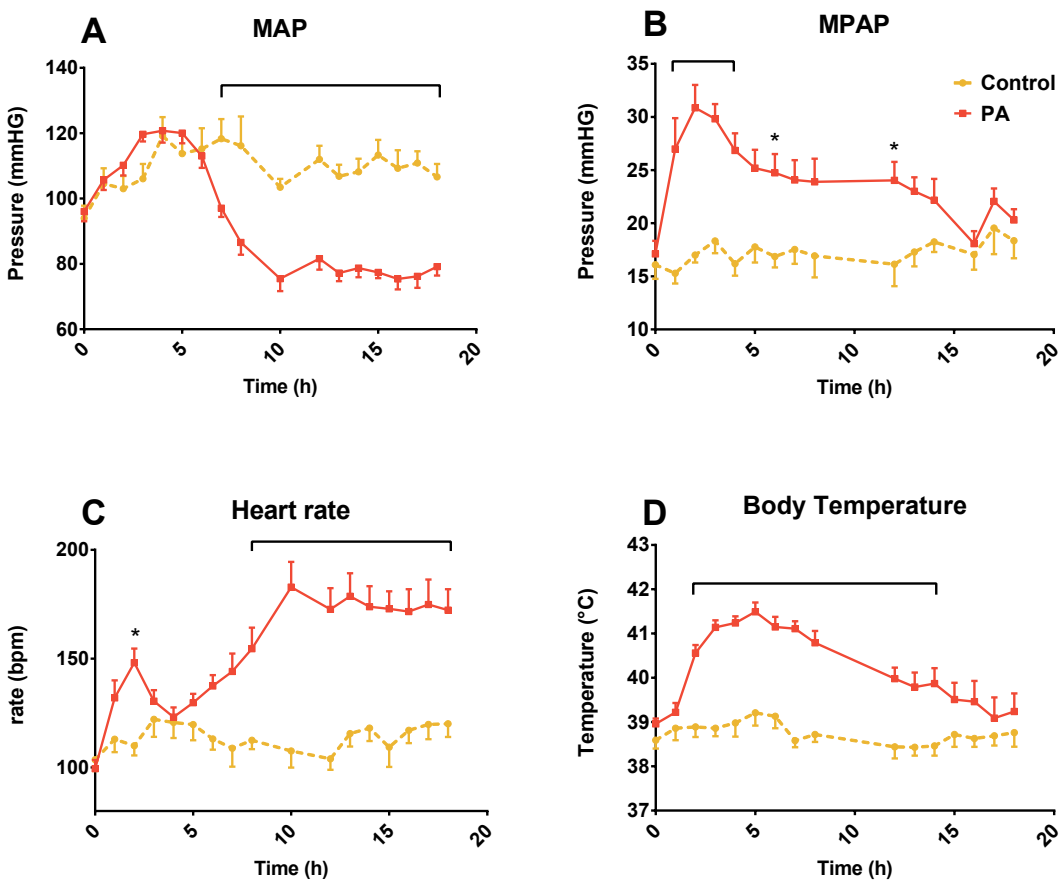

Figure 3. Hemodynamics and body temperature

Hemodynamic response on the Pseudomonas aeruginosa (PA) induced bacteremia in the pig. A: MAP is mean arterial pressure. B: MPAP is mean pulmonary arterial pressure. C: Heart rate. D: Body temperature. Control N=9; PA N=13. Values are mean \pm S.E.M. Statistics: Two-Way ANOVA comparison; when appropriate a post-hoc Bonferroni multiple comparison test was done; hook or *: $p<0.05$.
A. $\quad$ MAP: Interaction $p<0.0001$, Time effect $p<0.0001$, PA effect $p<0.0001$.
B. MPAP: Interaction $p=0.0021$, Time effect $p=0.0053$, PA effect $p<0.0001$.
C. $\quad H R$ : Interaction $p<0.0001$, Time effect $p<0.0001$, PA effect $p<0.0001$.
D. Body Temp: Interaction $p<0.0001$, Time effect $p<0.0001$, PA effect $p<0.0001$. 
was used to determine the clinical septic state. Within 6 hours after start PA, a septic state developed, as reflected by hyperthermia and cardiovascular changes (Figure 3). The period of 12-18h was characterized by severe sepsis caused by disturbed cardiovascular function, decreased portal drained viscera plasma flow as well as moderate villous injury in the small intestines (Figure 4). No lung, kidney or liver failure occurred during the study period. Significant metabolic changes such as enhanced protein breakdown, hypocalcemia and hypocholesterolemia were found. We concluded that the new Pseudomonas aeruginosa induced porcine sepsis model is a clinically relevant model to study metabolic changes during acute severe sepsis and instrumentation enables study of complex multi-organ metabolism.

The use of stable isotopes in vivo is considered to be the "gold standard" to study metabolism of substrates throughout the body and tissues to obtain knowledge on the rate of use of these substrates in metabolic pathways (16). Implementation of more advanced models needs to be done carefully. Therefore in Chapter 7 , we compared the use of two different stable tracer models to obtain knowledge of whole body protein breakdown in a disease state like severe sepsis (17). The primed-continuous (PC) phenylalanine (PHE) stable isotope infusion methodology is often used as a proxy for measuring whole body protein breakdown (WbPB) in sepsis. It is unclear, if WbPB data obtained by an easy-to-use single intra-venous PHE isotope pulse administration (PULSE) are comparable to those by PC. The non-compartmental analysis with the $\mathrm{PC}$ is greatly limited by the necessary assumption that production appears in the sampled compartment (i.e. plasma) into which the tracer is also administered (16) (Figure 5). This is not the case for amino acids. Therefore, compartmental modeling with the decay curve of the PULSE could provide us more insight in the real WbPB in sepsis. Seventeen hours after sepsis induction, we compared the Whole body rate of appearance (WbRa) of PHE obtained by PC (L-[ring $\left.\left.{ }^{13} \mathrm{C}_{6}\right]-\mathrm{PHE}\right)$ and PULSE(L-[ $\left.\left.{ }^{15} \mathrm{~N}\right]-\mathrm{PHE}\right)$ in arterial plasma using LC-MS/MS and (non)-compartmental modeling. PULSE-WbRa was highly correlated with PC-WbRa and WbPB independent of the septic state (Figure 6). PULSE-WbRa was 1.6 times higher than PC-WbRa. Compartmental and non-compartmental PULSE modeling provided comparable WbRa values, although compartmental modeling was more sensitive. Both PULSE-WbRa and PC-WbRa are an underestimation of the real WbPB. WbPB was elevated in sepsis. With PULSE, sepsis was characterized by an increase of the metabolic shunting (amount of PHE flux between intra- and extra-cellular pool that is not irreversible lost). Membrane transport capacity was the same. We concluded that both PC and PULSE-methods are able to assess changes in WbRa of plasma PHE reflecting WbPB changes with high sensitivity, independent of the (patho-) physiological state. The WbRa obtained from the easy-to-use (non-) compartmental PULSE reflects better the real WbPB than PC-WbRa. With PULSE compartmental analysis, we conclude that the membrane transport capacity for amino acids is not compromised in severe sepsis. 

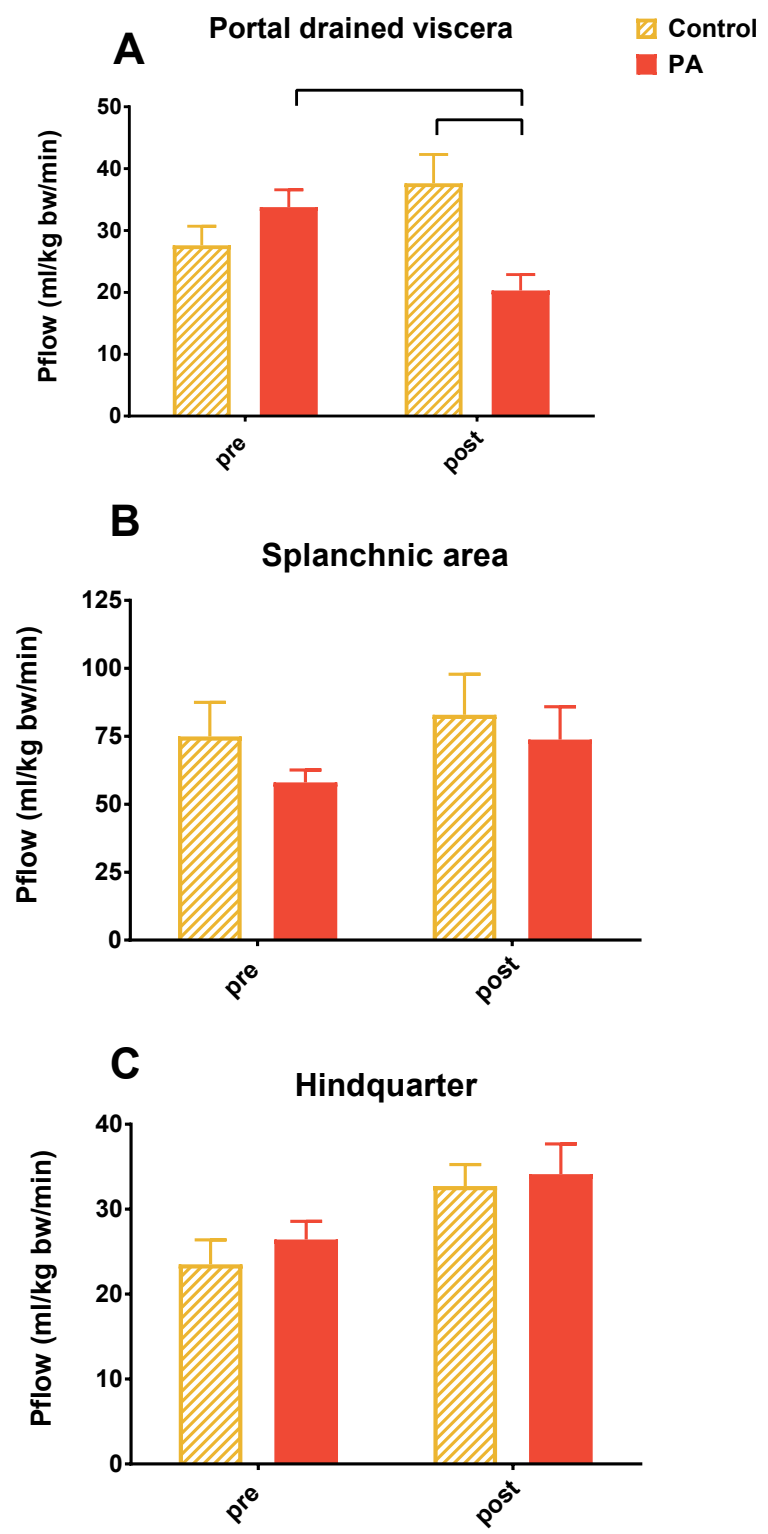

Figure 4. Organ Plasma flow

Organ plasma flows changes before and after Pseudomonas aeruginosa (PA) induced bacteremia in the pig. "Pre" is average plasma flow one hour before induction bacteremia. "Post" is average plasma flow between 14 and 18 hours after start induction bacteremia. Control $\mathrm{N}=9$; Sepsis $N=12$. Values are mean \pm S.E.M.. Statistics: Two-Way ANOVA repeated measures for time, when appropriate a post-hoc Tukey multiple comparison test; hook: $p<0.05$.
A. Portal drained viscera: Interaction $p=0.0008$, Time effect $p=0.5682$, PA effect $p=0.1297$
B. Splanchnic area: Interaction $p=0.7225$, Time effect $p=0.2849$, PA effect $p=0.2992$
C. Hindquarter: Interaction $p=0.6024$, Time effect $p<0.0001$, PA effect $p=0.2992$ 
PC

(non-compartmental)

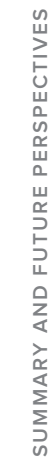

266

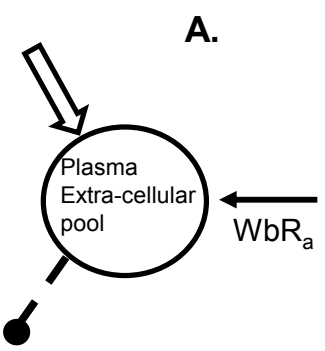

PULSE

(compartmental)

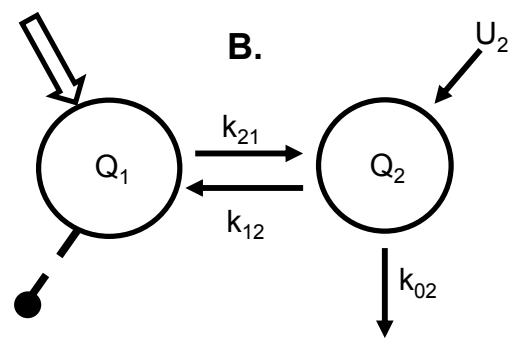

C.

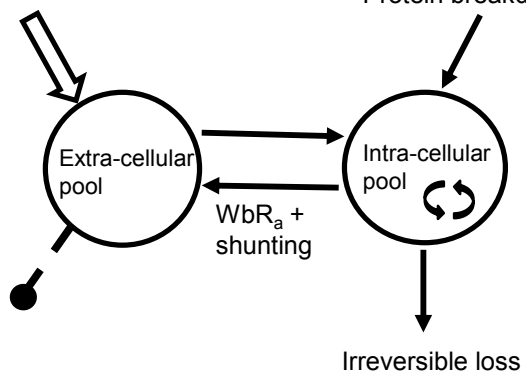

Figure 5: Calculation models for whole body protein breakdown

Two calculation models for whole body protein breakdown (WbPB) by determination of whole body rate of appearance (WbRa) of phenylalanine (Phe). (A) Non-compartmental model using a primed-continuous infusion of $\mathrm{L}-\left[\mathrm{ring}-{ }^{13} \mathrm{C}_{6}\right]-\mathrm{Phe}(\mathrm{PC})$. WbRa is the tracer infusion rate divided by the fraction of tracer found in plasma/extra cellular pool (TTR=tracer/tracee ratio) in a tracer steady state (B) Compartmental model using bolus infusion, L- $\left[{ }^{15} \mathrm{~N}\right]-$ Phe (PULSE) and SAAMII computer modeling. Computer parameters: $\mathrm{Q}=$ pool sizes of the compartments; $\mathrm{k}_{21}=$ rate parameter to pool 2, from pool 1; $k_{02}$ rate parameter of irreversible loss from pool 2; $U_{2}$ : rate of appearance of Phe in pool 2. (C) Physiological assignment of SAAMII computer model: Flux $\left(F_{12}\right)$ of Phe from the intra-cellular pool to the extra cellular pool represents the $k_{21}$ times $Q_{1}$, and is equal to $F_{21}$ in physiological steady state; $U_{2}\left(=F_{20}\right)$ represents WbPB; Flux of Phe from the intra-cellular pool to hydroxylation and protein synthesis represents the irreversible loss $\left(F_{02}\right.$ $\left.=k_{02} Q_{2}\right) ; W b R_{a}$ of Phe is the fraction of Phe from protein breakdown that appears in $Q_{1}$ that is not irreversible lost; The amount of Phe flux between $Q_{1}$ and $Q_{2}$ that is not irreversible lost is shunt back to $Q_{1}\left(F_{21}-W b R_{a}\right)$. 
A

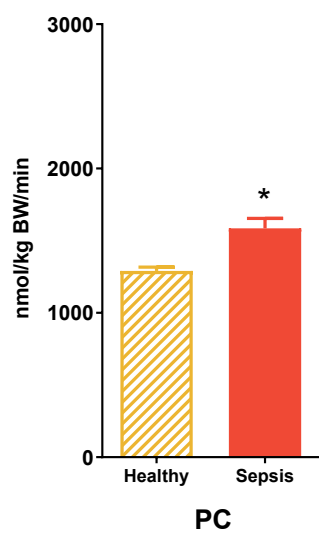

B

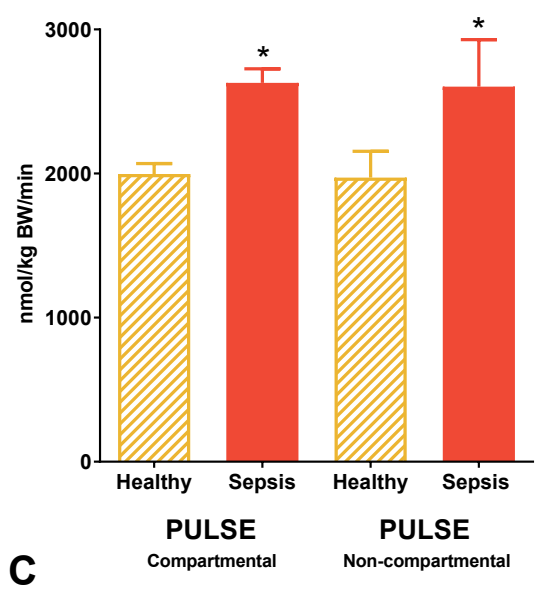

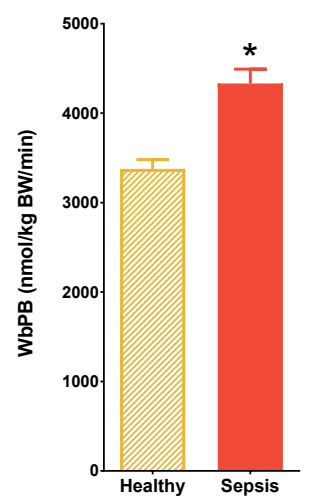

Figure 6. Effect of sepsis on whole body rate of appearance (WbRa) of phenylalanine (Phe) using two different tracer methods

WbRa as proxy for whole body protein breakdown in sepsis and healthy pigs. Calculated with A. PC tracer method: primed-continuous infusion of L-[ring $-{ }^{13} \mathrm{C}_{6}$ ]-Phe. Or B. with the PULSE tracer method: bolus infusion of $\mathrm{L}-\left[{ }^{15} \mathrm{~N}\right]-\mathrm{Ph}$. Compartmental and non-compartmental data analyses. C: Effect of sepsis on whole body protein breakdown (WbPB). WbPB data obtained with compartmental modeling (PULSE method). Data expressed as mean \pm SEM. Healthy $n=9$, Sepsis $n=13$. Statistics: sepsis compared to Healthy, unpaired t-test. *) significance $p<0.05$.

\section{Section 3: Interorgan balance studies in disease models.}

In this section we described the use of stable tracers to unravel different metabolic routes in disease states. Chapter 8 and 9 discusses arginine-nitric oxide metabolism in a model of acute liver failure in unconscious pigs. Chapter 8 provides a review of existing literature considering the different aspect of arginine de novo and nitric oxide (NO) production in disease states (18). Arginine is a conditionally essential amino acid in healthy adults, and therefore there is no specific 
nutritional requirement. However, dietary arginine is required in neonates, infants, and in certain conditions and diseases. L-arginine was identified as the precursor for NO. Although first discovered in endothelial cells, NO appeared to be a ubiquitous molecule present in a variety of cells including cells from the cardiovascular and nervous systems and also inflammatory cells. As such, NO has many physiological functions, and the relationship between arginine availability and NO production emphasizes the functional relevance of arginine.

In Chapter 9, we studied the arginine- NO metabolic route in acute liver failure (ALF) in the pig, because the hyperdynamic circulation is believed to be the result of overproduction of nitric oxide (NO) in the splanchnic circulation (19). However, it has been suggested that arginine concentrations (the substrate for $\mathrm{NO}$ ) are believed to be decreased in ALF, limiting substrate availability for NO production. To characterize the metabolic fate of arginine in early-phase ALF, we systematically assessed its interorgan transport and metabolism and measured the endogenous NO synthase inhibitor asymmetric dimethylarginine (ADMA) in a porcine model of ALF. Female adult pigs $(23-30 \mathrm{~kg}$ ) were randomized to a sham or hepatic devascularization ALF procedure for $6 \mathrm{~h}$. We measured plasma arginine, citrulline, ornithine levels; arginase activity, NO, and ADMA (Figure 7). Whole body metabolic rates and interorgan flux measurements were calculated using stable isotope-labeled amino acids. Plasma arginine decreased $>85 \%$ of the basal level at $t=6 \mathrm{~h}$, whereas citrulline and ornithine progressively increased in ALF. No difference was found between the groups in the whole body rate of appearance of arginine or NO. However, ALF induced a significant increase in de novo arginine synthesis. Interorgan data showed that citrulline net intestinal production and renal consumption was related to net renal production of arginine and ornithine. Both plasma arginase activity and plasma ADMA levels significantly increased in ALF. In this model of early-phase ALF, arginine deficiency or higher ADMA levels do not limit whole body NO production.

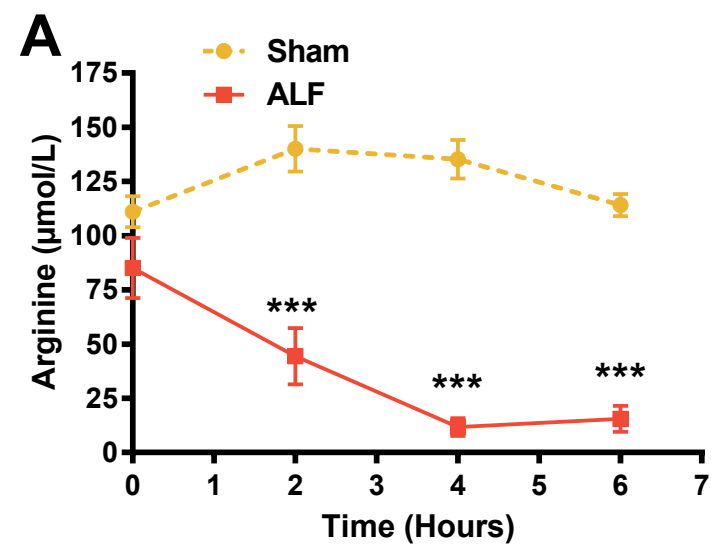



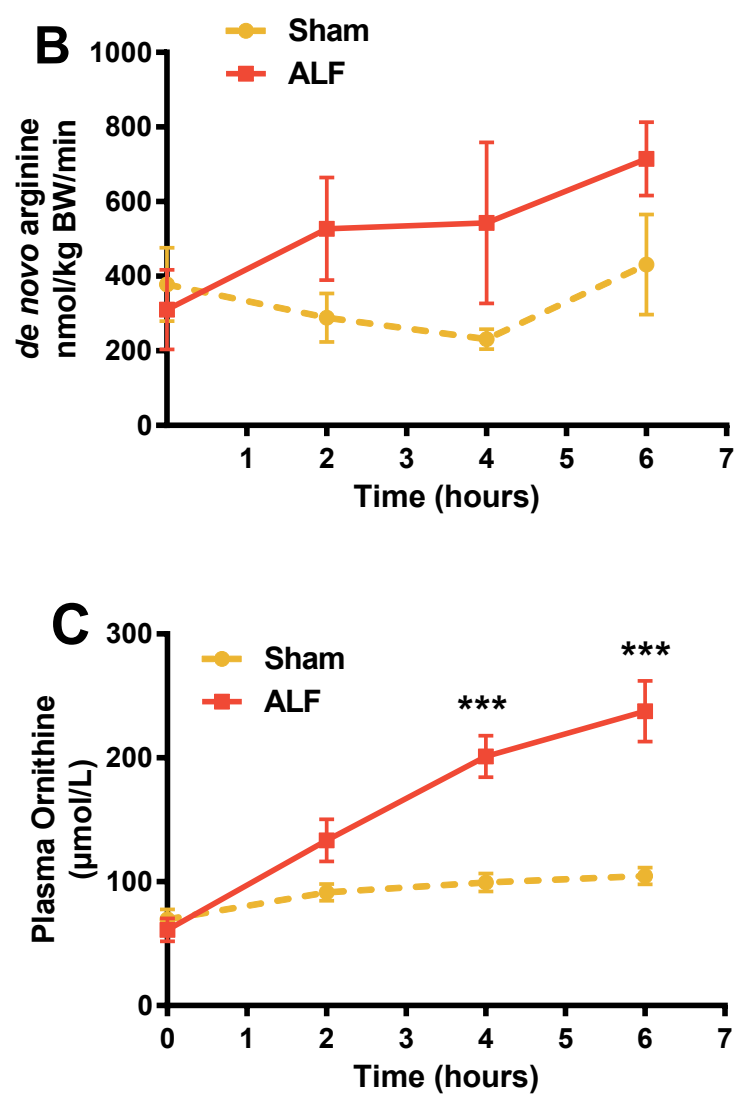

D

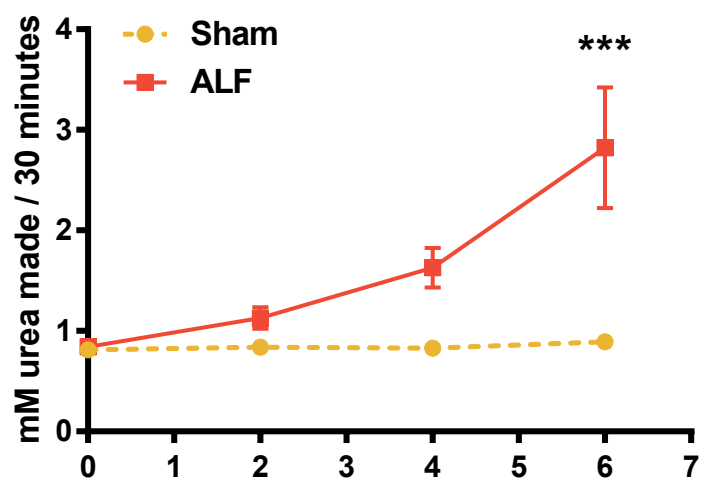

Figure 7. Plasma arginine (A) concentration, de novo arginine (B), Plasma ornithine (C) and plasma arginase activity (D) in Sham-operated and acute liver failure (ALF) pigs throughout the experimental period

There is a significant increase in the plasma ornithine levels with a corresponding significant increase in the plasma arginase activity at the end of the experimental period. $\left.{ }^{*}\right) \mathrm{p}<0.05 ;{ }^{* * *}$ ) $p<0.001 ; 2$-way ANOVA. Data shown are means \pm SE. 
Therefore, we conclude that arginine deficiency is caused by arginase-related arginine clearance in which arginine production is stimulated de novo.

In Chapter 10 and 11 we studied multi-organ metabolic disturbances in the Pseudomonas aeruginosa (PA) induced severe sepsis model. Maintenance of gut integrity has long been recognized as crucial for survival in sepsis but alterations in gut protein metabolism have not previously been documented. Therefore in Chapter 10, we studied the effect of a PA induced sepsis, on fractional protein synthesis (FSR) and breakdown rates (FBR) in jejunal mucosa in a fasted conscious state. We determined FSR by the measurement of the incorporation of stable isotope labeled amino acid (primed continuous infusion, $L$-[ring- ${ }^{13} \mathrm{C}_{6}$ ]-phenylalanine) into tissue protein, and FBR using the relation between blood arterial enrichment and intracellular enrichment in consecutive mucosal biopsies after a pulse of L-[ $\left.{ }^{15} \mathrm{~N}\right]$ phenylalanine. Additionally, we determined the FSR of other splanchnic tissue (jejunum, ileum, liver) and compared them with muscle and lung tissue. We found in this sham controlled acute severe sepsis pig model that jejunal mucosal protein turnover is reduced with both decreased FSR and FBR (Figure 8). We also found that FSR was unchanged in ileum and muscle, while it was higher in the liver and tended to be higher in the lung (Figure 9. In conclusion, our data suggest that jejunal mucosal protein metabolism is diminished in acute severe sepsis. Comparison with other tissues indicates that the most serious acute changes in severe sepsis occur in the gut rather than muscle.

In Chapter 11 inter- and multi-organ amino acid (AA) kinetics in sepsis was studied. We studied in our septic pig model the metabolic pathways that are involved in net catabolism in severe sepsis, by measuring AA substrate fluxes with multiple stable amino acid tracers on whole body (Wb) level by measuring rate of appearances (Ra)
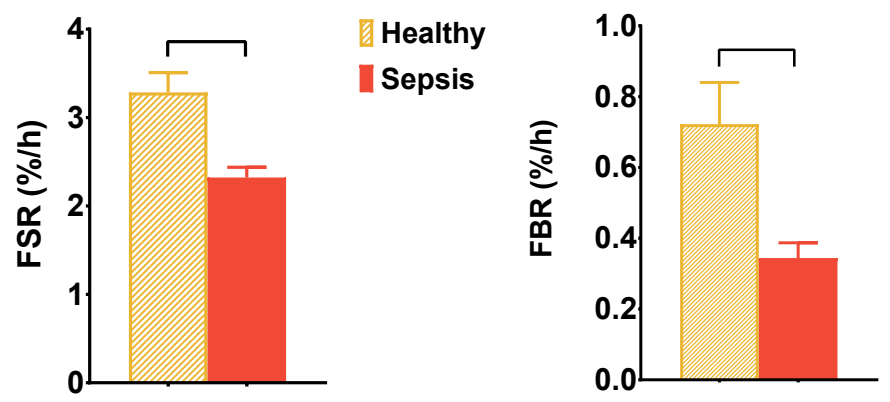

Figure 8. Fractional Synthesis Rate (FSR) and fractional breakdown rate (FBR) of protein in mucosa

Expressed as mean \pm SE in percent per hour. Post-absorptive state. FSR: Healthy $N=9$, Sepsis $\mathrm{N}=11$. FBR: Healthy $\mathrm{N}=8$, Sepsis $\mathrm{N}=9$ Statistics: Student t-test; Hook: $p<0.05$. 


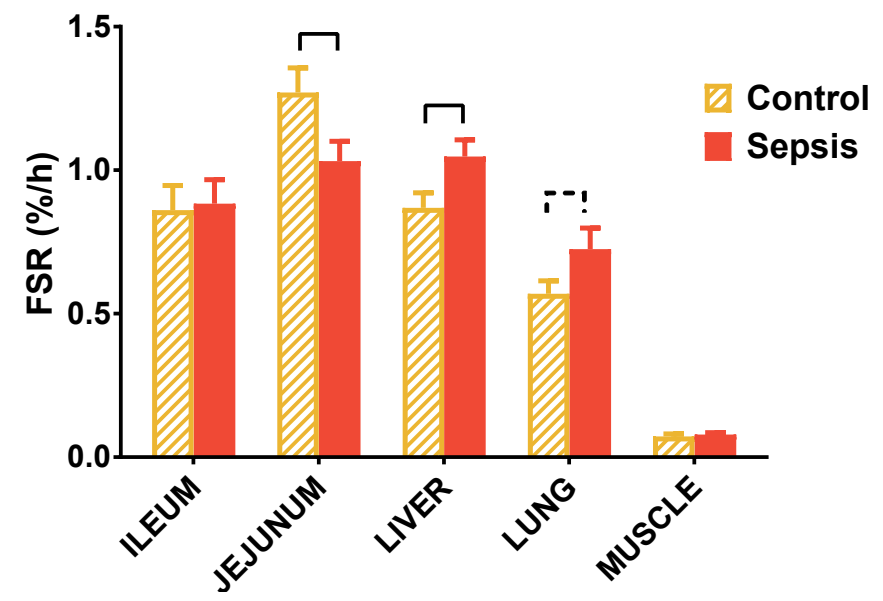

Figure 9. Fractional Synthesis Rate (FSR) of protein in tissues

Expressed as mean \pm SE in percent per hour. Post-absorptive state; Healthy $N=9$; Sepsis $N=11$. Statistics: Two-way ANOVA, sepsis effect $p=0.673$, tissue effect $p<0.0001$, interaction 0,0189 ; Post-hoc test Holm-Sidak: Hook: p<0.05; Dashed hook: tendency $\mathrm{p}<0.1$.

of $A A$, and $A A$ net balances (NB), PB and protein synthesis (PS) across the portal drained viscera (PDV), splanchnic area (SPL), liver and hindquarter (HQ). We measured before (baseline; BL) and 12 to 18 hours (POST) after start of sepsis induction by PA. We established in this sham controlled acute severe sepsis pig model that enhanced WbPB, WbPS, Wb net catabolic state and myofibrillar muscle breakdown occurs in sepsis. Across PDV, both PB and PS are decreased. The liver showed substantial increased uptake (3 times higher than control) of amino acids and severe increase of ureagenesis. Overall the SPL and HQ NB could not explain the enhanced whole body PB (Figure 10). Interacting glutamate, glutamine and alanine multiorgan pathways showed that severe systemic glutamate decrease (50\% of Control), in humans related to enhanced mortality, was caused by decreased glutamate release of the liver to the systemic plasma pool that could not be compensated by interacting glutamine and alanine multiorgan pathways (Figure 11). We conclude that whole body enhanced protein turnover and net catabolism cannot be explained by significant changes in protein metabolism in one single or multiple measured organs, despite prominent changes in muscle and the SPL area protein metabolism. The PDV is not responsible for the enhanced whole body protein turnover and thus other organ/tissue/proteins must be involved. Further tracer studies on tissue/ protein level will be helpful to delineate this. On the basis of the present results we hypothesize that in the acute phase of severe sepsis, the overall metabolic downregulated PDV and the compromised liver are key metabolic factors in sepsis. 


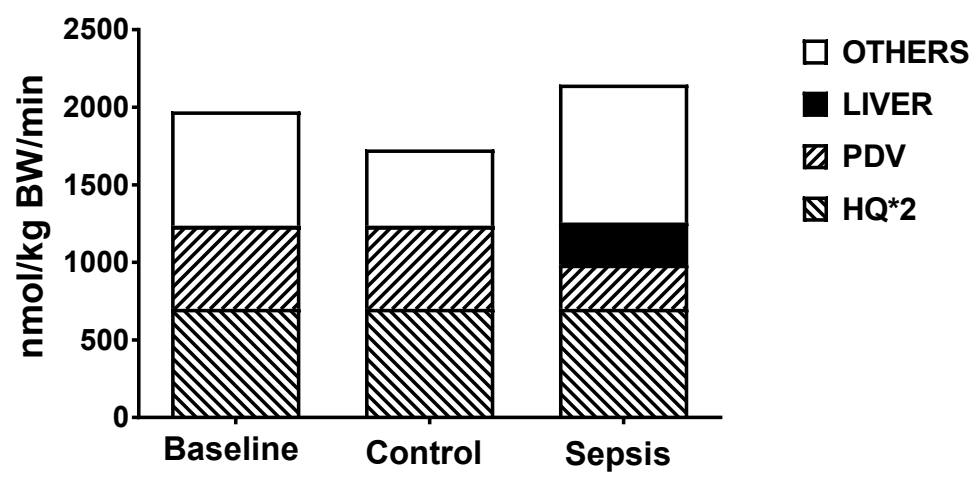

Figure 10. Contribution of organ Phe production to whole body rate of appearance of Phe (hypothetical)

Before (Baseline) and post infusion of Pseudomonas aeruginosa. Height of the bar = whole body rate of appearance of PHE corrected with factor of 1.6 (17). HQ is hindquarter (muscle compartment), $\mathrm{HQ}^{*} 2$ = whole body muscle compartment. PDV is Portal drained viscera.

\section{Summary of conclusions}

- To study factors that lead to enhanced, reduced or modified absorption kinetics, a sophisticated model in multi-catheterized pigs along with the use of isotopes that can calculate absorption and gut metabolism is necessary.

- Data coming from a catheterized model that enables simultaneously liver, portal drained viscera, spleen, liver, kidney, and hindquarter flux measurement of many metabolic substances in the pig are highly translational to humans due to the remarkable physiological similarity of the pig to humans.

- Protein quality of a meal is related to the postprandial anabolic response of the intestine.

- Systemic bio-availability of potential bioactive lacto-peptides are mainly determined by the digestion rate of the meal, the quality of protein, and fiber contents.

- The presented Pseudomonas aeruginosa induced porcine sepsis model is a new clinically relevant model for acute severe sepsis and instrumentation enables study complex multi-organ metabolism.

- The whole body rate of appearance of phenylalanine in plasma obtained by the easy-to-use (non-)compartmental stable tracer pulse infusion approach, reflects better the real whole body protein breakdown than the traditional primedcontinuous infusion. 

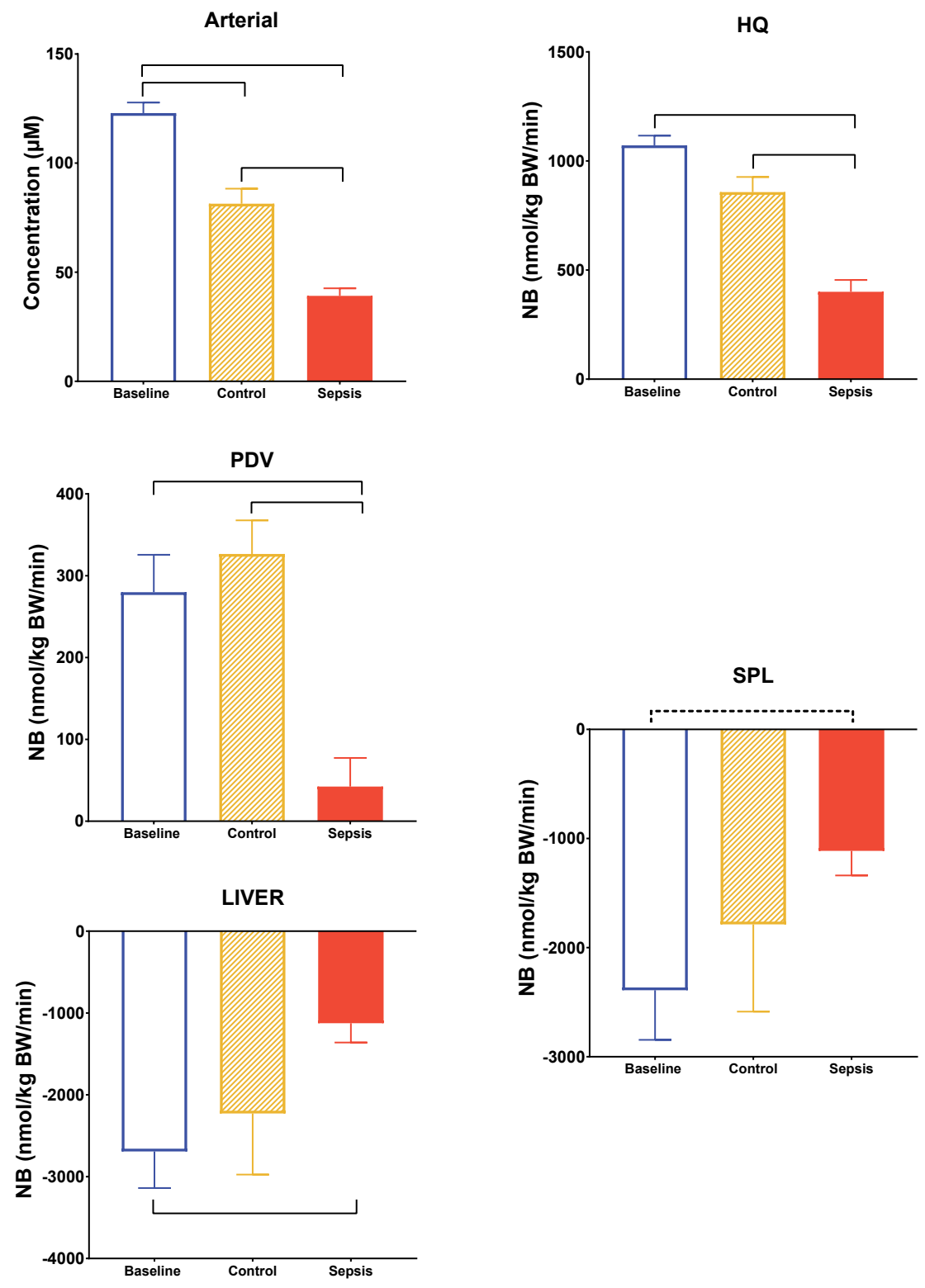

Figure 11. Glutamate arterial concentrations and organ net balances (NB)

Before (Baseline) and post infusion of Pseudomonas aeruginosa. HQ is hindquarter (muscle compartment), PDV is Portal drained viscera, SPL is splanchnic area. Control $n=9$, Sepsis $n=13$. Statistics: unpaired Student t-test, Significance $p<0.05$ (hook), Tendency $p<0.1$ (dashed hook). 
- Membrane transport capacity for amino acids is not compromised in severe sepsis.

- Nitric Oxide (NO) has many physiological functions, and the relationship between arginine availability and NO production emphasizes the functional relevance of arginine.

- In early-phase of acute liver failure, arginine deficiency or higher ADMA levels do not limit whole body NO production. Therefore, we conclude that arginine deficiency is caused by arginase-related arginine clearance in which arginine production is stimulated de novo.

- Jejunal mucosal protein metabolism is diminished in acute severe sepsis. Comparison with other tissues indicates that the most serious acute metabolic changes in severe sepsis occur in the gut rather than the muscle.

- Enhanced whole body protein turnover and net catabolism in acute severe sepsis cannot be explained by significant protein metabolism changes in one single or multiple measured organs, despite prominent changes in muscle and the splanchnic area protein metabolism. We hypothesize that in the acute phase of severe sepsis, the overall metabolic downregulated portal drained viscera and the compromised liver are key metabolic factors.

\section{Future perspectives}

\section{Multiorgan and transorgan research}

In the present dissertation, I presented several examples of multiorgan and transorgan metabolic research. Presented data illustrates an process of ongoing insight in the complex dynamics of nutritional substrate fluxes in (patho)physiological conditions, development of new and improved approaches of measurement due to the availability of advanced and improved analytical and tracer modeling technologies. However depending on the scientific question, future improvements like measurement of other organs, tissues, proteins, macronutrients, disease states with a variety of tracer models other than described in this dissertation, could be considered to continue the process of unraveling quantitatively highly compartmentalized, dynamic (disturbed) substrate metabolism and potential testing of new nutritional interventions.

\section{Organs}

Data showed that transorgan and multi-organ substrate flux measurements play an important role to understand whole body metabolic balances. It is clear that both 
in a health or disease state the gut and liver have a crucial role in the systemic bioavailability of nutrients which is highly dynamic and complex. Our knowledge about substrate metabolism and trafficking across the lung is however very limited and need to be considered in future studies, especially in disease states in which complex multiorgan substrate trafficking and lung and multiorgan failure is expected. We think that can be done by implantation of a Swan Ganz catheter to accommodate afferent blood sampling (pulmonary artery) for the lung.

\section{Tissues and proteins}

For protein metabolism, measuring absolute substrate balances across organs is the gold standard to understand protein breakdown and synthesis in certain areas, but it can be a challenge to obtain valid measurements in organs with a high level of metabolism and blood flow where arterial venous differences are small. Therefore, additional fractional synthesis and breakdown rate measurement on tissue/protein level could provide extra information with the use of for instance a triple pulse measurement (20).

\section{Macronutrients}

We showed the interacting role of other macronutrients like carbohydrates and fats during feeding on protein/peptide/amino acid absorption kinetics. However, in carbohydrate and fat metabolism, knowledge of interorgan dynamics are still limited (21-23). For instance, our knowledge of interorgan dynamics of carbohydrate and fat metabolism can be expanded by unraveling disturbed organ metabolism in diseases like obesity or metabolic syndrome and develop new/improved nutritional strategies.

\section{Nutritional intervention}

In disease states like acute severe sepsis, we showed major changes in the splanchnic area (low mucosal protein turnover, high amino acid wasting in the liver) indicating that early support of gut metabolism should be imminent to prevent or attenuate gut dysfunction. In the current clinical practice, early provision of enteral nutrition via the gastrointestinal tract to critically ill patients has been established as a standard marker of quality of care for critically ill patients $(7,24)$. However, due to complexity of the disease states (often the occurrence of multi-organ failure) in these patients, there is still a lot of challenges to overcome for adequate diagnostics (e.g. personalized requirements to determine dosing), adequate delivery (e.g. volume in time when gastro-intestinal intolerance occur), and optimal composition (e.g. the use of supplementation of specific micro and/or macro nutrients). Therefore, we conclude that multiorgan and transorgan measurements will be a valuable necessity in future (clinical) metabolic/nutritional research to collect supporting information for new/ improved clinical nutritional strategies. 


\section{Role of the pig as multi-organ and transorgan model in metabolic research}

Although the use of an animal for human (clinical) research can be controversial, experiments in animal models are relevant and essential, due to the fact that it is extremely difficult to perform studies in human subjects to the multitude of ethical issues and the limitations of invasive procedures. However, the principles of the 3Rs (reduction, refinement, replacement) are ongoing considerations for future studies.

\section{Reduction}

As described in the present dissertation, the pig is a clinically relevant large animal model to study multiorgan metabolism, but the invasive multi-catheterization that is required for this, demands for intensive post-surgical care (chapter 3 ) and highly skilled personnel. However, the surgical implantation of the catheters, easy adaptation of the pig to a small cage when performing experiments and the size of the animal ensures that we can measure over time and in a physiological relevant conscious condition in the post-absorptive and/or postprandial state. With the implementation of the use of existing and new commercial available stable tracers and new combined multiple stable tracer measurements with diverse "high throughput" mass spectrometry applications, we have the opportunity to study more scientific questions simultaneously in the same animal and therefore reduce the amount of animals needed.

\section{Refinement}

Despite widespread discussions in literature, the perfect clinically relevant animal model does not exist. Most of the time, concessions need to be made for practical and ethical reasons. For instance, with the presented pig model (20-30kg, female), you could argue that the pig is still very young (and female only) and therefore is not representing the humans to whom we want to translate the data to. We made these concessions for practical reasons (handling, catheter protection with the used harness), but with the knowledge that the development stage of the gastro intestinal tract in these animals is already mature (25). Therefore, we can still translate the obtained data to a broader age range. Nevertheless, if older age is critical for the scientific question to solve, the presented catheter model could also be used in long-term studies in older minipigs. Future efforts to refine for instance disease animal models like sepsis as described in chapter 6 and liver failure in chapter 9, will increase the quality of the data deducted from these studies and therefore its translational validity.

\section{Replacement}

Ongoing development of new multi stable tracer model approaches and technologies that are able to measure on systemic level, different organ related metabolic 
fluxes in for instance the splanchnic area (digestion, gut absorption, gut extraction, liver extraction of amino acids) simultaneously, can be translational to humans and could therefore potentially be replaced in humans. The pig could play a crucial role to validate these new models.

\section{General conclusion}

With the use of a unique highly clinical relevant catheterized pig model in health and certain disease states like severe sepsis and acute liver failure, we are able to unravel complex interaction between multiple specialized organs for transportation, digestion, absorption, redistribution, utilization, conversion, breakdown of nutritional substrates during post-prandial and/or post-absorptive stage(s). Presented results indicates the complex interactions of organs in the splanchnic area are highly dynamic. In feeding condition, food macronutrient composition and quality (e.g. amino acid profile of the used protein) are major factors in splanchnic utilization and systemic bioavailability. In pathophysiological conditions like acute severe sepsis the splanchnic area is highly vulnerable and therefore more quickly disturbed than muscle compartments. Together with ongoing new developments in the use of stable tracer models, the multi-organ metabolic research in the pig will continue to be of high value in the future for quantitative substrate flux measurements that are needed to support new nutritional strategies in health and disease to keep our body in nutritional balance.

\section{References}

1. Rosenblatt S, Clowes GH, Jr., George BC, Hirsch E, Lindberg B. Exchange of amino acids by muscle and liver in sepsis. Arch Surg. 1983;118(2):167-75.

2. Hartl WH, Jauch KW. Metabolic self-destruction in critically ill patients: origins, mechanisms and therapeutic principles. Nutrition. 2014;30(3):261-7.

3. Klaude M, Mori M, Tjader I, Gustafsson T, Wernerman J, Rooyackers O. Protein metabolism and gene expression in skeletal muscle of critically ill patients with sepsis. Clin Sci (Lond). 2012;122(3):133-42.

4. Rooyackers O, Kouchek-Zadeh R, Tjader I, Norberg A, Klaude M, Wernerman J. Whole body protein turnover in critically ill patients with multiple organ failure. Clin Nutr. 2015;34(1):95-100.

5. Coker RH, Wolfe RR. Bedrest and sarcopenia. Curr Opin Clin Nutr Metab Care. 2012;15(1):7-11.

6. Heyland DK, Stapleton RD, Mourtzakis M, Hough CL, Morris P, Deutz NE, et al. Combining nutrition and exercise to optimize survival and recovery from critical illness: Conceptual and methodological issues. Clin Nutr. 2016;35(5):1196-206.

7. Deutz NE, Matheson EM, Matarese LE, Luo M, Baggs GE, Nelson JL, et al. Readmission and mortality in malnourished, older, hospitalized adults treated with a specialized 
oral nutritional supplement: A randomized clinical trial. Clin Nutr. 2016;35(1):18-26.

8. Jalan R, Olde Damink SW, Hayes PC, Deutz NE, Lee A. Pathogenesis of intracranial hypertension in acute liver failure: inflammation, ammonia and cerebral blood flow. J Hepatol. 2004;41(4):613-20.

9. Olde Damink SW, Deutz NE, Dejong CH, Soeters PB, Jalan R. Interorgan ammonia metabolism in liver failure. Neurochem Int. 2002;41(2-3):177-88.

10. Ytrebo LM, Sen S, Rose C, Ten Have GA, Davies NA, Hodges S, et al. Interorgan ammonia, glutamate, and glutamine trafficking in pigs with acute liver failure. Am J Physiol Gastrointest Liver Physiol. 2006;291(3):G373-81.

11. Ten Have GA, Engelen MP, Luiking YC, Deutz NE. Absorption kinetics of Amino Acids, Peptides, and Intact Proteins. Int J Sport Nutr Exerc Metab. 2007;17(Supplement):S23-S36.

12. Ten Have GA, Bost MC, Suyk-Wierts JC, van den Bogaard AE, Deutz NE. Simultaneous measurement of metabolic flux in portally-drained viscera, liver, spleen, kidney and hindquarter in the conscious pig. Lab Anim. 1996;30(4):347-58.

13. Ten Have GA, Engelen MP, Soeters PB, Deutz NE. Absence of post-prandial gut anabolism after intake of a low quality protein meal. Clin Nutr. 2012;31(2):273-82.

14. Ten Have GA, van der Pijl PC, Kies AK, Deutz NE. Enhanced Lacto-Tri-Peptide BioAvailability by Co-Ingestion of Macronutrients. PLoS One. 2015;10(6):e0130638.

15. Ten Have GAM, Deutz RCl, Engelen M, Wolfe RR, Deutz NEP. Characteristics of a Pseudomonas aeruginosa induced porcine sepsis model for multi-organ metabolic flux measurements. Lab Anim. 2017:23677217718003.

16. Wolfe RR, Chinkes DL. Isotope Tracers in Metabolic Research: Principles and Practise of Kinetic Analysis. New York: Wiley; 2005. 274 p.

17. Ten Have GA, Engelen MP, Wolfe RR, Deutz NE. Phenylalanine isotope pulse method to measure effect of sepsis on protein breakdown and membrane transport in the pig. Am J Physiol Endocrinol Metab. 2017:ajpendo 003512016.

18. Luiking YC, Ten Have GA, Wolfe RR, Deutz NE. Arginine de novo and nitric oxide production in disease states. Am J Physiol Endocrinol Metab. 2012;303(10):E1177-89.

19. Sharma V, Ten Have GA, Ytrebo L, Sen S, Rose CF, Dalton RN, et al. Nitric Oxide and L-Arginine metabolism in a devascularized porcine model of Acute Liver Failure. American journal of physiology Gastrointestinal and liver physiology. 2012.

20. Bosoi CR, Oliveira MM, Ochoa-Sanchez R, Tremblay M, Ten Have GA, Deutz NE, et al. The bile duct ligated rat: A relevant model to study muscle mass loss in cirrhosis. Metab Brain Dis. 2017;32(2):513-8.

21. Mann J, Cummings JH, Englyst HN, Key T, Liu S, Riccardi G, et al. FAO/WHO scientific update on carbohydrates in human nutrition: conclusions. Eur J Clin Nutr. 2007;61 Suppl 1:S132-7.

22. Kalish BT, Fell GL, Nandivada P, Puder M. Clinically Relevant Mechanisms of Lipid Synthesis, Transport, and Storage. JPEN J Parenter Enteral Nutr. 2015;39(1 Suppl):8S-17S.

23. Bischoff SC, Boirie Y, Cederholm T, Chourdakis M, Cuerda C, Delzenne NM, et al. Towards a multidisciplinary approach to understand and manage obesity and related diseases. Clin Nutr. 2016.

24. Ridley E, Gantner D, Pellegrino V. Nutrition therapy in critically ill patients- a review of current evidence for clinicians. Clin Nutr. 2015;34(4):565-71.

25. Guilloteau P, Zabielski R, Hammon HM, Metges CC. Nutritional programming of gastrointestinal tract development. Is the pig a good model for man? Nutr Res Rev. 2010;23(1):4-22. 
CHAPTER 13 


\section{Samenvatting}

Een gezond lichaam heeft voedingsstoffen nodig voor een gebalanceerde stofwisseling. Zowel in gevoede- als in nuchtere toestand vinden complexe interacties tussen organen plaats om transport, vertering, absorptie, herdistributie, verbruik, omzetting en afbraak van voedingssubstraten mogelijk te maken. Deze interacties zijn dynamisch van aard en verstoord in pathofysiologische condities zoals bloedvergiftiging (sepsis) en leverfalen (1-10). Om stoornissen in de stofwisseling te kunnen ontrafelen zijn kwantitatieve en dynamische gegevens van substraattransport nodig. Deze kennis draagt bij aan de ontwikkeling van nieuwe voedingsstrategieën ter verbetering van de stofwisseling en ter ondersteuning van medische behandelingen. Vanwege ethische dilemma's en de benodigde invasieve procedures is het meten van de transportcapaciteit (hoeveelheid transport in de tijd) van substraten op (inter)orgaan niveau moeilijk uitvoerbaar in gezonde mensen en in het bijzonder in ernstige zieke patiënten. Klinisch relevante diermodellen zijn daarom essentieel om (patho)fysiologische transport- en stofwisselingroutes tussen organen op een gecontroleerde en herhaalbare manier te kunnen bestuderen. Diermodellen maken het gebruik van invasieve technieken mogelijk en daardoor de uitvoering van meerdere metingen binnen een dier tijdens voeding en ziekte. Vanwege de opvallende metabole gelijkenissen tussen varken en mens is het varkensmodel geliefd in biomedisch voedingsonderzoek en onderzoek naar metabole ziektebeelden.

Dit proefschrift is een weergave van stofwisselingsonderzoek, met name aan varkens met acute bloedvergiftiging veroorzaakt door de Pseudomonas aeruginosa bacterie. Door middel van hoogwaardige stabiele tracer methoden wordt het stofwisselingstransport van voornamelijk aminozuren en eiwitten, hun metabolieten en de interactie met andere macronutriënten op (inter)orgaanniveau getoond. Daarnaast presenteert dit proefschrift een studie van een onder anesthesie gedevasculariseerd leverfalen model.

Deel één brengt de huidige kennis van absorptie(snelheden) van eiwitten, peptiden, en aminozuren in kaart (hoofdstuk 2) (11). De dunne darm gedraagt zich als een intermediair tussen het darmlumen en de rest van het lichaam. Het transport van aminozuren uit een eiwitmaaltijd wordt gecontroleerd en gereguleerd door de dunne darm. Vervolgens gaan de aminozuren via de vena porta naar de lever en de grote circulatie. Na inname van een gebalanceerde eiwitmaaltijd gebruikt de darm 30-50\% van de aminozuren uit de voeding voor opbouw van een tijdelijke eiwitopslagplaats die vervolgens gebruikt kan worden in de nuchtere toestand. Ook de afwezigheid van een verschil in absorptie van eiwitten, peptiden en aminozuren 
komt aan bod; andere factoren leiden tot veranderingen in absorptie kinetiek van deze voedingsstoffen. Om het effect van deze factoren te bestuderen op de darmstofwisseling is een geavanceerd gekatheteriseerd varkensmodel nodig dat gebruik maakt van stabiele tracers. Hoofdstuk 3 beschrijft zo'n model dat gelijktijdig de substraattransporten over de lever, portaal gedraineerde organen (darm, milt), de nier en de achterbenen (welke voornamelijk bestaan uit spieren) meet (12). De stofwisselingsgegevens afkomstig van dit varkensmodel kunnen gemakkelijk vertaald worden naar die van de mens. Verder laten hoofdstuk 4 en 5 zien dat de kwaliteit van het eiwit in een maaltijd bepalend is voor hoeveel eiwitopbouw er uiteindelijk plaatsvindt in de darm (hoofdstuk 4) (13). Tenslotte toont dit proefschrift dat voornamelijk de verteringssnelheid en kwaliteit van het eiwit in de voeding, en de aanwezigheid van vezels de beschikbaarheid in de grote circulatie van potentieel bioactieve lacto-peptiden bepalen (hoofdstuk 5) (14).

Deel twee beschrijft een nieuw (door Pseudomonas aeruginosa bacterie veroorzaakt) varkens bloedvergiftigingsmodel dat bruikbaar is als een nieuw klinisch relevant model voor acute ernstige sepsis. Het inbrengen van de verschillende katheters in dit model geeft de mogelijkheid om complexe orgaanstofwisselingsroutes in dit ziektebeeld te bestuderen (hoofdstuk 6) (15). Hoofdstuk 7 betreft de vergelijking van twee stabiele tracermethoden die gebruikt kunnen worden om eiwitafbraak op heel lichaamsniveau te meten. De conclusie luidt dat de mate van verschijning van het aminozuur phenylalanine in het bloed met behulp van een eenmalige tracerpulse injectie een betere waarde geeft van de echte eiwitafbraaksnelheid dan een meer traditioneel gebruikte methode waarbij de tracer met een constante snelheid wordt geïnfundeerd (16). Bovendien blijkt de membraan transportcapaciteit voor aminozuren niet verminderd bij ernstige sepsis.

Deel drie beschrijt het gebruik van stabiele tracers om verschillende stofwisselingsroutes in ziektebeelden te kunnen ontrafelen. Een review beschrijft de vele fysiologische functies van stikstofoxide (nitric oxide, NO) en de relaties tussen de NO productie en de beschikbaarheid en functionele relevantie van het aminozuur arginine (bouwstof van NO) (hoofdstuk 8) (17). Hoofdstuk 9 toont dat in een vroege fase van acuut leverfalen het amino zuur arginine deficiënt is en de waarde van asymmetrisch dimethylarginine (ADMA, endogene NO synthese remmer) verhoogd. Dit resulteert echter niet in een verminderde NO productie. Uit grondige bestudering van de stofwisseling tussen organen blijkt dat de arginine deficiëntie veroorzaakt wordt door een verhoogde arginase activiteit buiten de lever, terwijl de NO productie op peil blijft door een verhoogde arginine productie in de nier (18).

Hoofdstukken 10 en 11 laten zien dat de eiwitstofwisseling in de darmmucosa verminderd is bij acute ernstige bloedvergiftiging. Vergelijking van deze resultaten met die van andere weefsels toont dat de grootste veranderingen in de stofwisseling 
tijdens bloedvergiftiging plaatsvinden in de darm en niet in de spier (hoofdstuk 10). Ook blijkt dat de verhoogde eiwitstofwisseling en afbraak op lichaamsniveau niet verklaard kunnen worden door de bestudeerde veranderingen in stofwisseling in de organen, ondanks duidelijke veranderingen gevonden in de spiereiwitstofwisseling en aminozuurstofwisseling in het darm-lever gebied (hoofdstuk 11). De conclusie op basis van de resultaten beschreven in het proefschrift luidt dat in de acute fase van ernstige bloedvergiftiging de stofwisselingsvertraging in de darm en de verstoorde leverstofwisseling belangrijke metabole factoren zijn in het verdere beloop van de aandoening.

\section{Conclusie}

Het beschreven klinisch relevante gekatheteriseerd varkensmodel stelt onderzoekers in staat om inzicht te krijgen in de stofwisseling in gezonde toestand en tijdens ziekte zoals ernstige bloedvergiftiging en acuut leverfalen. De beschreven resultaten laten zien dat er complexe relaties zijn tussen de organen in het darm-lever gebied en dat deze relaties zeer dynamisch zijn. In gevoede toestand zijn de samenstelling en kwaliteit van macronutriënten in voeding (zoals de aminozuursamenstelling van het eiwit) belangrijke factoren die de beschikbaarheid van voedingsnutriënten in de circulatie beïnvloeden. In pathofysiologische condities zoals tijdens ernstige bloedvergiftiging is het darm-lever gebied zeer kwetsbaar en de stofwisseling hier eerder verstoord dan in een spier compartiment. In combinatie met nieuwe stabiele tracermethoden zal het metabool onderzoek op (inter) orgaanniveau in het varken nieuwe belangrijke inzichten blijven genereren in de toekomst. Kwantitatieve en dynamische transportmetingen van substraten zijn noodzakelijk om nieuwe voedingsstrategieën tijdens ziekte te ontwikkelen met als doel de stofwisseling in balans te houden.

\section{Referenties}

1. Rosenblatt S, Clowes GH, Jr., George BC, Hirsch E, Lindberg B. Exchange of amino acids by muscle and liver in sepsis. Arch Surg. 1983;118(2):167-75.

2. Hartl WH, Jauch KW. Metabolic self-destruction in critically ill patients: origins, mechanisms and therapeutic principles. Nutrition. 2014;30(3):261-7.

3. Klaude M, Mori M, Tjader I, Gustafsson T, Wernerman J, Rooyackers O. Protein metabolism and gene expression in skeletal muscle of critically ill patients with sepsis. Clin Sci (Lond). 2012;122(3):133-42.

4. Rooyackers O, Kouchek-Zadeh R, Tjader I, Norberg A, Klaude M, Wernerman J. Whole body protein turnover in critically ill patients with multiple organ failure. Clin Nutr. 2015;34(1):95-100.

5. Coker RH, Wolfe RR. Bedrest and sarcopenia. Curr Opin Clin Nutr Metab Care. 2012;15(1):7-11.

6. Heyland DK, Stapleton RD, Mourtzakis M, Hough CL, Morris P, Deutz NE, et 
al. Combining nutrition and exercise to optimize survival and recovery from critical illness: Conceptual and methodological issues. Clin Nutr. 2016;35(5):1196-206.

7. Deutz NE, Matheson EM, Matarese LE, Luo M, Baggs GE, Nelson JL, et al. Readmission and mortality in malnourished, older, hospitalized adults treated with a specialized oral nutritional supplement: A randomized clinical trial. Clin Nutr. 2016;35(1):18-26.

8. Jalan R, Olde Damink SW, Hayes PC, Deutz NE, Lee A. Pathogenesis of intracranial hypertension in acute liver failure: inflammation, ammonia and cerebral blood flow. J Hepatol. 2004;41(4):613-20.

9. Olde Damink SW, Deutz NE, Dejong CH, Soeters PB, Jalan R. Interorgan ammonia metabolism in liver failure. Neurochem Int. 2002;41(2-3):177-88.

10. Ytrebo LM, Sen S, Rose C, Ten Have GA, Davies NA, Hodges S, et al. Interorgan ammonia, glutamate, and glutamine trafficking in pigs with acute liver failure. Am J Physiol Gastrointest Liver Physiol. 2006;291(3):G373-81.

11. Ten Have GA, Engelen MP, Luiking YC, Deutz NE. Absorption kinetics of Amino Acids, Peptides, and Intact Proteins. Int J Sport Nutr Exerc Metab. 2007;17(Supplement):S23-S36.

12. Ten Have GA, Bost MC, Suyk-Wierts JC, van den Bogaard AE, Deutz NE. Simultaneous measurement of metabolic flux in portally-drained viscera, liver, spleen, kidney and hindquarter in the conscious pig. Lab Anim. 1996;30(4):347-58.

13. Ten Have GA, Engelen MP, Wolfe RR, Deutz NE. Severely Compromised Anabolic Response to Nutrition in a Pseudomonas aeroginosa (PM) Induced Hyper-dynamic SepsisRecovery Pig Model. The FASEB Journal. 2016;30(1 Supplement):682.16-16.

14. Ten Have GA, van der Pijl PC, Kies AK, Deutz NE. Enhanced Lacto-Tri-Peptide BioAvailability by Co-Ingestion of Macronutrients. PLoS One. 2015;10(6):e0130638.

15. Ten Have GAM, Deutz RCl, Engelen M, Wolfe RR, Deutz NEP. Characteristics of a Pseudomonas aeruginosa induced porcine sepsis model for multi-organ metabolic flux measurements. Lab Anim. 2017:23677217718003.

16. Ten Have GA, Engelen MP, Wolfe RR, Deutz NE. Phenylalanine isotope pulse method to measure effect of sepsis on protein breakdown and membrane transport in the pig. Am J Physiol Endocrinol Metab. 2017:ajpendo 003512016.

17. Luiking YC, Ten Have GA, Wolfe RR, Deutz NE. Arginine de novo and nitric oxide production in disease states. Am J Physiol Endocrinol Metab. 2012;303(10):E1177-89.

18. Sharma V, Ten Have GA, Ytrebo L, Sen S, Rose CF, Dalton RN, et al. Nitric Oxide and L-Arginine metabolism in a devascularized porcine model of Acute Liver Failure. American journal of physiology Gastrointestinal and liver physiology. 2012. 
CHAPTER 14 


\section{Valorization}

A healthy human body needs nutrients to sustain its metabolic balance. To this end, complex interactions between specialized organs for transportation, digestion, absorption, redistribution, utilization, conversion, breakdown of nutritional substrates during post-prandial and/or post-absorptive stages must occur. These interactions are highly dynamic and subject to disturbances in many pathophysiological conditions (e.g. sepsis or liver failure) (1-10). To unravel (disturbed) complex nutritional substrate metabolism, quantitative and dynamic substrate flux measurements are needed. This knowledge will support the development of new nutritional strategies in health and disease, which are needed in light of many major worldwide nutritional problems:

\section{World Health Organization (WHO) malnutrition factsheet (May 2017) (11):}

Malnutrition, in all its forms, includes undernutrition (wasting, stunting, underweight), inadequate vitamins or minerals, overweight, obesity, and resulting diet-related non-communicable diseases.

- 1.9 billion adults are overweight or obese, while 462 million are underweight.

- 52 million children under 5 years of age are wasted, 17 million are severely wasted and 155 million are stunted, while 41 million are overweight or obese.

- Around $45 \%$ of deaths among children under 5 years of age are linked to undernutrition. These mostly occur in low- and middle-income countries. At the same time, in these same countries, rates of childhood overweight and obesity are rising.

- The developmental, economic, social, and medical impacts of the global burden of malnutrition are serious and lasting, for individuals and their families, for communities and for countries.

Malnutrition occurs in all countries and is considered one of the greatest global health challenges. Optimizing nutrition needs to start early in life to ensure longterm benefits. Poverty is considered a major risk factor for malnutrition and subsequently increases health care cost, reduces productivity and slows economic growth. Led by WHO and the Food and Agriculture Organization of the United Nations (FAO), the UN Decade of Action on Nutrition calls for policy action across several key areas. One of the areas is creating sustainable, resilient food systems for healthy diets. A healthy diet is a very generic term, and usually defined in terms 
of the needs of the organism, i.e. metabolic demands, and the dietary amount which will satisfy those needs, i.e. efficiency of utilization, thus: dietary requirement = metabolic demand/efficiency of utilization (12). Therefore, an appropriate diet in health is different from that in disease. For instance, we recently published that appropriate clinical nutrition is lowering mortality in hospitalized adults (7). In critically ill patients, muscle wasting is a life-threatening organ dysfunction that can be treated with appropriate nutrition $(13,14)$. To determine the "metabolic demand" and "efficiency of utilization", understanding of nutritional substrate metabolism, quantitative and dynamic substrate flux measurements in health and disease are needed and is the general focus of this dissertation.

Measuring complex fluxes and trafficking (amounts in time) of substrates in and between organs, is extremely difficult in humans due to the multitude of ethical issues and the limitations to perform invasive procedures, especially in pathophysiological conditions like in critically ill patients. Therefore, clinically relevant animal models are essential to study (patho) physiological metabolism in a controlled, repeatable way and to be able to use invasive techniques, providing multiple measurements and mechanistic data within the same animal in several nutritional and pathological stages. The pig, with its remarkable metabolic similarity to humans, is used often in biomedical research and more generally in the field of nutrition and associated metabolic disease states. Therefore the presented highly translational pig experiments generated important information that can be used for multiple purposes in the nutrition field.

We are aware that translational preclinical studies in the present dissertation are the "baby steps" in the journey to develop products and activities to solve worldwide nutritional health problems. However, the fact that eight of the ten described research projects in the present dissertation are published and received 184 citations (status $19^{\text {th }}$ of July 2017) indicates the value for the scientific community (15). Besides the scientific recognition, the societal relevance of this research was also recognized by the food industry, the ministry of economic affairs (The Netherlands) and the National Institutes of Health (USA). The findings of this dissertation can be used and translated in multiple ways for the food industry (development new products, improve consumers information), agriculture and environmental field (better understanding dietary protein quality in relation to meat production and emissions of nitrogen), clinical field (new food strategies, improvement design of clinical trials, better understanding of disturbed metabolism in critically ill patiënts, new clinical food products) and policy makers for general public (improved healthy food definitions/ claims/ information, importance of a healthy gut). 
Here an example:

In section 1 we presented research that shows the fate of amino acids (building blocks of protein) that are coming from dietary protein. We found that a high quality protein meal is healthy for your gut. In contrast with a low quality protein meal that breaks down the gut. Although these scientific findings were published a while ago, the discussion of dietary protein is currently a hot topic in the food social media. Online and on packages in the supermarket consumers are overwhelmed with beneficial health claims ("super foods") of protein. This however is misinformation if the protein involved is of low quality. For instance a gelatin pudding with $10 \%$ of low quality protein has no health benefits, this in contrast with $10 \%$ of high quality protein in yogurt. The "nutrition facts" sheet of a food package contains information of different types of fat and carbohydrates but protein quality grades are still missing. Therefore, I strongly recommend policy makers (US Food \& Drug Administration, European Food Safety Authority) to add the quality grade of the used food proteins to the "Nutrition Facts" sheet of a food package to ensure consumers are better informed and can make healthier choices.

In section 2 and 3 we presented with innovated techniques and preclinical models, new insights of how metabolic substrates are changed in critically ill pigs. The role of gut and liver metabolism on the disturbed whole body metabolism was studied in detail. This information gives physicians and (specialized) dieticians more knowledge how metabolic substrates behave in critically ill patients, which cannot be obtained with human research. These data are sound information for further design, planning and development of randomized controlled trials by physicians, specialized dieticians, scientists and the clinical nutrition industry, to improve nutritional interventions and strategies in critically ill humans.

\section{References}

1. Rosenblatt S, Clowes GH, Jr., George BC, Hirsch E, Lindberg B. Exchange of amino acids by muscle and liver in sepsis. Arch Surg. 1983;118(2):167-75.

2. Hartl WH, Jauch KW. Metabolic self-destruction in critically ill patients: origins, mechanisms and therapeutic principles. Nutrition. 2014;30(3):261-7.

3. Klaude M, Mori M, Tjader I, Gustafsson T, Wernerman J, Rooyackers O. Protein metabolism and gene expression in skeletal muscle of critically ill patients with sepsis. Clin Sci (Lond). 2012;122(3):133-42.

4. Rooyackers O, Kouchek-Zadeh R, Tjader I, Norberg A, Klaude M, Wernerman J. Whole body protein turnover in critically ill patients with multiple organ failure. Clin Nutr. 2015;34(1):95-100. 
5. Coker RH, Wolfe RR. Bedrest and sarcopenia. Curr Opin Clin Nutr Metab Care. 2012;15(1):7-11.

6. Heyland DK, Stapleton RD, Mourtzakis M, Hough CL, Morris P, Deutz NE, et al. Combining nutrition and exercise to optimize survival and recovery from critical illness: Conceptual and methodological issues. Clin Nutr. 2016;35(5):1196-206.

7. Deutz NE, Matheson EM, Matarese LE, Luo M, Baggs GE, Nelson JL, et al. Readmission and mortality in malnourished, older, hospitalized adults treated with a specialized oral nutritional supplement: A randomized clinical trial. Clin Nutr. 2016;35(1):18-26.

8. Jalan R, Olde Damink SW, Hayes PC, Deutz NE, Lee A. Pathogenesis of intracranial hypertension in acute liver failure: inflammation, ammonia and cerebral blood flow. J Hepatol. 2004;41(4):613-20.

9. Olde Damink SW, Deutz NE, Dejong CH, Soeters PB, Jalan R. Interorgan ammonia metabolism in liver failure. Neurochem Int. 2002;41(2-3):177-88.

10. Ytrebo LM, Sen S, Rose C, Ten Have GA, Davies NA, Hodges S, et al. Interorgan ammonia, glutamate, and glutamine trafficking in pigs with acute liver failure. Am J Physiol Gastrointest Liver Physiol. 2006;291(3):G373-81.

11. World Health Organization WHO. Malnutrion 2017. Available from: http://www.who. int/mediacentre/factsheets/malnutrition/en/.

12. World Health Organization WHO, Food and Agriculture Organization of the United Nations FAO, United Nations University UNU. Protein and amino acid requirements in human nutrition. 2007.

13. Cohen S, Nathan JA, Goldberg AL. Muscle wasting in disease: molecular mechanisms and promising therapies. Nat Rev Drug Discov. 2015;14(1):58-74.

14. Wischmeyer PE. The evolution of nutrition in critical care: how much, how soon? Crit Care. 2013;17 Suppl 1:S7.

15. Ten Have GAM. Available from: https://scholar.google.com/citations?user=KXj9EEY AAAAJ\&hl=en. 


\section{Biography}

Gabriëlla Adriana Maria ten Have was born on the second of March 1964 in VleutenDe Meern, The Netherlands. After high school graduation (VWO) in 1982 at the St. Bonifatius College in Utrecht, she began her advanced leveled professional research laboratorium education (HLO/Life Science, BSC) at Dr. Ir. W.L. Ghijsen institute (Utrecht, The Netherlands) and graduated in 1986. Although she had a broad interest in multiple disciplines (i.e. chemistry, medicine, physics, computer science), she specialized in biology, which gave her a strong fundamental education in conducting scientific research in the biomedical field.

During her nine-month internship in the research group of Prof. dr. F.P. Nijkamp at the Dept. of Pharmacology, Faculty of Veterinary Medicine, Utrecht University, The Netherlands, she had the opportunity to develop her professional skills by working on a pharmacological in vitro receptor detection method, which resulted in her first scientific publication (1). In 1986, she acquired her molecular biology skills as research assistant of Dr. A. Berns at the Dept. of Molecular Biology, Netherlands Cancer Institute, Amsterdam, The Netherlands. In 1987, she continued her research on pharmacological interactions of immune cells with endothelium in endotoxin disturbed pathophysiological conditions in animals using in vitro measurements. Together with Dr. A.J. van Oosterhout and Dr. D. van Heuven-Nolsen, she was involved in the validation, conduct, and design of new in vitro animal models, which resulted in four publications (2-5).

Her appointment in 1990 as research assistant at the Metabolic Research Centre (MRC) of Dr. N.E.P. Deutz and Prof. dr. P.B. Soeters at the Dept. of Surgery, Faculty of Medicine, Maastricht University, The Netherlands, gave her the opportunity to expand her research experience in the preclinical field. She worked with complex in vivo research models using large instrumented animals (pigs), and was heavily involved in the quantitative analysis of metabolic pathways, using isotopic tracers, and metabolic substrates by advanced analytical technology (HPLC and LC-MS/ MS). This work enhanced her ambition to conduct multidisciplinary translational research. She improved a catheterized in vivo pig model that is used for transorgan measurements of macronutrient fluxes, and introduced stable tracer models in multiple (patho)physiological clinical relevant conditions in international collaborative research (6-15). Alongside the multi-organ pig model, she developed with Dr. Deutz. a multi-organ metabolic flux model in mice. She was involved in the design and conduct of multiple (transgenic) in vivo mice/rat studies in (patho) physiological conditions with and without use of stable isotope metabolic tracer models in collaboration with (inter)national researchers (16-22). In the 25 years of research 
with Dr. Deutz, she gained metabolic research expertise which involved writing of scientific reviews, developing specific LC-MS/MS lab analytical methods, validation of new stable isotope model approaches, and developing a new clinically relevant sepsis model in the pig. Furthermore, she collaborated in human studies that involved stable isotope methods (23-28).

Ten Have's longstanding research experience gave her strong leadership, mentoring, and teaching skills which resulted in the present dissertation. In 2007, she moved with the metabolic research group of Dr. Deutz to the Center for Translational Research on Aging \& Longevity (CTRAL), at the Donald W. Reynolds Institute on Aging, University of Arkansas for Medical Sciences, Little Rock, AR, United States. In 2012, she subsequently moved to her current CTRAL employment at the Dept. of Health and Kinesiology, Texas A\&M University, College Station, TX, where she got the opportunity to gain further experience in managing and designing multidisciplinary laboratories. Furthermore, she was involved in the design and construction of the new Human Clinical Research Facility on Texas A\&M University campus in 2016. She is currently residing with her family in Montgomery, TX, United States. She aspires to become an independent researcher in the preclinical critical care metabolic research field. 


\section{Publications}

\section{https://www.ncbi.nlm.nih.gov/pubmed/?term=Ten+Have+GA}

1. Van Oosterhout AJ, Ten Have GA, Nijkamp FP. Endotoxin-induced reduction of beta-adrenoceptor number in guinea pig splenic lymphocyte membranes. Agents Actions. 1986;19(5-6):361-2.

2. Van Oosterhout AJ, Folkerts G, Ten Have GA, Nijkamp FP. Involvement of the spleen in the endotoxin-induced deterioration of the respiratory airway and lymphocyte betaadrenergic systems of the guinea pig. Eur J Pharmacol. 1988;147(3):421-9.

3. van Heuven-Nolsen D, Ten Have GA, Nijkamp FP. Increased reactivity to histamine in the coronary vascular system of the guinea-pig after endotoxin. Agents Actions. 1989;27(1-2):158-9.

4. van Heuven-Nolsen D, Ten Have GA, Nijkamp FP. Interaction between neutrophils and pig coronary artery increases histamine contractions. Agents Actions. 1990;30(1-2):195-7.

5. van Heuven-Nolsen D, Ten Have GA, Nijkamp FP. Neutrophils increase histamine contractions in pig coronary artery: a role for lipoxygenase products. $\mathrm{Br} \mathrm{J}$ Clin Pharmacol. 1990;30 Suppl 1:156S-8S.

6. Ten Have GA, Bost MC, Suyk-Wierts JC, van den Bogaard AE, Deutz NE. Simultaneous measurement of metabolic flux in portally-drained viscera, liver, spleen, kidney and hindquarter in the conscious pig. Lab Anim. 1996;30(4):347-58.

2947 7. Deutz NE, Ten Have GA, Soeters PB, Moughan PJ. Increased intestinal amino-acid retention from the addition of carbohydrates to a meal. Clin Nutr. 1995;14(6):354-64.

8. Ytrebo LM, Sen S, Rose C, Ten Have GA, Davies NA, Hodges S, et al. Interorgan ammonia, glutamate, and glutamine trafficking in pigs with acute liver failure. Am J Physiol Gastrointest Liver Physiol. 2006;291(3):G373-81.

9. Ten Have GA, Engelen MP, Soeters PB, Deutz NE. Absence of post-prandial gut anabolism after intake of a low quality protein meal. Clin Nutr. 2012;31(2):273-82.

10. Ten Have GA, van der Pijl PC, Kies AK, Deutz NE. Enhanced Lacto-Tri-Peptide BioAvailability by Co-Ingestion of Macronutrients. PLoS One. 2015;10(6):e0130638.

11. Ytrebo LM, Sen S, Rose C, Davies NA, Nedredal GI, Fuskevaag OM, et al. Systemic and regional haemodynamics in pigs with acute liver failure and the effect of albumin dialysis. Scand J Gastroenterol. 2006;41(11):1350-60.

12. Sharma V, Ten Have GA, Ytrebo L, Sen S, Rose CF, Dalton RN, et al. Nitric Oxide and L-Arginine metabolism in a devascularized porcine model of Acute Liver Failure. American journal of physiology Gastrointestinal and liver physiology. 2012.

13. van der Pijl PC, Kies AK, Ten Have GA, Duchateau GS, Deutz NE. Pharmacokinetics of proline-rich tripeptides in the pig. Peptides. 2008;29(12):2196-202.

14. Schooneman MG, Ten Have GA, van Vlies N, Houten SM, Deutz NE, Soeters MR. Transorgan fluxes in a porcine model reveal a central role for liver in acylcarnitine metabolism. Am J Physiol Endocrinol Metab. 2015;309(3):E256-64.

15. Eggink HM, van Nierop FS, Schooneman MG, Boelen A, Kalsbeek A, Koehorst M, et al. Transhepatic bile acid kinetics in pigs and humans. Clin Nutr. 2017.

16. Hallemeesch MM, Ten Have GA, Deutz NE. Metabolic flux measurements across portal drained viscera, liver, kidney and hindquarter in mice. Lab Anim. 2001;35(1):101-10. 
17. Braulio VB, Ten Have GA, Vissers YL, Deutz NE. Time course of nitric oxide production after endotoxin challenge in mice. Am J Physiol Endocrinol Metab. 2004;287(5):E912-8.

18. Boelens PG, Melis GC, van Leeuwen PA, ten Have GA, Deutz NE. Route of administration (enteral or parenteral) affects the contribution of L-glutamine to de novo L-arginine synthesis in mice: a stable-isotope study. Am J Physiol Endocrinol Metab. 2006;291(4):E683-90.

19. He Y, Hakvoort TB, Kohler SE, Vermeulen JL, de Waart DR, de Theije C, et al. Glutamine synthetase in muscle is required for glutamine production during fasting and extrahepatic ammonia detoxification. J Biol Chem. 2010;285(13):9516-24.

20. Bosoi CR, Oliveira MM, Ochoa-Sanchez R, Tremblay M, Ten Have GA, Deutz NE, et al. The bile duct ligated rat: A relevant model to study muscle mass loss in cirrhosis. Metab Brain Dis. 2017;32(2):513-8.

21. Kumar A, Davuluri G, deSilva RN, Engelen MP, TenHave G, Prayson R, et al. Ammonia lowering reverses sarcopenia of cirrhosis by restoring skeletal muscle proteostasis. Hepatology. 2017.

22. Ten Have GAM, Deutz Cl, Engelen MPKJ, Wolfe RR, Deutz NEP. Characteristics of a Pseudomonas aeruginosa induced porcine sepsis model for multi-organ metabolic flux measurements. Laboratory Animals. 2017:in press.

23. Ten Have GA, Engelen MP, Luiking YC, Deutz NE. Absorption kinetics of Amino Acids, Peptides, and Intact Proteins. Int J Sport Nutr Exerc Metab. 2007;17(Supplement):S23-S36.

24. Meesters RJ, van Eijk HM, ten Have GA, de Graaf AA, Venema K, van Rossum BE, et al. Application of liquid chromatography-mass spectrometry to measure the concentrations and study the synthesis of short chain fatty acids following stable isotope infusions. $J$ Chromatogr B Analyt Technol Biomed Life Sci. 2007;854(1-2):57-62.

25. Luiking YC, Ten Have GA, Wolfe RR, Deutz NE. Arginine de novo and nitric oxide production in disease states. Am J Physiol Endocrinol Metab. 2012;303(10):E1177-89.

26. Tsien C, Davuluri G, Singh D, Allawy A, Ten Have GA, Thapaliya S, et al. Metabolic and molecular responses to leucine-enriched branched chain amino acid supplementation in the skeletal muscle of alcoholic cirrhosis. Hepatology. 2015;61(6):2018-29.

27. Ten Have GA, Engelen MP, Wolfe RR, Deutz NE. Phenylalanine isotope pulse method to measure effect of sepsis on protein breakdown and membrane transport in the pig. Am J Physiol Endocrinol Metab. 2017:ajpendo 003512016.

28. Ten Have GAM, Deutz RCI, Engelen M, Wolfe RR, Deutz NEP. Characteristics of a Pseudomonas aeruginosa induced porcine sepsis model for multi-organ metabolic flux measurements. Lab Anim. 2017:23677217718003.

\section{Position and Employment}

1986 - 1987: Research assistant at the Department of Molecular Biology, NKI (Netherlands Cancer Institute) in Amsterdam. The Netherlands.

1987 - 1990: Research assistant at the Department of Pharmacology, faculty of Pharmacy, Utrecht University. The Netherlands.

1990 - 2003: Research assistant at the Metabolic Research Centre (MRC) of Dr. N.E.P. Deutz of the Department of Surgery, faculty of Medicine, Maastricht University, The Netherlands. 
2003 - 2007: Senior Instructor, at the Metabolic Research Centre (MRC) of Dr. N.E.P. Deutz of the department of Surgery, faculty of Medicine, Maastricht University, The Netherlands.

2007 - 2012: Research associate, Center for Translational Research on Aging \& Longevity. Donald W.Reynolds Institute on Aging. University of Arkansas for Medical Sciences, Little Rock, AR, USA

2012 - : Senior Research Associate, Center for Translational Research on Aging \& Longevity. Dept. Health and Kinesiology, Texas A\&M University, College Station, TX, USA

\section{Honors}

2012 ESPEN Research Fellowship Grant 2012

\section{Oral en Poster presentaties:}

1.Glutathione (GSH) Absolute Synthesis Rates (ASR) of Multiple Organs in a Pseudomonas aeroginosa (PM) induced Hyperdynamic Sepsis Pig Model, GA Ten Have, MP Engelen, RR Wolfe, NE Deutz, The FASEB Journal 30 (1 Supplement), 742.17-742.17

2. Severely Compromised Anabolic Response to Nutrition in a Pseudomonas aeroginosa (PM) Induced Hyper-dynamic Sepsis-Recovery Pig Model,GA Ten Have, MP Engelen, RR Wolfe, NE Deutz, The FASEB Journal 30 (1 Supplement), 682.16-682.16

3. Increased muscle myofibrillar protein breakdown rates using 3-methyl-histidine (tau-mHIS) stable isotopes in a Pseudomonas aeroginosa (PM) induced hyperdynamic sepsis pig model, GA Ten Have, MP Engelen, RR Wolfe, NE Deutz, The FASEB Journal 30 (1 Supplement), Ib761-|b761,

4. SUN-PP026: Reduced Protein Synthesis and Breakdown in Jejunal Mucosal Biopsies in a Live Bacteria Induced Hyperdynamic Sepsis Pig Model, GA Ten Have, MP Engelen, RR Wolfe, J Thaden, NE Deutz, Clinical Nutrition 34, S33

5. OR004: Protein Fractional Synthesis Rates (FSR) of Multiple Organs in a Pseudomonas aeruginosa (PM) Induced Hyperdynamic Sepsis Pig Model,GA Ten Have, MP Engelen, RR Wolfe, NE Deutz,Clinical Nutrition 34, S1

6. Reduced lung glutamate (GLU) production is the cause of decreased systemic glutamate availability in hyperdynamic sepsis, GA Ten Have, MP Engelen, RR Wolfe, NE Deutz,The FASEB Journal 26 (1 Supplement), 715.3-715.3,

7. Muscle breakdown determines Arginine (ARG) availability during hyperdynamic sepsis in the pig, GA Ten Have, MP Engelen, RR Wolfe, NE Deutz, The FASEB Journal 26 (1 Supplement), 43.7-43.7

8. Using the phenylalanine (PHE) stable isotope pulse method to measure intracellular protein breakdown and metabolic shunting in the context of sepsis in the pig, GA Ten Have, MP Engelen, RR Wolfe, NE Deutz, The FASEB Journal 26 (1 Supplement), 42.1-42.1 
9. Decreased circulating arginine in ALF mice does not compromise whole body NO production, GAM Ten Have, R Jalan, NEP Deutz, EUR J GASTROEN HEPAT 19 (10), A16-A16

10. Decreased circulating arginine in ALF mice does not compromise whole body NO production, GAM Ten Have, R Jalan, NEP Deutz, The FASEB Journal 20 (5), A1041

11. Characterization of hepatic encephalopathy (HE) in an acetaminophen (APAP) induced acute liver failure (ALF) mouse model, GAM Ten Have, MGW Van den Heuvel, A Blokland, R Jalan, NEP Deutz, FEDERATION AMER SOC EXP BIOL 19 (5), A1048-A1048

12. Neuropsychological and behavioural characterisation of a fully-reversible acetaminopheninduced model of hepatic encephalopathy (HE) in mice, GA Ten Have, A Blokland, R Jalan, NE Deutz, JOHN WILEY \& SONS INC 42 (4), 358A-359A

13. Evidence for marked disturbance in whole body and brain glutamate (GLU) metabolism with no alteration of glutamine II (GLN) metabolism in an acetaminophen induced fulminant hepatic encephalopathy (HE) mouse model. GA Ten Have, R Jalan, NE Deutz.JOHN WILEY \& SONS INC 42 (4), 364A-365A 


\section{Dankwoord / Thank you}

Eindelijk is het zover, "het boekje" is klaar! Ik kijk terug op mooie, leerzame, dynamische jaren, waarin bijzonder interessant wetenschappelijk onderzoek, artsen, wetenschappers en vele promovendi mij hebben geïnspireerd om deze mijlpaal te gaan halen. ledereen die mij de afgelopen jaren geholpen heeft, wil ik hartelijk bedanken en in het bijzonder de volgende mensen.

Allereerst dank aan alle proefdierverzorgers van de Universiteit Maastricht, die met hun kritische zorgzame blik altijd klaar stonden voor de varkens en hun onderzoekers. Hun zorg was essentieel voor het verkrijgen van betrouwbare gegevens voor dit proefschrift.

First of all thanks to all animal care takers of Arkansas Children Hospital (Little Rock, AR, USA), University Hospital of North Norway (Tromsø, Norway) for their essential 24/7 animal care and support to researchers, to ensure that the generated data from the pig studies described in the present thesis are reliable.

Dank aan mijn beide promotors, Prof. dr. N.E.P. Deutz en Prof. dr. S.W.M. Olde Damink en copromotor Dr. M.P.K.J. Engelen.

Beste Mick, ondanks dat promoveren niet mijn primaire taak was binnen jouw research groep, heb je me altijd gestimuleerd om mij verder te ontwikkelen in de wetenschap. Jouw enthousiasme, oog voor het medische detail bij het ontwikkelen van (dier)modellen en het verkrijgen en analyseren van (tracer)data om het onderste uit de wetenschappelijke "kan" met gegevens te halen, waren een inspiratie voor mijn verdere ontwikkeling. Je hebt me (en mijn gezin) tot twee keer toe in het "diepe" gegooid...., maar ons nooit laten verdrinken. Het heeft ons zeer waardevolle ervaringen op wereldniveau opgeleverd. Hartelijk dank voor je vertrouwen al die jaren, je eeuwige bereidheid en beschikbaarheid voor overleg, je steun, en aansporing. Ik hoop dat we nog lang mogen samenwerken en dat je mijn mentor kan zijn in mijn verdere wetenschappelijke loopbaan.

Beste Steven, ondanks dat jij wat later in mijn promotie traject stapte, ken ik je toch al heel wat jaren gedurende de verschillende samenwerking projecten met Mick. Jouw onuitputtelijke wetenschappelijke energie is aanstekelijk en een inspiratie voor anderen die naast een andere carrière, een wetenschappelijk loopbaan er op na willen houden. Bedankt voor de ondersteuning bij de laatste loodjes van het proefschrift. Ik hoop dat onze samenwerking nog lang zal voortduren. 
Beste Mariëlle. Hartelijk dank voor jouw hulp bij de data interpretatie en het schrijven van menig manuscript. Jouw eindeloos geduld om het manuscript nog beter leesbaar te maken is een voorbeeld geweest voor mij. Daarnaast zijn we met onze gezinnen vertrokken uit Nederland en hebben we onze kinderen zien opgroeien in een ander werelddeel. Dat heeft een speciale band opgeleverd en heel veel steun voor mijn gezinsleven. Bovenal dank hiervoor.

De leden van de beoordelingscommissie, Prof. dr. W.H. Lamers, Prof. dr. L.J.C. van Loon, Dr. M. van de Poll, Prof. dr. O. Rooijackers en Dr. M.R. Soeters wil ik hartelijk danken voor het beoordelen van het manuscript. Prof. dr. P.B. Soeters, Prof. dr. R.R. Wolfe en Prof. dr. S. Heeneman, wil ik bedanken voor het zitting nemen in de corona.

Beste Prof. dr. Peter Soeters. U heeft aan de wieg gestaan van het multi orgaan stofwisselings onderzoek in het varken. Hartelijk dank voor uw mentorschap voor onze onderzoeksgroep in de beginjaren en het mogen doorontwikkelen van uw gekatheteriseerde varkensmodel. Ik voel me vereerd dat u zitting heeft willen nemen in de corona.

Dear Prof. dr. Bob Wolfe. I want to thank you for your support and collaboration with our research with the septic pig model. Your teaching skills in tracer technologies and your knowledge to translate and summarize complex (tracer) data and metabolism in understandable language, were a great inspiration for me. It is a great honor for me that you are joining the corona during the defense of my dissertation. I hope you are able to continue to mentor our research.

Onderzoek in varkens is teamwork. Bedankt Ayhan, Ruud, Karin, Jolien, Renske, Bea, Jolien, Merel, Heleen, Santoesha en Lisa. Zonder jullie hulp en enorme inzet bij de operaties, dierenverzorging en de experimenten had ik de varkensproeven niet kunnen uitvoeren op de Universiteit Maastricht, Arkansas Children Hospital en Texas A\&M University. Er resteert nog een enorme schat aan monsters, voor toekomstige analyse en publicaties in de komende jaren.

Research with pigs is a team effort. Thank you Daniëlle and Cristina. Without your help and enormous effort during the surgeries, animal care and experiments, pig studies would not have been possible. Results of samples that you helped to collect will be analyzed and published in the coming years.

Some research studies in pigs were an international effort. Thank you Dr. Lars Ytrebo for your kind hospitality in Tromsø (Norway) and the opportunity to work with your team and the international researchers Dr. Rajiv Jalan (UK), Dr. Nathan Davies (UK) and Dr. Chris Rose (Canada) to obtain experience in international scientific collaborations. The scientific drive in this team was contagious. It was and is still a pleasure to work with all of you, and look forward to any future collaboration. 
Zonder een hoogwaardig analytisch-chemisch lab met hoogwaardige HPLC en LCMS expertise, goede analytische systeem experts, applicatie-ontwikkelaars en operators had ik geen betrouwbare onderzoeksresultaten gehad om te publiceren. Bedankt Hans, Marion, Dennis S, Dennis H, Dennis R, Jean en Roland voor de goede samenwerking en het omhoog brengen van mijn analytisch-chemische kennis.

Without an analytical chemical lab with benchmark HPLC and LCMS expertise, excellent analytical application experts, developers and operators, my research would not have yielded in high quality data used for this thesis and publications. My thanks to you, John and Joshua, for all your efforts to generate important and reliable data for the present dissertation.

Bedankt alle co-auteurs voor de hulp bij het tot standkoming van menig manuscript in dit proefschrift in welke vorm dan ook. Met name heb ik veel geleerd en genoten van de nauwe samenwerking met Joyce, May, Pieter, Arie en Yvette.

Vele promovendi en onderzoekers hebben mij geïnspireerd om zelf ook dit traject te bewandelen. Jullie waren mijn leerschool. Het helpen met het aanleren van vaardigheden, het opzetten van nieuwe protocollen, samenwerking, soms ploeteren, delen van frustraties en samen publiceren waren, nu terugkijkend, belangrijke en onmisbare ervaringen. Jullie hebben nog steeds een speciaal plekje in mijn hart. Bedankt Nel, Ivo, Maaike Br., Marcella, Yvonne, Martijn, Sylvia, Cindy, Ludy, Karin, Maaike Be, Carlijn en Marieke.

Dank aan al het ondersteunend personeel in de Heelkunde van toen en nu, met name Monique, Mo en Livia. Jullie zijn de olie in de machine. Zonder jullie inzet, zou alles vast lopen.

Thanks to my (ex)co-workers/friends and others who made me and my family feel welcome and helped develop happy memories living in the United States. Especially Julia and Tony, thank you both for your enormous support with the startup of a new family life on the other side of the world. Your hospitality is an example for a lot of people. Also thanks to Laura, Sabine, Dillon, Gerdien, Barbara, Fari, Sunday, Agatha, Clayton, Liz, nurses, students and all others. We will continue to make more great memories.

Beste Renate. Het is me een genoegen om samen met jouw dit promotie traject te gaan afronden. Ik heb veel met je meegemaakt in en buiten het lab. Binnen ons gezin wordt je beschouwd als een goede vriendin. We zullen je helaas niet meer zo vaak zien. Ik hoop van harte dat je weer je draai kan vinden in Nederland en heel veel succes in je verdere loopbaan en privé leven. 
Lieve familie, vrienden en vriendinnen. Door de grote afstand tussen ons, zien en spreken we elkaar niet zo frequent. Gelukkig helpt de social media, internet tegenwoordig om de afstand virtueel kleiner te maken. Door de drukte van een promotietraject ben ik me bewust van dat ik menig relatie nogal heb verwaarloosd. Echter mijn gezin en ik, hebben met groot genoegen mogen ervaren dat goede familie en vrienden banden niet zomaar breken. Bovenal dank voor jullie ondersteuning en begrip. Ik hoop dat vele van jullie mijn promotie dag zullen aangrijpen om elkaar weer even te zien en te spreken.

En dan kom ik aan bij mijn paranimfen. Lieve Anja, vanaf onze studietijd ben je al een fantastische vriendin, en samen met jouw Raymond, delen Andre en ik vele mooie herinneringen. Zodra ik je weer ziet, zelfs na lange tijd, breng je ontspanning en een glimlach op mijn mond. En dat is wel eens nodig tijdens een promotietraject. Ik ben daarom erg blij en dankbaar dat je mijn paranimf wil zijn. Bedankt voor je inzet om de laatste loodjes soepel te laten verlopen. Lieve Eva, mijn oudste telg. Ik heb je op zien groeien tot een zeer getalenteerde mooie jonge vrouw die niet bang is voor deze grote wereld. Ik was vereerd en verheugd dat je de enorme klus om dit boekje er mooi uit te laten zien, op je wilde nemen tijdens je drukke studie. Enorme dank hiervoor. Ik ben trots dat je naast me wil staan tijdens mijn promotie.

Lieve mama, dat dit proefschrift hier ligt heb ik, meer dan wie dan ook, aan jou en papa te danken. Jullie stimulans om door te leren (in een tijd dat dat niet vanzelfsprekend was) en in je werk altijd een stapje harder te lopen dan wat strikt noodzakelijk is, heeft mij geholpen om mij verder te blijven door ontwikkelen. Helaas kan papa deze dag niet meer meemaken, maar ik hoop dat u op uw hoge leeftijd (91 jaar) mag genieten van deze dag.

Zonder een liefdevol thuis geen promotie. Promoveren vraagt nog al wat privé uurtjes van het thuisfront. Lieve Mats, mijn jongste, maar zeker niet mijn kleinste telg. Het was genieten om jouw te zien opgroeien tot een charmante intelligente jonge man met grootse plannen voor volgend jaar in college. Bedankt voor je steun en heel veel succes en geluk toegewenst in het leerproces om op eigen benen te staan. Lieve André, "last but not least". Ik had me geen beter "maatje" kunnen bedenken. Al meer dan 25 jaar, jouw onvoorwaardelijke steun om met mij mee te gaan "all over the world". Waar we ook belandden, je inzet om een Hollands gezellig, veilig en een mooi "huis en tuin" te creëren, is bewonderingswaardig. Jouw relativeringsvermogen heeft menig kreukel binnen het promotietraject glad gestreken. Bedankt voor het waarmaken van deze belangrijke mijlpaal in mijn leven. Ik hoop dat we binnenkort als "empty nesters" wat uurtjes kunnen in halen. 


